\section{To: (Receiving Organiżation)} Distribution

5. Proj./Prog./Dept./Div.:

Tanks 241-T-201, 241-T-202, . 241-T-203, 241-T-204/Waste

Management/DAI/Process

Engineering

\section{Originator Remarks:}

This document is being released into the supporting document system for retrievability purposes. This document is superseding. the following documents: HNF-SO-WM-ER-726, Rev. 0, HNF-SD-WM-ER-727, Rev. 0, HNF-SD-WM-ER-728, Rev. 0 , and. HNF-SD-WM-ER-729, Rev. 0.

11. Receiver Remarks: 11A. Design Baseline Document? [] Yes [X] No For release.
6. Design Authority/ Design Agent/Cog. Brett C. Simpson

\section{From: (originating Organization) Data Assessment and Interpretation}

4. Related EDT No.:

N/A

7. Purchase Order No.:

N/A
9. Equip./Component No.: $\mathrm{N} / \mathrm{A}$

10. System/Bldg./Facility:

241-T-201, 241-T-202, $241-T-203,241-T-204$

12. Major Assm. Dwg. No.:

. N/A

13. Permit/Permit Application No.: $N / A$

14. Required Response Date: $02 / 17 / 98$

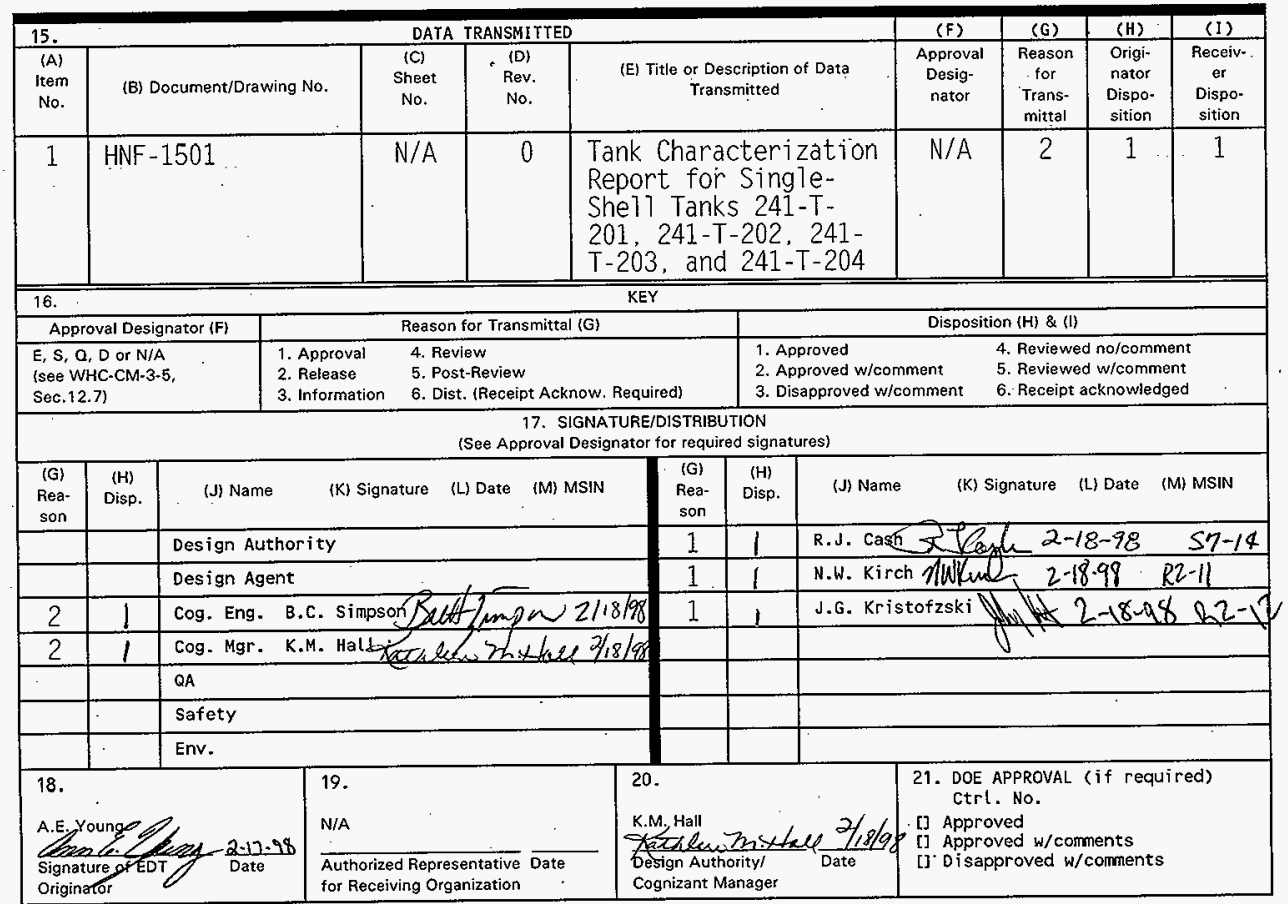


HNF-1501, Rev. 0

\title{
Tank Characterization Report for Single-Shell Tanks 241-T-201, 241-T-202, 241-T-203, and 241-T-204
}

Brett C. Simpson

Lockheed Martin Hanford Corp. . Richland, WA 99352

U.S. Department of Energy Contract DE-AC06-87RL10930

\author{
EDT/ECN: EDT-622427 UC: 2070 \\ Org Code: 74620 Charge Code: N4G4C
}

B\&R Code: EW 3120074 Total Pages: 307

Key Words: Waste Characterization, Single-She11 Tank, SST, Tank 241-T201, Tank T-201, T-201. Tank 241-T-202, Tank T-202, T-202, Tank 241-T203, Tank T-203, T-203, Tank 241-T-204, Tank T-204, T-204, T Farm, Tank Characterization Report. TCR, Waste Inventory, TPA Milestone M-44

Abstract: This document summarizes the information on the historical uses, present status, and the sampling and analysis results of waste stored in Tanks 241-T-201, 241-T-202, 241-T-203, and 241-T-204. This report supports the requirements of the Tri-Party Agreement Milestone M-44-15B.

\begin{abstract}
TRADEMARK DISCLAIMER. Reference herein to any specific commercial product, process, or service by trade name, trademark, manufacturer, or otherwise, does not necessarily constitute or imply its endorsement, recommendation, or favoring by the United States Government or any agency thereof or its contractors or subcontractors.
\end{abstract}

Printed in the United States of America. To obtain copies of this document, contact: WHC/BCS Document Control Services, P.0. Box 1970, Mailstop H6-08, Richland WA 99352, Phone (509) 372-2420; Fax (509) 376-4989.
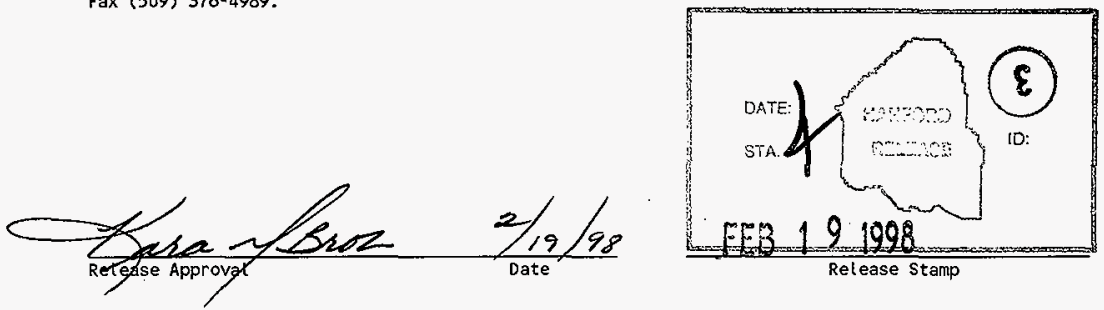

\section{Approved for Public Release}




\section{Tank Characterization Report for Single-Shell Tanks 241-T-201, T-202, T-203, and -T-204}

B. C. Simpson

Lockheed Martin Hanford Corp.

Date Published

February 1998

Prepared for the U.S. Department of Energy

Assistant Secretary for Environmental Management

Project Hanford Management Contractor for the

U.S. Department of Energy under Contract DE-AC06-96RL13200

Approved for public release; distribution is unlimited 


\section{CONTENTS}

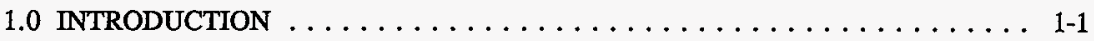

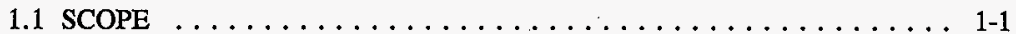

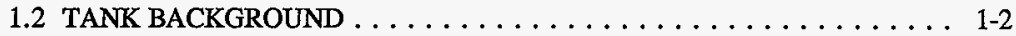

2.0 RESPONSE TO TECHNICAL ISSUES $\ldots \ldots \ldots \ldots \ldots \ldots \ldots \ldots \ldots$ 2-1

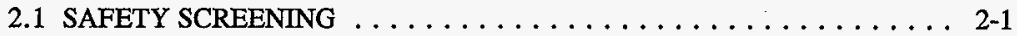

2.1.1 Exothermic Conditions (Energetics) $\ldots \ldots \ldots \ldots \ldots \ldots \ldots$ 2-1

2.1.2 Flammable Gas ................... 2-2

2.1 .3 Criticality ...................... 2-2

2.2 HAZARDOUS VAPOR SAFETY SCREENING $\ldots \ldots \ldots \ldots \ldots \ldots .2-3$

2.2.1 Flammable Gas . . . . . . . . . . . . . 2-3

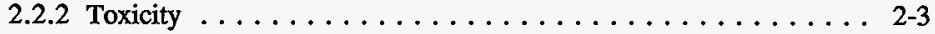

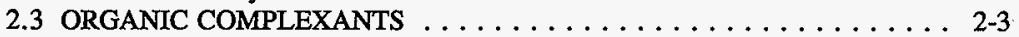

2.4 ORGANIC SOLVENTS . . . . . . . . . . . . . . . 2-4

2.5 OTHER TECHNICAL ISSUES $\ldots \ldots \ldots \ldots \ldots \ldots \ldots \ldots \ldots . \ldots \ldots$. . . . .

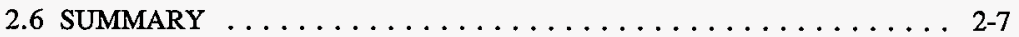

3.0 BEST-BASIS STANDARD INVENTORY ESTMMATE $\ldots \ldots \ldots \ldots \ldots \ldots$. $\ldots \ldots$

4.0 RECOMMENDATIONS $\ldots \ldots \ldots \ldots \ldots \ldots \ldots \ldots \ldots \ldots \ldots \ldots \ldots \ldots \ldots$ 4-1

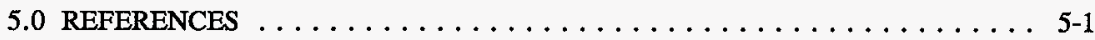

APPENDICES

APPENDIX A: HISTORICAL TANK INFORMATION $\ldots \ldots \ldots \ldots \ldots$ A-1

A1.0 CURRENT TANK STATUS $\ldots \ldots \ldots \ldots \ldots \ldots \ldots \ldots \ldots \ldots \ldots \ldots \ldots \ldots \ldots$

A2.0 TANK DESIGN AND BACKGROUND $\ldots \ldots \ldots \ldots \ldots \ldots \ldots \ldots$ A-4

A3.0 PROCESS KNOWLEDGE $\ldots \ldots \ldots \ldots \ldots \ldots \ldots \ldots \ldots \ldots \ldots$ A-13

A3.1 WASTE TRANSFER HISTORY $\ldots \ldots \ldots \ldots \ldots \ldots \ldots \ldots \ldots$ A-13

A3.2 HISTORICAL ESTIMATION OF TANK CONTENTS . . . . . . . . . A-14 


\section{CONTENTS (Continued)}

A4.0 SURVEILLANCE DATA $\ldots \ldots \ldots \ldots \ldots \ldots \ldots \ldots \ldots \ldots \ldots$ A-32

A4.1 SURFACE-LEVEL READINGS $\ldots \ldots \ldots \ldots \ldots \ldots \ldots \ldots \ldots$ A-32

A4.2 INTERNAL TANK TEMPERATURES $\ldots \ldots \ldots \ldots \ldots \ldots \ldots \ldots$ A-32

A4.3 TANK 241-T-201, -T-202, -T-203, AND -T-204 PHOTOGRAPHS $\ldots \ldots \ldots \ldots \ldots \ldots \ldots \ldots \ldots \ldots \ldots \ldots \ldots \ldots \ldots$

A5.0 APPENDIX A REFERENCES $\ldots \ldots \ldots \ldots \ldots \ldots \ldots \ldots \ldots \ldots$ A-42

APPENDIX B: SAMPLING OF 241-T-200 SERIES TANKS $\ldots \ldots \ldots \ldots \ldots \ldots$ B-1

B1.0 TANK SAMPLING OVERVIEW $\ldots \ldots \ldots \ldots \ldots \ldots \ldots \ldots \ldots$ B-3

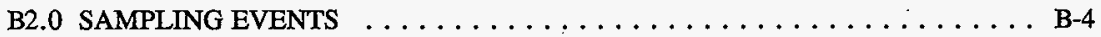

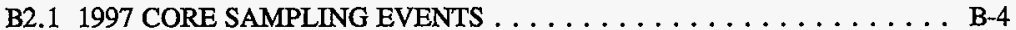

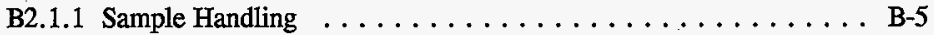

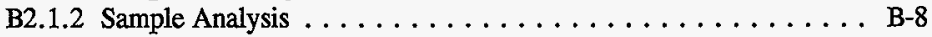

B2.1.3 Analytical Results . . . . . . . . . . . . B-16

B2.2 VAPOR PHASE MEASUREMENT . . . . . . . . . . . . . B-19

B2.3 DESCRIPTION OF HISTORICAL SAMPLING EVENT . . . . . . . . B B-19

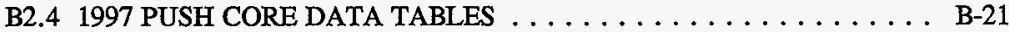

B3.0 ASSESSMENT OF CHARACTERIZATION RESULTS . . . . . . . . . B B-155

B3.1 FIELD OBSERVATIONS . . . . . . . . . . . . . . B-155

B3.2 QUALITY CONTROL ASSESSMENT $\ldots \ldots \ldots \ldots \ldots \ldots \ldots \ldots$. $\ldots \ldots$. $\ldots \ldots 5$

B3.3 DATA CONSISTENCY CHECKS . . . . . . . . . . . . . . B-156

B3.3.1 Comparison of Results from Different Analytical Methods . . . B-157

B3.3.2 Mass and Charge Balance . . . . . . . . . . . . B-158

B3.4 MEAN CONCENTRATIONS AND CONFIDENCE INTERVALS . . . . B-166

B4.0 APPENDIX B REFERENCES $\ldots \ldots \ldots \ldots \ldots \ldots \ldots \ldots \ldots$ B-177

APPENDIX C: STATISTICAL ANALYSIS FOR ISSUE RESOLUTION $\ldots \ldots \ldots$ C-1

C1.0 STATISTICS FOR THE SAFETY SCREENING

DATA QUALITY OBJECTIVE $\ldots \ldots \ldots \ldots \ldots \ldots \ldots \ldots \ldots \ldots$ C-3

C2.0 APPENDIX C REFERENCES $\ldots \ldots \ldots \ldots \ldots \ldots \ldots \ldots \ldots \ldots$ C-6 


\section{CONTENTS (Continued)}

APPENDIX D: EVALUATION TO ESTABLISH BEST-BASIS INVENTORY FOR THE T-200 SERTES SINGLE-SHELL, TANKS $\ldots \ldots \ldots \ldots \ldots$ D-1

D1.0 CHEMICAL INFORMATION SOURCES $\ldots \ldots \ldots \ldots \ldots \ldots \ldots \ldots$ D-3

D2.0 COMPARISON OF COMPONENT INVENTORY VALUES $\ldots \ldots \ldots \ldots \ldots$ D-4

D3.0 COMPONENT INVENTORY EVALUATION $\ldots \ldots \ldots \ldots \ldots \ldots \ldots$ D-8 D3.1 CONTRIBUTING WASTE TYPES $\ldots \ldots \ldots \ldots \ldots \ldots \ldots \ldots$ D-8

D3.2 EVALUATION OF FLOWSHEET INFORMATION . . . . . . . . . D-9

D3.3 ASSUMPTIONS FOR RECONCILING WASTE INVENTORIES . . . . . D-10 D3.4 BASIS FOR CALCULATIONS USED IN THE ENGINEERING EVALUATION AND SAMPLE-BASED ESTRMATES $\ldots \ldots \ldots \ldots$ D-11

D4.0 DEFINE THE BEST-BASIS AND ESTABLISH COMPONENT

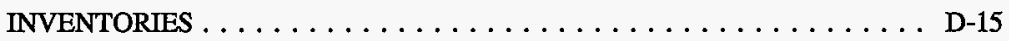

D5.0 APPENDIX D REFERENCES $\ldots \ldots \ldots \ldots \ldots \ldots \ldots \ldots \ldots \ldots \ldots \ldots$

APPENDIX E: BIBLIOGRAPHY FOR TANKS 241-T-201, 241-T-202, 241-T-203, AND $241-T-204 \ldots \ldots \ldots \ldots \ldots \ldots \ldots$. . . . . . . . . . . . . . 


\section{LIST OF FIGURES}

A2-1 Generic Riser Configuration for T-200 Series Tanks . . . . . . . . . . . A-6

A2-2 Tank 241-T-201 Cross Section and Schematic . . . . . . . . . . . . . . A-9

A2-3 Tank 241-T-202 Cross Section and Schematic . . . . . . . . . . . A-10

A2-4 Tank 241-T-203 Cross Section and Schematic . . . . . . . . . . A-11

A2-5 Tank 241-T-204 Cross Section and Schematic . . . . . . . . . . A-12

A3-1 Tank Layer Model $\ldots \ldots \ldots \ldots \ldots \ldots \ldots \ldots \ldots \ldots \ldots$ A-15

A4-1 Tank 241-T-201 Level History $\ldots \ldots \ldots \ldots \ldots \ldots \ldots \ldots \ldots \ldots$ A-34

A4-2 Tank 241-T-202 Level History $\ldots \ldots \ldots \ldots \ldots \ldots \ldots \ldots \ldots \ldots$ A-35

A4-3 Tank 241-T-203 Level History $\ldots \ldots \ldots \ldots \ldots \ldots \ldots \ldots \ldots \ldots$ A-36

A4-4 Tank 241-T-204 Level History $\ldots \ldots \ldots \ldots \ldots \ldots \ldots \ldots \ldots \ldots$. . . . . . . . . . . . .

A4-5 Tank 241-T-201 High Temperature Plot $\ldots \ldots \ldots \ldots \ldots \ldots \ldots \ldots$ A-38

A4-6 Tank 241-T-202 High Temperature Plot $\ldots \ldots \ldots \ldots \ldots \ldots \ldots \ldots$ A . . . . . .

A4-7 Tank 241-T-203 High Temperature Plot $\ldots \ldots \ldots \ldots \ldots \ldots \ldots \ldots$ A-40

A4-8 Tank 241-T-204 High Temperature Plot $\ldots \ldots \ldots \ldots \ldots \ldots \ldots \ldots$ A-41 


\section{LIST OF TABLES}

1-1 Summary of Recent Sampling $\ldots \ldots \ldots \ldots \ldots \ldots \ldots \ldots \ldots \ldots \ldots$ 1-2

1-2 Description of T-200 Series Tanks . . . . . . . . . . . . .

2-1 T-200 Criticality Thresholds and Results . . . . . . . . . . . 2-2

2-2 Comparisons of T-200 Analytical Results with Prediction Intervals . . . . . 2-5

2-3 Summary of Technical Issues $\ldots \ldots \ldots \ldots \ldots \ldots \ldots \ldots \ldots \ldots \ldots \ldots$

3-1 Best-Basis Inventory Estimates for Nonradioactive Components in T-200 Series Tanks . . . . . . . . . . . . . . . 3-1

3-2 Best-Basis Inventory Estimate for Radioactive Components in T-200 Series Tanks Decayed to January $1,1994 \ldots \ldots \ldots \ldots \ldots \ldots \ldots$. . . . . . . . .

4-1 Acceptance of Tanks 241-T-201, T-202, T-203, and T-204 Sampling and Analysis . . . . . . . . . . . . . 4-1

4-2 Acceptance of Evaluation of Characterization Data and Information for T-200 Series Tanks . . . . . . . . . . . . . . . . . 4-2

A1-1 Tank Contents Status Summary $\ldots \ldots \ldots \ldots \ldots \ldots \ldots \ldots$ A-4

A2-1 Tank 241-T-200 Series Tanks Risers and Nozzles . . . . . . . . . . A A-7

A3-1 Tank 241-T-201, -T-202, -T-203, and -T-204 Major Transfers $\ldots \ldots \ldots$ A-13

A3-2 241-T-201 Historical Tank Inventory Estimate . . . . . . . . . . A-16

A3-3 241-T-202 Historical Tank Inventory Estimate . . . . . . . . . . . A-20

A3-4 241-T-203 Historical Tank Inventory Estimate . . . . . . . . . . . . . A-24

A3-5 241-T-204 Historical Tank Inventory Estimate $\ldots \ldots \ldots \ldots \ldots \ldots \ldots$ A-28

B2-1 Integrated Data Quality Objective Requirements for T-200 Series Tanks . . . . B-4

B2-2 T-200 Series Tanks Subsampling Scheme and Sample Description . . . . . B B

B2-3 Analytical Procedures $\ldots \ldots \ldots \ldots \ldots \ldots \ldots \ldots \ldots \ldots$ B-9 


\section{LIST OF TABLES (Continued)}

B2-4 T-200 Series Sample Analysis Summary $\ldots \ldots \ldots \ldots \ldots \ldots \ldots \ldots$ B-10

B2-5 Analytical Tables . . . . . . . . . . . . . . . .

B2-6 Results of Headspace Measurements from T-200 Tanks . . . . . . . . . . B-19

B2-7 Historical Data Table $\ldots \ldots \ldots \ldots \ldots \ldots \ldots \ldots \ldots \ldots \ldots$ B-20

B2-8 Tank 241-T-201 Analytical Results: Aluminum . . . . . . . . . B B-21

B2-9 Tank 241-T-201 Analytical Results: Antimony $\ldots \ldots \ldots \ldots \ldots \ldots$ B-22

B2-10 Tank 241-T-201 Analytical Results: Arsenic . . . . . . . . . . . . B-23

B2-11 Tank 241-T-201 Analytical Results: Barium . . . . . . . . . . . B-24

B2-12 Tank 241-T-201 Analytical Results: Beryllium . . . . . . . . . B B-25

B2-13 Tank 241-T-201 Analytical Results: Bismuth . . . . . . . . . B-26

B2-14 Tank 241-T-201 Analytical Results: Boron . . . . . . . . . . . B-27

B2-15 Tank 241-T-201 Analytical Results: Cadmium . . . . . . . . . . B B-28

B2-16 Tank 241-T-201 Analytical Results: Calcium . . . . . . . . . . . . . . B-29

B2-17 Tank 241-T-201 Analytical Results: Cerium . . . . . . . . . . . B-30

B2-18 Tank 241-T-201 Analytical Results: Chromium . . . . . . . . . B-31

B2-19 Tank 241-T-201 Analytical Results: Cobalt . . . . . . . . . . . B B-32

B2-20 Tank 241-T-201 Analytical Results: Copper $\ldots \ldots \ldots \ldots \ldots \ldots \ldots$. . . . . .

B2-21 Tank 241-T-201 Analytical Results: Iron $\ldots \ldots \ldots \ldots \ldots \ldots \ldots$ B-34

B2-22 Tank 241-T-201 Analytical Results: Lanthanum . . . . . . . . . . . . B-35

B2-23 Tank 241-T-201 Analytical Results: Lead . . . . . . . . . . . . . . B-36

B2-24 Tank 241-T-201 Analytical Results: Lithium . . . . . . . . . . . B-37 


\section{LIST OF TABLES (Continued)}

B2-25 Tank 241-T-201 Analytical Results: Magnesium . . . . . . . . . . B-38

B2-26 Tank 241-T-201 Analytical Results: Manganese . . . . . . . . . . . B-39

B2-27 Tank 241-T-201 Analytical Results: Molybdenum . . . . . . . . . . B-40

B2-28 Tank 241-T-201 Analytical Results: Neodymium . . . . . . . . . . . B B-41

B2-29 Tank 241-T-201 Analytical Results: Nickel . . . . . . . . . . B-41

B2-30 Tank 241-T-201 Analytical Results: Phosphorus . . . . . . . . . . . . B-42

B2-31 Tank 241-T-201 Analytical Results: Potassium . . . . . . . . . . B-42

B2-32 Tank 241-T-201 Analytical Results: Samarium . . . . . . . . . B-43

B2-33 Tank 241-T-201 Analytical Results: Selenium . . . . . . . . . B B-43

B2-34 Tank 241-T-201 Analytical Results: Silicon . . . . . . . . . . . . . B-44

B2-35 Tank 241-T-201 Analytical Results: Silver . . . . . . . . . . . B-45

B2-36 Tank 241-T-201 Analytical Results: Sodium . . . . . . . . . . . B-46

B2-37 Tank 241-T-201 Analytical Results: Strontium . . . . . . . . . . . B-47

B2-38 Tank 241-T-201 Analytical Results: Sulfur . . . . . . . . . B B-48

B2-39 Tank 241-T-201 Analytical Results: Thallium . . . . . . . . . . . . . B-49

B2-40 Tank 241-T-201 Analytical Results: Titanium . . . . . . . . . . B-50

B2-41 Tank 241-T-201 Analytical Results: Total Uranium . . . . . . . . . . . B-51

B2-42 Tank 241-T-201 Analytical Results: Vanadium . . . . . . . . . B-52

B2-43 Tank 241-T-201 Analytical Results: Zinc . . . . . . . . . B-53

B2-44 Tank 241-T-201 Analytical Results: Zirconium. . . . . . . . . . B-54

B2-45 Tank 241-T-201 Analytical Results: Bromide $\ldots \ldots \ldots \ldots \ldots \ldots \ldots$ B-55 


\section{LIST OF TABLES (Continued)}

B2-46 Tank 241-T-201 Analytical Results: Chloride $\ldots \ldots \ldots \ldots \ldots \ldots \ldots$ B-56

B2-47 Tank 241-T-201 Analytical Results: Fluoride . . . . . . . . . . . . B-57

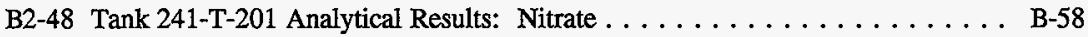

B2-49 Tank 241-T-201 Analytical Results: Nitrite . . . . . . . . . . . . B-59

B2-50 Tank 241-T-201 Analytical Results: Phosphate $\ldots \ldots \ldots \ldots \ldots \ldots$. . . . . . . .

B2-51 Tank 241-T-201 Analytical Results: Sulfate . . . . . . . . . . . . . B B-61

B2-52 Tank 241-T-201 Analytical Results: Oxalate $\ldots \ldots \ldots \ldots \ldots \ldots \ldots$ B-62

B2-53 Tank 241-T-201 Analytical Results: Bulk Density . . . . . . . . . . . . B B-62

B2-54 Tank 241-T-201 Analytical Results: Percent Water . . . . . . . . . . B B-63

B2-55 Tank 241-T-201 Analytical Results: Specific Gravity . . . . . . . . . . . B-63

B2-56 Tank 241-T-201 Analytical Results: Total Alpha, Drainable Liquid . . . . . B-64

B2-57 Tank 241-T-201 Analytical Results: Total Alpha, Solid . . . . . . . . . . B-64

B2-58 Tank 241-T-201 Analytical Results: Americium-241 . . . . . . . . . . B-64

B2-59 Tank 241-T-201 Analytical Results: Cesium-137 . . . . . . . . . B-65

B2-60 Tank 241-T-201 Analytical Results: Cobalt-60 . . . . . . . . . . . B-65

B2-61 Tank 241-T-201 Analytical Results: Europium-154 . . . . . . . . . . . B-65

B2-62 Tank 241-T-201 Analytical Results: Europium-155 . . . . . . . . . . . B-65

B2-63 Tank 241-T-201 Analytical Results: Strontium-89/90 . . . . . . . . B-66

B2-64 Tank 241-T-201 Analytical Results: Total Inorganic Carbon . . . . . . . . B-66

B2-65 Tank 241-T-201 Analytical Results: Total Organic Carbon . . . . . . . . . B B-66

B2-66 Tank 241-T-202 Analytical Results: Aluminum . . . . . . . . . . . . B-67 


\section{LIST OF TABLES (Continued)}

B2-67 Tank 241-T-202 Analytical Results: Antimony . . . . . . . . . . . B B-67

B2-68 Tank 241-T-202 Analytical Results: Arsenic . . . . . . . . . . . B B-68

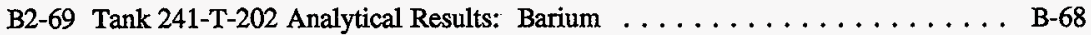

B2-70 Tank 241-T-202 Analytical Results: Beryllium . . . . . . . . . . . . . B-69

B2-71 Tank 241-T-202 Analytical Results: Bismuth . . . . . . . . . . . . . B-69

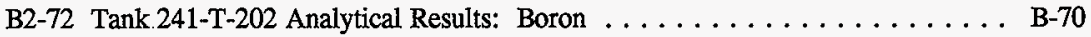

B2-73 Tank 241-T-202 Analytical Results: Cadmium . . . . . . . . . . . B-70

B2-74 Tank 241-T-202 Analytical Results: Calcium . . . . . . . . . . . B B-71

B2-75 Tank 241-T-202 Analytical Results: Cerium $\ldots \ldots \ldots \ldots \ldots \ldots \ldots$ B-71

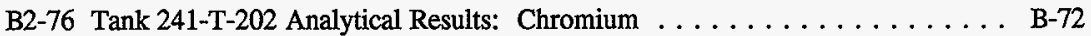

B2-77 Tank 241-T-202 Analytical Results: Cobalt . . . . . . . . . . B-72

B2-78 Tank 241-T-202 Analytical Results: Copper $\ldots \ldots \ldots \ldots \ldots \ldots \ldots$ B-73

B2-79 Tank 241-T-202 Analytical Results: Iron $\ldots \ldots \ldots \ldots \ldots \ldots \ldots$ B-73

B2-80 Tank 241-T-202 Analytical Results: Lanthanum . . . . . . . . . . . B-74

B2-81 Tank 241-T-202 Analytical Results: Lead . . . . . . . . . . . . . B-74

B2-82 Tank 241-T-202 Analytical Results: Lithium . . . . . . . . . . . . B-75

B2-83 Tank 241-T-202 Analytical Results: Magnesium . . . . . . . . . . . . B-75

B2-84 Tank 241-T-202 Analytical Results: Manganese . . . . . . . . . . . B-76

B2-85 Tank 241-T-202 Analytical Results: Molybdenum $\ldots \ldots \ldots \ldots \ldots \ldots$ B-76

B2-86 Tank 241-T-202 Analytical Results: Neodymium . . . . . . . . . . . . B-77

B2-87 Tank 241-T-202 Analytical Results: Nickel . . . . . . . . . . . . . B-77 


\section{LIST OF TABLES (Continued)}

B2-88 Tank 241-T-202 Analytical Results: Phosphorus . . . . . . . . . . . B-78

B2-89 Tank 241-T-202 Analytical Results: Potassium . . . . . . . . . B-78

B2-90 Tank 241-T-202 Analytical Results: Samarium . . . . . . . . . . . . B-79

B2-91 Tank 241-T-202 Analytical Results: Selenium . . . . . . . . . . . . B-79

B2-92 Tank 241-T-202 Analytical Results: Silicon . . . . . . . . . . . . . B-80

B2-93 Tank 241-T-202 Analytical Results: Silver . . . . . . . . . . . . . . B-80

B2-94 Tank 241-T-202 Analytical Results: Sodium . . . . . . . . . . . . . B-81

B2-95 Tank 241-T-202 Analytical Results: Strontium . . . . . . . . . . . . B-81

B2-96 Tank 241-T-202 Analytical Results: Sulfur . . . . . . . . . . . . B-82

B2-97 Tank 241-T-202 Analytical Results: Thallium . . . . . . . . . . . . B B-82

B2-98 Tank 241-T-202 Analytical Results: Titanium . . . . . . . . . . . B-83

B2-99 Tank 241-T-202 Analytical Results: Total Uranium . . . . . . . . . . . . B-83

B2-100 Tank 241-T-202 Analytical Results: Vanadium . . . . . . . . . . . B-84

B2-101 Tank 241-T-202 Analytical Results: Zinc . . . . . . . . . . . . . . B B-84

B2-102 Tank 241-T-202 Analytical Results: Zirconium . . . . . . . . . . B-85

B2-103 Tank 241-T-202 Analytical Results: Bromide . . . . . . . . . B B-85

B2-104 Tank 241-T-202 Analytical Results: Chloride . . . . . . . . . . B B-86

B2-105 Tank 241-T-202 Analytical Results: Fluoride . . . . . . . . . . B B-86

B2-106 Tank 241-T-202 Analytical Results: Nitrate . . . . . . . . . . . B-87

B2-107 Tank 241-T-202 Analytical Results: Nitrite . . . . . . . . . . . . B-87

B2-108 Tank 241-T-202 Analytical Results: Phosphate . . . . . . . . . . . B B-88 


\section{LIST OF TABLES (Continued)}

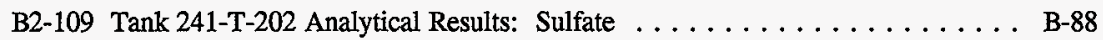

B2-110 Tank 241-T-202 Analytical Results: Oxalate . . . . . . . . . . . . B-89

B2-111 Tank 241-T-202 Analytical Results: Bulk Density . . . . . . . . . . . B-89

B2-112 Tank 241-T-202 Analytical Results: Percent Water . . . . . . . . . . . . B-90

B2-113 Tank 241-T-202 Analytical Results: Total Alpha . . . . . . . . . . . . B B-90

B2-114 Tank 241-T-202 Analytical Results: Americium-241 . . . . . . . . . . . B B-90

B2-115 Tank 241-T-202 Analytical Results: Cesium-137 . . . . . . . . . . . B-91

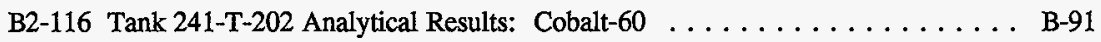

B2-117 Tank 241-T-202 Analytical Results: Europium-154 . . . . . . . . . B-91

B2-118 Tank 241-T-202 Analytical Results: Europium-155 . . . . . . . . . . B-91

B2-119 Tank 241-T-202 Analytical Results: Strontium-89/90 . . . . . . . . . . B-91

B2-120 Tank 241-T-202 Analytical Results: Total Inorganic Carbon . . . . . . . . . B-92

B2-121 Tank 241-T-202 Analytical Results: Total Organic Carbon . . . . . . . . . . B-92

B2-122 Tank 241-T-203 Analytical Results: Aluminum . . . . . . . . . . . B-92

B2-123 Tank 241-T-203 Analytical Results: Antimony . . . . . . . . . . B-93

B2-124 Tank 241-T-203 Analytical Results: Arsenic . . . . . . . . . . . . . B-95

B2-125 Tank 241-T-203 Analytical Results: Barium . . . . . . . . . . . B B-96

B2-126 Tank 241-T-203 Analytical Results: Beryllium . . . . . . . . . . . B-97

B2-127 Tank 241-T-203 Analytical Results: Bismuth . . . . . . . . . . . B B-98

B2-128 Tank 241-T-203 Analytical Results: Boron . . . . . . . . . . . . . B-99

B2-129 Tank 241-T-203 Analytical Results: Cadmium . . . . . . . . . . . B-100 


\section{LIST OF TABLES (Continued)}

B2-130 Tank 241-T-203 Analytical Results: Calcium . . . . . . . . . . . . B-101

B2-131 Tank 241-T-203 Analytical Results: Cerium . . . . . . . . . . . . . B-102

B2-132 Tank 241-T-203 Analytical Results: Chromium . . . . . . . . . . . . . B-103

B2-133 Tank 241-T-203 Analytical Results: Cobalt . . . . . . . . . . . . . B-104

B2-134 Tank 241-T-203 Analytical Results: Copper . . . . . . . . . . . . B-105

B2-135 Tank 241-T-203 Analytical Results: Iron . . . . . . . . . . . . . B-106

B2-136 Tank 241-T-203 Analytical Results: Lanthanum . . . . . . . . . . . . B-107

B2-137 Tank 241-T-203 Analytical Results: Lead . . . . . . . . . . . . . B-108

B2-138 Tank 241-T-203 Analytical Results: Lithium . . . . . . . . . . . . . . B-109

B2-139 Tank 241-T-203 Analytical Results: Magnesium . . . . . . . . . . . . B-110

B2-140 Tank 241-T-203 Analytical Results: Manganese . . . . . . . . . . . . . . . B-111

B2-141 Tank 241-T-203 Analytical Results: Molybdenum . . . . . . . . . . . B-112

B2-142 Tank 241-T-203 Analytical Results: Neodymium . . . . . . . . . . . B-113

B2-143 Tank 241-T-203 Analytical Results: Nickel . . . . . . . . . . . . . B-114

B2-144 Tank 241-T-203 Analytical Results: Phosphorus . . . . . . . . . . . . . B-114

B2-145 Tank 241-T-203 Analytical Results: Potassium . . . . . . . . . . . . B-115

B2-146 Tank 241-T-203 Analytical Results: Samarium . . . . . . . . . . B-116

B2-147 Tank 241-T-203 Analytical Results: Selenium . . . . . . . . . . . . . B-117

B2-148 Tank 241-T-203 Analytical Results: Silicon $\ldots \ldots \ldots \ldots \ldots \ldots \ldots \ldots$. . . . . . . .

B2-149 Tank 241-T-203 Analytical Results: Silver . . . . . . . . . . . . . B-119

B2-150 Tank 241-T-203 Analytical Results: Sodium . . . . . . . . . B-120 


\section{LIST OF TABLES (Continued)}

B2-151 Tank 241-T-203 Analytical Results: Strontium . . . . . . . . . . . B-121

B2-152 Tank 241-T-203 Analytical Results: Sulfur . . . . . . . . . . . B-122

B2-153 Tank 241-T-203 Analytical Results: Thallium . . . . . . . . . . . . B-123

B2-154 Tank 241-T-203 Analytical Results: Titanium . . . . . . . . . . . . . . B-124

B2-155 Tank 241-T-203 Analytical Results: Total Uranium . . . . . . . . . . . B-125

B2-156 Tank 241-T-203 Analytical Results: Vanadium . . . . . . . . . . B-126

B2-157 Tank 241-T-203 Analytical Results: Zinc . . . . . . . . . . . . B-127

B2-158 Tank 241-T-203 Analytical Results: Zirconium . . . . . . . . . . . . B-128

B2-159 Tank 241-T-203 Analytical Results: Bromide . . . . . . . . . . . . . B-129

B2-160 Tank 241-T-203 Analytical Results: Chloride . . . . . . . . . . . B-130

B2-161 Tank 241-T-203 Analytical Results: Fluoride . . . . . . . . . . . . B-131

B2-162 Tank 241-T-203 Analytical Results: Nitrate . . . . . . . . . . . . B-132

B2-163 Tank 241-T-203 Analytical Results: Nitrite $\ldots \ldots \ldots \ldots \ldots \ldots$. . . . . . . . .

B2-164 Tank 241-T-203 Analytical Results: Phosphate . . . . . . . . . . . . . B-134

B2-165 Tank 241-T-203 Analytical Results: Sulfate $\ldots \ldots \ldots \ldots \ldots \ldots \ldots$. . . . B-135

B2-166 Tank 241-T-203 Analytical Results: Oxalate . . . . . . . . . . . . B-136

B2-167 Tank 241-T-203 Analytical Results: Bulk Density . . . . . . . . . . . B-137

B2-168 Tank 241-T-203 Analytical Results: Exotherm - transition 1 . . . . . . . . . B-137

B2-169 Tank 241-T-203 Analytical Results: Percent Water . . . . . . . . . . . . B-138

B2-170 Tank 241-T-203 Analytical Results: Specific Gravity . . . . . . . . . . . B-139

B2-171 Tank 241-T-203 Analytical Results: Total Alpha . . . . . . . . . . . . . . B-139 


\section{LIST OF TABLES (Continued)}

B2-172 Tank 241-T-203 Analytical Results: Total Alpha . . . . . . . . . . . . . B-139

B2-173 Tank 241-T-203 Analytical Results: Americium-241 . . . . . . . . . . B-140

B2-174 Tank 241-T-203 Analytical Results: Cesium-137 . . . . . . . . . . . . B-140

B2-175 Tank 241-T-203 Analytical Results: Cobalt-60 . . . . . . . . . . . B-140

B2-176 Tank 241-T-203 Analytical Results: Europium-154 . . . . . . . . . . B-140

B2-177 Tank 241-T-203 Analytical Results: Europium-155 . . . . . . . . . . . B-140

B2-178 Tank 241-T-203 Analytical Results: Strontium-89/90 . . . . . . . . . B-141

B2-179 Tank 241-T-203 Analytical Results: Total Inorganic Carbon . . . . . . . . B-141

B2-180 Tank 241-T-203 Analytical Results: Total Organic Carbon . . . . . . . . . . B-141

B2-181 Tank 241-T-204 Analytical Results: Aluminum . . . . . . . . . . . . . B-141

B2-182 Tank 241-T-204 Analytical Results: Antimony . . . . . . . . . . . . B B-141

B2-183 Tank 241-T-204 Analytical Results: Arsenic . . . . . . . . . . . . . . B-142

B2-184 Tank 241-T-204 Analytical Results: Barium . . . . . . . . . . . . . B-142

B2-185 Tank 241-T-204 Analytical Results: Beryllium . . . . . . . . . . . B-142

B2-186 Tank 241-T-204 Analytical Results: Bismuth . . . . . . . . . . . . . B-142

B2-187 Tank 241-T-204 Analytical Results: Boron . . . . . . . . . . . . . . B-142

B2-188 Tank 241-T-204 Analytical Results: Cadmium . . . . . . . . . . . . B-143

B2-189 Tank 241-T-204 Analytical Results: Cerium . . . . . . . . . . . . . . B-143

B2-190 Tank 241-T-204 Analytical Results: Chromium . . . . . . . . . . . . . B-143

B2-191 Tank 241-T-204 Analytical Results: Cobalt . . . . . . . . . . . . . B-143

B2-192 Tank 241-T-204 Analytical Results: Copper . . . . . . . . . . . . B-143 


\section{LIST OF TABLES (Continued)}

B2-193 Tank 241-T-204 Analytical Results: Iron . . . . . . . . . . . . . . . B-144

B2-194 Tank 241-T-204 Analytical Results: Lanthanum . . . . . . . . . . . . B-144

B2-195 Tank 241-T-204 Analytical Results: Lead . . . . . . . . . . . . . . . B-144

B2-196 Tank 241-T-204 Analytical Results: Lithium . . . . . . . . . . . . . . B-144

B2-197 Tank 241-T-204 Analytical Results: Magnesium . . . . . . . . . . . . . B-144

B2-198 Tank 241-T-204 Analytical Results: Manganese . . . . . . . . . . . . . . B-145

B2-199 Tank 241-T-204 Analytical Results: Molybdenum . . . . . . . . . . . . B-145

B2-200 Tank 241-T-204 Analytical Results: Neodymium . . . . . . . . . . . . . B-145

B2-201 Tank 241-T-204 Analytical Results: Nickel . . . . . . . . . . . . B-145

B2-202 Tank 241-T-204 Analytical Results: Phosphorus . . . . . . . . . . . B-145

B2-203 Tank 241-T-204 Analytical Results: Potassium . . . . . . . . . . . B-146

B2-204 Tank 241-T-204 Analytical Results: Samarium . . . . . . . . . . . . B-146

B2-205 Tank 241-T-204 Analytical Results: Silicon . . . . . . . . . . . . . B-146

B2-206 Tank 241-T-204 Analytical Results: Silver . . . . . . . . . . . . . . B-146

B2-207 Tank 241-T-204 Analytical Results: Sodium . . . . . . . . . . . . B-146

B2-208 Tank 241-T-204 Analytical Results: Strontium . . . . . . . . . . . . B-147

B2-209 Tank 241-T-204 Analytical Results: Sulfur . . . . . . . . . . . . . . B-147

B2-210 Tank 241-T-204 Analytical Results: Thallium . . . . . . . . . . . . B-147

B2-211 Tank 241-T-204 Analytical Results: Titanium . . . . . . . . . . . . B-147

B2-212 Tank 241-T-204 Analytical Results: Total Uranium . . . . . . . . . . . B-147

B2-213 Tank 241-T-204 Analytical Results: Vanadium . . . . . . . . . . . B-148 


\section{LIST OF TABLES (Continued)}

B2-214 Tank 241-T-204 Analytical Results: Zinc . . . . . . . . . . . . . . . B-148

B2-215 Tank 241-T-204 Analytical Results: Zirconium . . . . . . . . . . . B-148

B2-216 Tank 241-T-204 Analytical Results: Bromide . . . . . . . . . . . . B-148

B2-217 Tank 241-T-204 Analytical Results: Chloride . . . . . . . . . . . . B-148

B2-218 Tank 241-T-204 Analytical Results: Fluoride . . . . . . . . . . . B-149

B2-219 Tank 241-T-204 Analytical Results: Nitrate . . . . . . . . . . . . . B-149

B2-220 Tank 241-T-204 Analytical Results: Nitrite . . . . . . . . . . . . B-149

B2-221 Tank 241-T-204 Analytical Results: Phosphate . . . . . . . . . . . . . B-149

B2-222 Tank 241-T-204 Analytical Results: Sulfate . . . . . . . . . . . . B-149

B2-223 Tank 241-T-204 Analytical Results: Oxalate . . . . . . . . . . . . . . . . B-150

B2-224 Tank 241-T-204 Analytical Results: Bulk Density . . . . . . . . . . . B-150

B2-225 Tank 241-T-204 Analytical Results: Percent Water . . . . . . . . . . . B-151

B2-226 Tank 241-T-204 Analytical Results: Total Alpha . . . . . . . . . . . . . B-152

B2-227 Tank 241-T-204 Analytical Results: Americium-241 . . . . . . . . . . . B-152

B2-228 Tank 241-T-204 Analytical Results: Cesium-137 . . . . . . . . . . . . B-152

B2-229 Tank 241-T-204 Analytical Results: Cobalt-60 . . . . . . . . . . . . B-153

B2-230 Tank 241-T-204 Analytical Results: Europium-154 . . . . . . . . . . B-153

B2-231 Tank 241-T-204 Analytical Results: Europium-155 . . . . . . . . . . B-153

B2-232 Tank 241-T-204 Analytical Results: Strontium-89/90 . . . . . . . . . B-153

B2-233 Tank 241-T-204 Analytical Results: Total Inorganic Carbon . . . . . . . . . B B-153 


\section{LIST OF TABLES (Continued)}

B2-234 Tank 241-T-204 Analytical Results: Total Organic Carbon . . . . . . . . . . B-154

B3-1 Comparison of Phosphate to Total Phosphorous . . . . . . . . . . . B-157

B3-2 Comparison of Sufate to Total Sulfur $\ldots \ldots \ldots \ldots \ldots \ldots$. . . . . . . . .

B3-3 Comparison of Oxalate to Total Organic Carbon . . . . . . . . . . . B-157

B3-4 241-T-201 Cation Mass and Charge Data . . . . . . . . . . . B-159

B3-5 241-T-201 Anion Mass and Charge Data . . . . . . . . . . . B-160

B3-6 Mass and Charge Balance Totals for Tank 241-T-201 . . . . . . . . . . B B-160

B3-7 241-T-202 Cation Mass and Charge Data . . . . . . . . . . . B-161

B3-8 241-T-202 Anion Mass and Charge Data . . . . . . . . . . . . B-162

B3-9 Mass and Charge Balance Totals for Tank 241-T-202 . . . . . . . . . . B-162

B3-10 241-T-203 Cation Mass and Charge Data . . . . . . . . . . . . B-163

B3-11 241-T-203 Anion Mass and Charge Data $\ldots \ldots \ldots \ldots \ldots \ldots \ldots \ldots$ B-164

B3-12 Mass and Charge Balance Totals for Tank 241-T-203 . . . . . . . . . B-164

B3-13 241-T-204 Cation Mass and Charge Data . . . . . . . . . . . . B-165

B3-14 241-T-204 Anion Mass and Charge Data . . . . . . . . . . . . B-166

B3-15 Mass and Charge Balance Totals for Tank 241-T-204 . . . . . . . . . . B-166

B3-16 Summary Statistics for Tank $241-\mathrm{T}-201 \ldots \ldots \ldots \ldots \ldots \ldots \ldots$ B-169 $\ldots \ldots \ldots$

B3-17 Summary Statistics for Tank $241-\mathrm{T}-202 \ldots \ldots \ldots \ldots \ldots \ldots \ldots$ B-171

B3-18 Summary Statistics for Tank $241-\mathrm{T}-203 \ldots \ldots \ldots \ldots \ldots \ldots$. . . . . . . . . . .

B3-19 Summary Statistics for Tank $241-\mathrm{T}-204 \ldots \ldots \ldots \ldots \ldots \ldots \ldots$ B-175 


\section{LIST OF TABLES (Continued)}

C1-1 95 Percent Confidence Interval Upper Limits for Alpha for Tanks 241-T-201, $-\mathrm{T}-202,-\mathrm{T}-203$, and $-\mathrm{T}-204 \ldots \ldots \ldots \ldots \ldots \ldots \ldots \ldots \ldots \ldots . . . \ldots \ldots$

D2-1 Engineering and Hanford Defined Waste-Based Inventory Estimates for Tank 241-T-201 Nonradioactive Components . . . . . . . . . . . . . D D-4

D2-2 Engineering and Hanford Defined Waste-based Inventory Estimates for Radioactive Components for Tank . . . . . . . . . . . . . D-5

D2-3 Engineering and Hanford Defined Waste-based Inventory Estimates for Tank 241-T-202 Nonradioactive Components . . . . . . . . . . . . . D-5

D2-4 Engineering and Hanford Defined Waste-based Inventory Estimates for Radioactive Components in Tank $241-\mathrm{T}-202 \ldots \ldots \ldots \ldots$. . . . . . . . . . .

D2-5 Engineering and Hanford Defined Waste-based Inventory Estimates for Tank 241-T-203 Nonradioactive Components . . . . . . . . . . . . . . D-7

D2-6 Engineering Assessment- and Hanford Defined Waste-Based Inventory Estimates for Tank 241-T-204 Nonradioactive Components . . . . . . . . . . . D-7

D3-1 Technical Flowsheet and Hanford Defined Waste Streams . . . . . . . . . . D-10

D3-2 Comparison of Selected Component Inventory Estimates for T-200 Series Tanks . . . . . . . . . . . . . . . . . . . . . . D-12

D4-1 Best-Basis Inventory Estimates for Nonradioactive Components in T-200 Series Tanks . . . . . . . . . . . . . . . D-16

D4-2 Best-Basis Inventory Estimate for Radioactive Components in T-200 Series Tanks Decayed to January $1,1994 \ldots \ldots \ldots \ldots \ldots$. . . . . . . . . D-18 
HNF-1501 Rev. 0

\section{LIST OF TERMS}

$\mathrm{Btu} / \mathrm{hr}$

Ci

$\mathrm{Ci} / \mathrm{L}$

CI

$\mathrm{cm}$

DQO

DSC

$\mathrm{ft}$

$\mathrm{ft}^{2}$

$\mathrm{g}$

$\mathrm{g} / \mathrm{cm}^{3}$

$\mathrm{g} / \mathrm{L}$

$\mathrm{g} / \mathrm{mL}$

GEA

HDW

HTCE

IC

ICP

ICP/AES

in.

$\mathrm{J} / \mathrm{g}$

$\mathrm{kg}$

$\mathrm{kg} / \mathrm{L}$

$\mathrm{kgal}$

kL

kW

LFL

LL

m

$\mathrm{mg}$

$\mathrm{m}^{2}$

$M$

$\mathrm{mL}$

MOU

$\mathrm{mm}$

n/a

NA

NR

$n / r$

PHMC
British thermal units per hour

curie

curies per liter

confidence interval

centimeter

data quality objective

differential scanning calorimetry

feet

square feet

gram

grams per cubic centimeter

grams per liter

grams per milliliter

gamma energy analysis

Hanford defined waste

historical tank content estimate

ion chromatography

inductively coupled plasma

inductively coupled plasma/atomic emission spectroscopy

inch

joules per gram

kilogram

kilograms per liter

kilogallon

kiloliter

kilowatt

lower flammability limit

lower limit

meter

milligram

square meters

moles

milliliter

Memorandum of Understanding

millimeter

not applicable

not available

not requested

not reported

Project Hanford Management Contractor 


\section{LIST OF TERMS (Continued)}

$\begin{array}{ll}\text { ppm } & \text { parts per million } \\ \text { QC } & \text { quality control } \\ \text { RPD } & \text { relative percent difference } \\ \text { SMM } & \text { supernatant mixing model } \\ \text { SpG } & \text { specific gravity } \\ \text { TCR } & \text { tank characterization report } \\ \text { TGA } & \text { thermogravimetric analysis } \\ \text { TTC } & \text { total inorganic carbon } \\ \text { TLM } & \text { tank layer model } \\ \text { TOC } & \text { total organic carbon } \\ \text { TWRS } & \text { Tank Waste Remediation System } \\ \text { UL } & \text { upper limit } \\ \text { W } & \text { watt } \\ \text { WSTRS } & \text { Waste Status and Transaction Record Summary } \\ \text { wt } \% & \text { weight percent } \\ \% & \text { percent } \\ { }^{\circ} \mathrm{C} & \text { degrees Celsius } \\ { }^{\circ} \mathrm{F} & \text { degrees Fahrenheit } \\ \mu \mathrm{Ci} / \mathrm{g} & \text { microcuries per gram } \\ \mu \mathrm{Ci} / \mathrm{mL} & \text { microcuries per milliliter } \\ \mu \mathrm{eq} / \mathrm{g} & \text { microequivalents per gram } \\ \mu \mathrm{g} / \mathrm{g} & \text { micrograms per gram } \\ & \end{array}$




\subsection{INTRODUCTION}

A major function of the Tank Waste Remediation System (TWRS) is to characterize waste in support of waste management and disposal activities at the Hanford Site. Analytical data from sampling and analysis, in addition to other available information about a tank are compiled and maintained in a tank characterization report (TCR). This report and its appendices serve as the TCR for the single-shell tank series consisting of tanks 241-T-201, -T-202, -T-203, and -T-204.

The objectives of this report are 1) to use characterization data in response to technical issues associated with T-200 series tank waste and 2) to provide a standard characterization of this waste in terms of a best-basis inventory estimate. Section 2.0 summarizes the response to technical issues, Section 3.0 shows the best-basis inventory estimate, Section 4.0 makes recommendations about the safety status of the tank and additional sampling needs. The appendices contain supporting data and information. This report supports the requirements of the Hanford Federal Facility Agreement and Consent Order (Ecology et al. 1997) Milestone M-44-15B, change request M-44-97-03, to "issue characterization deliverables consistent with Waste Information Requirements Document developed for 1998."

\subsection{SCOPE}

The characterization information in this report originated from sample analyses and known historical sources. The results of recent sample events will be used to fulfill the requirements of the data quality objectives (DQOs). The sampling and analytical protocols for each tank are documented in a specific tank sampling and analysis plan (Hu [1997], Bell [1997], Schreiber [1997c], and Winkleman [1997]). The results of the 1997 sampling events are reported in the specific laboratory data package for each tank (Nuzum [1997a], Esch [1997], Steen [1997], and Nuzum [1997b]). Other information can be used to support conclusions derived from these results.

Appendix A contains historical information for tanks 241-T-201 to T-204, including surveillance information, records pertaining to waste transfers and tank operations, and expected tank contents derived from a process knowledge-based computer program. Appendix B summarizes sampling events (see Table 1-1), sample data obtained before 1989, and the most current sampling results. The laboratory analyses performed on each tank satisfied the data requirements specified in Brown et al. (1997). Appendix C reports the statistical analysis and numerical manipulation of data used in issue resolution. Appendix D contains the evaluation to establish the best basis for the inventory estimate and the statistical analysis performed for this evaluation. Appendix $\mathrm{E}$ is a bibliography that resulted from an in-depth literature search of all known information sources applicable to tanks 241-T-201, -T-202, -T-203, and -T-204. The reports listed in Appendix E are available in the Tank Characterization and Safety Resource Center. 
Table 1-1. Summary of Recent Sampling.

\begin{tabular}{|c|c|c|c|c|}
\hline STample & Phasse & 26.10, & 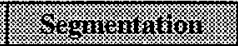 & 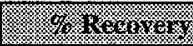 \\
\hline $\begin{array}{l}\text { Combustible gas } \\
\text { test } \\
\text { (Each tank) }\end{array}$ & Gas & $\begin{array}{l}\text { Tank headspace, } \\
\text { Riser } 3,4.6 \mathrm{~m} \\
(15 \mathrm{ft}) \text { below top of } \\
\text { riser }\end{array}$ & $\mathrm{n} / \mathrm{a}$ & $n / a$ \\
\hline $\begin{array}{l}\text { Push Core } 192 \\
241-T-201\end{array}$ & Solid/liquid & Riser 3 & $\begin{array}{l}8 \text { segments; } 5 \\
\text { liquid, } 3 \text { solid. } \\
\text { Upper half and } \\
\text { lower half on } \\
\text { solids }\end{array}$ & $90 \%$ \\
\hline $\begin{array}{l}\text { Push Core } 191 \\
241-T-202\end{array}$ & Solid/sludge & Riser 3 & $\begin{array}{l}5 \text { segments, upper } \\
\text { half and lower half }\end{array}$ & $91 \%$ \\
\hline $\begin{array}{l}\text { Push Core } 190 \\
241-\mathrm{T}-203\end{array}$ & Solid/sludge & Riser 3 & $\begin{array}{l}9 \text { segments, upper } \\
\text { half and lower half }\end{array}$ & $98 \%$ \\
\hline $\begin{array}{l}\text { Push Core } 188 \\
\text { 241-T-204 }\end{array}$ & Solid/sludge & Riser 3 & $\begin{array}{l}10 \text { segments, } \\
\text { upper half and } \\
\text { lower half }\end{array}$ & $88 \%$ \\
\hline
\end{tabular}

Note:

$\mathbf{n} / \mathfrak{a}=$ not applicable

\subsection{TANK BACKGROUND}

Tanks 241-T-201, T-202, T-203, and T-204 are located in the 200 West Area T Tank Farm on the Hanford Site. Tank 241-T-201 is not part of any tank cascade. Tanks 241-T-202, T-203, and T-204 are not cascaded but are connected together by tie lines. The tanks went into service in 1952, receiving lanthanum fluoride (224) waste from T-Plant. The tanks were filled later that year. The only other transfer of waste associated with these tanks occurred in 1976 and 1977 when liquids were pumped from the tanks in support of stabilization efforts. Final stabilization occurred in 1981 (Brevick et al. 1997).

Table 1-2 summarizes the description of tanks 241-T-201, T-202, T-203, and T-204, based on Hanlon (1997). Each tank has an operating capacity of $208 \mathrm{~kL}$ (55 kgal). These tanks are not on the Watch List (Public Law 101-510). 
HNF-1501 Rev. 0

Table 1-2. Description of T-200 Series Tanks.

\begin{tabular}{|c|c|c|c|c|}
\hline \multirow{2}{*}{\multicolumn{4}{|c|}{ Type }} & \\
\hline & & & & Single-shell \\
\hline \multicolumn{4}{|l|}{ Constructed } & 1943-1944 \\
\hline \multicolumn{4}{|l|}{ In service } & 1952 \\
\hline \multicolumn{4}{|l|}{ Diameter } & $6.1 \mathrm{~m}(20 \mathrm{ft})$ \\
\hline \multicolumn{4}{|l|}{ Operating depth } & $7.8 \mathrm{~m}(25.5 \mathrm{ft})$ \\
\hline \multicolumn{4}{|l|}{ Capacity } & $208 \mathrm{~kL}(55 \mathrm{kgal})$ \\
\hline \multicolumn{4}{|l|}{ Bottom shape } & Dish \\
\hline \multicolumn{4}{|l|}{ Ventilation } & Passive \\
\hline \multicolumn{4}{|c|}{ 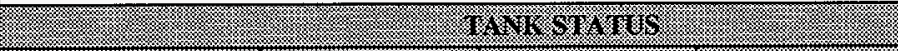 } & (1) \\
\hline \multicolumn{2}{|c|}{ (1) } & 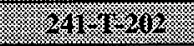 & $1.43: 18.2103$ & 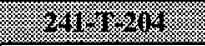 \\
\hline \multicolumn{2}{|c|}{\begin{tabular}{|l|l|} 
Waste classification & Noncomplexed \\
\end{tabular}} & Noncomplexed & Noncomplexed & Noncomplexed \\
\hline Total waste volume & $110 \mathrm{~kL}(29 \mathrm{kgal})$ & $79 \mathrm{~kL}(21 \mathrm{kgal})$ & $132 \mathrm{~kL}(35 \mathrm{kgal})$ & $144 \mathrm{~kL}(38 \mathrm{kgal})$ \\
\hline Supernatant volume & $3.8 \mathrm{~kL}(1 \mathrm{kgal})$ & $0 \mathrm{~kL}(0 \mathrm{kgal})$ & $0 \mathrm{~kL}(0 \mathrm{kgal})$ & $0 \mathrm{~kL}(0 \mathrm{kgal})$ \\
\hline Sludge volume & $106 \mathrm{~kL}(28 \mathrm{kgal})$ & $79 \mathrm{~kL}(21 \mathrm{kgal})$ & $132 \mathrm{~kL}(35 \mathrm{kgal})$ & $144 \mathrm{~kL}(38 \mathrm{kgal})$ \\
\hline $\begin{array}{l}\text { Drainable interstitial } \\
\text { liquid volume } \\
\end{array}$ & $11 \mathrm{~kL}(3 \mathrm{kgal})$ & $8 \mathrm{~kL}(2 \mathrm{kgal})$ & $15 \mathrm{~kL}(4 \mathrm{kgal})$ & $15 \mathrm{~kL}$ (4 kgal) \\
\hline Waste surface level & $4.1 \mathrm{~m}(162 \mathrm{in})$. & $2.7 \mathrm{~m}$ (105 in.) & $4.7 \mathrm{~m}$ (188 in.) & $4.9 \mathrm{~m}$ (194 in.) \\
\hline Avg. temperature ${ }^{1}$ & $17^{\circ} \mathrm{C}\left(62^{\circ} \mathrm{F}\right)$ & $17^{\circ} \mathrm{C}\left(62^{\circ} \mathrm{F}\right)$ & $17^{\circ} \mathrm{C}\left(62^{\circ} \mathrm{F}\right)$ & $17^{\circ} \mathrm{C}\left(63^{\circ} \mathrm{F}\right)$ \\
\hline Integrity & Sound & Sound & Sound & Sound \\
\hline \multirow[t]{2}{*}{ Watch List } & None & None & None & None \\
\hline & 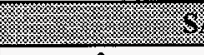 & 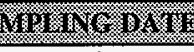 & & \\
\hline Core samples & $4 / 24 / 97^{2}$ & $4 / 21 / 97^{2}$ & $4 / 16 / 97^{2}$ & $3 / 27 / 97^{2}$ \\
\hline \multicolumn{5}{|c|}{ 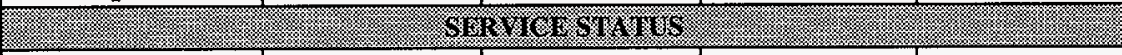 } \\
\hline Declared inactive & 1976 & 1976 & 1976 & 1976 \\
\hline Interim stabilization & 1981 & 1981 & 1981 & 1981 \\
\hline Intrusion prevention & 1981 & 1981 & 1981 & 1981 \\
\hline
\end{tabular}

Note:

${ }^{1}$ From March 1975 to April 1997

${ }^{2}$ Dates are shown in $\mathrm{mm} / \mathrm{dd} / \mathrm{yy}$ format. 
HNF-1501 Rev. 0

This page intentionally left blank. 


\subsection{RESPONSE TO TECHNICAC ISSUES}

Four technical issues have been identified for the T-200 series tanks (Brown et al. 1997 and Schreiber 1997b).

- Safety screening: Does the waste pose or contribute to any recognized potential safety problems?

- Hazardous vapor screening: Do hazardous storage conditions exist associated with gases and vapors in the tank?

- Organic complexants: Does the possibility exist for a point source ignition in the waste followed by a propagation of the reaction in the solid/liquid phase of the waste?

- Organic solvents: Does an organic solvent pool exist that may cause a fire or ignition of organic solvents in entrained waste solids?

Brown et al. (1997) provides the types of sampling and analysis used to address the above issues. Data from the analysis of push core samples, tank vapor space measurements, and available historical information provided the means to respond to the technical issues. Sections 2.1 and 2.2 present the response. See Appendix B for sample and analysis data for each tank.

\subsection{SAFETY SCREENING}

The data needed to screen the waste in the T-200 series tanks for potential safety problems are documented in Tank Safety Screening Data Quality Objective (Dukelow et al. 1995). These potential safety problems are exothermic conditions in the waste, flammable gases in the waste and/or tank headspace, and criticality conditions in the waste. Each condition is addressed separately below.

\subsubsection{Exothermic Conditions (Energetics)}

The first requirement outlined in the safety screening DQO (Dukelow et al. 1995) is to ensure that there are not sufficient exothermic constituents (organic or ferrocyanide) in the T-200 series tanks to pose a safety hazard. Because of this requirement, energetics in all four T-200 tanks (241-T-201, -T-202, -T-203, and -T-204) waste were evaluated. The safety screening DQO required that the waste sample profile be tested for energetics every $24 \mathrm{~cm}(9.5 \mathrm{in}$.) to determine whether the energetics exceeded the safety threshold limit. The threshold limit for energetics is $480 \mathrm{~J} / \mathrm{g}$ on a dry weight basis. 
Results obtained using differential scanning calorimetry (DSC) indicated that no sample obtained from any of the T-200 series tanks had mean exothermic reactions (on a dry-weight basis) exceeding the safety screening DQO limit. The maximum dry weight exotherm observed was $16.7 \mathrm{~J} / \mathrm{g}$ from tank 241-T-203, core 190, segment 9, lower half. Because of the low number of exothermic observations and their small magnitude, 95 percent confidence intervals on the means were not calculated. The other T-200 series tanks did not have any measurable energetics, therefore, no confidence interval could be calculated: These results indicate there is no energetics safety issue associated with these tanks.

\subsubsection{Flammable Gas}

Headspace measurements were taken from the sampling riser (riser 3 for each T-200 tank) before taking the push core samples. Flammable gas was not detected in any of the tank headspaces ( 0 percent of the lower flammability limit [LFL]) before sampling the four T-200 series tanks. These results are below the safety screening limit of 25 percent of the LFL, indicating no flammable gas safety issue associated with the tanks. Appendix B provides data for the March and April 1997 combustible gas measurement .

\subsubsection{Criticality}

The safety screening DQO threshold for criticality, based on the total alpha activity, is $1 \mathrm{~g} / \mathrm{L}$. Because total alpha activity is measured in $\mu \mathrm{Ci} / \mathrm{g}$ instead of $\mathrm{g} / \mathrm{L}$, the $1 \mathrm{~g} / \mathrm{L}$ limit is converted into units of $\mu \mathrm{Ci} / \mathrm{g}$ by assuming that all alpha decay originates from ${ }^{239} \mathrm{Pu}$. The safety limit threshold is $1 \mathrm{~g}{ }^{239} \mathrm{Pu}$ per liter of waste.

Table 2-1. T-200 Criticality Thresholds and Results.

\begin{tabular}{|c|c|c|c|c|}
\hline isin: & 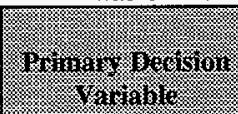 & 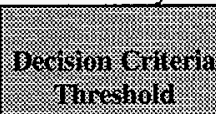 & 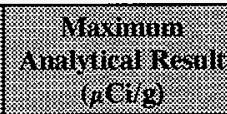 & 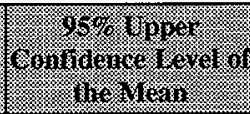 \\
\hline Criticality & Total alpha & $41 \mu \mathrm{Ci} / \mathrm{g}$ & $\begin{array}{l}\text { T-201: } 1.15 \\
\text { T-202: } 0.295 \\
\text { T-203: } 0.278 \\
\text { T-204: } 0.208\end{array}$ & $\begin{array}{l}\mathrm{T}-201: 1.63 \\
\mathrm{~T}-202: 0.409 \\
\mathrm{~T}-203: 0.469 \\
\mathrm{~T}-204: 0.549\end{array}$ \\
\hline
\end{tabular}

Assuming that all alpha is from ${ }^{239} \mathrm{Pu}$ and assuming a typical density of $1.27 \mathrm{~g} / \mathrm{mL}, 1 \mathrm{~g} / \mathrm{L}$ of ${ }^{239} \mathrm{Pu}$ is $48.4 \mu \mathrm{Ci} / \mathrm{g}$ of alpha activity. The largest total alpha activity result was $1.15 \mu \mathrm{Ci} / \mathrm{g}$ (core 192, segment 4, lower half). The highest upper limit to a 95 percent confidence interval 
on the mean observed in the T-200 tanks was $1.63 \mu \mathrm{Ci} / \mathrm{g}$, found in the same sample, indicating that the potential for a criticality event is extremely low. Therefore, criticality is not a concern for this tank. Appendix $\mathrm{C}$ contains the method used to calculate confidence limits and computational results.

\subsection{HAZARDOUS VAPOR SAFETY SCREENING}

The data required to support vapor screening are documented in Data Quality Objective for Tank Hazardous Vapor Safety Screening (Osborne and Buckley 1995). The vapor screening DQO addresses two issues: 1) does the vapor headspace exceed 25 percent of the LFL, and if so, what are the principal fuel components; and 2) does the potential exist for worker hazards associated with the toxicity of constituents in any fugitive vapor emissions from these tanks?

\subsubsection{Flammable Gas}

This is the same requirement as the safety screening flammability requirement. As noted previously, flammable gas was not detected in any of the T-200 tank headspaces ( 0 percent of the LFL) before sampling. Tanks 241-T-201, -T-202, and -T-204 are in Flammable Gas Facility Group 2; tank 241-T-203 is considered Flammable Gas Facility Group 3. There is no flammable gas hazard associated with these tanks.

\subsubsection{Toxicity}

The toxicity issue has been closed for all tanks (Hewitt 1996). Data from the March/April vapor surveillance sampling event indicates that for these four tanks, the ammonia level was 0 parts per million, and total organic carbon (TOC) was 0 parts per million. There is no vapor toxicity issue associated with these tanks.

\subsection{ORGANIC COMPLEXANTS}

The data required to support the issue of organic complexants are documented in Memorandum of Understanding for the Organic Complexant Safety Issue Data Requirements (Schreiber 1997b). Energetics by DSC and moisture analyses were conducted to address the organic complexants issue. All moisture analyses show the water content for these tanks is greater than 50 percent. Because no exotherms were detected by DSC analyses for tanks 241-T-201, $-\mathrm{T}-202$, and $-\mathrm{T}-204$, no further data were required to address the issue. According to the logic in Schreiber (1997b), these tanks are safe with respect to the organic complexants issue.

In tank 241-T-203, very small magnitude exotherms were observed. However, fewer than 25 percent of the samples examined had exotherms, very low levels of TOC were observed, 
and the water content was greater than 50 percent; therefore, this tank also is considered safe with respect to the organics complexant issue (Schreiber 1997a).

\subsection{ORGANIC SOLVENTS}

The data required to support the organic solvent screening issue are documented in the Data Quality Objective to Support Resolution of the Organic Solvent Safety Issue (Meacham et al. 1997). The DQO requires tank headspace samples be analyzed for total nonmethane organic compounds to determine whether the organic extractant pool in the tank is a hazard. The purpose of this assessment is to ensure that an organic solvent pool fire or ignition of organic solvents cannot occur. Analytical results showed no organics of any type were present in the headspace for any T-200 series tank. However, the tanks have not been sampled according to the protocols described in Meacham et al. (1997); therefore, no safety designation with regard to the organic solvent issue can be made.

\subsection{OTHER TECHNICAL ISSUES}

A factor in assessing tank safety is the heat generation and temperature of the waste. Heat is generated in the tanks from radioactive decay. Estimates of each tank's heat load based on the 1997 sample event can be made from sample data collected. Based on the analytical results, each tank had a heat load of less than $2 \mathrm{~W}$, because of the very low fission product content of this waste.

The heat load estimates based on the tank process history ranged from $0.165 \mathrm{~W}$ for tank 241-T-202 to $0.298 \mathrm{~W}$ for tank 241-T-204 (Agnew et al. 1997). The heat load estimate based on the tank headspace temperature ranged from $14 \mathrm{~W}(48 \mathrm{Btu} / \mathrm{hr})$ for tank 241-T-201 to $1,375 \mathrm{~W}(4,690 \mathrm{Btu} / \mathrm{hr}$ ) for tank 241-T-203 (Kummerer 1995). The 1,375 W estimate for tank 241-T-203 does not correspond with the other tank information and appears to be based on an errant thermocouple reading. All of these heat load estimates are quite low and are well below the limit of $11,700 \mathrm{~W}(40,000 \mathrm{Btu} / \mathrm{hr})$ that separates high- and low-heat-load tanks (Smith 1986).

Analysis of process history information suggests that the waste in each T-200 series tank is similar to the waste in other T-200 series tanks. It is also similar in composition to the B-200 series tanks. The sampling and analysis of the T-200 series tanks was designed to take advantage of this prior information. Both waste tank groups received lanthanum fluoride (224) waste only the B-200 series tanks received lanthanum fluoride (224) from B Plant, the T-200 series tanks received it from $\mathrm{T}$ Plant. The composition of this waste is distinctive from the other wastes in single-shell tanks. It has relatively high (weight percent) concentrations of lanthanum, bismuth, and manganese present, and low concentrations of fission products (usually near or below detection limits). The separations process did not vary from plant to 
plant, and no other transactions took place between these tanks and the rest of tank farms to alter or confound the waste stream composition; therefore, the variation in the waste in the $\mathrm{T}-200$ series tanks is believed to be relatively small.

The construction and fill method for each group of tanks (B-200 and T-200) appears similar. One tank in each group (241-B-201 and 241-T-201, respectively) was piped separately and received waste from a different path than others in the group. The other three tanks in each series were connected together by means of tie lines that allowed waste to transfer from one tank to another as they filled. Because of this configuration, waste in tanks 241-B-201 and 241-T-201 was anticipated to be different in composition from others in the group but still identifiable as lanthanum fluoride (224) waste.

Taking advantage of this prior information, the sampling and analysis of the T-200 series tanks was based on one core. Comprehensive composition information was obtained from the core composites from each tank and compared to predicted mean estimates and 90 percent prediction intervals derived from the B-200 series tank data (Engel et al. 1997). The spatial variability of the waste in the T-200 series tanks was assumed to be that observed in the B-200 series tanks.

The results of the comparison were generally quite favorable. Prediction intervals for 17 analytes were compared with analytical results from the four T-200 tanks. Table 2-2 shows the results. $\mathrm{A}$ " 1 " indicates that the mean core composite result fell within the prediction interval. A " 0 " indicates that the mean core composite result fell outside the prediction interval.

Table 2-2. Comparisons of T-200 Analytical Results with Prediction Intervals. (2 sheets)

\begin{tabular}{|c|c|c|c|c|c|}
\hline 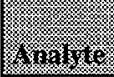 & 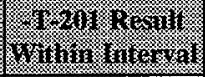 & 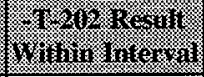 & (y) & 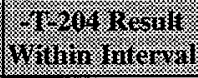 & 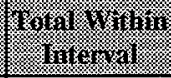 \\
\hline $\mathrm{Al}$ & 1 & 1 & 1 & 1 & $4 / 4$ \\
\hline $\mathrm{Bi}$ & 0 & 1 & 1 & 1 & $3 / 4$ \\
\hline $\mathrm{Ca}$ & 1 & 1 & 1 & 1 & $4 / 4$ \\
\hline $\mathrm{Cr}$ & 0 & 1 & 1 & 0 & $2 / 4$ \\
\hline${ }^{137} \mathrm{Cs}$ & 1 & 1 & 1 & 1 & $4 / 4$ \\
\hline $\mathrm{Fe}$ & 1 & 1 & 1 & 1 & $4 / 4$ \\
\hline$F$ & 1 & 1 & 1 & 1 & $4 / 4$ \\
\hline $\mathrm{H}_{2} \mathrm{O}$ & 1 & 1 & 1 & 1 & $4 / 4$ \\
\hline
\end{tabular}


Table 2-2. Comparisons of T-200 Analytical Results with Prediction Intervals. (2 sheets)

\begin{tabular}{|c|c|c|c|c|c|}
\hline 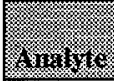 & 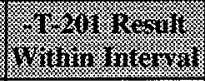 & f & 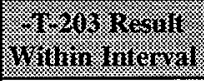 & 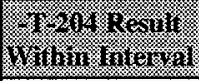 & 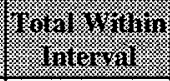 \\
\hline $\mathrm{La}$ & 0 & 1 & 1 & 1 & $3 / 4$ \\
\hline $\mathrm{Mn}$ & 0 & 1 & 1 & 1 & $3 / 4$ \\
\hline $\mathrm{Na}$ & 1 & 1 & 1 & 1 & $4 / 4$ \\
\hline $\mathrm{Ni}$ & 1 & 1 & 1 & 1 & $4 / 4$ \\
\hline $\mathrm{NO}_{3}$ & 1 & 1 & 1 & 1 & $4 / 4$ \\
\hline $\mathrm{PO}_{4}$ & 1 & 1 & 1 & 1 & $4 / 4$ \\
\hline $\mathrm{CO}_{3}$ & 1 & 1 & 1 & 1 & $4 / 4$ \\
\hline TOC & 1 & 1 & 1 & 1 & $4 / 4$ \\
\hline $\mathrm{U}$ & 1 & 1 & 1 & 1 & $4 / 4$ \\
\hline $\begin{array}{l}\text { Total } \\
\text { within } \\
\text { interval }\end{array}$ & $13 / 17$ & $17 / 17$ & $17 / 17$ & $16 / 17$ & $63 / 68$ \\
\hline $\begin{array}{l}\text { Percent } \\
\text { within } \\
\text { interval }\end{array}$ & 76.5 & 100 & 100 & 94.1 & 92.6 \\
\hline
\end{tabular}

In addition to having over 90 percent of the results fall within the prediction interval, 60 percent of the observations were within 50 percent of the predicted means, suggesting an identifiable process with distinct characteristics. Aluminum, calcium, and TOC concentrations in the B-200 series tanks were much higher than those observed in T-200 tanks, suggesting a difference attributable to each plant's operation. Bismuth, lanthanum, and manganese were observed to be higher in both tanks 241-B-201 and 241-T-201 than the other B-200 and T-200 tanks. Differences observed in tanks 241-B-201 and 241-T-201 as compared to the other B-200 and T-200 series tanks were anticipated because of the separate piping connection to their respective plants. 


\subsection{SUMMARY}

The results of all analyses performed to address potential safety issues showed that primary analyte(s) did not exceed safety decision threshold limits. The waste had little exothermic activity, had low total alpha concentration, no hazardous or flammable vapors were detected, and essentially no heat from radionuclide decay. The composition of the waste generally matched that expected from process history and the results from the B-200 series tanks. Table 2-3 summarizes the analyses results.

Table 2-3. Summary of Technical Issues.

\begin{tabular}{|c|c|c|}
\hline (2) & 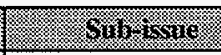 & Terantil \\
\hline \multirow[t]{3}{*}{$\begin{array}{l}\text { Safety } \\
\text { screening }\end{array}$} & Energetics & $\begin{array}{l}\text { No exotherms approaching or exceeding the threshold } \\
\text { value were observed in any sample. }\end{array}$ \\
\hline & Flammable gas & $\begin{array}{l}\text { Vapor measurement reported } 0 \text { percent of lower } \\
\text { flammability limit (combustible gas meter). }\end{array}$ \\
\hline & Criticality & All analyses well below $41 \mu \mathrm{Ci} / \mathrm{g}$ total alpha. \\
\hline \multirow{2}{*}{$\begin{array}{l}\text { Hazardous } \\
\text { vapor }\end{array}$} & Flammability & See safety screening - flammable gas \\
\hline & Toxicity & The toxicity issue has been closed for all tanks. ${ }^{1}$ \\
\hline \multirow{2}{*}{$\begin{array}{l}\text { Organic } \\
\text { Complexant }\end{array}$} & TOC & All measurements are less than $500 \mu \mathrm{g} / \mathrm{g}$ (wet). \\
\hline & Water content & All measurements are greater than 50 percent. \\
\hline $\begin{array}{l}\text { Organic } \\
\text { Solvents }\end{array}$ & $\begin{array}{l}\text { Total nonmethane } \\
\text { Hydrocarbons }\end{array}$ & No samples taken. \\
\hline
\end{tabular}

Note:

Hewitt (1966) 
HNF-1501 Rev. 0

This page intentionally left blank. 


\subsection{BEST-BASIS STANDARD INVENTORY ESTIMATE}

Information about the chemical and/or physical properties of tank wastes is used to perform safety analyses, engineering evaluations, and risk assessments associated with waste management activities, as well as to address regulatory issues. Waste management activities include overseeing tank farm operations and identifying, monitoring, and resolving safety issues associated with these operations and with the tank wastes. Disposal activities involve designing equipment, processes, and facilities for retrieving wastes and processing the wastes into a form that is suitable for long-term storage.

Chemical inventory information generally is derived using three approaches: 1) component inventories are estimated using the results of sample analyses, 2) component inventories are calculated using process knowledge and analytical information derived from related tanks, and 3) component inventories are predicted using a model based on process knowledge and historical information. The most recent model was developed by Los Alamos National Laboratory (Agnew et al. 1997). Information derived from these different approaches is inconsistent.

An effort is underway to provide waste inventory estimates that will serve as standard characterization information for the various waste management activities (Hodgson and LeClair 1996). Appendix D contains the complete narrative regarding the derivation of the inventory estimates shown in Tables 3-1 and 3-2.

Table 3-1. Best-Basis Inventory Estimates for Nonradioactive Components in T-200 Series Tanks (Effective May 31, 1997). (2 sheets)

\begin{tabular}{|c|c|c|c|c|c|c|}
\hline & & 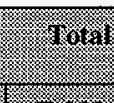 & yonis & & (3) & $\sqrt{4}$ \\
\hline Amates & I. 2 2) & 8202 & 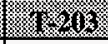 & 1.201 & . & Cominen: \\
\hline $\mathrm{Al}$ & 14.0 & 7.12 & 9.17 & 9.33 & $S$ & \\
\hline $\mathrm{Bi}$ & 16,600 & 4,040 & 7,940 & 8,960 & $S$ & \\
\hline $\mathrm{Ca}$ & 173 & 30.6 & 56.4 & 35.9 & $S$ & \\
\hline $\mathrm{Cl}$ & 151 & 68.3 & 107 & 117 & $S$ & \\
\hline $\mathrm{TIC}$ as $\mathrm{CO}_{3}$ & 564 & 1,025 & 1,290 & 1,220 & $\mathbf{S}$ & \\
\hline $\mathrm{Cr}$ & 746 & 371 & 618 & 781 & $S$ & \\
\hline $\mathrm{F}$ & 708 & 647 & 1,030 & 1,030 & $S$ & \\
\hline $\mathrm{Fe}$ & 1,380 & 751 & 1,110 & 703 & $S$ & \\
\hline $\mathrm{Hg}$ & 0 & 0 & 0 & 0 & M & \\
\hline $\mathrm{K}$ & 671 & 704 & 1,120 & 1,070 & $S$ & \\
\hline
\end{tabular}


Table 3-1. Best-Basis Inventory Estimates for Nonradioactive Components in T-200 Series Tanks (Effective May 31, 1997). (2 sheets)

\begin{tabular}{|c|c|c|c|c|c|c|}
\hline 等 & 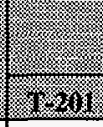 & 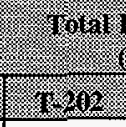 & 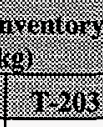 & 1.204 & 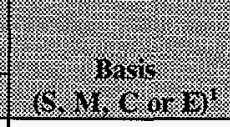 & : \\
\hline $\mathrm{La}$ & 3,470 & 1,240 & 1,910 & 2,000 & $\mathbf{S}$ & \\
\hline $\mathrm{Mn}$ & 6,180 & 1,460 & 2,620 & 2,450 & $\mathbf{S}$ & \\
\hline $\mathrm{Na}$ & 4,500 & 3,540 & 5,670 & 5,530 & $S$ & \\
\hline $\mathrm{Ni}$ & 87.8 & 13.0 & 24.1 & 42.0 & $S$ & \\
\hline $\mathrm{NO}_{2}$ & 43.8 & 51.7 & 48.1 & 49.4 & $S$ & \\
\hline $\mathrm{NO}_{3}$ & 6,730 & 6,470 & 10,500 & 9,610 & $S$ & \\
\hline $\mathrm{OH}$ & 14,600 & 3,640 & 6,690 & 6,640 & C & \\
\hline $\mathrm{Pb}$ & 29.6 & 5.72 & 3.39 & 54.0 & $S$ & \\
\hline $\mathrm{PO}_{4}$ & 1,940 & 721 & 1,190 & 1,310 & $S$ & \\
\hline $\mathrm{Si}$ & 259 & 184 & 261 & 261 & $S$ & \\
\hline $\mathrm{SO}_{4}$ & 38.6 & 109 & 71.4 & 63.2 & $s$ & \\
\hline $\mathrm{Sr}$ & 156 & 50.0 & 90.8 & 87.0 & $S$ & \\
\hline TOC & 42.4 & 34.2 & 68.4 & 54.3 & $S$ & \\
\hline $\mathrm{U}_{\text {TOTAL }}$ & 8.12 & 10.1 & 10.2 & 11.0 & $\mathrm{M}, \mathrm{S}, \mathrm{M}, \mathrm{M}$ & \\
\hline $\mathrm{Zr}$ & 0 & 0.2 & 0 & 0 & $S$ & \\
\hline
\end{tabular}

Note:

${ }^{1} \mathrm{~S}=$ Sample-based (see Appendix B), $\mathrm{M}=$ HDW model-based, $\mathrm{E}=$ Engineering assessment-based, and $\mathrm{C}=$ calculated by charge balance; includes oxides as hydroxides, not including $\mathrm{CO}_{3}, \mathrm{NO}_{2}, \mathrm{NO}_{3}, \mathrm{PO}_{4}, \mathrm{SO}_{4}$ and $\mathrm{SiO}_{3}$ 
Table 3-2. Best-Basis Inventory Estimate for Radioactive Components in T-200 Series Tanks Decayed to January 1, 1994 (Effective May 31, 1997). (2 sheets)

\begin{tabular}{|c|c|c|c|c|c|c|}
\hline & 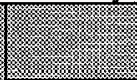 & 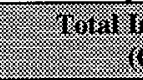 & Mernor & & & \\
\hline Arivatse & 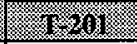 & $18.21 \%$ & 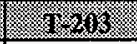 & 1.214 & 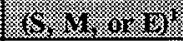 & Connmeri \\
\hline${ }^{3} \mathrm{H}$ & $2.86 \mathrm{E}+00$ & $2.02 \mathrm{E}+00$ & $3.34 \mathrm{E}+00$ & $3.57 \mathrm{E}+00$ & $\mathrm{E}$ & Based on B-201 \\
\hline${ }^{14} \mathrm{C}$ & $4.41 \mathrm{E}-02$ & $3.11 \mathrm{E}-02$ & $5.15 \mathrm{E}-02$ & $5.50 \mathrm{E}-02$ & E & Based on B-201 \\
\hline${ }^{59} \mathrm{Ni}$ & $9.56 \mathrm{E}-04$ & $6.76 \mathrm{E}-04$ & $1.12 \mathrm{E}-03$ & $1.19 \mathrm{E}-03$ & $E$ & Based on B-201 \\
\hline${ }^{60} \mathrm{Co}$ & 2.73E-01 & 1.93E-01 & $3.19 \mathrm{E}-01$ & 3.41E-01 & $\mathrm{E}$ & Based on B-201 \\
\hline${ }^{63} \mathrm{Ni}$ & 2.62E-02 & $1.85 \mathrm{E}-02$ & 3.06E-02 & $3.27 \mathrm{E}-02$ & E & Based on B-201 \\
\hline${ }^{79} \mathrm{Se}$ & $3.68 \mathrm{E}-05$ & $2.76 \mathrm{E}-05$ & $4.60 \mathrm{E}-05$ & $5.00 \mathrm{E}-05$ & $\mathrm{M}$ & \\
\hline${ }^{90} \mathrm{Sr}$ & $2.17 \mathrm{E}+01$ & $2.70 \mathrm{E}-01$ & $4.61 \mathrm{E}-01$ & $8.82 \mathrm{E}-01$ & $\mathrm{~S}$ & \\
\hline${ }^{90} \mathrm{Y}$ & $2.17 \mathrm{E}+01$ & $2.70 \mathrm{E}-01$ & $4.61 \mathrm{E}-01$ & 8.82E-01 & $\mathbf{S}$ & Based on ${ }^{90} \mathrm{Sr}$ \\
\hline${ }^{93 \mathrm{~m}} \mathrm{Nb}$ & $1.45 \mathrm{E}-04$ & $1.08 \mathrm{E}-04$ & 1.81E-04 & $1.96 \mathrm{E}-04$ & $\mathbf{M}$ & \\
\hline${ }^{93} \mathrm{Zr}$ & $1.75 \mathrm{E}-04$ & 1.31E-04 & $2.18 \mathrm{E}-04$ & 2.37E-04 & M & \\
\hline${ }^{99} \mathrm{Tc}$ & $1.21 \mathrm{E}-03$ & 9.09E-04 & 1.51E-03 & $1.64 \mathrm{E}-03$ & $\mathbf{M}$ & \\
\hline${ }^{106} \mathrm{Ru}$ & $4.20 \mathrm{E}-11$ & $3.15 \mathrm{E}-11$ & $5.25 \mathrm{E}-11$ & $5.70 \mathrm{E}-11$ & $\mathbf{M}$ & \\
\hline${ }^{113 m} \mathrm{Cd}$ & 4.89E-04 & $3.67 \mathrm{E}-04$ & 6.12E-04 & 6.64E-04 & $\mathbf{M}$ & \\
\hline${ }^{125} \mathrm{Sb}$ & $6.46 \mathrm{E}-05$ & $4.84 \mathrm{E}-05$ & $8.07 \mathrm{E}-05$ & $8.77 \mathrm{E}-05$ & $\mathbf{M}$ & \\
\hline${ }^{126} \mathrm{Sn}$ & $5.55 \mathrm{E}-05$ & $4.16 \mathrm{E}-05$ & $6.94 \mathrm{E}-05$ & $7.53 \mathrm{E}-05$ & $\mathbf{M}$ & \\
\hline${ }^{129} \mathbf{I}$ & 2.29E-06 & 1.72E-06 & $2.86 \mathrm{E}-06$ & $3.10 \mathrm{E}-06$ & $\mathbf{M}$ & \\
\hline${ }^{134} \mathrm{Cs}$ & $3.32 \mathrm{E}-01$ & $2.35 \mathrm{E}-01$ & $3.88 \mathrm{E}-01$ & $4.14 \mathrm{E}-01$ & $E$ & Based on B-201 \\
\hline${ }^{137 \mathrm{~m}} \mathrm{Ba}$ & $6.60 \mathrm{E}+00$ & $2.88 \mathrm{E}+00$ & $3.05 E+00$ & $1.40 \mathrm{E}+00$ & $\mathbf{S}$ & Based on ${ }^{137} \mathrm{Cs}$ \\
\hline${ }^{137} \mathrm{Cs}$ & $6.98 \mathrm{E}+00$ & $3.04 \mathrm{E}+00$ & $3.22 \mathrm{E}+00$ & $1.48 \mathrm{E}+00$ & $S$ & \\
\hline${ }^{151} \mathrm{Sm}$ & 0.139 & 0.104 & 0.174 & 0.189 & $\mathbf{M}$ & \\
\hline${ }^{152} \mathrm{Eu}$ & $1.82 \mathrm{E}-04$ & 1.37E-04 & $2.28 \mathrm{E}-04$ & $2.47 \mathrm{E}-04$ & $\mathrm{M}$ & \\
\hline${ }^{154} \mathrm{Eu}$ & 6.11E-01 & 4.32E- 01 & 7.14E-01 & 7.62E-01 & $E$ & Based on B-201 \\
\hline${ }^{155} \mathrm{Eu}$ & $4.57 \mathrm{E}-01$ & 3.23E-01 & 5.34E-01 & 5.71E-01 & $E$ & Based on B-201 \\
\hline${ }^{226} \mathrm{Ra}$ & $8.22 \mathrm{E}-09$ & $6.16 \mathrm{E}-09$ & 1.03E-08 & 1.12E-08 & $M$ & \\
\hline${ }^{227} \mathrm{Ac}$ & 4.34E-08 & 3.25E-08 & 5.42E-08 & $5.89 \mathrm{E}-08$ & $M$ & \\
\hline${ }^{228} \mathrm{Ra}$ & $5.28 \mathrm{E}-13$ & $3.96 \mathrm{E}-13$ & $6.61 \mathrm{E}-13$ & $7.17 \mathrm{E}-13$ & $\mathbf{M}$ & \\
\hline${ }^{229} \mathrm{Th}$ & $1.02 \mathrm{E}-10$ & $7.67 \mathrm{E}-11$ & $1.28 \mathrm{E}-10$ & $1.39 \mathrm{E}-10$ & M & \\
\hline${ }^{231} \mathrm{~Pa}$ & $1.00 \mathrm{E}-07$ & $7.51 \mathrm{E}-08$ & $1.25 \mathrm{E}-07$ & $1.36 \mathrm{E}-07$ & $\mathrm{M}$ & \\
\hline${ }^{232} \mathrm{Th}$ & $4.62 \mathrm{E}-14$ & $3.46 \mathrm{E}-14$ & $5.77 \mathrm{E}-14$ & $6.27 \mathrm{E}-14$ & $M$ & \\
\hline
\end{tabular}


Table 3-2. Best-Basis Inventory Estimate for Radioactive Components in T-200 Series Tanks Decayed to January 1, 1994 (Effective May 31, 1997). (2 sheets)

\begin{tabular}{|c|c|c|c|c|c|c|}
\hline & & 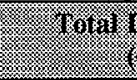 & 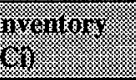 & & & \& \\
\hline anduse & 8,261 & (x) & $\sqrt{1}(1627)$ & 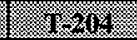 & 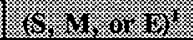 & Cranudin. \\
\hline${ }^{232} \mathrm{U}$ & $5.36 \mathrm{E}-08$ & 4.02E-08 & $6.70 \mathrm{E}-08$ & 7.27E-08 & $\mathbf{M}$ & \\
\hline${ }^{233} \mathrm{U}$ & $2.45 \mathrm{E}-09$ & $1.83 \mathrm{E}-09$ & $3.06 \mathrm{E}-09$ & 3.32E-09 & $\mathbf{M}$ & \\
\hline${ }^{234} \mathrm{U}$ & $2.67 \mathrm{E}-03$ & $2.00 \mathrm{E}-03$ & 3.34E-03 & $3.63 \mathrm{E}-03$ & $\mathbf{M}$ & \\
\hline${ }^{235} \mathrm{U}$ & $1.19 \mathrm{E}-04$ & $8.92 \mathrm{E}-05$ & $1.49 \mathrm{E}-04$ & 1.61E-04 & $\mathbf{M}$ & \\
\hline${ }^{236} \mathrm{U}$ & $2.33 \mathrm{E}-05$ & $1.75 \mathrm{E}-05$ & $2.91 \mathrm{E}-05$ & $3.16 \mathrm{E}-05$ & $\mathrm{M}$ & \\
\hline${ }^{237} \mathrm{~Np}$ & $7.51 \mathrm{E}-06$ & $5.63 \mathrm{E}-06$ & $9.39 \mathrm{E}-06$ & $1.02 \mathrm{E}-05$ & $\mathbf{M}$ & \\
\hline${ }^{238} \mathrm{Pu}$ & $4.85 \mathrm{E}-01$ & $3.43 \mathrm{E}-01$ & $5.67 \mathrm{E}-01$ & $6.06 \mathrm{E}-01$ & $\mathrm{E}$ & Based on B-201 \\
\hline${ }^{238} \mathrm{U}$ & $2.71 \mathrm{E}-03$ & $3.30 \mathrm{E}-03$ & $3.39 \mathrm{E}-03$ & $3.68 \mathrm{E}-03$ & $\mathbf{M}, \mathbf{S}, \mathbf{M}, \mathbf{M}$ & \\
\hline${ }^{239} \mathrm{Pu}$ & $9.96 \mathrm{E}+01$ & $2.07 \mathrm{E}+01$ & $3.05 E+01$ & $2.36 \mathrm{E}+01$ & $\mathrm{~S} / \mathrm{E}$ & Based on Alpha ${ }^{2}$ \\
\hline${ }^{240} \mathrm{Pu}$ & $6.40 \mathrm{E}+00$ & $1.30 \mathrm{E}+00$ & $1.40 \mathrm{E}+00$ & $1.50 \mathrm{E}+00$ & E & Based on Alpha ${ }^{2}$ \\
\hline${ }^{241} \mathrm{Am}$ & $4.32 \mathrm{E}+00$ & $3.06 \mathrm{E}+00$ & $5.85 \mathrm{E}+00$ & $4.25 \mathrm{E}+00$ & $E, E, S, S$ & $\begin{array}{l}\text { Engineering } \\
\text { based on B-201 }\end{array}$ \\
\hline${ }^{241} \mathrm{Pu}$ & $1.35 \mathrm{E}-02$ & $1.01 \mathrm{E}-02$ & $1.68 \mathrm{E}-02$ & 1.83E-02 & $\mathbf{M}$ & \\
\hline${ }^{222} \mathrm{Cm}$ & $3.70 \mathrm{E}-06$ & $2.78 \mathrm{E}-06$ & $4.63 \mathrm{E}-06$ & $5.03 \mathrm{E}-06$ & $\mathbf{M}$ & \\
\hline${ }^{242} \mathrm{Pu}$ & $6.23 \mathrm{E}-08$ & $4.67 \mathrm{E}-08$ & 7.79E-08 & 8.45E-08 & $\mathbf{M}$ & \\
\hline${ }^{243} \mathrm{Am}$ & $3.08 \mathrm{E}-09$ & 2.31E-09 & $3.86 \mathrm{E}-09$ & 4.19E-09 & $\mathbf{M}$ & \\
\hline${ }^{243} \mathrm{Cm}$ & $7.98 \mathrm{E}-08$ & 5.99E-08 & $9.98 \mathrm{E}-08$ & $1.08 \mathrm{E}-07$ & $\mathrm{M}$ & \\
\hline${ }^{244} \mathrm{Cm}$ & 7.84E-08 & $5.88 \mathrm{E}-08$ & $9.80 \mathrm{E}-08$ & $1.06 \mathrm{E}-07$ & $\mathbf{M}$ & \\
\hline
\end{tabular}

Notes:

${ }^{1} \mathbf{S}=$ sample-based, $\mathbf{M}=\mathrm{HDW}$ model-based, and $\mathbf{E}=$ engineering assessment-based

${ }^{2}$ Assumed total alpha was plutonium-based, and the ratio of ${ }^{239} \mathrm{Pu}$ to ${ }^{240} \mathrm{Pu}$ was 94 percent to 6 percent. The other plutonium contributors estimated by the HDW model fell within the uncertainty of the measurement. 


\subsection{RECOMMENDATIONS}

All analytical results for the safety screening DQO were far below safety notification limits. No hazardous or flammable vapors were detected. The sampling and analysis activities performed for the T-200 tank series (241-T-201, T-202, T-203, and T-204) are considered to have met all program requirements. The organic complexant, organic solvent, and flammable gas safety issues are expected to be closed in Fiscal Year 1998 as described in Milestone M-40-09. The Project Hanford Management Contractor (PHMC) TWRS Safety Program has determined that additional sampling is not required to close these issues for these tanks.

Table 4-1 summarizes the PHMC TWRS Program review status and acceptance of the sampling and analysis results reported in this TCR. All DQO issues required to be addressed by sampling and analysis are listed in column 1 of Table $4-1$. Column 2 indicates by "yes" or "no" whether the DQO requirements were met by the sampling and analysis activities performed. Column 3 indicates concurrence and acceptance by the program in PHMC TWRS that is responsible for the DQO that the sampling and analysis activities performed adequately meet the needs of the DQO. A "yes" or "no" in column 3 indicates acceptance or disapproval of the sampling and analysis information in the TCR. The waste was sampled and analyzed in accordance with the safety screening DQO and accepted by the responsible TWRS program.

Table 4-1. Acceptance of Tanks 241-T-201, T-202, T-203, and T-204 Sampling and Analysis.

\begin{tabular}{|c|c|c|}
\hline (1) & 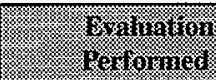 & 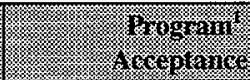 \\
\hline Safety screening DQO & Yes & Yes \\
\hline Hazardous vapor screening $D Q O$ & Yes & Yes \\
\hline Organic complexant MOU & Yes & Yes \\
\hline Organic solvent DQO & Yes $^{2}$ & Yes $^{2}$ \\
\hline
\end{tabular}

Notes:

MOU $=$ Memorandum of Understanding

${ }^{1}$ PHMC TWRS Program Office

${ }^{2}$ Documentation regarding the resolution of the organic solvent issue is pending. No further sampling in support of this issue is currently scheduled. 
Table 4-2 summarizes the status of PHMC TWRS Program review and acceptance of the evaluations and other characterization information contained in this report. The evaluations outlined in this report include those to determine whether the tank is safe, conditionally safe, or unsafe; and the best-basis inventory evaluation. Column 1 lists the different evaluations performed in this report. Columns 2 and 3 are in the same format as Table 4-1. The manner in which concurrence and acceptance are summarized is also the same as that in Table 4-1.

The safety categorization of the T-200 series tanks is listed as "safe" in Table 4-2, even though two cores were not obtained from each tank. The data from the B-200 series tanks used in interpreting the T-200 information, the available process information, and the consistency of the data between T-200 tanks did not indicate any safety problems and were considered sufficient by the PHMC TWRS Program Office to meet the intent of the safety screening DQO.

One final comment regarding the safety screening DQO needs to be made. The one-sided confidence intervals that were used to determine whether or not ${ }^{239} \mathrm{Pu}$ is below the DQO stated threshold limit were performed solely on each individual sample as required by the DQO.

Table 4-2. Acceptance of Evaluation of Characterization Data and Information for T-200 Series Tanks.

\begin{tabular}{|c|c|c|}
\hline$\sqrt{2} \sqrt{1258}$ & 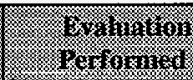 & 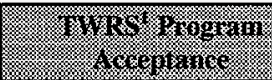 \\
\hline Safety categorization: SAFE & Yes & Yes \\
\hline
\end{tabular}

Note:

${ }^{1}$ PHMC TWRS Program Office 


\subsection{REFERENCES}

Agnew, S. F., J. Boyer, R. A. Corbin, T. B. Duran, J. R. Fitzpatrick, K. A. Jurgensen, T. P. Ortiz, and B. L. Young, 1997, Hanford Tank Chemical and Radionuclide Inventories: HDW Model Rev. 4, LA-UR-96-3860, Rev. 0, Los Alamos National Laboratory, Los Alamos, New Mexico.

Bell, K. E., 1997, Tank 241-T-202 Push Mode Core Sampling and Analysis Plan, HNF-SD-WM-TSAP-121, Rev. 0, Lockheed Martin Hanford Corp. for Fluor Daniel Hanford, Inc., Richland, Washington.

Brevick, C. H., J. L. Stroup, J. W. Funk, 1997, Historical Tank Content Estimate for the Northwest Quadrant of the Hanford 200 West Area, HNF-SD-WM-ER-351, Rev. 1, Fluor Daniel Northwest Inc. for Fluor Daniel Hanford, Inc., Richland, Washington.

Brown, T. M., J. W. Hunt, and L. J. Fergestrom, 1997, Tank Characterization Technical Sampling.Basis, HNF-SD-WM-TA-164, Rev. 3, Lockheed Martin Hanford Corp. for Fluor Daniel Hanford, Inc., Richland, Washington.

Dukelow, G. T., J. W. Hunt, H. Babad, and J. E. Meacham, 1995, Tank Safety Screening Data Quality Objective, WHC-SD-WM-SP-004, Rev. 2, Westinghouse Hanford Company, Richland, Washington.

Ecology, EPA, and DOE, 1997, Hanford Federal Facility Agreement and Consent Order, as amended, Washington State Department of Ecology, U.S. Environmental Protection Agency, and U.S. Department of Energy, Olympia, Washington.

Engel, D. W., T. A. Ferryman, K. M. Remund, D. S. Daly, G. Chen, S. A. Hartley, and B. C. Simpson, 1997, T-200 Series Tank Concentration Predictions, PNNL-11550, Pacific Northwest National Laboratory, Richland, Washington.

Esch, R. A., 1997, Tank 241-T-202, Core 191, Analytical Results for the Final Report, HNF-SD-WM-DP-253, Rev. 0, Waste Management of Hanford Inc., Richland, Washington.

Kummerer, M., 1995, Topical Report on Heat Removal Characteristics of Waste Storage Tanks, WHC-SD-WM-SARR-010, Rev. 1, Westinghouse Hanford Company, Richland, Washington.

Hanlon, B. M., 1997, Waste Tank Summary Report for Month Ending July, 31, 1997, HNF-EP-0182-112, Lockheed Martin Hanford Corp. for Fluor Daniel Hanford, Inc., Richland, Washington. 
Hewitt, E. R., 1996, Tank Waste Remediation System Resolution of Potentially Hazardous Vapor Issues, WHC-SD-TWR-RPT-001, Rev. 0, Westinghouse Hanford Company, Richland, Washington.

Hodgson, K. M., and M. D. LeClair, 1996, Work Plan for Defining a Standard Inventory Estimate for Wastes Stored in Hanford Site Underground Tanks, WHC-SD-WM-WP-311, Rev. 1, Westinghouse Hanford Company, Richland, Washington.

Hu, T. A., 1997, Tank 241-T-201 Push Mode Core Sampling and Analysis Plan, HNF-SD-WM-TSAP-130, Rev. 0, Lockheed Martin Hanford Corp. for Fluor Daniel Hanford, Inc, , Richland, Washington.

Meacham, J. E., D. L. Banning, M. R. Allen, and L. D. Muhlestein, 1997, Data Quality Objective to Support Resolution of the Organic Solvent Safety Issue, HNF-SD-WM-DQO-026, Rev. 0, Duke Engineering \& Services, Inc. for Fluor Daniel Hanford, Inc., Richland, Washington.

Nuzum, J. L., 1997a, Tank 241-T-201, Core 192, Analytical Results for the Final Report, HNF-SD-WM-DP-254, Rev. 0, Waste Management of Hanford Inc. for Fluor Daniel Hanford, Inc., Richland, Washington.

Nuzum, J. L. , 1997b, Tank 241-T-204, Core 188, Analytical Results for the Final Report, HNF-SD-WM-DP-255, Rev. 0, Waste Management of Hanford Inc. for Fluor Daniel Hanford, Inc., Richland, Washington.

Osborne, J. W., and L. L. Buckley, 1995, Data Quality Objectives for Tank Hazardous Vapor Safety Screening, WHC-SD-WM-DQO-002, Rev. 2, Westinghouse Hanford Company, Richland, Washington.

Public Law 101-510, 1990, "Safety Measures for Waste Tanks at Hanford Nuclear Reservation," Section 3137 of the National Defense Authorization Act for Fiscal Year 1991.

Schreiber, R. D. 1997a, “Meeting Minutes, Tank Data Review Committee,” September 22, Lockheed Martin Hanford Corp. for Fluor Daniel Hanford Inc., Richland, Washington.

Schreiber, R. D., 1997b, Memorandum of Understanding for the Organic Complexant Safety Issue Data Requirements, HNF-SD-WM-RD-060, Rev. 0, Lockheed Martin Hanford Corp. for Fluor Daniel Hanford, Inc., Richland, Washington.

Schreiber, R. D., 1997c, Tank 241-T-203 Push Mode Core Sampling and Analysis Plan, WHC-SD-WM-TSAP-118, Rev. 0A, Lockheed Martin Hanford Corp. for Fluor Daniel Hanford, Inc., Richland, Washington. 
Smith, D. A., 1986, Single-Shell Tank Isolation Safety Analysis Report, WHC-SD-WM-SAR-006, Rev. 2, Westinghouse Hanford Company, Richland, Washington.

Steen, F. H. , 1997, Tank 241-T-203, Core 190, Analytical Results for the Final Report, HNF-SD-WM-DP-247, Rev. 0, Waste Management of Hanford, Inc. for Fluor Daniel Hanford, Inc., Richland, Washington.

Winkleman, W. D., 1997, Tank 241-T-204 Push Core Sampling and Analysis Plan, HNF-SD-WM-TSAP-119, Rev. 0, Lockheed Martin Hanford Corp. for Fluor Daniel Hanford, Inc., Richland, Washington. 
HNF-1501 Rev. 0

This page intentionally left blank. 
HNF-1501 Rev. 0

APPENDIX A

HISTORICAL TANK INFORMATION

A-1 
HNF-1501 Rev. 0

This page intentionally left blank. 


\section{APPENDIX A \\ HISTORICAL TANK INFORMATION}

Appendix A describes the T-200 series tanks (241-T-201, -T-202, -T-203, and -T-204) based on historical information. For this report, historical information includes information about the fill history, waste types, surveillance, or modeling data about the tanks. This information is necessary for providing a balanced, comprehensive assessment of the sampling and analytical results.

This appendix contains the following information:

- Section A1.0: Current tank status, including the current waste levels and the tank stabilization and isolation status

- Section A2.0: Information about the tank design

- Section A3.0: Process knowledge about the tank, the waste transfer history, and the estimated contents of the tank based on modeling data

- Section A4.0: Surveillance data for the T-200 series tanks including surface-level readings, temperatures, and a description of the waste surface based on photographs

- Section A5.0: Appendix A References

\section{A1.0 CURRENT TANK STATUS}

As of July 31, 1997, tanks 241-T-201, -T-202, -T-203, and -T-204 contained an estimated $110,79,132$, and $144 \mathrm{~kL}(29,21,35$, and $38 \mathrm{kgal})$, respectively of noncomplexed waste (Hanlon 1997). The waste volumes were estimated using a manual tape surface-level gauge or a combination of manual tape, photographic evaluation, and sludge measurement devices. Table A1-1 shows the estimated volumes of the waste phases found in the tank. The solids volume for each tank was last updated in 1978 or 1981.

All tanks are sound and were declared inactive in 1976. Pumping to remove liquids started in 1977. The tanks were finally interim stabilized in 1981; intrusion prevention (interim isolation) was also completed in 1981 . The final stabilization designation was made administratively; therefore a small amount of liquid remained in tank 241-T-201. These tanks are passively ventilated, and none are on the Watch List (Public Law 101-510). 
Table A1-1. Tank Contents Status Summary.

\begin{tabular}{|c|c|c|c|c|}
\hline 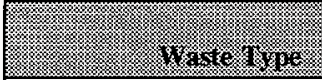 & 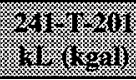 & 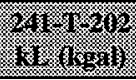 & 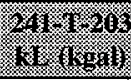 & 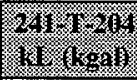 \\
\hline Total waste & $110(29)$ & $79(21)$ & $132(35)$ & $144(38)$ \\
\hline Supernatant & $3.8(1)$ & $0(0)$ & $0(0)$ & $0(0)$ \\
\hline Sludge & $106(28)$ & $79(21)$ & $132(35)$ & $144(38)$ \\
\hline Saltcake & $0(0)$ & $0(0)$ & $0(0)$ & $0(0)$ \\
\hline Drainable interstitial liquid & $11(3)$ & $8(2)$ & $15(4)$ & $15(4)$ \\
\hline Drainable liquid remaining & $15(4)$ & $8(2)$ & $15(4)$ & $15(4)$ \\
\hline Pumpable liquid remaining & $0(0)$ & $0(0)$ & $0(0)$ & $0(0)$ \\
\hline
\end{tabular}

\section{A2.0 TANK DESIGN AND BACKGROUND}

The 241-T Tank Farm is a first generation tank farm. Built between 1943 and 1944, it consists of twelve $2,010 \mathrm{~kL}(530 \mathrm{kgal})$ tanks(241-T-101 to $-\mathrm{T}-112)$ and four $208 \mathrm{~kL}$ (55 kgal) tanks (241-T-201 to -T-204). The tanks were designed for nonboiling waste with a maximum fluid temperature of $104{ }^{\circ} \mathrm{C}\left(220^{\circ} \mathrm{F}\right)$. Equipment to monitor and access the waste is sparse. A typical T Farm 200 series tank contains several risers that provide surface level access to the underground tank.

The T-200 series tanks are constructed of $0.3-\mathrm{m}(1-\mathrm{ft})$ thick reinforced concrete with a $0.64-\mathrm{cm}(0.25$-in.) thick mild carbon steel liner on the bottom and sides and a $30-\mathrm{cm}(12-\mathrm{in}$.) thick flat concrete top (Brevick et al. 1997a). The carbon steel liner has a $7.49 \mathrm{~m}$ ( $24.6 \mathrm{ft}$ )

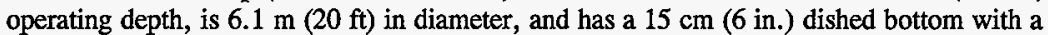

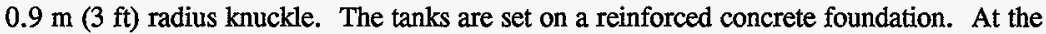
time of construction, the tanks were waterproofed on the sides with tar and a cement-like mixture. The tanks are covered with approximately $3.5 \mathrm{~m}(11.5 \mathrm{ft})$ of overburden. The T-200 series tanks have 8 risers. Usually two to three risers are available for intrusive tank activities. The risers range in diameter from $10 \mathrm{~cm}(4 \mathrm{in}$.) to $30 \mathrm{~cm}$ (12 in.). 
The four T-200 series tanks are at roughly the same elevation. Tank $241-\mathrm{T}-201$ is piped separately from the other $\mathrm{T}-200$ series tanks and received waste directly from $\mathrm{T}$ Plant. Tanks 241-T-204, T-203, and T-202 are connected to each other by a 7.6-cm (3-in.)-diameter line that enables waste transfers from one tank to the other. Because there is no vertical offset in the connection from one tank to the others, the tanks are not cascaded but rather tied together.

Figure A2-1 shows the riser configuration. Table A2-1 lists T-200 series tank risers, their diameters, and a brief description. Risers 3 and 7 (30.5 cm [12 in.]in diameter) are available for use in all of the T-200 series tanks. Riser $8(10 \mathrm{~cm} \mathrm{[4} \mathrm{in.]} \mathrm{in} \mathrm{diameter)} \mathrm{is} \mathrm{available} \mathrm{for} \mathrm{use}$ in tanks 241-T-201, -T-202, and -T-203. Figures A2-2, A2-3, A2-4, and A2-5 shows a cross section of each tank with an approximate waste level and a schematic of the tank equipment. 
Figure A2-1. Generic Riser Configuration for T-200 Series Tanks.
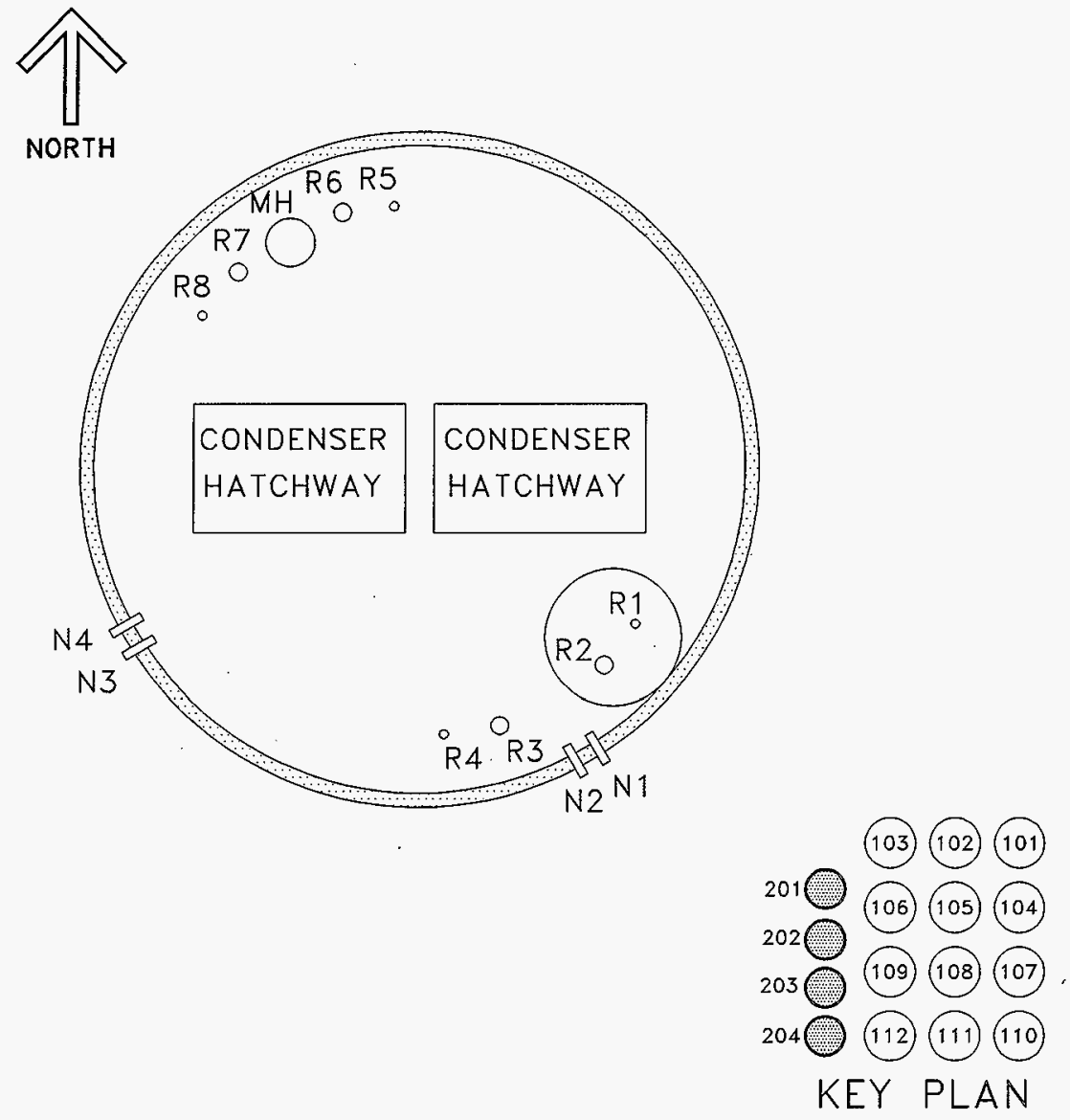
Table A2-1. Tank 241-T-200 Series Tanks Risers and Nozzles. ${ }^{1}$ (2 sheets)

\begin{tabular}{|c|c|c|c|}
\hline Hing & 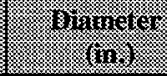 & (3anis & II. \\
\hline 1 & 4 & all & Not used; weather covered \\
\hline 2 & 12 & all & Salt well; weather covered \\
\hline \multirow[b]{2}{*}{$3^{2}$} & \multirow[b]{2}{*}{12} & $\mathrm{~T}-201$ & Not used; available for sampling \\
\hline & & $\begin{array}{l}\text { T-202 } \\
\text { T-203 } \\
T-204\end{array}$ & Blind flange; available for sampling \\
\hline 4 & 4 & all & Level gauge \\
\hline \multirow[t]{2}{*}{5} & \multirow[t]{2}{*}{4} & $\begin{array}{l}\text { T-201 } \\
\text { T-202 } \\
\text { T-203 }\end{array}$ & Thermocouple \\
\hline & & $\mathrm{T}-204$ & Blind flange \\
\hline 6 & 12 & all & Air filter \\
\hline $7^{2}$ & 12 & all & B-222 Observation port \\
\hline \multirow[t]{2}{*}{$8^{2}$} & \multirow[t]{2}{*}{4} & $\begin{array}{l}\mathrm{T}-201 \\
\mathrm{~T}-202 \\
\mathrm{~T}-203\end{array}$ & Thermocouple \\
\hline & & $\mathrm{T}-204$ & Blind flange \\
\hline \multirow{4}{*}{$\mathrm{N} 1$} & \multirow{4}{*}{3} & $\mathrm{~T}-201$ & Line $\mathrm{V}-711$ blanked in diversion box $241-\mathrm{T}-252$ \\
\hline & & $\mathrm{T}-202$ & Line V-713 blanked in diversion box $241-\mathrm{T}-252$ \\
\hline & & $\mathrm{T}-203$ & Line V-715 blanked in diversion box $241-\mathrm{T}-252$ \\
\hline & & $\mathrm{T}-204$ & Line V-717 blanked in diversion box $241-\mathrm{T}-252$ \\
\hline \multirow{4}{*}{ N2 } & \multirow{4}{*}{3} & $\mathrm{~T}-201$ & Line V-712 blanked in diversion box $241-T-252$ \\
\hline & & $\mathrm{T}-202$ & Line V-714 blanked in diversion box $241-\mathrm{T}-252$ \\
\hline & & $\mathrm{T}-203$ & Line $\mathrm{V}-716$ blanked in diversion box $241-\mathrm{T}-252$ \\
\hline & & T-204 & Line V-718 blanked in diversion box $241-T-252$ \\
\hline
\end{tabular}


Table A2-1. Tank 241-T-200 Series Tanks Risers and Nozzles. ${ }^{1}$ (2 sheets)

\begin{tabular}{|c|c|c|c|}
\hline (minger & mineres: & tanin & 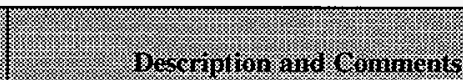 \\
\hline \multirow{3}{*}{ N3 } & \multirow{3}{*}{3} & $\mathrm{~T}-201$ & Not used; capped \\
\hline & & T-202 & Inlet \\
\hline & & T-204 & Not used \\
\hline \multirow{3}{*}{ N4 } & \multirow{3}{*}{3} & $\mathrm{~T}-201$ & Line to sump $216-\mathrm{T}-32$ \\
\hline & & T-202 & Overflow \\
\hline & & T-204 & Inlet \\
\hline
\end{tabular}

Notes:

${ }^{1}$ Brevick et al. (1997a), Alstad (1993), Lipnicki (1997), and Tran (1993)

${ }^{2}$ Denotes riser tentatively available for sampling. Applies to all T-200 series tanks except for riser 8, which is not tentatively available for sampling for tank 241-T-204. 
Figure A2-2. Tank 241-T-201 Cross Section and Schematic.

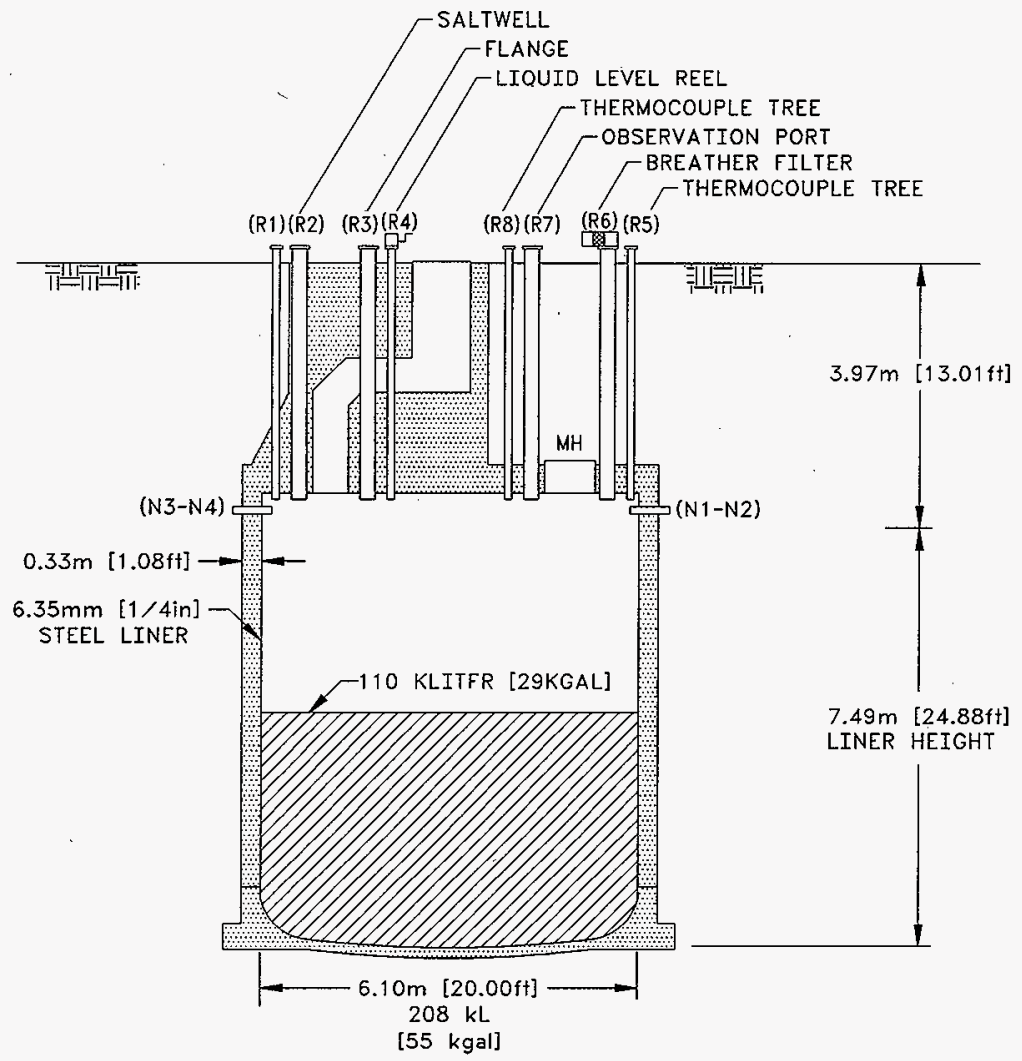


Figure A2-3. Tank 241-T-202 Cross Section and Schematic.

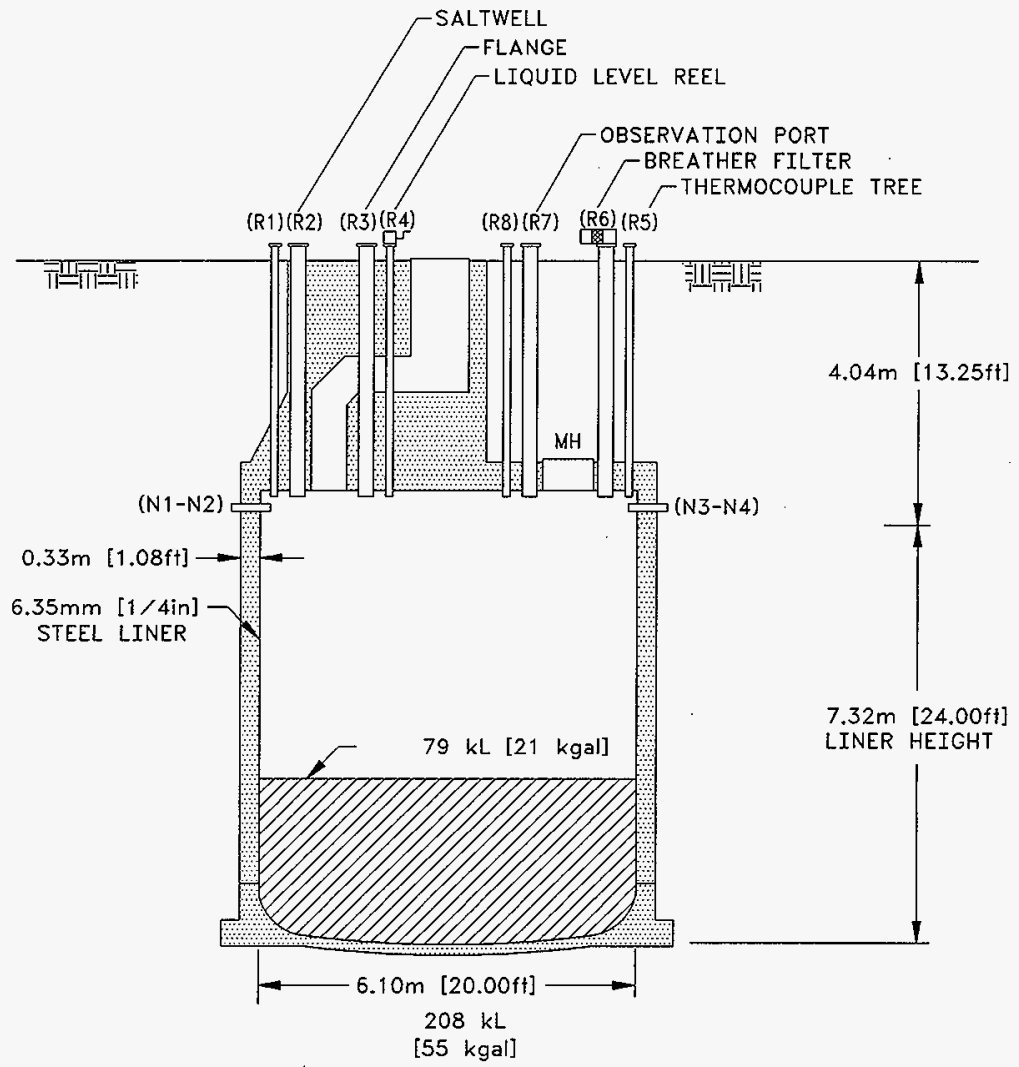


Figure A2-4. Tank 241-T-203 Cross Section and Schematic.

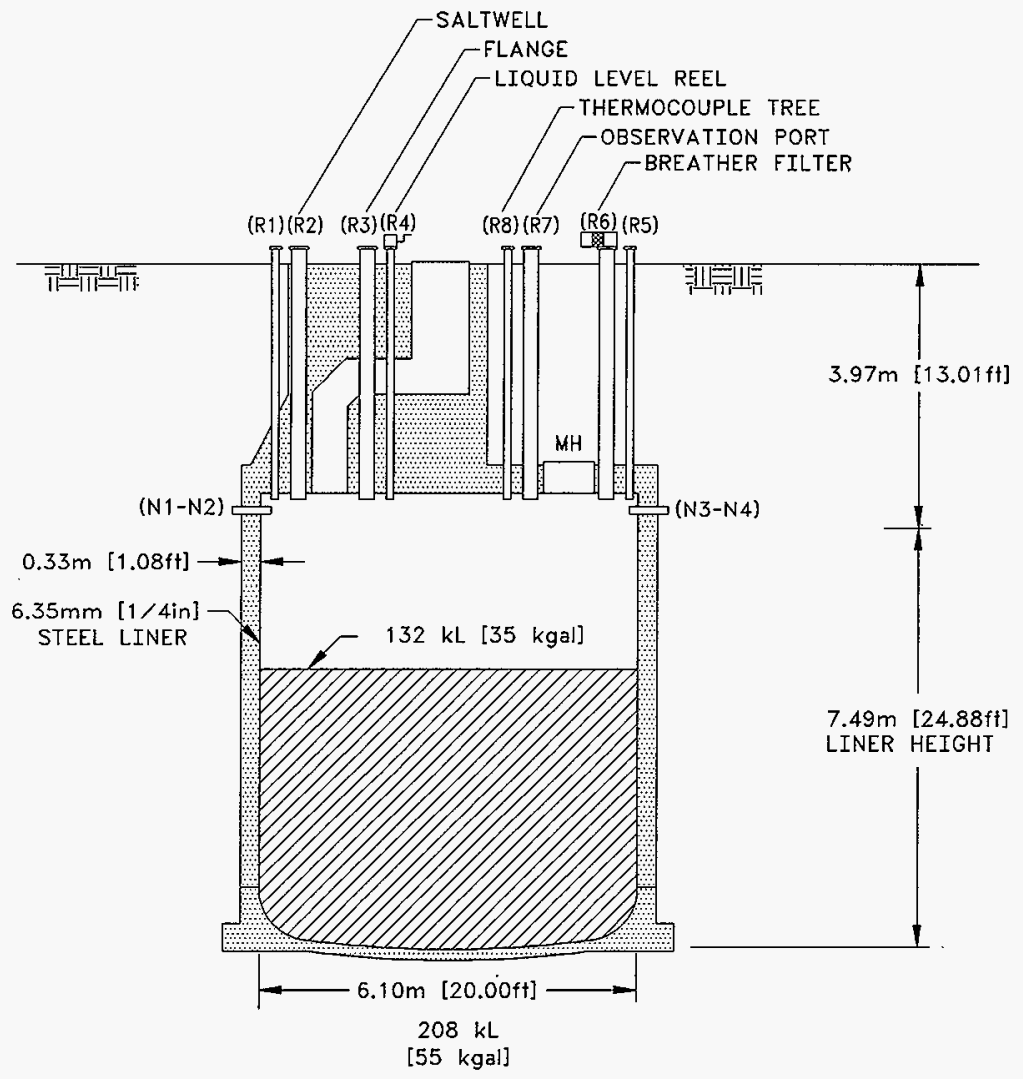


Figure A2-5. Tank 241-T-204 Cross Section and Schematic.

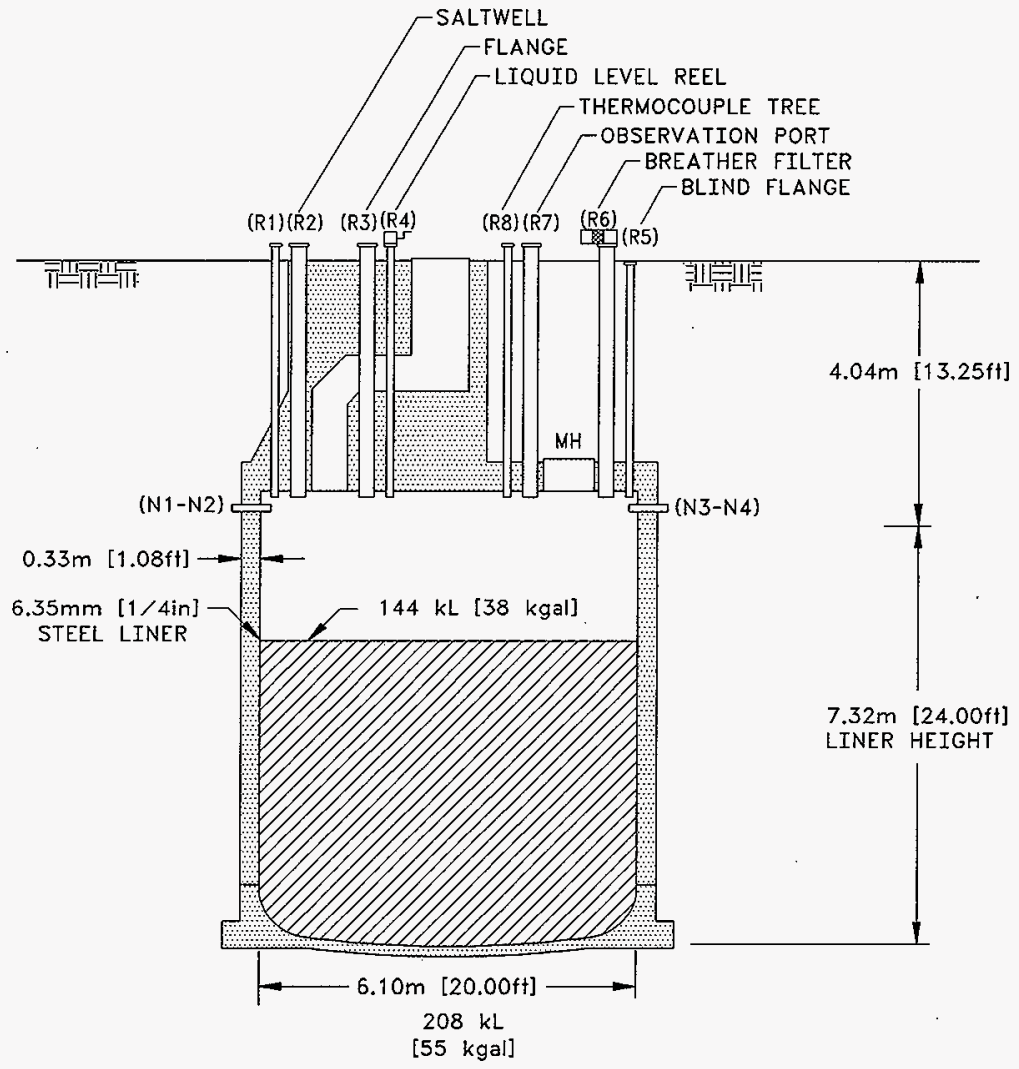




\section{A3.0 PROCESS KNOWLEDGE}

The sections below provide information about the transfer history of the T-200 series tanks, describe the process wastes that made up the transfers, and estimate the current tank contents based on transfer history.

\section{A3.1 WASTE TRANSFER HISTORY}

Table A3-1 summarizes the waste transfer history of tanks 241-T-201 to -T-204.

Tanks 241-T-201, -T-202, -T-203, and -T-204 are located in the 200 West Area T Tank Farm on the Hanford Site. Tank 241-T-201 is not part of any tank cascade. Tanks 241-T-202, -T-203, and -T-204 are not cascaded but are connected together by tie lines The tanks went into service in 1952, receiving lanthanum fluoride (224) waste from T-Plant. The tanks were filled later that year. The only other transfers of waste associated with these tanks occurred in 1976,1977 , and 1981 when liquids were pumped from the tank in support of stabilization efforts.

Table A3-1. Tank 241-T-201, -T-202, -T-203, and -T-204 Major Transfers. ${ }^{1}$ (2 sheets)

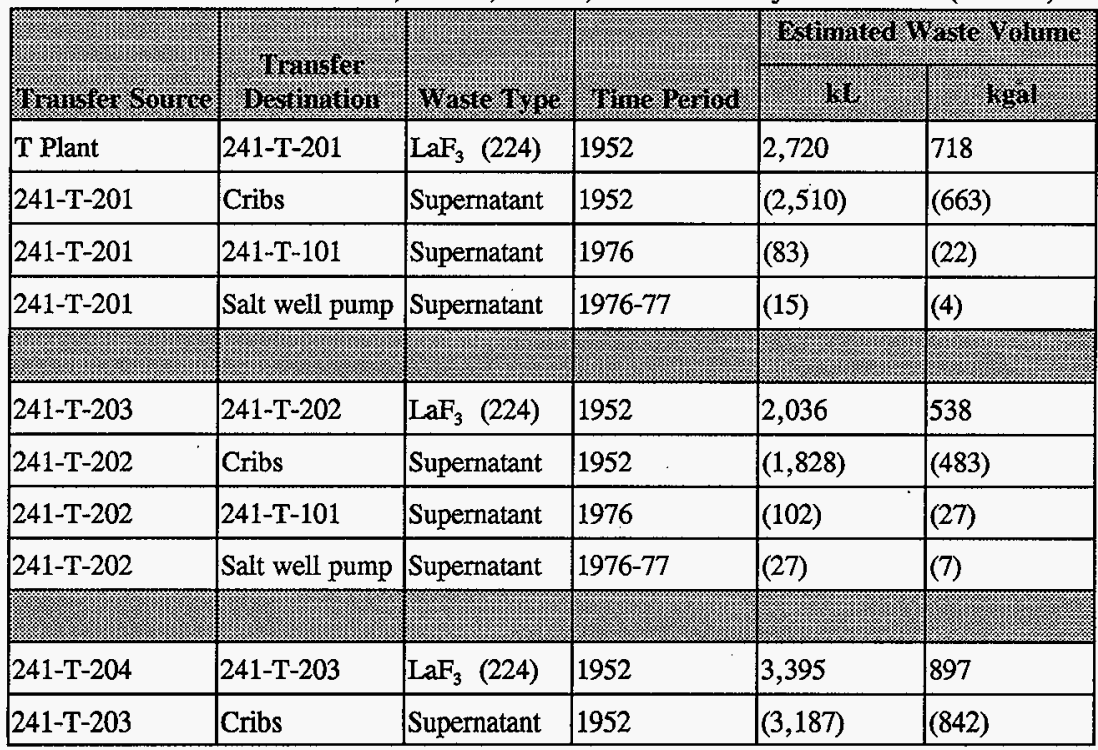


Table A3-1. Tank 241-T-201, -T-202, -T-203, and -T-204 Major Transfers. ${ }^{1}$ (2 sheets)

\begin{tabular}{|c|c|c|c|c|c|}
\hline flom & Maln & Hor & 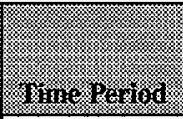 & $\frac{6}{4}$ & 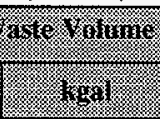 \\
\hline $241-T-203$ & $241-\mathrm{T}-101$ & Supernatant & 1976 & (34) & (9) \\
\hline $241-T-203$ & Salt well Pump & Supernatant & $1976-77$ & (42) & (11) \\
\hline T Plant & $241-\mathrm{T}-204$ & $\mathrm{LaF}_{3}$ (224) & 1952 & 3,687 & 974 \\
\hline $241-T-201$ & Cribs & Supernatant & 1952 & $(3,478)$ & (919) \\
\hline $241-\mathrm{T}-201$ & $241-\mathrm{T}-101$ & Supernatant & 1976 & (23) & (6) \\
\hline $241-T-201$ & Salt well Pump & Supernatant & $1976-77$ & (42) & (11) \\
\hline
\end{tabular}

Note:

${ }^{1}$ Agnew (1997b)

\section{A3.2 HISTORICAL ESTIMATION OF TANK CONTENTS}

The historical transfer data used for this estimate are from the following sources:

- The Waste Status and Transaction Record Summary: WSTRS, Rev. 4, (Agnew et al. 1997b) is a tank-by-tank quarterly summary spreadsheet of waste transactions.

- The Hanford Tank Chemical and Radionuclide Inventories: HDW Model Rev. 4 (Agnew et al. 1997a) contains the Hanford defined waste (HDW) list, the supernatant mixing model (SMM), the tank layer model (TLM), and the historical tank content estimate (HTCE).

- The HDW list is comprised of approximately 50 waste types defined by concentration for major analyses/compounds for sludge and supernatant layers.

- The TLM defines the sludge and saltcake layers in each tank using waste composition and waste transfer information.

- The SMM is a subroutine within the HDW model that calculates the volume and composition of certain supernatant blends and concentrates. 
Using these records, the TLM defines the sludge and saltcake layers in each tank. The SMM uses information from the WSTRS, the TLM, and the HDW list to describe the supernatants and concentrates in each tank. Together the WSTRS, TLM, SMM, and HDW list determine the inventory estimate for each tank. These model predictions are considered estimates that require further evaluation using analytical data.

Based on Agnew et al. (1997a), all T-200 tanks contain only lanthanum fluoride (224) waste. Figure A3-1 is a graphical representation of the estimated waste type and volume for the tank layer. The historical tank content estimate model predicts this waste is mostly water, and contains over 1 weight percent of sodium, nitrate, iron, oxalate, fluoride, hydroxide, and carbonate. Additionally, over 0.1 weight percent of bismuth, calcium, potassium, phosphate, and a trace quantity of plutonium are anticipated to be found. Very low concentrations of cesium and strontium are expected in this waste. Table A3-2 shows the historical estimate of the expected waste constituents and their concentrations.

Figure A3-1. Tank Layer Model.

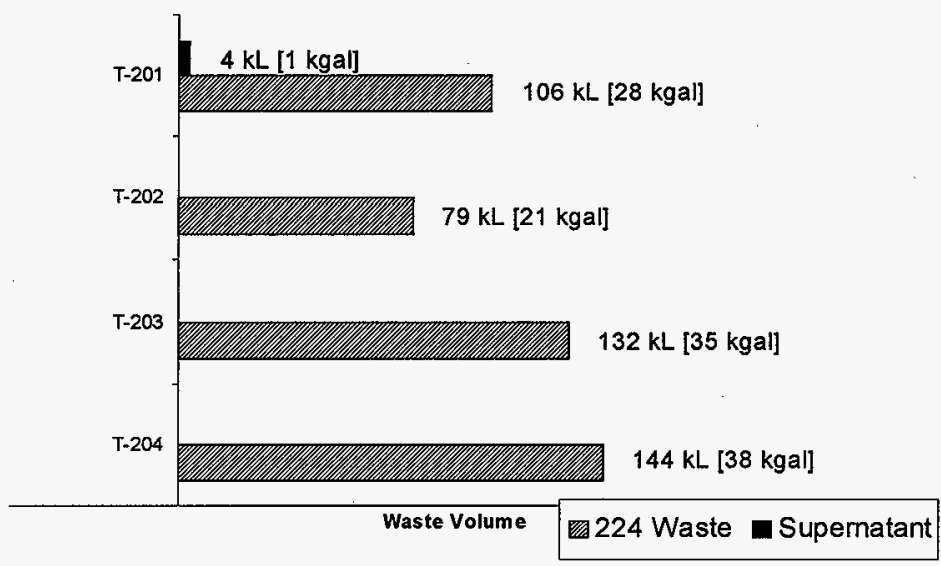


Table A3-2. 241-T-201 Historical Tank Inventory Estimate. ${ }^{1,2}$ (4 sheets)

\begin{tabular}{|c|c|c|c|c|c|}
\hline - & 8602 & 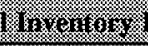 & Stiminare & & \\
\hline 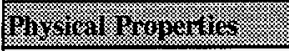 & স্যে & (3) & (1): & 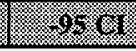 & 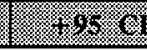 \\
\hline Total waste & $1.32 \mathrm{E}+05 \mathrm{~kg}$ & $\mathrm{~g}(29.0 \mathrm{kgal})$ & & & \\
\hline Heat load & $2.20 \mathrm{E}-04 \mathrm{~kW}$ & $(0.750 \mathrm{Btu}$ & /hr) & $1.64 \mathrm{E}-04$ & 2.71E-04 \\
\hline Bulk density ${ }^{3}$ & $1.20\left(\mathrm{~g} / \mathrm{cm}^{3}\right)$ & & & 1.15 & 1.26 \\
\hline Water wt $\%^{3}$ & 69.5 & & & 63.2 & 75.9 \\
\hline TOC wt\% C (wet) ${ }^{3}$ & 2.05 & & & 1.98 & 2.08 \\
\hline 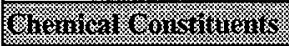 & 13 & (1) & (x) & 1850. & 1.952 \\
\hline $\mathrm{Na}^{+}$ & 4.09 & $7.83 \mathrm{E}+04$ & $1.03 \mathrm{E}+04$ & 2.92 & 5.87 \\
\hline $\mathrm{Al}^{3+}$ & 0 & 0 & 0 & 0 & 0 \\
\hline $\mathrm{Fe}^{3+}$ (total Fe) & 0.350 & $1.63 \mathrm{E}+04$ & $2.15 \mathrm{E}+03$ & 0.329 & 0.372 \\
\hline $\mathrm{Cr}^{3+}$ & $5.84 \mathrm{E}-03$ & 253 & 33.3 & 4.37E-03 & 7.20E-03 \\
\hline $\mathrm{Bi}^{3+}$ & $5.36 \mathrm{E}-02$ & $9.34 \mathrm{E}+03$ & $1.23 \mathrm{E}+03$ & $1.60 \mathrm{E}-02$ & $7.50 \mathrm{E}-02$ \\
\hline $\mathrm{La}^{3+}$ & $3.26 \mathrm{E}-03$ & 378 & 49.8 & $2.44 \mathrm{E}-03$ & $4.02 \mathrm{E}-03$ \\
\hline $\mathrm{Hg}^{2+}$ & 0 & 0 & 0 & 0 & 0 \\
\hline $\mathrm{Zr}$ (as $\left.\mathrm{ZrO}(\mathrm{OH})_{2}\right)$ & 0 & 0 & 0 & 0 & 0 \\
\hline $\mathrm{Pb}^{2+}$ & 0 & 0 & 0 & 0 & 0 \\
\hline $\mathrm{Ni}^{2+}$ & $1.37 \mathrm{E}-03$ & 67.2 & 8.85 & $1.03 \mathrm{E}-03$ & $6.38 \mathrm{E}-03$ \\
\hline $\mathrm{Sr}^{2+}$ & 0 & 0 & 0 & 0 & 0 \\
\hline $\mathrm{Mn}^{4+}$ & $4.38 \mathrm{E}-03$ & 200 & 26.4 & $3.27 \mathrm{E}-03$ & $5.40 \mathrm{E}-03$ \\
\hline $\mathrm{Ca}^{2+}$ & 0.236 & $7.88 \mathrm{E}+03$ & $1.04 \mathrm{E}+03$ & 0.152 & 0.320 \\
\hline $\mathrm{K}^{+}$ & 0.198 & $6.46 \mathrm{E}+03$ & 851 & 0.148 & 0.245 \\
\hline $\mathrm{OH}^{-}$ & 1.06 & $1.51 \mathrm{E}+04$ & $1.99 \mathrm{E}+03$ & 1.000 & 1.13 \\
\hline $\mathrm{NO}^{3-}$ & 1.18 & $6.11 \mathrm{E}+04$ & $8.05 \mathrm{E}+03$ & 0.885 & 1.46 \\
\hline $\mathrm{NO}^{2-}$ & 3.09E-03 & 119 & 15.6 & $1.70 \mathrm{E}-03$ & $4.79 \mathrm{E}-03$ \\
\hline $\mathrm{CO}_{3}^{2-}$ & 0.236 & $1.18 \mathrm{E}+04$ & $1.55 \mathrm{E}+03$ & 0.152 & 0.320 \\
\hline $\mathrm{PO}_{4}{ }^{3-}$ & $8.11 \mathrm{E}-02$ & $6.42 \mathrm{E}+03$ & 846 & $3.66 \mathrm{E}-02$ & 0.109 \\
\hline $\mathrm{SO}_{4}^{2-}$ & $2.58 \mathrm{E}-03$ & 206 & 27.2 & $1.93 \mathrm{E}-03$ & $3.18 \mathrm{E}-03$ \\
\hline $\mathrm{Si}\left(\right.$ as $\left.\mathrm{SiO}_{3}{ }^{2-}\right)$ & 0 & 0 & 0 & 0 & 0 \\
\hline $\mathrm{F}^{-}$ & 0.975 & $1.54 \mathrm{E}+04$ & $2.03 E+03$ & 0.173 & 2.79 \\
\hline $\mathrm{Cl}^{-}$ & 2.29E-02 & 676 & 89.1 & $1.71 \mathrm{E}-02$ & $2.83 \mathrm{E}-02$ \\
\hline
\end{tabular}


Table A3-2. 241-T-201 Historical Tank Inventory Estimate., ${ }^{1,2}$ (4 sheets)

\begin{tabular}{|c|c|c|c|c|c|}
\hline 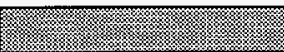 & & 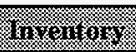 & s.timinge & & \\
\hline 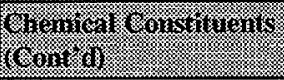 & 14 & (1) & $\frac{18}{8.8}$ & 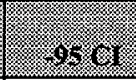 & iz: \\
\hline $\mathrm{C}_{6} \mathrm{H}_{5} \mathrm{O}_{7}{ }^{3-}$ & 0 & 0 & 0 & 0 & 0 \\
\hline EDTA $^{4-}$ & 0 & 0 & 0 & 0 & 0 \\
\hline HEDTA $^{3-}$ & 0 & 0 & 0 & 0 & 0 \\
\hline Glycolate $^{-}$ & 0 & 0 & 0 & 0 & 0 \\
\hline Acetate & 0 & 0 & 0 & 0 & 0 \\
\hline Oxalate $^{2-}$ & 1.03 & $7.52 \mathrm{E}+04$ & $9.90 \mathrm{E}+03$ & 0.978 & 1.05 \\
\hline DBP & 0 & 0 & 0 & 0 & 0 \\
\hline Butanol & 0 & 0 & 0 & 0 & 0 \\
\hline $\mathrm{NH}_{3}$ & $1.18 \mathrm{E}-07$ & $1.68 \mathrm{E}-03$ & $2.21 \mathrm{E}-04$ & $5.05 \mathrm{E}-08$ & $2.17 \mathrm{E}-07$ \\
\hline $\mathrm{Fe}(\mathrm{CN})_{6}^{4}$ & 0 & 0 & 0 & 0 & 0 \\
\hline 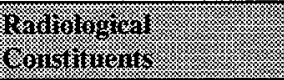 & 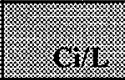 & 318 & $87^{3}$ & $\frac{96.01}{(2.11)}$ & 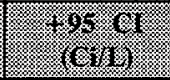 \\
\hline${ }^{3} \mathrm{H}$ & $5.13 \mathrm{E}-09$ & 4.27E-06 & 5.63E-04 & $2.84 \mathrm{E}-09$ & $7.79 \mathrm{E}-09$ \\
\hline${ }^{14} \mathrm{C}$ & 1.59E-09 & $1.32 \mathrm{E}-06$ & $1.75 \mathrm{E}-04$ & 1.19E-09 & $1.96 \mathrm{E}-09$ \\
\hline${ }^{59} \mathrm{Ni}$ & $4.52 \mathrm{E}-10$ & $3.76 \mathrm{E}-07$ & $4.96 \mathrm{E}-05$ & $3.38 \mathrm{E}-10$ & $2.10 \mathrm{E}-09$ \\
\hline${ }^{63} \mathrm{Ni}$ & 4.17E-08 & $3.47 \mathrm{E}-05$ & $4.57 \mathrm{E}-03$ & 3.11E-08 & $1.94 \mathrm{E}-07$ \\
\hline${ }^{60} \mathrm{Co}$ & $5.10 \mathrm{E}-10$ & $4.25 \mathrm{E}-07$ & $5.60 \mathrm{E}-05$ & $3.82 \mathrm{E}-10$ & $6.29 \mathrm{E}-10$ \\
\hline${ }^{79} \mathrm{Se}$ & $3.35 \mathrm{E}-10$ & $2.79 \mathrm{E}-07$ & $3.68 \mathrm{E}-05$ & $2.51 \mathrm{E}-10$ & $4.14 \mathrm{E}-10$ \\
\hline${ }^{90} \mathrm{Sr}$ & $1.66 \mathrm{E}-04$ & 0.138 & 18.2 & $1.24 \mathrm{E}-04$ & $2.05 \mathrm{E}-04$ \\
\hline${ }^{90} \mathrm{Y}$ & $1.66 \mathrm{E}-04$ & 0.138 & 18.2 & $1.24 \mathrm{E}-04$ & $2.05 \mathrm{E}-04$ \\
\hline${ }^{93} \mathrm{Zr}$ & 1.59E-09 & 1.33E-06 & $1.75 \mathrm{E}-04$ & 1.19E-09 & $1.96 \mathrm{E}-09$ \\
\hline${ }^{93 \mathrm{~m}} \mathrm{Nb}$ & $1.32 \mathrm{E}-09$ & $1.10 \mathrm{E}-06$ & $1.45 \mathrm{E}-04$ & $9.85 \mathrm{E}-10$ & $1.62 \mathrm{E}-09$ \\
\hline${ }^{99} \mathrm{Tc}$ & $1.10 \mathrm{E}-08$ & $9.20 \mathrm{E}-06$ & $1.21 \mathrm{E}-03$ & $8.25 \mathrm{E}-09$ & $1.36 \mathrm{E}-08$ \\
\hline${ }^{106} \mathrm{Ru}$ & $3.83 \mathrm{E}-16$ & 3.19E-13 & $4.20 \mathrm{E}-11$ & $2.86 \mathrm{E}-16$ & 4.72E-16 \\
\hline${ }^{113 \mathrm{~m}} \mathrm{Cd}$ & $4.46 \mathrm{E}-09$ & 3.71E-06 & 4.89E-04 & 3.33E-09 & $5.50 \mathrm{E}-09$ \\
\hline${ }^{125} \mathrm{Sb}$ & $5.88 \mathrm{E}-10$ & 4.90E-07 & $6.46 \mathrm{E}-05$ & $4.40 \mathrm{E}-10$ & $7.26 \mathrm{E}-10$ \\
\hline${ }^{126} \mathrm{Sn}$ & $5.06 \mathrm{E}-10$ & $4.21 \mathrm{E}-07$ & $5.55 \mathrm{E}-05$ & $3.78 \mathrm{E}-10$ & 6.24E-10 \\
\hline${ }^{129} \mathrm{I}$ & $2.08 \mathrm{E}-11$ & $1.74 \mathrm{E}-08$ & $2.29 \mathrm{E}-06$ & $1.56 \mathrm{E}-11$ & $2.57 \mathrm{E}-11$ \\
\hline${ }^{134} \mathrm{Cs}$ & $2.54 \mathrm{E}-11$ & $2.11 \mathrm{E}-08$ & $2.78 \mathrm{E}-06$ & $1.90 \mathrm{E}-11$ & 3.13E-11 \\
\hline
\end{tabular}


Table A3-2. 241-T-201 Historical Tank Inventory Estimate. ${ }^{1,2}$ (4 sheets)

\begin{tabular}{|c|c|c|c|c|c|}
\hline (:) & 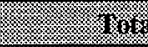 & linention & 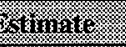 & & \\
\hline Riviologicul : & & & & 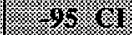 & 1.9 .95 .11 \\
\hline 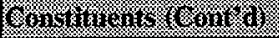 & 18.211 & $2.16 \% 8$ & 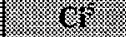 & .18141 & I \\
\hline${ }^{137} \mathrm{Cs}$ & $1.88 \mathrm{E}-04$ & 0.157 & 20.7 & 1.41E-04 & 2.32E-04 \\
\hline${ }^{137 \mathrm{~m}} \mathrm{Ba}$ & $1.78 \mathrm{E}-04$ & 0.148 & 19.6 & 1.33E-04 & $2.20 \mathrm{E}-04$ \\
\hline${ }^{151} \mathrm{Sm}$ & $1.27 \mathrm{E}-06$ & $1.06 \mathrm{E}-03$ & 0.139 & $9.49 \mathrm{E}-07$ & $1.56 \mathrm{E}-06$ \\
\hline${ }^{152} \mathrm{Eu}$ & $1.66 \mathrm{E}-09$ & $1.38 \mathrm{E}-06$ & $1.82 \mathrm{E}-04$ & $1.65 \mathrm{E}-09$ & $1.67 \mathrm{E}-09$ \\
\hline${ }^{154} \mathrm{Eu}$ & 8.19E-09 & $6.82 \mathrm{E}-06$ & $8.99 \mathrm{E}-04$ & $6.12 \mathrm{E}-09$ & $1.01 \mathrm{E}-08$ \\
\hline${ }^{155} \mathrm{Eu}$ & $1.50 \mathrm{E}-07$ & $1.25 \mathrm{E}-04$ & $1.64 \mathrm{E}-02$ & $1.49 \mathrm{E}-07$ & $1.51 \mathrm{E}-07$ \\
\hline${ }^{226} \mathrm{Ra}$ & 7.49E-14 & $6.24 \mathrm{E}-11$ & $8.22 \mathrm{E}-09$ & 5.60E-14 & $9.23 \mathrm{E}-14$ \\
\hline${ }^{228} \mathrm{Ra}$ & $4.81 \mathrm{E}-18$ & $4.01 \mathrm{E}-15$ & $5.28 \mathrm{E}-13$ & $4.77 \mathrm{E}-18$ & $4.85 \mathrm{E}-18$ \\
\hline${ }^{227} \mathrm{Ac}$ & $3.95 \mathrm{E}-13$ & $3.29 \mathrm{E}-10$ & $4.34 \mathrm{E}-08$ & $2.95 \mathrm{E}-13$ & $4.87 \mathrm{E}-13$ \\
\hline${ }^{231} \mathrm{~Pa}$ & 9.12E-13 & $7.60 \mathrm{E}-10$ & $1.00 \mathrm{E}-07$ & $6.82 \mathrm{E}-13$ & 1.12E-12 \\
\hline${ }^{229} \mathrm{Th}$ & 9.31E-16 & $7.76 \mathrm{E}-13$ & $1.02 \mathrm{E}-10$ & $9.23 \mathrm{E}-16$ & $9.39 \mathrm{E}-16$ \\
\hline${ }^{232} \mathrm{Th}$ & $4.21 \mathrm{E}-19$ & $3.51 \mathrm{E}-16$ & $4.62 \mathrm{E}-14$ & $3.15 \mathrm{E}-19$ & $5.19 \mathrm{E}-19$ \\
\hline${ }^{232} \mathrm{U}$ & $4.88 \mathrm{E}-13$ & $4.07 \mathrm{E}-10$ & $5.36 \mathrm{E}-08$ & $3.65 \mathrm{E}-13$ & $6.02 \mathrm{E}-13$ \\
\hline${ }^{233} \mathrm{U}$ & $2.23 \mathrm{E}-14$ & $1.86 \mathrm{E}-11$ & $2.45 \mathrm{E}-09$ & $1.67 \mathrm{E}-14$ & $2.75 \mathrm{E}-14$ \\
\hline${ }^{234} \mathrm{U}$ & $2.43 \mathrm{E}-08$ & $2.03 \mathrm{E}-05$ & $2.67 \mathrm{E}-03$ & $1.82 \mathrm{E}-08$ & $3.00 \mathrm{E}-08$ \\
\hline${ }^{235} \mathrm{U}$ & $1.08 \mathrm{E}-09$ & 9.02E-07 & $1.19 \mathrm{E}-04$ & $8.10 \mathrm{E}-10$ & $1.34 \mathrm{E}-09$ \\
\hline${ }^{236} \mathrm{U}$ & $2.12 \mathrm{E}-10$ & $1.77 \mathrm{E}-07$ & 2.33E-05 & $1.59 \mathrm{E}-10$ & $2.62 \mathrm{E}-10$ \\
\hline${ }^{238} \mathrm{U}$ & 2.47E- 08 & $2.06 \mathrm{E}-05$ & $2.71 \mathrm{E}-03$ & $1.85 \mathrm{E}-08$ & 3.05E-08 \\
\hline${ }^{237} \mathrm{~Np}$ & $6.84 \mathrm{E}-11$ & 5.70E-08 & 7.51E-06 & 5.11E-11 & 8.44E-11 \\
\hline${ }^{238} \mathrm{Pu}$ & 2.92E-09 & $2.43 \mathrm{E}-06$ & $3.21 \mathrm{E}-04$ & $2.18 \mathrm{E}-09$ & $3.60 \mathrm{E}-09$ \\
\hline${ }^{239} \mathrm{Pu}$ & 4.23E-07 & $3.52 \mathrm{E}-04$ & $4.64 \mathrm{E}-02$ & $3.16 \mathrm{E}-07$ & $5.21 \mathrm{E}-07$ \\
\hline${ }^{240} \mathrm{Pu}$ & $3.71 \mathrm{E}-08$ & 3.09E-05 & $4.07 \mathrm{E}-03$ & $2.77 \mathrm{E}-08$ & $4.58 \mathrm{E}-08$ \\
\hline${ }^{241} \mathrm{Pu}$ & $1.23 \mathrm{E}-07$ & $1.02 \mathrm{E}-04$ & $1.35 \mathrm{E}-02$ & $9.17 \mathrm{E}-08$ & $1.51 \mathrm{E}-07$ \\
\hline${ }^{242} \mathrm{Pu}$ & $5.67 \mathrm{E}-13$ & $4.73 \mathrm{E}-10$ & $6.23 \mathrm{E}-08$ & $4.24 \mathrm{E}-13$ & $7.00 \mathrm{E}-13$ \\
\hline${ }^{241} \mathrm{Am}$ & 3.46E-09 & $2.88 \mathrm{E}-06$ & $3.80 \mathrm{E}-04$ & $2.59 \mathrm{E}-09$ & $4.26 \mathrm{E}-09$ \\
\hline${ }^{243} \mathrm{Am}$ & $2.81 \mathrm{E}-14$ & $2.34 \mathrm{E}-11$ & $3.08 \mathrm{E}-09$ & $2.10 \mathrm{E}-14$ & $3.47 \mathrm{E}-14$ \\
\hline${ }^{242} \mathrm{Cm}$ & 3.38E-11 & 2.81E-08 & 3.70E-06 & $3.35 \mathrm{E}-11$ & $3.40 \mathrm{E}-11$ \\
\hline${ }^{243} \mathrm{Cm}$ & $7.27 \mathrm{E}-13$ & $6.06 \mathrm{E}-10$ & $7.98 \mathrm{E}-08$ & 7.21E-13 & 7.33E-13 \\
\hline${ }^{244} \mathrm{Cm}$ & $7.14 \mathrm{E}-13$ & $5.95 E-10$ & $7.84 \mathrm{E}-08$ & $5.34 \mathrm{E}-13$ & $8.81 \mathrm{E}-13$ \\
\hline
\end{tabular}


Table A3-2. 241-T-201 Historical Tank Inventory Estimate. ${ }^{1,2}$ (4 sheets)

\begin{tabular}{|c|c|c|c|c|c|}
\hline \multicolumn{6}{|c|}{ 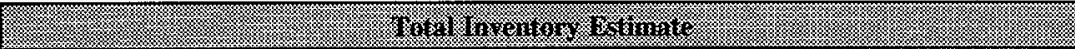 } \\
\hline ro: & 18 & . $10 \mathrm{~g}$ & 1868 & 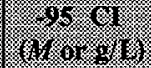 & . \\
\hline 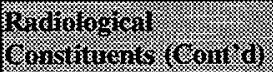 & 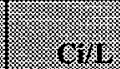 & 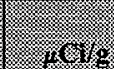 & 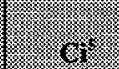 & $(2.95)$ & 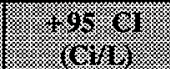 \\
\hline $\mathrm{Pu}$ & $\begin{array}{l}6.97 \mathrm{E}-06 \\
(\mathrm{~g} / \mathrm{L})\end{array}$ & --- & $7.65 \mathrm{E}-04$ & $5.21 \mathrm{E}-06$ & $8.59 \mathrm{E}-06$ \\
\hline $\mathrm{U}$ & 3.11E-04 & 61.7 & 8.13 & 2.33E-04 & $3.84 \mathrm{E}-04$ \\
\hline
\end{tabular}

Notes:

$\mathrm{CI}=$ confidence interval

${ }^{1}$ Agnew et al. (1997a)

${ }^{2}$ These predictions have not been validated and should be used with caution.

${ }^{3}$ This is the volume average for density, mass average water wt\%, and TOC wt\% carbon.

${ }^{4}$ Differences exist among the inventories in this column and the inventories calculated from the two sets of concentrations.

sUnknowns in tank solids inventory are assigned by the TLM. 
HNF-1501 Rev. 0

Table A3-3. 241-T-202 Historical Tank Inventory Estimate. ${ }^{1,2}$ (4 sheets)

\begin{tabular}{|c|c|c|c|c|c|}
\hline \multicolumn{6}{|c|}{ 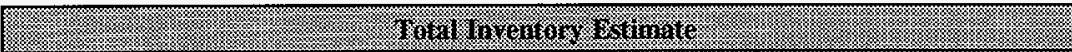 } \\
\hline \multirow{2}{*}{\multicolumn{6}{|c|}{ 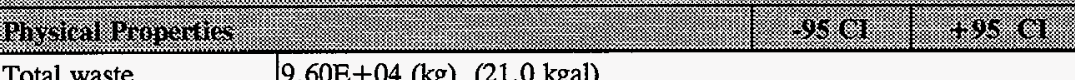 }} \\
\hline & & & & & \\
\hline Heat load & $1.65 \mathrm{E}-04(\mathrm{~kW})$ & $(0.563 \mathrm{Btu}$ & hr) & 1.23E-04 & 2.03E-04 \\
\hline Bulk density $^{3}$ & $1.21\left(\mathrm{~g} / \mathrm{cm}^{3}\right)$ & & & 1.15 & 1.26 \\
\hline Water wt $\%^{3}$ & 68.6 & & & 62.1 & 75.2 \\
\hline TOC wt\% C (wet) ${ }^{3}$ & 2.11 & & & 2.03 & 2.14 \\
\hline 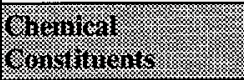 & 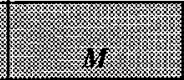 & (2) & 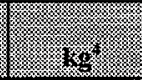 & 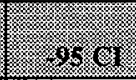 & 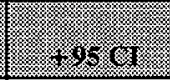 \\
\hline $\mathrm{Na}^{+}$ & 4.23 & $8.06 \mathrm{E}+04$ & $7.74 \mathrm{E}+03$ & 3.03 & 6.08 \\
\hline $\mathrm{Al}^{3+}$ & 0 & 0 & 0 & 0 & 0 \\
\hline $\mathrm{Fe}^{3+}$ (total Fe) & 0.363 & $1.68 \mathrm{E}+04$ & $1.61 \mathrm{E}+03$ & 0.341 & 0.385 \\
\hline $\mathrm{Cr}^{3+}$ & $6.05 \mathrm{E}-03$ & 260 & 25.0 & $4.52 \mathrm{E}-03$ & $7.46 \mathrm{E}-03$ \\
\hline $\mathrm{Bi}^{3+}$ & $5.55 \mathrm{E}-02$ & $9.61 \mathrm{E}+03$ & 923 & $1.66 \mathrm{E}-02$ & $7.77 \mathrm{E}-02$ \\
\hline $\mathrm{La}^{3+}$ & $3.38 \mathrm{E}-03$ & 389 & 37.3 & $2.53 \mathrm{E}-03$ & 4.17E-03 \\
\hline $\mathrm{Hg}^{2+}$ & 0 & 0 & 0 & 0 & 0 \\
\hline $\mathrm{Zr}\left(\right.$ as $\left.\mathrm{ZrO}(\mathrm{OH})_{2}\right)$ & 0 & 0 & 0 & 0 & 0 \\
\hline $\mathrm{Pb}^{2+}$ & 0 & 0 & 0 & 0 & 0 \\
\hline $\mathrm{Ni}^{2+}$ & $1.42 \mathrm{E}-03$ & 69.2 & 6.64 & $1.06 \mathrm{E}-03$ & $6.61 \mathrm{E}-03$ \\
\hline $\mathrm{Sr}^{2+}$ & 0 & 0 & 0 & 0 & 0 \\
\hline $\mathrm{Mn}^{4+}$ & $4.54 \mathrm{E}-03$ & 206 & 19.8 & 3.39E-03 & $5.59 \mathrm{E}-03$ \\
\hline $\mathrm{Ca}^{2+}$ & 0.244 & $8.11 \mathrm{E}+03$ & 779 & 0.157 & 0.332 \\
\hline $\mathrm{K}^{+}$ & 0.205 & $6.65 \mathrm{E}+03$ & 638 & 0.154 & 0.253 \\
\hline $\mathrm{OH}^{-}$ & 1.10 & $1.55 \mathrm{E}+04$ & $1.49 \mathrm{E}+03$ & 1.04 & 1.17 \\
\hline $\mathrm{NO}^{3-}$ & 1.23 & $6.29 \mathrm{E}+04$ & $6.04 \mathrm{E}+03$ & 0.917 & 1.51 \\
\hline $\mathrm{NO}^{2-}$ & $3.20 \mathrm{E}-03$ & 122 & 11.7 & $1.76 \mathrm{E}-03$ & $4.97 \mathrm{E}-03$ \\
\hline $\mathrm{CO}_{3}^{2-}$ & 0.244 & $1.21 \mathrm{E}+04$ & $1.17 \mathrm{E}+03$ & 0.157 & 0.332 \\
\hline $\mathrm{PO}_{4}^{3-}$ & $8.40 \mathrm{E}-02$ & $6.61 \mathrm{E}+03$ & 634 & $3.79 \mathrm{E}-02$ & 0.113 \\
\hline $\mathrm{SO}_{4}^{2-}$ & $2.67 \mathrm{E}-03$ & 212 & 20.4 & 1.99E-03 & $3.29 \mathrm{E}-03$ \\
\hline $\mathrm{Si} \mathrm{(as} \mathrm{SiO}_{3}{ }^{2-}$ ) & 0 & 0 & 0 & 0 & 0 \\
\hline $\mathbf{F}$ & 1.01 & $1.59 \mathrm{E}+04$ & $1.53 \mathrm{E}+03$ & 0.180 & 2.89 \\
\hline $\mathrm{Cl}^{-}$ & $2.37 \mathrm{E}-02$ & 696 & 66.8 & $1.77 \mathrm{E}-02$ & $2.93 \mathrm{E}-02$ \\
\hline
\end{tabular}


Table A3-3. 241-T-202 Historical Tank Inventory Estimate. ${ }^{1,2}$ (4 sheets)

\begin{tabular}{|c|c|c|c|c|c|}
\hline (2: & & intenting & Shinate & & \\
\hline 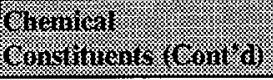 & 18 & $\sqrt{3}$ & 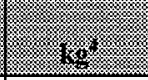 & . $810 \%$ & 18.206 \\
\hline $\mathrm{C}_{6} \mathrm{H}_{5} \mathrm{O}_{7}^{3-}$ & 0 & 0 & 0 & 0 & 0 \\
\hline EDTA $^{4}$ & 0 & 0 & 0 & 0 & 0 \\
\hline HEDTA $^{3-}$ & 0 & 0 & 0 & 0 & 0 \\
\hline Glycolate & 0 & 0 & 0 & 0 & 0 \\
\hline Acetate & 0 & 0 & 0 & 0 & 0 \\
\hline Oxalate $^{2-}$ & 1.06 & $7.74 \mathrm{E}+04$ & $7.43 \mathrm{E}+03$ & 1.01 & 1.09 \\
\hline DBP & 0 & 0 & 0 . & 0 & 0 \\
\hline Butanol & 0 & 0 & 0 & 0 & 0 \\
\hline $\mathrm{NH}_{3}$ & 1.23E-07 & $1.73 \mathrm{E}-03$ & $1.66 \mathrm{E}-04$ & $5.23 \mathrm{E}-08$ & $2.25 \mathrm{E}-07$ \\
\hline $\mathrm{Fe}(\mathrm{CN})_{6}{ }^{4}$ & 0 & 0 & 0 & 0 & 0 \\
\hline $\begin{array}{l}\text { Radiviogral } \\
\text { Canintinems }\end{array}$ & 1 & 1.18 & 12 & 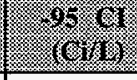 & H: \\
\hline${ }^{3} \mathrm{H}$ & 5.31E-09 & 4.40E-06 & $4.22 \mathrm{E}-04$ & $2.94 \mathrm{E}-09$ & 8.07E-09 \\
\hline${ }^{14} \mathrm{C}$ & $1.65 \mathrm{E}-09$ & $1.36 \mathrm{E}-06$ & 1.31E-04 & 1.23E-09 & 2.03E-09 \\
\hline${ }^{59} \mathrm{Ni}$ & $4.68 \mathrm{E}-10$ & $3.88 \mathrm{E}-07$ & 3.72E-05 & $3.50 \mathrm{E}-10$ & 2.17E-09 \\
\hline${ }^{63} \mathrm{Ni}$ & 4.32E-08 & $3.57 \mathrm{E}-05$ & 3.43E-03 & $3.23 \mathrm{E}-08$ & $2.01 \mathrm{E}-07$ \\
\hline${ }^{60} \mathrm{Co}$ & $5.29 \mathrm{E}-10$ & $4.38 \mathrm{E}-07$ & $4.20 \mathrm{E}-05$ & $3.95 \mathrm{E}-10$ & $6.52 \mathrm{E}-10$ \\
\hline${ }^{79} \mathrm{Se}$ & $3.47 \mathrm{E}-10$ & $2.88 \mathrm{E}-07$ & $2.76 \mathrm{E}-05$ & $2.60 \mathrm{E}-10$ & $4.28 \mathrm{E}-10$ \\
\hline${ }^{90} \mathrm{Sr}$ & $1.72 \mathrm{E}-04$ & 0.142 & 13.7 & $1.29 \mathrm{E}-04$ & 2.12E-04 \\
\hline${ }^{90} \mathrm{Y}$ & 1.72E-04 & 0.142 & 13.7 & $1.29 \mathrm{E}-04$ & $2.12 \mathrm{E}-04$ \\
\hline${ }^{93} \mathrm{Zr}$ & $1.65 \mathrm{E}-09$ & 1.37E-06 & $1.31 \mathrm{E}-04$ & 1.23E-09 & 2.03E-09 \\
\hline${ }^{93 \mathrm{~m}} \mathrm{Nb}$ & $1.36 \mathrm{E}-09$ & $1.13 \mathrm{E}-06$ & $1.08 \mathrm{E}-04$ & $1.02 \mathrm{E}-09$ & 1.68E-09 \\
\hline${ }^{99} \mathrm{Tc}$ & $1.14 \mathrm{E}-08$ & $9.47 \mathrm{E}-06$ & $9.09 \mathrm{E}-04$ & $8.55 \mathrm{E}-09$ & $1.41 \mathrm{E}-08$ \\
\hline${ }^{106} \mathrm{Ru}$ & $3.96 \mathrm{E}-16$ & $3.28 \mathrm{E}-13$ & $3.15 \mathrm{E}-11$ & $2.96 \mathrm{E}-16$ & $4.89 \mathrm{E}-16$ \\
\hline${ }^{113 m} \mathrm{Cd}$ & 4.62E-09 & $3.82 \mathrm{E}-06$ & 3.67E-04 & 3.45E-09 & 5.69E-09 \\
\hline${ }^{125} \mathrm{Sb}$ & $6.10 \mathrm{E}-10$ & $5.05 \mathrm{E}-07$ & $4.84 \mathrm{E}-05$ & $4.56 \mathrm{E}-10$ & $7.52 \mathrm{E}-10$ \\
\hline${ }^{126} \mathrm{Sn}$ & $5.24 \mathrm{E}-10$ & 4.34E-07 & $4.16 \mathrm{E}-05$ & $3.92 \mathrm{E}-10$ & $6.46 \mathrm{E}-10$ \\
\hline${ }^{129} I$ & $2.16 \mathrm{E}-11$ & $1.79 \mathrm{E}-08$ & $1.72 \mathrm{E}-06$ & 1.61E-11 & $2.66 \mathrm{E}-11$ \\
\hline${ }^{134} \mathrm{Cs}$ & $2.63 \mathrm{E}-11$ & $2.17 \mathrm{E}-08$ & $2.09 \mathrm{E}-06$ & $1.96 \mathrm{E}-11$ & $3.24 \mathrm{E}-11$ \\
\hline
\end{tabular}


Table A3-3. 241-T-202 Historical Tank Inventory Estimate. ${ }^{1,2}$ (4 sheets)

\begin{tabular}{|c|c|c|c|c|c|}
\hline & & Tryertors & 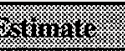 & & \\
\hline 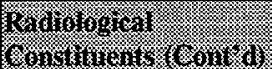 & $\sqrt{8 .+3}$ & 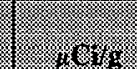 & $86^{3}$ & 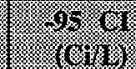 & 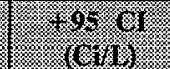 \\
\hline${ }^{137} \mathrm{Cs}$ & $1.95 \mathrm{E}-04$ & 0.162 & 15.5 & 1.46E-04 & $2.41 \mathrm{E}-04$ \\
\hline${ }^{137 \mathrm{~m}} \mathrm{Ba}$ & $1.85 \mathrm{E}-04$ & 0.153 & 14.7 & $1.38 \mathrm{E}-04$ & $2.28 \mathrm{E}-04$ \\
\hline${ }^{151} \mathrm{Sm}$ & $1.31 \mathrm{E}-06$ & $1.09 \mathrm{E}-03$ & 0.104 & 9.82E-07 & $1.62 \mathrm{E}-06$ \\
\hline${ }^{152} \mathrm{Eu}$ & $1.72 \mathrm{E}-09$ & $1.42 \mathrm{E}-06$ & $1.37 \mathrm{E}-04$ & $1.71 \mathrm{E}-09$ & 1.73E-09 \\
\hline${ }^{154} \mathrm{Eu}$ & $8.48 \mathrm{E}-09$ & 7.02E-06 & $6.74 \mathrm{E}-04$ & 6.34E-09 & $1.05 \mathrm{E}-08$ \\
\hline${ }^{155} \mathrm{Eu}$ & $1.55 \mathrm{E}-07$ & $1.29 \mathrm{E}-04$ & $1.23 \mathrm{E}-02$ & $1.54 \mathrm{E}-07$ & $1.56 \mathrm{E}-07$ \\
\hline${ }^{226} \mathrm{Ra}$ & 7.76E-14 & $6.42 \mathrm{E}-11$ & $6.16 \mathrm{E}-09$ & $5.80 \mathrm{E}-14$ & $9.56 \dot{\mathrm{E}}-14$ \\
\hline${ }^{228} \mathrm{Ra}$ & $4.99 \mathrm{E}-18$ & $4.13 \mathrm{E}-15$ & $3.96 \mathrm{E}-13$ & 4.94E-18 & 5.03E-18 \\
\hline${ }^{227} \mathrm{Ac}$ & $4.09 \mathrm{E}-13$ & 3.39E-10 & $3.25 \mathrm{E}-08$ & $3.06 \mathrm{E}-13$ & $5.05 \mathrm{E}-13$ \\
\hline${ }^{231} \mathrm{~Pa}$ & $9.44 \mathrm{E}-13$ & 7.82E-10 & $7.51 \mathrm{E}-08$ & 7.06E-13 & $1.16 \mathrm{E}-12$ \\
\hline${ }^{229} \mathrm{Th}$ & $9.65 \mathrm{E}-16$ & 7.99E-13 & 7.67E-11 & 9.57E-16 & $9.72 \mathrm{E}-16$ \\
\hline${ }^{232} \mathrm{Th}$ & $4.36 \mathrm{E}-19$ & 3.61E-16 & $3.46 \mathrm{E}-14$ & $3.26 \mathrm{E}-19$ & 5.37E-19 \\
\hline${ }^{232} \mathrm{U}$ & $5.06 \mathrm{E}-13$ & 4.19E-10 & $4.02 \mathrm{E}-08$ & $3.78 \mathrm{E}-13$ & $6.24 \mathrm{E}-13$ \\
\hline${ }^{233} \mathrm{U}$ & $2.31 \mathrm{E}-14$ & $1.91 \mathrm{E}-11$ & $1.83 \mathrm{E}-09$ & $1.72 \mathrm{E}-14$ & $2.85 \mathrm{E}-14$ \\
\hline${ }^{234} \mathrm{U}$ & $2.52 \mathrm{E}-08$ & $2.09 \mathrm{E}-05$ & $2.00 \mathrm{E}-03$ & $1.89 \mathrm{E}-08$ & $3.11 \mathrm{E}-08$ \\
\hline${ }^{235} \mathrm{U}$ & $1.12 \mathrm{E}-09$ & 9.29E-07 & $8.92 \mathrm{E}-05$ & $8.39 \mathrm{E}-10$ & $1.38 \mathrm{E}-09$ \\
\hline${ }^{236} \mathrm{U}$ & $2.20 \mathrm{E}-10$ & $1.82 \mathrm{E}-07$ & $1.75 \mathrm{E}-05$ & $1.64 \mathrm{E}-10$ & $2.71 \mathrm{E}-10$ \\
\hline${ }^{238} \mathrm{U}$ & $2.56 \mathrm{E}-08$ & $2.12 \mathrm{E}-05$ & $2.03 \mathrm{E}-03$ & 1.91E-08 & $3.16 \mathrm{E}-08$ \\
\hline${ }^{237} \mathrm{~Np}$ & 7.09E-11 & $5.87 \mathrm{E}-08$ & 5.63E-06 & $5.30 \mathrm{E}-11$ & 8.74E-11 \\
\hline${ }^{238} \mathrm{Pu}$ & 3.03E-09 & 2.51E-06 & 2.40E-04 & $2.26 \mathrm{E}-09$ & $3.73 \mathrm{E}-09$ \\
\hline${ }^{239} \mathrm{Pu}$ & 4.38E-07 & 3.63E-04 & $3.48 \mathrm{E}-02$ & 3.27E-07 & $5.40 \mathrm{E}-07$ \\
\hline${ }^{240} \mathrm{Pu}$ & 3.84E-08 & $3.18 \mathrm{E}-05$ & $3.06 \mathrm{E}-03$ & $2.87 \mathrm{E}-08$ & 4.74E-08 \\
\hline${ }^{241} \mathrm{Pu}$ & $1.27 \mathrm{E}-07$ & $1.05 \mathrm{E}-04$ & 1.01E-02 & $9.50 \mathrm{E}-08$ & $1.57 \mathrm{E}-07$ \\
\hline${ }^{242} \mathrm{Pu}$ & $5.88 \mathrm{E}-13$ & $4.87 \mathrm{E}-10$ & $4.67 \mathrm{E}-08$ & $4.39 \mathrm{E}-13$ & $7.25 \mathrm{E}-13$ \\
\hline${ }^{241} \mathrm{Am}$ & $3.58 \mathrm{E}-09$ & 2.97E-06 & $2.85 \mathrm{E}-04$ & $2.68 \mathrm{E}-09$ & 4.42E-09 \\
\hline${ }^{243} \mathrm{Am}$ & $2.91 \mathrm{E}-14$ & $2.41 \mathrm{E}-11$ & 2.31E-09 & $2.18 \mathrm{E}-14$ & $3.59 \mathrm{E}-14$ \\
\hline${ }^{242} \mathrm{Cm}$ & $3.50 \mathrm{E}-11$ & $2.89 \mathrm{E}-08$ & 2.78E-06 & $3.47 \mathrm{E}-11$ & $3.52 \mathrm{E}-11$ \\
\hline${ }^{243} \mathrm{Cm}$ & 7.53E-13 & $6.24 \mathrm{E}-10$ & 5.99E-08 & 7.47E-13 & 7.59E-13 \\
\hline${ }^{244} \mathrm{Cm}$ & $7.40 \mathrm{E}-13$ & $6.13 \mathrm{E}-10$ & $5.88 \mathrm{E}-08$ & $5.53 \mathrm{E}-13$ & $9.12 \mathrm{E}-13$ \\
\hline
\end{tabular}


Table A3-3. 241-T-202 Historical Tank Inventory Estimate. ${ }^{1,2}$ (4 sheets)

\begin{tabular}{|c|c|c|c|c|c|}
\hline 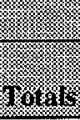 & 4. & incento & $\frac{1}{4}$ & 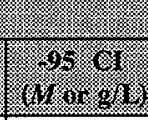 & 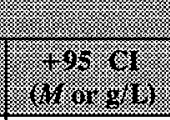 \\
\hline $\mathbf{P u}$ & $\begin{array}{l}7.22 \mathrm{E}-06 \\
\mathrm{~g} / \mathrm{L})\end{array}$ & - & $5.74 \mathrm{E}-04$ & 5.39E-06 & 8.90E-06 \\
\hline $\mathbf{U}$ & 3.22E-04 & 63.5 & 6.09 & $2.41 \mathrm{E}-04$ & $3.97 \mathrm{E}-04$ \\
\hline
\end{tabular}

Notes:

$\mathrm{CI}=$ confidence interval

${ }^{1}$ Agnew et al. (1997a)

${ }^{2}$ These predictions have not been validated and should be used with caution.

${ }^{3}$ This is the volume average for density, mass average water wt\% and TOC wt\% carbon.

${ }^{4}$ Differences exist among the inventories in this columan and the inventories calculated from the two sets of concentrations.

${ }^{5}$ Unknowns in tank solids inventory are assigned by the TLM. 
Table A3-4. 241-T-203 Historical Tank Inventory Estimate. ${ }^{1,2}$ (4 sheets)

\begin{tabular}{|c|c|c|c|c|c|}
\hline s. & 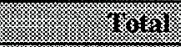 & 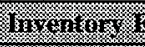 & 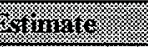 & & 3.8 \\
\hline 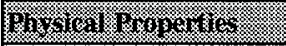 & 4 & X) & 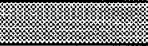 & II & 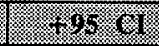 \\
\hline Total waste & $1.60 \mathrm{E}+05(\mathrm{~kg})$ & ) $(35.0 \mathrm{kgal}$ & & & \\
\hline Heat load & $2.75 \mathrm{E}-04(\mathrm{~kW})$ & (0.938 Bto & $\mathrm{a} / \mathrm{hr})$ & $2.05 \mathrm{E}-04$ & $3.39 \mathrm{E}-04$ \\
\hline Bulk density ${ }^{3}$ & $1.21\left(\mathrm{~g} / \mathrm{cm}^{3}\right)$ & & & 1.15 & 1.26 \\
\hline Water wt $\%^{3}$ & 68.6 & & & 62.1 & 75.2 \\
\hline TOC wt\% C (wet) ${ }^{3}$ & 2.11 & & & 2.03 & 2.14 \\
\hline 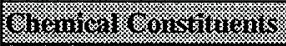 & 10 & 2011 & (16) & , & 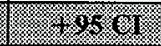 \\
\hline $\mathrm{Na}^{+}$ & 4.23 & $8.06 \mathrm{E}+04$ & $1.29 \mathrm{E}+04$ & 3.03 & 6.08 \\
\hline $\mathrm{Al}^{3+}$ & 0 & 0 & 0 & 0 & 0 \\
\hline $\mathrm{Fe}^{3+}($ total $\mathrm{Fe})$ & 0.363 & $1.68 \mathrm{E}+04$ & $2.68 \mathrm{E}+03$ & 0.341 & 0.385 \\
\hline $\mathrm{Cr}^{3+}$ & $6.05 \mathrm{E}-03$ & 260 & 41.7 & $4.52 \mathrm{E}-03$ & $7.46 \mathrm{E}-03$ \\
\hline $\mathrm{Bi}^{3+}$ & $5.55 \mathrm{E}-02$ & $9.61 \mathrm{E}+03$ & $1.54 \mathrm{E}+03$ & $1.66 \mathrm{E}-02$ & $7.77 \mathrm{E}-02$ \\
\hline $\mathrm{La}^{3+}$ & $3.38 \mathrm{E}-03$ & 389 & 62.2 & $2.53 \mathrm{E}-03$ & $4.17 \mathrm{E}-03$ \\
\hline $\mathrm{Hg}^{2+}$ & 0 & 0 & 0 & 0 & 0 \\
\hline $\mathrm{Zr}\left(\right.$ as $\left.\mathrm{ZrO}(\mathrm{OH})_{2}\right)$ & 0 & 0 & 0 & 0 & 0 \\
\hline $\mathrm{Pb}^{2+}$ & 0 & 0 & 0 & 0 & 0 \\
\hline $\mathrm{Ni}^{2+}$ & 1.42E-03 & 69.2 & 11.1 & $1.06 \mathrm{E}-03$ & $6.61 \mathrm{E}-03$ \\
\hline $\mathrm{Sr}^{2+}$ & 0 & 0 & 0 & 0 & 0 \\
\hline $\mathrm{Mn}^{4+}$ & $4.54 \mathrm{E}-03$ & 206 & 33.0 & $3.39 \mathrm{E}-03$ & $5.59 \mathrm{E}-03$ \\
\hline $\mathrm{Ca}^{2+}$ & 0.244 & $8.11 E+03$ & $1.30 \mathrm{E}+03$ & 0.157 & 0.332 \\
\hline $\mathbf{K}^{+}$ & 0.205 & $6.65 \mathrm{E}+03$ & $1.06 \mathrm{E}+03$ & 0.154 & 0.253 \\
\hline $\mathrm{OH}^{-}$ & 1.10 & $1.55 \mathrm{E}+04$ & $2.48 \mathrm{E}+03$ & 1.04 & 1.17 \\
\hline $\mathrm{NO}^{3-}$ & 1.23 & $6.29 \mathrm{E}+04$ & $1.01 \mathrm{E}+04$ & 0.917 & 1.51 \\
\hline $\mathrm{NO}^{2-}$ & $3.20 \mathrm{E}-03$ & 122 & 19.5 & $1.76 \mathrm{E}-03$ & $4.97 \mathrm{E}-03$ \\
\hline $\mathrm{CO}_{3}^{2-}$ & 0.244 & $1.21 \mathrm{E}+04$ & $1.94 \mathrm{E}+03$ & 0.157 & 0.332 \\
\hline $\mathrm{PO}_{4}^{3-}$ & $8.40 \mathrm{E}-02$ & $6.61 \mathrm{E}+03$ & $1.06 \mathrm{E}+03$ & $3.79 \mathrm{E}-02$ & 0.113 \\
\hline $\mathrm{SO}_{4}^{2-}$ & $2.67 \mathrm{E}-03$ & 212 & 34.0 & 1.99E-03 & $3.29 \mathrm{E}-03$ \\
\hline $\mathrm{Si}\left(\right.$ as $\left.\mathrm{SiO}_{3}{ }^{2-}\right)$ & 0 & 0 & 0 & 0 & 0 \\
\hline$F$ & 1.01 & $1.59 \mathrm{E}+04$ & $2.54 \mathrm{E}+03$ & 0.180 & 2.89 \\
\hline $\mathrm{Cl}^{-}$ & $2.37 \mathrm{E}-02$ & 696 & 111 & $1.77 \mathrm{E}-02$ & $2.93 \mathrm{E}-02$ \\
\hline
\end{tabular}


HNF-1501 Rev. 0

Table A3-4. 241-T-203 Historical Tank Inventory Estimate. ${ }^{1,2}$ (4 sheets)

\begin{tabular}{|c|c|c|c|c|c|}
\hline$=0$ & (2) & Win ent & 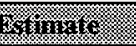 & & \\
\hline 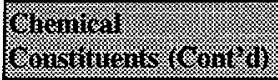 & (2) & 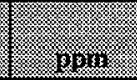 & $18=18$ & 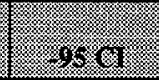 & 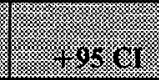 \\
\hline $\mathrm{C}_{6} \mathrm{H}_{5} \mathrm{O}_{7}^{3-}$ & 0 & 0 & 0 & 0 & 0 \\
\hline EDTA $^{4}$ & 0 & 0 & 0 & 0 & 0 \\
\hline HEDTA $^{3-}$ & 0 & 0 & 0 & 0 & 0 \\
\hline Glycolate & 0 & 0 & 0 & 0 & 0 \\
\hline Acetate- & 0 & 0 & 0 & 0 & 0 \\
\hline Oxalate $^{2-}$ & 1.06 & $7.74 \mathrm{E}+04$ & $1.24 \mathrm{E}+04$ & 1.01 & 1.09 \\
\hline DBP & 0 & 0 & 0 & 0 & 0 \\
\hline Butanol & 0 & 0 & 0 & 0 & 0 \\
\hline $\mathrm{NH}_{3}$ & $1.23 \mathrm{E}-07$ & $1.73 \mathrm{E}-03$ & $2.76 \mathrm{E}-04$ & 5.23E-08 & $2.25 \mathrm{E}-07$ \\
\hline $\mathrm{Fe}(\mathrm{CN})_{6}{ }^{4}$ & 0 & 0 & 0 & 0 & 0 \\
\hline Pordologror & 8 & 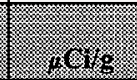 & (2) & 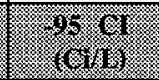 & $(=0.98 .10$ \\
\hline${ }^{3} \mathbf{H}$ & 5.31E-09 & 4.40E-06 & $7.04 \mathrm{E}-04$ & $2.94 \mathrm{E}-09$ & $8.07 \mathrm{E}-09$ \\
\hline${ }^{14} \mathrm{C}$ & $1.65 \mathrm{E}-09$ & 1.36E-06 & $2.18 \mathrm{E}-04$ & $1.23 \mathrm{E}-09$ & 2.03E-09 \\
\hline${ }^{59} \mathrm{Ni}$ & $4.68 \mathrm{E}-10$ & $3.88 \mathrm{E}-07$ & $6.20 \mathrm{E}-05$ & $3.50 \mathrm{E}-10$ & $2.17 \mathrm{E}-09$ \\
\hline${ }^{63} \mathrm{Ni}$ & 4.32E-08 & $3.57 \mathrm{E}-05$ & $5.72 \mathrm{E}-03$ & $3.23 \mathrm{E}-08$ & $2.01 \mathrm{E}-07$ \\
\hline${ }^{60} \mathrm{Co}$ & $5.29 \mathrm{E}-10$ & 4.38E-07 & 7.00E-05 & 3.95E-10 & $6.52 \mathrm{E}-10$ \\
\hline${ }^{79} \mathrm{Se}$ & 3.47E-10 & $2.88 \mathrm{E}-07$ & $4.60 \mathrm{E}-05$ & $2.60 \mathrm{E}-10$ & 4.28E-10 \\
\hline${ }^{90} \mathrm{Sr}$ & $1.72 \mathrm{E}-04$ & 0.142 & 22.8 & $1.29 \mathrm{E}-04$ & 2.12E-04 \\
\hline${ }^{90} \mathrm{Y}$ & $1.72 \mathrm{E}-04$ & 0.142 & 22.8 & $1.29 \mathrm{E}-04$ & 2.12E-04 \\
\hline${ }^{93} \mathrm{Zr}$ & $1.65 \mathrm{E}-09$ & 1.37E-06 & 2.18E-04 & 1.23E-09 & $2.03 \mathrm{E}-09$ \\
\hline${ }^{93 \mathrm{~m}} \mathrm{Nb}$ & $1.36 \mathrm{E}-09$ & $1.13 \mathrm{E}-06$ & $1.81 \mathrm{E}-04$ & $1.02 \mathrm{E}-09$ & $1.68 \mathrm{E}-09$ \\
\hline${ }^{99} \mathrm{Tc}$ & 1.14E-08 & 9.47E-06 & 1.51E-03 & $8.55 \mathrm{E}-09$ & 1.41E-08 \\
\hline${ }^{106} \mathrm{Ru}$ & $3.96 \mathrm{E}-16$ & $3.28 \mathrm{E}-13$ & $5.25 \mathrm{E}-11$ & $2.96 \mathrm{E}-16$ & $4.89 \mathrm{E}-16$ \\
\hline${ }^{113 \mathrm{~m}} \mathrm{Cd}$ & 4.62E-09 & 3.82E-06 & $6.12 \mathrm{E}-04$ & 3.45E-09 & 5.69E-09 \\
\hline${ }^{125} \mathrm{Sb}$ & $6.10 \mathrm{E}-10$ & $5.05 \mathrm{E}-07$ & 8.07E-05 & $4.56 \mathrm{E}-10$ & $7.52 \mathrm{E}-10$ \\
\hline${ }^{126} \mathrm{Sn}$ & $5.24 \mathrm{E}-10$ & 4.34E-07 & $6.94 \mathrm{E}-05$ & $3.92 \mathrm{E}-10$ & $6.46 \mathrm{E}-10$ \\
\hline${ }^{129} \mathbf{I}$ & $2.16 \mathrm{E}-11$ & 1.79E-08 & 2.86E-06 & 1.61E-11 & $2.66 \mathrm{E}-11$ \\
\hline${ }^{134} \mathrm{Cs}$ & $2.63 \mathrm{E}-11$ & $2.17 \mathrm{E}-08$ & $3.48 \mathrm{E}-06$ & $1.96 \mathrm{E}-11$ & 3.24E-11 \\
\hline
\end{tabular}


Table A3-4. 241-T-203 Historical Tank Inventory Estimate. ${ }^{1,2}$ (4 sheets)

\begin{tabular}{|c|c|c|c|c|c|}
\hline & 8 & Irrention: & Sitininter: & & \\
\hline Krolinibgical : & & & & 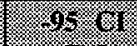 & 1.193 .0$. \\
\hline Sonstitivens (coitit) & (1) & A & 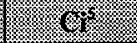 & 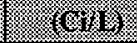 & $(\ln (1))$ \\
\hline${ }^{137} \mathrm{Cs}$ & $1.95 \mathrm{E}-04$ & 0.162 & 25.8 & $1.46 \mathrm{E}-04$ & 2.41E-04 \\
\hline${ }^{137 m} \mathrm{Ba}$ & $1.85 \mathrm{E}-04$ & 0.153 & 24.4 & $1.38 \mathrm{E}-04$ & $2.28 \mathrm{E}-04$ \\
\hline${ }^{151} \mathrm{Sm}$ & $1.31 \mathrm{E}-06$ & $1.09 \mathrm{E}-03$ & 0.174 & 9.82E-07 & $1.62 \mathrm{E}-06$ \\
\hline${ }^{152} \mathrm{Eu}$ & 1.72E-09 & $1.42 \mathrm{E}-06$ & $2.28 \mathrm{E}-04$ & 1.71E-09 & $1.73 \mathrm{E}-09$ \\
\hline${ }^{154} \mathrm{Eu}$ & $8.48 \mathrm{E}-09$ & $7.02 \mathrm{E}-06$ & $1.12 \mathrm{E}-03$ & $6.34 \mathrm{E}-09$ & 1.05E-08 \\
\hline${ }^{155} \mathrm{Eu}$ & $1.55 \mathrm{E}-07$ & $1.29 \mathrm{E}-04$ & $2.06 \mathrm{E}-02$ & $1.54 \mathrm{E}-07$ & $1.56 \mathrm{E}-07$ \\
\hline${ }^{226} \mathrm{Ra}$ & 7.76E-14 & $6.42 \mathrm{E}-11$ & $1.03 \mathrm{E}-08$ & $5.80 \mathrm{E}-14$ & $9.56 \mathrm{E}-14$ \\
\hline${ }^{228} \mathrm{Ra}$ & $4.99 \mathrm{E}-18$ & $4.13 \mathrm{E}-15$ & $6.61 \mathrm{E}-13$ & $4.94 \mathrm{E}-18$ & $5.03 \mathrm{E}-18$ \\
\hline${ }^{227} \mathrm{Ac}$ & $4.09 \mathrm{E}-13$ & $3.39 \mathrm{E}-10$ & 5.42E-08 & $3.06 \mathrm{E}-13$ & $5.05 \mathrm{E}-13$ \\
\hline${ }^{231} \mathrm{~Pa}$ & $9.44 \mathrm{E}-13$ & $7.82 \mathrm{E}-10$ & $1.25 \mathrm{E}-07$ & $7.06 \mathrm{E}-13$ & $1.16 \mathrm{E}-12$ \\
\hline${ }^{229} \mathrm{Th}$ & $9.65 \mathrm{E}-16$ & $7.99 \mathrm{E}-13$ & $1.28 \mathrm{E}-10$ & $9.57 \mathrm{E}-16$ & 9.72E-16 \\
\hline${ }^{232} \mathrm{Th}$ & $4.36 \mathrm{E}-19$ & $3.61 \mathrm{E}-16$ & $5.77 \mathrm{E}-14$ & $3.26 \mathrm{E}-19$ & $5.37 \mathrm{E}-19$ \\
\hline${ }^{232} \mathrm{U}$ & $5.06 \mathrm{E}-13$ & 4.19E-10 & $6.70 \mathrm{E}-08$ & $3.78 \mathrm{E}-13$ & $6.24 \mathrm{E}-13$ \\
\hline${ }^{233} \mathrm{U}$ & $2.31 \mathrm{E}-14$ & $1.91 \mathrm{E}-11$ & $3.06 \mathrm{E}-09$ & $1.72 \mathrm{E}-14$ & $2.85 \mathrm{E}-14$ \\
\hline${ }^{234} \mathrm{U}$ & $2.52 \mathrm{E}-08$ & $2.09 \mathrm{E}-05$ & $3.34 \mathrm{E}-03$ & $1.89 \mathrm{E}-08$ & 3.11E-08 \\
\hline${ }^{235} \mathrm{U}$ & 1.12E-09 & $9.29 \mathrm{E}-07$ & 1.49E-04 & 8.39E-10 & $1.38 \mathrm{E}-09$ \\
\hline${ }^{236} \mathrm{U}$ & $2.20 \mathrm{E}-10$ & $1.82 \mathrm{E}-07$ & $2.91 \mathrm{E}-05$ & $1.64 \mathrm{E}-10$ & 2.71E-10 \\
\hline${ }^{238} \mathrm{U}$ & $2.56 \mathrm{E}-08$ & $2.12 \mathrm{E}-05$ & 3.39E-03 & $1.91 \mathrm{E}-08$ & $3.16 \mathrm{E}-08$ \\
\hline${ }^{237} \mathrm{~Np}$ & $7.09 \mathrm{E}-11$ & 5.87E-08 & $9.39 \mathrm{E}-06$ & 5.30E-11 & 8.74E-11 \\
\hline${ }^{238} \mathrm{Pu}$ & 3.03E-09 & $2.51 \mathrm{E}-06$ & $4.01 \mathrm{E}-04$ & $2.26 \mathrm{E}-09$ & 3.73E-09 \\
\hline${ }^{239} \mathrm{Pu}$ & 4.38E-07 & 3.63E-04 & $5.80 \mathrm{E}-02$ & 3.27E-07 & 5.40E-07 \\
\hline${ }^{240} \mathrm{Pu}$ & $3.84 \mathrm{E}-08$ & $3.18 \mathrm{E}-05$ & $5.09 \mathrm{E}-03$ & 2.87E-08 & 4.74E-08 \\
\hline${ }^{241} \mathrm{Pu}$ & $1.27 \mathrm{E}-07$ & $1.05 \mathrm{E}-04$ & $1.68 \mathrm{E}-02$ & $9.50 \mathrm{E}-08$ & $1.57 \mathrm{E}-07$ \\
\hline${ }^{242} \mathrm{Pu}$ & $5.88 \mathrm{E}-13$ & $4.87 \mathrm{E}-10$ & 7.79E-08 & $4.39 \mathrm{E}-13$ & $7.25 \mathrm{E}-13$ \\
\hline${ }^{241} \mathrm{Am}$ & 3.58E-09 & $2.97 \mathrm{E}-06$ & 4.74E-04 & $2.68 \mathrm{E}-09$ & 4.42E-09 \\
\hline${ }^{243} \mathrm{Am}$ & 2.91E-14 & $2.41 \mathrm{E}-11$ & $3.86 \mathrm{E}-09$ & $2.18 \mathrm{E}-14$ & 3.59E-14 \\
\hline${ }^{242} \mathrm{Cm}$ & $3.50 \mathrm{E}-11$ & $2.89 \mathrm{E}-08$ & $4.63 \mathrm{E}-06$ & 3.47E-11 & $3.52 \mathrm{E}-11$ \\
\hline${ }^{243} \mathrm{Cm}$ & $7.53 \mathrm{E}-13$ & $6.24 \mathrm{E}-10$ & $9.98 \mathrm{E}-08$ & $7.47 \mathrm{E}-13$ & $7.59 \mathrm{E}-13$ \\
\hline${ }^{244} \mathrm{Cm}$ & $7.40 \mathrm{E}-13$ & $6.13 \mathrm{E}-10$ & $9.80 \mathrm{E}-08$ & $5.53 \mathrm{E}-13$ & $9.12 \mathrm{E}-13$ \\
\hline
\end{tabular}


Table A3-4. 241-T-203 Historical Tank Inventory Estimate. ${ }^{1,2}$ (4 sheets)

\begin{tabular}{|c|c|c|c|c|c|}
\hline \multicolumn{6}{|c|}{ 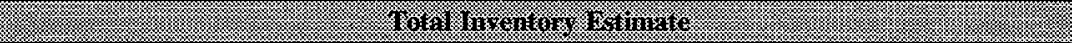 } \\
\hline (36) & 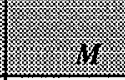 & 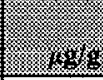 & 83 & 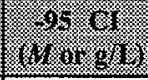 & 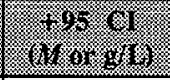 \\
\hline $\mathrm{Pu}$ & $\begin{array}{l}7.22 \mathrm{E}-06 \\
(\mathrm{~g} / \mathrm{L})\end{array}$ & --- & $9.56 \mathrm{E}-04$ & 5.39E-06 & $8.90 \mathrm{E}-06$ \\
\hline $\mathrm{U}$ & $3.22 \mathrm{E}-04$ & 63.5 & 10.2 & $2.41 \mathrm{E}-04$ & 3.97E-04 \\
\hline
\end{tabular}

Notes:

$\mathrm{CI}=$ confidence interval

${ }^{1}$ Agnew et al. (1997a)

${ }^{2}$ These predictions have not been validated and should be used with caution.

${ }^{3}$ This is the volume average for density, mass average water wt\% and TOC wt\% carbon.

"Differences exist among the inventories in this column and the inventories calculated from the two sets of concentrations.

SUnknowns in tank solids inventory are assigned by the TLM. 
Table A3-5. 241-T-204 Historical Tank Inventory Estimate. ${ }^{1,2}$ (4 sheets)

\begin{tabular}{|c|c|c|c|c|c|}
\hline \multicolumn{6}{|c|}{ 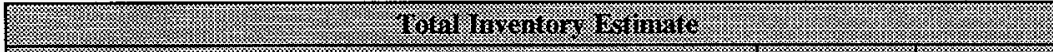 } \\
\hline \multicolumn{4}{|c|}{ 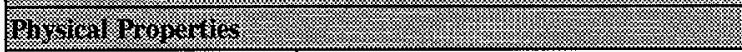 } & 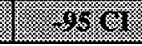 & 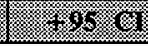 \\
\hline Total waste & $1.74 \mathrm{E}+05(\mathrm{~kg})$ & ) $(38.0 \mathrm{kgal}$ & & & \\
\hline Heat load & 2.98E-04 (kW) & $(1.02 \mathrm{Btu} /$ & & 2.23E-04 & $3.68 \mathrm{E}-04$ \\
\hline Bulk density ${ }^{3}$ & $1.21\left(\mathrm{~g} / \mathrm{cm}^{3}\right)$ & & & 1.15 & 1.26 \\
\hline Water wt $\%^{3}$ & 68.6 & & & 62.1 & 75.2 \\
\hline TOC wt $\% \mathrm{C}$ (wet) $^{3}$ & 2.11 & & & 2.03 & 2.14 \\
\hline 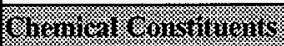 & 18 & (1) & 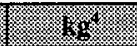 & 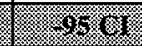 & (19.8. \\
\hline $\mathrm{Na}^{+}$ & 4.23 & $8.06 \mathrm{E}+04$ & $1.40 \mathrm{E}+04$ & 3.03 & 6.08 \\
\hline $\mathrm{Al}^{3+}$ & 0 & 0 & 0 & 0 & 0 \\
\hline $\mathrm{Fe}^{3+}($ total $\mathrm{Fe})$ & 0.363 & $1.68 \mathrm{E}+04$ & $2.91 \mathrm{E}+03$ & 0.341 & 0.385 \\
\hline $\mathrm{Cr}^{3+}$ & $6.05 \mathrm{E}-03$ & 260 & 45.2 & $4.52 \mathrm{E}-03$ & $7.46 \mathrm{E}-03$ \\
\hline $\mathrm{Bi}^{3+}$ & $5.55 \mathrm{E}-02$ & $9.61 \mathrm{E}+03$ & $1.67 \mathrm{E}+03$ & $1.66 \mathrm{E}-02$ & $7.77 \mathrm{E}-02$ \\
\hline $\mathrm{La}^{3+}$ & $3.38 \mathrm{E}-03$ & 389 & 67.5 & 2.53E-03 & $4.17 \mathrm{E}-03$ \\
\hline $\mathrm{Hg}^{2+}$ & 0 & 0 & 0 & 0 & 0 \\
\hline $\mathrm{Zr}\left(\right.$ as $\left.\mathrm{ZrO}(\mathrm{OH})_{2}\right)$ & 0 & 0 & 0 & 0 & 0 \\
\hline $\mathrm{Pb}^{2+}$ & 0 & 0 & 0 & 0 & 0 \\
\hline $\mathrm{Ni}^{2+}$ & $1.42 \mathrm{E}-03$ & 69.2 & 12.0 & $1.06 \mathrm{E}-03$ & $6.61 \mathrm{E}-03$ \\
\hline $\mathrm{Sr}^{2+}$ & 0 & 0 & 0 & 0 & 0 \\
\hline $\mathrm{Mn}^{4+}$ & $4.54 \mathrm{E}-03$ & 206 & 35.8 & 3.39E-03 & $5.59 \mathrm{E}-03$ \\
\hline $\mathrm{Ca}^{2+}$ & 0.244 & $8.11 \mathrm{E}+03$ & $1.41 \mathrm{E}+03$ & 0.157 & 0.332 \\
\hline $\mathrm{K}^{+}$ & 0.205 & $6.65 \mathrm{E}+03$ & $1.15 \mathrm{E}+03$ & 0.154 & 0.253 \\
\hline$\overline{\mathrm{OH}^{-}}$ & 1.10 & $1.55 \mathrm{E}+04$ & $2.69 \mathrm{E}+03$ & 1.04 & 1.17 \\
\hline $\mathrm{NO}^{3-}$ & 1.23 & $6.29 E+04$ & $1.09 \mathrm{E}+04$ & 0.917 & 1.51 \\
\hline $\mathrm{NO}^{2-}$ & 3.20E-03 & 122 & 21.2 & $1.76 \mathrm{E}-03$ & $4.97 \mathrm{E}-03$ \\
\hline $\mathrm{CO}_{3}{ }^{2-}$ & 0.244 & $1.21 \mathrm{E}+04$ & $2.11 \mathrm{E}+03$ & 0.157 & 0.332 \\
\hline $\mathrm{PO}_{4}^{3-2}$ & $8.40 \mathrm{E}-02$ & $6.61 \mathrm{E}+03$ & $1.15 \mathrm{E}+03$ & $3.79 \mathrm{E}-02$ & 0.113 \\
\hline $\mathrm{SO}_{4}^{2-}$ & $2.67 \mathrm{E}-03$ & 212 & 36.9 & $1.99 \mathrm{E}-03$ & $3.29 \mathrm{E}-03$ \\
\hline $\mathrm{Si}\left(\right.$ as $\left.\mathrm{SiO}_{3}{ }^{2-}\right)$ & 0 & 0 & 0 & 0 & 0 \\
\hline $\mathrm{F}^{-}$ & 1.01 & $1.59 \mathrm{E}+04$ & $2.76 \mathrm{E}+03$ & 0.180 & 2.89 \\
\hline $\mathrm{Cl}^{-}$ & $2.37 \mathrm{E}-02$ & 696 & 121 & $1.77 \mathrm{E}-02$ & $2.93 \mathrm{E}-02$ \\
\hline $\mathrm{C}_{6} \mathrm{H}_{5} \mathrm{O}_{7}{ }^{3-}$ & 0 & 0 & 0 & 0 & 0 \\
\hline
\end{tabular}


Table A3-5. 241-T-204 Historical Tank Inventory Estimate. ${ }^{1,2}$ (4 sheets)

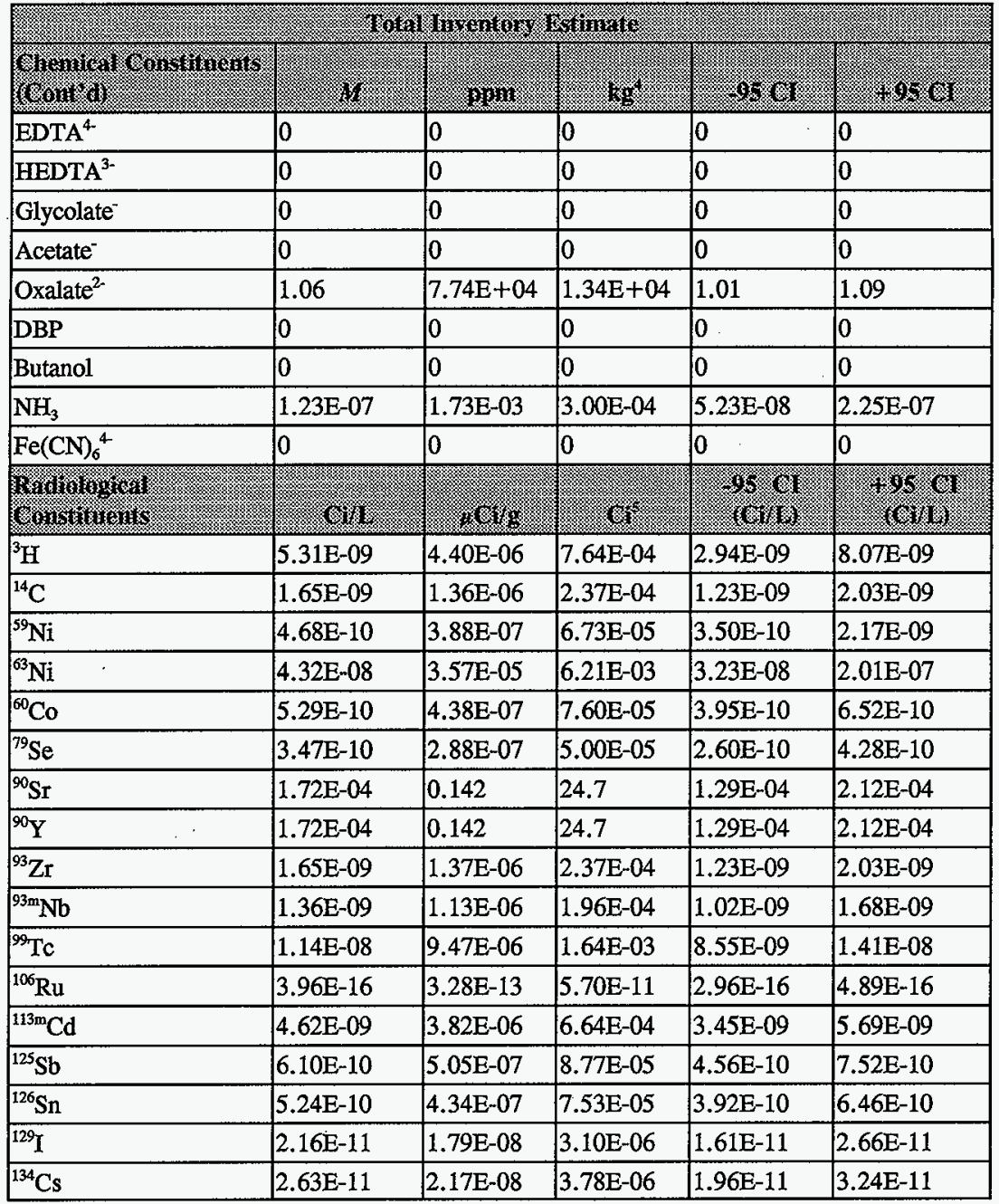


Table A3-5. 241-T-204 Historical Tank Inventory Estimate., ${ }^{1,2}$ (4 sheets)

\begin{tabular}{|c|c|c|c|c|c|}
\hline$\sqrt{18}$ & 18 & Inrenter & 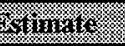 & & \\
\hline 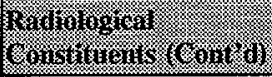 & 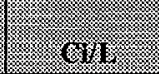 & 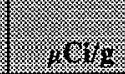 & 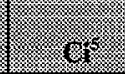 & 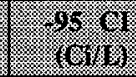 & (3) \\
\hline${ }^{137} \mathrm{Cs}$ & 1.95E-04 & 0.162 & 28.1 & $1.46 \mathrm{E}-04$ & 2.41E-04 \\
\hline${ }^{137 \mathrm{~m}} \mathrm{Ba}$ & $1.85 \mathrm{E}-04$ & 0.153 & 26.5 & $1.38 \mathrm{E}-04$ & 2.28E-04 \\
\hline${ }^{151} \mathrm{Sm}$ & 1.31E-06 & $1.09 \mathrm{E}-03$ & 0.189 & 9.82E-07 & $1.62 \mathrm{E}-06$ \\
\hline${ }^{152} \mathrm{Eu}$ & $1.72 \mathrm{E}-09$ & $1.42 \mathrm{E}-06$ & 2.47E-04 & $1.71 \mathrm{E}-09$ & $1.73 \mathrm{E}-09$ \\
\hline${ }^{154} \mathrm{Eu}$ & 8.48E-09 & $7.02 \mathrm{E}-06$ & $1.22 \mathrm{E}-03$ & $6.34 \mathrm{E}-09$ & $1.05 \mathrm{E}-08$ \\
\hline${ }^{155} \mathrm{Eu}$ & 1.55E-07 & $1.29 \mathrm{E}-04$ & 2.23E-02 & 1.54E-07 & $1.56 \mathrm{E}-07$ \\
\hline${ }^{226} \mathrm{Ra}$ & $7.76 \mathrm{E}-14$ & $6.42 \mathrm{E}-11$ & $1.12 \mathrm{E}-08$ & $5.80 \mathrm{E}-14$ & $9.56 \mathrm{E}-14$ \\
\hline${ }^{228} \mathrm{Ra}$ & $4.99 \mathrm{E}-18$ & $4.13 \mathrm{E}-15$ & $7.17 \mathrm{E}-13$ & $4.94 \mathrm{E}-18$ & $5.03 \mathrm{E}-18$ \\
\hline${ }^{229} \mathrm{Ac}$ & $4.09 \mathrm{E}-13$ & $3.39 \mathrm{E}-10$ & $5.89 \mathrm{E}-08$ & $3.06 \mathrm{E}-13$ & $5.05 \mathrm{E}-13$ \\
\hline${ }^{231} \mathrm{~Pa}$ & $9.44 \mathrm{E}-13$ & $7.82 \mathrm{E}-10$ & $1.36 \mathrm{E}-07$ & $7.06 \mathrm{E}-13$ & $1.16 \mathrm{E}-12$ \\
\hline${ }^{229} \mathrm{Th}$ & $9.65 \mathrm{E}-16$ & $7.99 \mathrm{E}-13$ & $1.39 \mathrm{E}-10$ & 9.57E-16 & $9.72 \mathrm{E}-16$ \\
\hline${ }^{232} \mathrm{Th}$ & $4.36 \mathrm{E}-19$ & $3.61 \mathrm{E}-16$ & 6.27E-14 & $3.26 \mathrm{E}-19$ & 5.37E-19 \\
\hline${ }^{232} \mathrm{U}$ & $5.06 \mathrm{E}-13$ & $4.19 \mathrm{E}-10$ & 7.27E-08 & $3.78 \mathrm{E}-13$ & $6.24 \mathrm{E}-13$ \\
\hline${ }^{233} \mathrm{U}$ & 2.31E-14 & $1.91 \mathrm{E}-11$ & 3.32E-09 & $1.72 \mathrm{E}-14$ & $2.85 \mathrm{E}-14$ \\
\hline${ }^{234} \mathrm{U}$ & $2.52 \mathrm{E}-08$ & $2.09 \mathrm{E}-05$ & $3.63 \mathrm{E}-03$ & $1.89 \mathrm{E}-08$ & $3.11 \mathrm{E}-08$ \\
\hline${ }^{235} U$ & $1.12 \mathrm{E}-09$ & $9.29 \mathrm{E}-07$ & $1.61 \mathrm{E}-04$ & $8.39 \mathrm{E}-10$ & 1.38E-09 \\
\hline${ }^{236} \mathrm{U}$ & $2.20 \mathrm{E}-10$ & $1.82 \mathrm{E}-07$ & $3.16 \mathrm{E}-05$ & $1.64 \mathrm{E}-10$ & $2.71 \mathrm{E}-10$ \\
\hline${ }^{238} \mathrm{U}$ & $2.56 \mathrm{E}-08$ & $2.12 \mathrm{E}-05$ & $3.68 \mathrm{E}-03$ & $1.91 \mathrm{E}-08$ & $3.16 \mathrm{E}-08$ \\
\hline${ }^{237} \mathrm{~Np}$ & $7.09 \mathrm{E}-11$ & $5.87 \mathrm{E}-08$ & $1.02 \mathrm{E}-05$ & $5.30 \mathrm{E}-11$ & $8.74 \mathrm{E}-11$ \\
\hline${ }^{238} \mathrm{Pu}$. & 3.03E-09 & $2.51 \mathrm{E}-06$ & $4.35 \mathrm{E}-04$ & 2.26E-09 & 3.73E-09 \\
\hline${ }^{239} \mathrm{Pu}$ & 4.38E-07 & 3.63E-04 & $6.30 \mathrm{E}-02$ & 3.27E-07 & 5.40E-07 \\
\hline${ }^{240} \mathrm{Pu}$ & $3.84 \mathrm{E}-08$ & $3.18 \mathrm{E}-05$ & $5.53 \mathrm{E}-03$ & 2.87E-08 & $4.74 \mathrm{E}-08$ \\
\hline${ }^{24 !} \mathrm{Pu}$ & 1.27E-07 & $1.05 \mathrm{E}-04$ & $1.83 \mathrm{E}-02$ & $9.50 \mathrm{E}-08$ & $1.57 \mathrm{E}-07$ \\
\hline${ }^{242} \mathrm{Pu}$ & $5.88 \mathrm{E}-13$ & $4.87 \mathrm{E}-10$ & $8.45 \mathrm{E}-08$ & $4.39 \mathrm{E}-13$ & $7.25 \mathrm{E}-13$ \\
\hline${ }^{241} \mathrm{Am}$ & 3.58E-09 & $2.97 \mathrm{E}-06$ & $5.15 \mathrm{E}-04$ & 2.68E-09 & $4.42 E-09$ \\
\hline${ }^{243} \mathrm{Am}$ & $2.91 \mathbf{E}-14$ & $2.41 \mathrm{E}-11$ & 4.19E-09 & $2.18 \mathrm{E}-14$ & $3.59 \mathrm{E}-14$ \\
\hline${ }^{242} \mathrm{Cm}$ & 3.50E-11 & $2.89 \mathrm{E}-08$ & $5.03 \mathrm{E}-06$ & $3.47 \mathrm{E}-11$ & $3.52 \mathrm{E}-11$ \\
\hline${ }^{243} \mathrm{Cm}$ & $7.53 \mathrm{E}-13$ & $6.24 \mathrm{E}-10$ & $1.08 \mathrm{E}-07$ & $7.47 \mathrm{E}-13$ & $7.59 \mathrm{E}-13$ \\
\hline${ }^{244} \mathrm{Cm}$ & $7.40 \mathrm{E}-13$ & $6.13 E-10$ & $1.06 \mathrm{E}-07$ & $5.53 \mathrm{E}-13$ & 9.12E-13 \\
\hline
\end{tabular}


Table A3-5. 241-T-204 Historical Tank Inventory Estimate. ${ }^{1,2}$ (4 sheets)

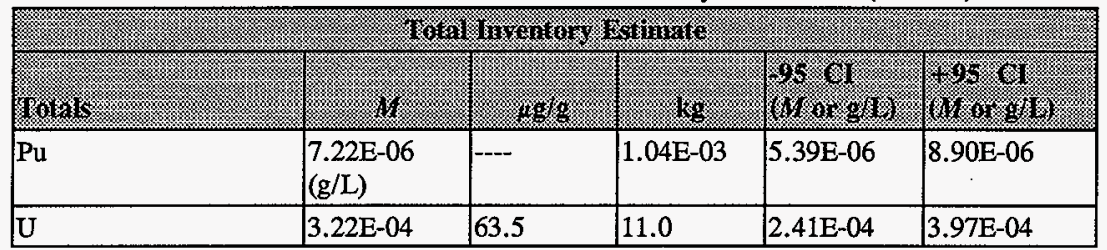

Notes:

CI $=$ confidence interval

${ }^{1}$ Agnew et al. (1997a)

${ }^{2}$ These predictions have not been validated and should be used with caution.

${ }^{3}$ This is the volume average for density, mass average water wt $\%$ and TOC wt $\%$ carbon.

${ }^{4}$ Differences exist among the inventories in this column and the inventories calculated from the two sets of concentrations.

${ }^{5}$ Unknowns in tank solids inventory are assigned by the TLM. 


\section{A4.0 SURVEILANCE DATA}

The surveillance efforts in place on the T-200 series tanks consist of surface-level measurements (liquid and solid) and temperature monitoring inside the tank (waste and headspace). Surveillance data provide the basis for determining tank integrity. Liquid-level measurements can indicate whether the tank has a major leak. Solid surface-level measurements can indicate physical changes in and consistencies of the solid layers of a tank.

\section{A4.1 SURFACE-LEVEL, READINGS}

None of the T-200 series tanks are considered leakers. A manual tape is used to monitor the surface level. Riser 4 was used in all tanks as the location of the manual tape. The surface-level plots for each tank indicate a steady waste level from January 1991 to January 1996. The waste surface level on April 1, 1997, was $412.12 \mathrm{~cm}$ (162.5 in.), $266.07 \mathrm{~cm}$ (104.75 in.), $478.16 \mathrm{~cm}$ (188.25 in.), and $492.76 \mathrm{~cm}$ (194 in:) for tanks 241-T-201, -T-202, -T-203, and -T-204, respectively. Figures A4-1 to A4-4 are level history graphs of the volume measurements. The T-200 series tanks have no liquid observation wells, but they have three identified dry wells.

\section{A4.2 INTERNAL TANK TEMPERATURES}

Each T-200 series tank has a single thermocouple tree with 11 thermocouples to monitor the waste temperature through risers 5, 5, 8, and 8 for tanks 241-T-201, T-202, T-203, and $\mathrm{T}-204$, respectively. The configuration of the thermocouple tree (that is, the spacing of the thermocouples and the distance from the end of the tree to the bottom of the tank) is unclear.

Intermittent tank data for each tank was recorded from 1975 to 1991 and was available from the surveillance analysis computer system. Within this time span, several large breaks occurred in the temperature data sequence for all thermocouples. Continuous temperature data has been available from approximately mid-1994 to January 1998.

The average tank temperature for each tank is 16 to $17^{\circ} \mathrm{C}\left(62\right.$ to $\left.63{ }^{\circ} \mathrm{F}\right)$, the minimums range from 6 to $11^{\circ} \mathrm{C}\left(44\right.$ to $\left.52{ }^{\circ} \mathrm{F}\right)$, and the maximum ranges from 22 to $27^{\circ} \mathrm{C}\left(72\right.$ to $\left.81{ }^{\circ} \mathrm{F}\right)$. For plots of the thermocouple readings, refer to the supporting document for the HTCE (Brevick et al. 1997a). Figures A4-5 to A4-8 are graphs of the weekly high temperature. 


\section{A4.3 TANK 241-T-201, -T-202, -T-203, AND -T-204 PHOTOGRAPHS}

Each tank has a separate photograph. The waste in tank 241-T-201 appears different from the other T-200 series tanks. It has a dried, cracked, rust-brown surface. There is a blue-black region that appears to have standing liquid present. The other T-200 series tanks appear to lack any free liquid and have dried, cracked gray-brown or gray-black surfaces.

A temperature probe, salt well screen, and a manual tape are also visible in the various photographs. The photographs were taken in 1986 and 1989 (Brevick et al. 1997b). 
Figure A4-1. Tank 241-T-201 Level History.

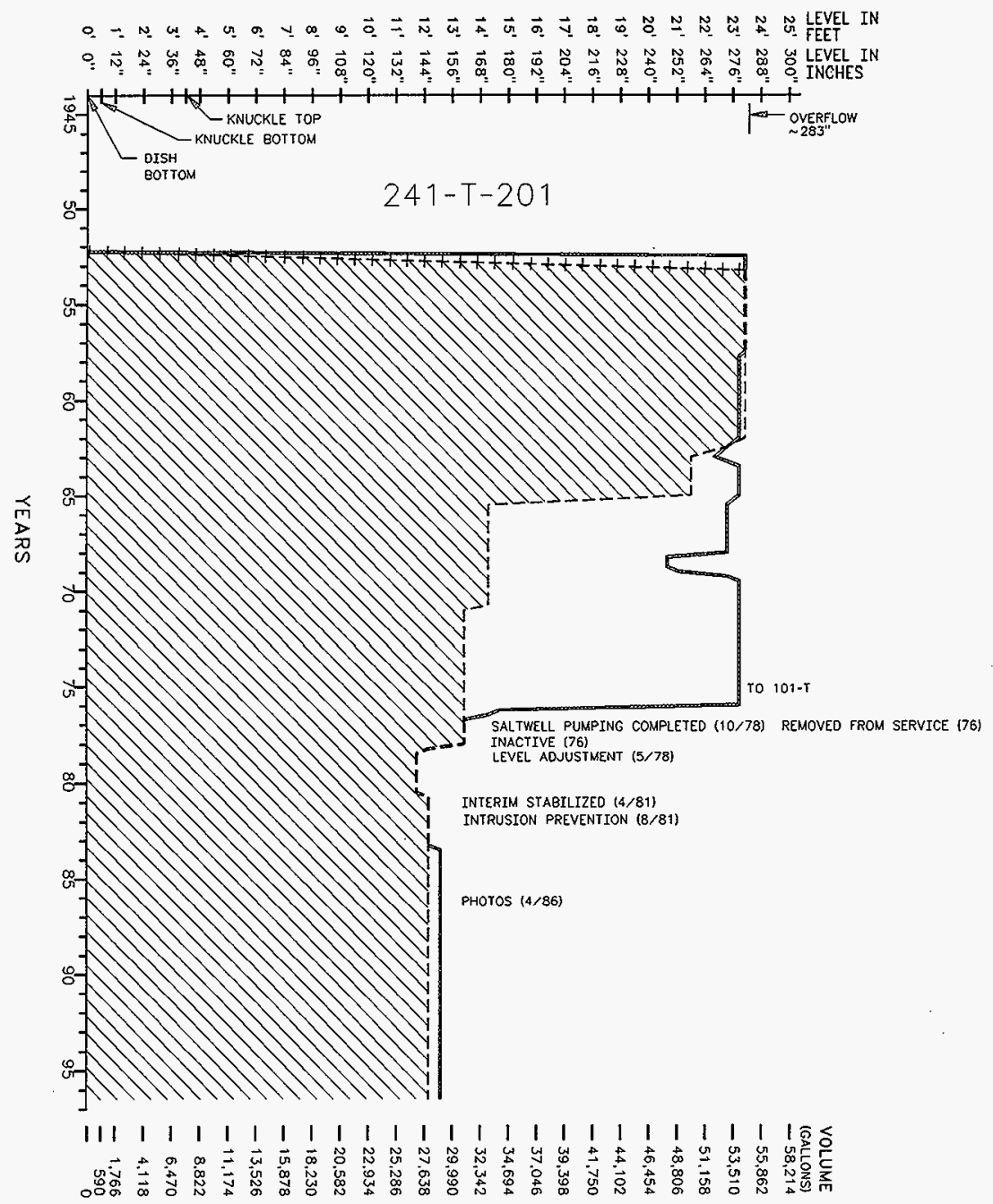


Figure A4-2. Tank 241-T-202 Level History.

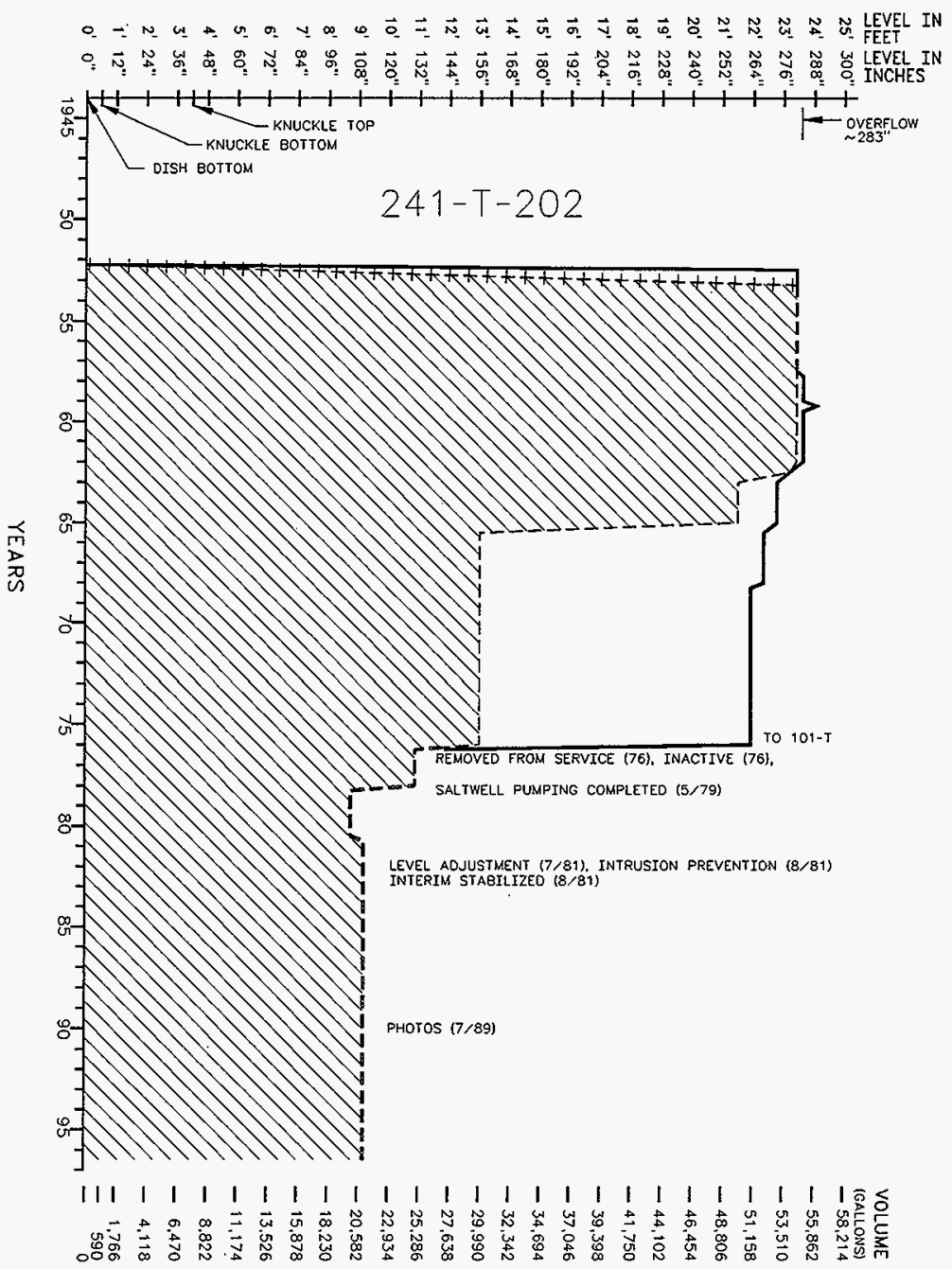


Figure A4-3. Tank 241-T-203 Level History.

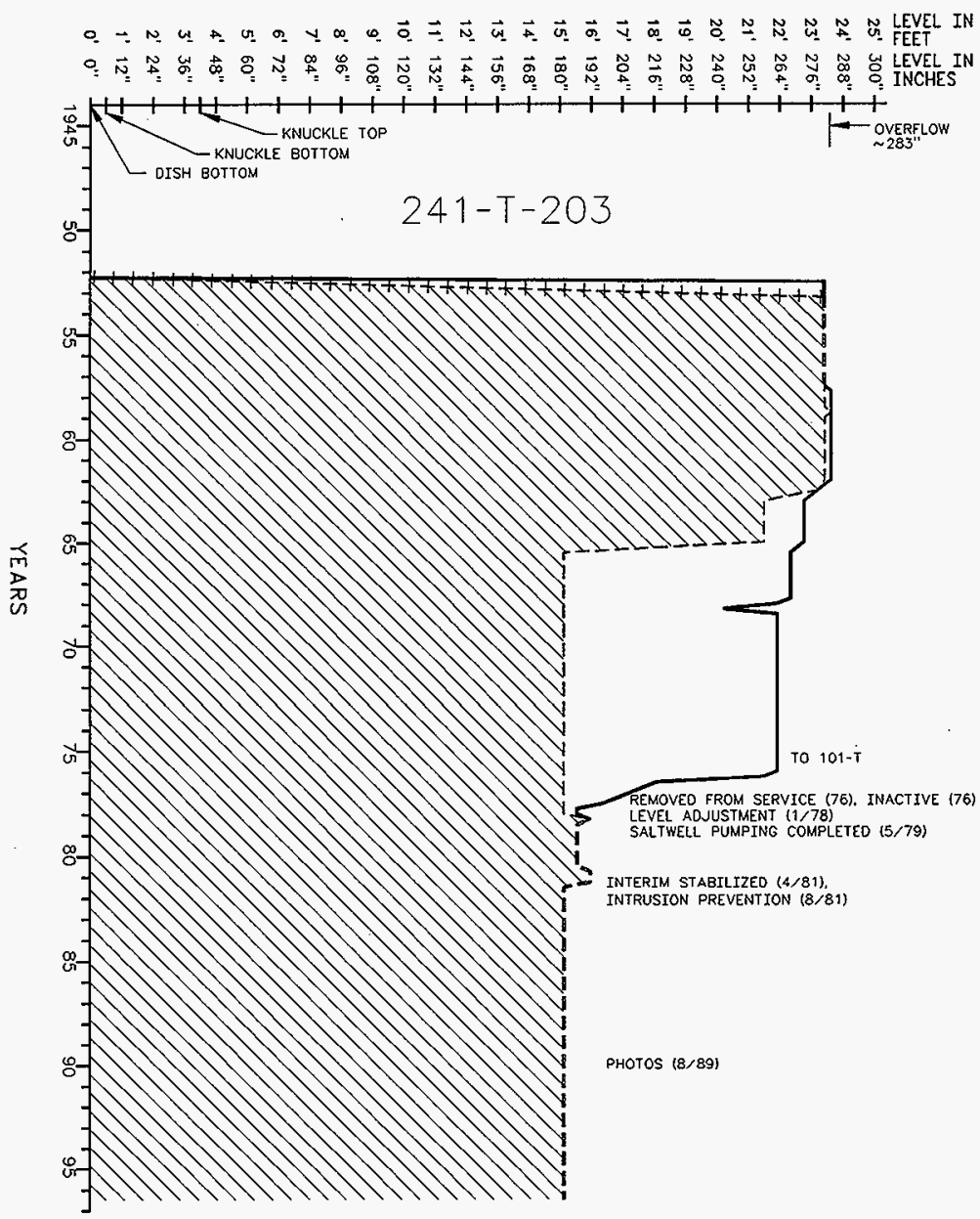

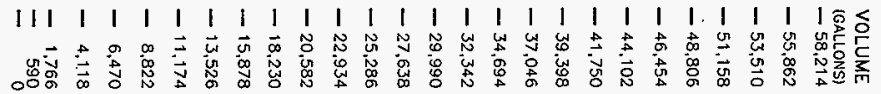


Figure A4-4. Tank 241-T-204 Level History.

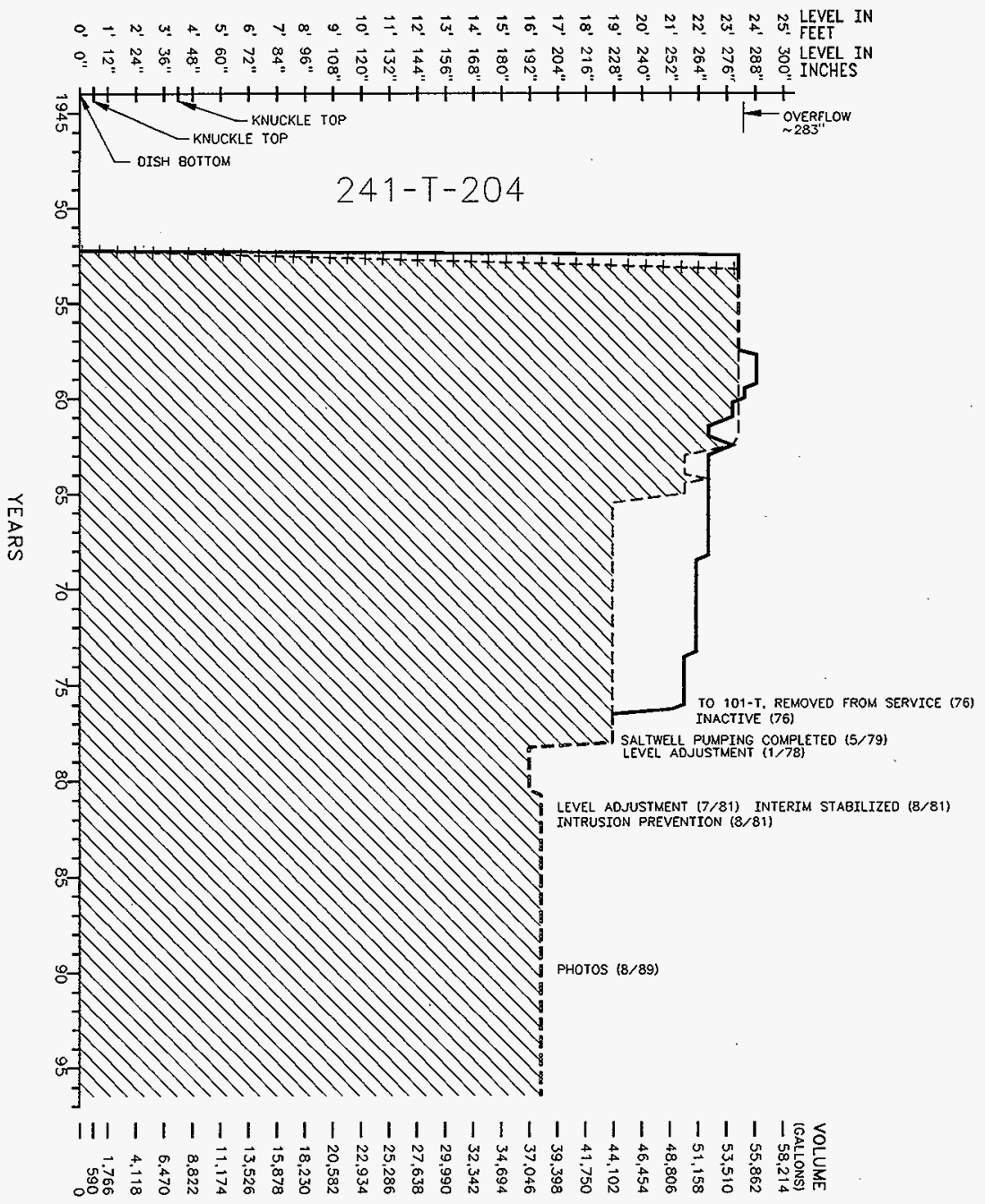


Figure A4-5. Tank 241-T-201 High Temperature Plot.

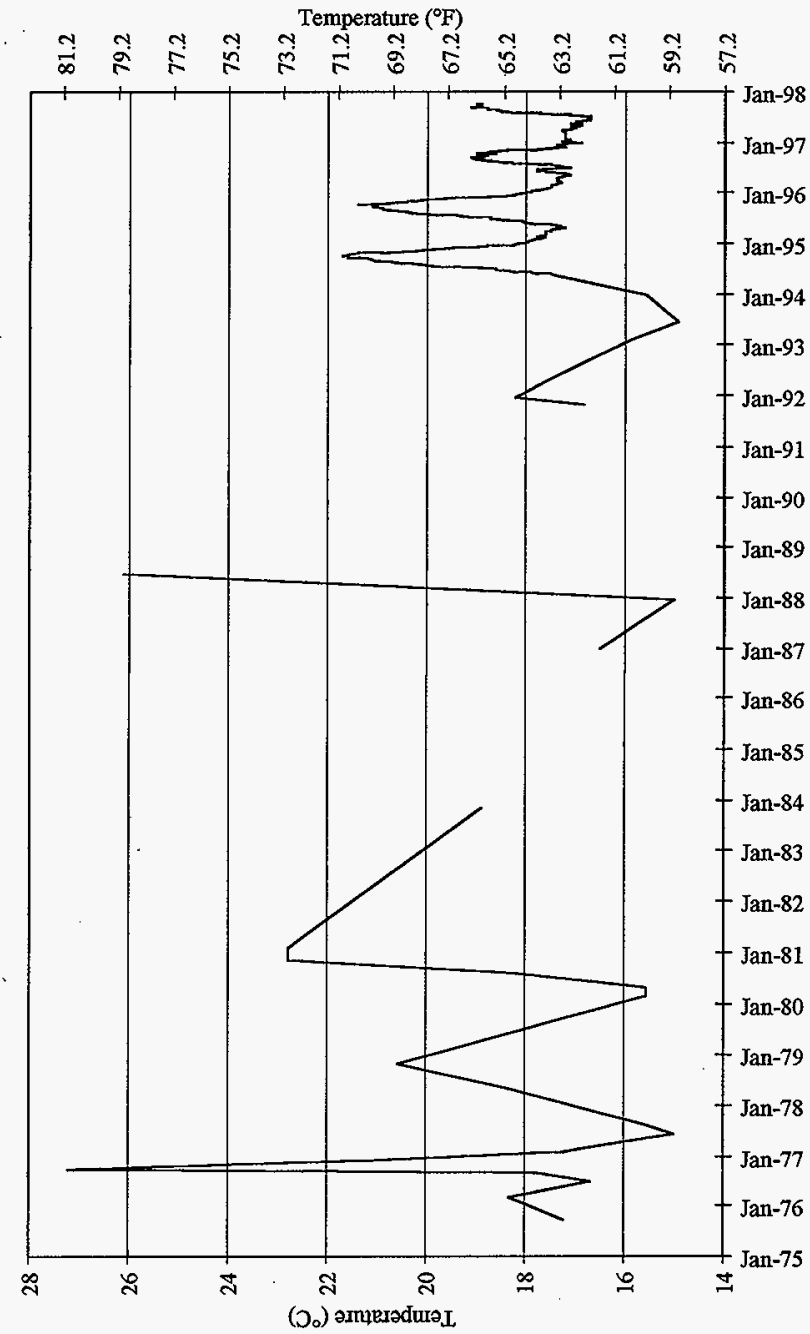


Figure A4-6. Tank 241-T-202 High Temperature Plot.

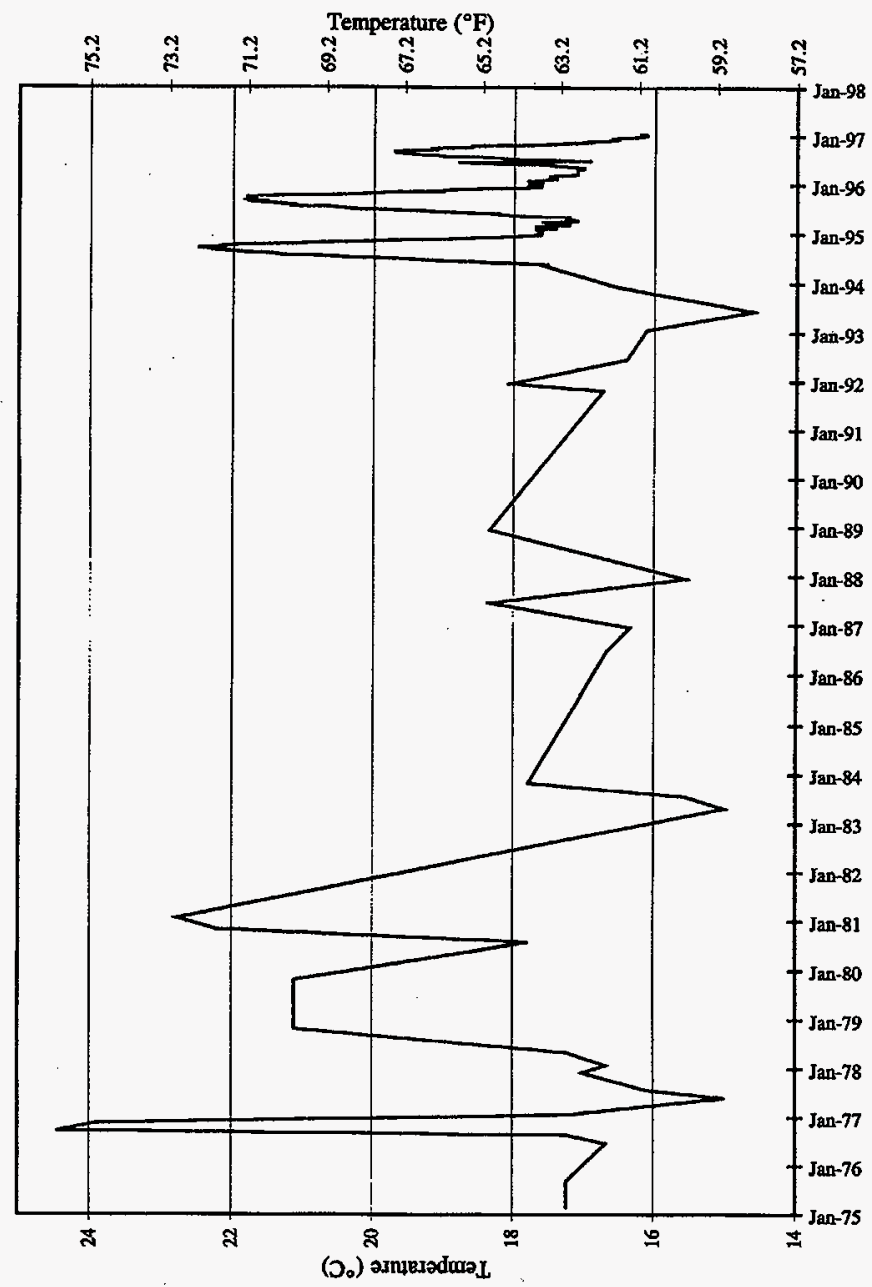


Figure A4-7. Tank 241-T-203 High Temperature Plot.

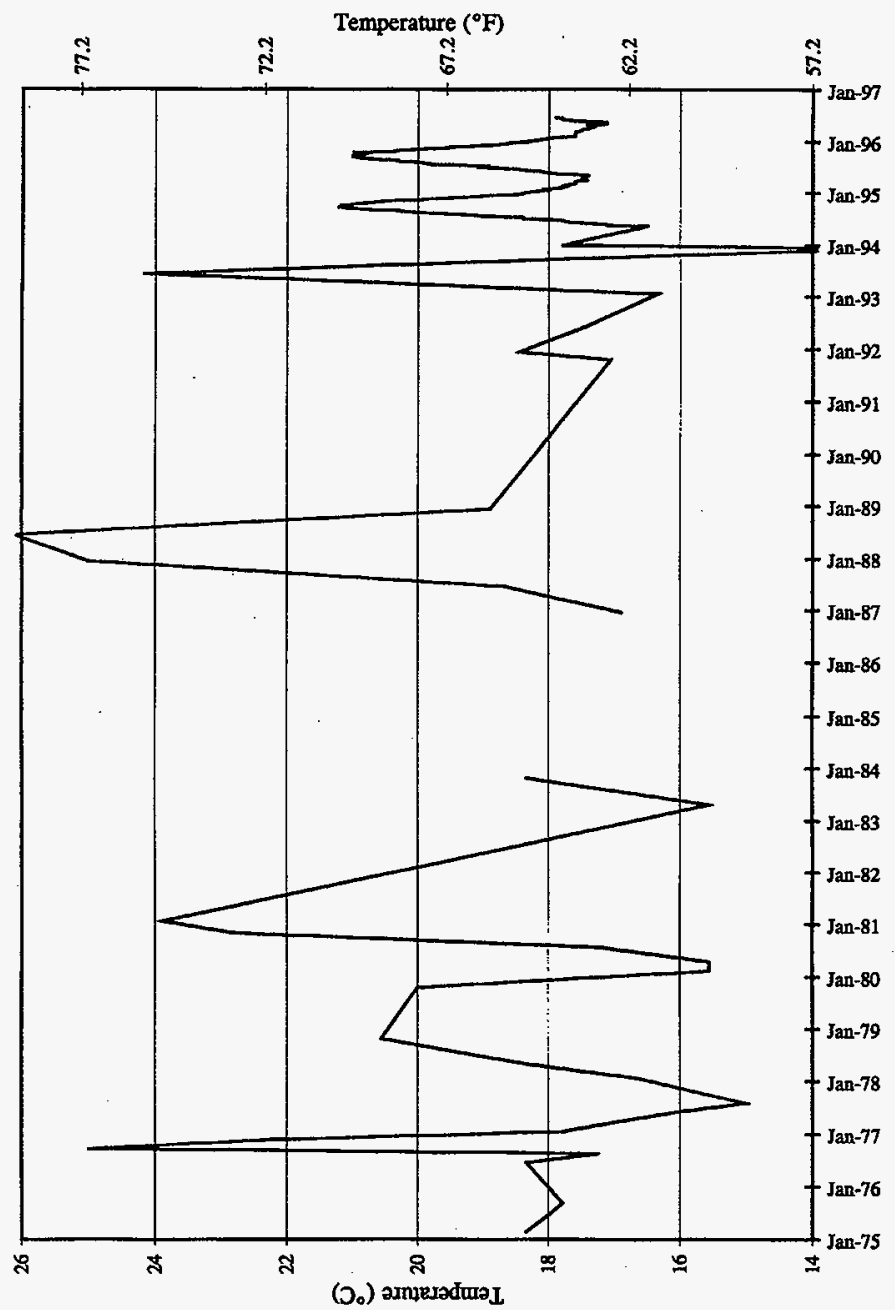


Figure A4-8. Tank 241-T-204 High Temperature Plot.

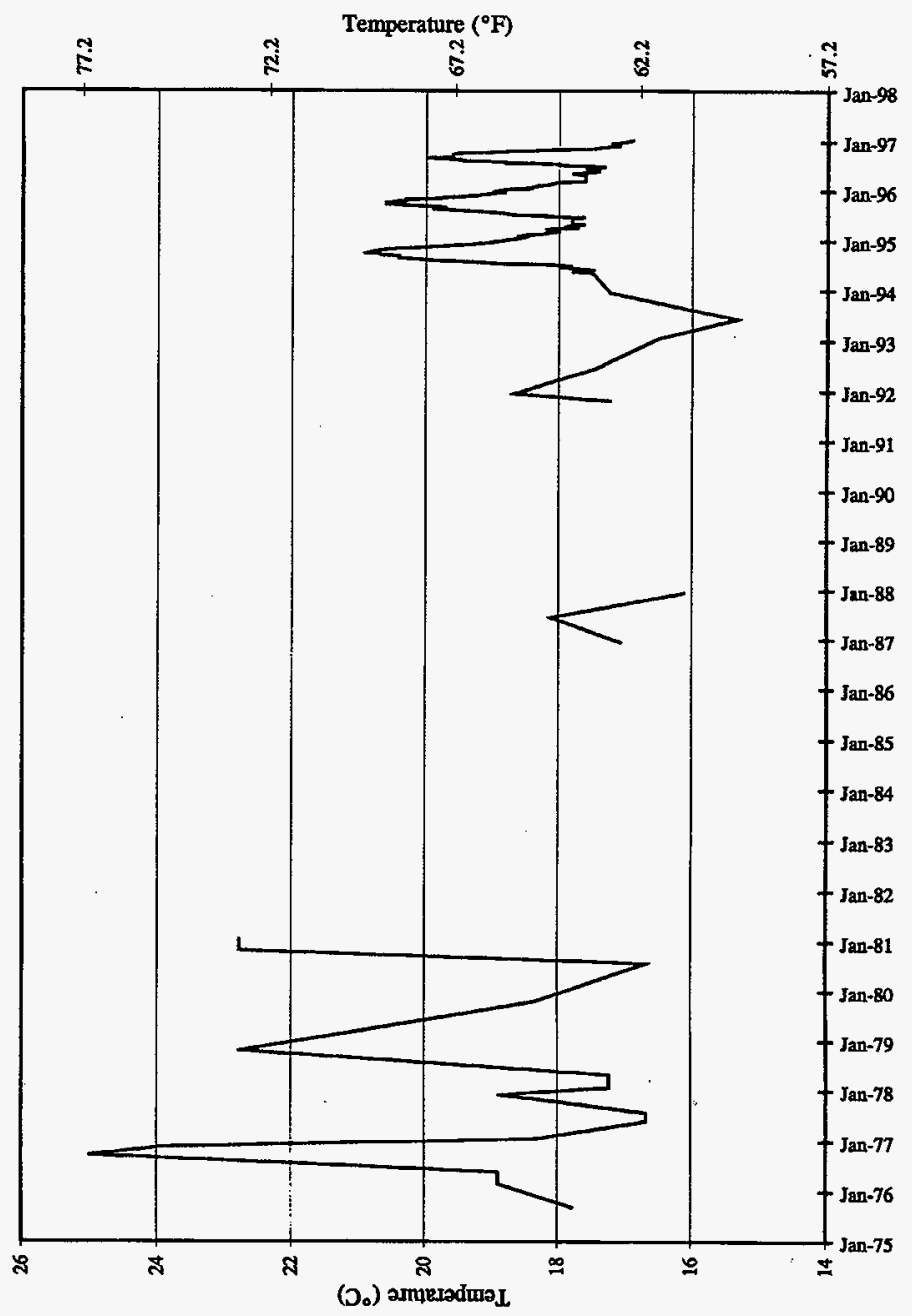




\section{A5.0 APPENDIX A REFERENCES}

Agnew, S. F., J. Boyer, R. A. Corbin, T. B. Duran, J. R. Fitzpatrick, K. A. Jurgensen, T. P. Ortiz, and B. L. Young, 1997a, Hanford Tank Chemical and Radionuclide Inventories: HDW Model Rev. 4, LA-UR-96-3860, Rev. 0, Los Alamos National Laboratory, Los Alamos, New Mexico.

Agnew, S. F., P. Baca, R. A. Corbin, T. B. Duran, and K. A. Jurgensen, 1997b, Waste Status and Transaction Record Summary (WSTRS) Rev. 4, LA-UR-97-311, Rev. 0, Los Alamos National Laboratory, Los Alamos, New Mexico.

Alstad, A. T., 1993, Riser Configuration Document for Single-Shell Waste Tanks, WHC-SD-RE-TI-053, Rev. 9, Westinghouse Hanford Company, Richland, Washington.

Brevick, C. H., J. L. Stroup, and J. W. Funk, 1997a, Supporting Document for the Historical Tank Content Estimate for T-Tank Farm, HNF-SD-WM-ER-320, Rev. 1, Fluor Daniel Northwest, Inc. for Fluor Daniel Hanford, Inc., Richland, Washington.

Brevick, C. H., J. L. Stroup, and J. W. Funk, 1997b, Historical Tank Content Estimate for the Northwest Quadrant of the Hanford 200 West Area, HNF-SD-WM-ER-351 Rev. 1, Fluor Daniel Northwest, Inc. for Fluor Daniel Hanford, Inc., Richland, Washington.

Hanlon, B. M.; 1997, Waste Tank Summary Report for Month Ending July 31, 1997, HNF-EP-0182-112, Lockheed Martin Hanford Corp. for Fluor Daniel Hanford, Inc., Richland, Washington.

Hewitt, E. R., 1996, Tank Waste Remediation System Resolution of Potentially Hazardous Vapor Issues, WHC-SD-TWR-RPT-001, Rev. 0, Westinghouse Hanford Company, Richland, Washington.

Lipnicki, J., 1997, Waste Tank Risers Available for Sampling, HNF-SD-WM-TI-710, Rev. 4, Numatec Hanford Corporation for Fluor Daniel Hanford, Inc., Richland, Washington.

Public Law 101-510, 1990, "Safety Measures for Waste Tanks at Hanford Nuclear Reservation," Section 3137 of National Defense Authorization Act for Fiscal Year 1991.

Tran, T. T., 1993, Thermocouple Status Single-Shell \& Double-Shell Waste Tanks, WHC-SD-WM-TI-553, Rev. 0, Westinghouse Hanford Company, Richland, Washington. 
HNF-1501 Rev. 0

APPENDIX B

SAMPLING OF 241-T-200 SERIES TANKS

(241-T-201, 241-T-202, 241-T-203, AND 241-T-204) 
HNF-1501 Rev. 0

This page intentionally left blank. 


\section{APPENDIX B \\ SAMPLING OF 241-T-200 SERIES TANKS \\ (241-T-201, 241-T-202, 241-T-203, AND 241-T-204)}

Appendix B provides sampling and analysis information for each known sampling event for T-200 series tanks and assesses the sample results. It includes the following.

- Section B1.0: Tank Sampling Overview

- Section B2.0: Sampling Events

- Section B3.0: Assessment of Characterization Results

- Section B4.0: Appendix B References

Future sampling information for these tanks will be appended to the above list.

\section{B1.0 TANK SAMPLING OVERVIEW}

This section describes the March/April 1997 sampling and analysis events for tanks 241-T-201, -T-202, -T-203, and -T-204. Core samples and vapro phase samples were taken to satisfy the requirements of the Tank Safety Screening Data Quality Objective (Dukelow et al. 1995). Vapor phase measurements were also used to satisfy the requirements of the Memorandum of Understandting for the Organic Compexant Safety Issue Data Requirements (Schreiber 1997a) and the Data Quality Objective to Support Resolution of the Organic Solvent Safety Issue (Meacham et al. 1997). The sampling and analyses were performed in accordance with the Tank 241-T-201 Push Mode Core Sampling and Analysis Plan (Hu 1997), Tank 241-T-202 Push Mode Core Sampling and Analysis Plan (Bell 1997), Tank 241-T-203 Push Mode Core Sampling and Analysis Plan (Schreiber 1997b), and the Tank. 241-T-204 Push Mode Core Sampling and Analysis Plan (Winkleman 1997). There were also two letters of instruction regarding the analysis of the composite core samples (Hall 1997a and Hall 1997b). Further discussions of the sampling and analysis procedures can be found in the Tank Characterization Reference Guide (DeLorenzo et al. 1994). The only previous analytical data for these tanks comes from a sample from tank 241-T-204 taken and analyzed in 1978 (Horton 1978). No samples from any other T-200 series tanks have been documented. 
'L66I '8 KETN 076 ITIV uroy SIOJEJoqeT S-ZZZ

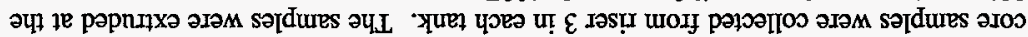

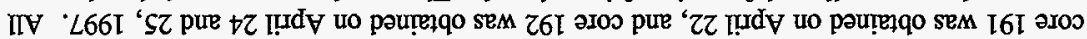

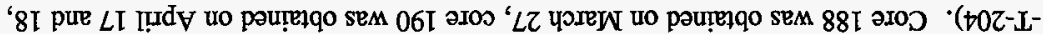

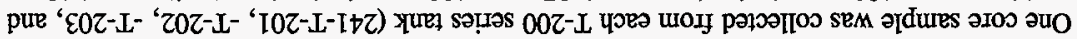

SINAAG DNITdWVS THOS L66I I'78

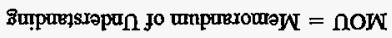

:ฆ⿴囗N

\begin{tabular}{|c|c|c|c|}
\hline 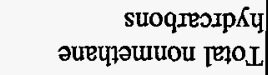 & səןdures rode $\Lambda$ & 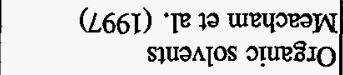 & \\
\hline 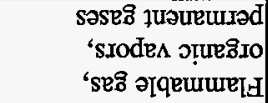 & 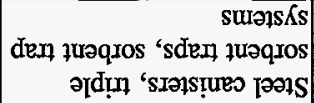 & 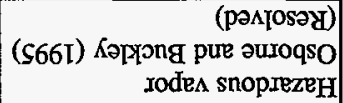 & $\begin{array}{r}\text { suṭdures } \\
\text { Iode } \Lambda\end{array}$ \\
\hline 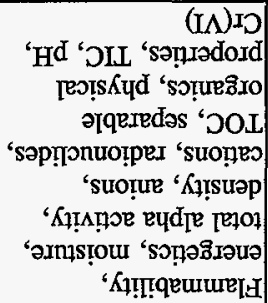 & 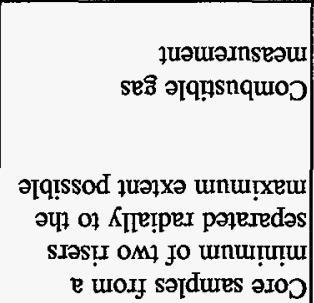 & 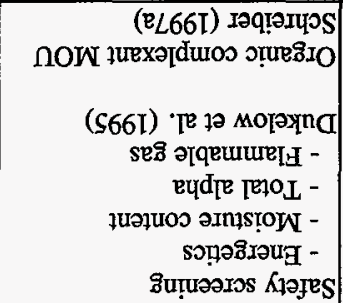 & $\begin{array}{r}\text { su!̣dures } \\
\text { əroo } \\
\text { əpour usn } \\
\end{array}$ \\
\hline 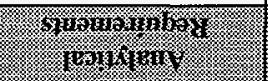 & 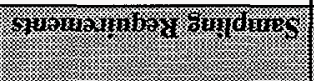 & 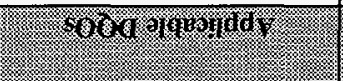 & mingil \\
\hline
\end{tabular}

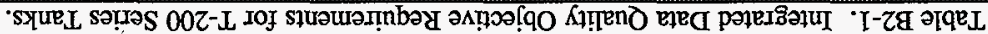

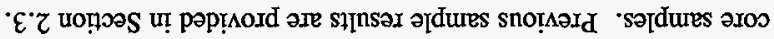

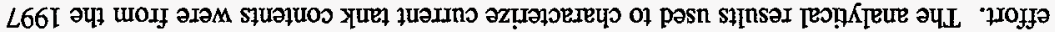

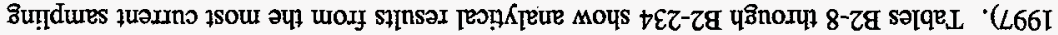

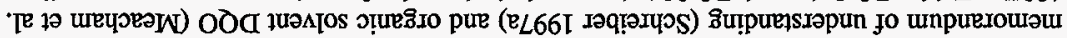

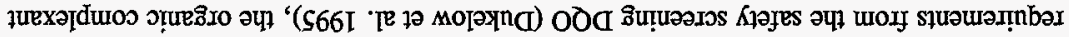

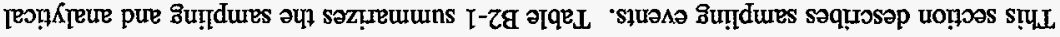

SLN'A MNITINFS 0*Zg 
Core sampling was used because of the depth of the waste and the expectation that a full vertical profile of the waste would be obtained from each tank. The core sample from tank 241-T-201, however, may not have recovered a full vertical profile of waste. The waste depth was expected to be approximately $4.1 \mathrm{~m}$ (161 in.). Based on observations from extrusion, several samples (segments 2-5) from this tank have large amounts of drainable liquid present where solids are believed to be. This suggests that there may be a local depression or anomaly beneath the riser, biasing the samples from that tank.

A vertical profile is used to satisfy the safety screening DQO (Dukelow et al. 1995). Safety screening analyses include: total alpha and bulk density to determine criticality, DSC to ascertain the fuel energy value, and thermogravimetric analysis (TGA) to obtain the total moisture content. In addition, combustible gas meter readings in the tank headspace were performed to measure flammability. Table B2-1 summarizes the sampling and analytical requirements from the safety screening $D Q O$.

\section{B2.1.1 Sample Handling}

The core samples obtained from each tank were taken from riser 3 . The total mass of solids obtained from each tank ranged from $1,100 \mathrm{~g}$ to $3,300 \mathrm{~g}$; liquids were obtained in substantial quantity from only one tank. The solids were uniformly black in color, and had varying degrees of moisture and physical consistency. Visual inspection of all of the extruded samples indicated that they contained substantial moisture. Table B2-2 shows the extrusion observations.

Table B2-2. T-200 Series Tanks Subsampling Scheme and Sample Description. ${ }^{1}$ (3 sheets)

\begin{tabular}{|c|c|c|c|c|}
\hline 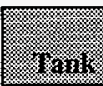 & (ainginer & 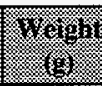 & 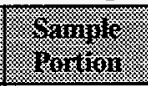 & 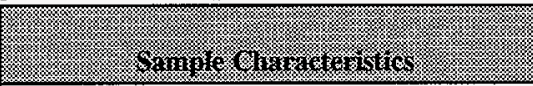 \\
\hline $\mathrm{T}-201$ & $192-01$ & 268.6 & \begin{tabular}{|l|}
$\begin{array}{l}\text { Drainable } \\
\text { liquid }\end{array}$ \\
\end{tabular} & $\begin{array}{l}\text { Pale yellow and slightly cloudy. No organic } \\
\text { layer. }\end{array}$ \\
\hline T-201 & $192-02$ & 248.2 & $\begin{array}{l}\text { Drainable } \\
\text { liquid }\end{array}$ & $\begin{array}{l}\text { Drainable liquid was black and opaque. } \\
\text { Settled black solids present. No organic layer. }\end{array}$ \\
\hline $\mathrm{T}-201$ & $192-03$ & 280.4 & $\begin{array}{l}\text { Drainable } \\
\text { liquid }\end{array}$ & $\begin{array}{l}\text { Drainable liquid was black and opaque. } \\
\text { Settled black solids present. No organic layer. }\end{array}$ \\
\hline T-201 & 192-04 & 252.0 & $\begin{array}{l}\text { Drainable } \\
\text { liquid }\end{array}$ & $\begin{array}{l}\text { Drainable liquid was black and opaque. } \\
\text { Settled black solids present. No organic layer. }\end{array}$ \\
\hline T-201 & $192-05$ & 272.9 & $\begin{array}{l}\text { Drainable } \\
\text { liquid }\end{array}$ & $\begin{array}{l}\text { Drainable liquid was black and opaque. } \\
\text { Settled black solids present. No organic layer. }\end{array}$ \\
\hline \multirow[t]{2}{*}{ T-201 } & \multirow[t]{2}{*}{$192-06$} & 198.3 & Lower half & Black solids resembling a wet sludge. \\
\hline & & 177.9 & Upper half & Black solids resembling a wet sludge. \\
\hline
\end{tabular}


Table B2-2. T-200 Series Tanks Subsampling Scheme and Sample Description. ${ }^{1}$ (3 sheets)

\begin{tabular}{|c|c|c|c|c|}
\hline 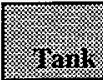 & Oningr & Weghi: & S 8901010 & 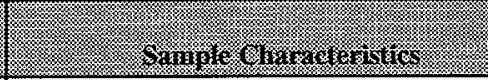 \\
\hline \multirow[t]{2}{*}{$\mathrm{T}-201$} & \multirow[t]{2}{*}{$192-07$} & 187.5 & Lower half & Black solids resembling a wet sludge. \\
\hline & & 200.3 & Upper half & Black solids resembling a wet sludge. \\
\hline \multirow[t]{2}{*}{$\mathrm{T}-201$} & \multirow[t]{2}{*}{$192-08$} & 166.9 & Lower half & Black solids resembling a wet sludge. \\
\hline & & 208.7 & Upper half & Black solids resembling a wet sludge. \\
\hline \multirow[t]{2}{*}{ T-202 } & \multirow[t]{2}{*}{$191-01$} & 172.0 & Lower half & Black solids resembling a wet sludge. \\
\hline & & 119.0 & Upper half & Black solids resembling a sludge/slurry. \\
\hline \multirow[t]{2}{*}{$\mathrm{T}-202$} & \multirow[t]{2}{*}{$191-02$} & 138.0 & Lower half & Black solids resembling a sludge/slurry. \\
\hline & & 156.3 & Upper half & Black solids resembling a sludge/slurry. \\
\hline \multirow[t]{2}{*}{$\mathrm{T}-202$} & \multirow[t]{2}{*}{$191-03$} & 168.9 & Lower half & Black solids resembling a wet sludge. \\
\hline & & 48.4 & Upper half & Black solids resembling a wet sludge. \\
\hline \multirow[t]{2}{*}{ T-202 } & \multirow[t]{2}{*}{$191-04$} & 158.2 & Lower half & Black solids resembling a dry sludge. \\
\hline & & 148.8 & Upper half & Black solids resembling a dry sludge. \\
\hline \multirow[t]{2}{*}{$\mathrm{T}-202$} & \multirow[t]{2}{*}{$191-05$} & 256.9 & Lower half & Black solids resembling a dry sludge. \\
\hline & & 86.4 & Upper half & Black solids resembling a wet sludge. \\
\hline \multirow[t]{2}{*}{ T-203 } & \multirow[t]{2}{*}{$190-01$} & 155.4 & Lower half & Black solids resembling a wet sludge. \\
\hline & & 170.4 & Upper half & Black solids resembling a wet sludge. \\
\hline \multirow[t]{3}{*}{$\mathrm{T}-203$} & \multirow[t]{3}{*}{$190-01 R$} & 137.8 & Lower half & Black solids resembling a sludge/slurry. \\
\hline & & & & $55.6 \mathrm{~g}$ drainable liquid, no organic layer. \\
\hline & & 109.6 & Upper half & Black solids resembling a sludge/slurry. \\
\hline \multirow[t]{2}{*}{$\mathrm{T}-203$} & \multirow[t]{2}{*}{$190-02$} & 167.3 & Lower half & Black solids resembling a wet sludge. \\
\hline & & 189.1 & Upper half & Black solids resembling a wet sludge. \\
\hline \multirow[t]{2}{*}{$\mathrm{T}-203$} & \multirow[t]{2}{*}{$190-03$} & 130.3 & Lower half & Black solids resembling a wet sludge. \\
\hline & & 138.4 & Upper half & Black solids resembling a wet sludge. \\
\hline \multirow[t]{2}{*}{ T-203 } & \multirow[t]{2}{*}{$190-04$} & 182.4 & Lower half & Black solids resembling a wet sludge. \\
\hline & & 176.3 & Upper half & Black solids resembling a wet sludge. \\
\hline \multirow[t]{2}{*}{$\mathrm{T}-203$} & \multirow[t]{2}{*}{$190-05$} & 171.6 & Lower half & Black solids resembling a wet sludge. \\
\hline & & 191.4 & Upper half & Black solids resembling a wet sludge. \\
\hline \multirow[t]{2}{*}{$\mathrm{T}-203$} & \multirow[t]{2}{*}{$190-06$} & $183: 7$ & Lower half & Black solids resembling a wet sludge. \\
\hline & & 169.8 & Upper half & Black solids resembling a wet sludge. \\
\hline \multirow[t]{2}{*}{ T-203 } & \multirow[t]{2}{*}{$190-07$} & 158.1 & Lower half & Black solids resembling a wet sludge. \\
\hline & & 183.6 & Upper half & Black solids resembling a wet studge. \\
\hline
\end{tabular}


Table B2-2. T-200 Series Tanks Subsampling Scheme and Sample Description. ${ }^{1}$ (3 sheets)

\begin{tabular}{|c|c|c|c|c|}
\hline Ianis & 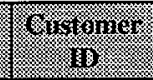 & Hivennt: & Ormule: & 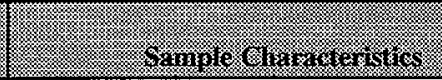 \\
\hline \multirow[t]{2}{*}{$\mathrm{T}-203$} & \multirow[t]{2}{*}{$190-08$} & 175.0 & Lower half & Black solids resembling a wet sludge. \\
\hline & & 168.1 & Upper half & Black solids resembling a wet sludge. \\
\hline \multirow[t]{2}{*}{$\mathrm{T}-203$} & \multirow[t]{2}{*}{$190-09$} & 185.0 & Lower half & Black solids resembling a wet sludge. \\
\hline & & 169.2 & Upper half & Black solids resembling a wet sludge. \\
\hline \multirow[t]{2}{*}{$\mathrm{T}-204$} & \multirow[t]{2}{*}{$97-60^{2}$} & 150.3 & Lower half & Black solids resembling a wet sludge. \\
\hline & & 161.2 & Upper half & Black solids resembling a wet sludge. \\
\hline \multirow[t]{2}{*}{ T-204 } & \multirow[t]{2}{*}{$97-61$} & 94.2 & Lower half & Black solids resembling a wet sludge. \\
\hline & & \begin{tabular}{|l} 
no \\
sample
\end{tabular} & Upper half & \\
\hline \multirow[t]{2}{*}{ T-204 } & \multirow[t]{2}{*}{$97-62$} & 216.6 & Lower half & Black solids resembling a wet sludge. \\
\hline & & $\begin{array}{l}\text { no } \\
\text { sample }\end{array}$ & Upper half & \\
\hline \multirow[t]{2}{*}{$\mathrm{T}-204$} & \multirow[t]{2}{*}{$97-63$} & 166.5 & Lower half & Black solids resembling a dry sludge. \\
\hline & & 189.4 & Upper half & Black solids resembling a dry sludge. \\
\hline \multirow[t]{2}{*}{$\mathrm{T}-204$} & \multirow[t]{2}{*}{$97-64$} & 178.1 & Lower half & Black solids resembling a dry sludge. \\
\hline & & 177.3 & Upper half & Black solids resembling a dry sludge. \\
\hline \multirow[t]{2}{*}{$\mathrm{T}-204$} & \multirow[t]{2}{*}{$97-65$} & 166.6 & Lower half & Black solids resembling a dry sludge. \\
\hline & & 194.8 & Upper half & Black solids resembling a dry sludge. \\
\hline \multirow[t]{2}{*}{$\mathrm{T}-204$} & \multirow[t]{2}{*}{$97-66$} & 183.2 & Lower half & Black solids resembling a dry sludge. \\
\hline & & 172.7 & Upper half & Black solids resembling a dry sludge. \\
\hline \multirow[t]{2}{*}{$\mathrm{T}-204$} & \multirow[t]{2}{*}{$97-67$} & 193.1 & Lower half & Black solids resembling a dry sludge. \\
\hline & & 159.5 & Upper half & Black solids resembling a dry sludge. \\
\hline \multirow[t]{2}{*}{$\mathrm{T}-204$} & \multirow[t]{2}{*}{$97-68$} & 153.7 & Lower half & Black solids resembling a dry sludge. \\
\hline & & 173.9 & Upper half & Black solids resembling a dry sludge. \\
\hline \multirow[t]{2}{*}{$\mathrm{T}-204$} & \multirow[t]{2}{*}{$97-69$} & 151.9 & Lower half & Black solids resembling a dry sludge. \\
\hline & & 195.3 & Upper half & Black solids resembling a dry sludge. \\
\hline
\end{tabular}

Note:

${ }^{1}$ Nuzum (1997a), Esch (1997), Steen (1997), and Nuzum (1997b)

${ }^{2}$ Sample 97060 through 97069 (Customer ID. 97-60 through 97-69) corresponds to core 188. 
The samples obtained did not visually differ from each other substantially except in one case. Tank 241-T-201 had several samples (segments 1-5) that were mostly drainable liquid. The liquids were mostly black in color from the presence of suspended solids. Segment 1 of tank 241-T-201 did not have any suspended solids. The solid samples (segments 6-8) were divided into subsegments before analysis. The drainable liquids were allowed to settle. Composites were created from the solid segments and the settled solids and clarified liquor of the drainable liquid. All composites were analyzed separately. Although the solids from the drainable liquid in tank 241-T-201 were composited and analyzed, they were not included in the statistical analysis of the data because of the degree of contamination from supernatant.

The remaining T-200 series solid samples were divided into subsegments, homogenized, subsampled, and composited for further laboratory analyses and archiving. Analyses were performed on both the segment and core composite level.

\section{B2.1.2 Sample Analysis}

The analyses performed on the core samples were limited to those required by the safety screening DQO and specific assays used to evaluate the predictions made from the B-200 series data. The analyses required by the safety screening DQO included analyses for thermal properties by DSC, moisture content by TGA, and content of fissile material by total alpha activity analysis. The additional assays selected to evaluate the predictions consisted of a full set of ion chromatography (IC) and inductively coupled plasma (ICP) analytes on core composite samples to determine the presence of selected analytes, which in turn, helped to determine the efficacy of the predictions made.

Differential scanning calorimetry and TGA were performed on samples ranging in mass from $4.661 \mathrm{mg}$ to $52.100 \mathrm{mg}$. Quality control (QC) tests included performing the analyses in duplicate and using standards.

Total alpha activity measurements were performed on samples that had been fused in a solution of potassium then dissolved in acid. The resulting solution was dried on a counting planchet and counted in an alpha proportional counter. Quality control tests included standards, spikes, blanks, and duplicate analyses.

Ion chromatography was performed on samples that had been prepared by water digestion. Quality control tests included standards, spikes, blanks, and duplicate analyses. The sampling and analysis plans (Bell [1997], Hu [1997], Schreiber [1997b], and Winkelman [1997]) required measuring a select few analytes but reporting the full suite of $\mathrm{IC}$ analytes. 
Inductively coupled plasma atomic emission spectroscopy (ICP/AES) was performed either on samples that had been prepared by a fusion procedure followed by dissolution in acid or acid dissolution alone. Quality control tests included standards, blanks, spikes, and duplicate analyses. The sampling and analysis plans required analyzing a select few analytes and reporting the full suite of ICP elements.

All reported analyses were performed according to approved laboratory procedures. Table B2-3 lists procedure numbers and applicable analyses. Table B2-4 is a summary of the sample portions, sample numbers, and analyses performed on each sample.

Table B2-3. Analytical Procedures.

\begin{tabular}{|c|c|c|}
\hline 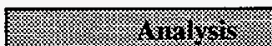 & Meknod: & W.credure Nun \\
\hline Energetics by DSC & Perkin Elmer ${ }^{1}$ & LA-514-114 \\
\hline Percent water by TGA & Perkin Elmer ${ }^{\circledR}$ & LA-514-114 \\
\hline Total alpha activity & Alpha proportional counter & LA-508-101 \\
\hline Flammable gas & Combustible gas analyzer & $\begin{array}{l}\text { WHC-IP-0030 IH } 1.4 \\
\text { and IH } 2.1^{2}\end{array}$ \\
\hline TOC/TIC & Coulometer & LA-342-100 \\
\hline Metals by ICP/AES & $\begin{array}{l}\text { Inductively coupled plasma } \\
\text { spectrometry }\end{array}$ & $\begin{array}{l}\text { LA-505-151 } \\
\text { LA-505-161 }\end{array}$ \\
\hline Anions by IC & Ion chromatograph & LA-533-105 \\
\hline Radionuclides & Gamma energy analysis & LA-548-121 \\
\hline${ }^{90} \mathrm{Sr}$ & Beta proportional counter & LA-220-101 \\
\hline Specific gravity & Direct & LA-510-112 \\
\hline Bulk density & Gravimetry & LO-160-103 \\
\hline
\end{tabular}

Note:

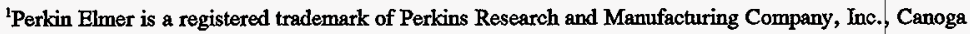
Park, California.

${ }^{2}$ WHC (1992) Safety Department Administrative Manuals, Westinghouse Hanford Company, Richland, Washington:

IH 1.4, Industrial Hygiene Direct Reading Instrument Survey

IH 2.1, Standard Operating Procedure, MSA Model 260 Combustible Gas and Oxygen Aaalyzer 
Table B2-4. T-200 Series Sample Analysis Summary. ${ }^{1}$ (6 sheets)

\begin{tabular}{|c|c|c|c|c|}
\hline s. & 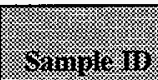 & 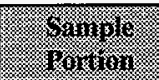 & Similor & 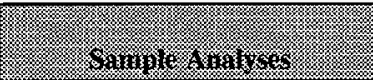 \\
\hline T-201 & $192-01$ & $\begin{array}{l}\text { Drainable } \\
\text { liquid }\end{array}$ & $\begin{array}{l}\text { S97T000833 } \\
\text { S97T001203 }\end{array}$ & $\begin{array}{l}\text { DSC/TGA, SpG } \\
\text { IC, ICP, total alpha }\end{array}$ \\
\hline $\mathrm{T}-201$ & $192-02$ & $\begin{array}{l}\text { Drainable } \\
\text { liquid }\end{array}$ & S97T000834 & $\begin{array}{l}\text { DSC/TGA, SpG } \\
\text { IC, ICP, total alpha }\end{array}$ \\
\hline T-201 & $192-03$ & $\begin{array}{l}\text { Drainable } \\
\text { liquid }\end{array}$ & $\begin{array}{l}\text { S97T000835 } \\
\text { S97T001259 }\end{array}$ & $\begin{array}{l}\text { DSC/TGA, SpG, IC, ICP total } \\
\text { alpha }\end{array}$ \\
\hline $\mathrm{T}-201$ & $192-04$ & $\begin{array}{l}\text { Drainable } \\
\text { liquid }\end{array}$ & S97T000836 & $\begin{array}{l}\text { DSC/TGA, SpG } \\
\text { IC, ICP, total alpha }\end{array}$ \\
\hline T-201 & $192-05$ & $\begin{array}{l}\text { Drainable } \\
\text { liquid }\end{array}$ & S97T000837 & $\begin{array}{l}\text { DSC/TGA, SpG } \\
\text { IC, ICP, total alpha }\end{array}$ \\
\hline \multirow[t]{2}{*}{$\mathrm{T}-201$} & \multirow[t]{2}{*}{ 192-06 } & Lower half & $\begin{array}{l}\text { S97T000894 } \\
\text { S97T000899 } \\
\text { S97T000900 } \\
\text { S97T000901 }\end{array}$ & $\begin{array}{l}\text { Density } \\
\text { DSC/TGA } \\
\text { IC } \\
\text { ICP, total alpha }\end{array}$ \\
\hline & & Upper half & $\begin{array}{l}\text { S97T000909 } \\
\text { S97T000921 } \\
\text { S97T000931 }\end{array}$ & $\begin{array}{l}\text { DSC/TGA } \\
\text { ICP } \\
\text { IC }\end{array}$ \\
\hline \multirow[t]{2}{*}{ T-201 } & \multirow[t]{2}{*}{$192-07$} & Lower half & $\begin{array}{l}\text { S97T000895 } \\
\text { S97T000910 } \\
\text { S97T000915 } \\
\text { S97T000919 } \\
\end{array}$ & $\begin{array}{l}\text { Density } \\
\text { DSC/TGA } \\
\text { IC } \\
\text { ICP, total alpha }\end{array}$ \\
\hline & & Upper half & $\begin{array}{l}\text { S97T000911 } \\
\text { S97T000916 } \\
\text { S97T000922 }\end{array}$ & $\begin{array}{l}\text { DSC/TGA } \\
\text { ICP } \\
\text { IC } \\
\end{array}$ \\
\hline \multirow[t]{2}{*}{ T-201 } & \multirow[t]{2}{*}{$192-08$} & Lower half & $\begin{array}{l}\text { S97T000896 } \\
\text { S97T000912 } \\
\text { S97T000917 } \\
\text { S97T000920 }\end{array}$ & $\begin{array}{l}\text { Density } \\
\text { DSC/TGA } \\
\text { IC } \\
\text { ICP, total alpha }\end{array}$ \\
\hline & & Upper half & $\begin{array}{l}\text { S97T000913 } \\
\text { S97T000918 } \\
\text { S97T000923 }\end{array}$ & $\begin{array}{l}\text { DSC/TGA } \\
\text { ICP } \\
\text { IC }\end{array}$ \\
\hline T-201 & $\begin{array}{l}192 \\
\text { Composite }\end{array}$ & $\begin{array}{l}\text { Drainable } \\
\text { liquid settled } \\
\text { solids }\end{array}$ & $\begin{array}{l}\text { S97T001252 } \\
\text { S97T001253 } \\
\text { S97T001256 } \\
\text { S97T001257 } \\
\text { S97T001754 }\end{array}$ & $\begin{array}{l}\text { Density } \\
\text { DSC/TGA, TIC/TOC } \\
\text { ICP } \\
\text { IC } \\
\text { GEA, Sr-90 }\end{array}$ \\
\hline
\end{tabular}


Table B2-4. T-200 Series Sample Analysis Summary. ${ }^{1}$ (6 sheets)

\begin{tabular}{|c|c|c|c|c|}
\hline watir & 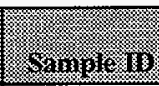 & 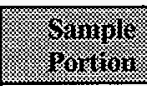 & 3amivic: & 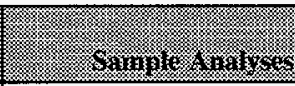 \\
\hline $\mathrm{T}-201$ & $\begin{array}{l}192 \\
\text { Composite }\end{array}$ & Solid & $\begin{array}{l}\text { S97T001245 } \\
\text { S97T001246 } \\
\text { S97T001250 } \\
\text { S97T001251 } \\
\text { S97T001753 }\end{array}$ & $\begin{array}{l}\text { Density } \\
\text { DSC/TGA, TIC/TOC } \\
\text { ICP } \\
\text { IC } \\
\text { GEA, Sr-90 }\end{array}$ \\
\hline \multirow[t]{2}{*}{ T-202 } & \multirow[t]{2}{*}{$191-01$} & Lower half & \begin{tabular}{|l|} 
S97T000786 \\
S97T000802 \\
S97T000939 \\
S97T000944 \\
S97T000954 \\
\end{tabular} & $\begin{array}{l}\text { Density } \\
\text { DSC/TGA } \\
\text { total alpha } \\
\text { ICP } \\
\text { IC } \\
\end{array}$ \\
\hline & & Upper half & $\begin{array}{l}\text { S97T000803 } \\
\text { S97T000945 } \\
\text { S97T000955 }\end{array}$ & $\begin{array}{l}\text { DSC/TGA } \\
\text { ICP } \\
\text { IC } \\
\end{array}$ \\
\hline \multirow[t]{2}{*}{$\mathrm{T}-202$} & \multirow[t]{2}{*}{ 191-02 } & Lower half & \begin{tabular}{|l|} 
S97T000787 \\
S97T000804 \\
S97T000940 \\
S97T000946 \\
S97T000956 \\
\end{tabular} & $\begin{array}{l}\text { Density } \\
\text { DSC/TGA } \\
\text { total alpha } \\
\text { ICP } \\
\text { IC } \\
\end{array}$ \\
\hline & & Upper half & $\begin{array}{l}\text { S97T000805 } \\
\text { S97T000947 } \\
\text { S97T000957 } \\
\end{array}$ & $\begin{array}{l}\text { DSC/TGA } \\
\text { ICP } \\
\text { IC } \\
\end{array}$ \\
\hline \multirow[t]{2}{*}{$\mathrm{T}-202$} & \multirow[t]{2}{*}{ 191-03 } & Lower half & $\begin{array}{l}\text { S97T000788 } \\
\text { S97T000806 } \\
\text { S97T000941 } \\
\text { S97T000948 } \\
\text { S97T000958 } \\
\end{array}$ & $\begin{array}{l}\text { Density } \\
\text { DSC/TGA } \\
\text { total alpha } \\
\text { ICP } \\
\text { IC } \\
\end{array}$ \\
\hline & & Upper half & $\begin{array}{l}\text { S97T000807 } \\
\text { S97T000949 } \\
\text { S97T000959 }\end{array}$ & $\begin{array}{l}\text { DSC/TGA } \\
\text { ICP } \\
\text { IC } \\
\end{array}$ \\
\hline \multirow[t]{2}{*}{ T-202 } & \multirow[t]{2}{*}{$191-04$} & Lower half & $\begin{array}{l}\text { S97T000789 } \\
\text { S97T000808 } \\
\text { S97T000942 } \\
\text { S97T000950 } \\
\text { S97T000960 } \\
\end{array}$ & $\begin{array}{l}\text { Density } \\
\text { DSC/TGA } \\
\text { total alpha } \\
\text { ICP } \\
\text { IC } \\
\end{array}$ \\
\hline & & Upper half & $\begin{array}{l}\text { S97T000809 } \\
\text { S97T000951 } \\
\text { S97T000961 }\end{array}$ & $\begin{array}{l}\text { DSC/TGA } \\
\text { ICP } \\
\text { IC } \\
\end{array}$ \\
\hline
\end{tabular}


Table B2-4. T-200 Series Sample Analysis Summary. ${ }^{1}$ (6 sheets)

\begin{tabular}{|c|c|c|c|c|}
\hline lank & Samivic 10 & 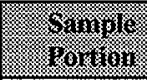 & Sinimis & 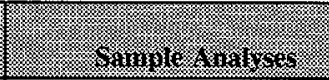 \\
\hline \multirow[t]{2}{*}{ T-202 } & \multirow[t]{2}{*}{$191-05$} & Lower half & $\begin{array}{l}\text { S97T000790 } \\
\text { S97T000810 } \\
\text { S97T000943 } \\
\text { S97T000952 } \\
\text { S97T000962 } \\
\end{array}$ & $\begin{array}{l}\text { Density } \\
\text { DSC/TGA } \\
\text { total alpha } \\
\text { ICP } \\
\text { IC }\end{array}$ \\
\hline & & Upper half & $\begin{array}{l}\text { S97T000811 } \\
\text { S97T000953 } \\
\text { S97T000963 }\end{array}$ & $\begin{array}{l}\text { DSC/TGA } \\
\text { ICP } \\
\text { IC }\end{array}$ \\
\hline $\mathrm{T}-202$ & $\begin{array}{l}191 \\
\text { Composite }\end{array}$ & Solid & $\begin{array}{l}\text { S97T001087 } \\
\text { S97T001127 } \\
\text { S97T001129 } \\
\text { S97T001673 } \\
\text { S97T001805 }\end{array}$ & $\begin{array}{l}\text { Density } \\
\text { GEA, Sr-90 } \\
\text { IC } \\
\text { DSC/TGA, TIC/TOC } \\
\text { ICP }\end{array}$ \\
\hline \multirow[t]{2}{*}{$\mathrm{T}-203$} & \multirow[t]{2}{*}{$190-01$} & Lower half & $\begin{array}{l}\text { S97T000657 } \\
\text { S97T000665 } \\
\text { S97T000759 } \\
\text { S97T000768 }\end{array}$ & $\begin{array}{l}\text { Density } \\
\text { DSC/TGA } \\
\text { ICP, total alpha } \\
\text { IC }\end{array}$ \\
\hline & & Upper half & $\begin{array}{l}\text { S97T000664 } \\
\text { S97T000735 } \\
\text { S97T000736 }\end{array}$ & $\begin{array}{l}\text { DSC/TGA } \\
\text { ICP } \\
\text { IC }\end{array}$ \\
\hline \multirow[t]{3}{*}{$T-203$} & \multirow[t]{3}{*}{$190-01 R$} & Lower half & \begin{tabular}{|l|} 
S97T000699 \\
S97T000711 \\
S97T000760 \\
S97T000769 \\
\end{tabular} & \begin{tabular}{|l} 
Density \\
DSC/TGA \\
ICP, total alpha \\
IC \\
\end{tabular} \\
\hline & & Upper half & $\begin{array}{l}\text { S97T000705 } \\
\text { S97T000737 } \\
\text { S97T000746 } \\
\end{array}$ & \begin{tabular}{|l} 
DSC/TGA \\
ICP \\
IC \\
\end{tabular} \\
\hline & & $\begin{array}{l}\text { Drainable } \\
\text { liquid }\end{array}$ & S97T000733 & $\begin{array}{l}\text { DSC/TGA, total alpha, IC, } \\
\text { ICP, SpG }\end{array}$ \\
\hline \multirow[t]{2}{*}{$T-203$} & \multirow[t]{2}{*}{$190-02$} & Lower half & $\begin{array}{l}\text { S97T000661 } \\
\text { S97T000669 } \\
\text { S97T000757 } \\
\text { S97T000758 } \\
\end{array}$ & \begin{tabular}{|l} 
Density \\
DSC/TGA \\
ICP, total alpha \\
IC \\
\end{tabular} \\
\hline & & Upper half & $\begin{array}{l}\text { S97T000666 } \\
\text { S97T000738 } \\
\text { S97T000747 } \\
\end{array}$ & \begin{tabular}{|l} 
DSC/TGA \\
ICP \\
IC \\
\end{tabular} \\
\hline
\end{tabular}


HNF-1501 Rev. 0

Table B2-4. T-200 Series Sample Analysis Summary. ${ }^{1}$ (6 sheets)

\begin{tabular}{|c|c|c|c|c|}
\hline (2) & sumpic Is & 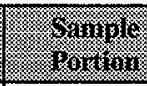 & Sumiler & 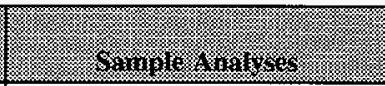 \\
\hline \multirow[t]{2}{*}{ T-203 } & \multirow[t]{2}{*}{$190-03$} & Lower half & $\begin{array}{l}\text { S97T000700 } \\
\text { S97T000712 } \\
\text { S97T000761 } \\
\text { S97T000770 }\end{array}$ & $\begin{array}{l}\text { Density } \\
\text { DSC/TGA } \\
\text { ICP, total alpha } \\
\text { IC }\end{array}$ \\
\hline & & Upper half & $\begin{array}{l}\text { S97T000706 } \\
\text { S97T000739 } \\
\text { S97T000748 }\end{array}$ & \begin{tabular}{|l|} 
DSC/TGA \\
ICP \\
IC \\
\end{tabular} \\
\hline \multirow[t]{2}{*}{ T-203 } & \multirow[t]{2}{*}{$190-04$} & Lower half & $\begin{array}{l}\text { S97T000662 } \\
\text { S97T000670 } \\
\text { S97T000762 } \\
\text { S97T000771 }\end{array}$ & $\begin{array}{l}\text { Density } \\
\text { DSC/TGA } \\
\text { ICP, total alpha } \\
\text { IC }\end{array}$ \\
\hline & & Upper half & $\begin{array}{l}\text { S97T000667 } \\
\text { S97T000740 } \\
\text { S97T000749 }\end{array}$ & $\begin{array}{l}\text { DSC/TGA } \\
\text { ICP } \\
\text { IC }\end{array}$ \\
\hline \multirow[t]{2}{*}{ T-203 } & \multirow[t]{2}{*}{$190-05$} & Lower half & $\begin{array}{l}\text { S97T000663 } \\
\text { S97T000671 } \\
\text { S97T000763 } \\
\text { S97T000772 }\end{array}$ & $\begin{array}{l}\text { Density } \\
\text { DSC/TGA } \\
\text { ICP, total alpha } \\
\text { IC }\end{array}$ \\
\hline & & Upper half & $\begin{array}{l}\text { S97T000668 } \\
\text { S97T000741 } \\
\text { S97T000750 }\end{array}$ & $\begin{array}{l}\text { DSC/TGA } \\
\text { ICP } \\
\text { IC }\end{array}$ \\
\hline \multirow[t]{2}{*}{ T-203 } & \multirow[t]{2}{*}{$190-06$} & Lower half & $\begin{array}{l}\text { S97T000701 } \\
\text { S97T000713 } \\
\text { S97T000764 } \\
\text { S97T000773 }\end{array}$ & $\begin{array}{l}\text { Density } \\
\text { DSC/TGA } \\
\text { ICP, total alpha } \\
\text { IC }\end{array}$ \\
\hline & & Upper half & $\begin{array}{l}\text { S97T000707 } \\
\text { S97T000742 } \\
\text { S97T000751 }\end{array}$ & $\begin{array}{l}\text { DSC/TGA } \\
\text { ICP } \\
\text { IC }\end{array}$ \\
\hline \multirow[t]{2}{*}{ T-203 } & \multirow[t]{2}{*}{$190-07$} & Lower half & $\begin{array}{l}\text { S97T000702 } \\
\text { S97T000714 } \\
\text { S97T000765 } \\
\text { S97T000774 } \\
\end{array}$ & $\begin{array}{l}\text { Density } \\
\text { DSC/TGA } \\
\text { ICP, total alpha } \\
\text { IC } \\
\end{array}$ \\
\hline & & Upper half & $\begin{array}{l}\text { S97T000708 } \\
\text { S97T000743 } \\
\text { S97T000752 }\end{array}$ & $\begin{array}{l}\text { DSC/TGA } \\
\text { ICP } \\
\text { IC }\end{array}$ \\
\hline
\end{tabular}


Table B2-4. T-200 Series Sample Analysis Summary. ${ }^{1}$ (6 sheets)

\begin{tabular}{|c|c|c|c|c|}
\hline (2.1. & 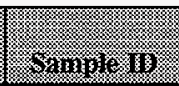 & Vimpilo & H: vamples: & 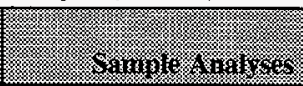 \\
\hline \multirow[t]{2}{*}{ T-203 } & \multirow[t]{2}{*}{$190-08$} & Lower half & \begin{tabular}{|l|} 
S97T000703 \\
S97T000715 \\
S97T000766 \\
S97T000775 \\
\end{tabular} & \begin{tabular}{|l|} 
Density \\
DSC/TGA \\
ICP, total alpha \\
IC \\
\end{tabular} \\
\hline & & Upper half & $\begin{array}{l}\text { S97T000709 } \\
\text { S97T000744 } \\
\text { S97T000753 }\end{array}$ & $\begin{array}{l}\text { DSC/TGA } \\
\text { ICP } \\
\text { IC }\end{array}$ \\
\hline \multirow[t]{2}{*}{ T-203 } & \multirow[t]{2}{*}{$190-09$} & Lower half & $\begin{array}{l}\text { S97T000704 } \\
\text { S97T000716 } \\
\text { S97T000767 } \\
\text { S97T000776 }\end{array}$ & $\begin{array}{l}\text { Density } \\
\text { DSC/TGA } \\
\text { ICP, total alpha } \\
\text { IC }\end{array}$ \\
\hline & & Upper half & $\begin{array}{l}\text { S97T000710 } \\
\text { S97T000745 } \\
\text { S97T000754 }\end{array}$ & $\begin{array}{l}\text { DSC/TGA } \\
\text { ICP } \\
\text { IC }\end{array}$ \\
\hline T-203 & $\begin{array}{l}190 \\
\text { Composite }\end{array}$ & Solid & $\begin{array}{l}\text { S97T001012 } \\
\text { S97T001013 } \\
\text { S97T001016 } \\
\text { S97T001017 } \\
\text { S97T001734 }\end{array}$ & $\begin{array}{l}\text { Density } \\
\text { DSC/TGA, TIC/TOC } \\
\text { IC } \\
\text { ICP } \\
\text { GEA, Sr-90 }\end{array}$ \\
\hline \multirow[t]{2}{*}{$\mathrm{T}-204$} & \multirow[t]{2}{*}{$\begin{array}{l}97-60 \\
\text { (Segment 1) }\end{array}$} & Lower half & $\begin{array}{l}\text { S97T000494 } \\
\text { S97T000498 } \\
\text { S97T000587 }\end{array}$ & $\begin{array}{l}\text { Density } \\
\text { DSC/TGA } \\
\text { total alpha }\end{array}$ \\
\hline & & Upper half & S97T000499 & DSC/TGA \\
\hline \multirow[t]{2}{*}{ T-204 } & \multirow[t]{2}{*}{$\begin{array}{l}97-61 \\
\text { (Segment 2) }\end{array}$} & Lower half & $\begin{array}{l}\text { S97T000570 } \\
\text { S97T000574 } \\
\text { S97T000588 }\end{array}$ & \begin{tabular}{|l} 
Density \\
DSC/TGA \\
total alpha \\
\end{tabular} \\
\hline & & Upper half & None & \\
\hline \multirow[t]{2}{*}{ T-204 } & \multirow[t]{2}{*}{$\begin{array}{l}97-62 \\
\text { (Segment 3) }\end{array}$} & Lower half & $\begin{array}{l}\text { S97T000571 } \\
\text { S97T000575 } \\
\text { S97T000589 } \\
\end{array}$ & $\begin{array}{l}\text { Density } \\
\text { DSC/TGA } \\
\text { total alpha }\end{array}$ \\
\hline & & Upper half & None & \\
\hline \multirow[t]{2}{*}{ T-204 } & \multirow[t]{2}{*}{$\begin{array}{l}97-63 \\
\text { (Segment 4) }\end{array}$} & Lower half & $\begin{array}{l}\text { S97T000572 } \\
\text { S97T000576 } \\
\text { S97T000590 }\end{array}$ & $\begin{array}{l}\text { Density } \\
\text { DSC/TGA } \\
\text { total alpha }\end{array}$ \\
\hline & & Upper half & S97T000580 & DSC/TGA \\
\hline
\end{tabular}


Table B2-4. T-200 Series Sample Analysis Summary. ${ }^{1}$ (6 sheets)

\begin{tabular}{|c|c|c|c|c|}
\hline (1) & 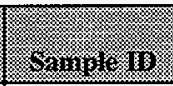 & (3imple: & S & 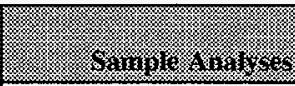 \\
\hline \multirow[t]{2}{*}{ T-204 } & \multirow[t]{2}{*}{$\begin{array}{l}97-64 \\
\text { (Segment 5) }\end{array}$} & Lower half & $\begin{array}{l}\text { S97T000609 } \\
\text { S97T000603 } \\
\text { S97T000621 }\end{array}$ & $\begin{array}{l}\text { Density } \\
\text { DSC/TGA } \\
\text { total alpha }\end{array}$ \\
\hline & & Upper half & S97T000633 & DSC/TGA \\
\hline \multirow[t]{2}{*}{$\mathrm{T}-204$} & \multirow[t]{2}{*}{$\begin{array}{l}97-65 \\
\text { (Segment 6) }\end{array}$} & Lower half & $\begin{array}{l}\text { S97T000610 } \\
\text { S97T000604 } \\
\text { S97T000622 }\end{array}$ & $\begin{array}{l}\text { Density } \\
\text { DSC/TGA } \\
\text { total alpha }\end{array}$ \\
\hline & & Upper half & S97T000634 & DSC/TGA \\
\hline \multirow[t]{2}{*}{ T-204 } & \multirow[t]{2}{*}{$\begin{array}{l}97-66 \\
\text { (Segment } 7 \text { ) }\end{array}$} & Lower half & $\begin{array}{l}\text { S97T000611 } \\
\text { S97T000605 } \\
\text { S97T000623 }\end{array}$ & $\begin{array}{l}\text { Density } \\
\text { DSC/TGA } \\
\text { total alpha }\end{array}$ \\
\hline & & Upper half & S97T000635 & DSC/TGA \\
\hline \multirow[t]{2}{*}{$\mathrm{T}-204$} & \multirow[t]{2}{*}{$\begin{array}{l}97-67 \\
\text { (Segment 8) }\end{array}$} & Lower half & $\begin{array}{l}\text { S97T000612 } \\
\text { S97T000606 } \\
\text { S97T000624 }\end{array}$ & $\begin{array}{l}\text { Density } \\
\text { DSC/TGA } \\
\text { total alpha }\end{array}$ \\
\hline & & Upper half & S97T000636 & DSC/TGA \\
\hline \multirow[t]{2}{*}{$\mathrm{T}-204$} & \multirow[t]{2}{*}{$\begin{array}{l}97-68 \\
\text { (Segment 9) }\end{array}$} & Lower half & $\begin{array}{l}\text { S97T000613 } \\
\text { S97T000607 } \\
\text { S97T000625 }\end{array}$ & $\begin{array}{l}\text { Density } \\
\text { DSC/TGA } \\
\text { total alpha }\end{array}$ \\
\hline & & Upper half & S97T000637 & DSC/TGA \\
\hline \multirow[t]{2}{*}{$T-204$} & \multirow[t]{2}{*}{$\begin{array}{l}97-69 \\
\text { (Segment 10) }\end{array}$} & Lower half & $\begin{array}{l}\text { S97T000614 } \\
\text { S97T000608 } \\
\text { S97T000626 } \\
\end{array}$ & $\begin{array}{l}\text { Density } \\
\text { DSC/TGA } \\
\text { total alpha }\end{array}$ \\
\hline & & Upper half & S97T000638 & DSC/TGA \\
\hline T-204 & Composite & Solid & $\begin{array}{l}\text { S97T001191 } \\
\text { S97T001197 } \\
\text { S97T001198 } \\
\text { S97T001199 } \\
\text { S97T001200 } \\
\end{array}$ & $\begin{array}{l}\text { Density } \\
\text { DSC/TGA, TIC/TOC } \\
\text { GEA, Sr-90 } \\
\text { ICP } \\
\text { IC } \\
\end{array}$ \\
\hline
\end{tabular}

Notes:

GEA = gamma energy analysis

SpG = specific gravity

${ }^{1}$ Nuzum (1997a), Esch (1997), Steen 1997, and Nuzum (1997b) 


\section{B2.1.3 Analytical Results}

This section summarizes the sampling and analytical results associated with the March/April 1997 sampling and analysis of tanks 241-T-201, -T-202, -T-203, and -T-204. Table B2-5 indexes the total alpha activity, percent water, energetics, IC, and ICP analytical results associated with these tanks. These results are documented in Nuzum 1997a, Esch 1997, Steen 1997, and Nuzum 1997.

Table B2-5. Analytical Tables.

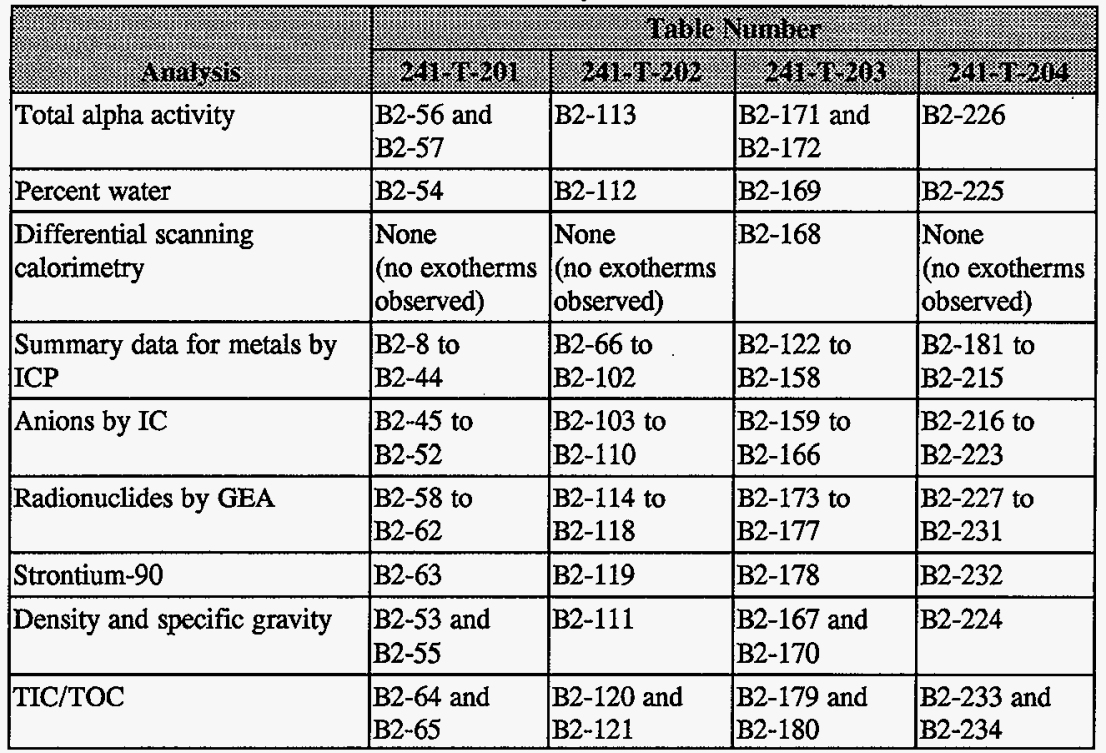

The four QC parameters assessed in conjunction with the T-200 series tank samples were standard recoveries, spike recoveries, duplicate analyses (relative percent differences), and blanks. The QC criteria are specified in the respective sampling and analysis plans for each tank (Hu 1997, Bell 1997, Schreiber 1997b, and Winkleman 1997). The only QC parameter for which limits are not specified in the sampling and analysis plans is blank contamination. The limits for blanks are set forth in guidelines followed by the laboratory, and all data results 
in this report have met those guidelines. Sample and duplicate pairs, in which any QC parameter was outside these limits, are footnoted in the sample mean column of the following data summary tables with an $\mathrm{a}, \mathrm{b}, \mathrm{c}, \mathrm{d}, \mathrm{e}, \mathrm{f}$, or $\mathrm{g}$ as follows.

- “a” indicates the standard recovery was below the QC limit.

- " $\mathrm{b}$ " indicates the standard recovery was above the QC limit.

- $" \mathrm{c}$ " indicates the spike recovery was below the QC limit.

- " $\mathrm{d}$ " indicates the spike recovery was above the QC limit.

- $\quad \mathrm{e}$ " indicates the RPD was above the QC limit.

- " $\mathrm{f}$ " indicates blank contamination.

- $\quad \mathrm{g}$ " indicates this is a tentatively identified compound.

In the analytical tables in this section, the "Mean" is the average of result and duplicate values. All values, including those below the detection level $(<)$, were averaged. If both sample and duplicate values were nondetected, the mean is expressed as a nondetected value. If one value was detected and the other was not, the mean is expressed as a detected value. If both values were detected, the mean is expressed as a detected value.

B2.1.3.1 Total Alpha Activity. Analyses for total alpha activity were performed on the segment-level samples recovered from all T-200 tanks. The samples were prepared by fusion digestion and were analyzed in duplicate. The results were averaged and reported as one value. These samples were relatively low in activity, and many results were not above detection limits. The highest result returned was $1.15 \mu \mathrm{Ci} / \mathrm{g}$.

B2.1.3.2 Thermogravimetric Analysis. Thermogravimetric analysis measures the mass of a sample as its temperature is increased at a constant rate. An gas (usually air or nitrogen) is passed over the sample during heating to remove any released gases. A decrease in the weight of a sample during TGA represents a loss of gaseous matter from the sample, through evaporation or through a reaction that forms gas phase products. The moisture content is estimated by assuming that all TGA sample weight loss up to a certain temperature (typically 150 to $200^{\circ} \mathrm{C}$ [ 300 to $\left.390^{\circ} \mathrm{F}\right]$ ) is caused by water evaporation. The temperature limit for moisture loss is chosen by the operator at an inflection point on the TGA plot. For the $\mathrm{T}-200$ series tanks, $200^{\circ} \mathrm{C}$ was selected as the threshold for determining water content. Other volatile matter fractions can often be differentiated by inflection points as well. 
B2.1.3.3 Differential Scanning Calorimetry. In a DSC analysis, heat absorbed or emitted by a substance is measured while the sample is heated at a constant rate. A gas (usually air or nitrogen) is passed over the sample material to remove any gases being released. The onset temperature for an endothermic or exothermic event is determined graphically.

The DSC analyses for these tanks were performed using a Mettler ${ }^{1}$ DSC 20 instrument or a Perkin-Elmer DSC 7 instrument. For tanks 241-T-201, -T-202, and -T-204, no exothermic reactions were noted; therefore, an upper limit of a 95 percent confidence interval on the mean for each sample was not calculated in most cases. The transitions observed represent endothermic events, which are principally caused by water evaporation.

B2.1.3.4 Inductively Coupled Plasma. Samples were prepared by fusion or acid digestion. Phosphorus and sulfur were analyzed as a solubility check for the phosphate and sulfate results reported from IC analyses. The liquid samples from tank 241-T-201 were analyzed directly. The full range of analytes was reported. The samples were analyzed in duplicate. The results were averaged and reported as one value. The potassium and nickel results for the ICP fusion analyses should be disregarded, because the samples were prepared in a nickel crucible by fusion using potassium hydroxide. In comparing acid and fusion digestion data from tank 241-T-203, there appear to be solubility related biases. Most of the quantified fusion analytes (bismuth, chromium, lanthanum, sodium, and strontium) are 20 to 30 percent higher in concentration than the acid results. For two analytes, iron and manganese, that behavior is reversed. The observed bias for iron is the same magnitude, however, the acid-based manganese value is triple the fusion value.

In addition, some irregular behavior was noted for some minor analytes. In tank 241-T-202, there appears to be an unusual pattern of quantified results followed by less than detection limits for arsenic, copper, lead, and thallium. Low levels of these analytes were also observed in tank 241-T-204. The presence of these trace analytes was not anticipated. These results may be from an unidentified interference or instrument error. These observations are not present in sufficient quantities to be statistically assessed. Furthermore, because they are not present in elevated concentrations, they do not impact the overall interpretation of the analytical results or calculation of inventory.

B2.1.3.5 Ion Chromotography. Samples were prepared by water digestion. The liquid samples were analyzed directly after being allowed to settle. The samples were analyzed in duplicate. The results were averaged and reported as one value. The full range of analytes was reported.

\footnotetext{
${ }^{1}$ Mettler is a registered trademark of Mettler Electronics, Anaheim, California.
} 
B2.1.3.6 Gamma Energy Analysis. Gamma energy analyses were performed on selected composite-level samples recovered from some of the T-200 tanks. The samples were prepared by fusion digestion and were analyzed in duplicate. The results were averaged and reported as one value. These samples were relatively low in activity, most results were not above the detection limit.

B2.1.3.7 Strontium-90 Analysis. A chemical separation and beta count was performed on selected fusion prepared composite-level samples. The results were averaged and reported as one value. These samples were relatively low in activity, most results were not above the detection limit.

\section{B2.2 VAPOR PHASE MEASUREMENT}

Before each sampling event, a vapor phase measurement was taken. These measurements supported the safety screening DQO (Dukelow et al. 1995) and the organic solvents DQO (Meacham et al. 1997). The vapor phase screening was taken for flammability issues. The vapor phase measurements were taken $15 \mathrm{ft}$ below riser 3 in the headspace of the tank, and results were obtained in the field (that is, no gas sample was sent to the laboratory for analysis). Table B2-6 shows the results of the vapor phase measurements.

Table B2-6. Results of Headspace Measurements from T-200 Tanks.

\begin{tabular}{|l|l|l|l|l|l|}
\hline & & & & \\
\hline
\end{tabular}

\section{B2.3 DESCRIPTION OF HISTORICAL SAMPLING EVENT}

Sampling data for tank 241-T-204 have been obtained for one sample obtained in 1978 and reported on December 4, 1978 (Horton 1978). The data are presented in Table B2-7. Pre-1989 analytical data have not been validated and should be used with caution.

No information was available regarding sample handling for this tank or the reason for the sampling. The sample was reported as being black, soft, and tar-like. 
Table B2-7. Historical Data Table. ${ }^{1}$

\begin{tabular}{|c|c|c|}
\hline 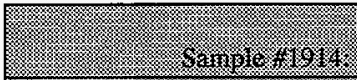 & 4yy & sit. \\
\hline 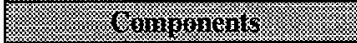 & 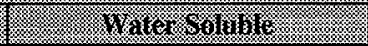 & (1) \\
\hline $\mathrm{Al}^{2}$ & $<0.002 \%$ & $0.02 \%$ \\
\hline $\mathrm{Bi}^{3+}$ & $0.006 \%$ & \\
\hline $\mathrm{CO}_{3}{ }^{2-}$ & $0.8 \%$ & \\
\hline $\mathrm{CrO}_{4}^{-}$ & $0.1 \%$ & \\
\hline $\mathrm{Cl}^{-}$ & $0.06 \%$ & \\
\hline$F$ & NR & \\
\hline $\mathrm{Fe}^{2}$ & 0.002 & $3.7 \%$ \\
\hline $\mathrm{Hg}^{2}$ & $0.004 \%$ & \\
\hline $\mathrm{K}^{+}$ & $2.7 \%$ & \\
\hline $\mathrm{La}^{3+}$ & NR & \\
\hline $\mathrm{Mn}^{2}$ & $<0.0004 \%$ & \\
\hline $\mathrm{Ni}^{2+}$ & NR & \\
\hline $\mathrm{NO}_{2}^{-}$ & $0.06 \%$ & \\
\hline $\mathrm{NO}_{3}^{-}$ & $5.0 \%$ & $0.3 \%$ \\
\hline $\mathrm{Na}^{+}$ & $3.0 \%$ & \\
\hline $\mathrm{OH}^{-}$ & $0.2 \%$ & \\
\hline $\mathrm{PO}_{4}^{3-}$ & $0.6 \%$ & $0.7 \%$ \\
\hline $\mathrm{SO}_{4}^{2-}$ & $<0.1 \%$ & $<0.1 \%$ \\
\hline $\mathrm{SiO}_{2}^{2-}$ & 0.008 & $2.7 \%$ \\
\hline $\mathrm{U}^{2}$ & $1.08 \mathrm{E}-07 \mathrm{~g} / \mathrm{g}$ & $1.10 \mathrm{E}-06 \mathrm{~g} / \mathrm{g}$ \\
\hline $\mathrm{Pu}^{2}$ & $6.02 \mathrm{E}-09 \mathrm{~g} / \mathrm{g}$ & $1.23 \mathrm{E}-06 \mathrm{~g} / \mathrm{g}$ \\
\hline $\mathrm{Am}^{2}$ & $5.43 \mathrm{E}-12 \mathrm{~g} / \mathrm{g}$ & \\
\hline${ }^{89+90} \mathrm{Sr}$ & $0.086 \mu \mathrm{Ci} / \mathrm{g}$ & $0.137 \mu \mathrm{Ci} / \mathrm{g}$ \\
\hline${ }^{137} \mathrm{Cs}$ & $0.009 \mu \mathrm{Ci} / \mathrm{g}$ & $0.012 \mu \mathrm{Ci} / \mathrm{g}$ \\
\hline${ }^{155} \mathrm{Eu}^{2}$ & $\mathrm{NR}$ & \\
\hline TOC & $0.6 M$ & \\
\hline Water solubility & $20 \%$ & \\
\hline Bulk density & $1.07 \mathrm{~g} / \mathrm{cm}^{2}$ & \\
\hline Percent water & 73.0 & \\
\hline
\end{tabular}

Notes:

NR $=$ Not requested (analysis)

${ }^{1}$ These data have not been validated and should be used with caution.

${ }^{2}$ All oxidation states 


\section{B2.4 1997 PUSH CORE DATA TABLES}

Tables B2-8 to B2-234 show the data gathered from the T-200 series tanks.

Table B2-8. Tank 241-T-201 Analytical Results: Aluminum (ICP).

\begin{tabular}{|c|c|c|c|c|c|}
\hline Sample & 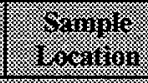 & $\begin{array}{l}\text { Samply } \\
\text { Portrinos }\end{array}$ & isteritin & 3.8. & 1818201 \\
\hline \multicolumn{3}{|c|}{ 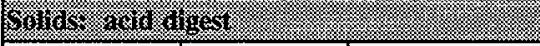 } & 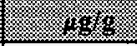 & 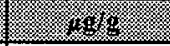 & 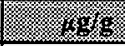 \\
\hline S97T001250 & \multirow[t]{2}{*}{ Core 192} & Solid composite & 105 & 96.2 & 101 \\
\hline S97T001256 & & Solid composite & 80 & 75.7 & 77.8 \\
\hline \multicolumn{2}{|c|}{ Horldis r r.sing } & (s- & 1.945 & 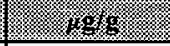 & 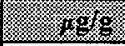 \\
\hline S97T000921 & \multirow[t]{2}{*}{ 192: 6} & Upper half & $<1,110$ & 1,170 & $<1,140$ \\
\hline S97T000901 & & Lower half & $<1,020$ & $<996$ & $<1,010$ \\
\hline S97T000922 & \multirow[t]{2}{*}{ 192: 7} & Upper half & $<1,000$ & $<1,010$ & $<1,010$ \\
\hline S97T000919 & & Lower half & $<1,040$ & $<1,020$ & $<1,030$ \\
\hline S97T000923 & \multirow[t]{2}{*}{ 192: 8} & Upper half & $<1,020$ & $<1,040$ & $<1,030$ \\
\hline S97T000920 & & Lower half & $<1,030$ & $<1,010$ & $<1,020$ \\
\hline 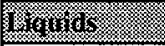 & \multicolumn{2}{|c|}{18} & r & 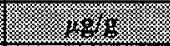 & 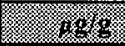 \\
\hline S97T001203 & 192: 1 & Drainable liquid & $<7.27$ & $<7.27$ & $<7.27$ \\
\hline S97T000834 & 192: 2 & Drainable liquid & $<1.83$ & $<1.83$ & $<1.83$ \\
\hline S97T000835 & 192: 3 & Drainable liquid & $<1.83$ & $<1.83$ & $<1.83$ \\
\hline S97T000836 & 192: 4 & Drainable liquid & $<1.83$ & $<1.83$ & $<1.83$ \\
\hline S97T000837 & $192: 5$ & Drainable liquid & $<1.83$ & $<1.83$ & $<1.83$ \\
\hline
\end{tabular}


Table B2-9. Tank 241-T-201 Analytical Results: Antimony (ICP).

\begin{tabular}{|c|c|c|c|c|c|}
\hline $\begin{array}{l}\text { Sample } \\
\text { Vanilor }\end{array}$ & 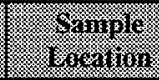 & $\begin{array}{l}\text { Smingle } \\
\text { Tomant }\end{array}$ & inguin: & 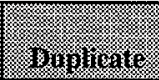 & Viln \\
\hline Sulin & (yess & & (1098. & $(8.69 \mathrm{~g}$ & $3.8186 \%$ \\
\hline S97T001250 & \multirow[t]{2}{*}{ Core 192} & Solid composite & $<46$ & $<45.8$ & $<45.9$ \\
\hline S97T001256 & & Solid composite & $<11.6$ & $<11.4$ & $<11.5$ \\
\hline \multicolumn{3}{|l|}{ 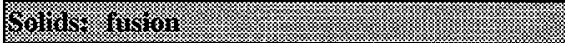 } & 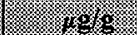 & $\sqrt{18} 8 \mathrm{~g}$ & 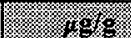 \\
\hline S97T000921 & \multirow[t]{2}{*}{$192: 6$} & Upper half & $<1,330$ & $<1,310$ & $<1,320$ \\
\hline S97T000901 & & Lower half & $<1,220$ & $<1,200$ & $<1,210$ \\
\hline S97T000922 & 192: 7 & Upper half & $<1,210$ & $<1,210$ & $<1,210$ \\
\hline S97T000919 & & Lower half & $<1,250$ & $<1,220$ & $<1,240$ \\
\hline S97T000923 & \multirow[t]{2}{*}{$192: 8$} & Upper half & $<1,220$ & $<1,250$ & $<1,240$ \\
\hline S97T000920 & & Lower half & $<1,230$ & $<1,220$ & $<1,230$ \\
\hline \multicolumn{3}{|l|}{ Fy,its: } & .898 & 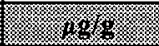 & 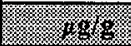 \\
\hline S97T001203 & 192: 1 & Drainable liquid & $<8.71$ & 17.1 & $<12.9^{\mathrm{QC}: e}$ \\
\hline S97T000834 & 192: 2 & Drainable liquid & $<2.2$ & $<2.2$ & $<2.2$ \\
\hline S97T000835 & 192: 3 & Drainable liquid & $<2.2$ & $<2.2$ & $<2.2$ \\
\hline S97T000836 & 192: 4 & Drainable liquid & $<2.2$ & $<2.2$ & $<2.2$ \\
\hline S97T000837 & 192: 5 & Drainable liquid & $<2.2$ & $<2.2$ & $<2.2$ \\
\hline
\end{tabular}


Table B2-10. Tank 241-T-201 Analytical Results: Arsenic (ICP).

\begin{tabular}{|c|c|c|c|c|c|}
\hline Maminils & 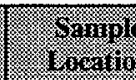 & Thingl & 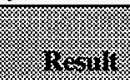 & Burticin & Whan \\
\hline \multicolumn{3}{|c|}{ 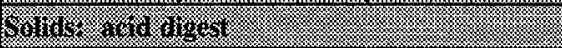 } & $\sqrt{3}+48$ & 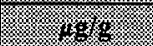 & (1) \\
\hline S97T001250 & Core 192 & Solid composite & $<76.7$ & $<76.4$ & $<76.6$ \\
\hline S97T001256 & & Solid composite & $<19.3$ & $<19.1$ & $<19.2$ \\
\hline \multicolumn{3}{|c|}{ 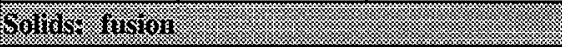 } & 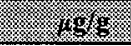 & 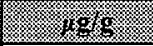 & 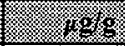 \\
\hline S97T000921 & \multirow[t]{2}{*}{ 192: 6} & Upper half & $<2,220$ & $<2,190$ & $<2,210$ \\
\hline S97T000901 & & Lower half & $<2,040$ & $<1,990$ & $<2,020$ \\
\hline S97T000922 & \multirow[t]{2}{*}{ 192: 7} & Upper half & $<2,010$ & $<2,020$ & $<2,020$ \\
\hline S97T000919 & & Lower half & $<2,080$ & $<2,040$ & $<2,060$ \\
\hline S97T000923 & \multirow[t]{2}{*}{ 192: 8} & Upper half & $<2,030$ & $<2,090$ & $<2,060$ \\
\hline S97T000920 & & Lower half & $<2,060$ & $<2,030$ & $<2,050$ \\
\hline (Mulits: & \multicolumn{2}{|c|}{3} & 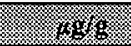 & 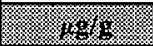 & 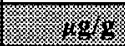 \\
\hline S97T001203 & 192: 1 & Drainable liquid & $<14.5$ & $<14.5$ & $<14.5$ \\
\hline S97T000834 & 192: 2 & Drainable liquid & $<3.67$ & $<3.67$ & $<3.67$ \\
\hline S97T000835 & $192: 3$ & Drainable liquid & $<3.67$ & $<3.67$ & $<3.67$ \\
\hline S97T000836 & 192: 4 & Drainable liquid & $<3.67$ & $<3.67$ & $<3.67$ \\
\hline S97T000837 & $192: 5$ & Drainable liquid & $<3.67$ & $<3.67$ & $<3.67$ \\
\hline
\end{tabular}




\begin{tabular}{|c|c|c|c|c|c|}
\hline$\varepsilon 8^{\circ} \mathrm{I}>$ & $\mathcal{E} 8^{\circ} I>$ & $\varepsilon 8^{\circ} \mathrm{I}>$ & p!̣nb!̣ गqRựeId & $s: 26 \mathrm{I}$ & $\angle E 8000$ LL6S \\
\hline$E 8^{\circ} \mathrm{I}>$ & $\varepsilon 8^{\circ} I>$ & $\varepsilon 8^{\prime} I>$ & p!̣nb!̣ əqquụexa & $\nabla: 26 \mathrm{I}$ & $9 \varepsilon 8000 \mathrm{~L} \angle \mathrm{S}$ \\
\hline$\varepsilon 8^{\circ} \mathrm{I}>$ & $\mathcal{E} 8^{\circ} \mathrm{I}>$ & $\varepsilon 8^{\circ} \mathrm{I}>$ & 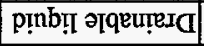 & $\varepsilon: 26 \mathrm{~T}$ & SE8000LL6S \\
\hline$\varepsilon 8^{\circ} I>$ & $\varepsilon 8^{\circ} I>$ & $\varepsilon 8^{\circ} \mathrm{I}>$ & 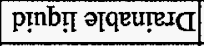 & $Z: 26 \mathrm{I}$ & $t \varepsilon 8000 \mathrm{~L} L 6 \mathrm{~S}$ \\
\hline$\angle Z^{\circ} L>$ & $\angle Z^{\circ} L>$ & $\angle Z \angle>$ & 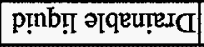 & I :26I & E0ZIOOLL6S \\
\hline 7\% & 76 & 806 & & & 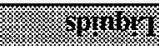 \\
\hline $020^{6} I>$ & $010^{6} I>$ & $0 \varepsilon 0^{\prime} I>$ & JाEप JəMOT & \multirow[b]{2}{*}{$8: 26 \mathrm{I}$} & $026000 \mathrm{~J} \angle 6 \mathrm{~S}$ \\
\hline $0 E 0^{\circ} T>$ & $0+0^{6} \mathrm{~T}>$ & $020^{6} \Upsilon \mathrm{T}$ & JाEq xadd & & $\varepsilon 26000 \mathrm{~L} / 6 \mathrm{~S}$ \\
\hline $0 E 0^{6} I>$ & $0 z 0^{\prime} I>$ & $0+0^{6} I>$ & I[еप دәмот] & \multirow[b]{2}{*}{$\angle: 26 \mathrm{I}$} & $6 \mathrm{I} 6000 \mathrm{~L} \angle 6 \mathrm{~S}$ \\
\hline $010^{6} \mathrm{I}>$ & $010^{6} \mathrm{~T}>$ & $000^{6} \mathrm{~T}>$ & Iाeч radd & & $226000 \mathrm{~L} L 6 \mathrm{~S}$ \\
\hline OIO'I> & $966>$ & $020^{\circ} \mathrm{I}>$ & J[Еप IOMOT] & \multirow[b]{2}{*}{$9: 26 \mathrm{I}$} & I06000LL6S \\
\hline $00 I^{6} I>$ & $060^{6} \mathrm{I}>$ & OII'I $>$ & JाЕप $\operatorname{Iədd}_{\Omega}$ & & $I Z 6000 \mathrm{~L} \angle 6 \mathrm{~S}$ \\
\hline (6) & 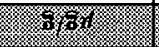 & (3/ & & & 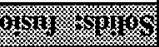 \\
\hline $0 z$ & $\varepsilon \cdot 0 z$ & $9.6 \mathrm{I}$ & ət!soduros p!Ios & \multirow[b]{2}{*}{261 ә } & 9SZI00L $\angle 6 S$ \\
\hline 709 & $9^{\circ}$ IS & {$[\cdot 19$} & əł!̣soduros p!IOS & & $0 S Z I 00 \mathrm{~L} \angle \mathrm{S}$ \\
\hline $3 \% \%$ & 640 & ingin: & & \multicolumn{2}{|c|}{ 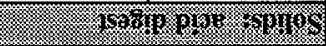 } \\
\hline 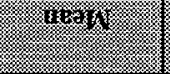 & 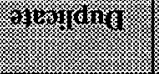 & (19: & 119110. & aristin & 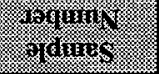 \\
\hline
\end{tabular}

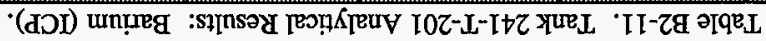


Table B2-12. Tank 241-T-201 Analytical Results: Beryllium (ICP).

\begin{tabular}{|c|c|c|c|c|c|}
\hline Simple & $\begin{array}{l}\text { Sample } \\
\text { Sorantion }\end{array}$ & 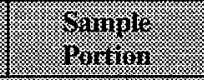 & Resin: & Bupluatr & il. \\
\hline \multicolumn{2}{|c|}{ 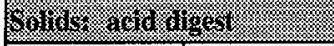 } & 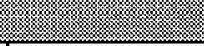 & 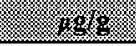 & 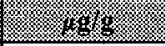 & 48 \\
\hline S97T001250 & Core 192 & Solid composite & $<3.84$ & $<3.82$ & $<3.83$ \\
\hline S97T001256 & & Solid composite & $<0.964$ & $<0.953$ & $<0.958$ \\
\hline \multicolumn{3}{|c|}{ Soligh- insion. } & 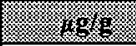 & 1. & (1.96. \\
\hline S97T000921 & \multirow[t]{2}{*}{ 192: 6} & Upper half & $<111$ & $<109$ & $<110$ \\
\hline S97T000901 & & Lower half & $<102$ & $<99.6$ & $<101$ \\
\hline S97T000922 & \multirow[t]{2}{*}{ 192: 7} & Upper half & $<100$ & $<101$ & $<101$ \\
\hline S97T000919 & & Lower half & $<104$ & $<102$ & $<103$ \\
\hline S97T000923 & \multirow[t]{2}{*}{ 192: 8} & Upper half & $<102$ & $<104$ & $<103$ \\
\hline S97T000920 & & Lower half & $<103$ & $<101$ & $<102$ \\
\hline Heining : & & (1) & ( & 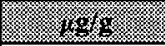 & - 198 \\
\hline S97T001203 & 192: 1 & Drainable liquid & $<0.727$ & $<0.727$ & $<0.727$ \\
\hline S97T000834 & 192: 2 & Drainable liquid & $<0.183$ & $<0.183$ & $<0.183$ \\
\hline S97T000835 & 192: 3 & Drainable liquid & $<0.183$ & $<0.183$ & $<0.183$ \\
\hline S97T000836 & 192: 4 & Drainable liquid & $<0.183$ & $<0.183$ & $<0.183$ \\
\hline S97T000837 & 192: 5 & Drainable liquid & $<0.183$ & $<0.183$ & $<0.183$ \\
\hline
\end{tabular}


HNF-1501 Rev, 0

Table B2-13. Tank 241-T-201 Analytical Results: Bismuth (ICP).

\begin{tabular}{|c|c|c|c|c|c|}
\hline Sample & S Somples: & f: Sample & Resin: & 3ilspincuse & 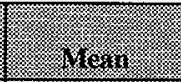 \\
\hline \multicolumn{3}{|c|}{ 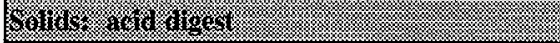 } & 4.848 & (39) & 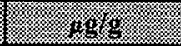 \\
\hline S97T001250 & \multirow[t]{2}{*}{ Core 192} & Solid composite & $1.28 \mathrm{E}+05$ & $1.10 \mathrm{E}+05$ & $1.19 \mathrm{E}+05^{\mathrm{eC}: \mathrm{d}}$ \\
\hline S97T001256 & & Solid composite & 19700 & 20500 & $20100^{\mathrm{QC:c}}$ \\
\hline \multicolumn{3}{|l|}{ 501368\% Mison: } & $\sqrt{1.48}$ & 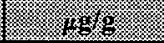 & (3) \\
\hline S97T000921 & \multirow[t]{2}{*}{ 192: 6} & Upper half & $91,800.00$ & $90,300.00$ & $91,100.00$ \\
\hline S97T000901 & & Lower half & $1.11 \mathrm{E}+05$ & $1.12 \mathrm{E}+05$ & $1.12 \mathrm{E}+05$ \\
\hline S97T000922 & \multirow[t]{2}{*}{ 192: 7} & Upper half & $1.03 \mathrm{E}+05$ & 60,100 & $81,600^{\mathrm{eC:}:}$ \\
\hline S97T000919 & & Lower half & 92,900 & 97,300 & 95,100 \\
\hline S97T000923 & \multirow[t]{2}{*}{ 192: 8} & Upper half & 90,300 & 87,900 & 89,100 \\
\hline S97T000920 & & Lower half & 79,100 & 75,700 & 77,400 \\
\hline Mrials: & $\sqrt{3}$ & (1) & 4.89 & 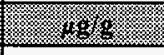 & 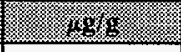 \\
\hline S97T001203 & 192: 1 & Drainable liquid & $<14.5$ & $<14.5$ & $<14.5$ \\
\hline S97T000834 & 192: 2 & Drainable liquid & $<3.67$ & $<3.67$ & $<3.67$ \\
\hline S97T000835 & 192: 3 & Drainable liquid & 9.78 & $<3.67$ & $<6.73^{\text {eC:0 }}$ \\
\hline S97T000836 & 192: 4 & Drainable liquid & 6.39 & $<3.67$ & $<5.03^{\mathrm{QC}: \mathrm{e}}$ \\
\hline S97T000837 & 192: 5 & Drainable liquid & $<3.67$ & 4.22 & $<3.94$ \\
\hline
\end{tabular}


Table B2-14. Tank 241-T-201 Analytical Results: Boron (ICP).

\begin{tabular}{|c|c|c|c|c|c|}
\hline $\begin{array}{l}\text { Sample } \\
\text { Vrimber }\end{array}$ & t) Gample & Somple & n-sill & Buplisate & irien \\
\hline \multicolumn{3}{|c|}{ 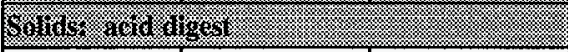 } & 1.938 & $4.08 \%$ & 2. $13 \mathrm{~g}$ \\
\hline S97T001250 & \multirow[t]{2}{*}{ Core 192} & Solid composite & 165 & 178 & 172 \\
\hline S97T001256 & & Solid composite & 87.2 & 117 & $102^{\mathrm{QC}: c}$ \\
\hline \multicolumn{3}{|c|}{ boling. rusion: } & 1.860 & 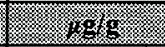 & 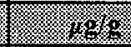 \\
\hline S97T000921 & \multirow[t]{2}{*}{ 192: 6} & Upper half & $<1,110$ & $<1,090$ & $<1,100$ \\
\hline S97T000901 & & Lower half & $<1,020$ & $<996$ & $<1,010$ \\
\hline S97T000922 & \multirow[t]{2}{*}{ 192: 7} & Upper half & $<1,000$ & $<1,010$ & $<1,010$ \\
\hline S97T000919 & & Lower half & $<1,040$ & $<1,020$ & $<1,030$ \\
\hline S97T000923 & \multirow[t]{2}{*}{ 192: 8} & Upper half & $<1,020$ & $<1,040$ & $<1,030$ \\
\hline S97T000920 & & Lower half & $<1,030$ & $<1,010$ & $<1,020$ \\
\hline Manis: & : : : & (1) & $8.6 \%$ & 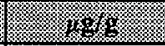 & 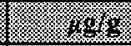 \\
\hline S97T001203 & 192: 1 & Drainable liquid & $<7.27$ & $<7.27$ & $<7.27$ \\
\hline S97T000834 & 192: 2 & Drainable liquid & 1.97 & 1.88 & 1.93 \\
\hline S97T000835 & 192: 3 & Drainable liquid & 2.32 & 2.3 & 2.31 \\
\hline S97T000836 & 192: 4 & Drainable liquid & 2.74 & 3.04 & 2.89 \\
\hline S97T000837 & $192: 5$ & Drainable liquid & 2.09 & 1.94 & 2.01 \\
\hline
\end{tabular}




\begin{tabular}{|c|c|c|c|c|c|}
\hline ह8! $\mathrm{I}^{\circ}>$ & $\varepsilon 8 I^{\circ} 0>$ & E\&I०0> & pịnḅ़ ग[qeuṬexa & $\varsigma: 26 \mathrm{I}$ & $\angle E 8000 L \angle 6 S$ \\
\hline$\varepsilon 8 \mathrm{I}^{\circ} 0>$ & $\varepsilon 8 I^{\circ} 0>$ & $\varepsilon 8 I^{\circ} 0>$ & p!̣nb!̣ әтqеu़ฺexa & $\nabla: 26 \mathrm{~T}$ & $9 \varepsilon 8000 \mathrm{I} L 6 \mathrm{~S}$ \\
\hline$\varepsilon 8 I^{\circ} 0>$ & $\varepsilon 8 I^{\circ} 0>$ & $\varepsilon 8 I^{\circ} 0>$ & 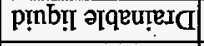 & $\varepsilon: 261$ & SE8000LL6S \\
\hline$\varepsilon 8 \mathrm{I}^{\circ} 0>$ & $\varepsilon 8 \tau^{\circ} 0>$ & E8I $0>$ & p!nḅ̣n әqеurexa & $z: 26 \mathrm{I}$ & $t \varepsilon 8000 \mathrm{~L} L 6 \mathrm{~S}$ \\
\hline$\angle Z L^{\circ} 0>$ & $\angle Z L^{\circ} 0>$ & $\angle Z L^{\circ} O>$ & p!̣nb!̣ गqqeựeId & $I: Z 6 I$ & E0ZT00.JL6S \\
\hline (5) & 97for & 64) & (: & (4) & 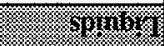 \\
\hline zor $>$ & IOI $>$ & $\varepsilon 0 I>$ & न[Еप IәмOT & \multirow[b]{2}{*}{$8: 26 \mathrm{I}$} & $026000 \times \angle 6 S$ \\
\hline$\varepsilon 0$ EO $>$ & $\pm 0 \mathrm{I}>$ & ZOI $>$ & झाषप Iadd & & $\varepsilon Z 6000 \mathrm{I} L 6 \mathrm{~S}$ \\
\hline EOI $>$ & $\mathrm{zor}>$ & tor $>$ & J[EY دәMOT] & \multirow[b]{2}{*}{$L: 26 \mathrm{I}$} & $6 \mathrm{I} 6000 \mathrm{~L} \angle 6 \mathrm{~S}$ \\
\hline I0โ> & IOI> & $00 \mathrm{~T}>$ & ғॅеч Iədd & & $226000 \mathrm{~L} \angle 6 \mathrm{~S}$ \\
\hline IOI $>$ & $9 \cdot 66>$ & ZOI $>$ & JाEY IәMOT & \multirow[b]{2}{*}{$9: 26 I$} & $\mathrm{I} 06000 \mathrm{~L} \angle 6 \mathrm{~S}$ \\
\hline OII $>$ & $60 I>$ & III $>$ & Jास् Iədd & & $I Z 6000 \mathrm{I} \angle 6 \mathrm{~S}$ \\
\hline $8 \%$ & (5) & 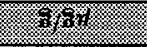 & & & 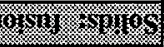 \\
\hline $896^{\circ} 0>$ & $E S 6^{\circ} 0>$ & $\not 96^{\circ} 0>$ & શI!̣oduros p!Ios & \multirow[b]{2}{*}{ 26I $210 \mathrm{D}$} & 9SZI00IL6S \\
\hline $\mathcal{E} 8^{\bullet} \mathcal{E}>$ & $Z 8^{\circ} \varepsilon>$ & $\nabla 8^{\circ} \varepsilon>$ & əి!̣soduros p!̣os & & OSZIOOLL6S \\
\hline 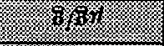 & 16in & 860 & & \multicolumn{2}{|c|}{ 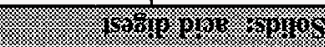 } \\
\hline 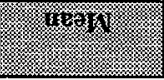 & oryoriding & 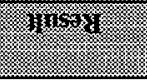 & 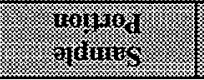 & 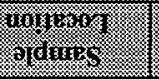 & Jisganify \\
\hline
\end{tabular}

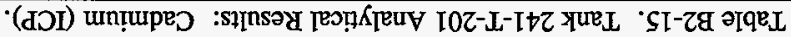


Table B2-16. Tank 241-T-201 Analytical Results: Calcium (ICP).

\begin{tabular}{|c|c|c|c|c|c|}
\hline Samile & Sarapts & Sample: & 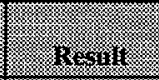 & miplinater & 1ran. \\
\hline \multicolumn{2}{|c|}{ 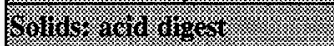 } & (4) & f & 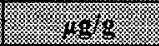 & f \\
\hline S97T001250 & \multirow[t]{2}{*}{ Core 192} & Solid composite & 1,360 & 1,120 & $1,240^{\mathrm{oC}: \mathrm{d}}$ \\
\hline S97T001256 & & Solid composite & 294 & 304 & 299 \\
\hline \multicolumn{3}{|c|}{ boligh 6usini: } & $\sqrt{1 . g \mathrm{~g}}$ & 8.8 & 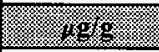 \\
\hline S97T000921 & \multirow[t]{2}{*}{ 192: 6} & Upper half & $<2,220$ & $<2,190$ & $<2,210$ \\
\hline S97T000901 & & Lower half & $<2,040$ & $<1,990$ & $<2,020$ \\
\hline S97T000922 & \multirow[t]{2}{*}{$192: 7$} & Upper half & $<2,010$ & $<2,020$ & $<2,020$ \\
\hline S97T000919 & & Lower half & $<2,080$ & $<2,040$ & $<2,060$ \\
\hline S97T000923 & \multirow[t]{2}{*}{ 192: 8} & Upper half & $<2,030$ & $<2,090$ & $<2,060$ \\
\hline S97T000920 & & Lower half & $<2,060$ & $<2,030$ & $<2,050$ \\
\hline \multicolumn{3}{|l|}{ Lanin } & $146 \mathrm{~s}$ & . & 8.183 \\
\hline S97T001203 & 192: 1 & Drainable liquid & $<14.5$ & $<14.5$ & $<14.5$ \\
\hline S97T000834 & 192: 2 & Drainable liquid & $<3.67$ & $<3.67$ & $<3.67$ \\
\hline S97T000835 & 192: 3 & Drainable liquid & $<3.67$ & $<3.67$ & $<3.67$ \\
\hline S97T000836 & 192: 4 & Drainable liquid & $<3.67$ & $<3.67$ & $<3.67$ \\
\hline S97T000837 & 192: 5 & Drainable liquid & $<3.67$ & $<3.67$ & $<3.67$ \\
\hline
\end{tabular}


Table B2-17. Tank 241-T-201 Analytical Results: Cerium (ICP).

\begin{tabular}{|c|c|c|c|c|c|}
\hline Sample & 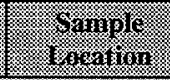 & Samplo & iresiat & Huplinate & . \\
\hline \multicolumn{3}{|c|}{ 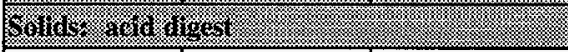 } & 3.98 & 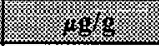 & 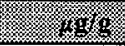 \\
\hline S97T001250 & Core 192 & Solid composite & 92.1 & $<76.4$ & $<84.3$ \\
\hline S97T001256 & & Solid composite & 19.5 & 20.4 & 19.9 \\
\hline \multicolumn{3}{|c|}{ Lonifs vision: } & 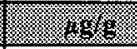 & 1.96 & 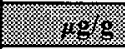 \\
\hline S97T000921 & \multirow[t]{2}{*}{ 192: 6} & Upper half & $<2,220$ & $<2,190$ & $<2,210$ \\
\hline S97T000901 & & Lower half & $<2,040$ & $<1,990$ & $<2,020$ \\
\hline S97T000922 & \multirow[t]{2}{*}{ 192: 7} & Upper half & $<2,010$ & $<2,020$ & $<2,020$ \\
\hline S97T000919 & & Lower half & $<2,080$ & $<2,040$ & $<2,060$ \\
\hline S97T000923 & \multirow[t]{2}{*}{ 192: 8} & Upper half & $<2,030$ & $<2,090$ & $<2,060$ \\
\hline S97T000920 & & Lower half & $<2,060$ & $<2,030$ & $<2,050$ \\
\hline \multicolumn{3}{|l|}{ 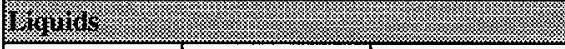 } & $1.18 \%$ & 1498 & 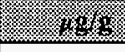 \\
\hline S97T001203 & 192: 1 & Drainable liquid & $<14.5$ & $<14.5$ & $<14.5$ \\
\hline S97T000834 & 192: 2 & Drainable liquid & $<3.67$ & $<3.67$ & $<3.67$ \\
\hline S97T000835 & 192: 3 & Drainable liquid & $<3.67$ & $<3.67$ & $<3.67$ \\
\hline S97T000836 & 192: 4 & Drainable liquid & $<3.67$ & $<3.67$ & $<3.67$ \\
\hline S97T000837 & 192: 5 & Drainable liquid & $<3.67$ & $<3.67$ & $<3.67$ \\
\hline
\end{tabular}


HNF-1501 Rev, 0

Table B2-18. Tank 241-T-201 Analytical Results: Chromium (ICP).

\begin{tabular}{|c|c|c|c|c|c|}
\hline $\begin{array}{l}\text { Simiplos: } \\
\text { Nimingr? }\end{array}$ & 4 Timinli & Sample & resulin: & Minplicats. & (1) \\
\hline \multicolumn{3}{|c|}{ 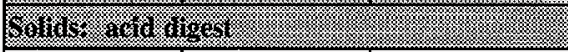 } & s. & 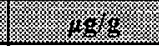 & 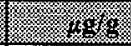 \\
\hline S97T001250 & \multirow[t]{2}{*}{ Core 192} & Solid composite & 5,650 & 5,050 & $5,350^{\mathrm{eC}: \mathrm{d}}$ \\
\hline S97T001256 & & Solid composite & 1,560 & 1,620 & $1,590^{\mathrm{eC}: \mathrm{c}}$ \\
\hline \multicolumn{2}{|l|}{ 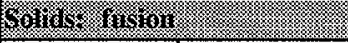 } & (3) & (1.58) & 1.89 .9 & 14. \\
\hline S97T000921 & \multirow[t]{2}{*}{ 192: 6} & Upper half & 4,030 & 4,080 & 4,060 \\
\hline S97T000901 & & Lower half & 5,360 & 5,390 & 5,380 \\
\hline S97T000922 & \multirow[t]{2}{*}{ 192: 7} & Upper half & 4,970 & 5,100 & 5,040 \\
\hline S97T000919 & & Lower half & 5,110 & 5,020 & 5,070 \\
\hline S97T000923 & \multirow[t]{2}{*}{ 192: 8} & Upper half & 2,770 & 2,810 & 2,790 \\
\hline S97T000920 & & Lower half & 2,900 & 2,880 & 2,890 \\
\hline (1) & $\sqrt{3}$ & & $13.26 \%$ & 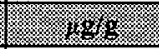 & $\sqrt{12} 8$ \\
\hline S97T001203 & 192: 1 & Drainable liquid & 4.84 & 4.88 & 4.86 \\
\hline S97T000834 & 192: 2 & Drainable liquid & 4.91 & 4.93 & 4.92 \\
\hline S97T000835 & 192: 3 & Drainable liquid & 5.32 & 4.58 & 4.95 \\
\hline S97T000836 & 192: 4 & Drainable liquid & 5.84 & 5.58 & 5.71 \\
\hline S97T000837 & 192: 5 & Drainable liquid & 3.35 & 3.46 & 3.4 \\
\hline
\end{tabular}




\begin{tabular}{|c|c|c|c|c|c|}
\hline$\nabla \varepsilon L^{\circ} 0>$ & $\nabla \varepsilon L^{\circ} 0>$ & $\nabla \varepsilon L^{\circ} 0>$ & 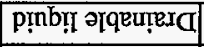 & $s: 26 \mathrm{I}$ & $\angle E 8000 \mathrm{~L} L 6 \mathrm{~S}$ \\
\hline$\nabla \varepsilon L O>$ & $\nabla \varepsilon L^{\circ} 0>$ & $\nabla \varepsilon L^{\circ} 0>$ & p!̣nb!̣ गqqu!̣esa & $t: 26 \mathrm{I}$ & $9 \varepsilon 8000 \mathrm{LL} 6 \mathrm{~S}$ \\
\hline$t \varepsilon L \cdot 0>$ & $t \varepsilon L^{\circ} 0>$ & $\nabla \varepsilon L \cdot 0>$ & 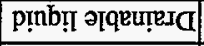 & $\varepsilon: 26 \mathrm{I}$ & SE8000LL6S \\
\hline$\downarrow \varepsilon L^{\circ} 0>$ & $t \varepsilon L^{\circ} 0>$ & $+\varepsilon L^{\circ} 0>$ & 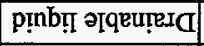 & $z: 26 \mathrm{I}$ & $t \varepsilon 8000 \mathrm{I} L 6 \mathrm{~S}$ \\
\hline II'S & $S I^{\prime} S$ & $90^{\circ} \mathrm{S}$ & p!̣nb!̣ әqqeựerd & $\mathrm{I}: \mathrm{Z6I}$ & EOZIOOLL6S \\
\hline 6 & 8.6\% & 1610\% & & & - Jiniling \\
\hline $60 t>$ & $90 t>$ & ZIt $>$ & न[ЕЧ دәMOT] & . & $0 Z 6000 \mathrm{~J} / 6 \mathrm{~S}$ \\
\hline ZTt> & LIt $>$ & $L 0 t>$ & Jाeq Iədđ & $8: 26 \mathrm{I}$ & $\varepsilon \tau 6000 \mathrm{~L} / 6 \mathrm{~S}$ \\
\hline ZIt $>$ & $L 0 t>$ & $9 I t>$ & I[Rप JәMOT] & & $6 \mathrm{I} 6000 \mathrm{~J} L 6 \mathrm{~S}$ \\
\hline$\varepsilon 0 t>$ & $t 0 t>$ & $20 t>$ & JॉE् Iədd & $L: Z 6 \mathrm{I}$ & $226000 \mathrm{~L} \angle 6 \mathrm{~S}$ \\
\hline$\varepsilon 0 t>$ & $86 \varepsilon>$ & $80 t>$ & गएप ГәмОТ & & I06000IL6S \\
\hline$I t D>$ & $8 \varepsilon \phi>$ & $t+t>$ & fleप Iədd & $9: 26 \mathrm{I}$ & IZ6000J L6S \\
\hline 8. & 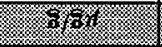 & 7\% & & $3=$ & 0911189198 \\
\hline $78^{\circ} \varepsilon>$ & $18^{\circ} \varepsilon>$ & $98^{\circ} \varepsilon>$ & әt!soduros p!IOS & & 9SZIO0L $\angle 6 \mathrm{~S}$ \\
\hline$\varepsilon^{*} \mathcal{S I}>$ & $\varepsilon^{\cdot} S I>$ & $\mathcal{E} \mathfrak{S I >}$ & əl!̣soduos p!ros & 261 210 & $0 \subseteq Z 100 L \angle 6 S$ \\
\hline 䇥 & 4.67 & 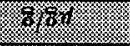 & & (5) & 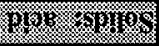 \\
\hline (11.4 & 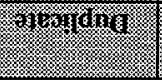 & (1) & 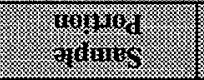 & 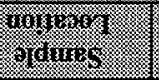 & of dings \\
\hline
\end{tabular}

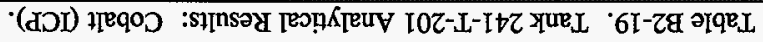


HNF-1501 Rev. 0

Table B2-20. Tank 241-T-201 Analytical Results: Copper (ICP).

\begin{tabular}{|c|c|c|c|c|c|}
\hline Siminger & Sariples & Shimple: & IIesin: & 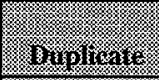 & Wejan \\
\hline \multicolumn{3}{|c|}{ 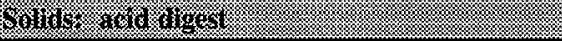 } & .848 & (1) & 38 \\
\hline S97T001250 & \multirow[t]{2}{*}{ Core 192} & Solid composite & $<7.67$ & $<7.64$ & $<7.65$ \\
\hline S97T001256 & & Solid composite & $<1.93$ & $<1.91$ & $<1.92$ \\
\hline \multicolumn{3}{|c|}{ 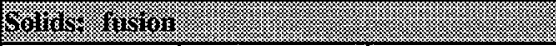 } & 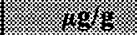 & 1.093 & 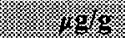 \\
\hline S97T000921 & \multirow[t]{2}{*}{ 192: 6} & Upper half & $<222$ & $<219$ & $<221$ \\
\hline S97T000901 & & Lower half & $<204$ & $<199$ & $<202$ \\
\hline S97T000922 & \multirow[t]{2}{*}{$192: 7$} & Upper half & $<201$ & $<202$ & $<202$ \\
\hline S97T000919 & & Lower half & $<208$ & $<204$ & $<206$ \\
\hline S97T000923 & \multirow[t]{2}{*}{ 192: 8} & Upper half & $<203$ & $<209$ & $<206$ \\
\hline S97T000920 & & Lower half & $<206$ & $<203$ & $<205$ \\
\hline byurus & $1: 0.20$ & 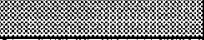 & 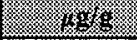 & 2.1868 & 1808 \\
\hline S97T001203 & 192: 1 & Drainable liquid & $<1.45$ & $<1.45$ & $<1.45$ \\
\hline S97T000834 & 192: 2 & Drainable liquid & $<0.367$ & $<0.367$ & $<0.367$ \\
\hline S97T000835 & 192: 3 & Drainable liquid & $<0.367$ & $<0.367$ & $<0.367$ \\
\hline S97T000836 & 192: 4 & Drainable liquid & $<0.367$ & $<0.367$ & $<0.367$ \\
\hline S97T000837 & 192: 5 & Drainable liquid & $<0.367$ & $<0.367$ & $<0.367$ \\
\hline
\end{tabular}




\begin{tabular}{|c|c|c|c|c|c|}
\hline$\varepsilon 8^{\circ} I>$ & $\varepsilon 8^{\circ} \mathrm{I}>$ & $\varepsilon 8^{\circ} I>$ & 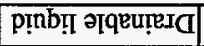 & $s: 26 \mathrm{I}$ & $\angle \varepsilon 8000 \mathrm{~L} L 6 \mathrm{~S}$ \\
\hline$\varepsilon 8^{\circ} I>$ & $\varepsilon 8^{\circ} \mathrm{I}>$ & $\varepsilon 8^{\circ} I>$ & 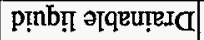 & $\downarrow: 26 \mathrm{I}$ & $9 \varepsilon 8000 \mathrm{~L} L 6 \mathrm{~S}$ \\
\hline$\varepsilon 8^{\circ} I>$ & $\mathcal{E} 8^{\circ} I>$ & $\varepsilon 8^{\circ} I>$ & pinb!̣ әqqựera & $\varepsilon: 26 \mathrm{~T}$ & SE8000.JL6S \\
\hline $\mathcal{E} 8^{\circ} I>$ & $\varepsilon 8^{\circ} I>$ & $\varepsilon 8^{\prime} I>$ & p!nb!̣া әqвu!rad & $z: 26 \mathrm{I}$ & $t \varepsilon 8000 \mathrm{~L} L 6 \mathrm{~S}$ \\
\hline$L Z^{\circ} L>$ & $\angle Z \cdot L>$ & $L Z^{\circ} L>$ & p!nb!̣ әqеu!̣ı & $I: 26 \mathrm{I}$ & E0zlo0L $\angle 6 \mathrm{~S}$ \\
\hline 3) & (96.6\% & 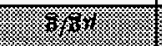 & & & - $>61516$ \\
\hline $0 Z I^{2} t$ & $096^{\circ} \varepsilon$ & $08 z^{6} t$ & Ј[Еप ІәмОТ & \multirow[b]{2}{*}{$8: 26 I$} & $026000 \mathrm{U} \angle 6 \mathrm{~S}$ \\
\hline $0: 000<t^{6} t$ & $020^{\circ} \mathrm{s}$ & $016^{6} \varepsilon$ & Fाeप Iədd & & $\varepsilon Z 6000 \mathrm{~L} \angle 6 \mathrm{~S}$ \\
\hline $026^{6} 8$ & $088^{6} 8$ & $096^{\prime} 8$ & ग[Еч РәмОТ] & \multirow[b]{2}{*}{$L: 26 \mathrm{I}$} & $6 \mathrm{~L} 6000 \mathrm{~L} \angle 6 \mathrm{~S}$ \\
\hline : & $08 L^{6} t$ & $026^{\circ} \mathrm{L}$ & नाеч Іәdd & & $\tau \tau 6000 \mathrm{~L} \angle \mathrm{S}$ \\
\hline $0 z \varepsilon^{\prime} 6$ & $00 \varepsilon^{\prime} 6$ & $0 t \varepsilon^{6} 6$ & IाRY IOMOT & \multirow[b]{2}{*}{$9: 26 \mathrm{I}$} & $106000 \mathrm{~L} L 6 \mathrm{~S}$ \\
\hline $0 \varepsilon 9^{\prime} 8$ & $009^{6} 8$ & $099^{\prime} 8$ & 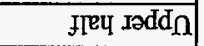 & & $I Z 6000 \mathrm{~L} \angle 6 \mathrm{~S}$ \\
\hline 6. & 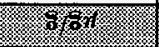 & 39in & & 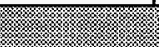 & OXsin soyos \\
\hline $0: 000 \mathcal{E} 0^{6} \mathrm{Z}$ & $0<0^{\circ} \mathrm{z}$ & $066^{6} \mathrm{I}$ & əItsoduros p!IOS & \multirow[b]{2}{*}{ 乙6I ә.०ว } & $9 S Z 100 \amalg L 6 S$ \\
\hline$p: 03098^{6} 6$ & $0 z \varepsilon^{\prime} 6$ & $00 t^{6} 0 \mathrm{I}$ & əి!̣soduros p!̣os & & OSZI00LL6S \\
\hline $28 \%$ & 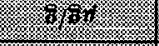 & 86 & & \multicolumn{2}{|c|}{ (5) } \\
\hline minul: & 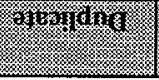 & 1964 & 401 & 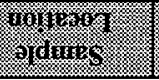 & 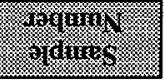 \\
\hline
\end{tabular}

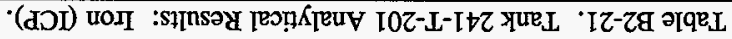


Table B2-22. Tank 241-T-201 Analytical Results: Lanthanum (ICP).

\begin{tabular}{|c|c|c|c|c|c|}
\hline $\begin{array}{l}\text { comagles } \\
\text { Mininer }\end{array}$ & S8magle & Siringle & I Restui: & 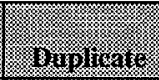 & 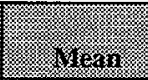 \\
\hline \multicolumn{2}{|c|}{ 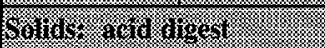 } & & (3) & 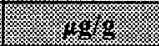 & (1) \\
\hline S97T001250 & \multirow[t]{2}{*}{ Core 192} & Solid composite & 27,500 & 22,200 & $24,900^{\mathrm{eC}: \mathrm{d}, \mathrm{c}}$ \\
\hline S97T001256 & & Solid composite & 5,910 & 6,090 & $6,000^{\alpha c: c}$ \\
\hline \multicolumn{2}{|c|}{ 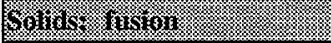 } & 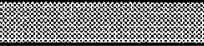 & 1) & 8.80 .60 & 148 \\
\hline S97T000921 & \multirow[t]{2}{*}{$192: 6$} & Upper half & 15,300 & 14,800 & 15,100 \\
\hline S97T000901 & & Lower half & 15,900 & 17,500 & 16,700 \\
\hline S97T000922 & \multirow[t]{2}{*}{ 192: 7} & Upper half & 21,000 & 19,600 & 20,300 \\
\hline S97T000919 & & Lower half & 21,200 & 22,300 & 21,800 \\
\hline S97T000923 & \multirow[t]{2}{*}{$192: 8$} & Upper half & 14,900 & 14,800 & 14,900 \\
\hline S97T000920 & & Lower half & 20,100 & 19,400 & 19,800 \\
\hline \multicolumn{2}{|l|}{ Mopinas } & & 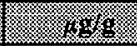 & 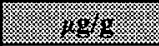 & 8.98 \\
\hline S97T001203 & 192: 1 & Drainable liquid & $<7.27$ & $<7.27$ & $<7.27$ \\
\hline S97T000834 & 192: 2 & Drainable liquid & $<1.83$ & $<1.83$ & $<1.83$ \\
\hline S97T000835 & $192: 3$ & Drainable liquid & 3.03 & $<1.83$ & $<2.43^{\mathrm{QC}: \mathrm{e}}$ \\
\hline S97T000836 & 192: 4 & Drainable liquid & $<1.83$ & $<1.83$ & $<1.83$ \\
\hline S97T000837 & $192: 5$ & Drainable liquid & $<1.83$ & $<1.83$ & $<1.83$ \\
\hline
\end{tabular}


HINF-1501 Rev. 0

Table B2-23. Tank 241-T-201 Analytical Results: Lead (ICP).

\begin{tabular}{|c|c|c|c|c|c|}
\hline Saripies & Syoringer & S Somitur & $\sqrt[3]{1} \cdot 5$ & 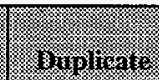 & $\sqrt{11}$ \\
\hline \multicolumn{3}{|c|}{ 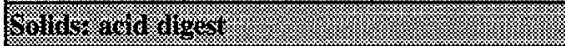 } & - & 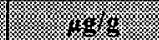 & (1) \\
\hline S97T001250 & Core 192 & Solid composite & 125 & 299 & $212^{\mathrm{QC}: \mathrm{e}}$ \\
\hline S97T001256 & & Solid composite & 288 & 300 & 294 \\
\hline \multicolumn{3}{|c|}{ 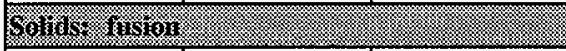 } & 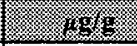 & 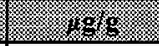 & . \\
\hline S97T000921 & \multirow[t]{2}{*}{ 192: 6} & Upper half & $<2,220$ & $<2,190$ & $<2,210$ \\
\hline S97T000901 & & Lower half & $<2,040$ & $<1,990$ & $<2,020$ \\
\hline S97T000922 & \multirow[t]{2}{*}{ 192: 7} & Upper half & $<2,010$ & $<2,020$ & $<2,020$ \\
\hline S97T000919 & & Lower half & $<2,080$ & $<2,040$ & $<2,060$ \\
\hline S97T000923 & \multirow[t]{2}{*}{ 192: 8} & Upper half & $<2,030$ & $<2,090$ & $<2,060$ \\
\hline S97T000920 & & Lower half & $<2,060$ & $<2,030$ & $<2,050$ \\
\hline \multicolumn{3}{|c|}{ Munius } & $8.8 \mathrm{~g}$ & 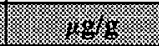 & 1.898 \\
\hline S97T001203 & 192: 1 & Drainable liquid & $<14.5$ & $<14.5$ & $<14.5$ \\
\hline S97T000834 & 192: 2 & Drainable liquid & $<3.67$ & $<3.67$ & $<3.67$ \\
\hline S97T000835 & 192: 3 & Drainable liquid & $<3.67$ & $<3.67$ & $<3.67$ \\
\hline S97T000836 & 192: 4 & Drainable liquid & $<3.67$ & $<3.67$ & $<3.67$ \\
\hline S97T000837 & $192: 5$ & Drainable liquid & $<3.67$ & $<3.67$ & $<3.67$ \\
\hline
\end{tabular}




\begin{tabular}{|c|c|c|c|c|c|}
\hline$\angle 9 \varepsilon^{\circ} 0>$ & $\angle 9 \varepsilon^{\circ} 0>$ & $\angle 9 \varepsilon^{\circ} 0>$ & p!nb!n əlqeu!exd & $s: 26 \mathrm{I}$ & $\angle E 8000 \mathrm{~L} \angle 6 \mathrm{~S}$ \\
\hline$\angle 9 \varepsilon^{\circ} 0>$ & $\angle 9 \varepsilon^{\circ} 0>$ & $\angle 9 \varepsilon^{\circ} 0>$ & p!nb!̣ əqqựeı & $t: 26 \mathrm{I}$ & $9 \varepsilon 8000 . L L 6 S$ \\
\hline$\angle 9 \varepsilon^{\circ} 0>$ & $\angle 9 \varepsilon^{\circ} 0>$ & $\angle 9 \varepsilon^{\circ} 0>$ & p!̣nb!̣ ग|qeựexa & $\varepsilon: 26 \mathrm{I}$ & SE8000IL6S \\
\hline$\angle 9 \varepsilon^{\circ} 0>$ & $\angle 9 \varepsilon^{\circ} 0>$ & $\angle 9 \varepsilon^{\circ} 0>$ & p!̣nb!̣ əqqeu!̣eI & $2: 26 \mathrm{I}$ & $\downarrow \varepsilon 8000 \mathrm{~L} L 6 \mathrm{~S}$ \\
\hline$S t^{\circ} I>$ & St. I $>$ & $S_{t} \cdot I>$ & 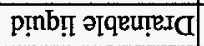 & $\mathrm{I}: 26 \mathrm{I}$ & E0ZI00LL6S \\
\hline 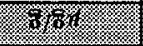 & 7\% & 6,69 & . & & 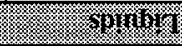 \\
\hline soz> & $\varepsilon 0 \tau>$ & $90 z>$ & JाEY IOMOT & \multirow[b]{2}{*}{$8: 26 \mathrm{I}$} & $026000 . \angle 6 \mathrm{~S}$ \\
\hline $90 z>$ & $60 z>$ & $\varepsilon 0 z>$ & Ifeq rodd & & $\varepsilon 26000 \mathrm{~L} L 6 \mathrm{~S}$ \\
\hline $90 z>$ & toz> & $80 Z>$ & J[ЕЧ IəMOT & \multirow[b]{2}{*}{$L: 26 \mathrm{I}$} & $6 \mathrm{I} 6000 \mathrm{~L} \angle 6 \mathrm{~S}$ \\
\hline$z 0 z>$ & $z 0 \tau>$ & roz> & IГеप Iәdd & & $2 Z 6000 \mathrm{~L} L 6 \mathrm{~S}$ \\
\hline$z 0 z>$ & $66 I>$ & $t 0 z>$ & गएеप IəMOT & \multirow[b]{2}{*}{$9: 26 \mathrm{I}$} & I06000I $\angle 6 \mathrm{~S}$ \\
\hline IZZ> & $6 I Z>$ & $z z \tau>$ & freq Iədd $\Omega$ & & $126000 \mathrm{~L} \angle 6 \mathrm{~S}$ \\
\hline 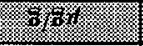 & (7) & 83 & (3) & \multicolumn{2}{|c|}{ 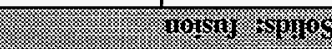 } \\
\hline $26^{\circ} I>$ & $I 6^{\circ} I>$ & $\varepsilon 6^{\circ} \mathrm{I}>$ & ว!!soduros p!IOS & & 9SZI00L $\angle 6 \mathrm{~S}$ \\
\hline $59^{\circ} L>$ & $t 9^{\circ} L>$ & $\angle 9 \angle>$ & ग!!soduos p!̣os & 261 әมоว & 0SZI00LL6S \\
\hline 7\% & 3.611 & 6. & & \multicolumn{2}{|c|}{ (3) } \\
\hline 13 & 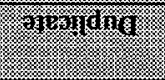 & 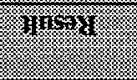 & viogiod & 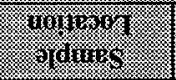 & (2) \\
\hline
\end{tabular}

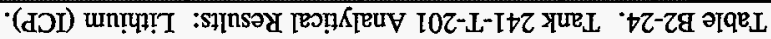


Table B2-25. Tank 241-T-201 Analytical Results: Magnesium (ICP).

\begin{tabular}{|c|c|c|c|c|c|}
\hline Sample & - S Sariple & Sample & resintin & Miphinese & 1 Iran \\
\hline \multicolumn{3}{|c|}{ 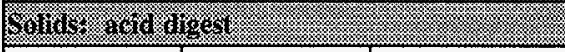 } & (3) & 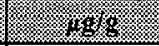 & 8.1896 \\
\hline S97T001250 & \multirow[t]{2}{*}{ Core 192} & Solid composite & 349 & 296 & 323 \\
\hline S97T001256 & & Solid composite & 61.4 & 60.8 & 61.1 \\
\hline \multicolumn{2}{|c|}{ 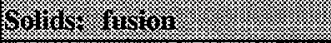 } & 18 & 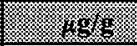 & 1.893 & (3. \\
\hline S97T000921 & \multirow[t]{2}{*}{ 192: 6} & Upper half & $<2,220$ & $<2,190$ & $<2,210$ \\
\hline S97T000901 & & Lower half & $<2,040$ & $<1,990$ & $<2,020$ \\
\hline S97T000922 & \multirow[t]{2}{*}{ 192: 7} & Upper half & $<2,010$ & $<2,020$ & $<2,020$ \\
\hline S97T000919 & & Lower half & $<2,080$ & $<2,040$ & $<2,060$ \\
\hline S97T000923 & \multirow[t]{2}{*}{ 192: 8} & Upper half & $<2,030$ & $<2,090$ & $<2,060$ \\
\hline S97T000920 & & Lower half & $<2,060$ & $<2,030$ & $<2,050$ \\
\hline \multicolumn{3}{|l|}{ Hunis: } & 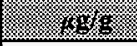 & 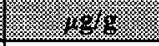 & (3) \\
\hline S97T001203 & 192: 1 & Drainable liquid & $<14.5$ & $<14.5$ & $<14.5$ \\
\hline S97T000834 & 192: 2 & Drainable liquid & $<3.67$ & $<3.67$ & $<3.67$ \\
\hline S97T000835 & 192: 3 & Drainable liquid & $<3.67$ & $<3.67$ & $<3.67$ \\
\hline S97T000836 & 192: 4 & Drainable liquid & $<3.67$ & $<3.67$ & $<3.67$ \\
\hline S97T000837 & 192: 5 & Drainable liquid & $<3.67$ & $<3.67$ & $<3.67$ \\
\hline
\end{tabular}


Table B2-26. Tank 241-T-201 Analytical Results: Manganese (ICP).

\begin{tabular}{|c|c|c|c|c|c|}
\hline Sample & 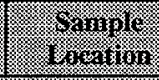 & H: & resesili & 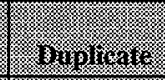 & Wean \\
\hline \multicolumn{3}{|c|}{ 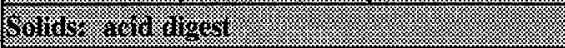 } & 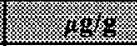 & (2) & 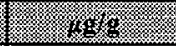 \\
\hline S97T001250 & Core 192 & Solid composite & 48,500 & 40,000 & $44,300^{\mathrm{eC}: \mathrm{d}}$ \\
\hline S97T001256 & & Solid composite & 6,960 & 7,310 & $7,140^{\mathrm{eC}: \mathrm{c}}$ \\
\hline \multicolumn{2}{|l|}{ 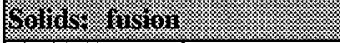 } & (3) & Y $/ 28$ & (1) & 1.8. \\
\hline S97T000921 & \multirow[t]{2}{*}{ 192: 6} & Upper half & 25,400 & 24,500 & 25,000 \\
\hline S97T000901 & & Lower half & 29,500 & 29,900 & 29,700 \\
\hline S97T000922 & \multirow[t]{2}{*}{ 192: 7} & Upper half & 15,600 & 6,760 & $11,200^{\mathrm{QC}: \mathrm{c}}$ \\
\hline S97T000919 & & Lower half & 33,000 & 33,200 & 33,100 \\
\hline S97T000923 & \multirow[t]{2}{*}{ 192: 8} & Upper half & 5,320 & 9,700 & $7,510^{\mathrm{ec}: \mathrm{e}}$ \\
\hline S97T000920 & & Lower half & 24,700 & 24,000 & 24,400 \\
\hline monges: & & 8 & 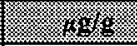 & 8.1698 & 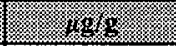 \\
\hline S97T001203 & 192: 1 & Drainable liquid & $<1.45$ & $<1.45$ & $<1.45$ \\
\hline S97T000834 & 192: 2 & Drainable liquid & $<0.367$ & $<0.367$ & $<0.367$ \\
\hline S97T000835 & 192: 3 & Drainable liquid & 2.83 & $<0.367$ & $<1.6^{\mathrm{QC}: \mathrm{e}}$ \\
\hline S97T000836 & 192: 4 & Drainable liquid & 1.74 & 0.777 & $1.26^{\mathrm{QC}: c}$ \\
\hline S97T000837 & $192: 5$ & Drainable liquid & 0.655 & 0.906 & $0.781^{\mathrm{QC}: e}$ \\
\hline
\end{tabular}


Table B2-27. Tank 241-T-201 Analytical Results: Molybdenum (ICP).

\begin{tabular}{|c|c|c|c|c|c|}
\hline Qumple & Samplie & I) varingle & resulin: & 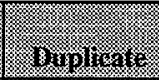 & Jean \\
\hline \multicolumn{3}{|c|}{ 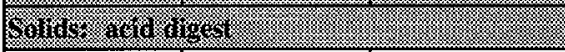 } & 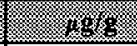 & 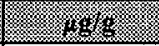 & $8.8 \mathrm{~g} / \mathrm{g}$ \\
\hline S97T001250 & \multirow{2}{*}{ Core 192} & Solid composite & $<38.4$ & $<38.2$ & $<38.3$ \\
\hline S97T001256 & & Solid composite & $<9.64$ & $<9.53$ & $<9.59$ \\
\hline \multicolumn{3}{|l|}{ 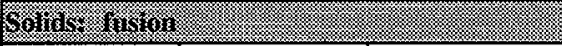 } & 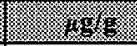 & 8.80 & 8.186 \\
\hline S97T000921 & \multirow[t]{2}{*}{ 192: 6} & Upper half & $<1,110$ & $<1,090$ & $<1,100$ \\
\hline \$97T000901 & & Lower half & $<1,020$ & $<, 996$ & $<1,010$ \\
\hline S97T000922 & \multirow[t]{2}{*}{ 192: 7} & Upper half & $<1,000$ & $<1,010$ & $<1,010$ \\
\hline S97T000919 & & Lower half & $<1,040$ & $<1,020$ & $<1,030$ \\
\hline S97T000923 & \multirow[t]{2}{*}{ 192: 8} & Upper half & $<1,020$ & $<1,040$ & $<1,030$ \\
\hline S97T000920 & & Lower half & $<1,030$ & $<1,010$ & $<1,020$ \\
\hline Truins & 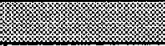 & 18 & 8.898. & 1.86 .9 & 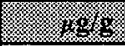 \\
\hline S97T001203 & 192: 1 & Drainable liquid & $<7.27$ & $<7.27$ & $<7.27$ \\
\hline S97T000834 & 192: 2 & Drainable liquid & $<1.83$ & $<1.83$ & $<1.83$ \\
\hline S97T000835 & 192: 3 & Drainable liquid & $<1.83$ & $<1.83$ & $<1.83$ \\
\hline S97T000836 & 192: 4 & Drainable liquid & $<1.83$ & $<1.83$ & $<1.83$ \\
\hline S97T000837 & 192: 5 & Drainable liquid & $<1.83$ & $<1.83$ & $<1.83$ \\
\hline
\end{tabular}




\begin{tabular}{|c|c|c|c|c|c|}
\hline$\downarrow \varepsilon L 0>$ & $\downarrow \mathcal{E} L O>$ & $Ð \varepsilon L L^{\circ} O>$ & 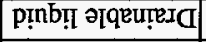 & $S: Z 6 \mathrm{I}$ & $\angle E 8000 \mathrm{~L} \angle 6 \mathrm{~S}$ \\
\hline$\nabla \mathcal{E} L O>$ & $\downarrow \mathcal{E} L L^{\circ} 0>$ & $\nabla \varepsilon L O>$ & 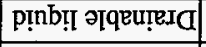 & $t: 26 \mathrm{I}$ & $9 \varepsilon 8000 \mathrm{~L} L 6 \mathrm{~S}$ \\
\hline$† E L O O$ & $\nabla \mathcal{\nabla} L^{\circ} O>$ & $\downarrow \varepsilon L O>$ & p!̣b!̣ әтquureIC] & $\mathcal{E}: 26 \mathrm{I}$ & SE8000LL6S \\
\hline$\downarrow \mathcal{E} L L^{\circ} 0>$ & $\downarrow \mathcal{E} L^{\circ} 0>$ & $\nabla \mathcal{E} L O>$ & 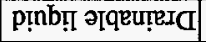 & $Z: 26 \mathrm{I}$ & $\triangleright \varepsilon 8000 \mathrm{~L} L 6 \mathrm{~S}$ \\
\hline $68^{\circ} \tau>$ & $68^{\circ} Z>$ & $68^{\circ} z>$ & p!nb!̣ әqqụeIC & $\mathrm{I}: 26 \mathrm{I}$ & E0ZI00LL6S \\
\hline whor & (1) & (6) & r & 1 & (1) \\
\hline SZI & $8 Z I$ & ZZI & วł!̣oduros p!̣os & & 9SZI00JL6S \\
\hline OE9 & $66 \mathrm{~S}$ & 099 & әุ!soduos p!̣os & 26I $210 \mathrm{D}$ & 0SZI00LL6S \\
\hline 6) & $8 \%$ & 6 & & 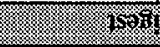 & 10\% \\
\hline ateris & 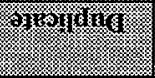 & 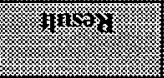 & 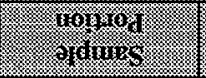 & arom & aginging \\
\hline
\end{tabular}

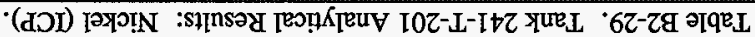

\begin{tabular}{|c|c|c|c|c|c|}
\hline$\angle 9^{\circ} \mathcal{E}>$ & $\angle 9^{\circ} \varepsilon>$ & $\angle 9^{\circ} \varepsilon>$ & p!̣nb!I əqqeụes] & $s: 26 \mathrm{I}$ & $\angle E 8000 \mathrm{~L} \angle 6 \mathrm{~S}$ \\
\hline$\angle 9^{\circ} \varepsilon>$ & $\angle 9^{\circ} \varepsilon>$ & $\angle 9^{\circ} \varepsilon>$ & p!̣nb!̣ әqеu!eı & $t: 26 \mathrm{I}$ & $9 \varepsilon 8000 \mathrm{~L} L 6 \mathrm{~S}$ \\
\hline$\angle 9^{\circ} \varepsilon>$ & $\angle 9^{\circ} \mathcal{E}>$ & $\angle 9^{\circ} \varepsilon>$ & p!nb!̣ әqеu!̣exa & $\varepsilon: 26 \mathrm{I}$ & SE8000JL6S \\
\hline$\angle 9^{\circ} \varepsilon>$ & $\angle 9^{\circ} \varepsilon>$ & $\angle 9^{\circ} \varepsilon>$ & 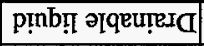 & $2: 26 \mathrm{I}$ & $t E 8000 \mathrm{~L} L 6 \mathrm{~S}$ \\
\hline$s+t>$ & $S \cdot t I>$ & $c^{*}+I>$ & p!̣nb!n әाqeu!̣eId & $\mathrm{I}: 26 \mathrm{I}$ & E0ZIOOL $\angle 6 S$ \\
\hline G & 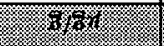 & $8.1 \%$ & ) & 48 & 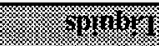 \\
\hline $0 S 0^{6} z>$ & $0 \varepsilon 0^{6} Z>$ & $090^{\prime} z>$ & Ғ[ЕЧ ІәMOT & \multirow[b]{2}{*}{$8: 26 I$} & $026000 \mathrm{~L} \angle 6 \mathrm{~S}$ \\
\hline $090^{\circ} Z>$ & $060^{\circ} \mathrm{z}>$ & $0 \varepsilon 0^{6} Z>$ & Iाएप Iədd & & $\varepsilon Z 6000 L L 6 S$ \\
\hline $090^{\circ} z>$ & $0+0^{6} z>$ & $080^{6} z>$ & J[Ец دәмOT & \multirow[b]{2}{*}{$L: 26 \mathrm{I}$} & $6 \mathrm{I} 6000 \mathrm{~J} \angle 6 \mathrm{~S}$ \\
\hline $0 z 0^{\circ} \mathrm{Z>}$ & $020^{\prime} \mathrm{Z}>$ & 0I0'そ> & ЈГеч дәdd & & $226000 \mathrm{~L} L 6 \mathrm{~S}$ \\
\hline $0 Z 0^{6} Z>$ & $066^{\prime} I>$ & $0+0^{6} \tau>$ & JГEY IOMOT & \multirow[b]{2}{*}{$9: 26 \mathrm{I}$} & I06000I $\angle 6 \mathrm{~S}$ \\
\hline $0 I Z^{6} Z>$ & $06 I^{\prime} z>$ & $0 z z^{6} \tau>$ & J[EY Iədd & & $I 26000 \mathrm{~L} \angle 6 \mathrm{~S}$ \\
\hline 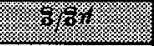 & (6) & (5) & & \multicolumn{2}{|c|}{ 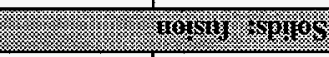 } \\
\hline $2.61>$ & I.6I > & $\varepsilon^{\circ} 6 \mathrm{I}>$ & әт!soduos p!̣OS & & 9SZI00L $\angle 6 S$ \\
\hline $9^{\circ} 9 L>$ & $t^{\circ} 9 L>$ & $L 9 L>$ & Il!̣soduos p!Ios & 261 əro) & 0SZI00L $\angle 6 S$ \\
\hline 16 & 8 & 6/ & & \multicolumn{2}{|c|}{ (5) } \\
\hline 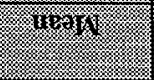 & II) & 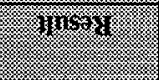 & 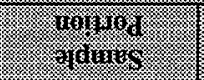 & 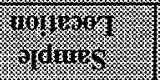 & Sogkiny \\
\hline
\end{tabular}

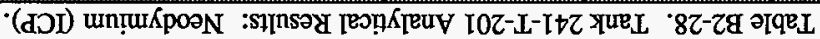


Table B2-30. Tank 241-T-201 Analytical Results: Phosphorus (ICP).

\begin{tabular}{|c|c|c|c|c|c|}
\hline Samiplcs & Sirange & Saminle & 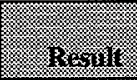 & (8) & lis. \\
\hline \multicolumn{3}{|c|}{ 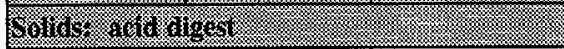 } & 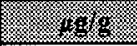 & 8 & (1.8. \\
\hline S97T001250 & \multirow[t]{2}{*}{ Core 192} & Solid composite & 5,040 & 4,070 & $4,560^{\mathrm{eC}: \mathrm{d}, \mathrm{e}}$ \\
\hline S97T001256 & & Solid composite & 1,300 & 1,330 & 1,320 \\
\hline \multicolumn{2}{|c|}{ 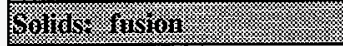 } & 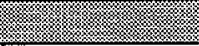 & 169. & 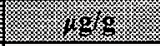 & 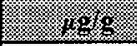 \\
\hline S97T000921 & \multirow[t]{2}{*}{ 192: 6} & Upper half & $<4,440$ & $<4,380$ & $<4,410$ \\
\hline S97T000901 & & Lower half & $<4,080$ & $<3,980$ & $<4,030$ \\
\hline \$97T000922 & \multirow[t]{2}{*}{ 192: 7} & Upper half & $<4,020$ & $<4,040$ & $<4,030$ \\
\hline S97T000919 & & Lower half & $<4,160$ & $<4,070$ & $<4,120$ \\
\hline S97T000923 & \multirow[t]{2}{*}{ 192: 8} & Upper half & $<4,070$ & $<4,170$ & $<4,120$ \\
\hline S97T000920 & & Lower half & $<4,120$ & $<4,060$ & $<4,090$ \\
\hline \multicolumn{2}{|l|}{ (1) nums: } & 4 & 1.8969 & $2.19 \mathrm{gz}$ & . \\
\hline S97T001203 & 192: 1 & Drainable liquid & 176 & 178 & 177 \\
\hline S97T000834 & 192: 2 & Drainable liquid & 173 & 173 & 173 \\
\hline S97T000835 & 192: 3 & Drainable liquid & 145 & 144 & 145 \\
\hline S97T000836 & 192: 4 & Drainable liquid & 140 & 143 & 141 \\
\hline S97T000837 & $192: 5$ & Drainable liquid & 137 & 137 & 137 \\
\hline
\end{tabular}

Table B2-31. Tank 241-T-201 Analytical Results: Potassium (ICP).

\begin{tabular}{|c|c|c|c|c|c|}
\hline S) & 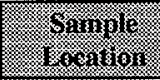 & Sringli & esesintin & 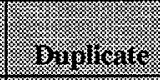 & . 1 lean \\
\hline \multicolumn{3}{|c|}{ 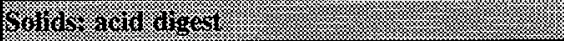 } & 8 & 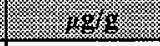 & 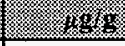 \\
\hline S97T001250 & \multirow[t]{2}{*}{ Core 192} & Solid composite & 5,010 & 4,600 & $4,810^{\mathrm{ec}: \mathrm{d}}$ \\
\hline S97T001256 & & Solid composite & 4,070 & 3,920 & $4,000^{\mathrm{eC}: \mathrm{c}}$ \\
\hline Ninuris: & 18 & : & (x) & 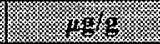 & $1 \% 5$ \\
\hline S97T001203 & 192: 1 & Drainable liquid & 3,090 & 3,060 & $3,080^{\mathrm{eC}: \mathrm{c}}$ \\
\hline S97T000834 & 192: 2 & Drainable liquid & 2,940 & 2,980 & 2,960 \\
\hline S97T000835 & 192: 3 & Drainable liquid & 2,690 & 2,710 & 2,700 \\
\hline S97T000836 & 192: 4 & Drainable liquid & 2,820 & 2,830 & 2,830 \\
\hline S97T000837 & 192: 5 & Drainable liquid & 2,910 & 2,910 & 2,910 \\
\hline
\end{tabular}


HNF-1501 Rev. 0

Table B2-32. Tank 241-T-201 Analytical Results: Samarium (ICP).

\begin{tabular}{|c|c|c|c|c|c|}
\hline Jamigle: & Simine & Simple & resill & Hinlmats & (1) \\
\hline \multicolumn{3}{|c|}{ Solidist id digert } & 8.98 & 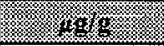 & 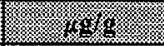 \\
\hline S97T001250 & \multirow[t]{2}{*}{ Core 192} & Solid composite & $<76.7$ & $<76.4$ & $<76.6$ \\
\hline S97T001256 & & Solid composite & $<19.3$ & $<19.1$ & $<19.2$ \\
\hline \multicolumn{3}{|c|}{ 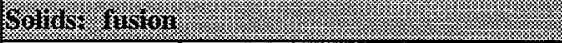 } & 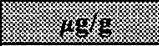 & 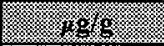 & (3) \\
\hline S97T000921 & \multirow[t]{2}{*}{ 192: 6} & Upper half & $<2,220$ & $<2,190$ & $<2,210$ \\
\hline S97T000901 & & Lower half & $<2,040$ & $<1,990$ & $<2,020$ \\
\hline \$97T000922 & \multirow[t]{2}{*}{ 192: 7} & Upper half & $<2,010$ & $<2,020$ & $<2,020$ \\
\hline S97T000919 & & Lower half & $<2,080$ & $<2,040$ & $<2,060$ \\
\hline S97T000923 & \multirow[t]{2}{*}{ 192: 8} & Upper half & $<2,030$ & $<2,090$ & $<2,060$ \\
\hline \$97T000920 & & Lower half & $<2,060$ & $<2,030$ & $<2,050$ \\
\hline Mirina: & $4=$ & 1 & $8,8,8 \mathrm{~g}$ & (1) & 1.98 \\
\hline S97T001203 & 192: 1 & Drainable liquid & $<14.5$ & $<14.5$ & $<14.5$ \\
\hline S97T000834 & 192: 2 & Drainable liquid & $<3.67$ & $<3.67$ & $<3.67$ \\
\hline S97T000835 & 192: 3 & Drainable liquid & $<3.67$ & $<3.67$ & $<3.67$ \\
\hline S97T000836 & 192: 4 & Drainable liquid & $<3.67$ & $<3.67$ & $<3.67$ \\
\hline S97T000837 & 192: 5 & Drainable liquid & $<3.67$ & $<3.67$ & $<3.67$ \\
\hline
\end{tabular}

Table B2-33. Tank 241-T-201 Analytical Results: Selenium (ICP). (2 sheets)

\begin{tabular}{|c|c|c|c|c|c|}
\hline Sarmule & 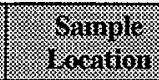 & $\frac{\text { Sarmule }}{\text { rotriou }}$ & sering: & (3) & risun \\
\hline \multicolumn{3}{|c|}{ 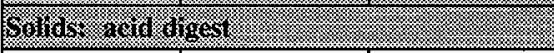 } & 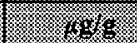 & (3.8 & 1.963 \\
\hline S97T001250 & \multirow[t]{2}{*}{ Core 192} & Solid composite & $<76.7$ & $<76.4$ & $<76.6$ \\
\hline S97T001256 & & Solid composite & $<19.3$ & $<19.1$ & $<19.2$ \\
\hline \multicolumn{2}{|l|}{ 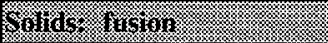 } & & (1.9. & 486 & 488 \\
\hline S97T000921 & \multirow[t]{2}{*}{ 192: 6} & Upper half & $<2,220$ & $<2,190$ & $<2,210$ \\
\hline S97T000901 & & Lower half & $<2,040$ & $<1,990$ & $<2,020$ \\
\hline S97T000922 & \multirow[t]{2}{*}{ 192: 7} & Upper half & $<2,010$ & $<2,020$ & $<2,020$ \\
\hline S97T000919 & & Lower half & $<2,080$ & $<2,040$ & $<2,060$ \\
\hline S97T000923 & \multirow[t]{2}{*}{ 192: 8} & Upper half & $<2,030$ & $<2,090$ & $<2,060$ \\
\hline S97T000920 & & Lower half & $<2,060$ & $<2,030$ & $<2,050$ \\
\hline
\end{tabular}


Table B2-33. Tank 241-T-201 Analytical Results: Selenium (ICP). (2 sheets)

\begin{tabular}{|c|c|c|c|c|c|}
\hline 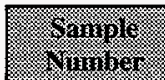 & Sompres & 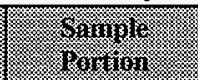 & 16esinit & Hoiplinat & 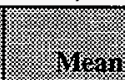 \\
\hline 1801405 & $\$:$ & 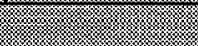 & 10 & 1098 & 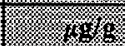 \\
\hline S97T000834 & 192: 2 & Drainable liquid & $<3.67$ & $<3.67$ & $<3.67$ \\
\hline S97T000835 & 192: 3 & Drainable liquid & $<3.67$ & $<3.67$ & $<3.67$ \\
\hline S97T000836 & 192: 4 & Drainable liquid & $<3.67$ & $<3.67$ & $<3.67$ \\
\hline S97T000837 & 192: 5 & Drainable liquid & $<3.67$ & $<3.67$ & $<3.67$ \\
\hline
\end{tabular}

Table B2-34. Tank 241-T-201 Analytical Results: Silicon (ICP).

\begin{tabular}{|c|c|c|c|c|c|}
\hline Shmoli & Samile & 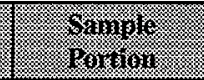 & gravil: & Binilleates & (1) \\
\hline \multicolumn{3}{|c|}{ 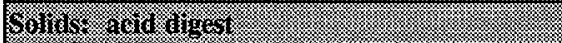 } & 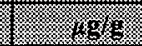 & 1.96 & 148 \\
\hline S97T001250 & \multirow[t]{2}{*}{ Core 192} & Solid composite & 1,970 & 1,740 & $1,860^{\text {QC:bld }}$ \\
\hline S97T001256 & & Solid composite & 1,440 & 1,220 & $1,330^{\mathrm{QC:b}}$ \\
\hline \multicolumn{2}{|l|}{ (1) } & 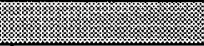 & 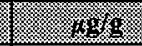 & (1) & 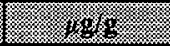 \\
\hline S97T000921 & \multirow[t]{2}{*}{ 192: 6} & Upper half & $<1,110$ & 4,030 & $<2,570^{\mathrm{eC}: \mathrm{e}}$ \\
\hline S97T000901 & & Lower half & $<1,020$ & 1,140 & $<1,080$ \\
\hline S97T000922 & \multirow[t]{2}{*}{ 192: 7} & Upper half & 1,050 & 3,800 & $2,430^{\mathrm{QC:0}}$ \\
\hline S97T000919 & & Lower half & 1,160 & 1,570 & $1,370^{\mathrm{QC:e}}$ \\
\hline S97T000923 & \multirow[t]{2}{*}{ 192: 8} & Upper half & 2,540 & 1,190 & $1,870^{\mathrm{QC}: e}$ \\
\hline S97T000920 & & Lower half & 1,250 & 1,230 & 1,240 \\
\hline \multicolumn{2}{|l|}{ Wirunitis } & 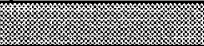 & 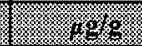 & 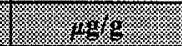 & 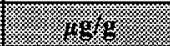 \\
\hline S97T001203 & 192: 1 & Drainable liquid & 27.1 & 31.8 & 29.5 \\
\hline S97T000834 & 192: 2 & Drainable liquid & 40.2 & 38.8 & 39.5 \\
\hline S97T000835 & 192: 3 & Drainable liquid & 42 & 42.2 & 42.1 \\
\hline S97T000836 & 192: 4 & Drainable liquid & 45 & 46 & 45.5 \\
\hline S97T000837 & $192: 5$ & Drainable liquid & 27.7 & 32.9 & 30.3 \\
\hline
\end{tabular}


Table B2-35. Tank 241-T-201 Analytical Results: Silver (ICP).

\begin{tabular}{|c|c|c|c|c|c|}
\hline Samplet & Somicis & Squgle & 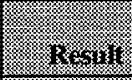 & 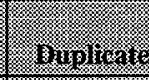 & .1312 .11 \\
\hline \multicolumn{2}{|c|}{ 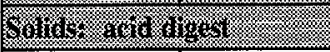 } & : & 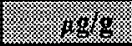 & 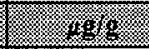 & 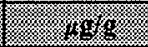 \\
\hline S97T001250 & \multirow[t]{2}{*}{ Core 192} & Solid composite & $<7.67$ & $<7.64$ & $<7.65^{\mathrm{QC}: a, \mathrm{c}}$ \\
\hline S97T001256 & & Solid composite & 2.26 & 2.21 & $2.23^{\mathrm{QC}: \mathrm{a}, \mathrm{c}}$ \\
\hline \multicolumn{3}{|l|}{ 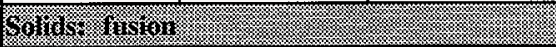 } & f & $8.8 \mathrm{~g}$ & t \\
\hline S97T000921 & \multirow[t]{2}{*}{ 192: 6} & Upper half & $<222$ & $<219$ & $<221$ \\
\hline S97T000901 & & Lower half & $<204$ & $<199$ & $<202$ \\
\hline S97T000922 & \multirow[t]{2}{*}{ 192: 7} & Upper half & $<201$ & $<202$ & $<202$ \\
\hline S97T000919 & & Lower half & $<208$ & $<204$ & $<206$ \\
\hline S97T000923 & \multirow[t]{2}{*}{ 192: 8} & Upper half & $<203$ & $<209$ & $<206$ \\
\hline S97T000920 & & Lower half & $<206$ & $<203$ & $<205$ \\
\hline liginas & \multicolumn{2}{|c|}{ (2) } & 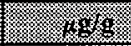 & 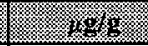 & 18 \\
\hline S97T001203 & 192: 1 & Drainable liquid & $<1.45$ & $<1.45$ & $<1.45^{\mathrm{QC:c}}$ \\
\hline S97T000834 & 192: 2 & Drainable liquid & 1.32 & 1.32 & 1.32 \\
\hline S97T000835 & 192: 3 & Drainable liquid & 1.2 & 1.22 & 1.21 \\
\hline S97T000836 & 192: 4 & Drainable liquid & 1.27 & 1.28 & 1.28 \\
\hline S97T000837 & 192: 5 & Drainable liquid & 1.32 & 1.33 & $1.32^{\mathrm{QC}: \mathrm{c}}$ \\
\hline
\end{tabular}


Table B2-36. Tank 241-T-201 Analytical Results: Sodium (ICP).

\begin{tabular}{|c|c|c|c|c|c|}
\hline Sample & Samples & 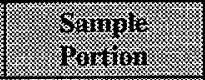 & resull: & ryolutar & 11:an: \\
\hline Solmos. & 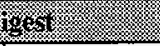 & 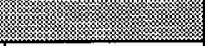 & 13. & $3.1918 \mathrm{~s}=$ & 8.1968 \\
\hline S97T001250 & \multirow[t]{2}{*}{ Core 192} & Solid composite & 33,900 & 30,700 & $32,300^{\mathrm{eC}: \mathrm{d}}$ \\
\hline S97T001256 & & Solid composite & 24,900 & 23,700 & $24,300^{\mathrm{QC:c}}$ \\
\hline 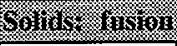 & 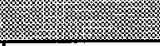 & 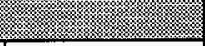 & 2486 & (1) & 3.98 \\
\hline S97T000921 & \multirow[t]{2}{*}{$192: 6$} & Upper half & 28,100 & 27,400 & 27,800 \\
\hline S97T000901 & & Lower half & 27,000 & 27,000 & 27,000 \\
\hline S97T000922 & \multirow[t]{2}{*}{ 192: 7} & Upper half & 25,800 & 28,300 & 27,100 \\
\hline S97T000919. & & Lower half & 27,600 & 27,500 & 27,600 \\
\hline S97T000923 & \multirow[t]{2}{*}{ 192: 8} & Upper half & 28,100 & 27,900 & 28,000 \\
\hline S97T000920 & & Lower half & 28,700 & 27,600 & 28,200 \\
\hline \multicolumn{3}{|l|}{ 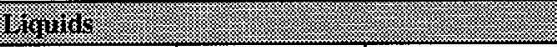 } & (1.8.8. & 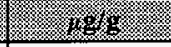 & 1 $18 \mathrm{~g}$ \\
\hline S97T001203 & 192: 1 & Drainable liquid & 19,100 & 18,800 & $19,000^{\mathrm{QC}: \mathrm{c}}$ \\
\hline S97T000834 & 192: 2 & Drainable liquid & 18,600 & 18,700 & 18,600 \\
\hline S97T000835 & 192: 3 & Drainable liquid & 16,700 & 16,900 & 16,800 \\
\hline S97T000836 & 192: 4 & Drainable liquid & 17,700 & 17,800 & 17,800 \\
\hline S97T000837 & $192: 5$ & Drainable liquid & 18,400 & 18,300 & $18,300^{\mathrm{QC:c}}$ \\
\hline
\end{tabular}




\begin{tabular}{|c|c|c|c|c|c|}
\hline$\angle 9 \varepsilon^{\circ} 0>$ & $\angle 9 E O>$ & $\angle 9 E^{\circ} 0>$ & p!nb! әq әqugxa & $s: 26 \mathrm{I}$ & $\angle E 8000 \mathrm{LL6S}$ \\
\hline$\angle 9 \varepsilon^{\circ} 0>$ & $\angle 9 \varepsilon^{\circ} 0>$ & $\angle 9 \varepsilon^{\circ} 0>$ & ptnbṭ әqвựeI & $t: 26 \mathrm{I}$ & $9 \varepsilon 8000 \mathrm{~L} L 6 \mathrm{~S}$ \\
\hline$\angle 9 \varepsilon^{\circ} 0>$ & $\angle 9 \varepsilon^{\circ} 0>$ & $\angle 9 \varepsilon^{\circ} 0>$ & 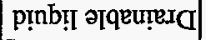 & $\mathcal{E}: 26 \mathrm{I}$ & SE8000LL6S \\
\hline$\angle 9 \varepsilon^{\circ} 0>$ & $\angle 9 \varepsilon^{\circ} 0>$ & $\angle 9 \varepsilon^{\circ} 0>$ & pinb!̣ әqеựexa & $2: 261$ & $t E 8000 \mathrm{~L} \angle 6 \mathrm{~S}$ \\
\hline$S t^{\circ} I>$ & St. I> & $S t^{\circ} I>$ & 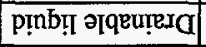 & $\mathrm{I}: \mathrm{ZGI}$ & E0ZIO0LL6S \\
\hline 1) & (4) & (6) & (ব) & & 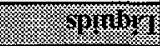 \\
\hline $\mathrm{I} 8 \mathrm{~L}$ & $0 L L$ & I6L & गुEप IOMOT & \multirow[b]{2}{*}{$8: 26 \mathrm{I}$} & $026000 \mathrm{~L} L 6 \mathrm{~S}$ \\
\hline $92 L$ & $6 \mathrm{IL}$ & $\tau \varepsilon L$ & Jూ飞प Iadd $\Omega$ & & $\varepsilon Z 6000 \mathrm{I} \angle 6 \mathrm{~S}$ \\
\hline 808 & 008 & 9I8 & 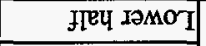 & \multirow[b]{2}{*}{$L: 26 \mathrm{I}$} & $6 \mathrm{I} 6000 \mathrm{~L} \angle 6 \mathrm{~S}$ \\
\hline$z \varepsilon 6$ & $0 S_{6}$ & $\varepsilon I 6$ & Jाey Iadd & & $276000 \mathrm{~L} L 6 \mathrm{~S}$ \\
\hline $06 I^{6} \mathrm{I}$ & $0 I Z^{6} \mathrm{~T}$ & $0 L I^{6} \mathrm{~T}$ & fleप دәMOT & \multirow[b]{2}{*}{$9: 26 \mathrm{I}$} & I06000IL6S \\
\hline 858 & $\tau \varepsilon 8$ & 788 & Jॉeч rədd & & IZ6000L $\angle 6 S$ \\
\hline 8 & 37\%? & 8) & (1) & 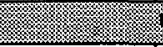 & 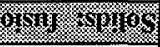 \\
\hline$t I I$ & $\mathcal{S I T}$ & ZII & ว!!soduos p!os & \multirow[b]{2}{*}{261 әIо० } & 9SZLO0LL6S \\
\hline$\partial^{\prime} p: D O Z O Z I^{6} \tau$ & 666 & $0+Z^{6} \mathrm{~T}$ & əุ!̣soduos p!̣OS & & 0SZI00I $\angle 6 S$ \\
\hline (j) & 6. & 7\% & 8 & \multicolumn{2}{|c|}{ 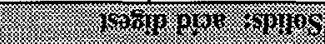 } \\
\hline rewin & भzingrigr & (3) & 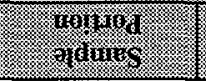 & 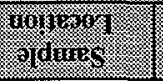 & 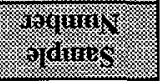 \\
\hline
\end{tabular}

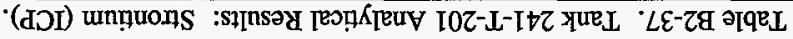


Table B2-38. Tank 241-T-201 Analytical Results: Sulfur (ICP).

\begin{tabular}{|c|c|c|c|c|c|}
\hline $\begin{array}{l}\text { Samples } \\
\text { Viminger }\end{array}$ & Yampir: & Somingls & Resiul & Amplinate & 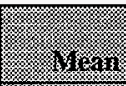 \\
\hline \multicolumn{2}{|c|}{ 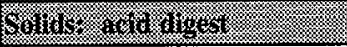 } & $\sqrt{2.8}$ & 2.1 .4 & 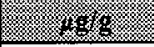 & 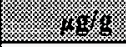 \\
\hline S97T001250 & \multirow[t]{2}{*}{ Core 192} & Solid composite & 105 & 79.4 & $92.2^{Q \mathrm{C:c}}$ \\
\hline S97T001256 & & Solid composite & 57.8 & 54.8 & 56.3 \\
\hline \multicolumn{2}{|c|}{ 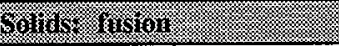 } & 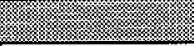 & (1) & $1.9 \mathrm{gg}$ & f \\
\hline S97T000921 & \multirow[t]{2}{*}{ 192: 6} & Upper half & $<2,220$ & $<2,190$ & $<2,210$ \\
\hline S97T000901 & & Lower half & $<2,040$ & $<1,990$ & $<2,020$ \\
\hline S97T000922 & \multirow[t]{2}{*}{ 192: 7} & Upper half & $<2,010$ & $<2,020$ & $<2,020$ \\
\hline S97T000919 & & Lower half & $<2,080$ & $<2,040$ & $<2,060$ \\
\hline S97T000923 & \multirow[t]{2}{*}{ 192: 8} & Upper half & $<2,030$ & $<2,090$ & $<2,060$ \\
\hline S97T000920 & & Lower half & $<2,060$ & $<2,030$ & $<2,050$ \\
\hline \multicolumn{3}{|l|}{ Loung: } & 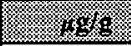 & 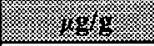 & 18.89. \\
\hline S97T001203 & 192: 1 & Drainable liquid & 26.8 & 26.6 & 26.7 \\
\hline S97T000834 & 192: 2 & Drainable liquid & 29.5 & 28.8 & 29.2 \\
\hline S97T000835 & 192: 3 & Drainable liquid & 26.5 & 25.9 & 26.2 \\
\hline S97T000836 & 192: 4 & Drainable liquid & 26.6 & 28.3 & 27.5 \\
\hline S97T000837 & 192: 5 & Drainable liquid & 28.3 & 28.1 & 28.2 \\
\hline
\end{tabular}


Table B2-39. Tank 241-T-201 Analytical Results: Thallium (ICP).

\begin{tabular}{|c|c|c|c|c|c|}
\hline Sample & Sormigle & Samill & (2) & Duphicur. & 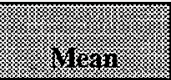 \\
\hline \multicolumn{3}{|c|}{ 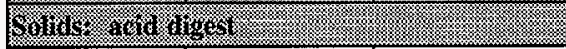 } & 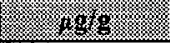 & 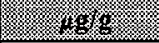 & 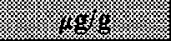 \\
\hline S97T001250 & \multirow[t]{2}{*}{ Core 192} & Solid composite & $<153$ & $<153$ & $<153$ \\
\hline S97T001256 & & Solid composite & $<38.6$ & $<38.1$ & $<38.4$ \\
\hline \multicolumn{3}{|c|}{ Sontas . } & 18. & 8.898 & 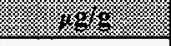 \\
\hline S97T000921 & \multirow[t]{2}{*}{ 192: 6} & Upper half & $<4,440$ & $<4,380$ & $<4,410$ \\
\hline S97T000901 & & Lower half & $<4,080$ & $<3,980$ & $<4,030$ \\
\hline S97T000922 & \multirow[t]{2}{*}{ 192: 7} & Upper half & $<4,020$ & $<4,040$ & $<4,030$ \\
\hline S97T000919 & & Lower half & $<4,160$ & $<4,070$ & $<4,120$ \\
\hline S97T000923 & \multirow[t]{2}{*}{ 192: 8} & Upper half & $<4,070$ & $<4,170$ & $<4,120$ \\
\hline S97T000920 & & Lower half & $<4,120$ & $<4,060$ & $<4,090$ \\
\hline \multicolumn{2}{|l|}{ Wrinas: } & (2) & 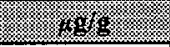 & $1.8 \mathrm{~g}$ & 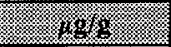 \\
\hline S97T001203 & 192: 1 & Drainable liquid & $<28.9$ & $<28.9$ & $<28.9$ \\
\hline S97T000834 & 192: 2 & Drainable liquid & $<7.34$ & $<7.34$ & $<7.34$ \\
\hline S97T000835 & 192: 3 & Drainable liquid & $<7.34$ & $<7.34$ & $<7.34$ \\
\hline S97T000836 & 192: 4 & Drainable liquid & $<7.34$ & $<7.34$ & $<7.34$ \\
\hline S97T000837 & 192: 5 & Drainable liquid & $<7.34$ & $<7.34$ & $<7.34$ \\
\hline
\end{tabular}


Table B2-40. Tank 241-T-201 Analytical Results: Titanium (ICP).

\begin{tabular}{|c|c|c|c|c|c|}
\hline$\frac{\text { Sanple }}{\text { Mininer }}$ & 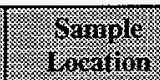 & Simule & resille & 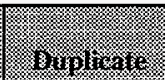 & vicar \\
\hline \multicolumn{3}{|c|}{ 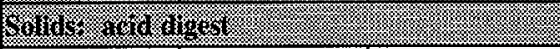 } & $\sqrt{18} 8 \%$ & (3) & 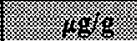 \\
\hline S97T001250 & \multirow[t]{2}{*}{ Core 192} & Solid composite & 10.1 & 9.74 & 9.92 \\
\hline S97T001256 & & Solid composite & 3.34 & 4.13 & $3.73^{\mathrm{QC}: \mathrm{c}}$ \\
\hline \multicolumn{3}{|c|}{ 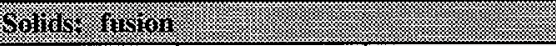 } & (15) & 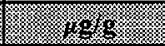 & 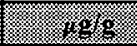 \\
\hline S97T000921 & \multirow[t]{2}{*}{$192: 6$} & Upper half & $<222$ & $<219$ & $<221$ \\
\hline S97T000901 & & Lower half & $<204$ & $<199$ & $<202$ \\
\hline S97T000922 & \multirow[t]{2}{*}{ 192: 7} & Upper half & $<201$ & $<202$ & $<202$ \\
\hline S97T000919 & & Lower half & $<208$ & $<204$ & $<206$ \\
\hline S97T000923 & \multirow[t]{2}{*}{ 192: 8} & Upper half & $<203$ & $<209$ & $<206$ \\
\hline S97T000920 & & Lower half & $<206$ & $<203$ & $<205$ \\
\hline \multicolumn{2}{|l|}{ Wriaro: } & স4: & 8.168 . & 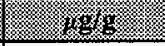 & $=2,03$ \\
\hline S97T001203 & 192: 1 & Drainable liquid & $<1.45$ & $<1.45$ & $<1.45$ \\
\hline S97T000834 & 192: 2 & Drainable liquid & $<0.367$ & $<0.367$ & $<0.367$ \\
\hline S97T000835 & 192: 3 & Drainable liquid & $<0.367$ & $<0.367$ & $<0.367$ \\
\hline S97T000836 & 192: 4 & Drainable liquid & $<0.367$ & $<0.367$ & $<0.367$ \\
\hline S97T000837 & $192: 5$ & Drainable liquid & $<0.367$ & $<0.367$ & $<0.367$ \\
\hline
\end{tabular}


Table B2-41. Tank 241-T-201 Analytical Results: Total Uranium (ICP).

\begin{tabular}{|c|c|c|c|c|c|}
\hline Suminis & S Sample & $\begin{array}{l}\text { Samile } \\
\text { Iortion }\end{array}$ & 7resing & 10uplicatic: & 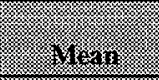 \\
\hline \multicolumn{3}{|c|}{ 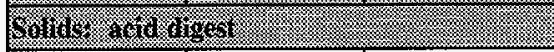 } & 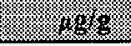 & $=4.48=$ & 4.1486 \\
\hline S97T001250 & \multirow[t]{2}{*}{ Core 192} & Solid composite & $<384$ & $<382$ & $<383$ \\
\hline S97T001256 & & Solid composite & $<96.4$ & $<95.3$ & $<95.8$ \\
\hline \multicolumn{2}{|l|}{ 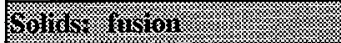 } & (2:- & 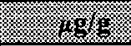 & 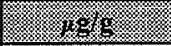 & $18 \mathrm{grg}$ \\
\hline S97T000921 & \multirow[t]{2}{*}{ 192: 6} & Upper half & $<11,100$ & $<10,900$ & $<11,000$ \\
\hline S97T000901 & & Lower half & $<10,200$ & $<9,960$ & $<10,100$ \\
\hline S97T000922 & \multirow[t]{2}{*}{ 192: 7} & Upper half & $<10,000$ & $<10,100$ & $<10,100$ \\
\hline S97T000919 & & Lower half & $<10,400$ & $<10,200$ & $<10,300$ \\
\hline S97T000923 & \multirow[t]{2}{*}{ 192: 8} & Upper half & $<10,200$ & $<10,400$ & $<10,300$ \\
\hline S97T000920 & & Lower half & $<10,300$ & $<10,100$ & $<10,200$ \\
\hline \multicolumn{2}{|l|}{ (19mis: } & (1) & 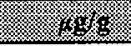 & 18.85 & 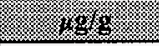 \\
\hline S97T001203 & 192: 1 & Drainable liquid & $<71.9$ & $<71.9$ & $<71.9$ \\
\hline S97T000834 & 192: 2 & Drainable liquid & $<18.3$ & $<18.3$ & $<18.3$ \\
\hline S97T000835 & 192: 3 & Drainable liquid & $<18.3$ & $<18.3$ & $<18.3$ \\
\hline S97T000836 & 192: 4 & Drainable liquid & $<18.3$ & $<18.3$ & $<18.3$ \\
\hline S97T000837 & 192: 5 & Drainable liquid & $<18.3$ & $<18.3$ & $<18.3$ \\
\hline
\end{tabular}


HNF-1501 Rev. 0

Table B2-42. Tank 241-T-201 Analytical Results: Vanadium (ICP).

\begin{tabular}{|c|c|c|c|c|c|}
\hline $\begin{array}{l}\text { Samule } \\
\text { Niminer }\end{array}$ & 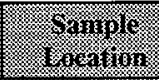 & sonjer & rosing & Mriplizatis: & 18003 \\
\hline \multicolumn{3}{|c|}{ Soldur arit rigert } & 3.198 & 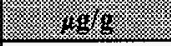 & 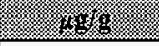 \\
\hline \$97T001250 & \multirow[t]{2}{*}{ Core 192} & Solid composite & $<38.4$ & $<38.2$ & $<38.3$ \\
\hline S97T001256 & & Solid composite & $<9.64$ & $<9.53$ & $<9.59$ \\
\hline \multicolumn{3}{|l|}{ 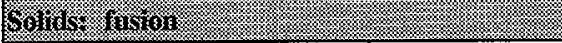 } & 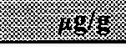 & 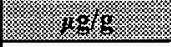 & 4.98 \\
\hline S97T000921 & \multirow[t]{2}{*}{ 192: 6} & Upper half & $<1,110$ & $<1,090$ & $<1,100$ \\
\hline S97T000901 & & Lower half & $<1,020$ & $<996$ & $<1,010$ \\
\hline S97T000922 & \multirow[t]{2}{*}{ 192: 7} & Upper half & $<1,000$ & $<1,010$ & $<1,010$ \\
\hline S97T000919 & & Lower half & $<1,040$ & $<1,020$ & $<1,030$ \\
\hline S97T000923 & \multirow[t]{2}{*}{ 192: 8} & Upper half & $<1,020$ & $<1,040$ & $<1,030$ \\
\hline S97T000920 & & Lower half & $<1,030$ & $<1,010$ & $<1,020$ \\
\hline \multicolumn{3}{|l|}{ I Lemings: } & 4.186 & 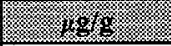 & 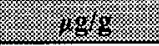 \\
\hline S97T001203 & 192: 1 & Drainable liquid & $<7.27$ & $<7.27$ & $<7.27$ \\
\hline S97T000834 & 192: 2 & Drainable liquid & $<1.83$ & $<1.83$ & $<1.83$ \\
\hline S97T000835 & 192: 3 & Drainable liquid & $<1.83$ & $<1.83$ & $<1.83$ \\
\hline S97T000836 & 192: 4 & Drainable liquid & $<1.83$ & $<1.83$ & $<1.83$ \\
\hline S97T000837 & $192: 5$ & Drainable liquid & $<1.83$ & $<1.83$ & $<1.83$ \\
\hline
\end{tabular}


Table B2-43. Tank 241-T-201 Analytical Results: Zinc (ICP).

\begin{tabular}{|c|c|c|c|c|c|}
\hline Sarniple & 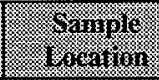 & Tringle & (8sint) & Biplinats: & 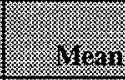 \\
\hline \multicolumn{2}{|c|}{ 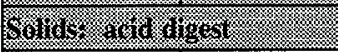 } & " & 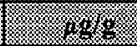 & 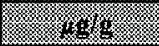 & 4.48 \\
\hline S97T001250 & \multirow[t]{2}{*}{ Core 192} & Solid composite & 105 & 83.9 & $94.5^{\propto \mathrm{CC}: \mathrm{e}}$ \\
\hline S97T001256 & & Solid composite & 66.3 & 68 & 67.2 \\
\hline \multicolumn{2}{|l|}{ 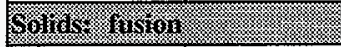 } & & 1.98 & 3.98 & 8.89 \\
\hline S97T000921 & \multirow[t]{2}{*}{ 192: 6} & Upper half & $<222$ & $<219$ & $<221$ \\
\hline S97T000901 & & Lower half & $<204$ & $<199$ & $<202$ \\
\hline S97T000922 & \multirow[t]{2}{*}{ 192: 7} & Upper half & $<201$ & $<202$ & $<202$ \\
\hline S97T000919 & & Lower half & $<208$ & $<204$ & $<206$ \\
\hline S97T000923 & \multirow[t]{2}{*}{ 192: 8} & Upper half & $<203$ & $<209$ & $<206$ \\
\hline S97T000920 & & Lower half & $<206$ & $<203$ & $<205$ \\
\hline \multicolumn{3}{|l|}{ Wuar } & 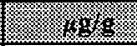 & 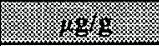 & 3.186 \\
\hline S97T001203 & 192: 1 & Drainable liquid & $<1.45$ & $<1.45$ & $<1.45$ \\
\hline S97T000834 & 192: 2 & Drainable liquid & $<0.367$ & $<0.367$ & $<0.367$ \\
\hline S97T000835 & 192: 3 & Drainable liquid & 0.381 & $<0.367$ & $<0.374$ \\
\hline S97T000836 & 192: 4 & Drainable liquid & $<0.367$ & $<0.367$ & $<0.367$ \\
\hline S97T000837 & $192: 5$ & Drainable liquid & $<0.367$ & $<0.367$ & $<0.367$ \\
\hline
\end{tabular}


Table B2-44. Tank 241-T-201 Analytical Results: Zirconium (ICP).

\begin{tabular}{|c|c|c|c|c|c|}
\hline $\begin{array}{l}\text { Samine } \\
\text { Jiniber }\end{array}$ & 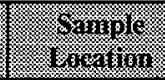 & Single: & res.1.11 & 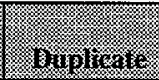 & 19uris: \\
\hline \multicolumn{3}{|c|}{ foridx } & 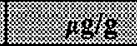 & 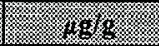 & 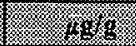 \\
\hline \$97T001250 & \multirow[t]{2}{*}{ Core 192} & Solid composite & $<7.67$ & $<7.64$ & $<7.65$ \\
\hline S97T001256 & & Solid composite & 2.21 & 2.4 & 2.3 \\
\hline \multicolumn{3}{|l|}{$5011 \% 8.863100$} & 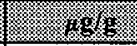 & 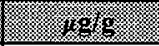 & (1.98 \\
\hline S97T000921 & \multirow[t]{2}{*}{ 192: 6} & Upper half & $<222$ & $<219$ & $<221$ \\
\hline S97T000901 & & Lower half & $<204$ & $<199$ & $<202$ \\
\hline S97T000922 & \multirow[t]{2}{*}{ 192: 7} & Upper half & $<201$ & $<202$ & $<202$ \\
\hline \$97T000919 & & Lower half & $<208$ & $<204$ & $<206$ \\
\hline S97T000923 & \multirow[t]{2}{*}{ 192: 8} & Upper half & $<203$ & $<209$ & $<206$ \\
\hline S97T000920 & & Lower half & $<206$ & $<203$ & $<205$ \\
\hline Livining & 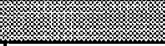 & : & 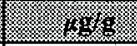 & 18 & (1) \\
\hline S97T001203 & 192: 1 & Drainable liquid & $<1.45$ & $<1.45$ & $<1.45$ \\
\hline S97T000834 & 192: 2 & Drainable liquid & $<0.367$ & $<0.367$ & $<0.367$ \\
\hline S97T000835 & 192: 3 & Drainable liquid & $<0.367$ & $<0.367$ & $<0.367$ \\
\hline S97T000836 & 192: 4 & Drainable liquid & $<0.367$ & $<0.367$ & $<0.367$ \\
\hline S97T000837 & 192: 5 & Drainable liquid & $<0.367$ & $<0.367$ & $<0.367$ \\
\hline
\end{tabular}


HNF-1501 Rev. 0

Table B2-45. Tank 241-T-201 Analytical Results: Bromide (IC).

\begin{tabular}{|c|c|c|c|c|c|}
\hline Sininiter & Simples & Simple: & gesin: & Dimilisar: & 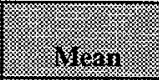 \\
\hline Euras & 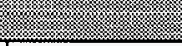 & 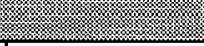 & $8.7,0.6$ & 3.48 & 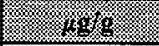 \\
\hline S97T001203 & 192: 1 & Drainable liquid & $<191$ & $<191$ & $<191$ \\
\hline S97T000834 & 192: 2 & Drainable liquid & $<99.9$ & $<100$ & $<100$ \\
\hline S97T000835 & 192: 3 & Drainable liquid & $<99.9$ & $<100$ & $<100$ \\
\hline S97T000836 & 192: 4 & Drainable liquid & $<191$ & $<191$ & $<191$ \\
\hline S97T000837 & $192: 5$ & Drainable liquid & $<191$ & $<191$ & $<191$ \\
\hline \multicolumn{3}{|c|}{ 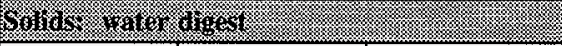 } & 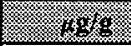 & 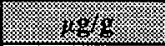 & 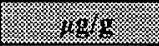 \\
\hline S97T000931 & \multirow[t]{2}{*}{ 192: 6} & Upper half & $<302$ & $<299$ & $<300$ \\
\hline S97T000900 & & Lower half & $<278$ & $<280$ & $<279$ \\
\hline S97T000916 & \multirow[t]{2}{*}{$192: 7$} & Upper half & $<270$ & $<277$ & $<273$ \\
\hline S97T000915 & & Lower half & $<273$ & $<265$ & $<269$ \\
\hline S97T000918 & \multirow[t]{2}{*}{ 192: 8} & Upper half & $<270$ & $<279$ & $<274$ \\
\hline S97T000917 & & Lower half & $<276$ & $<272$ & $<274$ \\
\hline S97T001251 & \multirow[t]{2}{*}{ Core 192} & Solid composite & $<279$ & $<277$ & $<278$ \\
\hline S97T001257 & & Solid composite & $<272$ & $<274$ & $<273$ \\
\hline
\end{tabular}


Table B2-46. Tank 241-T-201 Analytical Results: Chloride (IC).

\begin{tabular}{|c|c|c|c|c|c|}
\hline Saminle: & Y, Saminte & $\begin{array}{l}\text { Samplo } \\
\text { lavinition }\end{array}$ & 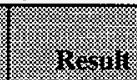 & ouplinut & 11 ara \\
\hline maxiss = & $2 z_{2}$ & (2) & 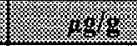 & 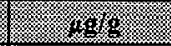 & 8.868 \\
\hline S97T001203 & 192: 1 & Drainable liquid & 677 & 712 & 694 \\
\hline S97T000834 & 192: 2 & Drainable liquid & 728 & 741 & 735 \\
\hline S97T000835 & 192: 3 & Drainable liquid & 655 & 632 & 644 \\
\hline S97T000836 & 192: 4 & Drainable liquid & 650 & 645 & 647 \\
\hline S97T000837 & 192: 5 & Drainable liquid & 696 & 727 & 711 \\
\hline 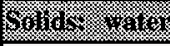 & dis & (2) & $\sqrt{3}, 48$ & $1=1894$ & 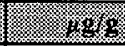 \\
\hline S97T000931 & \multirow[t]{2}{*}{ 192: 6} & Upper half & 976 & 983 & 980 \\
\hline S97T000900 & & Lower half & 1,000 & 940 & 972 \\
\hline S97T000916 & \multirow[t]{2}{*}{$192: 7$} & Upper half & 902 & 923 & 912 \\
\hline S97T000915 & & Lower half & 954 & 1,010 & 982 \\
\hline S97T000918 & \multirow[t]{2}{*}{ 192: 8} & Upper half & 1,030 & 1,000 & 1,010 \\
\hline S97T000917 & & Lower half & 1,020 & 951 & 983 \\
\hline S97T001251 & \multirow[t]{2}{*}{ Core 192} & Solid composite & 1,060 & 1,090 & 1,080 \\
\hline S97T001257 & & Solid composite & 829 & 850 & 840 \\
\hline
\end{tabular}


Table B2-47. Tank 241-T-201 Analytical Results: Fluoride (IC).

\begin{tabular}{|c|c|c|c|c|c|}
\hline 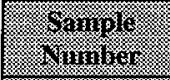 & 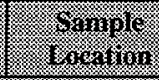 & Samplo & riresulli: & Simolicate & 14. \\
\hline Ha aldy & (3) & (3) & 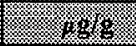 & 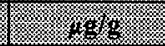 & 1.498 \\
\hline S97T001203 & 192: 1 & Drainable liquid & 3,010 & 3,050 & 3,030 \\
\hline S97T000834 & 192: 2 & Drainable liquid & 3,220 & 3,150 & 3,190 \\
\hline S97T000835 & 192: 3 & Drainable liquid & 2,820 & 2,760 & 2,790 \\
\hline S97T000836 & $192: 4$ & Drainable liquid & 3,080 & 3,080 & 3,080 \\
\hline S97T000837 & 192: 5 & Drainable liquid & 3,320 & 3,440 & 3,380 \\
\hline 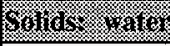 & aiget & (3) & $\sqrt{18}$ & 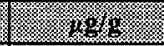 & $\sqrt{18}$ \\
\hline S97T000931 & \multirow[t]{2}{*}{ 192: 6} & Upper half & 4,640 & 4,710 & 4,670 \\
\hline S97T000900 & & Lower half & 4,770 & 4,400 & 4,590 \\
\hline S97T000916 & \multirow[t]{2}{*}{ 192: 7} & Upper half & 4,820 & 4,920 & 4,870 \\
\hline S97T000915 & & Lower half & 4,560 & 4,560 & 4,560 \\
\hline S97T000918 & \multirow[t]{2}{*}{ 192: 8} & Upper half & 5,220 & 5,170 & 5,200 \\
\hline S97T000917 & & Lower half & 5,420 & 5,010 & 5,210 \\
\hline S97T001251 & \multirow[t]{2}{*}{ Core 192} & Solid composite & 5,030 & 5,120 & 5,080 \\
\hline S97T001257 & & Solid composite & 4,290 & 4,300 & 4,300 \\
\hline
\end{tabular}


Table B2-48. Tank 241-T-201 Analytical Results: Nitrate (IC).

\begin{tabular}{|c|c|c|c|c|c|}
\hline Samples & Sample & f Somplos & Resilis & Buplicat & . \\
\hline \multicolumn{3}{|c|}{ Hatros v } & 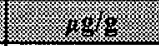 & 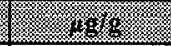 & S \\
\hline S97T001203 & 192: 1 & Drainable liquid & 34,300 & 33,900 & 34,100 \\
\hline S97T000834 & 192: 2 & Drainable liquid & 34,800 & 34,700 & 34,800 \\
\hline S97T000835 & 192: 3 & Drainable liquid & 31,200 & 30,900 & 31,000 \\
\hline S97T000836 & 192: 4 & Drainable liquid & 32,200 & 31,800 & 32,000 \\
\hline S97T000837 & $192: 5$ & Drainable liquid & 34,000 & 34,500 & 34,200 \\
\hline \multicolumn{3}{|c|}{ 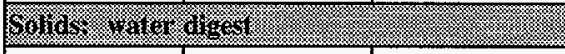 } & 8898 & 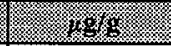 & 1.86 \\
\hline S97T000931 & \multirow[t]{2}{*}{ 192: 6} & Upper half & 45,600 & 46,800 & 46,200 \\
\hline S97T000900 & & Lower half & 44,300 & 43,600 & 44,000 \\
\hline S97T000916 & \multirow[t]{2}{*}{ 192: 7} & Upper half & 40,300 & 41,000 & 40,600 \\
\hline S97T000915 & & Lower half & 43,300 & 43,500 & 43,400 \\
\hline S97T000918 & \multirow[t]{2}{*}{ 192: 8} & Upper half & 43,700 & 43,800 & 43,700 \\
\hline S97T000917 & & Lower half & 45,400 & 42,500 & 43,900 \\
\hline S97T001251 & \multirow[t]{2}{*}{ Core 192} & Solid composite & 47,100 & 49,500 & 48,300 \\
\hline S97T001257 & & Solid composite & 42,100 & 42,400 & 42,300 \\
\hline
\end{tabular}


HNF-1501 Rev. 0

Table B2-49. Tank 241-T-201 Analytical Results: Nitrite (IC).

\begin{tabular}{|c|c|c|c|c|c|}
\hline $\begin{array}{l}\text { Sample } \\
\text { Ninniter }\end{array}$ & S.rangles & $\begin{array}{l}\text { Sample } \\
\text { Partion }\end{array}$ & restil & Burlicutits & 4i:an: \\
\hline \multicolumn{3}{|c|}{ Wrangs } & (196\% & 3. & 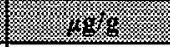 \\
\hline S97T001203 & 192: 1 & Drainable liquid & 223 & 224 & 224 \\
\hline S97T000834 & 192: 2 & Drainable liquid & 165 & 167 & 166 \\
\hline S97T000835 & 192: 3 & Drainable liquid & 140 & 148 & 144 \\
\hline S97T000836 & 192: 4 & Drainable liquid & 344 & 334 & 339 \\
\hline S97T000837 & 192: 5 & Drainable liquid & 335 & 332 & 334 \\
\hline \multicolumn{3}{|c|}{ 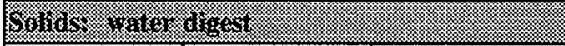 } & 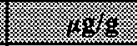 & (3) & 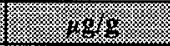 \\
\hline S97T000931 & \multirow[t]{2}{*}{ 192: 6} & Upper half & 317 & 317 & 317 \\
\hline S97T000900 & & Lower half & 317 & 298 & 307 \\
\hline S97T000916 & \multirow[t]{2}{*}{ 192: 7} & Upper half & 464 & 485 & 475 \\
\hline S97T000915 & & Lower half & 302 & 296 & 299 \\
\hline S97T000918 & \multirow[t]{2}{*}{$192: 8$} & Upper half & 513 & 516 & 514 \\
\hline S97T000917 & & Lower half & 500 & 484 & 492 \\
\hline S97T001251 & \multirow[t]{2}{*}{ Core 192} & Solid composite & 309 & 318 & 314 \\
\hline S97T001257 & & Solid composite & 289 & 302 & 295 \\
\hline
\end{tabular}


HNF-1501 Rev. 0

Table B2-50. Tank 241-T-201 Analytical Results: Phosphate (IC).

\begin{tabular}{|c|c|c|c|c|c|}
\hline $\begin{array}{l}\text { Sampis } \\
\text { Muninier }\end{array}$ & Siample & Simile & nestits & Hollinat: & Minin \\
\hline \multicolumn{3}{|c|}{ Tyrias } & $(1.9 \mathrm{~g}$ & $10.8 \mathrm{~g}$ & 8.68 \\
\hline S97T001203 & 192: 1 & Drainable liquid & 545 & 499 & 522 \\
\hline S97T000834 & 192: 2 & Drainable liquid & 530 & 508 & 519 \\
\hline S97T000835 & 192: 3 & Drainable liquid & 444 & 431 & 437 \\
\hline S97T000836 & 192: 4 & Drainable liquid & 468 & 430 & 449 \\
\hline S97T000837 & 192: 5 & Drainable liquid & 528 & 463 & 495 \\
\hline \multicolumn{2}{|c|}{ 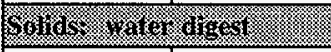 } & 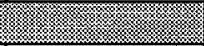 & $\sqrt{3.38}$ & 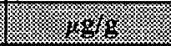 & 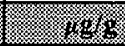 \\
\hline S97T000931 & \multirow[t]{2}{*}{ 192: 6} & Upper half & 1,220 & 1,160 & 1,190 \\
\hline S97T000900 & & Lower half & 554 & 680 & $617^{\mathrm{QC}: e}$ \\
\hline S97T000916 & \multirow[t]{2}{*}{ 192: 7} & Upper half & 379 & 618 & $498^{\mathrm{QC}: \mathrm{e}}$ \\
\hline S97T000915 & & Lower half & 577 & 417 & $497^{\text {QC:e }}$ \\
\hline S97T000918 & \multirow[t]{2}{*}{ 192: 8} & Upper half & 368 & 348 & 358 \\
\hline S97T000917 & & Lower half & 316 & 478 & $397^{\text {QC:c }}$ \\
\hline S97T001251 & \multirow[t]{2}{*}{ Core 192} & Solid composite & 366 & 520 & $443^{\mathrm{OC}: \mathrm{c}}$ \\
\hline S97T001257 & & Solid composite & 1,130 & 1,110 & 1,120 \\
\hline
\end{tabular}


HNF-1501 Rev. 0

Table B2-51. Tank 241-T-201 Analytical Results: Sulfate (IC).

\begin{tabular}{|c|c|c|c|c|c|}
\hline Sample & Samile & S5rn116: & gesili: & Bigligate & 1ran \\
\hline Pyas = & (3) & 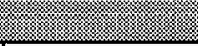 & 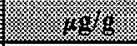 & $3.89 \mathrm{~g}=$ & 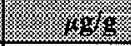 \\
\hline S97T001203 & 192: 1 & Drainable liquid & $<211$ & $<211$ & $<211$ \\
\hline S97T000834 & 192: 2 & Drainable liquid & $<110$ & $<110$ & $<110$ \\
\hline S97T000835 & 192: 3 & Drainable liquid & $<110$ & $<110$ & $<110$ \\
\hline S97T000836 & 192: 4 & Drainable liquid & $<211$ & $<211$ & $<211$ \\
\hline S97T000837 & $192: 5$ & Drainable liquid & $<211$ & $<211$ & $<211$ \\
\hline 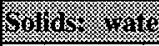 & (1) & 8.8 & 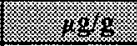 & 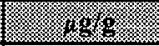 & $4.1 \%$ \\
\hline S97T000931 & \multirow[t]{2}{*}{ 192: 6} & Upper half & 963 & $<331$ & $<647^{\text {QC:e }}$ \\
\hline S97T000900 & & Lower half & $<307$ & 392 & $<349^{\text {QC:e }}$ \\
\hline S97T000916 & \multirow[t]{2}{*}{ 192: 7} & Upper half & 464 & 527 & 496 \\
\hline S97T000915 & & Lower half & $<302$ & 981 & $<641^{\mathrm{QC}: c}$ \\
\hline S97T000918 & \multirow[t]{2}{*}{ 192: 8} & Upper half & 727 & 596 & 661 \\
\hline S97T000917 & & Lower half & 397 & 559 & $478^{\mathrm{QC}: \mathrm{c}}$ \\
\hline S97T001251 & \multirow[t]{2}{*}{ Core 192} & Solid composite & $<308$ & 1,250 & $<779^{\mathrm{QC:O}}$ \\
\hline S97T001257 & & Solid composite & 1,080 & $<303$ & $<693^{Q \mathrm{QC:e}}$ \\
\hline
\end{tabular}


Table B2-52. Tank 241-T-201 Analytical Results: Oxalate (IC).

\begin{tabular}{|c|c|c|c|c|c|}
\hline Simples & (O) Samples & Somile: & resin: & 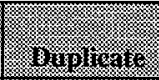 & Mran \\
\hline Mands & (1) & 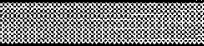 & (198\% & 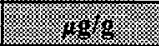 & (1) \\
\hline S97T001203 & 192: 1 & Drainable liquid & $<160$ & $<160$ & $<160$ \\
\hline S97T000834 & 192: 2 & Drainable liquid & $<84$ & 97.1 & $<90.5$ \\
\hline S97T000835 & 192: 3 & Drainable liquid & $<84$ & $<84.2$ & $<84.1$ \\
\hline S97T000836 & 192: 4 & Drainable liquid & $<160$ & $<160$ & $<160$ \\
\hline S97T000837 & 192: 5 & Drainable liquid & $<160$ & $<160$ & $<160$ \\
\hline 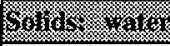 & aligey, & 8 & 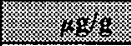 & 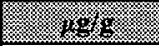 & 8 \\
\hline S97T000931 & \multirow[t]{2}{*}{ 192: 6} & Upper half & 1,020 & 1,120 & 1,070 \\
\hline S97T000900 & & Lower half & 1,080 & 984 & 1,030 \\
\hline S97T000916 & \multirow[t]{2}{*}{ 192: 7} & Upper half & 644 & 721 & 682 \\
\hline S97T000915 & & Lower half & 1,030 & 988 & 1,010 \\
\hline S97T000918 & \multirow[t]{2}{*}{ 192: 8} & Upper half & 769 & 742 & 755 \\
\hline S97T000917 & & Lower half & 809 & 722 & 766 \\
\hline S97T001251 & \multirow[t]{2}{*}{ Core 192} & Solid composite & 1,150 & 1,140 & 1,140 \\
\hline S97T001257 & & Solid composite & $<228$ & $<230$ & $<229$ \\
\hline
\end{tabular}

Table B2-53. Tank 241-T-201 Analytical Results: Bulk Density.

\begin{tabular}{|c|c|c|c|c|c|}
\hline Siniolo & Goringle & Yimale & Resuan: & Punlegre & Menin \\
\hline Sulus & 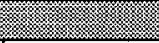 & U. & estru & $\mathrm{g} / \mathrm{n}$ & 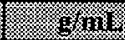 \\
\hline S97T000894 & 192: 6 & Lower half & 1.39 & N/A & 1.39 \\
\hline S97T000895 & 192: 7 & Lower half & 1.28 & N/A & 1.28 \\
\hline S97T000896 & 192: 8 & Lower half & 1.27 & N/A & 1.27 \\
\hline S97T001245 & \multirow[t]{2}{*}{ Core 192} & Solid composite & 1.27 & N/A & 1.27. \\
\hline S97T001252 & & Solid composite & 1.13 & N/A & 1.13 \\
\hline
\end{tabular}




\begin{tabular}{|c|c|c|c|c|c|}
\hline $90^{\circ} \mathrm{I}$ & $\angle 0^{\circ} \mathrm{I}$ & $90^{\circ} \mathrm{I}$ & p!̣nb!̣ ə|qeựer & $s: 26 \mathrm{I}$ & $\angle E 8000 \mathrm{~L} L 6 \mathrm{~S}$ \\
\hline $90^{\circ} \mathrm{T}$ & $S 0^{\circ} \mathrm{T}$ & $90^{\circ} \mathrm{T}$ & 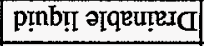 & $t: 26 \mathrm{I}$ & $9 \varepsilon 8000 \mathrm{~L} \angle 6 \mathrm{~S}$ \\
\hline $\mathrm{SO}^{\circ} \mathrm{T}$ & $S 0^{\circ} \mathrm{I}$ & $90^{\circ} \mathrm{T}$ & 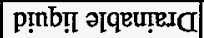 & $\varepsilon: 26 \mathrm{I}$ & SE8000LL6S \\
\hline $90^{\circ} \mathrm{I}$ & $90^{\circ} \mathrm{I}$ & $\mathrm{SO}^{\circ} \mathrm{I}$ & pụnb!̣ əqеụera & $2: 26 \mathrm{I}$ & $+\varepsilon 8000 \mathrm{~L} \angle 6 \mathrm{~S}$ \\
\hline $\mathrm{SO}^{\circ} \mathrm{T}$ & $\mathrm{SO}^{\circ} \mathrm{I}$ & SO. I & pụnbñ əqqựexa & $\mathrm{I}: 26 \mathrm{I}$ & EE8000L L6S \\
\hline ( & 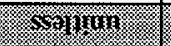 & 2851411 & & & T.110101010 \\
\hline rive & 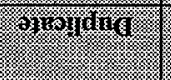 & $1914.8 \%$ & inofiod & 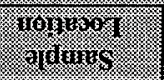 & 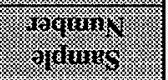 \\
\hline
\end{tabular}

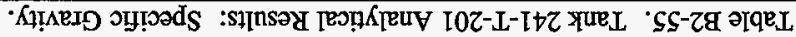

\begin{tabular}{|c|c|c|c|c|c|}
\hline $9^{\circ} 58$ & $\varepsilon<8$ & $6 \mathcal{E}^{\circ}$ & 21!sodwo p!̣os & & ESZI00LL6S \\
\hline $9^{\circ}[9$ & 6.29 & $\varepsilon 09$ & $\begin{array}{r}\text { əq!soduros } \\
\text { गp!ा०S }\end{array}$ & $26 \mathrm{I}$ วI0 & $9+Z I 00 \mathrm{~L} L 6 \mathrm{~S}$ \\
\hline$L^{*} I L$ & $\varepsilon^{\cdot} \cdot L$ & $I^{\prime} z L$ & IГеप गәMOT] & \multirow[b]{2}{*}{$8: 26 \mathrm{~T}$} & ZI6000LL6S \\
\hline$\varepsilon^{*} 0 L$ & 6.69 & $\angle \cdot 0 L$ & न[धч Iədd $\Omega$ & & $\varepsilon \mathrm{I} 6000 \mathrm{~L} L 6 \mathrm{~S}$ \\
\hline $6^{\circ}+9$ & $\varepsilon ' 99$ & s. $\varepsilon 9$ & Jाеप دәмоT & \multirow[b]{2}{*}{$L: 26 \mathrm{I}$} & $0 \mathrm{I} 6000 \mathrm{~L} L 6 \mathrm{~S}$ \\
\hline$\varepsilon 9$ & $\varsigma^{\prime} 09$ & 9.59 & माe् Iədd & & I I6000L $\angle 6 \mathrm{~S}$ \\
\hline $5 \$ 9$ & $8 \div 9$ & $\varepsilon \varepsilon^{\circ} 99$ & नाЕप IOMOT & \multirow[b]{2}{*}{9 : Z6I } & $668000 \mathrm{I} \angle 6 \mathrm{~S}$ \\
\hline$L \cdot 69$ & 769 & 0.04 & $2 / \tau$ Iadd $\Omega$ & & $606000 \mathrm{~L} \angle 6 \mathrm{~S}$ \\
\hline 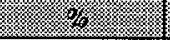 & (1) & (5) & & & 80168 \\
\hline I6 & I6 & I6 & prnb!̣ әqqeựexa & $\mathrm{s}: 26 \mathrm{I}$ & $\angle E 8000 \mathrm{~L} \angle 6 \mathrm{~S}$ \\
\hline$\varepsilon \cdot 16$ & I'โ6 & 716 & p!̣nb!̣ әqquா̣eI & $t: 26 \mathrm{I}$ & $9 \varepsilon 8000 \mathrm{~L} \angle 6 \mathrm{~S}$ \\
\hline $8^{\circ} \mathrm{I} 6$ & 8.16 & $8^{\circ} 16$ & 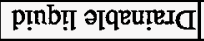 & $\varepsilon: 26 \mathrm{I}$ & SE8000IL6S \\
\hline I'I6 & I6 & $2^{\prime} 16$ & pịnb!̣ әqеựera & $2: 261$ & $\dagger \varepsilon 8000 \mathrm{~L} \angle 6 \mathrm{~S}$ \\
\hline I6 & I6 & I'I6 & ptnb!̣ əqqeựerd & $\mathrm{I}: 26 \mathrm{I}$ & $\varepsilon \varepsilon 8000 \mathrm{~L} \angle 6 \mathrm{~S}$ \\
\hline 4 & 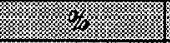 & 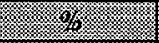 & & & 4 - \\
\hline 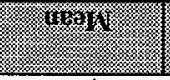 & 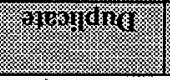 & 1795ry & Iroy & 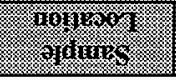 & orouging \\
\hline
\end{tabular}

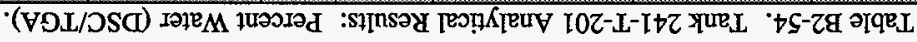


Table B2-56. Tank 241-T-201 Analytical Results: Total Alpha, Drainable Liquid (Alpha Rad).

\begin{tabular}{|c|c|c|c|c|c|}
\hline Sinazile & $\begin{array}{l}\text { Sample } \\
\text { Socto }\end{array}$ & Proning & Henul: & OHiplisater & Meri: \\
\hline risunds: & & স্যে & (6) Wu & 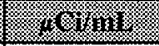 & 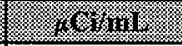 \\
\hline \$97T001203 & 192: 1 & Drainable liquid & $1.05 \mathrm{E}-05$ & $8.96 \mathrm{E}-06$ & $9.73 \mathrm{E}-06$ \\
\hline S97T000834 & 192: 2 & Drainable liquid & $7.96 \mathrm{E}-06$ & $9.41 \mathrm{E}-06$ & $8.68 \mathrm{E}-06$ \\
\hline S97T001259 & 192: 3 & Drainable liquid & $8.49 \mathrm{E}-06$ & $8.33 \mathrm{E}-06$ & $8.41 \mathrm{E}-06$ \\
\hline S97T000836 & 192: 4 & Drainable liquid & $<3.46 \mathrm{E}-05$ & $<6.26 \mathrm{E}-05$ & $<4.86 \mathrm{E}-05^{\text {ec: }}$ \\
\hline \$97T000837 & 192: 5 & Drainable liquid & $<5.59 \mathrm{E}-05$ & $<7.61 \mathrm{E}-05$ & $<6.60 \mathrm{E}-05^{\mathrm{QC}: \mathrm{f}}$ \\
\hline
\end{tabular}

Table B2-57. Tank 241-T-201 Analytical Results: Total Alpha, Solid (Alpha).

\begin{tabular}{|c|c|c|c|c|c|}
\hline $\begin{array}{l}\text { Sample } \\
\text { Surnoer }\end{array}$ & 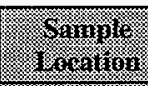 & $\begin{array}{l}\text { Sarnole } \\
\text { romiton }\end{array}$ & is & B) & $\sqrt{18}$ \\
\hline 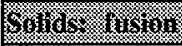 & স & (4) & 1. & 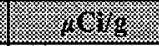 & . \\
\hline \$97T000901 & 192: 6 & Lower half & 0.632 & 0.628 & 0.63 \\
\hline S97T000919 & 192: 7 & Lower half & 0.598 & 0.565 & 0.581 \\
\hline S97T000920 & 192: 8 & Lower half & 1.15 & 0.966 & 1.06 \\
\hline
\end{tabular}

Table B2-58. Tank 241-T-201 Analytical Results: Americium-241 (GEA).

\begin{tabular}{|c|c|c|c|c|c|}
\hline Tamigle & Sumilo & Promition & rigumil & 3inglicar. & . \\
\hline 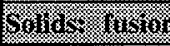 & & 4 & 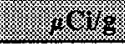 & $.1 .61,8$ & (6) \\
\hline S97T001753 & \multirow[t]{2}{*}{ Core 192} & Solid composite & $<0.0346$ & $<0.0293$ & $<0.0319$ \\
\hline S97T001754 & & Solid composite & $<0.0205$ & $<0.0207$ & $<0.0206$ \\
\hline
\end{tabular}


Table B2-59. Tank 241-T-201 Analytical Results: Cesium-137 (GEA).

\begin{tabular}{|c|c|c|c|c|c|}
\hline Samples & Saringlic & 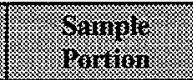 & ingusint: & Buplitira & Mean \\
\hline $801116 \%$. & ly & 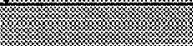 & 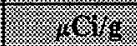 & 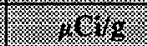 & 48 \\
\hline S97T001753 & \multirow[t]{2}{*}{ Core 192} & Solid composite & 0.0481 & 0.0433 & 0.0457 \\
\hline S97T001754 & & Solid composite & $<0.00847$ & 0.0123 & $<0.0104^{\mathrm{QC}: \mathrm{c}}$ \\
\hline
\end{tabular}

Table B2-60. Tank 241-T-201 Analytical Results: Cobalt-60 (GEA).

\begin{tabular}{|c|c|c|c|c|c|}
\hline 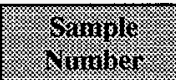 & 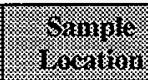 & / Somolitis & nesilit & Oinplicre & 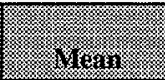 \\
\hline 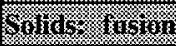 & & & 1018 & (1) & 1 \\
\hline S97T001753 & \multirow[t]{2}{*}{ Core 192} & Solid composite & $<0.00634$ & $<0.00566$ & $<0.006$ \\
\hline S97T001754 & & Solid composite & $<0.00452$ & $<0.00437$ & $<0.00444$ \\
\hline
\end{tabular}

Table B2-61. Tank 241-T-201 Analytical Results: Europium-154 (GEA).

\begin{tabular}{|c|c|c|c|c|c|}
\hline $\begin{array}{c}\text { Samile } \\
\text { Wmaber }\end{array}$ & S & $\begin{array}{l}\text { Sranule } \\
\text { Portion }\end{array}$ & iresulis & Buplicure & IIenin \\
\hline 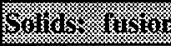 & & & 40 & 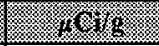 & 等, \\
\hline S97T001753 & \multirow[t]{2}{*}{ Core 192} & Solid composite & $<0.0182$ & $<0.0186$ & $<0.0184$ \\
\hline S97T001754 & & Solid composite & $<0.0135$ & $<0.0144$ & $<0.014$ \\
\hline
\end{tabular}

Table B2-62. Tank 241-T-201 Analytical Results: Europium-155 (GEA).

\begin{tabular}{|c|c|c|c|c|c|}
\hline Sompin & S. Samile & S.minle: & (x) & Bvilinate & 1018 \\
\hline Soling ning & 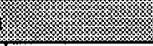 & $x^{\prime 4}$ & 1) & 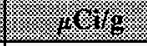 & 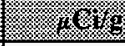 \\
\hline S97T001753 & \multirow[t]{2}{*}{ Core 192} & Solid composite & $<0.0146$ & $<0.0139$ & $<0.0143$ \\
\hline S97T001754 & & Solid composite & $<0.0107$ & $<0.0104$ & $<0.0105$ \\
\hline
\end{tabular}


Table B2-63. Tank 241-T-201 Analytical Results: Strontium-89/90.

\begin{tabular}{|c|c|c|c|c|c|}
\hline Sample & 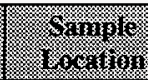 & $\begin{array}{l}\text { Somple } \\
\text { Sormor }\end{array}$ & Jcoulit & ormplocis & Yren \\
\hline S. & (1) & 4. & $8,764 \mathrm{~g}=$ & (1) & (1) \\
\hline S97T001753 & \multirow[t]{2}{*}{ Core 192} & Solid composite & 0.137 & 0.145 & 0.141 \\
\hline S97T001754 & & Solid composite & 0.0495 & 0.0117 & $0.0306^{Q C: e}$ \\
\hline
\end{tabular}

Table B2-64. Tank 241-T-201 Analytical Results: Total Inorganic Carbon.

\begin{tabular}{|c|c|c|c|c|c|}
\hline Sinovis & 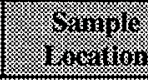 & 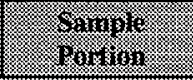 & r8esulin: & Banolicate & 3yeris \\
\hline S69uid s & "艹 & $(3)(3)$ & (13. & 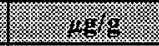 & $16 \mathrm{~g} \cdot \mathrm{s}$ \\
\hline S97T001246 & Core 192 & Solid composite & 828. & 791 & 810 \\
\hline S97T001253 & & Solid composite & 1,800 & 1,860 & 1,830 \\
\hline
\end{tabular}

Table B2-65. Tank 241-T-201 Analytical Results: Total Organic Carbon.

\begin{tabular}{|c|c|c|c|c|c|}
\hline 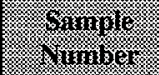 & $\begin{array}{l}\text { Samile } \\
\text { 4 }\end{array}$ & Simpis & Rerulu & 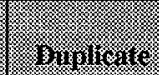 & ligen \\
\hline S101306 & י & 桨: & 1,828 & 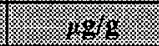 & (18 \\
\hline S97T001246 & Core 192 & Solid composite & 303 & 304 & $304^{\text {eC:c }}$ \\
\hline S97T001253 & & Solid composite & 156 & 110 & $133^{\mathrm{QC}: \mathrm{c}}$ \\
\hline
\end{tabular}


Table B2-66. Tank 241-T-202 Analytical Results: Aluminum (ICP).

\begin{tabular}{|c|c|c|c|c|c|}
\hline Saminger & 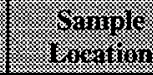 & oquingles & Resul & 16ipling & . \\
\hline \multicolumn{2}{|c|}{ 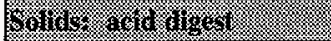 } & 8 & 18 & 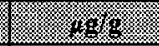 & 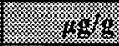 \\
\hline S97T000945 & \multirow[t]{2}{*}{ 191: 1} & Upper half & 71.9 & 97.3 & $84.6^{\mathrm{eC}: \mathrm{e}}$ \\
\hline \$97T000944 & & Lower half & 70.8 & 57.9 & $64.3^{\mathrm{QC}: \mathrm{c}}$ \\
\hline S97T000947 & \multirow[t]{2}{*}{ 191: 2} & Upper half & 64.4 & 84.6 & $74.5^{\mathrm{eC}: \mathrm{e}}$ \\
\hline S97T000946 & & Lower half & 68.9 & 58.9 & 63.9 \\
\hline S97T000949 & \multirow[t]{2}{*}{ 191: 3} & Upper half & 112 & 88 & $100^{\mathrm{QC:c}}$ \\
\hline S97T000948 & & Lower half & 46.2 & 54.2 & 50.2 \\
\hline S97T000951 & \multirow[t]{2}{*}{ 191: 4} & Upper half & 63.3 & 60.1 & 61.7 \\
\hline S97T000950 & & Lower half & 59.6 & 73.2 & $66.4^{\mathrm{QC}: \mathrm{a}, \mathrm{c}}$ \\
\hline S97T000953 & \multirow[t]{2}{*}{ 191: 5} & Upper half & 62 & 71.1 & 66.5 \\
\hline S97T000952 & & Lower half & 120 & 84 & $102^{\mathrm{QC}: \mathrm{a}, \mathrm{e}}$ \\
\hline S97T001805 & Core 191 & Solid composite & 76.4 & 68 & 72.2 \\
\hline
\end{tabular}

Table B2-67. Tank 241-T-202 Analytical Results: Antimony (ICP).

\begin{tabular}{|c|c|c|c|c|c|}
\hline Samisil: & $\begin{array}{l}\text { Sumile } \\
\text { Losition: }\end{array}$ & $\begin{array}{l}\text { Sarmine } \\
\text { rovion }\end{array}$ & resulu & Bingingate & yearin \\
\hline \multicolumn{3}{|c|}{ Silib naridigery } & $8.48+8$ & $=1.828$ & 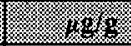 \\
\hline S97T000945 & \multirow[t]{2}{*}{ 191: 1} & Upper half & $<17.2$ & $<17.1$ & $<17.1$ \\
\hline S97T000944 & & Lower half & $<11.8$ & $<11.8$ & $<11.8^{\mathrm{QC:a}}$ \\
\hline S97T000947 & \multirow[t]{2}{*}{ 191: 2} & Upper half & 27.6 & $<17.1$ & $<22.4^{\mathrm{QC:}: 0}$ \\
\hline S97T000946 & & Lower half & $<12.2$ & $<12$ & $<12.1^{\mathrm{QC:A}}$ \\
\hline S97T000949 & \multirow[t]{2}{*}{ 191: 3} & Upper half & 31.7 & 25 & $28.4^{\mathrm{QC}: c}$ \\
\hline S97T000948 & & Lower half & $<11.5$ & $<11.5$ & $<11.5^{\text {QC:a }}$ \\
\hline S97T000951 & \multirow[t]{2}{*}{ 191: 4} & Upper half & 20.4 & 28.5 & $24.4^{\mathrm{eC}: \mathrm{e}}$ \\
\hline S97T000950 & & Lower half & $<12$ & $<12.2$ & $<12.1^{\mathrm{QC}: \mathrm{a}}$ \\
\hline S97T000953 & \multirow[t]{2}{*}{ 191: 5} & Upper half & 35.5 & 25.2 & $30.4^{\text {eC:e }}$ \\
\hline S97T000952 & & Lower half & $<12.1$ & $<12.2$ & $<12.1^{\mathrm{QC}: \mathrm{a}}$ \\
\hline S97T001805 & Core 191 & Solid composite & $<12.1$ & $<12.1$ & $<12.1$. \\
\hline
\end{tabular}


Table B2-68. Tank 241-T-202 Analytical Results: Arsenic (ICP).

\begin{tabular}{|c|c|c|c|c|c|}
\hline Sample: & 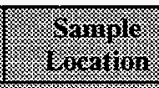 & $\begin{array}{l}\text { samile } \\
\text { rortion }\end{array}$ & Resinil & Hopllivare & $4 y_{2 n}$ \\
\hline \multicolumn{2}{|c|}{ 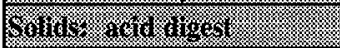 } & (1) & 3.148 & 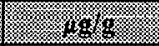 & 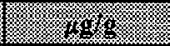 \\
\hline S97T000945 & \multirow[t]{2}{*}{ 191: 1} & Upper half & 155 & 167 & 161 \\
\hline S97T000944 & & Lower half & $<19.6$ & $<19.7$ & $<19.6$ \\
\hline S97T000947 & \multirow[t]{2}{*}{ 191: 2} & Upper half & 196 & 188 & 192 \\
\hline S97T000946 & & Lower half & $<20.3$ & $<20.1$ & $<20.2$ \\
\hline S97T000949 & \multirow[t]{2}{*}{ 191: 3} & Upper half & 188 & 187 & 188 \\
\hline S97T000948 & & Lower half & $<19.2$ & $<19.2$ & $<19.2$ \\
\hline S97T000951 & \multirow[t]{2}{*}{ 191: 4} & Upper half & 229 & 221 & 225 \\
\hline S97T000950 & & Lower half & $<20$ & $<20.3$ & $<20.1$ \\
\hline S97T000953 & \multirow[t]{2}{*}{ 191: 5} & Upper half & 171 & 173 & 172 \\
\hline S97T000952 & & Lower half & $<20.2$ & $<20.3$ & $<20.3$ \\
\hline S97T001805 & Core 191 & Solid composite & $<20.2$ & $<20.1$ & $<20.1$ \\
\hline
\end{tabular}

Table B2-69. Tank 241-T-202 Analytical Results: Barium (ICP)

\begin{tabular}{|c|c|c|c|c|c|}
\hline STrmiler & 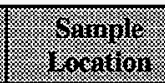 & $\begin{array}{l}\text { Simpis! } \\
\text { Iortor }\end{array}$ & (imesulu & shyilicate & . \\
\hline \multicolumn{3}{|c|}{ 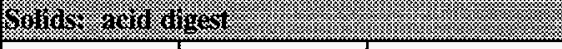 } & 8,13868 & (1) & 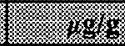 \\
\hline S97T000945 & \multirow[t]{2}{*}{ 191: 1} & Upper half & 1,610 & 1,640 & 1,630 \\
\hline S97T000944 & & Lower half & 1,530 & 1,480 & 1,510 \\
\hline S97T000947 & \multirow[t]{2}{*}{ 191: 2} & Upper half & 1,590 & 1,610 & 1,600 \\
\hline S97T000946 & & Lower half & 1,790 & 1,950 & 1,870 \\
\hline S97T000949 & \multirow[t]{2}{*}{ 191: 3} & Upper half & 1,940 & 1,980 & 1,960 \\
\hline S97T000948 & & Lower half & 1,970 & 2,080 & 2,030 \\
\hline S97T000951 & \multirow[t]{2}{*}{ 191: 4} & Upper half & 1,080 & 1,030 & 1,060 \\
\hline S97T000950 & & Lower half & 22.6 & 22.2 & $22.4^{\mathrm{eC:}: \mathrm{a}}$ \\
\hline S97T000953 & \multirow[t]{2}{*}{$191: 5$} & Upper half & 67 & 65.6 & 66.3 \\
\hline S97T000952 & & Lower half & 23.4 & 25.5 & $24.4^{\mathrm{QC:A}}$ \\
\hline S97T001805 & Core 191 & Solid composite & 1,250 & 1,280 & 1,270 \\
\hline
\end{tabular}


Table B2-70. Tank 241-T-202 Analytical Results: Beryllium (ICP).

\begin{tabular}{|c|c|c|c|c|c|}
\hline Samples & 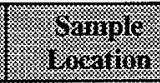 & I Somition & (Ix) & 3130191048 & 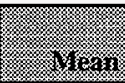 \\
\hline \multicolumn{3}{|c|}{ Soldas ard } & ( & 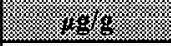 & 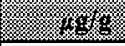 \\
\hline S97T000945 & \multirow[t]{2}{*}{ 191: 1} & Upper half & $<1.43$ & $<1.43$ & $<1.43$ \\
\hline S97T000944 & & Lower half & $<0.982$ & $<0.987$ & $<0.984$ \\
\hline S97T000947 & \multirow[t]{2}{*}{ 191: 2} & Upper half & $<1.45$ & $<1.43$ & $<1.44$ \\
\hline S97T000946 & & Lower half & $<1.01$ & $<1$ & $<1$ \\
\hline S97T000949 & \multirow[t]{2}{*}{ 191: 3} & Upper half & $<1.45$ & $<1.42$ & $<1.44$ \\
\hline S97T000948 & & Lower half & $<0.959$ & $<0.958$ & $<0.958$ \\
\hline S97T000951 & \multirow[t]{2}{*}{ 191: 4} & Upper half & $<1.45$ & $<1.44$ & $<1.44$ \\
\hline S97T000950 & & Lower half & $<1$ & $<1.01$ & $<1$ \\
\hline S97T000953 & \multirow[t]{2}{*}{$191: 5$} & Upper half & $<1.42$ & $<1.4$ & $<1.41$ \\
\hline S97T000952 & & Lower half & $<1.01$ & $<1.02$ & $<1.02$ \\
\hline S97T001805 & Core 191 & Solid composite & $<1.01$ & $<1.01$ & $<1.01$ \\
\hline
\end{tabular}

Table B2-71. Tank 241-T-202 Analytical Results: Bismuth (ICP).

\begin{tabular}{|c|c|c|c|c|c|}
\hline Gamjie & 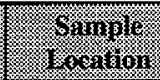 & Sample & 2. & Byinionse & 16eris: \\
\hline \multicolumn{2}{|c|}{ 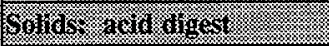 } & 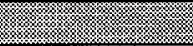 & 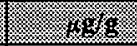 & 4.186 & 1.840 \\
\hline S97T000945 & \multirow[t]{2}{*}{ 191: 1} & Upper half & 29,600 & 30,600 & 30,100 \\
\hline S97T000944 & & Lower half & 28,900 & 28,100 & $28,500^{\mathrm{QC:a,d}}$ \\
\hline S97T000947 & \multirow[t]{2}{*}{ 191: 2} & Upper half & 34,300 & 34,300 & 34,300 \\
\hline S97T000946 & & Lower half & 34,300 & 36,700 & $35,500^{\mathrm{QC:a}}$ \\
\hline S97T000949 & \multirow[t]{2}{*}{ 191: 3} & Upper half & 36,800 & 36,400 & 36,600 \\
\hline S97T000948 & & Lower half & 35,000 & 36,400 & $35,700^{\mathrm{QC:} \mathrm{a}}$ \\
\hline S97T000951 & \multirow[t]{2}{*}{ 191: 4} & Upper $1 / 2$ & 44,500 & None & 44,500 \\
\hline S97T000950 & & Lower half & 42,700 & 40,700 & $41,700^{\mathrm{QC:}:}$ \\
\hline S97T000953 & \multirow[t]{2}{*}{ 191: 5} & Upper half & 35,200 & 34,900 & 35,100 \\
\hline S97T000952 & & Lower half & 41,100 & 41,700 & $41,400^{\mathrm{QC}: a}$ \\
\hline S97T001805 & Core 191 & Solid composite & 40,800 & 41,100 & $41,000^{\mathrm{QC}: \mathrm{d}}$ \\
\hline
\end{tabular}


Table B2-72. Tank 241-T-202 Analytical Results: Boron (ICP).

\begin{tabular}{|c|c|c|c|c|c|}
\hline Sorvine & 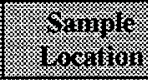 & 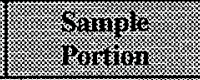 & Iresint: & 101211026 & Viran \\
\hline \multicolumn{3}{|c|}{ boling acra biger: } & 2.1989 & 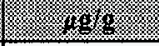 & 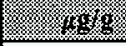 \\
\hline S97T000945 & \multirow[t]{2}{*}{ 191: 1} & Upper half & 123 & 170 & $147^{\mathrm{QC}: c}$ \\
\hline S97T000944 & & Lower half & 182 & 157 & 170 \\
\hline S97T000947 & \multirow[t]{2}{*}{ 191: 2} & Upper half & 132 & 132 & 132 \\
\hline S97T000946 & & Lower half & 188 & 161 & 175 \\
\hline S97T000949 & \multirow[t]{2}{*}{ 191: 3} & Upper half & 141 & 168 & 155 \\
\hline S97T000948 & & Lower half & 83.2 & 150 & $117^{\mathrm{QC}: \mathrm{e}}$ \\
\hline S97T000951 & \multirow[t]{2}{*}{ 191: 4} & Upper half & 171 & 136 & $154^{\mathrm{eC}: \mathrm{o}}$ \\
\hline S97T000950 & & Lower half & 164 & 220 & $192^{\mathrm{QC}: e}$ \\
\hline S97T000953 & \multirow[t]{2}{*}{ 191: 5} & Upper half & 118 & 114 & 116 \\
\hline S97T000952 & & Lower half & 162 & 157 & 160 \\
\hline S97T001805 & Core 191 & Solid composite & 154 & 162 & 158 \\
\hline
\end{tabular}

Table B2-73. Tank 241-T-202 Analytical Results: Cadmium (ICP).

\begin{tabular}{|c|c|c|c|c|c|}
\hline Simule & 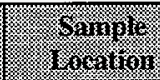 & 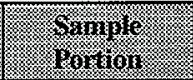 & Mesili & 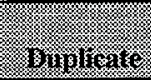 & Nigarir \\
\hline \multicolumn{3}{|c|}{ 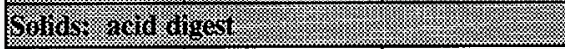 } & 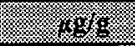 & 1.968 & 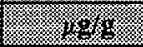 \\
\hline S97T000945 & \multirow[t]{2}{*}{ 191: 1} & Upper half & $<1.43$ & $<1.43$ & $<1.43$ \\
\hline S97T000944 & & Lower half & $<0.982$ & $<0.987$ & $<0.984^{\mathrm{QC:}: \mathrm{a}}$ \\
\hline S97T000947 & \multirow[t]{2}{*}{ 191: 2} & Upper half & $<1.45$ & $<1.43$ & $<1.44$ \\
\hline S97T000946 & & Lower half & $<1.01$ & 1.06 & $<1.04^{\mathrm{QC: \textrm {R }}}$ \\
\hline S97T000949 & \multirow[t]{2}{*}{ 191: 3} & Upper half & $<1.45$ & $<1.42$ & $<1.44$ \\
\hline S97T000948 & & Lower half & 1.57 & 1.51 & $1.54^{\mathrm{eC:a}}$ \\
\hline S97T000951 & \multirow[t]{2}{*}{ 191: 4} & Upper half & $<1.45$ & $<1.44$ & $<1.44$ \\
\hline S97T000950 & & Lower half & $<1$ & $<1.01$ & $<1^{\mathrm{QC}: \mathrm{a}}$ \\
\hline S97T000953 & \multirow[t]{2}{*}{$191: 5$} & Upper half & $<1.42$ & $<1.4$ & $<1.41$ \\
\hline S97T000952 & & Lower half & $<1.01$ & $<1.02$ & $<1.02^{\mathrm{QC:a}}$ \\
\hline S97T001805 & Core 191 & Solid composite & $<1.01$ & $<1.01$ & $<1.01$ \\
\hline
\end{tabular}


Table B2-74. Tank 241-T-202 Analytical Results: Calcium (ICP).

\begin{tabular}{|c|c|c|c|c|c|}
\hline 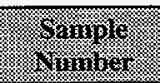 & viminle & Samgle & restit & Bunplizare & 1842418 \\
\hline \multicolumn{3}{|c|}{ 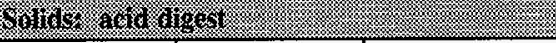 } & (1) & 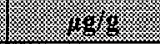 & 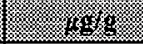 \\
\hline S97T000945 & \multirow[t]{2}{*}{ 191: 1} & Upper half & 284 & 330 & 307 \\
\hline S97T000944 & & Lower half & 294 & 236 & $265^{\mathrm{QC:c,e}}$ \\
\hline \$97T000947 & \multirow[t]{2}{*}{ 191: 2} & Upper half & 278 & 271 & 275 \\
\hline S97T000946 & & Lower half & 246 & 243 & 245 \\
\hline S97T000949 & \multirow[t]{2}{*}{ 191: 3} & Upper half & 315 & 335 & 325 \\
\hline S97T000948 & & Lower half & 172 & 270 & $221^{\mathrm{QC}: e}$ \\
\hline S97T000951 & \multirow[t]{2}{*}{ 191: 4} & Upper half & 213 & 211 & $212^{\mathrm{eC:b}}$ \\
\hline S97T000950 & & Lower half & 202 & 230 & 216 \\
\hline S97T000953 & \multirow[t]{2}{*}{ 191: 5} & Upper half & 208 & 212 & $210^{\mathrm{QC}: \mathrm{b}}$ \\
\hline S97T000952 & & Lower half & 252 & 258 & 255 \\
\hline S97T001805 & Core 191 & Solid composite & 323 & 297 & 310 \\
\hline
\end{tabular}

Table B2-75. Tank 241-T-202 Analytical Results: Cerium (ICP).

\begin{tabular}{|c|c|c|c|c|c|}
\hline $\begin{array}{l}\text { Surnole } \\
\text { virniser }\end{array}$ & Sampla & Sampole & itsul & (t) & keari: \\
\hline \multicolumn{3}{|c|}{ 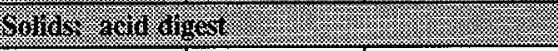 } & 8.1898 & 4.1846 & 1.880 \\
\hline S97T000945 & \multirow[t]{2}{*}{ 191: 1} & Upper half & 47.5 & 62.9 & $55.2^{\mathrm{OC}: \mathrm{e}}$ \\
\hline S97T000944 & & Lower half & 44.6 & 44.2 & 44.4 \\
\hline S97T000947 & \multirow[t]{2}{*}{ 191: 2} & Upper half & 57.2 & 53.1 & 55.2 \\
\hline S97T000946 & & Lower half & 33.9 & 36.8 & 35.3 \\
\hline S97T000949 & \multirow[t]{2}{*}{ 191: 3} & Upper half & 38 & 62.6 & $50.3^{\mathrm{OC:0}}$ \\
\hline S97T000948 & & Lower half & 40.1 & 42.3 & 41.2 \\
\hline S97T000951 & \multirow[t]{2}{*}{ 191: 4} & Upper half & 57.2 & 66.3 & 61.8 \\
\hline S97T000950 & & Lower half & 57.7 & 54.8 & $56.3^{\mathrm{QC}: a}$ \\
\hline S97T000953 & \multirow[t]{2}{*}{ 191: 5} & Upper half & 61.1 & 59.1 & 60.1 \\
\hline S97T000952 & & Lower half & 59.9 & 59.8 & $59.8^{\text {QC:a }}$ \\
\hline S97T001805 & Core 191 & Solid composite & 53.2 & 54.2 & 53.7 \\
\hline
\end{tabular}


Table B2-76. Tank 241-T-202 Analytical Results: Chromium (ICP).

\begin{tabular}{|c|c|c|c|c|c|}
\hline Saminer & (Singule & sinuple & Tesal: & 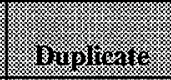 & (3) \\
\hline \multicolumn{3}{|c|}{ 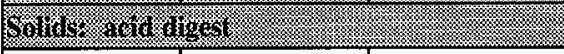 } & 1948 & (1) 1796 & 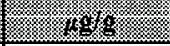 \\
\hline S97T000945 & \multirow[t]{2}{*}{ 191: 1} & Upper half & 1,580 & 1,660 & 1,620 \\
\hline S97T000944 & & Lower half & 1,620 & 1,540 & 1,580 \\
\hline S97T000947 & \multirow[t]{2}{*}{ 191: 2} & Upper half & 3,480 & 3,420 & 3,450 \\
\hline S97T000946 & & Lower half & 3,270 & 3,540 & 3,410 \\
\hline S97T000949 & \multirow[t]{2}{*}{ 191: 3} & Upper half & 3,170 & 3,220 & 3,200 \\
\hline S97T000948 & & Lower half & 3,350 & 3,510 & 3,430 \\
\hline S97T000951 & \multirow[t]{2}{*}{ 191: 4} & Upper half & 4,730 & 4,470 & 4,600 \\
\hline S97T000950 & & Lower half & 3,930 & 3,830 & $3,880^{\mathrm{eC}: \mathrm{a}}$ \\
\hline S97T000953 & \multirow[t]{2}{*}{ 191: 5} & Upper half & 3,330 & 3,300 & 3,320 \\
\hline S97T000952 & & Lower half & 3,760 & 3,700 & $3,730^{\mathrm{eC:a}}$ \\
\hline S97T001805 & Core 191 & Solid composite & 3,750 & 3,760 & 3,760 \\
\hline
\end{tabular}

Table B2-77. Tank 241-T-202 Analytical Results: Cobalt (ICP).

\begin{tabular}{|c|c|c|c|c|c|}
\hline 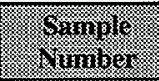 & 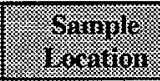 & 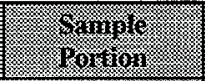 & . & Bingingante. & vian \\
\hline \multicolumn{3}{|c|}{ Solido ado digest } & (1) 18969 & 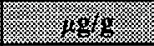 & 1.184 \\
\hline S97T000945 & \multirow[t]{2}{*}{ 191: 1} & Upper half & 9.55 & 8.07 & 8.81 \\
\hline S97T000944 & & Lower half & $<3.93$ & $<3.95$ & $<3.94^{\mathrm{QC}: \mathrm{a}}$ \\
\hline S97T000947 & \multirow[t]{2}{*}{ 191: 2} & Upper half & 8.34 & $<5.71$ & $<7.03^{\text {QC:c }}$ \\
\hline S97T000946 & & Lower half & $<4.05$ & $<4.02$ & $<4.04^{\mathrm{QC: \textrm {a }}}$ \\
\hline S97T000949 & \multirow[t]{2}{*}{ 191: 3} & Upper half & $<5.78$ & $<5.68$ & $<5.73$ \\
\hline S97T000948 & & Lower half & $<3.83$ & $<3.83$ & $<3.83^{\mathrm{QC: \textrm {a }}}$ \\
\hline S97T000951 & \multirow[t]{2}{*}{ 191: 4} & Upper half & 6.55 & 7.04 & 6.8 \\
\hline S97T000950 & & Lower half & $<4$ & $<4.06$ & $<4.03^{\mathrm{QC:a}}$ \\
\hline S97T000953 & \multirow[t]{2}{*}{ 191: 5} & Upper half & 7.26 & 9.11 & $8.18^{\mathrm{QC:O}}$ \\
\hline S97T000952 & & Lower half & $<4.05$ & $<4.07$ & $<4.06^{\mathrm{QC:A}}$ \\
\hline S97T001805 & Core 191 & Solid composite & $<4.04$ & $<4.03$ & $<4.04$ \\
\hline
\end{tabular}


Table B2-78. Tank 241-T-202 Analytical Results: Copper (ICP).

\begin{tabular}{|c|c|c|c|c|c|}
\hline Sinivils: & S Sumile & $\begin{array}{l}\text { Sampice } \\
\text { Yoman }\end{array}$ & Resing & Binincat so & Merr. \\
\hline \multicolumn{3}{|c|}{ 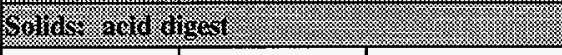 } & 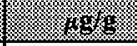 & 1394 & 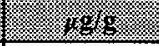 \\
\hline S97T000945 & \multirow[t]{2}{*}{ 191: 1} & Upper half & 5.22 & 5.89 & 5.55 \\
\hline S97T000944 & & Lower half & 3.37 & $<1.97$ & $<2.67^{\mathrm{QC}: \mathrm{a}, \mathrm{c}, \mathrm{c}}$ \\
\hline S97T000947 & \multirow[t]{2}{*}{ 191: 2} & Upper half & 6.23 & 7.76 & $7^{\mathrm{QC}: \mathrm{c}}$ \\
\hline S97T000946 & & Lower half & $<2.03$ & $<2.01$ & $<2.02^{\mathrm{QC}: \mathrm{a}}$ \\
\hline S97T000949 & \multirow[t]{2}{*}{ 191: 3} & Upper half & 10.8 & 9.11 & 9.96 \\
\hline S97T000948 & & Lower half & $<1.92$ & $<1.92$ & $<1.92^{\mathrm{QC:A}}$ \\
\hline S97T000951 & \multirow[t]{2}{*}{ 191: 4} & Upper half & 7.1 & 6.33 & 6.71 \\
\hline S97T000950 & & Lower half & $<2$ & $<2.03$ & $<2.01^{\mathrm{QC:a}}$ \\
\hline S97T000953 & \multirow[t]{2}{*}{ 191: 5} & Upper half & 5.06 & 4.79 & 4.92 \\
\hline S97T000952 & & Lower half & $<2.02$ & $<2.03$ & $<2.02^{\mathrm{QC}: a}$ \\
\hline S97T001805 & Core 191 & Solid composite & $<2.02$ & $<2.01$ & $<2.01$ \\
\hline
\end{tabular}

Table B2-79. Tank 241-T-202 Analytical Results: Iron (ICP).

\begin{tabular}{|c|c|c|c|c|c|}
\hline Sampil & Sarminté & 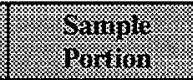 & resulit & Bripilicats & $\sqrt{1.28}$ \\
\hline \multicolumn{2}{|c|}{ 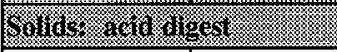 } & & rig. & $4.48 \mathrm{~g}$ & 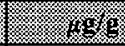 \\
\hline S97T000945 & \multirow[t]{2}{*}{ 191: 1} & Upper half & 2,090 & 2,670 & $2,380^{\mathrm{QC}: 0}$ \\
\hline S97T000944 & & Lower half & 1,970 & 1,760 & $1,870^{\mathrm{eC}: \mathrm{a}, \mathrm{c}}$ \\
\hline S97T000947 & \multirow[t]{2}{*}{ 191: 2} & Upper half & 5,950 & 10,300 & $8,130^{\mathrm{eC}: \mathrm{c}}$ \\
\hline S97T000946 & & Lower half & 4,380 & 4,560 & $4,470^{\mathrm{eC}: \mathrm{a}}$ \\
\hline S97T000949 & \multirow[t]{2}{*}{ 191: 3} & Upper half & 28,600 & 19,200 & $2,3900^{\mathrm{QC}: \mathrm{c}}$ \\
\hline S97T000948 & & Lower half & 2,790 & 3,740 & $3,270^{\mathrm{eC}: \mathrm{a}, \mathrm{e}}$ \\
\hline S97T000951 & \multirow[t]{2}{*}{ 191: 4} & Upper half & 3,910 & 2,730 & $3,320^{\mathrm{eC}: \mathrm{e}}$ \\
\hline S97T000950 & & Lower half & 2,760 & 2,600 & $2,680^{\mathrm{QC}: \mathrm{a}}$ \\
\hline S97T000953 & \multirow[t]{2}{*}{ 191: 5} & Upper half & 2,610 & 2,430 & 2,520 \\
\hline S97T000952 & & Lower half & 2,700 & 2,730 & $2,720^{\mathrm{OC}: \mathrm{a}}$ \\
\hline S97T001805 & Core 191 & Solid composite & 9,700 & 5,530 & $7,620^{\mathrm{eC}: d, \mathrm{e}}$ \\
\hline
\end{tabular}


Table B2-80. Tank 241-T-202 Analytical Results: Lanthanum (ICP).

\begin{tabular}{|c|c|c|c|c|c|}
\hline Sample: & (1) Samile & $\begin{array}{l}\text { Sample } \\
\text { enortion }\end{array}$ & rictsin: & gimplicat & Mir:2r: \\
\hline \multicolumn{3}{|c|}{ 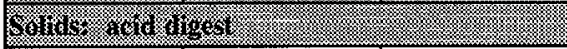 } & 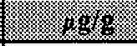 & 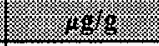 & -4.48 \\
\hline S97T000945 & \multirow[t]{2}{*}{ 191: 1} & Upper half & 8,840 & 9,060 & 8,950 \\
\hline S97T000944 & & Lower half & 9,550 & 9,330 & 9,440 \\
\hline S97T000947 & \multirow[t]{2}{*}{ 191: 2} & Upper half & 10,500 & 10,600 & 10,600 \\
\hline S97T000946 & & Lower half & 9,410 & 10,200 & 9,810 \\
\hline S97T000949 & \multirow[t]{2}{*}{ 191: 3} & Upper half & 10,700 & 10,700 & 10,700 \\
\hline S97T000948 & & Lower half & 11,300 & 11,800 & 11,600 \\
\hline S97T000951 & \multirow[t]{2}{*}{ 191: 4} & Upper half & 13,100 & 12,500 & 12,800 \\
\hline S97T000950 & & Lower half & 11,900 & 11,400 & $11,700^{\mathrm{QC:a}}$ \\
\hline S97T000953 & \multirow[t]{2}{*}{ 191: 5} & Upper half & 9,490 & 9,320 & 9,410 \\
\hline S97T000952 & & Lower half & 11,900 & 12,100 & $12,000^{\propto \mathrm{C}: a}$ \\
\hline S97T001805 & Core 191 & Solid composite & 12,500 & 12,600 & $12,600^{\mathrm{QC}: \mathrm{d}}$ \\
\hline
\end{tabular}

Table B2-81. Tank 241-T-202 Analytical Results: Lead (ICP)

\begin{tabular}{|c|c|c|c|c|c|}
\hline Shampie & 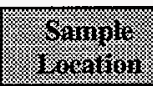 & 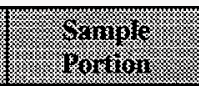 & irsing & Brivicate & (1) \\
\hline \multicolumn{3}{|c|}{ 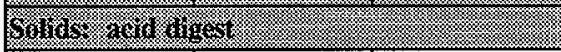 } & 18. & 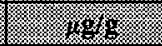 & 璱: \\
\hline S97T000945 & \multirow[t]{2}{*}{ 191: 1} & Upper half & 48 & 58.8 & $53.4^{\mathrm{QC:c}}$ \\
\hline S97T000944 & & Lower half & $<19.6$ & $<19.7$ & $<19.6^{\text {QC:a,c }}$ \\
\hline S97T000947 & \multirow[t]{2}{*}{ 191: 2} & Upper half & 198 & 201 & 200 \\
\hline S97T000946 & & Lower half & $<20.3$ & $<20.1$ & $<20.2^{\mathrm{QC:}: \mathrm{a}}$ \\
\hline S97T000949 & \multirow[t]{2}{*}{ 191: 3} & Upper half & 190 & 153 & $172^{\mathrm{eC}: e}$ \\
\hline S97T000948 & & Lower half & $<19.2$ & $<19.2$ & $<19.2^{\mathrm{QC: \textrm {a }}}$ \\
\hline S97T000951 & \multirow[t]{2}{*}{ 191: 4} & Upper half & 55.9 & 43.5 & $49.7^{\mathrm{QC:e}}$ \\
\hline S97T000950 & & Lower half & $<20$ & $<20.3$ & $<20.1^{\mathrm{OC:a}}$ \\
\hline S97T000953 & \multirow[t]{2}{*}{ 191: 5} & Upper half & 122 & 116 & 119 \\
\hline S97T000952 & & Lower half & 248 & 309 & $279^{\mathrm{CC}: \mathrm{a}, \mathrm{e}}$ \\
\hline S97T001805 & Core 191 & Solid composite & 85 & 30.9 & $58^{0 \mathrm{CO}: 0}$ \\
\hline
\end{tabular}


HNF-1501 Rev. 0

Table B2-82. Tank 241-T-202 Analytical Results: Lithium (ICP).

\begin{tabular}{|c|c|c|c|c|c|}
\hline Somples: & f Somplis & Sarnole & 3ising & linplicarc & 1. \\
\hline \multicolumn{3}{|c|}{ 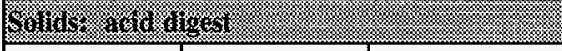 } & 1888 & 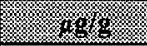 & 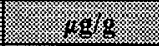 \\
\hline S97T000945 & \multirow[t]{2}{*}{ 191: 1} & Upper half & $<2.86$ & $<2.85$ & $<2.86$ \\
\hline S97T000944 & & Lower half & $<1.96$ & $<1.97$ & $<1.96$ \\
\hline S97T000947 & \multirow[t]{2}{*}{ 191: 2} & Upper half & $<2.9$ & $<2.86$ & $<2.88$ \\
\hline S97T000946 & & Lower half & $<2.03$ & $<2.01$ & $<2.02$ \\
\hline S97T000949 & \multirow[t]{2}{*}{ 191: 3} & Upper half & $<2.89$ & $<2.84$ & $<2.87$ \\
\hline S97T000948 & & Lower half & $<1.92$ & $<1.92$ & $<1.92$ \\
\hline S97T000951 & \multirow[t]{2}{*}{ 191: 4} & Upper half & $<2.9$ & $<2.88$ & $<2.89$ \\
\hline S97T000950 & & Lower half & $<2$ & $<2.03$ & $<2.01$ \\
\hline S97T000953 & \multirow[t]{2}{*}{ 191: 5} & Upper half & $<2.85$ & $<2.79$ & $<2.82$ \\
\hline S97T000952 & & Lower half & $<2.02$ & $<2.03$ & $<2.02$ \\
\hline \$97T001805 & Core 191 & Solid composite & $<2.02$ & $<2.01$ & $<2.01$ \\
\hline
\end{tabular}

Table B2-83. Tank 241-T-202 Analytical Results: Magnesium (ICP).

\begin{tabular}{|c|c|c|c|c|c|}
\hline Simple & S Sample & 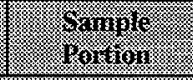 & Iesul: & 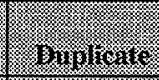 & neerin \\
\hline \multicolumn{3}{|c|}{ 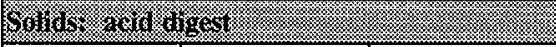 } & 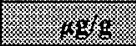 & 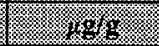 & 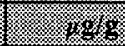 \\
\hline S97T000945 & \multirow[t]{2}{*}{ 191: 1} & Upper half & 58.6 & 81 & $69.8^{\mathrm{QC}: e}$ \\
\hline S97T000944 & & Lower half & 102 & 85.9 & $94^{\mathrm{QC}: \mathrm{a}}$ \\
\hline S97T000947 & \multirow[t]{2}{*}{ 191: 2} & Upper half & 67.8 & 146 & $107^{\mathrm{QC:c}}$ \\
\hline S97T000946 & & Lower half & 78.7 & 75.3 & $77^{\mathrm{QC}: \mathrm{a}}$ \\
\hline S97T000949 & \multirow[t]{2}{*}{ 191: 3} & Upper half & 97.6 & 126 & $112^{\mathrm{QC}: e}$ \\
\hline S97T000948 & & Lower half & 54.5 & 57.2 & $55.9^{\text {oC:a }}$ \\
\hline S97T000951 & \multirow[t]{2}{*}{ 191: 4} & Upper half & 52.5 & 46 & 49.3 \\
\hline S97T000950 & & Lower half & 66.8 & 68.1 & $67.4^{\mathrm{eC}: \mathrm{a}}$ \\
\hline S97T000953 & \multirow[t]{2}{*}{ 191: 5} & Upper half & 42.1 & 44.8 & 43.5 \\
\hline S97T000952 & & Lower half & 73.5 & 71.4 & $72.5^{\text {eC:a }}$ \\
\hline S97T001805 & Core 191 & Solid composite & 94.7 & 82.9 & 88.8 \\
\hline
\end{tabular}


Table B2-84. Tank 241-T-202 Analytical Results: Manganese (ICP).

\begin{tabular}{|c|c|c|c|c|c|}
\hline 9amiles & S Samples & simple & Skesin: & Tuplinat is & Mean: \\
\hline \multicolumn{3}{|c|}{ Soltur a did aiges } & 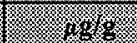 & $28 \%$ & 898 \\
\hline S97T000945 & \multirow[t]{2}{*}{ 191: 1} & Upper half & 8,420 & 8,840 & 8,630 \\
\hline S97T000944 & & Lower half & 10,100 & 9,860 & $9,980^{\mathrm{QC}: \mathrm{a}, \mathrm{d}}$ \\
\hline S97T000947 & \multirow[t]{2}{*}{ 191: 2} & Upper half & 9,720 & 9,780 & 9,750 \\
\hline S97T000946 & & Lower half & 10,400 & 11,300 & $10,900^{\mathrm{QC:a}}$ \\
\hline S97T000949 & \multirow[t]{2}{*}{ 191: 3} & Upper half & 9,980 & 10,100 & 10,000 \\
\hline S97T000948 & & Lower half & 11,500 & 12,100 & $11,800^{\mathrm{eC}: \mathrm{a}}$ \\
\hline S97T000951 & \multirow[t]{2}{*}{ 191: 4} & Upper half & 12,400 & 11,900 & 12,200 \\
\hline S97T000950 & & Lower half & 14,800 & 13,900 & $14,400^{\mathrm{QC:}: \mathrm{a}}$ \\
\hline S97T000953 & \multirow[t]{2}{*}{ 191: 5} & Upper half & 10,500 & 10,400 & 10,500 \\
\hline S97T000952 & & Lower half & 14,500 & 14,800 & $14,700^{\text {QC:a }}$ \\
\hline S97T001805 & Core 191 & Solid composite & 14,700 & 14,900 & $14,800^{\mathrm{eC}: \mathrm{d}}$ \\
\hline
\end{tabular}

Table B2-85. Tank 241-T-202 Analytical Results: Molybdenum (ICP).

\begin{tabular}{|c|c|c|c|c|c|}
\hline V Sampie & $\begin{array}{l}\text { Sinjulc } \\
\text { Hocation }\end{array}$ & Samile & Iresuin & Bilinicate & 1/ein \\
\hline \multicolumn{3}{|c|}{ 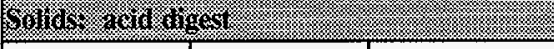 } & $8,86 \mathrm{~g}$ & 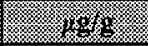 & 4 \\
\hline S97T000945 & \multirow[t]{2}{*}{ 191: 1} & Upper half & $<14.3$ & $<14.3$ & $<14.3$ \\
\hline S97T000944 & & Lower half & $<9.82$ & $<9.87$ & $<9.84^{\mathrm{QC}: \mathrm{a}}$ \\
\hline S97T000947 & \multirow[t]{2}{*}{ 191: 2} & Upper half & $<14.5$ & $<14.3$ & $<14.4$ \\
\hline S97T000946 & & Lower half & $<10.1$ & $<10$ & $<10.1^{\mathrm{CC:s}}$ \\
\hline S97T000949 & \multirow[t]{2}{*}{ 191: 3} & Upper half & $<14.5$ & $<14.2$ & $<14.3$ \\
\hline S97T000948 & & Lower half & $<9.59$ & $<9.58$ & $<9.59^{\mathrm{QC}: \mathrm{a}}$ \\
\hline S97T000951 & \multirow[t]{2}{*}{ 191: 4} & Upper half & $<14.5$ & $<14.4$ & $<14.4$ \\
\hline S97T000950 & & Lower half & $<10$ & $<10.1$ & $<10.1^{\mathrm{eC:a}}$ \\
\hline S97T000953 & \multirow[t]{2}{*}{ 191: 5} & Upper half & $<14.2$ & $<14$ & $<14.1$ \\
\hline S97T000952 & & Lower half & $<10.1$ & $<10.2$ & $<10.1^{\mathrm{QC:a}}$ \\
\hline S97T001805 & Core 191 & Solid composite & $<10.1$ & $<10.1$ & $<10.1$ \\
\hline
\end{tabular}


Table B2-86. Tank 241-T-202 Analytical Results: Neodymium (ICP).

\begin{tabular}{|c|c|c|c|c|c|}
\hline Suningle & 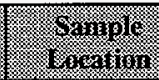 & Shmple: & riserail & . & W. \\
\hline \multicolumn{3}{|c|}{ 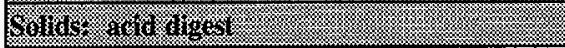 } & 18 & 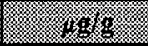 & $8.8 \%$ \\
\hline S97T000945 & \multirow[t]{2}{*}{ 191: 1} & Upper half & $<28.6$ & $<28.5$ & $<28.6$ \\
\hline \$97T000944 & & Lower half & $<19.6$ & $<19.7$ & $<19.6$ \\
\hline S97T000947 & \multirow[t]{2}{*}{ 191: 2} & Upper half & $<29$ & $<28.6$ & $<28.8$ \\
\hline S97T000946 & & Lower half & $<20.3$ & $<20.1$ & $<20.2$ \\
\hline S97T000949 & \multirow[t]{2}{*}{ 191: 3} & Upper half & $<28.9$ & $<28.4$ & $<28.6$ \\
\hline S97T000948 & & Lower half & $<19.2$ & $<19.2$ & $<19.2$ \\
\hline S97T000951 & \multirow[t]{2}{*}{ 191: 4} & Upper half & $<29$ & $<28.8$ & $<28.9$ \\
\hline S97T000950 & & Lower half & $<20$ & $<20.3$ & $<20.1^{\mathrm{QC:a}}$ \\
\hline S97T000953 & \multirow[t]{2}{*}{ 191: 5} & Upper half & $<28.5$ & $<27.9$ & $<28.2$ \\
\hline S97T000952 & & Lower half & $<20.2$ & $<20.3$ & $<20.3^{\mathrm{QC:a}}$ \\
\hline S97T001805 & Core 191 & Solid composite & $<20.2$ & $<20.1$ & $<20.1$ \\
\hline
\end{tabular}

Table B2-87. Tank 241-T-202 Analytical Results: Nickel (ICP).

\begin{tabular}{|c|c|c|c|c|c|}
\hline Siminge & 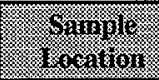 & Soming & 26rin: & Mulingite & 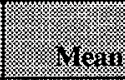 \\
\hline \multicolumn{2}{|c|}{ 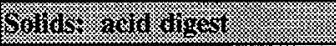 } & (3: & 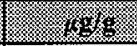 & (18, & 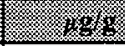 \\
\hline S97T000945 & \multirow{2}{*}{ 191: 1} & Upper half & 82.3 & 86.6 & 84.4 \\
\hline S97T000944 & & Lower half & 79.5 & 75.2 & 77.3 \\
\hline \$97T000947 & \multirow[t]{2}{*}{ 191: 2} & Upper half & 113 & 112 & 113 \\
\hline S97T000946 & & Lower half & 94.7 & 102 & 98.3 \\
\hline S97T000949 & \multirow[t]{2}{*}{ 191: 3} & Upper half & 104 & 101 & 103 \\
\hline S97T000948 & & Lower half & 92.9 & 96.4 & 94.7 \\
\hline S97T000951 & \multirow[t]{2}{*}{$191: 4$} & Upper half & 141 & 135 & 138 \\
\hline S97T000950 & & Lower half & 204 & 193 & $199^{\propto C ; a}$ \\
\hline S97T000953 & \multirow[t]{2}{*}{ 191: 5} & Upper half & 128 & 127 & 128 \\
\hline S97T000952 & & Lower half & 130 & 133 & $132^{\mathrm{\alpha C}: \mathrm{a}}$ \\
\hline S97T001805 & Core 191 & Solid composite & 131 & 133 & 132 \\
\hline
\end{tabular}


Table B2-88. Tank 241-T-202 Analytical Results: Phosphorus (ICP).

\begin{tabular}{|c|c|c|c|c|c|}
\hline $\begin{array}{l}\text { Sample: } \\
\text { Nanier: }\end{array}$ & Sample & Sampl: & Irsuril & Miplifire & $\sqrt{1}$ \\
\hline \multicolumn{3}{|c|}{ Solits ordanger } & 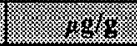 & 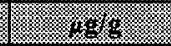 & 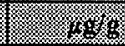 \\
\hline S97T000945 & \multirow[t]{2}{*}{ 191: 1} & Upper half & 2,370 & 2,440 & 2,410 \\
\hline S97T000944 & & Lower half & 2,330 & 2,250 & $2,290^{\mathrm{eC:a}}$ \\
\hline S97T000947 & \multirow[t]{2}{*}{ 191: 2} & Upper half & 2,690 & 2,670 & 2,680 \\
\hline S97T000946 & & Lower half & 1,910 & 2,040 & $1,980^{\mathrm{QC}: \mathrm{A}}$ \\
\hline S97T000949 & \multirow[t]{2}{*}{ 191: 3} & Upper half & 2,330 & 2,380 & 2,360 \\
\hline S97T000948 & & Lower half & 2,210 & 2,310 & $2,260^{\propto \mathrm{C}: a}$ \\
\hline S97T000951 & \multirow[t]{2}{*}{ 191: 4} & Upper half & 2,360 & 2,220 & 2,290 \\
\hline S97T000950 & & Lower half & 1,820 & 1,780 & $1,800^{\mathrm{QC}: \mathrm{a}}$ \\
\hline S97T000953 & \multirow[t]{2}{*}{ 191: 5} & Upper half & 1,660 & 1,690 & 1,680 \\
\hline S97T000952 & & Lower half & 1,900 & 1,920 & $1,910^{\mathrm{QC}: \mathrm{a}}$ \\
\hline S97T001805 & Core 191 & Solid composite & 2,370 & 2,410 & 2,390 \\
\hline
\end{tabular}

Table B2-89. Tank 241-T-202 Analytical Results: Potassium (ICP).

\begin{tabular}{|c|c|c|c|c|c|}
\hline Samilo & Samulfe & $\begin{array}{l}\text { Samile } \\
\text { Iornion }\end{array}$ & igesilin & 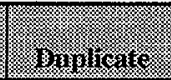 & ) \\
\hline \multicolumn{3}{|c|}{ 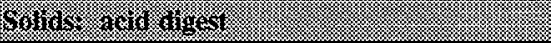 } & 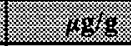 & 1.963: & 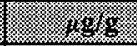 \\
\hline S97T000945 & \multirow[t]{2}{*}{ 191: 1} & Upper half & 7,390 & 7,500 & 7,450 \\
\hline S97T000944 & & Lower half & 8,150 & 7,480 & $7,820^{\mathrm{QC:c}}$ \\
\hline S97T000947 & \multirow[t]{2}{*}{ 191: 2} & Upper half & 7,110 & 7,170 & 7,140 \\
\hline S97T000946 & & Lower half & 6,970 & 7,140 & 7,060 \\
\hline S97T000949 & \multirow[t]{2}{*}{ 191: 3} & Upper half & 6,640 & 6,710 & 6,680 \\
\hline S97T000948 & & Lower half & 6,690 & 6,840 & 6,770 \\
\hline S97T000951 & \multirow[t]{2}{*}{ 191: 4} & Upper half & 6,540 & 6,190 & 6,370 \\
\hline S97T000950 & & Lower half & 6,270 & 6,300 & 6,290 QC:a \\
\hline S97T000953 & \multirow[t]{2}{*}{ 191: 5} & Upper half & 6,400 & 6,340 & 6,370 \\
\hline S97T000952 & & Lower half & 6,150 & 6,200 & $6,180^{\mathrm{eC:a}}$ \\
\hline S97T001805 & Core 191 & Solid composite & 7,120 & 7,150 & 7,140 \\
\hline
\end{tabular}


Table B2-90. Tank 241-T-202 Analytical Results: Samarium (ICP).

\begin{tabular}{|c|c|c|c|c|c|}
\hline $\begin{array}{l}\text { Sumples } \\
\text { Siminter }\end{array}$ & 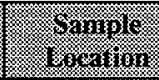 & 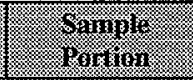 & 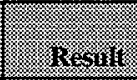 & Orplinate & $\sqrt{3} \cdot 19 \pi$ \\
\hline \multicolumn{3}{|c|}{ 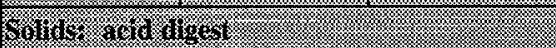 } & 4 & 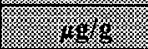 & 1.1 .89 .8 \\
\hline S97T000945 & \multirow[t]{2}{*}{ 191: 1} & Upper half & $<28.6$ & $<28.5$ & $<28.6$ \\
\hline S97T000944 & & Lower half & $<19.6$ & $<19.7$ & $<19.6^{\mathrm{QC}: \mathrm{a}}$ \\
\hline S97T000947 & \multirow[t]{2}{*}{ 191: 2} & Upper half & $<29$ & $<28.6$ & $<28.8$ \\
\hline S97T000946 & & Lower half & $<20.3$ & $<20.1$ & $<20.2^{\mathrm{QC}: a}$ \\
\hline S97T000949 & \multirow[t]{2}{*}{$191: 3$} & Upper half & $<28.9$ & $<28.4$ & $<28.6$ \\
\hline S97T000948 & & Lower half & $<19.2$ & $<19.2$ & $<19.2^{\text {eC:a }}$ \\
\hline S97T000951 & \multirow[t]{2}{*}{ 191: 4} & Upper half & $<29$ & $<28.8$ & $<28.9$ \\
\hline S97T000950 & & Lower half & $<20$ & $<20.3$ & $<20.1^{\mathrm{QC}: \mathrm{a}}$ \\
\hline S97T000953 & \multirow[t]{2}{*}{ 191: 5} & Upper half & $<28.5$ & $<27.9$ & $<28.2$ \\
\hline S97T000952 & & Lower half & $<20.2$ & $<20.3$ & $<20.3^{\text {ec:a }}$ \\
\hline S97T001805 & Core 191 & Solid composite & $<20.2$ & $<20.1$ & $<20.1$ \\
\hline
\end{tabular}

Table B2-91. Tank 241-T-202 Analytical Results: Selenium (ICP).

\begin{tabular}{|c|c|c|c|c|c|}
\hline Somingle & S Trumile & Throfle & revilit & 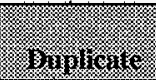 & $1 \operatorname{len}$ \\
\hline \multicolumn{2}{|c|}{ 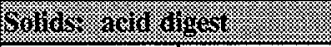 } & & $1 / 28 \%$ & 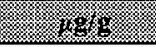 & . \\
\hline S97T000944 & 191: 1 & Lower half & $<19.6$ & $<19.7$ & $<19.6^{\text {eC:a }}$ \\
\hline S97T000946 & 191: 2 & Lower half & $<20.3$ & $<20.1$ & $<20.2^{\mathrm{OC}: \mathrm{a}}$ \\
\hline S97T000948 & 191: 3 & Lower half & $<19.2$ & $<19.2$ & $<19.2^{\mathrm{QC}: \mathrm{a}}$ \\
\hline S97T000950 & 191: 4 & Lower half & $<20$ & $<20.3$ & $<20.1^{\mathrm{QC:a}}$ \\
\hline S97T000952 & 191: 5 & Lower half & $<20.2$ & $<20.3$ & $<20.3^{\text {QC:a }}$ \\
\hline S97T001805 & Core 191 & Solid composite & $<20.2$ & $<20.1$ & $<20.1$ \\
\hline
\end{tabular}


Table B2-92. Tank 241-T-202 Analytical Results: Silicon (ICP).

\begin{tabular}{|c|c|c|c|c|c|}
\hline Yaming & 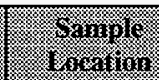 & 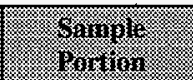 & 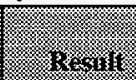 & 9.7. & 1. \\
\hline \multicolumn{3}{|c|}{ 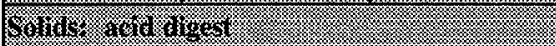 } & $\sqrt{1.968}$ & 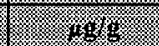 & $\sqrt{4}+9$ \\
\hline S97T000945 & \multirow[t]{2}{*}{ 191: 1} & Upper half & 1,740 & 1,950 & $1,850^{\mathrm{ec}: \mathrm{b}}$ \\
\hline S97T000944 & & Lower half & 1,680 & 1,530 & $1,610^{\mathrm{QC:b}, \mathrm{c}}$ \\
\hline S97T000947 & \multirow[t]{2}{*}{ 191: 2} & Upper half & 1,770 & 2,130 & $1,950^{\alpha \mathrm{C}: \mathrm{b}}$ \\
\hline S97T000946 & & Lower half & 1,350 & 1,200 & $1,280^{\text {QC:b }}$ \\
\hline S97T000949 & \multirow[t]{2}{*}{ 191: 3} & Upper half & 1,600 & 1,900 & $1,750^{\propto \mathrm{C:}: \mathrm{b}}$ \\
\hline S97T000948 & & Lower half & 1,330 & 1,450 & $1,390^{\text {ec:b }}$ \\
\hline S97T000951 & \multirow[t]{2}{*}{ 191: 4} & Upper half & 1,740 & 1,510 & $1,630^{\mathrm{QC}: \mathrm{b}}$ \\
\hline S97T000950 & & Lower half & 1,600 & 1,550 & $1,580^{\mathrm{eC}: \mathrm{b}}$ \\
\hline S97T000953 & \multirow[t]{2}{*}{ 191: 5} & Upper half & 1,550 & 1,740 & $1,650^{\mathrm{ec}: \mathrm{b}}$ \\
\hline S97T000952 & & Lower half & 1,530 & 1,670 & $1,600^{\mathrm{QC:b}}$ \\
\hline S97T001805 & Core 191 & Solid composite & 1,930 & 1,810 & $1,870^{\mathrm{eC}: \mathrm{b}}$ \\
\hline
\end{tabular}

Table B2-93. Tank 241-T-202 Analytical Results: Silver (ICP).

\begin{tabular}{|c|c|c|c|c|c|}
\hline Sominger & 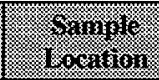 & $\begin{array}{l}\text { Samplo } \\
\text { prifion }\end{array}$ & resull & bingtionic & Merin \\
\hline \multicolumn{2}{|c|}{ Soldos avio ugest: } & : & 1.96 & 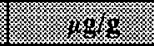 & 1.898 \\
\hline S97T000945 & \multirow[t]{2}{*}{ 191: 1} & Upper half & $<2.86$ & $<2.85$ & $<2.86$ \\
\hline S97T000944 & & Lower half & 3.03 & 2.89 & $2.96^{\mathrm{QC:A}}$ \\
\hline S97T000947 & \multirow[t]{2}{*}{ 191: 2} & Upper half & $<2.9$ & $<2.86$ & $<2.88$ \\
\hline S97T000946 & & Lower half & 2.78 & 2.96 & $2.87^{\mathrm{QC:R}}$ \\
\hline S97T000949 & \multirow[t]{2}{*}{ 191: 3} & Upper half & $<2.89$ & $<2.84$ & $<2.87$ \\
\hline S97T000948 & & Lower half & 3.19 & 3.09 & $3.14^{\mathrm{eC:a}}$ \\
\hline S97T000951 & \multirow[t]{2}{*}{ 191: 4} & Upper half & $<2.9$ & $<2.88$ & $<2.89$ \\
\hline S97T000950 & & Lower half & 2.34 & 2.24 & $2.29^{\mathrm{eC:a}}$ \\
\hline S97T000953 & \multirow[t]{2}{*}{ 191: 5} & Upper half & $<2.85$ & $<2.79$ & $<2.82$ \\
\hline \$97T000952 & & Lower half & 2.82 & 2.81 & $2.82^{\mathrm{QC:}: \mathrm{a}}$ \\
\hline S97T001805 & Core 191 & Solid composite & $<2.02$ & 2.45 & $<2.24$ \\
\hline
\end{tabular}


Table B2-94. Tank 241-T-202 Analytical Results: Sodium (ICP).

\begin{tabular}{|c|c|c|c|c|c|}
\hline 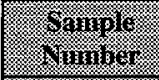 & Sominge & Sominon & iesull & 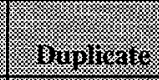 & Mean \\
\hline \multicolumn{3}{|c|}{ 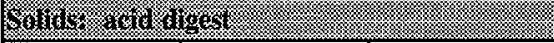 } & 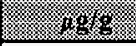 & (x) & 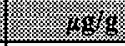 \\
\hline S97T000945 & \multirow[t]{2}{*}{ 191: 1} & Upper half & 37,100 & 37,400 & 37,300 \\
\hline S97T000944 & & Lower half & 39,600 & 36,300 & $38,000^{\mathrm{QC:c}}$ \\
\hline S97T000947 & \multirow[t]{2}{*}{ 191: 2} & Upper half & 36,400 & 36,400 & 36,400 \\
\hline S97T000946 & & Lower half & 35,100 & 36,200 & 35,700 \\
\hline S97T000949 & \multirow[t]{2}{*}{ 191: 3} & Upper half & 33,900 & 34,300 & 34,100 \\
\hline S97T000948 & & Lower half & 34,000 & 34,800 & 34,400 \\
\hline S97T000951 & \multirow[t]{2}{*}{ 191: 4} & Upper half & 34,000 & 31,900 & 33,000 \\
\hline S97T000950 & & Lower half & 32,500 & 32,400 & 32,500 \\
\hline S97T000953 & \multirow[t]{2}{*}{ 191: 5} & Upper half & 32,400 & 32,400 & 32,400 \\
\hline S97T000952 & & Lower half & 31,700 & 31,800 & 31,800 \\
\hline S97T001805 & Core 191 & Solid composite & 35,800 & 35,900 & $35,900^{\mathrm{QC:b}}$ \\
\hline
\end{tabular}

Table B2-95. Tank 241-T-202 Analytical Results: Strontium (ICP).

\begin{tabular}{|c|c|c|c|c|c|}
\hline Simule & $\begin{array}{l}\text { Sample } \\
\text { I }\end{array}$ & $\begin{array}{l}\text { Samile } \\
\text { porition }\end{array}$ & aresulu & Bumilicate & ilent \\
\hline \multicolumn{2}{|c|}{ 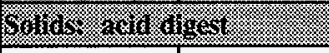 } & স্যে & $8.6 \% 8$ & 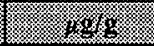 & 8.85 \\
\hline S97T000945 & \multirow[t]{2}{*}{ 191: 1} & Upper half & 649 & 664 & 657 \\
\hline S97T000944 & & Lower half & 271 & 255 & $263^{\mathrm{QC:2}}$ \\
\hline S97T000947 & \multirow[t]{2}{*}{ 191: 2} & Upper half & 424 & 421 & 423 \\
\hline S97T000946 & & Lower half & 435 & 473 & $454^{\mathrm{QC:a}}$ \\
\hline S97T000949 & \multirow[t]{2}{*}{ 191: 3} & Upper half & 475 & 487 & 481 \\
\hline S97T000948 & & Lower half & 465 & 486 & $476^{\mathrm{eC:a}}$ \\
\hline S97T000951 & \multirow[t]{2}{*}{ 191: 4} & Upper half & 579 & None & 579 \\
\hline S97T000950 & & Lower half & 459 & 503 & $481^{\text {QC:a }}$ \\
\hline S97T000953 & \multirow[t]{2}{*}{ 191: 5} & Upper half & 331 & 332 & 332 \\
\hline S97T000952 & & Lower half & 440 & 454 & $447^{\mathrm{QC:a}}$ \\
\hline S97T001805 & Core 191 & Solid composite & 493 & 501 & 497 \\
\hline
\end{tabular}


Table B2-96. Tank 241-T-202 Analytical Results: Sulfur (ICP).

\begin{tabular}{|c|c|c|c|c|c|}
\hline Sample & Soming & Saniple & Hesing & initivar: & $H^{H}=$ \\
\hline \multicolumn{2}{|c|}{ 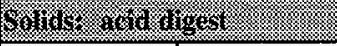 } & ) & 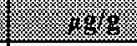 & 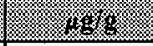 & 8.896 \\
\hline S97T000945 & \multirow[t]{2}{*}{ 191: 1} & Upper half & 288 & 297 & 293 \\
\hline S97T000944 & & Lower half & 375 & 348 & $362^{\mathrm{QC}: \mathrm{a}, \mathrm{c}}$ \\
\hline S97T000947 & \multirow[t]{2}{*}{ 191: 2} & Upper half & 257 & 257 & 257 \\
\hline S97T000946 & & Lower half & 322 & 329 & $326^{\mathrm{QC:a}}$ \\
\hline S97T000949 & \multirow[t]{2}{*}{ 191: 3} & Upper half & 231 & 241 & 236 \\
\hline S97T000948 & & Lower half & 301 & 309 & $305^{\mathrm{QC:a}}$ \\
\hline S97T000951 & \multirow[t]{2}{*}{ 191: 4} & Upper half & 135 & 130 & 133 \\
\hline S97T000950 & & Lower half & 234 & 238 & $236^{\mathrm{QC:a}}$ \\
\hline S97T000953 & \multirow[t]{2}{*}{ 191: 5} & Upper half & 135 & 138 & 137 \\
\hline S97T000952 & & Lower half & 202 & 206 & $204^{\mathrm{QC}: \mathrm{a}}$ \\
\hline S97T001805 & Core 191 & Solid composite & 299 & 304 & 302 \\
\hline
\end{tabular}

Table B2-97. Tank 241-T-202 Analytical Results: Thallium (ICP).

\begin{tabular}{|c|c|c|c|c|c|}
\hline $\begin{array}{l}\text { Sample } \\
\text { tiniver }\end{array}$ & 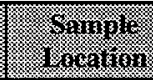 & Gample & ressill & Migingus & (8) \\
\hline \multicolumn{2}{|c|}{ SWhar wiflagest } & 4 & $8.68 \%$ & 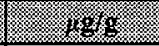 & .0 .68 \\
\hline S97T000945 & \multirow[t]{2}{*}{ 191: 1} & Upper half & 171 & 195 & 183 \\
\hline S97T000944 & & Lower half & $<39.3$ & $<39.5$ & $<39.4^{\mathrm{eC}: \mathrm{a}, \mathrm{c}}$ \\
\hline S97T000947 & \multirow[t]{2}{*}{ 191: 2} & Upper half & 201 & 197 & 199 \\
\hline S97T000946 & & Lower half & $<40.5$ & $<40.2$ & $<40.4^{\mathrm{QC:A}}$ \\
\hline S97T000949 & \multirow[t]{2}{*}{ 191: 3} & Upper half & 200 & 224 & 212 \\
\hline S97T000948 & & Lower half & $<38.3$ & $<38.3$ & $<38.3^{\text {QC:a }}$ \\
\hline S97T000951 & \multirow[t]{2}{*}{ 191: 4} & Upper half & 250 & 260 & 255 \\
\hline S97T000950 & & Lower half & $<40$ & $<40.6$ & $<40.3^{\mathrm{QC:a}}$ \\
\hline S97T000953 & \multirow[t]{2}{*}{$191: 5$} & Upper half & 213 & 212 & 213 \\
\hline S97T000952 & & Lower half & $<40.5$ & $<40.7$ & $<40.6^{\mathrm{QC:a}}$ \\
\hline S97T001805 & Core 191 & Solid composite & $<40.4$ & $<40.3$ & $<40.3$ \\
\hline
\end{tabular}


HNF-1501 Rev. 0

Table B2-98. Tank 241-T-202 Analytical Results: Titanium (ICP).

\begin{tabular}{|c|c|c|c|c|c|}
\hline $\begin{array}{l}\text { Sumple } \\
\text { Wminer }\end{array}$ & Gingule: & rompile & Resuris & Miplivale & Vilean \\
\hline \multicolumn{3}{|c|}{ 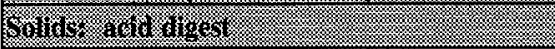 } & 1.968 & 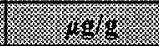 & 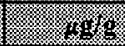 \\
\hline S97T000945 & \multirow[t]{2}{*}{ 191: 1} & Upper half & 3.03 & 3.88 & $3.46^{\mathrm{CC}: e}$ \\
\hline S97T000944 & & Lower half & 2.96 & 2.67 & $2.82^{\mathrm{QC:a,c}}$ \\
\hline S97T000947 & \multirow[t]{2}{*}{ 191: 2} & Upper half & 3.89 & 213 & $108^{\mathrm{QC:c}}$ \\
\hline S97T000946 & & Lower half & 3.4 & 3.89 & $3.65^{\mathrm{OC:a}}$ \\
\hline S97T000949 & \multirow[t]{2}{*}{ 191: 3} & Upper half & 7.25 & 4.65 & $5.95^{\mathrm{QC:e}}$ \\
\hline S97T000948 & & Lower half & 3.39 & 2.94 & $3.17^{\mathrm{QC:a}}$ \\
\hline S97T000951 & \multirow[t]{2}{*}{ 191: 4} & Upper half & 3.62 & 2.9 & $3.26^{\mathrm{QC}: \mathrm{e}}$ \\
\hline S97T000950 & & Lower half & 3.19 & 2.91 & $3.05^{\mathrm{QC:A}}$ \\
\hline S97T000953 & \multirow[t]{2}{*}{ 191: 5} & Upper half & $<2.85$ & $<2.79$ & $<2.82$ \\
\hline S97T000952 & & Lower half & 6.32 & 7.4 & $6.86^{\mathrm{QC:s}}$ \\
\hline S97T001805 & Core 191 & Solid composite & 4.86 & 4.5 & 4.68 \\
\hline
\end{tabular}

Table B2-99. Tank 241-T-202 Analytical Results: Total Uranium (ICP).

\begin{tabular}{|c|c|c|c|c|c|}
\hline Simingle & 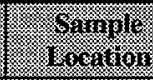 & 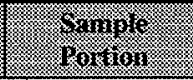 & irenili: & Bublicats & rienn \\
\hline \multicolumn{3}{|c|}{ 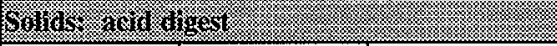 } & 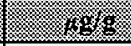 & 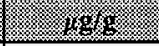 & 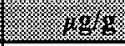 \\
\hline S97T000945 & \multirow[t]{2}{*}{ 191: 1} & Upper half & $<143$ & $<143$ & $<143$ \\
\hline S97T000944 & & Lower half & 216 & 208 & $212^{\mathrm{eC:a}}$ \\
\hline S97T000947 & \multirow[t]{2}{*}{ 191: 2} & Upper half & $<145$ & $<143$ & $<144$ \\
\hline S97T000946 & & Lower half & $<101$ & $<100$ & $<101^{\text {eC:a }}$ \\
\hline S97T000949 & \multirow[t]{2}{*}{ 191: 3} & Upper half & $<145$ & $<142$ & $<144$ \\
\hline S97T000948 & & Lower half & $<95.9$ & $<95.8$ & $<95.8^{\mathrm{QC}: \mathrm{a}}$ \\
\hline \multirow[t]{2}{*}{ S97T000951 } & \multirow[t]{2}{*}{ 191: 4} & Upper half & $<145$ & $<144$ & $<145$ \\
\hline & & Lower half & $<100$ & $<101$ & $<101^{\mathrm{QC:a}}$ \\
\hline & \multirow[t]{2}{*}{ 191: 5} & Upper half & $<142$ & $<140$ & $<141$ \\
\hline S97T000950 & & Lower half & $<101$ & $<102$ & $<102^{\mathrm{QC:a}}$ \\
\hline S97T000953 & Core 191 & Solid composite & 102 & $<101$ & $<102$ \\
\hline
\end{tabular}


Table B2-100. Tank 241-T-202 Analytical Results: Vanadium (ICP).

\begin{tabular}{|c|c|c|c|c|c|}
\hline Szmple & (smplo: & STanple & Res.11) & Hirplicas: & 118an \\
\hline \multicolumn{3}{|c|}{ 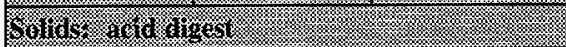 } & $\sqrt[1868]{186}$ & 1.89 .8 & (2) \\
\hline S97T000945 & \multirow[t]{2}{*}{ 191: 1} & Upper half & $<14.3$ & $<14.3$ & $<14.3$ \\
\hline S97T000944 & & Lower half & $<9.82$ & $<9.87$ & $<9.84^{\mathrm{QC}: \mathrm{a}}$ \\
\hline S97T000947 & \multirow[t]{2}{*}{ 191: 2} & Upper half & $<14.5$ & $<14.3$ & $<14.4$ \\
\hline S97T000946 & & Lower half & $<10.1$ & $<10$ & $<10.1^{\mathrm{QC}: \mathrm{a}}$ \\
\hline S97T000949 & \multirow[t]{2}{*}{$191: 3$} & Upper half & $<14.5$ & $<14.2$ & $<14.3$ \\
\hline S97T000948 & & Lower half & $<9.59$ & $<9.58$ & $<9.59^{\mathrm{QC:a}}$ \\
\hline S97T000951 & \multirow[t]{2}{*}{ 191: 4} & Upper half & $<14.5$ & $<14.4$ & $<14.4$ \\
\hline S97T000950 & & Lower half & $<10$ & $<10.1$ & $<10.1^{\mathrm{QC}: \mathrm{a}}$ \\
\hline S97T000953 & \multirow[t]{2}{*}{ 191: 5} & Upper half & $<14.2$ & $<14$ & $<14.1$ \\
\hline S97T000952 & & Lower half & $<10.1$ & $<10.2$ & $<10.1^{\mathrm{QC}: \mathrm{a}}$ \\
\hline S97T001805 & Core 191 & Solid composite & $<10.1$ & $<10.1$ & $<10.1$ \\
\hline
\end{tabular}

Table B2-101. Tank 241-T-202 Analytical Results: Zinc (ICP).

\begin{tabular}{|c|c|c|c|c|c|}
\hline Saminle & 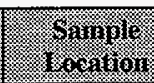 & Grunple & (restil: & 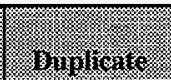 & 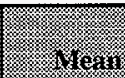 \\
\hline \multicolumn{2}{|c|}{ Sollids } & 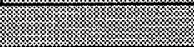 & $\frac{16}{1566}$ & $\sqrt{14968}$ & 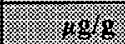 \\
\hline S97T000945 & \multirow[t]{2}{*}{ 191: 1} & Upper half & 16.2 & 18 & 17.1 \\
\hline S97T000944 & & Lower half & 14.7 & 12 & $13.3^{\mathrm{QC:a,c,e}}$ \\
\hline S97T000947 & \multirow[t]{2}{*}{ 191: 2} & Upper half & 14.9 & 16.5 & 15.7 \\
\hline S97T000946 & & Lower half & 9.51 & 11.2 & $10.4^{\mathrm{QC:a}}$ \\
\hline S97T000949 & \multirow[t]{2}{*}{ 191: 3} & Upper half & 18 & 22.4 & $20.2^{\mathrm{QC:e}}$ \\
\hline S97T000948 & & Lower half & 6.95 & 9.42 & $8.19^{\mathrm{QC:a,e}}$ \\
\hline S97T000951 & \multirow[t]{2}{*}{ 191: 4} & Upper half & 11.9 & 14.7 & $13.3^{\mathrm{QC}: e}$ \\
\hline S97T000950 & & Lower half & 12.9 & 12 & $12.4^{\mathrm{eC:a}}$ \\
\hline S97T000953 & \multirow[t]{2}{*}{ 191: 5} & Upper half & 19.2 & 18.7 & 18.9 \\
\hline S97T000952 & & Lower half & 51.8 & 55.7 & $53.8^{\mathrm{QC: \textrm {B }}}$ \\
\hline S97T001805 & Core 191 & Solid composite & 59.9 & 28.3 & $44.1^{\mathrm{QC}: e}$ \\
\hline
\end{tabular}


Table B2-102. Tank 241-T-202 Analytical Results: Zirconium (ICP).

\begin{tabular}{|c|c|c|c|c|c|}
\hline Gample & (3) & S Single: & icsulin & $\sin 11=$ & ( \\
\hline \multicolumn{2}{|c|}{ 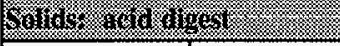 } & 4 & $18 \%$ & 48 & 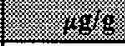 \\
\hline S97T000945 & \multirow[t]{2}{*}{ 191: 1} & Upper half & $<2.86$ & 3.3 & $<3.08$ \\
\hline S97T000944 & & Lower half & $<1.96$ & $<1.97$ & $<1.96$ \\
\hline S97T000947 & \multirow[t]{2}{*}{ 191: 2} & Upper half & $<2.9$ & 3.87 & $<3.38^{\text {QC:e }}$ \\
\hline S97T000946 & & Lower half & $<2.03$ & $<2.01$ & $<2.02$ \\
\hline S97T000949 & \multirow[t]{2}{*}{ 191: 3} & Upper half & $<2.89$ & $<2.84$ & $<2.87$ \\
\hline S97T000948 & & Lower half & $<1.92$ & $<1.92$ & $<1.92$ \\
\hline S97T000951 & \multirow[t]{2}{*}{ 191: 4} & Upper $1 / 2$ & 3.75 & None & 3.75 \\
\hline S97T000950 & & Lower half & $<2$ & $<2.03$ & $<2.01$ \\
\hline S97T000953 & \multirow[t]{2}{*}{ 191: 5} & Upper half & $<2.85$ & $<2.79$ & $<2.82$ \\
\hline \$97T000952 & & Lower half & $<2.02$ & $<2.03$ & $<2.02$ \\
\hline S97T001805 & Core 191 & Solid composite & $<2.02$ & $<2.01$ & $<2.01$ \\
\hline
\end{tabular}

Table B2-103. Tank 241-T-202 Analytical Results: Bromide (IC).

\begin{tabular}{|c|c|c|c|c|c|}
\hline Shanile & Sample & Somile & iresul & Inijilicat & yenin \\
\hline 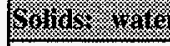 & 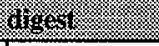 & (3) & 8.85 & ( & (1, \\
\hline S97T000955 & \multirow[t]{2}{*}{ 191: 1} & Upper half & $<518$ & $<520$ & $<519$ \\
\hline S97T000954 & & Lower half & $<277$ & $<281$ & $<279$ \\
\hline S97T000957 & \multirow[t]{2}{*}{ 191: 2} & Upper half & $<509$ & $<522$ & $<515$ \\
\hline S97T000956 & & Lower half & $<252$ & $<249$ & $<250$ \\
\hline S97T000959 & \multirow[t]{2}{*}{ 191: 3} & Upper half & $<522$ & $<534$ & $<528$ \\
\hline S97T000958 & & Lower half & $<265$ & $<268$ & $<266$ \\
\hline S97T000961 & \multirow[t]{2}{*}{ 191: 4} & Upper half & $<528$ & $<517$ & $<523$ \\
\hline S97T000960 & & Lower half & $<1,000$ & $<1,010$ & $<1,010$ \\
\hline S97T000963 & \multirow[t]{2}{*}{ 191: 5} & Upper half & $<518$ & $<523$ & $<520$ \\
\hline S97T000962 & & Lower half & $<1,030$ & $<1,040$ & $<1,040$ \\
\hline S97T001129 & Core 191 & Solid composite & $<303$ & $<303$ & $<303$ \\
\hline
\end{tabular}


Table B2-104. Tank 241-T-202 Analytical Results: Chloride (IC).

\begin{tabular}{|c|c|c|c|c|c|}
\hline Simples & Somales & somfinon: & riresuil & 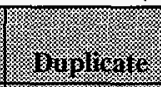 & (1.911 \\
\hline \multicolumn{2}{|c|}{ 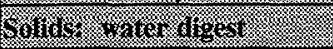 } & $\sqrt{2.8}$ & (1) & 10.46 & 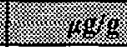 \\
\hline S97T000955 & \multirow[t]{2}{*}{ 191: 1} & Upper half & 706 & 719 & 713 \\
\hline S97T000954 & & Lower half & 658 & 602 & 630 \\
\hline S97T000957 & \multirow[t]{2}{*}{ 191: 2} & Upper half & 835 & 830 & 833 \\
\hline S97T000956 & & Lower half & 577 & 647 & 612 \\
\hline S97T000959 & \multirow[t]{2}{*}{ 191: 3} & Upper half & 756 & 779 & 768 \\
\hline S97T000958 & & Lower half & 636 & 568 & 602 \\
\hline S97T000961 & \multirow[t]{2}{*}{ 191: 4} & Upper half & 641 & 674 & 657 \\
\hline S97T000960 & & Lower half & 660 & 646 & 653 \\
\hline S97T000963 & \multirow[t]{2}{*}{ 191: 5} & Upper half & 696 & 788 & 742 \\
\hline S97T000962 & & Lower half & 840 & 570 & $705^{\mathrm{QC:}: 0}$ \\
\hline S97T001129 & Core 191 & Solid composite & 674 & 711 & 693 \\
\hline
\end{tabular}

Table B2-105. Tank 241-T-202 Analytical Results: Fluoride (IC).

\begin{tabular}{|c|c|c|c|c|c|}
\hline Surine & Sormilon & Saminic & isesing & Gublicate & Y 1 en: \\
\hline 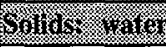 & Aiget : & 4 & 10.48 & (3. & 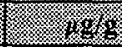 \\
\hline S97T000955 & \multirow[t]{2}{*}{ 191: 1} & Upper half & 6,780 & 6,590 & 6,690 \\
\hline S97T000954 & & Lower half & 6,240 & 5,920 & 6,080 \\
\hline S97T000957 & \multirow[t]{2}{*}{ 191: 2} & Upper half & 6,540 & 6,590 & 6,570 \\
\hline S97T000956 & & Lower half & 5,620 & 5,930 & 5,770 \\
\hline S97T000959 & \multirow[t]{2}{*}{$191: 3$} & Upper half & 6,300 & 6,540 & 6,420 \\
\hline S97T000958 & & Lower half & 5,620 & 5,580 & 5,600 \\
\hline S97T000961 & \multirow[t]{2}{*}{ 191: 4} & Upper half & 5,740 & 5,930 & 5,840 \\
\hline S97T000960 & & Lower half & 5,610 & 5,760 & 5,690 \\
\hline S97T000963 & \multirow[t]{2}{*}{ 191: 5} & Upper half & 6,010 & 5,970 & 5,990 \\
\hline S97T000962 & & Lower half & 5,720 & 5,680 & 5,700 \\
\hline S97T001129 & Core 191 & Solid composite & 6,650 & 6,470 & 6,560 \\
\hline
\end{tabular}


Table B2-106. Tank 241-T-202 Analytical Results: Nitrate (IC).

\begin{tabular}{|c|c|c|c|c|c|}
\hline Samiple: & s.minge & 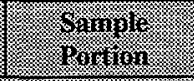 & mesili: & monilinare & 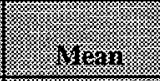 \\
\hline \multicolumn{3}{|c|}{ 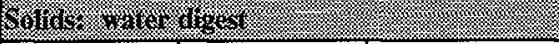 } & 48 & 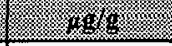 & 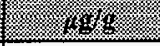 \\
\hline S97T000955 & \multirow[t]{2}{*}{ 191: 1} & Upper half & 65,000 & 64,800 & 64,900 \\
\hline S97T000954 & & Lower half & 67,600 & 64,900 & 66,200 \\
\hline S97T000957 & \multirow[t]{2}{*}{ 191: 2} & Upper half & 64,700 & 65,400 & 65,000 \\
\hline S97T000956 & & Lower half & 62,100 & 63,900 & 63,000 \\
\hline S97T000959 & \multirow[t]{2}{*}{ 191: 3} & Upper half & 61,500 & 62,000 & 61,700 \\
\hline S97T000958 & & Lower half & 63,100 & 61,500 & 62,300 \\
\hline S97T000961 & \multirow[t]{2}{*}{ 191: 4} & Upper half & 56,900 & 58,800 & 57,800 \\
\hline S97T000960 & & Lower half & 58,100 & 57,500 & 57,800 \\
\hline S97T000963 & \multirow[t]{2}{*}{ 191: 5} & Upper half & 60,600 & 60,100 & 60,400 \\
\hline S97T000962 & & Lower half & 57,000 & 57,600 & 57,300 \\
\hline S97T001129 & Core 191 & Solid composite & 66,200 & 65,000 & 65,600 \\
\hline
\end{tabular}

Table B2-107. Tank 241-T-202 Analytical Results: Nitrite (IC).

\begin{tabular}{|c|c|c|c|c|c|}
\hline $\begin{array}{l}\text { Sumples } \\
\text { Tumber }\end{array}$ & $\begin{array}{l}\text { wample } \\
\text { - ineation }\end{array}$ & 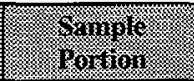 & nerin & 3irginge & (1) \\
\hline \multicolumn{3}{|c|}{ 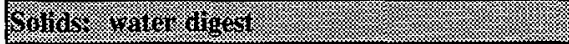 } & r & 18.86 & 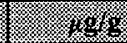 \\
\hline S97T000955 & \multirow{2}{*}{ 191: 1} & Upper half & 773 & 781 & 777 \\
\hline S97T000954 & & Lower half & 319 & 313 & 316 \\
\hline S97T000957 & \multirow[t]{2}{*}{ 191: 2} & Upper half & 758 & 772 & 765 \\
\hline S97T000956 & & Lower half & 303 & 295 & 299 \\
\hline S97T000959 & \multirow[t]{2}{*}{ 191: 3} & Upper half & 765 & 759 & 762 \\
\hline S97T000958 & & Lower half & 316 & 326 & 321 \\
\hline S97T000961 & \multirow[t]{2}{*}{ 191: 4} & Upper half & 695 & 705 & 700 \\
\hline S97T000960 & & Lower half & $<867$ & $<870$ & $<868$ \\
\hline S97T000963 & \multirow[t]{2}{*}{ 191: 5} & Upper half & 689 & 699 & 694 \\
\hline S97T000962 & & Lower half & $<890$ & $<899$ & $<895$ \\
\hline S97T001129 & Core 191 & Solid composite & 518 & 531 & 525 \\
\hline
\end{tabular}


Table B2-108. Tank 241-T-202 Analytical Results: Phosphate (IC).

\begin{tabular}{|c|c|c|c|c|c|}
\hline - Gimplo & 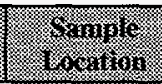 & S Simple & icrsin & 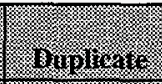 & (1) \\
\hline \multicolumn{2}{|c|}{ 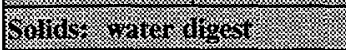 } & & 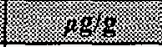 & 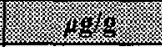 & 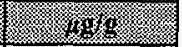 \\
\hline S97T000955 & \multirow[t]{2}{*}{ 191: 1} & Upper half & 1,910 & 1,870 & 1,890 \\
\hline S97T000954 & & Lower half & 1,920 & 1,940 & 1,930 \\
\hline S97T000957 & \multirow[t]{2}{*}{ 191: 2} & Upper half & 2,150 & 2,210 & 2,180 \\
\hline S97T000956 & & Lower half & 1,650 & 1,600 & 1,630 \\
\hline S97T000959 & \multirow[t]{2}{*}{ 191: 3} & Upper half & 1,790 & 1,790 & 1,790 \\
\hline S97T000958 & & Lower half & 1,730 & 1,710 & 1,720 \\
\hline S97T000961 & \multirow[t]{2}{*}{ 191: 4} & Upper half & 1,520 & 1,480 & 1,500 \\
\hline S97T000960 & & Lower half & 2,360 & 2,130 & 2,240 \\
\hline S97T000963 & \multirow[t]{2}{*}{ 191: 5} & Upper half & 1,590 & 1,730 & 1,660 \\
\hline S97T000962 & & Lower half & 2,200 & 2,040 & 2,120 \\
\hline S97T001129 & Core 191 & Solid composite & 1,880 & 1,810 & 1,840 \\
\hline
\end{tabular}

Table B2-109. Tank 241-T-202 Analytical Results: Sulfate (IC).

\begin{tabular}{|c|c|c|c|c|c|}
\hline $\begin{array}{l}\text { Sample } \\
\text { Sumber }\end{array}$ & 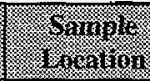 & Simple & (1) & Dublicute & (2) \\
\hline \multicolumn{3}{|c|}{ 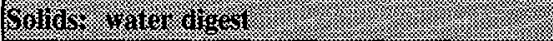 } & 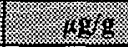 & 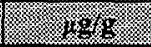 & 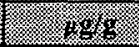 \\
\hline S97T000955 & \multirow[t]{2}{*}{ 191: 1} & Upper half & 1,560 & 1,570 & 1,570 \\
\hline S97T000954 & & Lower half & 1,230 & 1,150 & 1,190 \\
\hline S97T000957 & \multirow[t]{2}{*}{ 191: 2} & Upper half & 1,620 & 1,840 & 1,730 \\
\hline S97T000956 & & Lower half & 992 & 1,020 & 1,010 \\
\hline S97T000959 & \multirow[t]{2}{*}{ 191: 3} & Upper half & 1,680 & 1,970 & 1,830 \\
\hline S97T000958 & & Lower half & 1,120 & 1,350 & 1,230 \\
\hline S97T000961 & \multirow[t]{2}{*}{ 191: 4} & Upper half & 1,220 & 1,770 & $1,490^{\mathrm{ec}: \mathrm{e}}$ \\
\hline S97T000960 & & Lower half & $<1110$ & 1,390 & $<1250^{\mathrm{QC}: e}$ \\
\hline S97T000963 & \multirow[t]{2}{*}{ 191: 5} & Upper half & 1,500 & 1,830 & 1,670 \\
\hline S97T000962 & & Lower half & $<1140$ & 1,420 & $<1,280^{\text {QC:e }}$ \\
\hline S97T001129 & Core 191 & Solid composite & 1,150 & 1,060 & 1,110 \\
\hline
\end{tabular}


Table B2-110. Tank 241-T-202 Analytical Results: Oxalate (IC).

\begin{tabular}{|c|c|c|c|c|c|}
\hline 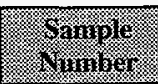 & (9) & 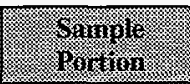 & f & Brolionic & 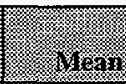 \\
\hline \multicolumn{2}{|c|}{ 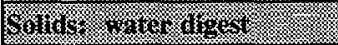 } & & $8 x$ & 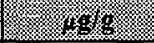 & 468 \\
\hline S97T000955 & \multirow[t]{2}{*}{ 191: 1} & Upper half & $<435$ & $<437$ & $<436$ \\
\hline S97T000954 & & Lower half & $<232$ & $<236$ & $<234$ \\
\hline S97T000957 & \multirow[t]{2}{*}{ 191: 2} & Upper half & $<427$ & $<439$ & $<433$ \\
\hline S97T000956 & & Lower half & 401 & 344 & 372 \\
\hline \$97T000959 & \multirow[t]{2}{*}{ 191: 3} & Upper half & $<438$ & $<449$ & $<444$ \\
\hline S97T000958 & & Lower half & 724 & 638 & 681 \\
\hline S97T000961 & \multirow[t]{2}{*}{ 191: 4} & Upper half & 474 & $<435$ & $<455$ \\
\hline S97T000960 & & Lower half & 1,050 & 1,810 & $1,430^{\mathrm{QC}: c}$ \\
\hline S97T000963 & \multirow[t]{2}{*}{ 191: 5} & Upper half & $<435$ & 458 & $<447$ \\
\hline S97T000962 & & Lower half & 1,530 & 1,480 & 1,510 \\
\hline S97T001129 & Core 191 & Solid composite & 475 & 490 & 482 \\
\hline
\end{tabular}

Table B2-111. Tank 241-T-202 Analytical Results: Bulk Density.

\begin{tabular}{|c|c|c|c|c|c|}
\hline 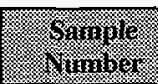 & $f_{1}$ & 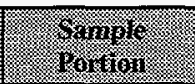 & 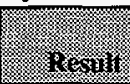 & 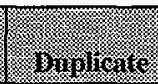 & ritien \\
\hline 13. & 17. & 事 & (1) & 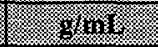 & 28, \\
\hline S97T000786 & 191: 1 & Lower half & 1.21 & N/A & 1.21 \\
\hline S97T000787 & 191: 2 & Lower half & 1.26 & N/A & 1.26 \\
\hline S97T000788 & 191: 3 & Lower half & 1.09 & N/A & 1.09 \\
\hline S97T000789 & 191: 4 & Lower half & 1.1 & N/A & 1.1 \\
\hline S97T000790 & $191: \overline{5}$ & Lower half & 1.25 & N/A & 1.25 \\
\hline \$97T001087 & Core 191 & Solid composite & 1.24 & N/A & 1.24 \\
\hline
\end{tabular}


Table B2-112. Tank 241-T-202 Analytical Results: Percent Water (DSC/TGA).

\begin{tabular}{|c|c|c|c|c|c|}
\hline Simiples & Simaple: & Simple & (1).311. & Migingate & . \\
\hline 601105 & 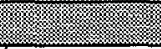 & 4 & 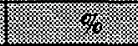 & 18 & (1) \\
\hline S97T000803 & \multirow[t]{2}{*}{ 191: 1} & Upper half & 79 & 78.6 & 78.8 \\
\hline S97T000802 & & Lower half & 77.2 & 78.3 & 77.8 \\
\hline S97T000805 & \multirow[t]{2}{*}{ 191: 2} & Upper half & 75.5 & 77.5 & 76.5 \\
\hline S97T000804 & & Lower half & 78.7 & 76.2 & 77.4 \\
\hline S97T000807 & \multirow[t]{2}{*}{$191: 3$} & Upper half & 76.5 & 68.8 & 72.7 \\
\hline S97T000806 & & Lower half & 76.3 & 73.3 & 74.8 \\
\hline S97T000809 & \multirow[t]{2}{*}{ 191: 4} & Upper half & 75.7 & 76.3 & 76 \\
\hline S97T000808 & & Lower half & 76.5 & 76.6 & 76.5 \\
\hline S97T000811 & \multirow[t]{2}{*}{$191: 5$} & Upper half & 63.7 & 79.4 & 71.5 \\
\hline S97T000810 & & Lower half & 76.6 & 75.2 & 75.9 \\
\hline S97T001673 & Core 191 & Solid composite & 73.6 & 72 & 72.8 \\
\hline
\end{tabular}

Table B2-113. Tank 241-T-202 Analytical Results: Total Alpha (Alpha).

\begin{tabular}{|c|c|c|c|c|c|}
\hline Sample & 8.ingie & 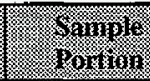 & rexsuly & Dupitrat & $\sqrt{16 \text { en }}$ \\
\hline 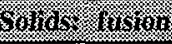 & & (4) & 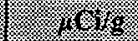 & $y=$ & 10.18 \\
\hline S97T000939 & 191: 1 & Lower half & 0.22 & 0.225 & 0.223 \\
\hline S97T000940 & 191: 2 & Lower half & 0.29 & 0.295 & 0.293 \\
\hline S97T000941 & 191: 3 & Lower half & 0.195 & 0.206 & 0.201 \\
\hline S97T000942 & 191: 4 & Lower half & 0.254 & 0.189 & 0.222 \\
\hline S97T000943 & $191: 5$ & Lower half & 0.164 & 0.185 & 0.175 \\
\hline
\end{tabular}

Table B2-114. Tank 241-T-202 Analytical Results: Americium-241 (GEA).

\begin{tabular}{|c|c|c|c|c|c|}
\hline Simpie & 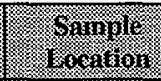 & (qanugle & Iresail: & Brablican & (redin \\
\hline 31165 . 14510 & ) & স্য & U. & 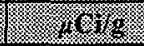 & 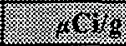 \\
\hline S97T001127 & Core 191 & Solid composite & $<0.121$ & $<0.108$ & $<0.114$ \\
\hline
\end{tabular}


Table B2-115. Tank 241-T-202 Analytical Results: Cesium-137 (GEA).

\begin{tabular}{|c|c|c|c|c|c|}
\hline (3) & Gonivio & (6) & II & 3) & (16) \\
\hline 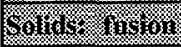 & & & 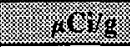 & 18. & 8. \\
\hline \$97T001127 & Core 191 & Solid composite & $<0.0206$ & $<0.0358$ & $<0.0282^{\text {QC:e }}$ \\
\hline
\end{tabular}

Table B2-116. Tank 241-T-202 Analytical Results: Cobalt-60 (GEA).

\begin{tabular}{|c|c|c|c|c|c|}
\hline Whinger & gampon & 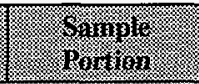 & ris $=310$ & (3) & \\
\hline 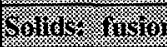 & & স স্যে: & $8 \%$ & 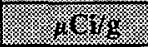 & $6 \%$ \\
\hline S97T001127 & Core 191 & Solid composite & $<0.0156$ & $<0.0178$ & $<0.0167$ \\
\hline
\end{tabular}

Table B2-117. Tank 241-T-202 Analytical Results: Europium-154 (GEA)

\begin{tabular}{|c|c|c|c|c|c|}
\hline 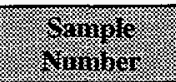 & \%oc. & 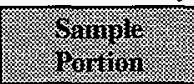 & 3esing & 3yolus & 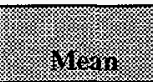 \\
\hline 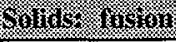 & & & (6) & (1) & 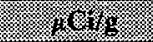 \\
\hline S97T001127 & Core 191 & Solid composite & $<0.0366$ & $<0.0461$ & $<0.0413^{\text {QC:e }}$ \\
\hline
\end{tabular}

Table B2-118. Tank 241-T-202 Analytical Results: Europium-155 (GEA).

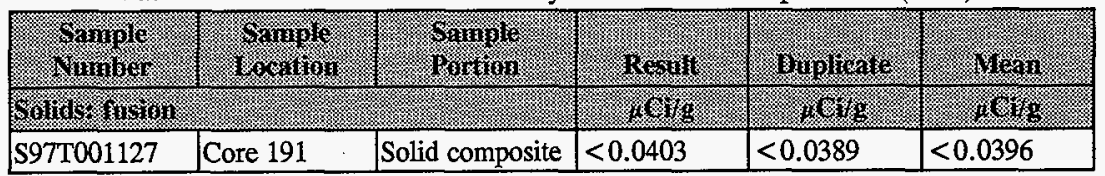

Table B2-119. Tank 241-T-202 Analytical Results: Strontium-89/90.

\begin{tabular}{|c|c|c|c|c|c|}
\hline Muninger & (4) & 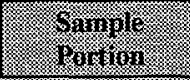 & (1) & 8170101426 & 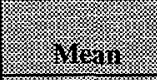 \\
\hline 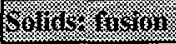 & & & (3) & (1) & 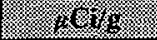 \\
\hline S97T001127 & Core 191 & Solid composite & 7.95E-04 & 0.00418 & $0.00249^{\mathrm{QC:e}}$ \\
\hline
\end{tabular}


Table B2-120. Tank 241-T-202 Analytical Results: Total Inorganic Carbon.

\begin{tabular}{|c|c|c|c|c|c|}
\hline Surnoles & Sample & S Single & 3icsint & on & (1) \\
\hline $60111 \%$ & & & 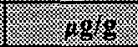 & 3.1 .18 & 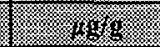 \\
\hline S97T001673 & Core 191 & Solid composite & 2,070 & 2,090 & 2,080 \\
\hline
\end{tabular}

Table B2-121. Tank 241-T-202 Analytical Results: Total Organic Carbon.

\begin{tabular}{|c|c|c|c|c|c|}
\hline Suritilger & 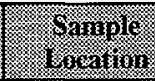 & gromple & Resi: & 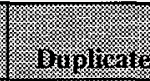 & 815an \\
\hline 601065 & & & 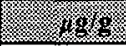 & 4.1648 & 4.486 \\
\hline \$97T001673 & Core 191 & Solid composite & 335 & 358 & 347 \\
\hline
\end{tabular}

Table B2-122. Tank 241-T-203 Analytical Results: Aluminum (ICP). (2 sheets)

\begin{tabular}{|c|c|c|c|c|c|}
\hline $\begin{array}{l}\text { Sample } \\
\text { Siminer }\end{array}$ & (S) Sarmole & 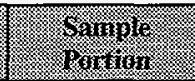 & 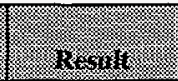 & 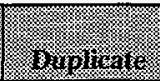 & Mean \\
\hline \multicolumn{2}{|c|}{ 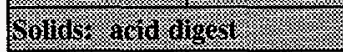 } & 4 & $=\sqrt{1.68}$ & $1.2 \mathrm{~g}$ & $1=8.896$ \\
\hline S97T001017 & Core 190 & Solid composite & 57.5 & 55 & 56.3 \\
\hline \multicolumn{2}{|c|}{ 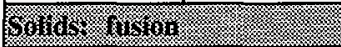 } & & $\sqrt{1}$ & 8.868 & (l) \\
\hline S97T000735 & \multirow[t]{2}{*}{ 190: 1} & Upper half & $<975$ & $<984$ & $<980$ \\
\hline S97T000759 & & Lower half & $<1,040$ & $<1,030$ & $<1,040$ \\
\hline S97T000737 & \multirow[t]{2}{*}{ 190: $1 \mathrm{R}$} & Upper half & $<1,010$ & $<1,000$ & $<1,010$ \\
\hline S97T000760 & & Lower half & 2,230 & $<929$ & $<1,580^{\mathrm{QC}: \mathrm{e}}$ \\
\hline S97T000738 & \multirow[t]{2}{*}{ 190: 2} & Upper half & $<976$ & $<999$ & $<988$ \\
\hline S97T000757 & & Lower half & $<904$ & $<881$ & $<893$ \\
\hline S97T000739 & \multirow[t]{2}{*}{ 190: 3} & Upper half & $<1,030$ & $<1,060$ & $<1,050$ \\
\hline S97T000761 & & Lower half & $<947$ & $<956$ & $<952$ \\
\hline S97T000740 & \multirow[t]{2}{*}{$190: 4$} & Upper half & $<931$ & $<945$ & $<938$ \\
\hline S97T000762 & & Lower half & $<989$ & $<981$ & $<985$ \\
\hline S97T000741 & \multirow[t]{2}{*}{$190: 5$} & Upper half & $<1,020$ & $<1,010$ & $<1,020$ \\
\hline S97T000763 & & Lower half & $<995$ & $<1000$ & $<998$ \\
\hline
\end{tabular}


Table B2-122. Tank 241-T-203 Analytical Results: Aluminum (ICP). (2 sheets)

\begin{tabular}{|c|c|c|c|c|c|}
\hline 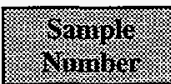 & \% & 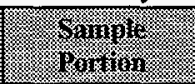 & 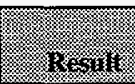 & mavingac & 庵: \\
\hline 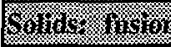 & & (3. & 8 & (3) & $8.18 \mathrm{~g}$ \\
\hline S97T000742 & \multirow[t]{2}{*}{ 190: 6} & Upper half & $<1,020$ & $<1,030$ & $<1,030$ \\
\hline S97T000764 & & Lower half & $<1,010$ & $<1,010$ & $<1,010$ \\
\hline \$97T000743 & \multirow[t]{2}{*}{ 190: 7} & Upper half & $<1,020$ & $<1,020$ & $<1,020$ \\
\hline \$97T000765 & & Lower half & $<1,030$ & $<1,010$ & $<1,020$ \\
\hline \$97T000744 & \multirow[t]{2}{*}{ 190: 8} & Upper half & $<1,030$ & $<1,010$ & $<1,020$ \\
\hline S97T000766 & & Lower half & $<1,030$ & $<1,010$ & $<1,020$ \\
\hline \$97T000745 & \multirow[t]{2}{*}{ 190: 9} & Upper half & $<1,020$ & $<1,020$ & $<1,020$ \\
\hline S97T000767 & & Lower half & $<1,010$ & $<1,010$ & $<1,010$. \\
\hline S97T001734 & Core 190 & Solid composite & $<978$ & $<974$ & $<976$ \\
\hline 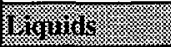 & & & 836 & 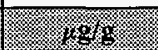 & 6 \\
\hline S97T000733 & 190: 1R & Drainable liquid & $<3.63$ & $<3.63$ & $<3.63$ \\
\hline
\end{tabular}

Table B2-123. Tank 241-T-203 Analytical Results: Antimony (ICP). (2 sheets)

\begin{tabular}{|c|c|c|c|c|c|}
\hline 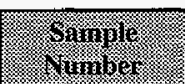 & 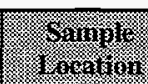 & 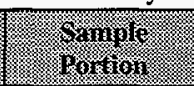 & hesinis & 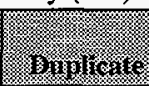 & r. \\
\hline Mink & $g_{6}$ & & (6) & (1) & s. \\
\hline S97T001017 & Core 190 & Solid composite & $<12$ & $<12$ & $<12$ \\
\hline 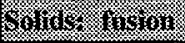 & & & 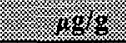 & 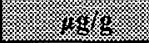 & 8,858 \\
\hline \$97T000735 & \multirow[t]{2}{*}{ 190: 1} & Upper half & $<1,170$ & $<1,180$ & $<1,180$ \\
\hline S97T000759 & & Lower half & $<1,250$ & $<1,240$ & $<1,250$ \\
\hline S97T000737 & \multirow[t]{2}{*}{ 190: 1R } & Upper half & $<1,210$ & $<1,200$ & $<1,210$ \\
\hline S97T000760 & & Lower half & $<1,180$ & $<1,110$ & $<1,150$ \\
\hline S97T000738 & \multirow[t]{2}{*}{ 190: 2} & Upper half & $<1,170$ & $<1,200$ & $<1,190$ \\
\hline S97T000757 & & Lower half & $<1,080$ & $<1,060$ & $<1,070$ \\
\hline \$97T000739 & \multirow[t]{2}{*}{ 190: 3} & Upper half & $<1,240$ & $<1,270$ & $<1,260$ \\
\hline S97T000761 & & Lower half & $<1,140$ & $<1,150$ & $<1,150$ \\
\hline
\end{tabular}


Table B2-123. Tank 241-T-203 Analytical Results: Antimony (ICP). (2 sheets)

\begin{tabular}{|c|c|c|c|c|c|}
\hline $\begin{array}{l}\text { Samile } \\
\text { Tminary }\end{array}$ & . & sample & (1) & Minolizan: & 19.2n \\
\hline 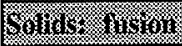 & & ( & 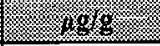 & 3.4968 & 1698 \\
\hline S97T000740 & \multirow[t]{2}{*}{ 190: 4} & Upper half & $<1,120$ & $<1,130$ & $<1,130$ \\
\hline S97T000762 & & Lower half & $<1,190$ & $<1,180$ & $<1,190$ \\
\hline S97T000741 & \multirow[t]{2}{*}{ 190: 5} & Upper half & $<1,230$ & $<1,220$ & $<1,230$ \\
\hline S97T000763 & & Lower half & $<1,190$ & $<1,200$ & $<1,200$ \\
\hline S97T000742 & \multirow[t]{2}{*}{ 190: 6} & Upper half & $<1,220$ & $<1,240$ & $<1,230$ \\
\hline S97T000764 & & Lower half & $<1,210$ & $<1,210$ & $<1,210$ \\
\hline S97T000743 & \multirow[t]{2}{*}{ 190: 7} & Upper half & $<1,230$ & $<1,220$ & $<1,230$ \\
\hline S97T000765 & & Lower half & $<1,230$ & $<1,210$ & $<1,220$ \\
\hline S97T000744 & \multirow[t]{2}{*}{ 190: 8} & Upper half & $<1,240$ & $<1,210$ & $<1,230$ \\
\hline S97T000766 & & Lower half & $<1,230$ & $<1,210$ & $<1,220$ \\
\hline S97T000745 & \multirow[t]{2}{*}{ 190: 9} & Upper half & $<1,230$ & $<1,220$ & $<1,230$ \\
\hline S97T000767 & & Lower half & $<1,210$ & $<1,220$ & $<1,220$ \\
\hline S97T001734 & Core 190 & Solid composite & $<1,170$ & $<1,170$ & $<1,170$ \\
\hline Manite: & & $\sqrt{2}$ & $108 \mathrm{~g}$ & 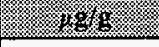 & 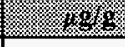 \\
\hline S97T000733 & 190: 1R & Drainable liquid & $<4.36$ & $<4.36$ & $<4.36$ \\
\hline
\end{tabular}


HNF-1501 Rev. 0

Table B2-124. Tank 241-T-203 Analytical Results: Arsenic (ICP).

\begin{tabular}{|c|c|c|c|c|c|}
\hline Sormplo & 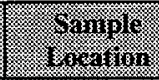 & Somplc: & Resin: & 3 applinare & (1) 14 \\
\hline \multicolumn{2}{|c|}{ 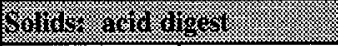 } & & 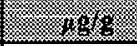 & 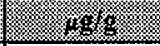 & (19.8. \\
\hline S97T001017 & Core 190 & Solid composite & $<20$ & $<20$ & $<20$ \\
\hline \multicolumn{2}{|c|}{ 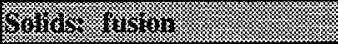 } & & 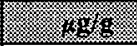 & 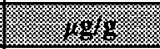 & 4.85 \\
\hline S97T000735 & \multirow[t]{2}{*}{ 190: 1} & Upper half & $<1,950$ & $<1,970$ & $<1,960$ \\
\hline S97T000759 & & Lower half & $<2,080$ & $<2,060$ & $<2,070$ \\
\hline S97T000737 & \multirow[t]{2}{*}{ 190: 1R } & Upper half & $<2,010$ & $<2,000$ & $<2,010$ \\
\hline S97T000760 & & Lower half & $<1,970$ & $<1,860$ & $<1,920$ \\
\hline S97T000738 & \multirow[t]{2}{*}{ 190: 2} & Upper half & $<1,950$ & $<2,000$ & $<1,980$ \\
\hline S97T000757 & & Lower half & $<1,810$ & $<1,760$ & $<1,790$ \\
\hline S97T000739 & \multirow[t]{2}{*}{ 190: 3} & Upper half & $<2,070$ & $<2,110$ & $<2,090$ \\
\hline S97T000761 & & Lower half & $<1,890$ & $<1,910$ & $<1,900$ \\
\hline S97T000740 & \multirow[t]{2}{*}{ 190: 4} & Upper half & $<1,860$ & $<1,890$ & $<1,880$ \\
\hline S97T000762 & & Lower half & $<1,980$ & $<1,960$ & $<1,, 970$ \\
\hline S97T000741 & \multirow[t]{2}{*}{ 190: 5} & Upper half & $<2,050$ & $<2,030$ & $<2,040$ \\
\hline S97T000763 & & Lower half & $<1,990$ & $<2,000$ & $<2,000$ \\
\hline S97T000742 & \multirow[t]{2}{*}{ 190: 6} & Upper half & $<2,040$ & $<2,060$ & $<2,050$ \\
\hline S97T000764 & & Lower half & $<2,010$ & $<2,020$ & $<2,020$ \\
\hline S97T000743 & \multirow[t]{2}{*}{ 190: 7} & Upper half & $<2,050$ & $<2,040$ & $<2,050$ \\
\hline S97T000765 & & Lower half & $<2,050$ & $<2,020$ & $<2,040$ \\
\hline S97T000744 & \multirow[t]{2}{*}{ 190: 8} & Upper half & $<2,070$ & $<2,020$ & $<2,050$ \\
\hline S97T000766 & & Lower half & $<2,060$ & $<2,020$ & $<2,040$ \\
\hline S97T000745 & \multirow[t]{2}{*}{ 190: 9} & Upper half & $<2,050$ & $<2,030$ & $<2,040$ \\
\hline S97T000767 & & Lower half & $<2,010$ & $<2,030$ & $<2,020$ \\
\hline S97T001734 & Core 190 & Solid composite & $<1,960$ & $<1,950$ & $<1,960$ \\
\hline Mritas & & & (1) & 1. 1,198 & 8,89 \\
\hline S97T000733 & 190: 1R & Drainable liquid & $<7.27$ & $<7.27$ & $<7.27$ \\
\hline
\end{tabular}




\begin{tabular}{|c|c|c|c|c|c|}
\hline$\varepsilon 9^{\circ} \varepsilon>$ & $\varepsilon 9^{\circ} \varepsilon>$ & $\mathcal{E} 9^{\circ} \mathcal{E}>$ & p!̣nb!l әqеu!eId & \&I :06I & EEL000LL6S \\
\hline (4) & s. & \% & & & (5) \\
\hline $9 \angle 6>$ & $\nabla \angle 6>$ & $8 L 6>$ & eqtsoduros pyos & 061 כ10ว & $t E L I 00 \mathrm{~L} L 6 \mathrm{~S}$ \\
\hline $0 \mathrm{LO} 0^{6} \mathrm{I}>$ & $010^{6} \mathrm{I}>$ & $010^{6} \mathrm{I}>$ & I[Eप دәMOT] & \multirow[b]{2}{*}{$6: 06 \mathrm{I}$} & $\angle 9 L 000 L \angle 6 \mathrm{~S}$ \\
\hline $020^{6} \mathrm{I}>$ & $020^{6} I>$ & $020^{6} I>$ & I[एप Iədd & & $S \nabla \angle 000 \mathrm{~L} L 6 \mathrm{~S}$ \\
\hline $020^{6} I>$ & $010^{6} \mathrm{I}>$ & $0 E 0^{6} I>$ & I[एบ ІәмоТ] & \multirow[b]{2}{*}{$8: 06 \mathrm{I}$} & 99L000LL6S \\
\hline $020^{6} I>$ & $0 \mathrm{I0} 0^{\circ} \mathrm{I}>$ & $0 \varepsilon 0^{6} \mathrm{I}>$ & I[एप Iәdd & & $\nabla t \angle 000 \mathrm{~L} L 6 \mathrm{~S}$ \\
\hline $020^{6} \mathrm{I}>$ & $0 \mathrm{LO} \mathrm{I}^{6} \mathrm{I}>$ & $0 \mathcal{E} 0^{6} I>$ & I[एप IәMOT] & \multirow[b]{2}{*}{$L: 06 \mathrm{I}$} & \$9L000LL6S \\
\hline $020^{6} I>$ & $020^{\circ} \mathrm{I}>$ & $020^{6} \mathrm{I}>$ & JॉЕप Iadd $\Omega$ & & $\varepsilon \rightarrow \angle 000 \mathrm{LL6S}$ \\
\hline $010^{6} \mathrm{I}>$ & $0 \mathrm{IO}^{6} \mathrm{I}>$ & $010^{6} \mathrm{I}>$ & I[Eप IәMOT] & \multirow[b]{2}{*}{$9: 06 \mathrm{~T}$} & $79 \angle 000 L \angle 6 S$ \\
\hline $0 \varepsilon 0^{6} I>$ & $0 E 0^{\circ} \mathrm{I}>$ & $020^{6} \mathrm{I}>$ & IГएप Iədd & & $z+\angle 000 \mathrm{~L} \angle 6 \mathrm{~S}$ \\
\hline $866>$ & $000^{6} \mathrm{I}>$ & $\$ 66>$ & Fाயप IәMOT & \multirow[b]{2}{*}{$\mathrm{S}: 06 \mathrm{I}$} & E9L000UL6S \\
\hline $020^{6} \mathrm{I}>$ & $010^{6} \mathrm{I}>$ & $020^{6} \mathrm{I}>$ & J[eप Iədd & & $I \nabla \angle 000 \mathrm{~L} L 6 \mathrm{~S}$ \\
\hline$\$ 86>$ & I86> & $686>$ & IIEU IəMOT & \multirow[b]{2}{*}{$\vdash: 06 \mathrm{I}$} & $29 \angle 000 \mathrm{~L} L 6 \mathrm{~S}$ \\
\hline $8 \varepsilon 6>$ & $S+6>$ & IE6> & Iाएप Iədd $\Omega$ & & $0+\angle 000 J \angle 6 \mathrm{~S}$ \\
\hline$z S 6>$ & $9 \$ 6>$ & $\angle t 6>$ & F[EY IOMOT & \multirow[b]{2}{*}{$\mathcal{E}: 06 \mathrm{~T}$} & I9L000LL6S \\
\hline $090^{6} I>$ & $090^{6} \mathrm{I}>$ & $0 E 0^{6} \mathrm{I}>$ & Ireप Iədd & & 6EL000LL6S \\
\hline$\varepsilon 68>$ & I88> & t06> & I[EY IәMOT] & \multirow[b]{2}{*}{$\tau: 06 \mathrm{I}$} & LSL000LL6S \\
\hline $886>$ & $666>$ & $9 \angle 6>$ & I[एप Iədd & & 8EL000.LL6S \\
\hline $9 S 6>$ & $626>$ & $\varepsilon 86>$ & ІІеप ІәмоТ & \multirow[b]{2}{*}{ \&I :06I } & 09L000LL6S \\
\hline $010^{6} I>$ & $000^{6} \mathrm{I}>$ & $010^{6} \mathrm{I}>$ & J[Еप $\lrcorner ә \mathrm{dd}_{\Omega}$ & & LELOOOLL6S \\
\hline $0+0^{6} I>$ & $0 \varepsilon 0^{6} I>$ & $070^{6} \mathrm{I}>$ & IlEप ІәмOT & \multirow[b]{2}{*}{ I :06I } & 6SLO00LL6S \\
\hline $086>$ & $786>$ & SL6> & IIEY Iədd $\Omega$ & & SELO00IL6S \\
\hline 6) & \% & 桨, & 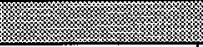 & \multirow{3}{*}{\multicolumn{2}{|c|}{ 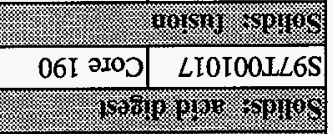 }} \\
\hline $8^{\circ} \nabla L$ & $\varepsilon 9 L$ & $\tau \cdot \varepsilon L$ & 21!̣soduros p!nOS & & \\
\hline (1) & (6) & \% & & & \\
\hline 48 & arexishag & 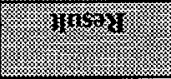 & 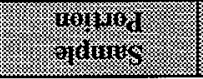 & $\operatorname{mog}$ & 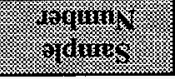 \\
\hline
\end{tabular}

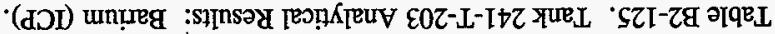


Table B2-126. Tank 241-T-203 Analytical Results: Beryllium (ICP).

\begin{tabular}{|c|c|c|c|c|c|}
\hline Samples: & Iaringle & H: Sumple & Resul & Dinglimate: & 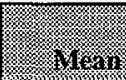 \\
\hline \multicolumn{3}{|c|}{ 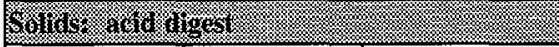 } & 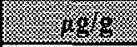 & 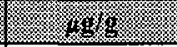 & .468 \\
\hline S97T001017 & Core 190 & Solid composite & $<0.998$ & $<0.998$ & $<0.998$ \\
\hline \multicolumn{2}{|c|}{ 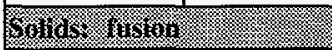 } & 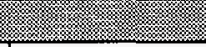 & 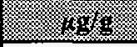 & 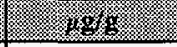 & 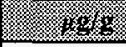 \\
\hline S97T000735 & \multirow[t]{2}{*}{ 190: 1} & Upper half & $<97.5$ & $<98.4$ & $<98$ \\
\hline S97T000759 & & Lower half & $<104$ & $<103$ & $<104$ \\
\hline S97T000737 & \multirow[t]{2}{*}{ 190: 1R } & Upper half & $<101$ & $<100$ & $<101$ \\
\hline S97T000760 & & Lower half & $<98.3$ & $<92.9$ & $<95.6$ \\
\hline S97T000738 & \multirow[t]{2}{*}{ 190: 2} & Upper half & $<97.6$ & $<99.9$ & $<98.8$ \\
\hline S97T000757 & & Lower half & $<90.4$ & $<88.1$ & $<89.3$ \\
\hline S97T000739 & \multirow[t]{2}{*}{$190: 3$} & Upper half & $<103$ & $<106$ & $<105$ \\
\hline S97T000761 & & Lower half & $<94.7$ & $<95.6$ & $<95.2$ \\
\hline S97T000740 & \multirow[t]{2}{*}{ 190: 4} & Upper half & $<93.1$ & $<94.5$ & $<93.8$ \\
\hline S97T000762 & & Lower half & $<98.9$ & $<98.1$ & $<98.5$ \\
\hline S97T000741 & \multirow[t]{2}{*}{ 190: 5} & Upper half & $<102$ & $<101$ & $<102$ \\
\hline S97T000763 & & Lower half & $<99.5$ & $<100$ & $<99.8$ \\
\hline S97T000742 & \multirow[t]{2}{*}{ 190: 6} & Upper half & $<102$ & $<103$ & $<103$ \\
\hline S97T000764 & & Lower half & $<101$ & $<101$ & $<101$ \\
\hline S97T000743 & \multirow[t]{2}{*}{ 190: 7} & Upper half & $<102$ & $<102$ & $<102$ \\
\hline \$97T000765 & & Lower half & $<103$ & $<101$ & $<102$ \\
\hline S97T000744 & \multirow[t]{2}{*}{ 190: 8} & Upper half & $<103$ & $<101$ & $<102$ \\
\hline S97T000766 & & Lower half & $<103$ & $<101$ & $<102$ \\
\hline S97T000745 & \multirow[t]{2}{*}{ 190: 9} & Upper half & $<102$ & $<102$ & $<102$ \\
\hline S97T000767 & & Lower half & $<101$ & $<101$ & $<101$ \\
\hline S97T001734 & Core 190 & Solid composite & $<97.8$ & $<97.4$ & $<97.6$ \\
\hline Lagnas & (1) & 4 & $2,8.88$ & 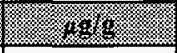 & 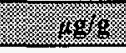 \\
\hline S97T000733 & 190: 1R & Drainable liquid & $<0.363$ & $<0.363$ & $<0.363$ \\
\hline
\end{tabular}


Table B2-127. Tank 241-T-203 Analytical Results: Bismuth (ICP).

\begin{tabular}{|c|c|c|c|c|c|}
\hline Samiplis: & S & A Sample & 3.8 & Giplinare & 145an \\
\hline \multicolumn{2}{|c|}{ 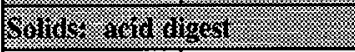 } & $\sqrt{18}$ & 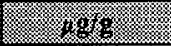 & 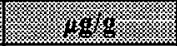 & 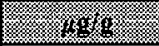 \\
\hline S97T001017 & Core 190 & Solid composite & 49,500 & 47,800 & $48,700^{\mathrm{Q}, \mathrm{Ccc}}$ \\
\hline \multicolumn{2}{|c|}{ 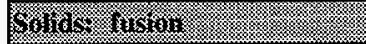 } & 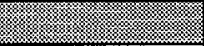 & . 186 & 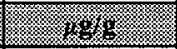 & (18) \\
\hline S97T000735 & \multirow[t]{2}{*}{ 190: 1} & Upper half & 29,000 & 27,100 & 28,100 \\
\hline S97T000759 & & Lower half & 36,300 & 34,100 & 35,200 \\
\hline S97T000737 & \multirow[t]{2}{*}{ 190: 1R } & Upper half & 25,000 & 26,000 & 25,500 \\
\hline S97T000760 & & Lower half & 29,300 & 31,100 & 30,200 \\
\hline S97T000738 & \multirow[t]{2}{*}{ 190: 2} & Upper half & 25,500 & 29,000 & 27,300 \\
\hline S97T000757 & & Lower half & 37,200 & 35,900 & 36,600 \\
\hline S97T000739 & \multirow[t]{2}{*}{ 190: 3} & Upper half & 36,200 & 33,900 & 35,100 \\
\hline S97T000761 & & Lower half & 46,000 & 41,400 & 43,700 \\
\hline S97T000740 & \multirow[t]{2}{*}{ 190: 4} & Upper half & 41,100 & 37,800 & 39,500 \\
\hline S97T000762 & & Lower half & 41,100 & 33,400 & $37,300^{\mathrm{QC:}: 0}$ \\
\hline S97T000741 & \multirow[t]{2}{*}{ 190: 5} & Upper half & 34,900 & 38,100 & 36,500 \\
\hline S97T000763 & & Lower half & 38,200 & 41,100 & 39,700 \\
\hline S97T000742 & \multirow[t]{2}{*}{ 190: 6} & Upper half & 41,200 & 39,800 & 40,500 \\
\hline S97T000764 & & Lower half & 37,400 & 35,800 & 36,600 \\
\hline S97T000743 & \multirow[t]{2}{*}{ 190: 7} & Upper half & 53,900 & 52,500 & 53,200 \\
\hline S97T000765 & & Lower half & 53,000 & 44,000 & 48,500 \\
\hline S97T000744 & \multirow[t]{2}{*}{$190: 8$} & Upper half & 54,200 & 59,000 & 56,600 \\
\hline S97T000766 & & Lower half & 53,000 & 63,100 & 58,100 \\
\hline S97T000745 & \multirow[t]{2}{*}{ 190: 9} & Upper half & 31,900 & 28,600 & 30,300 \\
\hline S97T000767 & & Lower half & 34,700 & 30,900 & 32,800 \\
\hline S97T001734 & Core 190 & Solid composite & 60,800 & 62,300 & 61,600 \\
\hline \multicolumn{2}{|c|}{ - Mquilit } & & 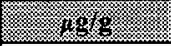 & (18. & 4898 \\
\hline S97T000733 & 190: 1R & Drainable liquid & $<7.27$ & $<7.27$ & $<7.27$ \\
\hline
\end{tabular}


Table B2-128. Tank 241-T-203 Analytical Results: Boron (ICP).

\begin{tabular}{|c|c|c|c|c|c|}
\hline Siminer & 9.80 .210 & 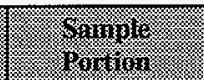 & $\operatorname{lng} 3(1)$ & ongivivatc: & . \\
\hline \multicolumn{2}{|c|}{ Soldes arid agrst: } & & (i) & 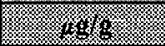 & 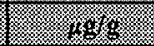 \\
\hline S97T001017 & Core 190 & Solid composite & 75.2 & 111 & $93.1^{\text {ec:e }}$ \\
\hline \multicolumn{2}{|c|}{ 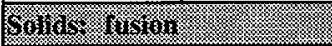 } & 4 & 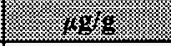 & 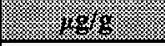 & 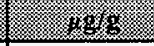 \\
\hline S97T000735 & \multirow[t]{2}{*}{ 190: 1} & Upper half & $<975$ & $<984$ & $<980$ \\
\hline S97T000759 & & Lower half & $<1,040$ & $<1,030$ & $<1,040$ \\
\hline S97T000737 & \multirow[t]{2}{*}{ 190: $1 R$} & Upper half & $<1,010$ & $<1,000$ & $<1,010$ \\
\hline S97T000760 & & Lower half & $<983$ & $<929$ & $<956$ \\
\hline S97T000738 & \multirow[t]{2}{*}{ 190: 2} & Upper half & $<976$ & $<999$ & $<988$ \\
\hline S97T000757 & & Lower half & $<904$ & $<881$ & $<893$ \\
\hline S97T000739 & \multirow[t]{2}{*}{ 190: 3} & Upper half & $<1,030$ & $<1,060$ & $<1,050$ \\
\hline S97T000761 & & Lower half & $<947$ & $<956$ & $<952$ \\
\hline S97T000740 & \multirow[t]{2}{*}{ 190: 4} & Upper half & $<931$ & $<945$ & $<938$ \\
\hline S97T000762 & & Lower half & $<989$ & $<981$ & $<985$ \\
\hline S97T000741 & \multirow[t]{2}{*}{ 190: 5} & Upper half & $<1,020$ & $<1,010$ & $<1,020$ \\
\hline S97T000763 & & Lower half & $<995$ & $<1,000$ & $<998$ \\
\hline S97T000742 & \multirow[t]{2}{*}{$190: 6$} & Upper half & $<1,020$ & $<1,030$ & $<1,030$ \\
\hline S97T000764 & & Lower half & $<1,010$ & $<1,010$ & $<1,010$ \\
\hline S97T000743 & \multirow[t]{2}{*}{ 190: 7} & Upper half & $<1,020$ & $<1,020$ & $<1,020$ \\
\hline S97T000765 & & Lower half & $<1,030$ & $<1,010$ & $<1,020$ \\
\hline S97T000744 & \multirow[t]{2}{*}{ 190: 8} & Upper half & $<1,030$ & $<1,010$ & $<1,020$ \\
\hline S97T000766 & & Lower half & $<1,030$ & $<1,010$ & $<1,020$ \\
\hline S97T000745 & \multirow[t]{2}{*}{ 190: 9} & Upper half & $<1,020$ & $<1,020$ & $<1,020$ \\
\hline S97T000767 & & Lower half & $<1,010$ & $<1,010$ & $<1,010$ \\
\hline S97T001734 & Core 190 & Solid composite & $<978$ & $<974$ & $<976$ \\
\hline Pinivin & & (3) & . & 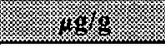 & . \\
\hline S97T000733 & 190: 1R & Drainable liquid & $<3.63$ & $<3.63$ & $<3.63$ \\
\hline
\end{tabular}


HNF-1501 Rev. 0

Table B2-129. Tank 241-T-203 Analytical Results: Cadmium (ICP).

\begin{tabular}{|c|c|c|c|c|c|}
\hline Sumilicer & Saringle & Simples & Resuis: & Biniliente & - \\
\hline \multicolumn{3}{|c|}{ 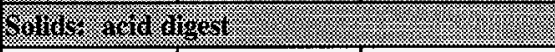 } & 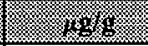 & 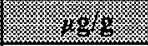 & 1.98 \\
\hline S97T001017 & Core 190 & Solid composite & $<0.998$ & $<0.998$ & $<0.998$ \\
\hline \multicolumn{2}{|l|}{ 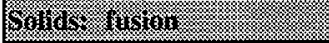 } & 18 & (1) & (4) & 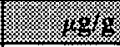 \\
\hline S97T000735 & \multirow[t]{2}{*}{ 190: 1} & Upper half & $<97.5$ & $<98.4$ & $<98$ \\
\hline S97T000759 & & Lower half & $<104$ & $<103$ & $<104$ \\
\hline S97T000737 & \multirow[t]{2}{*}{ 190: IR } & Upper half & $<101$ & $<100$ & $<101$ \\
\hline S97T000760 & & Lower half & $<98.3$ & $<92.9$ & $<95.6$ \\
\hline \$97T000738 & \multirow[t]{2}{*}{ 190: 2} & Upper half & $<97.6$ & $<99.9$ & $<98.8$ \\
\hline S97T000757 & & Lower half & $<90.4$ & $<88.1$ & $<89.3$ \\
\hline S97T000739 & \multirow[t]{2}{*}{ 190: 3} & Upper half & $<103$ & $<106$ & $<105$ \\
\hline S97T000761 & & Lower half & $<94.7$ & $<95.6$ & $<95.2$ \\
\hline S97T000740 & \multirow[t]{2}{*}{ 190: 4} & Upper half & $<93.1$ & $<94.5$ & $<93.8$ \\
\hline S97T000762 & & Lower half & $<98.9$ & $<98.1$ & $<98.5$ \\
\hline S97T000741 & \multirow[t]{2}{*}{ 190: 5} & Upper half & $<102$ & $<101$ & $<102$ \\
\hline S97T000763 & & Lower half & $<99.5$ & $<100$ & $<99.8$ \\
\hline S97T000742 & \multirow[t]{2}{*}{ 190: 6} & Upper half & $<102$ & $<103$ & $<103$ \\
\hline S97T000764 & & Lower half & $<101$ & $<101$ & $<101$ \\
\hline S97T000743 & \multirow[t]{2}{*}{ 190: 7} & Upper half & $<102$ & $<102$ & $<102$ \\
\hline S97T000765 & & Lower half & $<103$ & $<101$ & $<102$ \\
\hline S97T000744 & \multirow[t]{2}{*}{ 190: 8} & Upper half & $<103$ & $<101$ & $<102$ \\
\hline S97T000766 & & Lower half & $<103$ & $<101$ & $<102$ \\
\hline S97T000745 & \multirow[t]{2}{*}{ 190: 9} & Upper half & $<102$ & $<102$ & $<102$ \\
\hline S97T000767 & & Lower half & $<101$ & $<101$ & $<101$ \\
\hline S97T001734 & Core 190 & Solid composite & $<97.8$ & $<97.4$ & $<97.6$ \\
\hline Mirinidis & 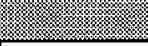 & 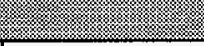 & $8.869=$ & . & 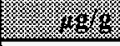 \\
\hline S97T000733 & 190: 1R & Drainable liquid & $<0.363$ & $<0.363$ & $<0.363$ \\
\hline
\end{tabular}


HNF-1501 Rev. 0

Table B2-130. Tank 241-T-203 Analytical Results: Calcium (ICP).

\begin{tabular}{|c|c|c|c|c|c|}
\hline Vinall: & Sarmiplo & (.). & nesili: & 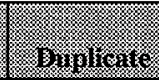 & Mran: \\
\hline \multicolumn{3}{|c|}{ 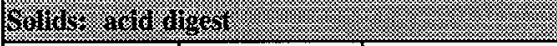 } & (1) & (1) & 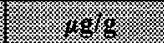 \\
\hline S97T001017 & Core 190 & Solid composite & 353 & 339 & 346 \\
\hline \multicolumn{2}{|c|}{ 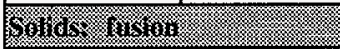 } & (1) & 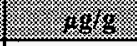 & 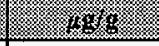 & 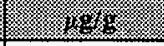 \\
\hline S97T000735 & \multirow[t]{2}{*}{$190: 1$} & Upper half & $<1,950$ & $<1,970$ & $<1,960$ \\
\hline S97T000759 & & Lower half & $<2,080$ & $<2,060$ & $<2,070$ \\
\hline S97T000737 & \multirow[t]{2}{*}{ 190: 1R } & Upper half & $<2,010$ & $<2,000$ & $<2,010$ \\
\hline S97T000760 & & Lower half & $<1,970$ & $<1,860$ & $<1,920$ \\
\hline S97T000738 & \multirow[t]{2}{*}{ 190: 2} & Upper half & $<1,950$ & $<2,000$ & $<1,980$ \\
\hline S97T000757 & & Lower half & $<1,810$ & $<1,760$ & $<1,790$ \\
\hline S97T000739 & \multirow[t]{2}{*}{ 190: 3} & Upper half & $<2,070$ & $<2,110$ & $<2,090$ \\
\hline S97T000761 & & Lower half & $<1,890$ & $<1,910$ & $<1,900$ \\
\hline S97T000740 & \multirow[t]{2}{*}{ 190: 4} & Upper half & $<1,860$ & $<1,890$ & $<1,880$ \\
\hline S97T000762 & & Lower half & $<1,980$ & $<1,960$ & $<1,970$ \\
\hline S97T000741 & \multirow[t]{2}{*}{ 190: 5} & Upper half & $<2,050$ & $<2,030$ & $<2,040$ \\
\hline S97T000763 & & Lower half & $<1,990$ & $<2,000$ & $<2,000$ \\
\hline S97T000742 & \multirow[t]{2}{*}{ 190: 6} & Upper half & $<2,040$ & $<2,060$ & $<2,050$ \\
\hline S97T000764 & & Lower half & $<2,010$ & $<2,020$ & $<2,020$ \\
\hline S97T000743 & \multirow[t]{2}{*}{ 190: 7} & Upper half & $<2,050$ & $<2,040$ & $<2,050$ \\
\hline S97T000765 & & Lower half & $<2,050$ & $<2,020$ & $<2,040$ \\
\hline S97T000744 & \multirow[t]{2}{*}{ 190: 8} & Upper half & $<2,070$ & $<2,020$ & $<2,050$ \\
\hline S97T000766 & & Lower half & $<2,060$ & $<2,020$ & $<2 ; 040$ \\
\hline S97T000745 & \multirow[t]{2}{*}{ 190: 9} & Upper half & $<2,050$ & $<2,030$ & $<2,040$ \\
\hline S97T000767 & & Lower half & $<2,010$ & $<2,030$ & $<2,020$ \\
\hline S97T001734 & Core 190 & Solid composite & $<1,960$ & $<1,950$ & $<1,960$ \\
\hline Virninds & (1) & 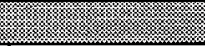 & 4.189 & (.1.9. & 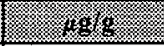 \\
\hline S97T000733 & 190: 1R & Drainable liquid & $<7.27$ & $<7.27$ & $<7.27$ \\
\hline
\end{tabular}


HNF-1501 Rev. 0

Table B2-131. Tank 241-T-203 Analytical Results: Cerium (ICP).

\begin{tabular}{|c|c|c|c|c|c|}
\hline Samplo & f Samples & 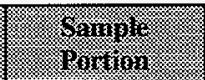 & (1) & 3450 inde & 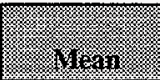 \\
\hline \multicolumn{3}{|c|}{ 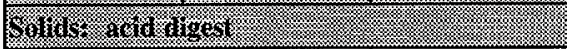 } & 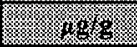 & $(1)$ & 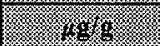 \\
\hline S97T001017 & Core 190 & Solid composite & 56.6 & 53.9 & 55.3 \\
\hline \multicolumn{2}{|c|}{ 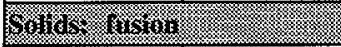 } & 8s & 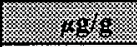 & ( 1948 & 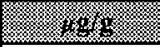 \\
\hline S97T000735 & \multirow[t]{2}{*}{ 190: 1} & Upper half & $<1,950$ & $<1,970$ & $<1,960$ \\
\hline S97T000759 & & Lower half & $<2,080$ & $<2,060$ & $<2,070$ \\
\hline S97T000737 & \multirow[t]{2}{*}{ 190: 1R } & Upper half & $<2,010$ & $<2,000$ & $<2,010$ \\
\hline S97T000760 & & Lower half & $<1,970$ & $<1,860$ & $<1,920$ \\
\hline S97T000738 & \multirow[t]{2}{*}{ 190: 2} & Upper half & $<1,950$ & $<2,000$ & $<1,980$ \\
\hline S97T000757 & & Lower half & $<1,810$ & $<1,760$ & $<1,790$ \\
\hline S97T000739 & \multirow[t]{2}{*}{ 190: 3} & Upper half & $<2,070$ & $<2,110$ & $<2,090$ \\
\hline S97T000761. & & Lower half & $<1,890$ & $<1,910$ & $<1,900$ \\
\hline S97T000740 & \multirow[t]{2}{*}{ 190: 4} & Upper half & $<1,860$ & $<1,890$ & $<1,880$ \\
\hline S97T000762 & & Lower half & $<1,980$ & $<1,960$ & $<1,970$ \\
\hline S97T000741 & \multirow[t]{2}{*}{ 190: 5} & Upper half & $<2,050$ & $<2,030$ & $<2,040$ \\
\hline S97T000763 & & Lower half & $<1,990$ & $<2,000$ & $<2,000$ \\
\hline S97T000742 & \multirow[t]{2}{*}{ 190: 6} & Upper half & $<2,040$ & $<2,060$ & $<2,050$ \\
\hline S97T000764 & & Lower half & $<2,010$ & $<2,020$ & $<2,020$ \\
\hline S97T000743 & \multirow[t]{2}{*}{ 190: 7} & Upper half & $<2,050$ & $<2,040$ & $<2,050$ \\
\hline S97T000765 & & Lower half & $<2,050$ & $<2,020$ & $<2,040$ \\
\hline S97T000744 & \multirow[t]{2}{*}{ 190: 8} & Upper half & $<2,070$ & $<2,020$ & $<2,050$ \\
\hline S97T000766 & & Lower half & $<2,060$ & $<2,020$ & $<2,040$ \\
\hline S97T000745 & \multirow[t]{2}{*}{ 190: 9} & Upper half & $<2,050$ & $<2,030$ & $<2,040$ \\
\hline S97T000767 & & Lower half & $<2,010$ & $<2,030$ & $<2,020$ \\
\hline S97T001734 & Core 190 & Solid composite & $<1,960$ & $<1,950$ & $<1,960$ \\
\hline Ginatas & 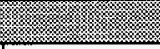 & 2 & . & . & 8.96 \\
\hline S97T000733 & 190: 1R & Drainable liquid & $<7.27$ & $<7.27$ & $<7.27$ \\
\hline
\end{tabular}


HNF-1501 Rev. 0

Table B2-132. Tank 241-T-203 Analytical Results: Chromium (ICP).

\begin{tabular}{|c|c|c|c|c|c|}
\hline Samill: & 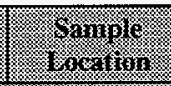 & $\begin{array}{l}\text { Sample: } \\
\text { goritorl }\end{array}$ & Iresuli: & Divilivis: & yrs. \\
\hline \multicolumn{3}{|c|}{ 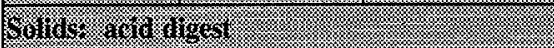 } & 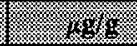 & 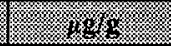 & 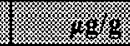 \\
\hline S97T001017 & Core 190 & Solid composite & 3,870 & 3,710 & $3,790^{\mathrm{QC:c}}$ \\
\hline \multicolumn{2}{|c|}{ 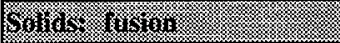 } & & 3.86 & 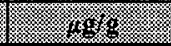 & $\sqrt{7}$ \\
\hline S97T000735 & \multirow[t]{2}{*}{ 190: 1} & Upper half & 2,670 & 2,640 & $2 ; 660$ \\
\hline S97T000759 & & Lower half & 2,530 & 2,610 & 2,570 \\
\hline S97T000737 & \multirow[t]{2}{*}{ 190: 1R } & Upper half & 1,770 & 1,770 & 1,770 \\
\hline S97T000760 & & Lower half & 3,090 & 3,060 & 3,080 \\
\hline S97T000738 & \multirow[t]{2}{*}{ 190: 2} & Upper half & 2,900 & 3,110 & 3,010 \\
\hline S97T000757 & & Lower half & 3,250 & 3,250 & 3,250 \\
\hline S97T000739 & \multirow[t]{2}{*}{ 190: 3} & Upper half & 3,040 & 2,980 & 3,010 \\
\hline S97T000761 & & Lower half & 3,650 & 3,640 & 3,650 \\
\hline S97T000740 & \multirow[t]{2}{*}{$190: 4$} & Upper half & 3,960 & 3,870 & 3,920 \\
\hline S97T000762 & & Lower half & 4,290 & 3,550 & 3,920 \\
\hline S97T000741 & \multirow[t]{2}{*}{ 190: 5} & Upper half & 3,360 & 3,440 & 3,400 \\
\hline S97T000763 & & Lower half & 4,040 & 4,320 & 4,180 \\
\hline S97T000742 & \multirow[t]{2}{*}{ 190: 6} & Upper half & 4,820 & 4,640 & 4,730 \\
\hline S97T000764 & & Lower half & 4,440 & 4,340 & 4,390 \\
\hline S97T000743 & \multirow[t]{2}{*}{ 190: 7} & Upper half & 3,550 & 3,400 & 3,480 \\
\hline S97T000765 & & Lower half & 4,540 & 4,460 & 4,500 \\
\hline S97T000744 & \multirow[t]{2}{*}{ 190: 8} & Upper half & 3,940 & 4,120 & 4,030 \\
\hline S97T000766 & & Lower half & 3,980 & 3,950 & 3,970 \\
\hline S97T000745 & \multirow[t]{2}{*}{ 190: 9} & Upper half & 3,750 & 3,500 & 3,630 \\
\hline S97T000767 & & Lower half & 3,750 & 3,830 & 3,790 \\
\hline S97T001734 & Core 190 & Solid composite & 4,700 & 4,850 & 4,780 \\
\hline Lraing & (2) & $\sqrt{2}$ & $f_{1}$ & 1.8 .8 & (3) \\
\hline S97T000733 & 190: 1R & Drainable liquid & 30.4 & 30.9 & 30.6 \\
\hline
\end{tabular}




\begin{tabular}{|c|c|c|c|c|c|}
\hline$S D^{\prime} I>$ & $S t^{\circ} I>$ & $s t^{*} I>$ & p!̣nb!̣ əqqụฺer & XI :06I & EELO000LL6S \\
\hline 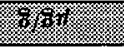 & 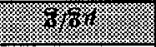 & F\% & & 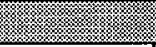 & (1) \\
\hline $06 \varepsilon>$ & $68 \varepsilon>$ & $I 6 \varepsilon>$ & วฺ!soduros płos & 06I әIO) & $\nabla E L I 00 L L 6 \mathrm{~S}$ \\
\hline$\varsigma_{0}>>$ & $90 \pitchfork>$ & $\varepsilon 0 \hbar>$ & I[EY IOMOT] & \multirow[b]{2}{*}{$6: 06 \mathrm{I}$} & $\angle 9 L 000 \mathrm{~L} \angle 6 \mathrm{~S}$ \\
\hline $80 t>$ & $90 \star>>$ & $60 t>$ & J[एप Iədd & & $S \forall \angle 000 L \angle 6 \mathrm{~S}$ \\
\hline $80 t>$ & sot> & IIt> & J[एบ IәмоT & \multirow[b]{2}{*}{$8: 06 \mathrm{I}$} & $99 \angle 000 L \angle 6 S$ \\
\hline $60 t>$ & $t 0 t>$ & $\varepsilon[t>$ & I[eप Iəddn & & $t \forall \angle 000 \mathrm{~L} L 6 \mathrm{~S}$ \\
\hline$L O t>$ & $\varepsilon 0 \pitchfork>$ & oIt> & ILU IәMOT] & \multirow[b]{2}{*}{$\angle: 06 \mathrm{I}$} & S9L000LL6S \\
\hline $60 t>$ & $80 t>$ & ort> & J[एu Iədd & & $\varepsilon t \angle 000 \mathrm{~L} L 6 \mathrm{~S}$ \\
\hline t0t> & sot> & $\varepsilon 0 t>$ & J[Еप ІәмОТ] & \multirow[b]{2}{*}{$9: 06 \mathrm{I}$} & $\$ 9 L 000 \mathrm{~L} L 6 \mathrm{~S}$ \\
\hline 0It> & ZIt> & $80 t>$ & J[एप Iәdd & & $\tau \vdash \angle 000 L \angle 6 S$ \\
\hline $00 t>$ & IOt> & $86 \varepsilon>$ & J[Eप IOMOT & \multirow[b]{2}{*}{$\mathcal{S}: 06 \mathrm{I}$} & E9L000LL6S \\
\hline$\angle O t>$ & sot> & $60 t>$ & I[एч Iədd $\Omega$ & & It $\angle 000 L \angle 6 S$ \\
\hline $76 \varepsilon>$ & $z 6 \varepsilon>$ & $96 \varepsilon>$ & I[Eप دәMO I] & \multirow[b]{2}{*}{$\downarrow: 06 \mathrm{I}$} & $29 \angle 000 \mathrm{LL} 6 \mathrm{~S}$ \\
\hline SLE> & $8 \angle \mathcal{E}>$ & $Z L \varepsilon>$ & J[eq Iədd & & $0 † \angle 000 L \angle 6 S$ \\
\hline $18 \varepsilon>$ & $z 8 \varepsilon>$ & $6 L \mathcal{}>$ & Ғाеप ІәмоТ & \multirow[b]{2}{*}{$\varepsilon: 06 \mathrm{I}$} & I9L000JL6S \\
\hline $6 \mathrm{It}>$ & $\varepsilon Z \downarrow>$ & $t[t>$ & Ғ[еप Iәddח] & & 6EL000LL6S \\
\hline $8 S \mathcal{E}>$ & $\varepsilon S E>$ & $z 9 \varepsilon>$ & f[еप IəMOT] & \multirow[b]{2}{*}{$\tau: 06 \mathrm{I}$} & $\angle S \angle 000 L \angle 6 S$ \\
\hline $96 \varepsilon>$ & $00 \triangleright>$ & $I 6 \mathcal{E}>$ & y[eप rəddn & & $8 \mathcal{E} \angle 000 \mathrm{LL} 6 \mathrm{~S}$ \\
\hline $\mathcal{E} 8 \mathcal{E}>$ & $\tau L \mathcal{E}>$ & $\varepsilon 6 \varepsilon>$ & II'U IOMOT & \multirow[b]{2}{*}{ yІ :06I } & $09 \angle 000 \mathrm{LL} 6 \mathrm{~S}$ \\
\hline $20 t>$ & $00 t>$ & $\varepsilon 0 \pitchfork>$ & IEप Iəddח & & LEL000LL6S \\
\hline tIt $>$ & 2It $>$ & SIt> & JIEप دәMOT & \multirow[b]{2}{*}{ I:06I } & 6SL000LL6S \\
\hline $26 \varepsilon>$ & $\mathcal{E} 6 \mathcal{E}>$ & $06 \varepsilon>$ & I[Eบ Iədd & & SEL000LL6S \\
\hline Fis & \% & (5) & 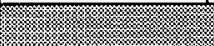 & \multicolumn{2}{|c|}{ (5) } \\
\hline $66^{\circ} \mathcal{E}>$ & $66^{\circ} \mathcal{E}>$ & $66^{\circ} \mathcal{E}>$ & ә!!soduos p!IOS & $06 I$ əroD & LIOI00LL6S \\
\hline 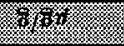 & 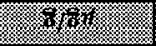 & 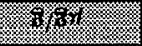 & & \multicolumn{2}{|c|}{ ( } \\
\hline 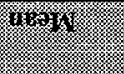 & (7) & His & 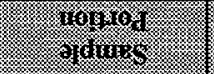 & oring & \% \\
\hline
\end{tabular}

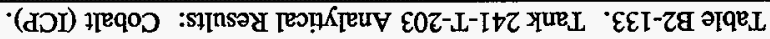


Table B2-134. Tank 241-T-203 Analytical Results: Copper (ICP).

\begin{tabular}{|c|c|c|c|c|c|}
\hline $\begin{array}{l}\text { Sample: } \\
\text { Sumber }\end{array}$ & $1=0$ & S Samples & nesinin & Bunicale & 11:an \\
\hline \multicolumn{2}{|c|}{ 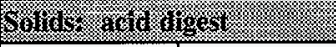 } & (1) & $=8 \%$ & 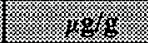 & 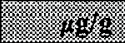 \\
\hline S97T001017 & Core 190 & Solid composite & $<2$ & $<2$ & $<2$ \\
\hline \multicolumn{2}{|c|}{ 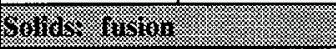 } & & F & (3) & 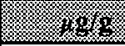 \\
\hline S97T000735 & \multirow[t]{2}{*}{ 190: 1} & Upper half & $<195$ & $<197$ & $<196$ \\
\hline S97T000759 & & Lower half & $<208$ & $<206$ & $<207$ \\
\hline S97T000737 & \multirow[t]{2}{*}{ 190: $1 R$} & Upper half & $<201$ & $<200$ & $<201$ \\
\hline S97T000760 & & Lower half & $<197$ & $<186$ & $<192$ \\
\hline S97T000738 & \multirow[t]{2}{*}{$190: 2$} & Upper half & $<195$ & $<200$ & $<198$ \\
\hline S97T000757 & & Lower half & $<181$ & $<176$ & $<179$ \\
\hline S97T000739 & \multirow[t]{2}{*}{ 190: 3} & Upper half & $<207$ & $<211$ & $<209$ \\
\hline S97T000761 & & Lower half & $<189$ & $<191$ & $<190$ \\
\hline S97T000740 & \multirow[t]{2}{*}{ 190: 4} & Upper half & $<186$ & $<189$ & $<188$ \\
\hline S97T000762 & & Lower half & $<198$ & $<196$ & $<197$ \\
\hline S97T000741 & \multirow[t]{2}{*}{ 190: 5} & Upper half & $<205$ & $<203$ & $<204$ \\
\hline S97T000763 & & Lower half & $<199$ & $<200$ & $<200$ \\
\hline S97T000742 & \multirow[t]{2}{*}{ 190: 6} & Upper half & $<204$ & $<206$ & $<205$ \\
\hline S97T000764 & & Lower half & $<201$ & $<202$ & $<202$ \\
\hline S97T000743 & \multirow[t]{2}{*}{ 190: 7} & Upper half & $<205$ & $<204$ & $<205$ \\
\hline S97T000765 & & Lower half & $<205$ & $<202$ & $<204$ \\
\hline S97T000744 & \multirow[t]{2}{*}{ 190: 8} & Upper half & $<207$ & $<202$ & $<205$ \\
\hline S97T000766 & & Lower half & $<206$ & $<202$ & $<204$ \\
\hline S97T000745 & \multirow[t]{2}{*}{ 190: 9} & Upper half & $<205$ & $<203$ & $<204$ \\
\hline S97T000767 & & Lower half & $<201$ & $<203$ & $<202$ \\
\hline S97T001734 & Core 190 & Solid composite & $<196$ & $<195$ & $<196$ \\
\hline Liandias & & $\sqrt{4}$ & 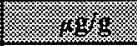 & $\frac{15}{3}$ & 8.1 .98 \\
\hline S97T000733 & 190: 1R & Drainable liquid & $<0.727$ & $<0.727$ & $<0.727$ \\
\hline
\end{tabular}


HNF-1501 Rev. 0

Table B2-135. Tank 241-T-203 Analytical Results: Iron (ICP).

\begin{tabular}{|c|c|c|c|c|c|}
\hline $\begin{array}{l}\text { Simingl: } \\
\text { Vimibyer: }\end{array}$ & Simple: & $\begin{array}{l}\text { Sampil } \\
\text { iortor }\end{array}$ & resuil: & Duritionte & 10 \\
\hline \multicolumn{3}{|c|}{ 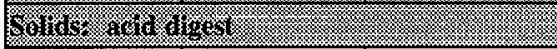 } & (3. & 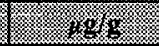 & 18 \\
\hline S97T001017 & Core 190 & Solid composite & 3,620 & 10,000 & $6,810^{\mathrm{eC}: \mathrm{d}, \mathrm{c}}$ \\
\hline \multicolumn{2}{|c|}{ 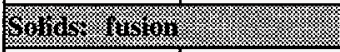 } & (l) & 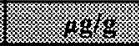 & 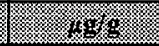 & S. \\
\hline S97T000735 & \multirow[t]{2}{*}{ 190: 1} & Upper half & 1,960 & 1,780 & 1,870 \\
\hline S97T000759 & & Lower half & 2,210 & 2,040 & 2,130 \\
\hline S97T000737 & \multirow[t]{2}{*}{ 190: 1R } & Upper half & 14,500 & 30,200 & $22,400^{\mathrm{QC:O}}$ \\
\hline S97T000760 & & Lower half & 2,110 & 1,800 & 1,960 \\
\hline S97T000738 & \multirow[t]{2}{*}{ 190: 2} & Upper half & 17,500 & 3,130 & $10,300^{\mathrm{QC:c}}$ \\
\hline S97T000757 & & Lower half & 2,270 & 2,010 & 2,140 \\
\hline S97T000739 & \multirow[t]{2}{*}{ 190: 3} & Upper half & 5,700 & 8,050 & $6,880^{\text {QC:e }}$ \\
\hline S97T000761 & & Lower half & 2,520 & 2,480 & 2,500 \\
\hline S97T000740 & \multirow[t]{2}{*}{ 190: 4} & Upper half & 2,710 & 4,220 & $3,470^{\mathrm{QC:e}}$ \\
\hline S97T000762 & & Lower half & 2,440 & 2,100 & 2,270 \\
\hline S97T000741 & \multirow[t]{2}{*}{ 190: 5} & Upper half & 1,860 & 1,930 & 1,900 \\
\hline S97T000763 & & Lower half & 2,230 & 2,340 & 2,290 \\
\hline S97T000742 & \multirow[t]{2}{*}{ 190: 6} & Upper half & 2,240 & 2,240 & 2,240 \\
\hline S97T000764 & & Lower half & 2,540 & 2,570 & 2,560 \\
\hline S97T000743 & \multirow[t]{2}{*}{ 190: 7} & Upper half & 3,890 & 3,780 & 3,840 \\
\hline S97T000765 & & Lower half & 3,080 & 2,810 & 2,950 \\
\hline S97T000744 & \multirow[t]{2}{*}{ 190: 8} & Upper half & 3,080 & 3,130 & 3,110 \\
\hline S97T000766 & & Lower half & 2,910 & 2,680 & 2,800 \\
\hline S97T000745 & \multirow[t]{2}{*}{ 190: 9} & Upper half & 2,150 & 1,660 & $1,910^{\mathrm{QC}: \mathrm{e}}$ \\
\hline S97T000767 & & Lower half & 2,320 & 2,200 & 2,260 \\
\hline S97T001734 & Core 190 & Solid composite & 5,900 & 4,160 & $5,030^{\mathrm{eC:c}}$ \\
\hline igrios & & (1) & 1.485 & 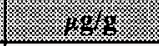 & 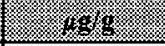 \\
\hline S97T000733 & 190: 1R & Drainable liquid & $<3.63$ & $<3.63$ & $<3.63$ \\
\hline
\end{tabular}


HNF-1501 Rev. 0

Table B2-136. Tank 241-T-203 Analytical Results: Lanthanum (ICP).

\begin{tabular}{|c|c|c|c|c|c|}
\hline Saminis: & h & $\begin{array}{l}\text { Shante } \\
\text { Portion }\end{array}$ & inesilin & Bintione & 17resin \\
\hline \multicolumn{3}{|c|}{ 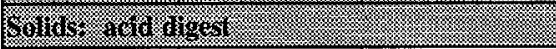 } & 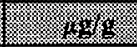 & 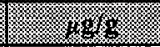 & 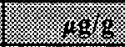 \\
\hline S97T001017 & Core 190 & Solid composite & 11,900 & 11,500 & $11,700^{\mathrm{eC}: c}$ \\
\hline \multicolumn{2}{|c|}{ 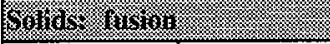 } & & 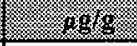 & 8.48968 & 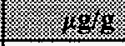 \\
\hline S97T000735 & \multirow[t]{2}{*}{ 190: 1} & Upper half & 8,680 & 8,630 & 8,660 \\
\hline S97T000759 & & Lower half & 9,630 & 9,890 & 9,760 \\
\hline S97T000737 & \multirow[t]{2}{*}{ 190: 1R } & Upper half & 8,370 & 8,330 & 8,350 \\
\hline S97T000760 & & Lower half & 7,090 & 7,500 & 7,300 \\
\hline S97T000738 & \multirow[t]{2}{*}{ 190: 2} & Upper half & 7,140 & 7,870 & 7,510 \\
\hline S97T000757 & & Lower half & 9,750 & 9,260 & 9,510 \\
\hline S97T000739 & \multirow[t]{2}{*}{ 190: 3} & Upper half & 10,000 & 9,720 & 9,860 \\
\hline S97T000761 & & Lower half & 11,800 & 11,800 & 11,800 \\
\hline S97T000740 & \multirow[t]{2}{*}{$190: 4$} & Upper half & 9,730 & 9,270 & 9,500 \\
\hline S97T000762 & & Lower half & 11,300 & 11,200 & 11,300 \\
\hline S97T000741 & \multirow[t]{2}{*}{$190: 5$} & Upper half & 9,850 & 10,100 & 9,980 \\
\hline S97T000763 & & Lower half & 12,000 & 11,100 & 11,600 \\
\hline S97T000742 & \multirow[t]{2}{*}{ 190: 6} & Upper half & 10,300 & 10,700 & 10,500 \\
\hline S97T000764 & & Lower half & 10,000 & 10,400 & 10,200 \\
\hline S97T000743 & \multirow[t]{2}{*}{ 190: 7} & Upper half & 10,800 & 10,900 & 10,900 \\
\hline S97T000765 & & Lower half & 9,740 & 9,560 & 9,650 \\
\hline S97T000744 & \multirow[t]{2}{*}{ 190: 8} & Upper half & 10,600 & 11,200 & 10,900 \\
\hline S97T000766 & & Lower half & 11,900 & 13,900 & 12,900 \\
\hline S97T000745 & \multirow[t]{2}{*}{ 190: 9} & Upper half & 9,290 & 9,760 & 9,530 \\
\hline S97T000767 & & Lower half & 10,400 & 9,910 & 10,200 \\
\hline S97T001734 & Core 190 & Solid composite & 14,000 & 14,400 & 14,200 \\
\hline levinitis & 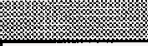 & & , 1989 & 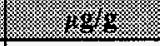 & 18 \\
\hline S97T000733 & 190: 1R & Drainable liquid & $<3.63$ & $<3.63$ & $<3.63$ \\
\hline
\end{tabular}


Table B2-137. Tank 241-T-203 Analytical Results: Lead (ICP).

\begin{tabular}{|c|c|c|c|c|c|}
\hline Sample & 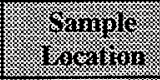 & 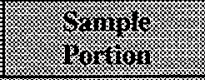 & eresili: & 3ytingares: & Merin \\
\hline \multicolumn{3}{|c|}{ 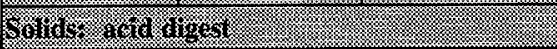 } & 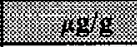 & 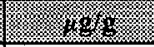 & 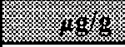 \\
\hline S97T001017 & Core 190 & Solid composite & $<20$ & 21.5 & $<20.8^{\mathrm{QC}: \mathrm{d}}$ \\
\hline \multicolumn{3}{|c|}{ 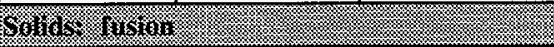 } & 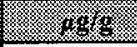 & 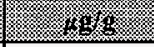 & 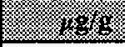 \\
\hline S97T000735 & \multirow[t]{2}{*}{ 190: 1} & Upper half & $<1,950$ & $<1,970$ & $<1,960$ \\
\hline S97T000759 & & Lower half & $<2,080$ & $<2,060$ & $<2,070$ \\
\hline S97T000737 & \multirow[t]{2}{*}{ 190: 1R } & Upper half & $<2,010$ & $<2,000$ & $<2,010$ \\
\hline S97T000760 & & Lower half & $<1,970$ & $<1,860$ & $<1,920$ \\
\hline S97T000738 & \multirow[t]{2}{*}{ 190: 2} & Upper half & $<1,950$ & $<2,000$ & $<1,980$ \\
\hline S97T000757 & & Lower half & $<1,810$ & $<1,760$ & $<1,790$ \\
\hline S97T000739 & \multirow[t]{2}{*}{ 190: 3} & Upper half & $<2,070$ & $<2,110$ & $<2,090$ \\
\hline S97T000761 & & Lower half & $<1,890$ & $<1,910$ & $<1,900$ \\
\hline S97T000740 & \multirow[t]{2}{*}{ 190: 4} & Upper half & $<1,860$ & $<1,890$ & $<1,880$ \\
\hline S97T000762 & & Lower half & $<1,980$ & $<1,960$ & $<1,970$ \\
\hline S97T000741 & \multirow[t]{2}{*}{ 190: 5} & Upper half & $<2,050$ & $<2,030$ & $<2,040$ \\
\hline S97T000763 & & Lower half & $<1,990$ & $<2,000$ & $<2,000$ \\
\hline S97T000742 & \multirow[t]{2}{*}{ 190: 6} & Upper half & $<2,040$ & $<2,060$ & $<2,050$ \\
\hline S97T000764 & & Lower half & $<2,010$ & $<2,020$ & $<2,020$ \\
\hline S97T000743 & \multirow[t]{2}{*}{ 190: 7} & Upper half & $<2,050$ & $<2,040$ & $<2,050$ \\
\hline S97T000765 & & Lower half & $<2,050$ & $<2,020$ & $<2,040$ \\
\hline S97T000744 & \multirow[t]{2}{*}{ 190: 8} & Upper half & $<2,070$ & $<2,020$ & $<2,050$ \\
\hline S97T000766 & & Lower half & $<2,060$ & $<2,020$ & $<2,040$ \\
\hline S97T000745 & \multirow[t]{2}{*}{ 190: 9} & Upper half & $<2,050$ & $<2,030$ & $<2,040$ \\
\hline S97T000767 & & Lower half & $<2,010$ & $<2,030$ & $<2,020$ \\
\hline S97T001734 & Core 190 & Solid composite & $<1,960$ & $<1,950$ & $<1,960$ \\
\hline Wgaras: & ) & 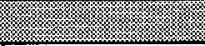 & (3) & 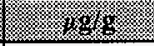 & 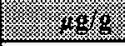 \\
\hline S97T000733 & 190: 1R & Drainable liquid & $<7.27$ & $<7.27$ & $<7.27$ \\
\hline
\end{tabular}


Table B2-138. Tank 241-T-203 Analytical Results: Lithium (ICP).

\begin{tabular}{|c|c|c|c|c|c|}
\hline $\begin{array}{l}\text { Samipto } \\
\text { Similier }\end{array}$ & 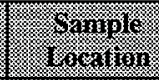 & Thruile & Iring: & Bitillicate: & (2) \\
\hline \multicolumn{3}{|c|}{ 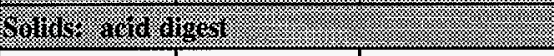 } & (1) & (-) & 4.45 \\
\hline S97T001017 & Core 190 & Solid composite & $<2$ & $<2$ & $<2$ \\
\hline \multicolumn{2}{|c|}{ 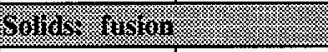 } & (2) & 3.46 & 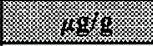 & 18.896 \\
\hline S97T000735 & \multirow[t]{2}{*}{ 190: 1} & Upper half & $<195$ & $<197$ & $<196$ \\
\hline S97T000759 & & Lower half & $<208$ & $<206$ & $<207$ \\
\hline S97T000737 & \multirow[t]{2}{*}{ 190: $1 R$} & Upper half & $<201$ & $<200$ & $<201$ \\
\hline S97T000760 & & Lower half & $<197$ & $<186$ & $<192$ \\
\hline S97T000738 & \multirow[t]{2}{*}{ 190: 2} & Upper half & $<195$ & $<200$ & $<198$ \\
\hline S97T000757 & & Lower half & $<181$ & $<176$ & $<179$ \\
\hline S97T000739 & \multirow[t]{2}{*}{ 190: 3} & Upper half & $<207$ & $<211$ & $<209$ \\
\hline S97T000761 & & Lower half & $<189$ & $<191$ & $<190$ \\
\hline S97T000740 & \multirow[t]{2}{*}{ 190: 4} & Upper half & $<186$ & $<189$ & $<188$ \\
\hline S97T000762 & & Lower half & $<198$ & $<196$ & $<197$ \\
\hline S97T000741 & \multirow[t]{2}{*}{ 190: 5} & Upper half & $<205$ & $<203$ & $<204$ \\
\hline S97T000763 & & Lower half & $<199$ & $<200$ & $<200$ \\
\hline S97T000742 & \multirow[t]{2}{*}{ 190: 6} & Upper half & $<204$ & $<206$ & $<205$ \\
\hline S97T000764 & & Lower half & $<201$ & $<202$ & $<202$ \\
\hline S97T000743 & \multirow[t]{2}{*}{ 190: 7} & Upper half & $<205$ & $<204$ & $<205$ \\
\hline S97T000765 & & Lower half & $<205$ & $<202$ & $<204$ \\
\hline S97T000744 & \multirow[t]{2}{*}{ 190: 8} & Upper half & $<207$ & $<202$ & $<205$ \\
\hline S97T000766 & & Lower half & $<206$ & $<202$ & $<204$ \\
\hline S97T000745 & \multirow[t]{2}{*}{ 190: 9} & Upper half & $<205$ & $<203$ & $<204$ \\
\hline S97T000767 & & Lower half & $<201$ & $<203$ & $<202$ \\
\hline S97T001734 & Core 190 & Solid composite & $<196$ & $<195$ & $<196$ \\
\hline 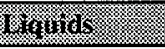 & & (2) & 1.898 & 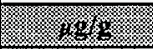 & 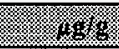 \\
\hline S97T000733 & 190: 1R & Drainable liquid & $<0.727$ & $<0.727$ & $<0.727$ \\
\hline
\end{tabular}


Table B2-139. Tank 241-T-203 Analytical Results: Magnesium (ICP).

\begin{tabular}{|c|c|c|c|c|c|}
\hline Vaminis & samile & 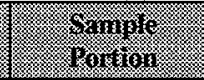 & igenain & 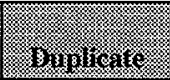 & (4) \\
\hline \multicolumn{3}{|c|}{ 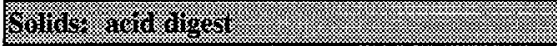 } & 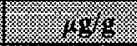 & $1.1 .89=0$ & (3) \\
\hline S97T001017 & Core 190 & Solid composite & 77.8 & 80.2 & 79 \\
\hline \multicolumn{2}{|c|}{ 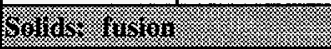 } & & 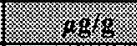 & 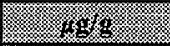 & 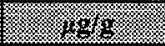 \\
\hline S97T000735 & \multirow[t]{2}{*}{ 190: 1} & Upper half & $<1,950$ & $<1,970$ & $<1,960$ \\
\hline S97T000759 & & Lower half & $<2,080$ & $<2,060$ & $<2,070$ \\
\hline S97T000737 & \multirow[t]{2}{*}{ 190: $1 \mathbf{R}$} & Upper half & $<2,010$ & $<2,000$ & $<2,010$ \\
\hline S97T000760 & & Lower half & $<1,970$ & $<1,860$ & $<1,920$ \\
\hline S97T000738 & \multirow[t]{2}{*}{ 190: 2} & Upper half & $<1,950$ & $<2,000$ & $<1,980$ \\
\hline S97T000757 & & Lower half & $<1,810$ & $<1,760$ & $<1,790$ \\
\hline S97T000739 & \multirow[t]{2}{*}{ 190: 3} & Upper half & $<2,070$ & $<2,110$ & $<2,090$ \\
\hline S97T000761 & & Lower half & $<1,890$ & $<1,910$ & $<1,900$ \\
\hline S97T000740 & \multirow[t]{2}{*}{ 190: 4} & Upper half & $<1,860$ & $<1,890$ & $<1,880$ \\
\hline S97T000762 & & Lower half & $<1,980$ & $<1,960$ & $<1,970$ \\
\hline S97T000741 & \multirow[t]{2}{*}{ 190: 5} & Upper half & $<2,050$ & $<2,030$ & $<2,040$ \\
\hline S97T000763 & & Lower half & $<1,990$ & $<2,000$ & $<2,000$ \\
\hline S97T000742 & \multirow[t]{2}{*}{ 190: 6} & Upper half & $<2,040$ & $<2,060$ & $<2,050$ \\
\hline S97T000764 & & Lower half & $<2,010$ & $<2,020$ & $<2,020$ \\
\hline S97T000743 & \multirow[t]{2}{*}{ 190: 7} & Upper half & $<2,050$ & $<2,040$ & $<2,050$ \\
\hline S97T000765 & & Lower half & $<2,050$ & $<2,020$ & $<2,040$ \\
\hline S97T000744 & \multirow[t]{2}{*}{$190: 8$} & Upper half & $<2,070$ & $<2,020$ & $<2,050$ \\
\hline S97T000766 & & Lower half & $<2,060$ & $<2,020$ & $<2,040$ \\
\hline S97T000745 & \multirow[t]{2}{*}{ 190: 9} & Upper half & $<2,050$ & $<2,030$ & $<2,040$ \\
\hline S97T000767 & & Lower half & $<2,010$ & $<2,030$ & $<2,020$ \\
\hline S97T001734 & Core 190 & Solid composite & $<1,960$ & $<1,950$ & $<1,960$ \\
\hline Hinas & & & 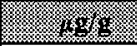 & 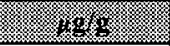 & 4.968 \\
\hline S97T000733 & 190: 1R & Drainable liquid & $<7.27$ & $<7.27$ & $<7.27$ \\
\hline
\end{tabular}


HNF-1501 Rev. 0

Table B2-140. Tank 241-T-203 Analytical Results: Manganese (ICP).

\begin{tabular}{|c|c|c|c|c|c|}
\hline Somule & Sample: & $\frac{\text { Samile }}{103 \text { tion }}$ & iensilu & Piginicate & 138.21 \\
\hline \multicolumn{3}{|c|}{ 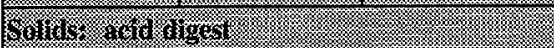 } & $8.8 \%$ & 18 & 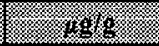 \\
\hline S97T001017 & Core 190 & Solid composite & 16,400 & 15,700 & $16,100^{\mathrm{eC:c}}$ \\
\hline I. & & & I) & 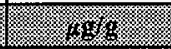 & 1) \\
\hline S97T000735 & \multirow[t]{2}{*}{ 190: 1} & Upper half & 9,230 & 9,080 & 9,160 \\
\hline S97T000759 & & Lower half & 10,700 & 10,800 & 10,800 \\
\hline S97T000737 & \multirow[t]{2}{*}{ 190: 1R } & Upper half & 8,040 & 8,110 & 8,080 \\
\hline S97T000760 & & Lower half & 7,940 & 8,180 & 8,060 \\
\hline S97T000738 & \multirow[t]{2}{*}{ 190: 2} & Upper half & 7,900 & 8,680 & 8,290 \\
\hline S97T000757 & & Lower half & 10,600 & 10,400 & 10,500 \\
\hline S97T000739 & \multirow[t]{2}{*}{ 190: 3} & Upper half & 10,200 & 9,970 & 10,100 \\
\hline S97T000761 & & Lower half & 11,700 & 11,700 & 11,700 \\
\hline S97T000740 & \multirow[t]{2}{*}{ 190: 4} & Upper half & 11,000 & 10,400 & 10,700 \\
\hline S97T000762 & & Lower half & 11,800 & 11,300 & 11,600 \\
\hline S97T000741 & \multirow[t]{2}{*}{$190: 5$} & Upper half & 10,700 & 10,900 & 10,800 \\
\hline S97T000763 & & Lower half & 12,300 & 11,500 & 11,900 \\
\hline S97T000742 & \multirow[t]{2}{*}{ 190: 6} & Upper half & 11,800 & 12,000 & 11,900 \\
\hline S97T000764 & & Lower half & 11,600 & 11,700 & 11,700 \\
\hline S97T000743 & \multirow[t]{2}{*}{ 190: 7} & Upper half & 12,700 & 12,800 & 12,800 \\
\hline S97T000765 & & Lower half & 12,900 & 12,200 & 12,600 \\
\hline S97T000744 & \multirow[t]{2}{*}{ 190: 8} & Upper half & 12,600 & 12,800 & 12,700 \\
\hline S97T000766 & & Lower half & 13,100 & 14,000 & 13,600 \\
\hline S97T000745 & \multirow[t]{2}{*}{ 190: 9} & Upper half & 10,800 & 6,150 & $8,480^{\circ \mathrm{C}: \mathrm{e}}$ \\
\hline S97T000767 & & Lower half & 8,180 & 7,280 & 7,730 \\
\hline S97T001734 & Core 190 & Solid composite & 7,250 & 3,400 & $5,330^{\mathrm{QC:0}}$ \\
\hline mougr & 1 & $\frac{1}{4}$ & ( & 17.98 & (1) \\
\hline S97T000733 & 190: 1R & Drainable liquid & $<0.727$ & $<0.727$ & $<0.727$ \\
\hline
\end{tabular}


HNF-1501 Rev. 0

Table B2-141. Tank 241-T-203 Analytical Results: Molybdenum (ICP).

\begin{tabular}{|c|c|c|c|c|c|}
\hline Simulis & 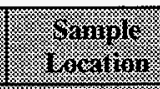 & $\begin{array}{l}\text { Sarmple } \\
\text { rorton }\end{array}$ & resin: & Bubleate & 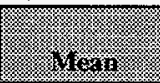 \\
\hline \multicolumn{2}{|c|}{ 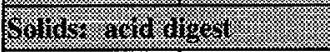 } & $\sqrt{8.28}$ & 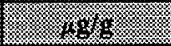 & 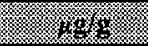 & 48 \\
\hline S97T001017 & Core 190 & Solid composite & $<9.98$ & $<9.98$ & $<9.98$ \\
\hline \multicolumn{2}{|c|}{ 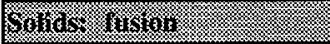 } & & 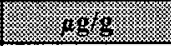 & 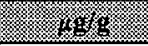 & 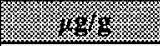 \\
\hline S97T000735 & \multirow[t]{2}{*}{ 190: 1} & Upper half & $<975$ & $<984$ & $<980$ \\
\hline S97T000759 & & Lower half & $<1040$ & $<1,030$ & $<1,040$ \\
\hline S97T000737 & \multirow[t]{2}{*}{ 190: 1R } & Upper half & $<1,010$ & $<1,000$ & $<1,010$ \\
\hline S97T000760 & & Lower half & $<983$ & $<929$ & $<956$ \\
\hline S97T000738 & \multirow[t]{2}{*}{ 190: 2} & Upper half & $<976$ & $<999$ & $<988$ \\
\hline S97T000757 & & Lower half & $<904$ & $<881$ & $<893$ \\
\hline S97T000739 & \multirow[t]{2}{*}{ 190: 3} & Upper half & $<1,030$ & $<1,060$ & $<1,050$ \\
\hline S97T000761 & & Lower half & $<947$ & $<956$ & $<952$ \\
\hline S97T000740 & \multirow[t]{2}{*}{ 190: 4} & Upper half & $<931$ & $<945$ & $<938$ \\
\hline S97T000762 & & Lower half & $<989$ & $<981$ & $<985$ \\
\hline S97T000741 & \multirow[t]{2}{*}{ 190: 5} & Upper half & $<1,020$ & $<1,010$ & $<1,020$ \\
\hline S97T000763 & & Lower half & $<995$ & $<1,000$ & $<998$ \\
\hline S97T000742 & \multirow[t]{2}{*}{ 190: 6} & Upper half & $<1,020$ & $<1,030$ & $<1,030$ \\
\hline S97T000764 & & Lower half & $<1,010$ & $<1,010$ & $<1,010$ \\
\hline S97T000743 & \multirow[t]{2}{*}{ 190: 7} & Upper half & $<1,020$ & $<1,020$ & $<1,020$ \\
\hline S97T000765 & & Lower half & $<1,030$ & $<1,010$ & $<1,020$ \\
\hline S97T000744 & \multirow[t]{2}{*}{ 190: 8} & Upper half & $<1,030$ & $<1,010$ & $<1,020$ \\
\hline S97T000766 & & Lower half & $<1,030$ & $<1,010$ & $<1,020$ \\
\hline S97T000745 & \multirow[t]{2}{*}{ 190: 9} & Upper half & $<1,020$ & $<1,020$ & $<1,020$ \\
\hline S97T000767 & & Lower half & $<1,010$ & $<1,010$ & $<1,010$ \\
\hline S97T001734 & Core 190 & Solid composite & $<978$ & $<974$ & $<976$ \\
\hline liliginid & & 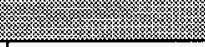 & 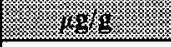 & 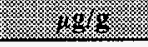 & 8.898 \\
\hline S97T000733 & 190: 1R & Drainable liquid & $<3.63$ & $<3.63$ & $<3.63$ \\
\hline
\end{tabular}


Table B2-142. Tank 241-T-203 Analytical Results: Neodymium (ICP).

\begin{tabular}{|c|c|c|c|c|c|}
\hline 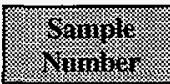 & 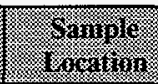 & $f_{1}$ & ir esulu & (5.) & 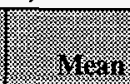 \\
\hline \multicolumn{2}{|c|}{ 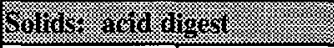 } & & (2) & (x) & 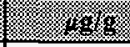 \\
\hline S97T001017 & Core 190 & Solid composite & $<20$ & $<20$ & $<20$ \\
\hline 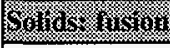 & & (4) & (r) & 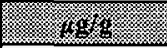 & 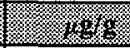 \\
\hline S97T000735 & \multirow[t]{2}{*}{ 190: 1} & Upper half & $<1,950$ & $<1,970$ & $<1,960$ \\
\hline \$97T000759 & & Lower half & $<2,080$ & $<2,060$ & $<2,070$ \\
\hline S97T000737 & \multirow[t]{2}{*}{ 190: 1R } & Upper half & $<2,010$ & $<2,000$ & $<2,010$ \\
\hline S97T000760 & & Lower half & $<1,970$ & $<1,860$ & $<1,920$ \\
\hline S97T000738 & \multirow[t]{2}{*}{ 190: 2} & Upper half & $<1,950$ & $<2,000$ & $<1,980$ \\
\hline S97T000757 & & Lower half & $<1,810$ & $<1,760$ & $<1,790$ \\
\hline S97T000739 & \multirow[t]{2}{*}{ 190: 3} & Upper half & $<2,070$ & $<2,110$ & $<2,090$ \\
\hline S97T000761 & & Lower half & $<1,890$ & $<1,910$ & $<1,900$ \\
\hline S97T000740 & \multirow[t]{2}{*}{ 190: 4} & Upper half & $<1,860$ & $<1,890$ & $<1,880$ \\
\hline s97T000762 & & Lower half & $<1,980$ & $<1,960$ & $<1,970$ \\
\hline S97T000741 & \multirow[t]{2}{*}{ 190: 5} & Upper half & $<2,050$ & $<2,030$ & $<2,040$ \\
\hline S97T000763 & & Lower half & $<1,990$ & $<2,000$ & $<2,000$ \\
\hline S97T000742 & \multirow[t]{2}{*}{ 190: 6} & Upper half & $<2,040$ & $<2,060$ & $<2,050$ \\
\hline S97T000764 & & Lower half & $<2,010$ & $<2,020$ & $<2,020$ \\
\hline \$97T000743 & \multirow[t]{2}{*}{ 190: 7} & Upper half & $<2,050$ & $<2,040$ & $<2,050$ \\
\hline S97T000765 & & Lower half & $<2,050$ & $<2,020$ & $<2,040$ \\
\hline \$97T000744 & \multirow[t]{2}{*}{ 190: 8} & Upper half & $<2,070$ & $<2,020$ & $<2,050$ \\
\hline \$97T000766 & & Lower half & $<2,060$ & $<2,020$ & $<2,040$ \\
\hline S97T000745 & \multirow[t]{2}{*}{ 190: 9} & Upper half & $<2,050$ & $<2,030$ & $<2,040$ \\
\hline S97T000767 & & Lower half & $<2,010$ & $<2,030$ & $<2,020$ \\
\hline S97T001734 & Core 190 & Solid composite & $<1,960$ & $<1,950$ & $<1,960$ \\
\hline 6) & (3) & & 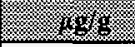 & 4 & 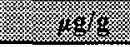 \\
\hline S97T000733 & 190: 1R & Drainable liquid & $<7.27$ & $<7.27$ & $<7.27$ \\
\hline
\end{tabular}


HNF-1501 Rev. 0

Table B2-143. Tank 241-T-203 Analytical Results: Nickel (ICP).

\begin{tabular}{|c|c|c|c|c|c|}
\hline $\begin{array}{l}\text { Sammle } \\
\text { Number }\end{array}$ & $\begin{array}{l}\text { Sampie } \\
\text { Sorifion }\end{array}$ & 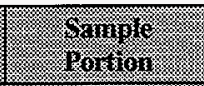 & incsul & Byilican & nitsin \\
\hline \multicolumn{3}{|c|}{ 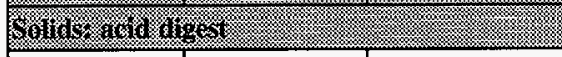 } & 4.486. & 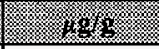 & t \\
\hline S97T001017 & Core 190 & Solid composite & 149 & 146 & 148 \\
\hline Manins & & & 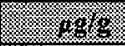 & . $68 \%$ & 486 \\
\hline S97T000733 & 190: 1R & Drainable liquid & $<1.45$ & $<1.45$ & $<1.45$ \\
\hline
\end{tabular}

Table B2-144. Tank 241-T-203 Analytical Results: Phosphorus (ICP). (2 sheets)

\begin{tabular}{|c|c|c|c|c|c|}
\hline Sample: & Samigle: & $\begin{array}{l}\text { Sample } \\
\text { lortion }\end{array}$ & 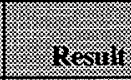 & 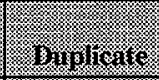 & Vilenis \\
\hline \multicolumn{3}{|c|}{ 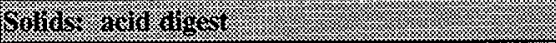 } & 18: & 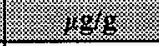 & 18 \\
\hline S97T001017 & Core 190 & Solid composite & 2,420 & 2,350 & $2,390^{\mathrm{QC:c}}$ \\
\hline \multicolumn{3}{|c|}{ 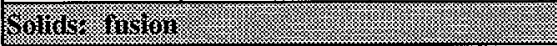 } & S. & $\sqrt{1.968 \%}$ & 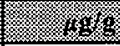 \\
\hline S97T000735 & \multirow[t]{2}{*}{ 190: 1} & Upper half & $<3,900$ & $<3,930$ & $<3,920$ \\
\hline S97T000759 & & Lower half & $<4,150$ & $<4,120$ & $<4,140$ \\
\hline S97T000737 & \multirow[t]{2}{*}{ 190: $1 \mathrm{R}$} & Upper half & $<4,030$ & $<4,000$ & $<4,020$ \\
\hline S97T000760 & & Lower half & $<3,930$ & $<3,720$ & $<3,830$ \\
\hline S97T000738 & \multirow[t]{2}{*}{ 190: 2} & Upper half & $<3,910$ & $<4,000$ & $<3,960$ \\
\hline S97T000757 & & Lower half & $<3,620$ & $<3,530$ & $<3,580$ \\
\hline S97T000739 & \multirow[t]{2}{*}{ 190: 3} & Upper half & $<4,140$ & $<4,230$ & $<4,190$ \\
\hline S97T000761 & & Lower half & $<3,790$ & $<3,820$ & $<3,810$ \\
\hline S97T000740 & \multirow[t]{2}{*}{ 190: 4} & Upper half & $<3,720$ & $<3,780$ & $<3,750$ \\
\hline S97T000762 & & Lower half & $<3,960$ & $<3,920$ & $<3,940$ \\
\hline S97T000741 & \multirow[t]{2}{*}{ 190: 5} & Upper half & $<4,090$ & $<4,050$ & $<4,070$ \\
\hline S97T000763 & & Lower half & $<3,980$ & $<4,010$ & $<4,000$ \\
\hline S97T000742 & \multirow[t]{2}{*}{ 190: 6} & Upper half & $<4,080$ & $<4,120$ & $<4,100$ \\
\hline S97T000764 & & Lower half & $<4,030$ & $<4,050$ & $<4,040$ \\
\hline S97T000743 & \multirow[t]{2}{*}{ 190: 7} & Upper half & $<4,100$ & $<4,080$ & $<4,090$ \\
\hline S97T000765 & & Lower half & $<4,100$ & $<4,030$ & $<4,070$ \\
\hline
\end{tabular}


Table B2-144. Tank 241-T-203 Analytical Results: Phosphorus (ICP). (2 sheets)

\begin{tabular}{|c|c|c|c|c|c|}
\hline S Smiple & 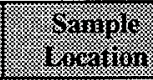 & 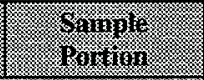 & Ressin & Bmolinat & Y) \\
\hline 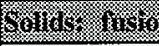 & 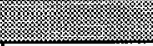 & $\sqrt{31}$ & 3.1868 & 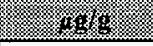 & 8.1596 \\
\hline S97T000744 & \multirow[t]{2}{*}{$190: 8$} & Upper half & $<4,130$ & $<4,040$ & $<4,090$ \\
\hline S97T000766 & & Lower half & $<4,110$ & $<4,050$ & $<4,080$ \\
\hline S97T000745 & \multirow[t]{2}{*}{ 190: 9} & Upper half & $<4,090$ & $<4,060$ & $<4,080$ \\
\hline S97T000767 & & Lower half & $<4,030$ & $<4,060$ & $<4,050$ \\
\hline S97T001734 & Core 190 & Solid composite & $<3,910$ & $<3,890$ & $<3,900$ \\
\hline \multicolumn{3}{|c|}{ Vinum } & 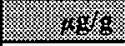 & 18.48 & 3.1864 \\
\hline S97T000733 & 190: 1R & Drainable liquid & 536 & 542 & 539 \\
\hline
\end{tabular}

Table B2-145. Tank 241-T-203 Analytical Results: Potassium (ICP).

\begin{tabular}{|c|c|c|c|c|c|}
\hline Samines & 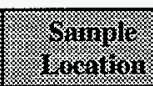 & $\begin{array}{l}\text { Sample } \\
\text { rotron }\end{array}$ & R 8 -s:11: & Hoplowate & 11sur \\
\hline \multicolumn{3}{|c|}{ 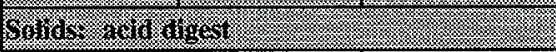 } & 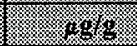 & 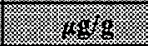 & 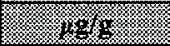 \\
\hline S97T001017 & Core 190 & Solid composite & 6,960 & 6,740 & $6,850^{\mathrm{QC:c}}$ \\
\hline 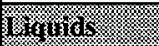 & 4 & & (1) & 168 & 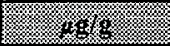 \\
\hline S97T000733 & 190: $1 \mathrm{R}$ & Drainable liquid & 6,020 & 6,040 & $6,030^{\mathrm{QC:d}}$ \\
\hline
\end{tabular}


Table B2-146. Tank 241-T-203 Analytical Results: Samarium (ICP).

\begin{tabular}{|c|c|c|c|c|c|}
\hline $\begin{array}{l}\text { Sample } \\
\text { ITmber }\end{array}$ & 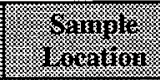 & $\begin{array}{l}\text { Sample } \\
\text { Tortion }\end{array}$ & Iresulit & inivicule: & $18+2 \pi$ \\
\hline \multicolumn{2}{|c|}{ 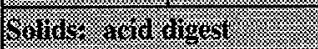 } & (3) & 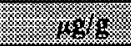 & 1.189 .4 & 4.856 \\
\hline S97T001017 & Core 190 & Solid composite & $<20$ & $<20$ & $<20$ \\
\hline \multicolumn{3}{|c|}{ 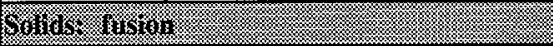 } & 4.896 & $8.1 .6 \%$ & (1.8. \\
\hline S97T000735 & \multirow[t]{2}{*}{ 190: 1} & Upper half & $<1,950$ & $<1,970$ & $<1,960$ \\
\hline \$97T000759 & & Lower half & $<2,080$ & $<2,060$ & $<2,070$ \\
\hline S97T000737 & \multirow[t]{2}{*}{ 190: 1R } & Upper half & $<2,010$ & $<2,000$ & $<2,010$ \\
\hline S97T000760 & & Lower half & $<1,970$ & $<1,860$ & $<1,920$ \\
\hline S97T000738 & \multirow[t]{2}{*}{ 190: 2} & Upper half & $<1,950$ & $<2,000$ & $<1,980$ \\
\hline S97T000757 & & Lower half & $<1,810$ & $<1,760$ & $<1,790$ \\
\hline \$97T000739 & \multirow[t]{2}{*}{ 190: 3} & Upper half & $<2,070$ & $<2,110$ & $<2,090$ \\
\hline S97T000761 & & Lower half & $<1,890$ & $<1,910$ & $<1,900$ \\
\hline S97T000740 & \multirow[t]{2}{*}{ 190: 4} & Upper half & $<1,860$ & $<1,890$ & $<1,880$ \\
\hline S97T000762 & & Lower half & $<1,980$ & $<1,960$ & $<1,970$ \\
\hline S97T000741 & \multirow[t]{2}{*}{ 190: 5} & Upper half & $<2,050$ & $<2,030$ & $<2,040$ \\
\hline S97T000763 & & Lower half & $<1,990$ & $<2,000$ & $<2,000$ \\
\hline S97T000742 & \multirow[t]{2}{*}{ 190: 6} & Upper half & $<2,040$ & $<2,060$ & $<2,050$ \\
\hline S97T000764 & & Lower half & $<2,010$ & $<2,020$ & $<2,020$ \\
\hline S97T000743 & \multirow[t]{2}{*}{ 190: 7} & Upper half & $<2,050$ & $<2,040$ & $<2,050$ \\
\hline S97T000765 & & Lower half & $<2,050$ & $<2,020$ & $<2,040$ \\
\hline S97T000744 & \multirow[t]{2}{*}{ 190: 8} & Upper half & $<2,070$ & $<2,020$ & $<2,050$ \\
\hline S97T000766 & & Lower half & $<2,060$ & $<2,020$ & $<2,040$ \\
\hline S97T000745 & \multirow[t]{2}{*}{ 190: 9} & Upper half & $<2,050$ & $<2,030$ & $<2,040$ \\
\hline S97T000767 & & Lower half & $<2,010$ & $<2,030$ & $<2,020$ \\
\hline S97T001734 & Core 190 & Solid composite & $<1,960$ & $<1,950$ & $<1,960$ \\
\hline 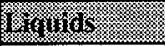 & & 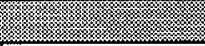 & . 148 & 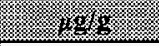 & 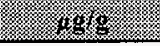 \\
\hline S97T000733 & 190: 1R & Drainable liquid & $<7.27$ & $<7.27$ & $<7.27$ \\
\hline
\end{tabular}


Table B2-147. Tank 241-T-203 Analytical Results: Selenium (ICP).

\begin{tabular}{|c|c|c|c|c|c|}
\hline $\begin{array}{l}\text { Saming } \\
\text { Vimint }\end{array}$ & Simple & Sornule & mesin: & Buplisats & wern \\
\hline \multicolumn{2}{|l|}{ 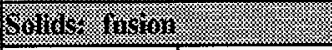 } & (:3) & 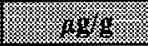 & 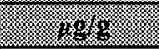 & $\sqrt{1.1868}$ \\
\hline S97T000735 & \multirow[t]{2}{*}{ 190: 1} & Upper half & $<1,950$ & $<1,970$ & $<1,960$ \\
\hline S97T000759 & & Lower half & $<2,080$ & $<2,060$ & $<2,070$ \\
\hline S97T000737 & \multirow[t]{2}{*}{ 190: 1R } & Upper half & $<2,010$ & $<2,000$ & $<2,010$ \\
\hline S97T000760 & & Lower half & $<1,970$ & $<1,860$ & $<1,920$ \\
\hline S97T000738 & \multirow[t]{2}{*}{ 190: 2} & Upper half & $<1,950$ & $<2,000$ & $<1,980$ \\
\hline S97T000757 & & Lower half & $<1,810$ & $<1,760$ & $<1,790$ \\
\hline S97T000739 & \multirow[t]{2}{*}{ 190: 3} & Upper half & $<2,070$ & $<2,110$ & $<2,090$ \\
\hline S97T000761 & & Lower half & $<1,890$ & $<1,910$ & $<1,900$ \\
\hline S97T000740 & \multirow[t]{2}{*}{ 190: 4} & Upper half & $<1,860$ & $<1,890$ & $<1,880$ \\
\hline S97T000762 & & Lower half & $<1,980$ & $<1,960$ & $<1,970$ \\
\hline S97T000741 & \multirow[t]{2}{*}{ 190: 5} & Upper half & $<2,050$ & $<2,030$ & $<2,040$ \\
\hline S97T000763 & & Lower half & $<1,990$ & $<2,000$ & $<2,000$ \\
\hline S97T000742 & \multirow[t]{2}{*}{ 190: 6} & Upper half & $<2,040$ & $<2,060$ & $<2,050$ \\
\hline S97T000764 & & Lower half & $<2,010$ & $<2,020$ & $<2,020$ \\
\hline S97T000743 & \multirow[t]{2}{*}{ 190: 7} & Upper half & $<2,050$ & $<2,040$ & $<2,050$ \\
\hline S97T000765 & & Lower half & $<2,050$ & $<2,020$ & $<2,040$ \\
\hline S97T000744 & \multirow[t]{2}{*}{ 190: 8} & Upper half & $<2,070$ & $<2,020$ & $<2,050$ \\
\hline S97T000766 & & Lower half & $<2,060$ & $<2,020$ & $<2,040$ \\
\hline S97T000745 & \multirow[t]{2}{*}{ 190: 9} & Upper half & $<2,050$ & $<2,030$ & $<2,040$ \\
\hline S97T000767 & & Lower half & $<2,010$ & $<2,030$ & $<2,020$ \\
\hline S97T001734 & Core 190 & Solid composite & $<1,960$ & $<1,950$ & $<1,960$ \\
\hline \multicolumn{2}{|l|}{ minutis } & 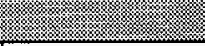 & (1) & 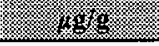 & 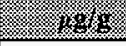 \\
\hline S97T000733 & 190: 1R & Drainable liquid & $<7.27$ & $<7.27$ & $<7.27$ \\
\hline
\end{tabular}


HNF-1501 Rev. 0

Table B2-148. Tank 241-T-203 Analytical Results: Silicon (ICP).

\begin{tabular}{|c|c|c|c|c|c|}
\hline Sinnale & Samaple & 8ringli, & (r. & 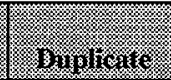 & ntar \\
\hline \multicolumn{3}{|c|}{ 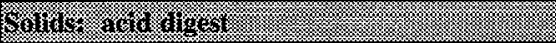 } & 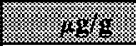 & 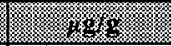 & 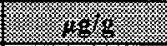 \\
\hline S97T001017 & Core 190 & Solid composite & 1,690 & 1510 & $1600^{\mathrm{QC:b}}$ \\
\hline \multicolumn{3}{|c|}{ 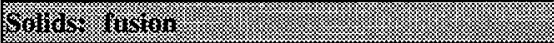 } & (1) & 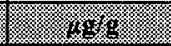 & (3.6\% \\
\hline S97T000735 & \multirow[t]{2}{*}{ 190: 1} & Upper half & $<975$ & 1,050 & $<1,010$ \\
\hline S97T000759 & & Lower half & $<1,040$ & $<1,030$ & $<1,040$ \\
\hline S97T000737 & \multirow[t]{2}{*}{ 190: $1 R$} & Upper half & 1,060 & $<1,000$ & $<1,030$ \\
\hline S97T000760 & & Lower half & 6,270 & $<929$ & $<3600^{\text {ec:e }}$ \\
\hline S97T000738 & \multirow[t]{2}{*}{ 190: 2} & Upper half & $<976$ & 1,190 & $<1,080$ \\
\hline S97T000757 & & Lower half & 2,210 & 1,100 & $1660^{\text {eC:e }}$ \\
\hline S97T000739 & \multirow[t]{2}{*}{ 190: 3} & Upper half & 1,060 & $<1,060$ & $<1,060$ \\
\hline S97T000761 & & Lower half & $<947$ & $<956$ & $<952$ \\
\hline S97T000740 & \multirow[t]{2}{*}{ 190: 4} & Upper half & $<931$ & $<945$ & $<938$ \\
\hline S97T000762 & & Lower half & $<989$ & 1,310 & $<1,150^{\mathrm{QC}: \mathrm{e}}$ \\
\hline S97T000741 & \multirow[t]{2}{*}{ 190: 5} & Upper half & $<1,020$ & $<1,010$ & $<1,020$ \\
\hline S97T000763 & & Lower half & $<995$ & $<1,000$ & $<998$ \\
\hline S97T000742 & \multirow[t]{2}{*}{ 190: 6} & Upper half & $<1,020$ & $<1,030$ & $<1,030$ \\
\hline S97T000764 & & Lower half & $<1,010$ & $<1,010$ & $<1,010$ \\
\hline S97T000743 & \multirow[t]{2}{*}{ 190: 7} & Upper half & $<1,020$ & $<1,020$ & $<1,020$ \\
\hline S97T000765 & & Lower half & $<1,030$ & 1,080 & $<1,060$ \\
\hline S97T000744 & \multirow[t]{2}{*}{ 190: 8} & Upper half & $<1,030$ & $<1,010$ & $<1,020$ \\
\hline S97T000766 & & Lower half & 1,100 & $<1,010$ & $<1,060$ \\
\hline S97T000745 & \multirow[t]{2}{*}{ 190: 9} & Upper half & $<1,020$ & $<1,020$ & $<1,020$ \\
\hline S97T000767 & & Lower half & 1,280 & $<1,010$ & $<1,150^{\text {ec:e }}$ \\
\hline S97T001734 & Core 190 & Solid composite & 1,570 & 1,770 & 1,670 \\
\hline Exaido & 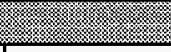 & 40 & $=1.98$ & . 1898 & 40.989 \\
\hline S97T000733 & 190: 1R & Drainable liquid & 35.8 & 38.8 & 37.3 \\
\hline
\end{tabular}


Table B2-149. Tank 241-T-203 Analytical Results: Silver (ICP).

\begin{tabular}{|c|c|c|c|c|c|}
\hline $\begin{array}{l}\text { Q.ample } \\
\text { Tumber }\end{array}$ & H Sampie & 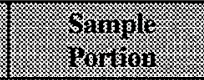 & insuls: & 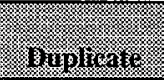 & 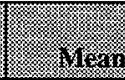 \\
\hline 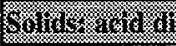 & ger & 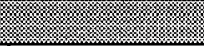 & 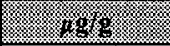 & 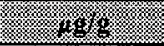 & . \\
\hline S97T001017 & Core 190 & Solid composite & 2.41 & $<2$ & $<2.21$ \\
\hline 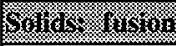 & 1 & 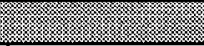 & 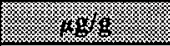 & 6.1048 & 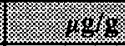 \\
\hline S97T000735 & \multirow[t]{2}{*}{ 190: 1} & Upper half & $<195$ & $<197$ & $<196$ \\
\hline S97T000759 & & Lower half & $<208$ & $<206$ & $<207$ \\
\hline S97T000737 & \multirow[t]{2}{*}{ 190: $1 R$} & Upper half & $<201$ & $<200$ & $<201$ \\
\hline S97T000760 & & Lower half & $<197$ & $<186$ & $<192$ \\
\hline S97T000738 & \multirow[t]{2}{*}{ 190: 2} & Upper half & $<195$ & $<200$ & $<198$ \\
\hline S97T000757 & & Lower half & $<181$ & $<176$ & $<179^{\mathrm{eC}: \mathrm{c}}$ \\
\hline S97T000739 & \multirow[t]{2}{*}{ 190: 3} & Upper half & $<207$ & $<211$ & $<209$ \\
\hline S97T000761 & & Lower half & $<189$ & $<191$ & $<190$ \\
\hline S97T000740 & \multirow[t]{2}{*}{ 190: 4} & Upper half & $<186$ & $<189$ & $<188$ \\
\hline S97T000762 & & Lower half & $<198$ & $<196$ & $<197$ \\
\hline S97T000741 & \multirow[t]{2}{*}{$190: 5$} & Upper half & $<205$ & $<203$ & $<204$ \\
\hline S97T000763 & & Lower half & $<199$ & $<200$ & $<200$ \\
\hline S97T000742 & \multirow[t]{2}{*}{ 190: 6} & Upper half & $<204$ & $<206$ & $<205$ \\
\hline S97T000764 & & Lower half & $<201$ & $<202$ & $<202$ \\
\hline S97T000743 & \multirow[t]{2}{*}{ 190: 7} & Upper half & $<205$ & $<204$ & $<205$ \\
\hline S97T000765 & & Lower half & $<205$ & $<202$ & $<204$ \\
\hline S97T000744 & \multirow[t]{2}{*}{ 190: 8} & Upper half & $<207$ & $<202$ & $<205$ \\
\hline S97T000766 & & Lower half & $<206$ & $<202$ & $<204$ \\
\hline S97T000745 & \multirow[t]{2}{*}{ 190: 9} & Upper half & $<205$ & $<203$ & $<204$ \\
\hline S97T000767 & & Lower half & $<201$ & $<203$ & $<202$ \\
\hline S97T001734 & Core 190 & Solid composite & $<196$ & $<195$ & $<196$ \\
\hline 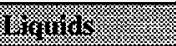 & $\sqrt{4}$ & (3) & 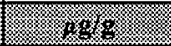 & 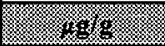 & $8.18 .68 \%$ \\
\hline S97T000733 & 190: 1R & Drainable liquid & 2.12 & 2.09 & 2.11 \\
\hline
\end{tabular}


HNF-1501 Rev. 0

Table B2-150. Tank 241-T-203 Analytical Results: Sodium (ICP).

\begin{tabular}{|c|c|c|c|c|c|}
\hline Samingres & S.minge & $\begin{array}{l}\text { Simple } \\
\text { Thidron }\end{array}$ & 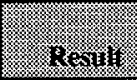 & 3ryilivate & 1ran \\
\hline \multicolumn{3}{|c|}{$60116 \%$ aridi $61 \mathrm{ges}$} & 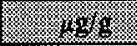 & 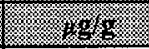 & 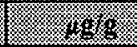 \\
\hline S97T001017 & Core 190 & Solid composite & 35,300 & 34,300 & $34,800^{\text {QC:c }}$ \\
\hline \multicolumn{3}{|c|}{ 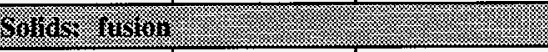 } & 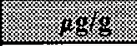 & 28.1898 & 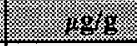 \\
\hline S97T000735 & \multirow[t]{2}{*}{ 190: 1} & Upper half & 36,600 & 36,900 & 36,800 \\
\hline S97T000759 & & Lower half & 36,000 & 36,900 & 36,500 \\
\hline S97T000737 & \multirow[t]{2}{*}{ 190: 1R } & Upper half & 36,600 & 36,000 & 36,300 \\
\hline S97T000760 & & Lower half & 36,700 & 36,400 & 36,600 \\
\hline S97T000738 & \multirow[t]{2}{*}{ 190: 2} & Upper half & 34,400 & 35,300 & 34,900 \\
\hline S97T000757 & & Lower half & 34,700 & 35,500 & 35,100 \\
\hline S97T000739 & \multirow[t]{2}{*}{ 190: 3} & Upper half & 31,800 & 32,400 & 32,100 \\
\hline S97T000761 & & Lower half & 33,100 & 33,400 & 33,300 \\
\hline S97T000740 & \multirow[t]{2}{*}{ 190: 4} & Upper half & 33,100 & 32,900 & 33,000 \\
\hline S97T000762 & & Lower half & 35,200 & 29,800 & 32,500 \\
\hline S97T000741 & \multirow[t]{2}{*}{ 190: 5} & Upper half & 33,800 & 33,500 & 33,700 \\
\hline S97T000763 & & Lower half & 33,700 & 35,100 & 34,400 \\
\hline \$97T000742 & \multirow[t]{2}{*}{ 190: 6} & Upper half & 34,500 & 35,200 & 34,900 \\
\hline S97T000764 & & Lower half & 34,100 & 33,900 & 34,000 \\
\hline S97T000743 & \multirow[t]{2}{*}{ 190: 7} & Upper half & 34,900 & 35,000 & 35,000 \\
\hline S97T000765 & & Lower half & 33,800 & 34,100 & 34,000 \\
\hline S97T000744 & \multirow[t]{2}{*}{ 190: 8} & Upper half & 34,100 & 34,600 & 34,400 \\
\hline S97T000766 & & Lower half & 34,600 & 34,400 & 34,500 \\
\hline S97T000745 & \multirow[t]{2}{*}{ 190: 9} & Upper half & 34,300 & 34,900 & 34,600 \\
\hline S97T000767 & & Lower half & 34,100 & 34,000 & 34,100 \\
\hline S97T001734 & Core 190 & Solid composite & 44,700 & 44,900 & 44,800 \\
\hline Matidis & & 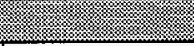 & 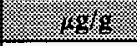 & f & 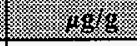 \\
\hline S97T000733 & 190: $1 \mathrm{R}$ & Drainable liquid & 29,600 & 29,600 & $29,600^{\mathrm{OC}: \mathrm{d}}$ \\
\hline
\end{tabular}


HNF-1501 Rev. 0

Table B2-151. Tank 241-T-203 Analytical Results: Strontium (ICP).

\begin{tabular}{|c|c|c|c|c|c|}
\hline Shmalc & 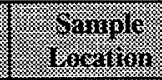 & $\begin{array}{l}\text { Samigle } \\
\text { rom }\end{array}$ & resili: & Bullionte & 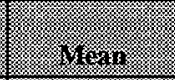 \\
\hline \multicolumn{2}{|c|}{ 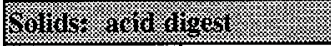 } & יר & 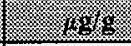 & 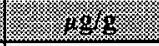 & $18-1.96$ \\
\hline S97T001017 & Core 190 & Solid composite & 570 & 544 & 557 \\
\hline \multicolumn{2}{|c|}{ W } & & 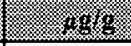 & 8.698 & 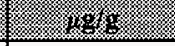 \\
\hline S97T000735 & \multirow[t]{2}{*}{$190: 1$} & Upper half & 409 & 412 & 411 \\
\hline S97T000759 & & Lower half & 437 & 478 & 458 \\
\hline S97T000737 & \multirow[t]{2}{*}{ 190: 1R } & Upper half & 419 & 413 & 416 \\
\hline S97T000760 & & Lower half & 255 & 284 & 270 \\
\hline S97T000738 & \multirow[t]{2}{*}{ 190: 2} & Upper half & 229 & 240 & 235 \\
\hline S97T000757 & & Lower half & 342 & 335 & 339 \\
\hline \$97T000739 & \multirow[t]{2}{*}{ 190: 3} & Upper half & 334 & 328 & 331 \\
\hline S97T000761 & & Lower half & 450 & 454 & 452 \\
\hline S97T000740 & \multirow[t]{2}{*}{ 190: 4} & Upper half & 358 & 340 & 349 \\
\hline S97T000762 & & Lower half & 639 & 487 & $563^{Q C: e}$ \\
\hline S97T000741 & \multirow[t]{2}{*}{ 190: 5} & Upper half & 515 & 529 & 522 \\
\hline S97T000763 & & Lower half & 507 & 640 & $574^{\mathrm{CO}: \mathrm{e}}$ \\
\hline \$97T000742 & \multirow[t]{2}{*}{ 190: 6} & Upper half & 556 & 560 & 558 \\
\hline S97T000764 & & Lower half & 587 & 603 & 595 \\
\hline S97T000743 & \multirow[t]{2}{*}{ 190: 7} & Upper half & 583 & 580 & 582 \\
\hline \$97T000765 & & Lower half & 589 & $589^{\circ}$ & 589 \\
\hline S97T000744 & \multirow[t]{2}{*}{ 190: 8} & Upper half & 729 & 748 & 739 \\
\hline S97T000766 & & Lower half & 671 & 780 & 726 \\
\hline S97T000745 & \multirow[t]{2}{*}{ 190: 9} & Upper half & 639 & 676 & 658 \\
\hline S97T000767 & & Lower half & 696 & 683 & 690 \\
\hline S97T001734 & Core 190 & Solid composite & 689 & 695 & 692 \\
\hline Nonge & 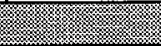 & (1) & 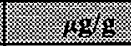 & 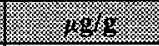 & (3) \\
\hline \$97T000733 & 190: 1R & Drainable liquid & $<0.727$ & $<0.727$ & $<0.727$ \\
\hline
\end{tabular}


Table B2-152. Tank 241-T-203 Analytical Results: Sulfur (ICP).

\begin{tabular}{|c|c|c|c|c|c|}
\hline S. & 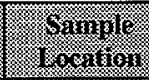 & $\begin{array}{l}\text { Sinple } \\
\text { Vortori }\end{array}$ & iresinis: & Gijoligals & ment \\
\hline \multicolumn{2}{|c|}{ 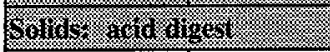 } & 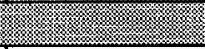 & 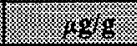 & $\sqrt{1.68}$ & 8.496 \\
\hline S97T001017 & Core 190 & Solid composite & 124 & 131 & 128 \\
\hline 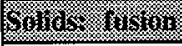 & & 2: & ( & 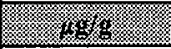 & 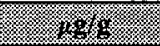 \\
\hline S97T000735 & \multirow[t]{2}{*}{ 190: 1} & Upper half & $<1,950$ & $<1,970$ & $<1,960$ \\
\hline S97T000759 & & Lower half & $<2,080$ & $<2,060$ & $<2,070$ \\
\hline S97T000737 & \multirow[t]{2}{*}{ 190: $1 \mathrm{R}$} & Upper half & $<2,010$ & $<2,000$ & $<2,010$ \\
\hline S97T000760 & & Lower half & $<1,970$ & $<1,860$ & $<1,920$ \\
\hline S97T000738 & \multirow[t]{2}{*}{ 190: 2} & Upper half & $<1,950$ & $<2,000$ & $<1,980$ \\
\hline S97T000757 & & Lower half & $<1,810$ & $<1,760$ & $<1,790$ \\
\hline S97T000739 & \multirow[t]{2}{*}{ 190: 3} & Upper half & $<2,070$ & $<2,110$ & $<2,090$ \\
\hline S97T000761 & & Lower half & $<1,890$ & $<1,910$ & $<1,900$ \\
\hline S97T000740 & \multirow[t]{2}{*}{ 190: 4} & Upper half & $<1,860$ & $<1,890$ & $<1,880$ \\
\hline S97T000762 & & Lower half & $<1,980$ & $<1,960$ & $<1,970$ \\
\hline S97T000741 & \multirow[t]{2}{*}{ 190: 5} & Upper half & $<2,050$ & $<2,030$ & $<2,040$ \\
\hline S97T000763 & & Lower half & $<1,990$ & $<2,000$ & $<2,000$ \\
\hline S97T000742 & \multirow[t]{2}{*}{ 190: 6} & Upper half & $<2,040$ & $<2,060$ & $<2,050$ \\
\hline S97T000764 & & Lower half & $<2,010$ & $<2,020$ & $<2,020$ \\
\hline S97T000743 & \multirow[t]{2}{*}{ 190: 7} & Upper half & $<2,050$ & $<2,040$ & $<2,050$ \\
\hline S97T000765 & & Lower half & $<2,050$ & $<2,020$ & $<2,040$ \\
\hline S97T000744 & \multirow[t]{2}{*}{ 190: 8} & Upper half & $<2,070$ & $<2,020$ & $<2,050$ \\
\hline S97T000766 & & Lower half & $<2,060$ & $<2,020$ & $<2,040$ \\
\hline S97T000745 & \multirow[t]{2}{*}{ 190: 9} & Upper half & $<2,050$ & $<2,030$ & $<2,040$ \\
\hline S97T000767 & & Lower half & $<2,010$ & $<2,030$ & $<2,020$ \\
\hline S97T001734 & Core 190 & Solid composite & $<1,960$ & $<1,950$ & $<1,960$ \\
\hline Ligua & & 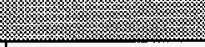 & 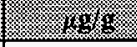 & 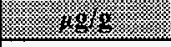 & 2.0898 \\
\hline S97T000733 & 190: 1R & Drainable liquid & 89.9 & 89.9 & 89.9 \\
\hline
\end{tabular}


Table B2-153. Tank 241-T-203 Analytical Results: Thallium (ICP).

\begin{tabular}{|c|c|c|c|c|c|}
\hline Sampt & Fi & fl & 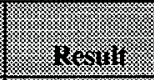 & Divinicale & , \\
\hline \multicolumn{3}{|c|}{ 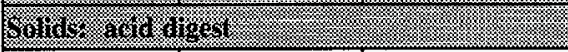 } & $4.86 \%$ & $3.868=8$ & $1.48 \mathrm{~s}$ \\
\hline \$97T001017 & Core 190 & Solid composite & $<39.9$ & $<39.9$ & $<39.9$ \\
\hline \multicolumn{2}{|c|}{ 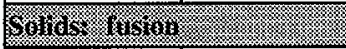 } & $\sqrt{4.83}$ & 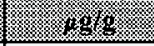 & $1.68 y_{8}$ & 1.8 .89 \\
\hline S97T000735 & \multirow[t]{2}{*}{ 190: 1} & Upper half & $<3,900$ & $<3,930$ & $<3,920$ \\
\hline S97T000759 & & Lower half & $<4,150$ & $<4,120$ & $<4,140$ \\
\hline S97T000737 & \multirow[t]{2}{*}{ 190: $1 \mathrm{R}$} & Upper half & $<4,030$ & $<4,000$ & $<4,020$ \\
\hline S97T000760 & & Lower half & $<3,930$ & $<3,720$ & $<3,830$ \\
\hline S97T000738 & \multirow[t]{2}{*}{$190: 2$} & Upper half & $<3,910$ & $<4,000$ & $<3,960$ \\
\hline S97T000757 & & Lower half & $<3,620$ & $<3,530$ & $<3,580$ \\
\hline \$97T000739 & \multirow[t]{2}{*}{ 190: 3} & Upper half & $<4,140$ & $<4,230$ & $<4,190$ \\
\hline S97T000761 & & Lower half & $<3,790$ & $<3,820$ & $<3,810$ \\
\hline S97T000740 & \multirow[t]{2}{*}{$190: 4$} & Upper half & $<3,720$ & $<3,780$ & $<3,750$ \\
\hline S97T000762 & & Lower half & $<3,960$ & $<3,920$ & $<3,940$ \\
\hline S97T000741 & \multirow[t]{2}{*}{ 190: 5} & Upper half & $<4,090$ & $<4,050$ & $<4,070$ \\
\hline S97T000763 & & Lower half & $<3,980$ & $<4,010$ & $<4,000$ \\
\hline S97T000742 & \multirow[t]{2}{*}{ 190: 6} & Upper half & $<4,080$ & $<4,120$ & $<4,100$ \\
\hline S97T000764 & & Lower half & $<4,030$ & $<4,050$ & $<4,040$ \\
\hline S97T000743 & \multirow[t]{2}{*}{ 190: 7} & Upper half & $<4,100$ & $<4,080$ & $<4,090$ \\
\hline S97T000765 & & Lower half & $<4,100$ & $<4,030$ & $<4,070$ \\
\hline S97T000744 & \multirow[t]{2}{*}{ 190: 8} & Upper half & $<4,130$ & $<4,040$ & $<4,090$ \\
\hline S97T000766 & & Lower half & $<4,110$ & $<4,050$ & $<4,080$ \\
\hline S97T000745 & \multirow[t]{2}{*}{ 190: 9} & Upper half & $<4,090$ & $<4,060$ & $<4,080$ \\
\hline S97T000767 & & Lower half & $<4,030$ & $<4,060$ & $<4,050$ \\
\hline S97T001734 & Core 190 & Solid composite & $<3,910$ & $<3,890$ & $<3,900$ \\
\hline Marmas = & (1) & 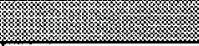 & 4.645 & 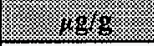 & 8.1 .8 \\
\hline S97T000733 & 190: 1R & Drainable liquid & $<14.5$ & $<14.5$ & $<14.5$ \\
\hline
\end{tabular}


Table B2-154. Tank 241-T-203 Analytical Results: Titanium (ICP).

\begin{tabular}{|c|c|c|c|c|c|}
\hline $\begin{array}{l}\text { Samples } \\
\text { Vomber }\end{array}$ & \& Samole & Sample & Iresill: & Yiringerite & iresin \\
\hline \multicolumn{3}{|c|}{ 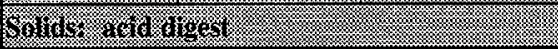 } & 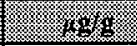 & 28 & 8.838 \\
\hline S97T001017 & Core 190 & Solid composite & 3.2 & 3.34 & 3.27 \\
\hline \multicolumn{3}{|c|}{ Solido: . } & 18. & (3) & 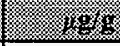 \\
\hline S97T000735 & \multirow[t]{2}{*}{ 190: 1} & Upper half & $<195$ & $<197$ & $<196$ \\
\hline S97T000759 & & Lower half & $<208$ & $<206$ & $<207$ \\
\hline S97T000737 & \multirow[t]{2}{*}{ 190: 1R } & Upper half & $<201$ & $<200$ & $<201$ \\
\hline S97T000760 & & Lower half & $<197$ & $<186$ & $<192$ \\
\hline S97T000738 & \multirow[t]{2}{*}{ 190: 2} & Upper half & $<195$ & $<200$ & $<198$ \\
\hline S97T000757 & & Lower half & $<181$ & $<176$ & $<179$ \\
\hline S97T000739 & \multirow[t]{2}{*}{ 190: 3} & Upper half & $<207$ & $<211$ & $<209$ \\
\hline S97T000761 & & Lower half & $<189$ & $<191$ & $<190$ \\
\hline S97T000740 & \multirow[t]{2}{*}{ 190: 4} & Upper half & $<186$ & $<189$ & $<188$ \\
\hline S97T000762 & & Lower half & $<198$ & $<196$ & $<197$ \\
\hline S97T000741 & \multirow[t]{2}{*}{ 190: 5} & Upper half & $<205$ & $<203$ & $<204$ \\
\hline S97T000763 & & Lower half & $<199$ & $<200$ & $<200$ \\
\hline S97T000742 & \multirow[t]{2}{*}{ 190: 6} & Upper half & $<204$ & $<206$ & $<205$ \\
\hline S97T000764 & & Lower half & $<201$ & $<202$ & $<202$ \\
\hline S97T000743 & \multirow[t]{2}{*}{ 190: 7} & Upper half & $<205$ & $<204$ & $<205$ \\
\hline S97T000765 & & Lower half & $<205$ & $<202$ & $<204$ \\
\hline S97T000744 & \multirow[t]{2}{*}{ 190: 8} & Upper half & $<207$ & $<202$ & $<205$ \\
\hline S97T000766 & & Lower half & $<206$ & $<202$ & $<204$ \\
\hline S97T000745 & \multirow[t]{2}{*}{ 190: 9} & Upper half & $<205$ & $<203$ & $<204$ \\
\hline S97T000767 & & Lower half & $<201$ & $<203$ & $<202$ \\
\hline S97T001734 & Core 190 & Solid composite & $<196$ & $<195$ & $<196$ \\
\hline (1) & ${ }_{2}$ & 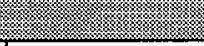 & 1385 & $4=84.48$ & 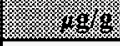 \\
\hline S97T000733 & 190: 1R & Drainable liquid & $<0.727$ & $<0.727$ & $<0.727$ \\
\hline
\end{tabular}


HNF-1501 Rev. 0

Table B2-155. Tank 241-T-203 Analytical Results: Total Uranium (ICP).

\begin{tabular}{|c|c|c|c|c|c|}
\hline Sumila & vaning & Sagniple & renul: & Bingligate. & 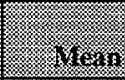 \\
\hline \multicolumn{2}{|c|}{ 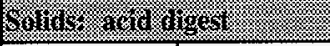 } & & (3. & 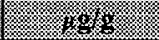 & 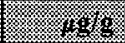 \\
\hline S97T001017 & Core 190 & Solid composite & $<99.8$ & $<99.8$ & $<99.8$ \\
\hline \multicolumn{2}{|l|}{ 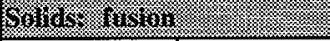 } & 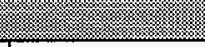 & 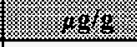 & 4.898 & 8.89 \\
\hline S97T000735 & \multirow[t]{2}{*}{ 190: 1} & Upper half & $<9,750$ & $<9,840$ & $<9,800$ \\
\hline \$97T000759 & & Lower half & $<10,400$ & $<10,300$ & $<10,400$ \\
\hline S97T000737 & \multirow[t]{2}{*}{ 190: $1 \mathrm{R}$} & Upper half & $<10,100$ & $<10,000$ & $<10,100$ \\
\hline S97T000760 & & Lower half & $<9,830$ & $<9,290$ & $<9,560$ \\
\hline S97T000738 & \multirow[t]{2}{*}{ 190: 2} & Upper half & $<9,760$ & $<9,990$ & $<9,880$ \\
\hline S97T000757 & & Lower half & $<9,040$ & $<8810$ & $<8,930$ \\
\hline S97T000739 & \multirow[t]{2}{*}{$190: 3$} & Upper half & $<10,300$ & $<10,600$ & $<10,500$ \\
\hline S97T000761 & & Lower half & $<9470$ & $<9,560$ & $<9,520$ \\
\hline S97T000740 & \multirow[t]{2}{*}{ 190: 4} & Upper half & $<9310$ & $<9,450$ & $<9,380$ \\
\hline S97T000762 & & Lower half & $<9890$ & $<9,810$ & $<9,850$ \\
\hline S97T000741 & \multirow[t]{2}{*}{$190: 5$} & Upper half & $<1,0200$ & $<10,100$ & $<10,200$ \\
\hline S97T000763 & & Lower half & $<9,950$ & $<10,000$ & $<9,980$ \\
\hline S97T000742 & \multirow[t]{2}{*}{ 190: 6} & Upper half & $<10,200$ & $<10,300$ & $<10,300$ \\
\hline S97T000764 & & Lower half & $<10,100$ & $<10,100$ & $<10,100$ \\
\hline S97T000743 & \multirow[t]{2}{*}{ 190: 7} & Upper half & $<10,200$ & $<10,200$ & $<10,200$ \\
\hline S97T000765 & & Lower half & $<10,300$ & $<10,100$ & $<10,200$ \\
\hline S97T000744 & \multirow[t]{2}{*}{ 190: 8} & Upper half & $<10,300$ & $<10,100$ & $<10,200$ \\
\hline S97T000766 & & Lower half & $<10,300$ & $<10,100$ & $<10,200$ \\
\hline S97T000745 & \multirow[t]{2}{*}{ 190: 9} & Upper half & $<10,200$ & $<10,200$ & $<10,200$ \\
\hline S97T000767 & & Lower half & $<10,100$ & $<10,100$ & $<10,100$ \\
\hline S97T001734 & Core 190 & Solid composite & $<9780$ & $<9,740$ & $<9760$ \\
\hline Liguinidy & 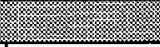 & & 1.96 & 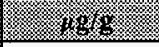 & 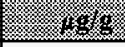 \\
\hline S97T000733 & 190: 1R & Drainable liquid & $<36.3$ & $<36.3$ & $<36.3$ \\
\hline
\end{tabular}


HNF-1501 Rev. 0

Table B2-156. Tank 241-T-203 Analytical Results: Vanadium (ICP).

\begin{tabular}{|c|c|c|c|c|c|}
\hline varupl: & I. S.mpile & 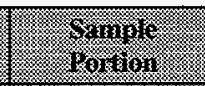 & 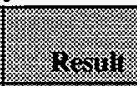 & Bubuteats & 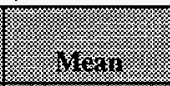 \\
\hline \multicolumn{3}{|c|}{ 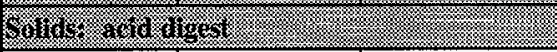 } & (3. & (1) & 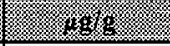 \\
\hline S97T001017 & Core 190 & Solid composite & $<9.98$ & $<9.98$ & $<9.98$ \\
\hline \multicolumn{2}{|c|}{ 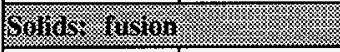 } & 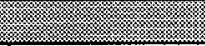 & 3.89 & 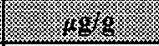 & 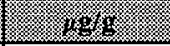 \\
\hline S97T000735 & \multirow[t]{2}{*}{ 190: 1} & Upper half & $<975$ & $<984$ & $<980$ \\
\hline S97T000759 & & Lower half & $<1,040$ & $<1,030$ & $<1,040$ \\
\hline S97T000737 & \multirow[t]{2}{*}{ 190: 1R } & Upper half & $<1,010$ & $<1,000$ & $<1,010$ \\
\hline S97T000760 & & Lower half & $<983$ & $<929$ & $<956$ \\
\hline S97T000738 & \multirow[t]{2}{*}{ 190: 2} & Upper half & $<976$ & $<999$ & $<988$ \\
\hline S97T000757 & & Lower half & $<904$ & $<881$ & $<893$ \\
\hline S97T000739 & \multirow[t]{2}{*}{ 190: 3} & Upper half & $<1,030$ & $<1,060$ & $<1,050$ \\
\hline S97T000761 & & Lower half & $<947$ & $<956$ & $<952$ \\
\hline S97T000740 & \multirow[t]{2}{*}{$190: 4$} & Upper half & $<931$ & $<945$ & $<938$ \\
\hline S97T000762 & & Lower half & $<989$ & $<981$ & $<985$ \\
\hline S97T000741 & \multirow[t]{2}{*}{ 190: 5} & Upper half & $<1,020$ & $<1,010$ & $<1,020$ \\
\hline S97T000763 & & Lower half & $<995$ & $<1,000$ & $<998$ \\
\hline S97T000742 & \multirow[t]{2}{*}{ 190: 6} & Upper half & $<1,020$ & $<1,030$ & $<1,030$ \\
\hline S97T000764 & & Lower half & $<1,010$ & $<1,010$ & $<1,010$ \\
\hline S97T000743 & \multirow[t]{2}{*}{ 190: 7} & Upper half & $<1,020$ & $<1,020$ & $<1,020$ \\
\hline S97T000765 & & Lower half & $<1,030$ & $<1,010$ & $<1,020$ \\
\hline S97T000744 & \multirow[t]{2}{*}{ 190: 8} & Upper half & $<1,030$ & $<1,010$ & $<1,020$ \\
\hline S97T000766 & & Lower half & $<1,030$ & $<1,010$ & $<1,020$ \\
\hline S97T000745 & \multirow[t]{2}{*}{ 190: 9} & Upper half & $<1,020$ & $<1,020$ & $<1,020$ \\
\hline S97T000767 & & Lower half & $<1,010$ & $<1,010$ & $<1,010$ \\
\hline S97T001734 & Core 190 & Solid composite & $<978$ & $<974$ & $<976$ \\
\hline Lroud & 4 & $\sqrt{4}$ & .1868 & 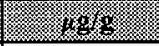 & 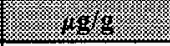 \\
\hline S97T000733 & 190: 1R & Drainable liquid & $<3.63$ & $<3.63$ & $<3.63$ \\
\hline
\end{tabular}


Table B2-157. Tank 241-T-203 Analytical Results: Zinc (ICP).

\begin{tabular}{|c|c|c|c|c|c|}
\hline $\begin{array}{l}\text { Sample: } \\
\text { Vimmbr: }\end{array}$ & S Yample & 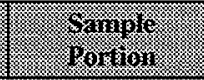 & Iesuin: & Bunilicate & 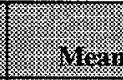 \\
\hline \multicolumn{2}{|c|}{ 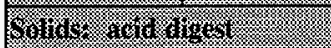 } & 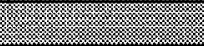 & 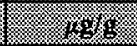 & $18=1.98$ & 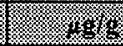 \\
\hline \$97T001017 & Core 190 & Solid composite & 20.7 & 20.5 & 20.6 \\
\hline \multicolumn{2}{|c|}{ 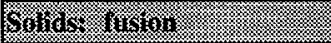 } & (1) & 1. & 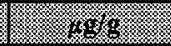 & 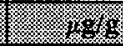 \\
\hline S97T000735 & \multirow[t]{2}{*}{ 190: 1} & Upper half & $<195$ & $<197$ & $<196$ \\
\hline S97T000759 & & Lower half & $<208$ & $<206$ & $<207$ \\
\hline S97T000737 & \multirow[t]{2}{*}{ 190: $1 \mathrm{R}$} & Upper half & $<201$ & $<200$ & $<201$ \\
\hline S97T000760 & & Lower half & $<197$ & $<186$ & $<192$ \\
\hline S97T000738 & \multirow[t]{2}{*}{ 190: 2} & Upper half & $<195$ & $<200$ & $<198$ \\
\hline S97T000757 & & Lower half & $<181$ & $<176$ & $<179$ \\
\hline S97T000739 & \multirow[t]{2}{*}{ 190: 3} & Upper half & $<207$ & $<211$ & $<209$ \\
\hline S97T000761 & & Lower half & $<189$ & $<191$ & $<190$ \\
\hline S97T000740 & \multirow[t]{2}{*}{ 190: 4} & Upper half & $<186$ & $<189$ & $<188$ \\
\hline S97T000762 & & Lower half & $<198$ & $<196$ & $<197$ \\
\hline S97T000741 & \multirow[t]{2}{*}{$190: 5$} & Upper half & $<205$ & $<203$ & $<204$ \\
\hline S97T000763 & & Lower half & $<199$ & $<200$ & $<200$ \\
\hline S97T000742 & \multirow[t]{2}{*}{ 190: 6} & Upper half & $<204$ & $<206$ & $<205$ \\
\hline S97T000764 & & Lower half & $<201$ & $<202$ & $<202$ \\
\hline S97T000743 & \multirow[t]{2}{*}{ 190: 7} & Upper half & $<205$ & $<204$ & $<205$ \\
\hline S97T000765 & & Lower half & $<205$ & $<202$ & $<204$ \\
\hline S97T000744 & \multirow[t]{2}{*}{ 190: 8} & Upper half & $<207$ & $<202$ & $<205$ \\
\hline S97T000766 & & Lower half & $<206$ & $<202$ & $<204$ \\
\hline S97T000745 & \multirow[t]{2}{*}{ 190: 9} & Upper half & $<205$ & $<203$ & $<204$ \\
\hline S97T000767 & & Lower half. & $<201$ & $<203$ & $<202$ \\
\hline S97T001734 & Core 190 & Solid composite & $<196$ & $<195$ & $<196$ \\
\hline Hownon & (1) & 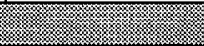 & $14,5 \mathrm{~g}$ & (1) & 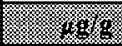 \\
\hline S97T000733 & 190: 1R & Drainable liquid & $<0.727$ & $<0.727$ & $<0.727$ \\
\hline
\end{tabular}


HINF-1501 Rev. 0

Table B2-158. Tank 241-T-203 Analytical Results: Zirconium (ICP).

\begin{tabular}{|c|c|c|c|c|c|}
\hline Samigl: & 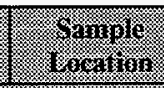 & 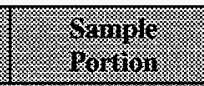 & 96531) & Buyluease & 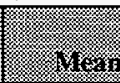 \\
\hline \multicolumn{2}{|c|}{ 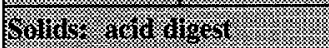 } & (1) & (3) & 3.1368 & 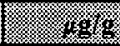 \\
\hline S97T001017 & Core 190 & Solid composite & $<2$ & $<2$ & $<2$ \\
\hline \multicolumn{2}{|c|}{ Sohas gungu: } & 4 & $\sqrt{18}+80$ & 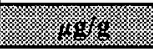 & 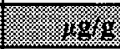 \\
\hline S97T000735 & \multirow[t]{2}{*}{ 190: 1} & Upper half & $<195$ & $<197$ & $<196$ \\
\hline S97T000759 & & Lower half & $<208$ & $<206$ & $<207$ \\
\hline S97T000737 & \multirow[t]{2}{*}{ 190: 1R } & Upper half & $<201$ & $<200$ & $<201$ \\
\hline S97T000760 & & Lower half & $<197$ & $<186$ & $<192$ \\
\hline S97T000738 & \multirow[t]{2}{*}{ 190: 2} & Upper half & $<195$ & $<200$ & $<198$ \\
\hline S97T000757 & & Lower half & $<181$ & $<176$ & $<179$ \\
\hline S97T000739 & \multirow[t]{2}{*}{ 190: 3} & Upper half & $<207$ & $<211$ & $<209$ \\
\hline S97T000761 & & Lower half & $<189$ & $<191$ & $<190$ \\
\hline S97T000740 & \multirow[t]{2}{*}{$190: 4$} & Upper half & $<186$ & $<189$ & $<188$ \\
\hline S97T000762 & & Lower half & $<198$ & $<196$ & $<197$ \\
\hline S97T000741 & \multirow[t]{2}{*}{$190: 5$} & Upper half & $<205$ & $<203$ & $<204$ \\
\hline S97T000763 & & Lower half & $<199$ & $<200$ & $<200$ \\
\hline S97T000742 & \multirow[t]{2}{*}{ 190: 6} & Upper half & $<204$ & $<206$ & $<205$ \\
\hline S97T000764 & & Lower half & $<201$ & $<202$ & $<202$ \\
\hline S97T000743 & \multirow[t]{2}{*}{ 190: 7} & Upper half & $<205$ & $<204$ & $<205$ \\
\hline S97T000765 & & Lower half & $<205$ & $<202$ & $<204$ \\
\hline S97T000744 & \multirow[t]{2}{*}{ 190: 8} & Upper half & $<207$ & $<202$ & $<205$ \\
\hline S97T000766 & & Lower half & $<206$ & $<202$ & $<204$ \\
\hline S97T000745 & \multirow[t]{2}{*}{ 190: 9} & Upper half & $<205$ & $<203$ & $<204$ \\
\hline S97T000767 & & Lower half & $<201$ & $<203$ & $<202$ \\
\hline S97T001734 & Core 190 & Solid composite & $<196$ & $<195$ & $<196$ \\
\hline Hiroinas & (1) & 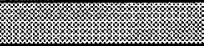 & (3) & (1.) & 8.1 .89 .9 \\
\hline S97T000733 & 190: 1R & Drainable liquid & $<0.727$ & $<0.727$ & $<0.727$ \\
\hline
\end{tabular}


HNF-1501 Rev. 0

Table B2-159. Tank 241-T-203 Analytical Results: Bromide (IC).

\begin{tabular}{|c|c|c|c|c|c|}
\hline S.mingle & Sominge & 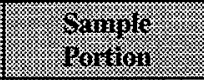 & resulin & Divilicate: & 14tan \\
\hline \multicolumn{3}{|c|}{ 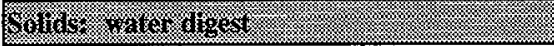 } & (3) & 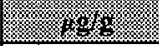 & 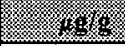 \\
\hline S97T000736 & \multirow[t]{2}{*}{ 190: 1} & Upper half & $<261$ & $<260$ & $<261$ \\
\hline S97T000768 & & Lower half & $<269$ & $<265$ & $<267$ \\
\hline S97T000746 & \multirow[t]{2}{*}{ 190: 1R } & Upper half & $<278$ & $<271$ & $<274$ \\
\hline S97T000769 & & Lower half & $<229$ & $<225$ & $<227$ \\
\hline S97T000747 & \multirow[t]{2}{*}{ 190: 2} & Upper half & $<262$ & $<259$ & $<260$ \\
\hline S97T000758 & & Lower half & $<275$ & $<270$ & $<273$ \\
\hline S97T000748 & \multirow[t]{2}{*}{ 190: 3} & Upper half & $<264$ & $<258$ & $<261$ \\
\hline S97T000770 & & Lower half & $<230$ & $<232$ & $<231$ \\
\hline S97T000749 & \multirow[t]{2}{*}{ 190: 4} & Upper half & $<256$ & $<246$ & $<251$ \\
\hline S97T000771 & & Lower half & $<264$ & $<267$ & $<266$ \\
\hline S97T000750 & \multirow[t]{2}{*}{ 190: 5} & Upper half & $<268$ & $<274$ & $<271$ \\
\hline S97T000772 & & Lower half & $<270$ & $<266$ & $<268$ \\
\hline S97T000751 & \multirow[t]{2}{*}{ 190: 6} & Upper half & $<519$ & $<525$ & $<522$ \\
\hline S97T000773 & & Lower half & $<274$ & $<270$ & $<272$ \\
\hline S97T000752 & \multirow[t]{2}{*}{ 190: 7} & Upper half & $<268$ & $<272$ & $<270$ \\
\hline S97T000774 & & Lower half & $<271$ & $<271$ & $<271$ \\
\hline \$97T000753 & \multirow[t]{2}{*}{ 190: 8} & Upper half & $<277$ & $<270$ & $<273$ \\
\hline S97T000775 & & Lower half & $<275$ & $<279$ & $<277$ \\
\hline $597 T 000754$ & \multirow[t]{2}{*}{ 190: 9} & Upper half & $<262$ & $<267$ & $<265$ \\
\hline S97T000776 & & Lower half & $<259$ & $<258$ & $<259$ \\
\hline S97T001016 & Core 190 & Solid composite & $<301$ & $<292$ & $<297$ \\
\hline Mininas & 18 & (1) & 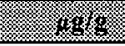 & 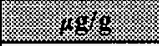 & 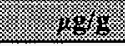 \\
\hline S97T000733 & 190: $1 \mathrm{R}$ & Drainable liquid & $<191$ & $<191$ & $<191$ \\
\hline
\end{tabular}


HNF-1501 Rev. 0

Table B2-160. Tank 241-T-203 Analytical Results: Chloride (IC).

\begin{tabular}{|c|c|c|c|c|c|}
\hline 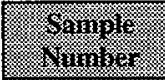 & 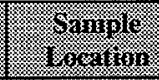 & 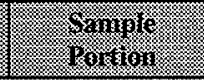 & $f_{3}$ & 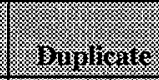 & 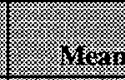 \\
\hline \multicolumn{2}{|c|}{ 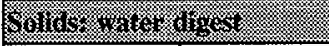 } & (2) & 1 & $8.8,84$ & 4. \\
\hline S97T000736 & \multirow[t]{2}{*}{ 190: 1} & Upper half & 659 & 642 & 651 \\
\hline S97T000768 & & Lower half & 577 & 632 & 605 \\
\hline S97T000746 & \multirow[t]{2}{*}{ 190: $1 \mathrm{R}$} & Upper half & 602 & 592 & 597 \\
\hline S97T000769 & & Lower half & 653 & 618 & 636 \\
\hline S97T000747 & \multirow[t]{2}{*}{$190: 2$} & Upper half & 599 & 641 & 620 \\
\hline S97T000758 & & Lower half & 621 & 577 & 599 \\
\hline S97T000748 & \multirow[t]{2}{*}{ 190: 3} & Upper half & 598 & 573 & 585 \\
\hline S97T000770 & & Lower half & 609 & 636 & 622 \\
\hline S97T000749 & \multirow[t]{2}{*}{ 190: 4} & Upper half & 618 & 596 & 607 \\
\hline S97T000771 & & Lower half & 607 & 618 & 612 \\
\hline S97T000750 & \multirow[t]{2}{*}{ 190: 5} & Upper half & 786 & 839 & 812 \\
\hline S97T000772 & & Lower half & 606 & 623 & 614 \\
\hline S97T000751 & \multirow[t]{2}{*}{ 190: 6} & Upper half & 461 & 500 & 481 \\
\hline S97T000773 & & Lower half & 546 & 549 & 548 \\
\hline S97T000752 & \multirow[t]{2}{*}{ 190: 7} & Upper half & 422 & 444 & 433 \\
\hline S97T000774 & & Lower half & 596 & 615 & 605 \\
\hline S97T000753 & \multirow[t]{2}{*}{ 190: 8} & Upper half & 591 & 444 & $518^{Q \mathrm{Q}: \varepsilon}$ \\
\hline S97T000775 & & Lower half & 614 & 607 & 611 \\
\hline S97T000754 & \multirow[t]{2}{*}{ 190: 9} & Upper half & 580 & 556 & 568 \\
\hline S97T000776 & & Lower half & 592 & 596 & 594 \\
\hline S97T001016 & Core 190 & Solid composite & 689 & 627 & 658 \\
\hline Lanite & 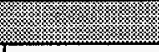 & & $\sqrt{1}+968$ & (2) 1.6 & 18 \\
\hline S97T000733 & 190: 1R & Drainable liquid & 498 & 519 & 509 \\
\hline
\end{tabular}


HNF-1501 Rev. 0

Table B2-161. Tank 241-T-203 Analytical Results: Fluoride (IC).

\begin{tabular}{|c|c|c|c|c|c|}
\hline Squnge: & 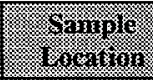 & 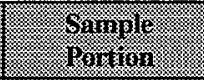 & R r-5.11 & loulicars & 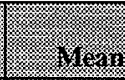 \\
\hline 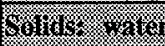 & 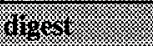 & 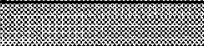 & 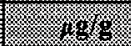 & 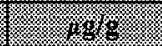 & 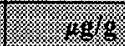 \\
\hline S97T000736 & \multirow[t]{2}{*}{ 190: 1} & Upper half & 7,190 & 6,900 & 7,050 \\
\hline S97T000768 & & Lower half & 6,220 & 6,440 & 6,330 \\
\hline S97T000746 & \multirow[t]{2}{*}{ 190: $1 R$} & Upper half & 6,600 & 6,710 & 6,650 \\
\hline S97T000769 & & Lower half & 6,460 & 6,110 & 6,290 \\
\hline S97T000747 & \multirow[t]{2}{*}{ 190: 2} & Upper half & 6,380 & 6,500 & 6,440 \\
\hline S97T000758 & & Lower half & 6,320 & 5,860 & 6,090 \\
\hline S97T000748 & \multirow[t]{2}{*}{$190: 3$} & Upper half & 6,120 & 6,170 & 6,150 \\
\hline S97T000770 & & Lower half & 6,120 & 6,240 & 6,180 \\
\hline S97T000749 & \multirow[t]{2}{*}{ 190: 4} & Upper half & 6,310 & 6,350 & 6,330 \\
\hline S97T000771 & & Lower half & 6,260 & 6,470 & 6,360 \\
\hline S97T000750 & \multirow[t]{2}{*}{ 190: 5} & Upper half & 6,750 & 6,860 & 6,810 \\
\hline S97T000772 & & Lower half & 6,650 & 6,550 & 6,600 \\
\hline S97T000751 & \multirow[t]{2}{*}{ 190: 6} & Upper half & 4,830 & 4,840 & 4,830 \\
\hline S97T000773 & & Lower half & 5,840 & 5,880 & 5,860 \\
\hline S97T000752 & \multirow[t]{2}{*}{ 190: 7} & Upper half & 4,540 & 4,610 & 4,580 \\
\hline S97T000774 & & Lower half & 6,710 & 6,670 & 6,690 \\
\hline S97T000753 & \multirow[t]{2}{*}{ 190: 8} & Upper half & 6,580 & 4,710 & $5,640^{\mathrm{QC}: \mathrm{c}}$ \\
\hline S97T000775 & & Lower half & 6,390 & 6,630 & 6,510 \\
\hline S97T000754 & \multirow[t]{2}{*}{ 190: 9} & Upper half & 6,220 & 6,090 & 6,160 \\
\hline S97T000776 & & Lower half & 6,150 & 6,210 & 6,180 \\
\hline S97T001016 & Core 190 & Solid composite & 6,320 & 6,350 & 6,340 \\
\hline Lomigs & 4 & 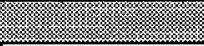 & 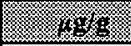 & $f_{1}=8.85$ & 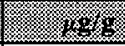 \\
\hline S97T000733 & 190: 1R & Drainable liquid & 5,110 & 4,990 & 5,050 \\
\hline
\end{tabular}


Table B2-162. Tank 241-T-203 Analytical Results: Nitrate (IC).

\begin{tabular}{|c|c|c|c|c|c|}
\hline Viminle: & U Sample & Saminges & (3esini: & (2) Bublicate & $4 \log n$ \\
\hline \multicolumn{2}{|c|}{ 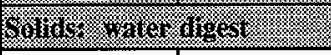 } & I. & . & 1.98 & 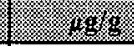 \\
\hline S97T000736 & \multirow[t]{2}{*}{ 190: 1} & Upper half & 63,600 & 62,900 & 63,300 \\
\hline S97T000768 & & Lower half & 62,700 & 62,700 & 62,700 \\
\hline S97T000746 & \multirow[t]{2}{*}{ 190: 1R } & Upper half & 63,300 & 61,800 & 62,500 \\
\hline S97T000769 & & Lower half & 66,300 & 62,700 & 64,500 \\
\hline S97T000747 & \multirow[t]{2}{*}{ 190: 2} & Upper half & 64,600 & 65,900 & 65,300 \\
\hline S97T000758 & & Lower half & 64,900 & 58,500 & 61,700 \\
\hline S97T000748 & \multirow[t]{2}{*}{$190: 3$} & Upper half & 63,200 & 60,900 & 62,100 \\
\hline S97T000770 & & Lower half & 62,400 & 63,700 & 63,000 \\
\hline S97T000749 & \multirow[t]{2}{*}{ 190: 4} & Upper half & 62,500 & 59,600 & 61,100 \\
\hline S97T000771 & & Lower half & 54,600 & 55,400 & 55,000 \\
\hline S97T000750 & \multirow[t]{2}{*}{ 190: 5} & Upper half & 59,400 & 61,100 & 60,200 \\
\hline S97T000772 & & Lower half & 57,600 & 58,200 & 57,900 \\
\hline S97T000751 & \multirow[t]{2}{*}{$190: 6$} & Upper half & 45,100 & 46,200 & 45,600 \\
\hline S97T000773 & & Lower half & 57,600 & 57,500 & 57,600 \\
\hline S97T000752 & \multirow[t]{2}{*}{ 190: 7} & Upper half & 43,600 & 44,500 & 44,100 \\
\hline S97T000774 & & Lower half & 57,600 & 58,800 & 58,200 \\
\hline S97T000753 & \multirow[t]{2}{*}{ 190: 8} & Upper half & 57,300 & 42,200 & $49,800^{\mathrm{QC}: 0}$ \\
\hline S97T000775 & & Lower half & 57,100 & 57,700 & 57,400 \\
\hline S97T000754 & \multirow[t]{2}{*}{ 190: 9} & Upper half & 62,200 & 59,500 & 60,900 \\
\hline S97T000776 & & Lower half & 59,300 & 60,200 & 59,700 \\
\hline S97T001016 & Core 190 & Solid composite & 63,700 & 65,200 & 64,400 \\
\hline Livangs & 4 & & 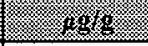 & $3.18 \mathrm{~g}$ & 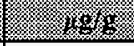 \\
\hline S97T000733 & 190: 1R & Drainable liquid & 53,000 & 53,100 & 53,000 \\
\hline
\end{tabular}


HNF-1501 Rev. 0

Table B2-163. Tank 241-T-203 Analytical Results: Nitrite (IC).

\begin{tabular}{|c|c|c|c|c|c|}
\hline $\begin{array}{l}\text { Somper: } \\
\text { Nimiler }\end{array}$ & Sumiles & 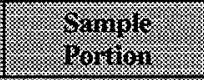 & 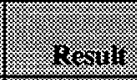 & 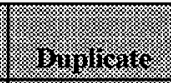 & I I tern \\
\hline \multicolumn{3}{|c|}{ 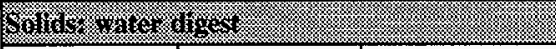 } & (1) & 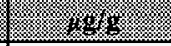 & 49 \\
\hline S97T000736 & \multirow[t]{2}{*}{ 190: 1} & Upper half & 497 & 489 & 493 \\
\hline S97T000768 & & Lower half & 322 & 309 & 316 \\
\hline S97T000746 & \multirow[t]{2}{*}{ 190: 1R } & Upper half & 336 & 338 & 337 \\
\hline S97T000769 & & Lower half & 267 & 253 & 260 \\
\hline S97T000747 & \multirow[t]{2}{*}{ 190: 2} & Upper half & 300 & 295 & 298 \\
\hline S97T000758 & & Lower half & 303 & 276 & 289 \\
\hline S97T000748 & \multirow[t]{2}{*}{ 190: 3} & Upper half & 272 & 271 & 272 \\
\hline S97T000770 & & Lower half & 246 & 239 & 242 \\
\hline S97T000749 & \multirow[t]{2}{*}{ 190: 4} & Upper half & 260 & 248 & 254 \\
\hline S97T000771 & & Lower half & 398 & 405 & 402 \\
\hline S97T000750 & \multirow[t]{2}{*}{ 190: 5} & Upper half & 418 & 435 & 426 \\
\hline S97T000772 & & Lower half & 399 & 425 & 412 \\
\hline S97T000751 & \multirow[t]{2}{*}{ 190: 6} & Upper half & $<448$ & $<454$ & $<451$ \\
\hline S97T000773 & & Lower half & 293 & 273 & 283 \\
\hline S97T000752 & \multirow[t]{2}{*}{ 190: 7} & Upper half & 255 & 257 & 256 \\
\hline S97T000774 & & Lower half & 407 & 398 & 402 \\
\hline S97T000753 & \multirow[t]{2}{*}{ 190: 8} & Upper half & 410 & 374 & 392 \\
\hline$\$ 97 T 000775$ & & Lower half & 418 & 412 & 415 \\
\hline S97T000754 & \multirow[t]{2}{*}{ 190: 9} & Upper half & 210 & 207 & 208 \\
\hline S97T000776 & & Lower half & 275 & 271 & 273 \\
\hline S97T001016 & Core 190 & Solid composite & 297 & 292 & 295 \\
\hline Mirinings: & (3) & י) & 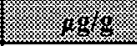 & 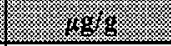 & 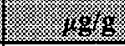 \\
\hline S97T000733 & 190: 1R & Drainable liquid & 250 & 258 & 254 \\
\hline
\end{tabular}


Table B2-164. Tank 241-T-203 Analytical Results: Phosphate (IC).

\begin{tabular}{|c|c|c|c|c|c|}
\hline Samine: & 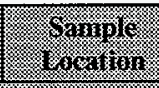 & is & is & J & 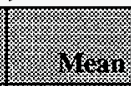 \\
\hline \multicolumn{3}{|c|}{ 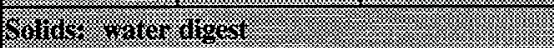 } & 1) & $8.86 \mathrm{~g}=$ & (4) \\
\hline S97T000736 & \multirow[t]{2}{*}{ 190: 1} & Upper half & 2,880 & 2,900 & 2,890 \\
\hline S97T000768 & & Lower half & 2,280 & 2,320 & 2,300 \\
\hline S97T000746 & \multirow[t]{2}{*}{ 190: $1 \mathbf{R}$} & Upper half & 2,620 & 2,660 & 2,640 \\
\hline S97T000769 & & Lower half & 2,190 & 1,810 & 2,000 \\
\hline S97T000747 & \multirow[t]{2}{*}{$190: 2$} & Upper half & 2,140 & 1,990 & 2,070 \\
\hline S97T000758 & & Lower half & 2,250 & 1,880 & 2,060 \\
\hline S97T000748 & \multirow[t]{2}{*}{ 190: 3} & Upper half & 2,220 & 2,160 & 2,190 \\
\hline \$97T000770 & & Lower half & 2,400 & 2,830 & 2,610 \\
\hline S97T000749 & \multirow[t]{2}{*}{ 190: 4} & Upper half & 2,290 & 2,220 & 2,250 \\
\hline S97T000771 & & Lower half & 2,200 & 2,280 & 2,240 \\
\hline S97T000750 & \multirow[t]{2}{*}{$190: 5$} & Upper half & 2,420 & 2,400 & 2,410 \\
\hline S97T000772 & & Lower half & 2,370 & 2,410 & 2,390 \\
\hline S97T000751 & \multirow[t]{2}{*}{ 190: 6} & Upper half & 1,880 & 1,780 & 1,830 \\
\hline S97T000773 & & Lower half & 2,070 & 2,260 & 2,170 \\
\hline S97T000752 & \multirow[t]{2}{*}{ 190: 7} & Upper half & 1,500 & 2,330 & $1,920^{\mathrm{ec}: t}$ \\
\hline S97T000774 & & Lower half & 2,310 & 2,270 & 2,290 \\
\hline S97T000753 & \multirow[t]{2}{*}{ 190: 8} & Upper half & 2,000 & 1,590 & $1,790^{\text {ec:e }}$ \\
\hline S97T000775 & & Lower half & 2,080 & 1,930 & 2,010 \\
\hline S97T000754 & \multirow[t]{2}{*}{ 190: 9} & Upper half & 2,500 & 3,160 & $2,830^{\mathrm{QC:e}}$ \\
\hline S97T000776 & & Lower half & 3,620 & 2,560 & $3,090^{\text {C::e }}$ \\
\hline S97T001016 & Core 190 & Solid composite & 2,290 & 3,530 & $2,910^{\propto C: e}$ \\
\hline Minging & & 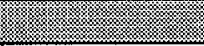 & (1.8. & 8.856 & 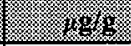 \\
\hline S97T000733 & 190: IR & Drainable liquid & 1,350 & 1,380 & 1,360 \\
\hline
\end{tabular}


HNF-1501 Rev. 0

Table B2-165. Tank 241-T-203 Analytical Results: Sulfate (IC).

\begin{tabular}{|c|c|c|c|c|c|}
\hline Siminiles & Sumple & $\begin{array}{l}\text { Srmple } \\
\text { Iorion }\end{array}$ & Reruly & Giviterive & 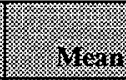 \\
\hline \multicolumn{2}{|c|}{ 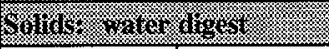 } & (x) & I & 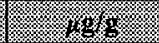 & 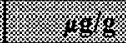 \\
\hline S97T000736 & \multirow[t]{2}{*}{ 190: 1} & Upper half & 607 & 613 & 610 \\
\hline S97T000768. & & Lower half & 432 & 450 & 441 \\
\hline S97T000746 & \multirow[t]{2}{*}{ 190: 1R } & Upper half & 337 & $<299$ & $<318$ \\
\hline S97T000769 & & Lower half & 388 & 294 & $341^{\propto C_{: e}}$ \\
\hline S97T000747 & \multirow[t]{2}{*}{ 190: 2} & Upper half & $<289$ & 387 & $<338^{\mathrm{QC:0}}$ \\
\hline S97T000758 & & Lower half & $<304$ & 329 & $<316$ \\
\hline S97T000748 & \multirow[t]{2}{*}{ 190: 3} & Upper half & $<292$ & $<284$ & $<288$ \\
\hline S97T000770 & & Lower half & 366 & $<256$ & $<311^{\mathrm{QC}: \mathrm{e}}$ \\
\hline S97T000749 & \multirow[t]{2}{*}{ 190: 4} & Upper half & $<283$ & $<272$ & $<278$ \\
\hline S97T000771 & & Lower half & 567 & 610 & 588 \\
\hline S97T000750 & \multirow[t]{2}{*}{ 190: 5} & Upper half & 637 & 688 & 663 \\
\hline S97T000772 & & Lower half & 816 & 621 & $718^{\text {eC:e }}$ \\
\hline S97T000751 & \multirow[t]{2}{*}{ 190: 6} & Upper half & $<572$ & $<580$ & $<576$ \\
\hline S97T000773 & & Lower half & $<302$ & 658 & $<480^{\text {QC:e }}$ \\
\hline S97T000752 & \multirow[t]{2}{*}{ 190: 7} & Upper half & $<296$ & $<301$ & $<298$ \\
\hline S97T000774 & & Lower half & 353 & 696 & $524^{\mathrm{QC}: e}$ \\
\hline S97T000753 & \multirow[t]{2}{*}{ 190: 8} & Upper half & 317 & 582 & $450^{\mathrm{eC}: \mathrm{e}}$ \\
\hline$\$ 97 T 000775$ & & Lower half & 352 & 733 & $542^{\mathrm{CC}_{\mathrm{C}} \mathrm{e}}$ \\
\hline S97T000754 & \multirow[t]{2}{*}{ 190: 9} & Upper half & 368 & 371 & 369 \\
\hline S97T000776 & & Lower half & 467 & 734 & $600^{\mathrm{eC}: 0}$ \\
\hline S97T001016 & Core 190 & Solid composite & $<333$ & 543 & $<438^{\text {ec:e }}$ \\
\hline Lariars: & & (1: & 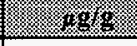 & (1, 389 & 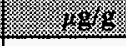 \\
\hline S97T000733 & 190: 1R & Drainable liquid & 309 & 365 & 337 \\
\hline
\end{tabular}


Table B2-166. Tank 241-T-203 Analytical Results: Oxalate (IC).

\begin{tabular}{|c|c|c|c|c|c|}
\hline Siringl & 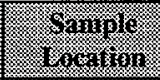 & $\begin{array}{l}\text { Cimple } \\
\text { oritoris }\end{array}$ & ignitu & Bimingure & 11.8. \\
\hline \multicolumn{3}{|c|}{ 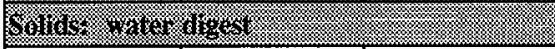 } & 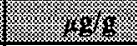 & 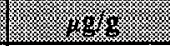 & 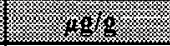 \\
\hline S97T000736 & \multirow[t]{2}{*}{ 190: 1} & Upper half & $<219$ & $<219$ & $<219$ \\
\hline S97T000768 & & Lower half & 546 & 554 & 550 \\
\hline$\$ 977000746$ & \multirow[t]{2}{*}{ 190: $1 R$} & Upper half & 302 & 265 & 283 \\
\hline S97T000769 & & Lower half & 750 & 757 & 754 \\
\hline S97T000747 & \multirow[t]{2}{*}{ 190: 2} & Upper half & 1,010 & 1,040 & 1,030 \\
\hline S97T000758 & & Lower half & 1,420 & 1,220 & 1,320 \\
\hline S97T000748 & \multirow[t]{2}{*}{ 190: 3} & Upper half & 1,430 & 1,510 & 1,470 \\
\hline S97T000770 & & Lower half & 1,620 & 1,690 & 1,660 \\
\hline S97T000749 & \multirow[t]{2}{*}{ 190: 4} & Upper half & 1,550 & 1,500 & 1,530 \\
\hline S97T000771 & & Lower half & $1 ; 150$ & 1,160 & 1,150 \\
\hline S97T000750 & \multirow[t]{2}{*}{ 190: 5} & Upper half & 1,300 & 1,370 & 1,330 \\
\hline S97T000772 & & Lower half & 1,340 & 1,260 & 1,300 \\
\hline S97T000751 & \multirow[t]{2}{*}{ 190: 6} & Upper half & 1,310 & 1,280 & 1,300 \\
\hline S97T000773 & & Lower half & 1,560 & 1,570 & 1,570 \\
\hline S97T000752 & \multirow[t]{2}{*}{ 190: 7} & Upper half & 1,250 & 1,260 & 1,260 \\
\hline S97T000774 & & Lower half & 1,260 & 1,300 & 1,280 \\
\hline S97T000753 & \multirow[t]{2}{*}{ 190: 8} & Upper half & 1,300 & 865 & $1,080^{\mathrm{eC}: \mathrm{e}}$ \\
\hline S97T000775 & & Lower half & 1,240 & 1,220 & 1,230 \\
\hline S97T000754 & \multirow[t]{2}{*}{ 190: 9} & Upper half & 1,600 & 1,590 & 1,590 \\
\hline S97T000776 & & Lower half & 1,520 & 1,550 & 1,540 \\
\hline S97T001016 & Core 190 & Solid composite & 1,410 & 1,330 & 1,370 \\
\hline Wirives: & & (3.: & 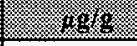 & 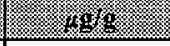 & ( \\
\hline S97T000733 & 190: 1R & Drainable liquid & $<160$ & $<160$ & $<160$ \\
\hline
\end{tabular}


HNF-1501 Rev. 0

Table B2-167. Tank 241-T-203 Analytical Results: Bulk Density.

\begin{tabular}{|c|c|c|c|c|c|}
\hline Ninim: & Sample & Strmife & renily & mingivaris: & $\operatorname{lin}$ \\
\hline Bomins & 4 & 2) & \% & ( & (1211 \\
\hline S97T000657 & 190: 1 & Lower half & 1.21 & N/A & 1.21 \\
\hline S97T000699 & 190: 1R & Lower half & 1.19 & N/A & 1.19 \\
\hline S97T000661 & 190: 2 & Lower half & 1.2 & N/A & 1.2 \\
\hline S97T000700 & 190: 3 & Lower half & 1.39 & N/A & 1.39 \\
\hline S97T000662 & 190: 4 & Lower half & 1.22 & $\mathrm{~N} / \mathrm{A}$ & 1.22 \\
\hline S97T000663 & 190: 5 & Lower half & 1.14 & N/A & 1.14 \\
\hline S97T000701 & 190: 6 & Lower half & 1.27 & N/A & 1.27 \\
\hline S97T000702 & 190: 7 & Lower half & 1.17 & N/A & 1.17 \\
\hline S97T000703 & 190: 8 & Lower half & 1.15 & N/A & 1.15 \\
\hline S97T000704 & 190: 9 & Lower half & 1.29 & N/A & 1.29 \\
\hline S97T001012 & Core 190 & Solid composite & 1.23 & N/A & 1.23 \\
\hline
\end{tabular}

Table B2-168. Tank 241-T-203 Analytical Results: Exotherm - transition 1 (DSC/TGA).

\begin{tabular}{|c|c|c|c|c|c|}
\hline Saminle & Stmple & $\begin{array}{l}\text { Samilie } \\
\text { - jomition }\end{array}$ & Resurin & 3)intinate & Hen: \\
\hline Oullas & & & 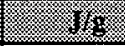 & 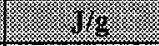 & $f^{\prime}$ \\
\hline S97T000713 & 190: 6 & Lower half & 2.44 & 3.38 & $2.91^{\mathrm{QC:e}}$ \\
\hline S97T000714 & 190: 7 & Lower half & 4.68 & 2.29 & $3.48^{\mathrm{QC} ; \mathrm{c}}$ \\
\hline S97T000710 & $190: 9$ & Upper half & 4.39 & 4.67 & 4.53 \\
\hline
\end{tabular}


HNF-1501 Rev. 0

Table B2-169. Tank 241-T-203 Analytical Results: Percent Water (DSC/TGA).

\begin{tabular}{|c|c|c|c|c|c|}
\hline Mample & Sumple: & $\begin{array}{l}\text { Sample } \\
\text { rombon: }\end{array}$ & Resil1 & Buninones & 14.21 \\
\hline Sinis: & (1) & 48 & 18 & . & 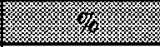 \\
\hline S97T000664 & \multirow[t]{2}{*}{ 190: 1} & Upper half & 77.8 & 76.7 & 77.3 \\
\hline S97T000665 & & Lower half & 74.1 & 75.6 & 74.8 \\
\hline S97T000705 & \multirow[t]{2}{*}{ 190: 1R } & Upper half & 79.8 & 80.9 & 80.4 \\
\hline S97T000711 & & Lower half & 83.8 & 82.9 & 83.3 \\
\hline S97T000666 & \multirow[t]{2}{*}{ 190: 2} & Upper half & 76.3 & 75.2 & 75.7 \\
\hline S97T000669 & & Lower half & 76.8 & 76.3 & 76.5 \\
\hline S97T000706 & \multirow[t]{2}{*}{ 190: 3} & Upper half & 78.1 & 78.3 & 78.2 \\
\hline S97T000712 & & Lower half & 69.5 & 76 & 72.8 \\
\hline S97T000667 & \multirow[t]{2}{*}{ 190: 4} & Upper half & 74.8 & 75.9 & 75.3 \\
\hline S97T000670 & & Lower half & 75.7 & 73 & 74.3 \\
\hline S97T000668 & \multirow[t]{2}{*}{ 190: 5} & Upper half & 78.1 & 77.8 & 78 \\
\hline S97T000671 & & Lower half & 76.9 & 76.5 & 76.7 \\
\hline \$97T000707 & \multirow[t]{2}{*}{ 190: 6} & Upper half & 76.6 & 76.2 & 76.4 \\
\hline S97T000713 & & Lower half & 75.2 & 75.9 & 75.6 \\
\hline S97T000708 & \multirow[t]{2}{*}{ 190: 7} & Upper half & 74.2 & 76.6 & 75.4 \\
\hline S97T000714 & & Lower half & 75 & 71.5 & 73.2 \\
\hline S97T000709 & \multirow[t]{2}{*}{ 190: 8} & Upper half & 75.4 & 76.4 & 75.9 \\
\hline S97T000715 & & Lower half & 75.8 & 75.9 & 75.8 \\
\hline S97T000710 & \multirow[t]{2}{*}{ 190: 9} & Upper half & 74.3 & 71.3 & 72.8 \\
\hline S97T000716 & & Lower half & 76.6 & 76.1 & 76.4 \\
\hline S97T001013 & Core 190 & Solid composite & 69.6 & 72.5 & 71 \\
\hline Hanite & 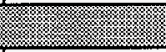 & (2):10: & 2 & 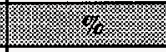 & $\sqrt{7.28}$ \\
\hline S97T000733 & 190: 1R & Drainable liquid & 85.3 & 85.2 & 85.3 \\
\hline
\end{tabular}


Table B2-170. Tank 241-T-203 Analytical Results: Specific Gravity.

\begin{tabular}{|c|c|c|c|c|c|}
\hline Simine & Sumple & S & oresull & B. & $\operatorname{ten} n$ \\
\hline Harmas: & 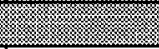 & 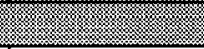 & 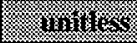 & ( Dnilless: & (1) \\
\hline S97T000733 & 190: 1R & Drainable liquid & 1.09 & 1.08 & 1.09 \\
\hline
\end{tabular}

Table B2-171. Tank 241-T-203 Analytical Results: Total Alpha (Alpha Rad).

\begin{tabular}{|c|c|c|c|c|c|}
\hline vinulary & Samples & 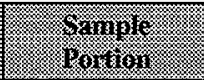 & resuli: & 70 & $178 \pi$ \\
\hline 14romos & & & 80.411 & 8.9 .1311 & 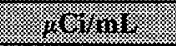 \\
\hline $97 \mathrm{~T} 000733$ & 190: 1R & Drainable liquid & $<8.48 \mathrm{E}-05$ & $<5.50 \mathrm{E}-05$ & $<6.99 \mathrm{E}-05^{\mathrm{QC}: f}$ \\
\hline
\end{tabular}

Table B2-172. Tank 241-T-203 Analytical Results: Total Alpha (Alpha).

\begin{tabular}{|c|c|c|c|c|c|}
\hline S Sampler: & S Sumple & $\begin{array}{l}\text { S.mugte } \\
\text { lom }\end{array}$ & Resulu & 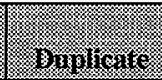 & 1128 \\
\hline \multicolumn{2}{|c|}{ 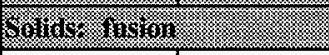 } & : & H. & 1.1010 & 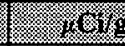 \\
\hline S97T000759 & 190: 1 & Lower half & 0.23 & 0.249 & 0.24 \\
\hline S97T000760 & 190: 1R & Lower half & 0.179 & 0.169 & 0.174 \\
\hline S97T000757 & $190: 2$ & Lower half & 0.247 & 0.206 & 0.226 \\
\hline S97T000761 & 190: 3 & Lower half & 0.311 & 0.247 & 0.279 \\
\hline S97T000762 & 190: 4 & Lower half & 0.201 & 0.181 & 0.191 \\
\hline S97T000763 & $190: 5$ & Lower half & 0.278 & 0.278 & 0.278 \\
\hline S97T000764 & 190: 6 & Lower half & 0.129 & 0.128 & 0.129 \\
\hline S97T000765 & 190: 7 & Lower half & 0.14 & 0.123 & 0.132 \\
\hline S97T000766 & 190: 8 & Lower half & 0.154 & 0.18 & 0.167 \\
\hline S97T000767 & 190: 9 & Lower half & 0.159 & 0.124 & 0.142 \\
\hline
\end{tabular}


HNF-1501 Rev. 0

Table B2-173. Tank 241-T-203 Analytical Results: Americium-241 (GEA).

\begin{tabular}{|c|c|c|c|c|c|}
\hline saming & 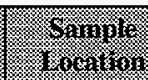 & $\begin{array}{l}\text { Sampole } \\
\text { Gortion }\end{array}$ & (2) & (8upliter & Mican \\
\hline 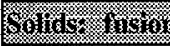 & & & 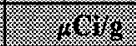 & 1. & 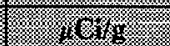 \\
\hline S97T001734 & Core 190 & Solid composite & 0.0359 & 0.0359 & 0.0359 \\
\hline
\end{tabular}

Table B2-174. Tank 241-T-203 Analytical Results: Cesium-137 (GEA).

\begin{tabular}{|c|c|c|c|c|c|}
\hline Siminge & 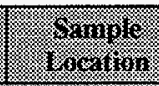 & $\begin{array}{l}\text { Yamie } \\
\text { Poction }\end{array}$ & resull & 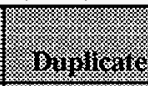 & Yterin \\
\hline 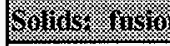 & & & $4 x$ & 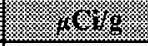 & $\sqrt{10} \times 8$ \\
\hline S97T001734 & Core 190 & Solid composite & $<0.0178$ & $<0.0183$ & $<0.0181$ \\
\hline
\end{tabular}

Table B2-175. Tank 241-T-203 Analytical Results: Cobalt-60 (GEA).

\begin{tabular}{|c|c|c|c|c|c|}
\hline $\begin{array}{l}\text { Samingle } \\
\text { Nunialer }\end{array}$ & 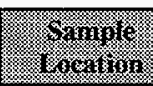 & $\begin{array}{l}\text { Sample } \\
\text { ronton }\end{array}$ & Resuli & 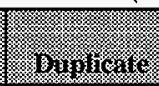 & $19 x_{n}$ \\
\hline 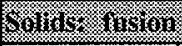 & & & 0.18 & $101 \% \mathrm{~g}$ & (3) \\
\hline S97T001734 & Core 190 & Solid composite & $<0.013$ & $<0.0122$ & $<0.0126$ \\
\hline
\end{tabular}

Table B2-176. Tank 241-T-203 Analytical Results: Europium-154 (GEA).

\begin{tabular}{|c|c|c|c|c|c|}
\hline $\begin{array}{l}\text { Sample } \\
\text { Vmanje }\end{array}$ & S.moringe & Samilit & isenvilu & 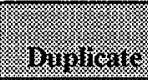 & 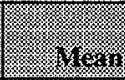 \\
\hline Solnat. & ( & & 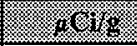 & 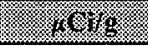 & $.85 \times 18$ \\
\hline S97T001734 & Core 190 & Solid composite & $<0.0432$ & $<0.0392$ & $<0.0412$ \\
\hline
\end{tabular}

Table B2-177. Tank 241-T-203 Analytical Results: Europium-155 (GEA).

\begin{tabular}{|c|c|c|c|c|c|}
\hline Sunjle & 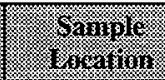 & lomontion & Revill & buglicate & 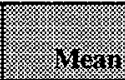 \\
\hline 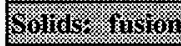 & & (3) & 80 & (5) & 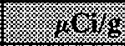 \\
\hline S97T001734 & Core 190 & Solid composite & $<0.0334$ & $<0.0337$ & $<0.0336$ \\
\hline
\end{tabular}


Table B2-178. Tank 241-T-203 Analytical Results: Strontium-89/90.

\begin{tabular}{|c|c|c|c|c|c|}
\hline Samingle & Siringlo & $\begin{array}{l}\text { Siminis } \\
\text { rowion }\end{array}$ & Resilit & Brybiricie & rirsm \\
\hline 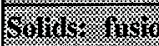 & X: & 1. & $101 \%$ & 1.9018 & 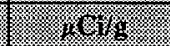 \\
\hline S97T001734 & Core 190 & Solid composite & 0.00246 & 0.00267 & 0.00257 \\
\hline
\end{tabular}

Table B2-179. Tank 241-T-203 Analytical Results: Total Inorganic Carbon.

\begin{tabular}{|c|c|c|c|c|c|}
\hline Siminler & Sulangle & $\begin{array}{l}\text { gampile } \\
\text { gombrom }\end{array}$ & Resul: & bivilitiste & Visan \\
\hline 6.0.16s & 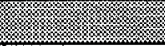 & (2) & (3) & 8.86 & 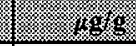 \\
\hline S97T001013 & Core 190 & Solid composite & 1,580 & 1,580 & 1,580 \\
\hline
\end{tabular}

Table B2-180. Tank 241-T-203 Analytical Results: Total Organic Carbon.

\begin{tabular}{|c|c|c|c|c|c|}
\hline Sample: & S & Soming & 1865n & Mapirar & Yran \\
\hline 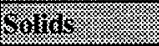 & (1) & & mg & 4.898 & 6498 \\
\hline S97T001013 & Core 190 & Solid composite & 418 & 423 & 421 \\
\hline
\end{tabular}

Table B2-181. Tank 241-T-204 Analytical Results: Aluminum (ICP).

\begin{tabular}{|c|c|c|c|c|c|}
\hline $\begin{array}{l}\text { sample } \\
\text { viniser }\end{array}$ & Shomples & $\begin{array}{l}\text { Simais } \\
\text { gortion }\end{array}$ & $U_{1}$ & (b) & (3) \\
\hline oblingr ario & 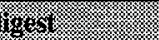 & (s: & $\sqrt{2}$ & 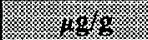 & 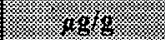 \\
\hline S97T001199 & Core 188 & Solid composite & 52.9 & 54.3 & 53.6 \\
\hline
\end{tabular}

Table B2-182. Tank 241-T-204 Analytical Results: Antimony (ICP).

\begin{tabular}{|c|c|c|c|c|c|}
\hline Sample & Sormolition & $\begin{array}{l}\text { Sample } \\
\text { I00 }\end{array}$ & ifesilin & Biviplicate & I: MEsin \\
\hline \multicolumn{3}{|c|}{ 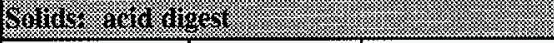 } & $10 \mathrm{~s}$ & 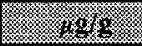 & 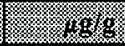 \\
\hline S97T001199 & Core 188 & Solid composite & 30.5 & 36.8 & 33.6 \\
\hline
\end{tabular}


HNF-1501 Rev, 0

Table B2-183. Tank 241-T-204 Analytical Results: Arsenic (ICP).

\begin{tabular}{|c|c|c|c|c|c|}
\hline 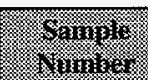 & S.juring & 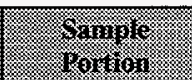 & mesilis: & Gupiricate & intar: \\
\hline \multicolumn{2}{|c|}{ 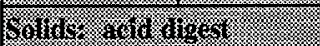 } & / & 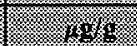 & (1) & its \\
\hline S97T001199 & Core 188 & Solid composite & 207 & 209 & 208 \\
\hline
\end{tabular}

Table B2-184. Tank 241-T-204 Analytical Results: Barium (ICP).

\begin{tabular}{|c|c|c|c|c|c|}
\hline Sunble & Sample: & 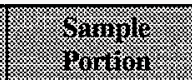 & resrin & 80101921 & 1menn \\
\hline \multicolumn{3}{|c|}{ 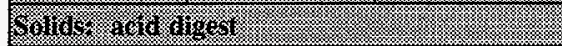 } & 4.869 & 14.486 & 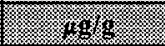 \\
\hline S97T001199 & Core 188 & Solid composite & $<14.3$ & $<14$ & $<14.2$ \\
\hline
\end{tabular}

Table B2-185. Tank 241-T-204 Analytical Results: Beryllium (ICP).

\begin{tabular}{|c|c|c|c|c|c|}
\hline Simile & $\begin{array}{l}\text { Gampin } \\
\text { resifion }\end{array}$ & 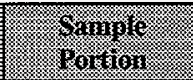 & resulu & bininicres & henis: \\
\hline 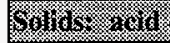 & (yctis & & , & 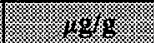 & 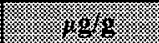 \\
\hline S97T001199 & Core 188 & Solid composite & $<1.43$ & $<1.4$ & $<1.42$ \\
\hline
\end{tabular}

Table B2-186. Tank 241-T-204 Analytical Results: Bismuth (ICP).

\begin{tabular}{|c|c|c|c|c|c|}
\hline Vamiles & $\begin{array}{l}\text { Samile } \\
\text { I cration. }\end{array}$ & Samivil & Resiul: & Eivilingrise & Kreari \\
\hline Golios 2 asid & Hges: & & $1358 \mathrm{~s}$ & r & 16968 \\
\hline S97T001199 & Core 188 & Solid composite & 51,100 & 51,900 & $51,500^{\mathrm{eC}: \mathrm{d}}$ \\
\hline
\end{tabular}

Table B2-187. Tank 241-T-204 Analytical Results: Boron (ICP).

\begin{tabular}{|c|c|c|c|c|c|}
\hline Shanile & 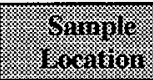 & orinulis & Alerill: & Burgisite & 1ren: \\
\hline \multicolumn{3}{|c|}{ 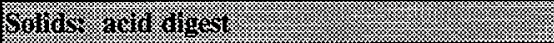 } & 138 & 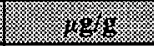 & 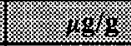 \\
\hline 597T001199 & Core 188 & Solid composite & 67.2 & 123 & $95.1^{\mathrm{QC:0}}$ \\
\hline
\end{tabular}


Table B2-188. Tank 241-T-204 Analytical Results: Cadmium (ICP).

\begin{tabular}{|c|c|c|c|c|c|}
\hline Sanples & $\begin{array}{l}\text { Sample } \\
\end{array}$ & 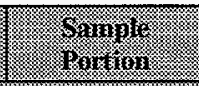 & Resill & Burpinizitio & $\sqrt{1}$ \\
\hline \multicolumn{3}{|c|}{ 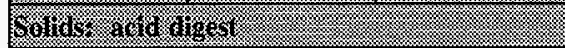 } & 1,818 & 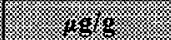 & 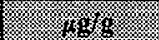 \\
\hline S97T001199 & Core 188 & Solid composite & $<1.43$ & $<1.4$ & $<1.42$ \\
\hline
\end{tabular}

Table B2-189. Tank 241-T-204 Analytical Results: Cerium (ICP).

\begin{tabular}{|c|c|c|c|c|c|}
\hline Sanivoler & 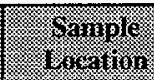 & Samble & Resull & Bumpinatro & 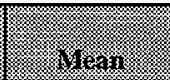 \\
\hline \multicolumn{2}{|c|}{ 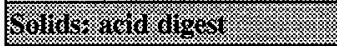 } & & 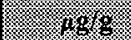 & . & 1698 \\
\hline S97T001199 & Core 188 & Solid composite & 61.6 & 64.3 & 63 \\
\hline
\end{tabular}

Table B2-190. Tank 241-T-204 Analytical Results: Chromium (ICP).

\begin{tabular}{|c|c|c|c|c|c|}
\hline comoles & 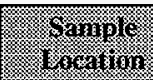 & romonilen & Rsesin: & Auplizar: & nean \\
\hline \multicolumn{3}{|c|}{ 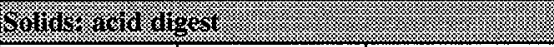 } & 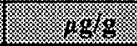 & 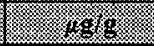 & $\sqrt{6898}$ \\
\hline S97T001199 & Core 188 & Solid composite & 4,510 & 4,470 & 4,490 \\
\hline
\end{tabular}

Table B2-191. Tank 241-T-204 Analytical Results: Cobalt (ICP).

\begin{tabular}{|c|c|c|c|c|c|}
\hline $\begin{array}{l}\text { sampir } \\
\text { Saniner }\end{array}$ & S Sampatan & $\begin{array}{l}\text { Shingle } \\
\text { Bartion }\end{array}$ & renin: & sultivets & 178sin \\
\hline 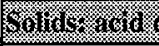 & gest: & & 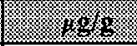 & 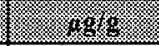 & $\sqrt[189]{85}$ \\
\hline S97T001199 & Core 188 & Solid composite & 6.12 & 6.92 & 6.52 \\
\hline
\end{tabular}

Table B2-192. Tank 241-T-204 Analytical Results: Copper (ICP).

\begin{tabular}{|c|c|c|c|c|c|}
\hline Gaminger & Sample & $\begin{array}{l}\text { Sample } \\
\text { Pritian }\end{array}$ & Resin: & Briplizare & Vear: \\
\hline Krinds & gert & & 8888 & 4.169 & 13 \\
\hline S97T001199 & Core 188 & Solid composite & 7.25 & 6.42 & 6.84 \\
\hline
\end{tabular}


Table B2-193. Tank 241-T-204 Analytical Results: Iron (ICP).

\begin{tabular}{|c|c|c|c|c|c|}
\hline 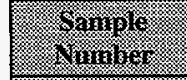 & I Sarmegle & 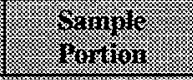 & Ifrovil: & 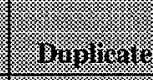 & 11 ean \\
\hline 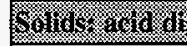 & (5-st: & 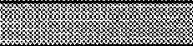 & (3) & 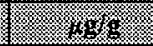 & 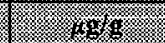 \\
\hline S97T001199 & Core 188 & Solid composite & 3,860 & 4,210 & $4,040^{\mathrm{QC:d}}$ \\
\hline
\end{tabular}

Table B2-194. Tank 241-T-204 Analytical Results: Lanthanum (ICP).

\begin{tabular}{|c|c|c|c|c|c|}
\hline grinule & T.jomple & $\begin{array}{l}\text { Shaniple } \\
\text { Protion }\end{array}$ & Resiti & Bunirate & Mean \\
\hline \multicolumn{3}{|c|}{ 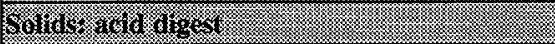 } & . & 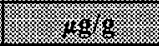 & $1,1, y_{\mathrm{g}}$ \\
\hline S97T001199 & Core 188 & Solid composite & 11,300 & 11,700 & $11,500^{\mathrm{eC}: \mathrm{d}}$ \\
\hline
\end{tabular}

Table B2-195. Tank 241-T-204 Analytical Results: Lead (ICP).

\begin{tabular}{|c|c|c|c|c|c|}
\hline Samin!e & $\begin{array}{l}\text { Gimiple } \\
\text { Sorition }\end{array}$ & I & resul & Biogilinate & Nean \\
\hline \multicolumn{3}{|c|}{ Soind a a d ligert } & 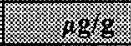 & 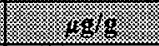 & .8968 \\
\hline S97T001199 & Core 188 & Solid composite & 308 & 312 & 310 \\
\hline
\end{tabular}

Table B2-196. Tank 241-T-204 Analytical Results: Lithium (ICP).

\begin{tabular}{|c|c|c|c|c|c|}
\hline Simpir & $\frac{13 m i g}{4}$ & Timole & Reurit & binilicine & 17.t. \\
\hline \multicolumn{3}{|c|}{ 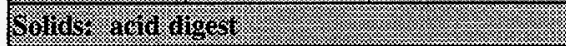 } & 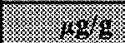 & 8.1 .98 & 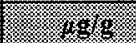 \\
\hline S97T001199 & Core 188 & Solid composite & $<2.86$ & $<2.81$ & $<2.84$ \\
\hline
\end{tabular}

Table B2-197. Tank 241-T-204 Analytical Results: Magnesium (ICP).

\begin{tabular}{|c|c|c|c|c|c|}
\hline Vinger & Sianili & Somule & Iresin & Ouplicats & 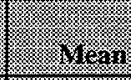 \\
\hline 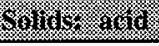 & 19est & $\sqrt{4-3}$ & 1468 & 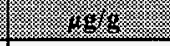 & ( \\
\hline S97T001199 & Core 188 & Solid composite & 35.1 & 34.1 & 34.6 \\
\hline
\end{tabular}


Table B2-198. Tank 241-T-204 Analytical Results: Manganese (ICP).

\begin{tabular}{|c|c|c|c|c|c|}
\hline Jamivle & 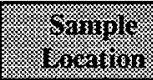 & I S Somple & iresull & 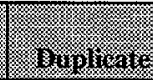 & Vyegr: \\
\hline \multicolumn{2}{|c|}{ 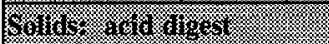 } & 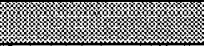 & 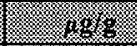 & 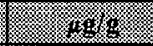 & 11940 \\
\hline S97T001199 & Core 188 & Solid composite & 14,100 & 14,000 & $14,100^{\mathrm{QC:d}}$ \\
\hline
\end{tabular}

Table B2-199. Tank 241-T-204 Analytical Results: Molybdenum (ICP).

\begin{tabular}{|c|c|c|c|c|c|}
\hline Siminges & f 8 amplo & Sompli & Iresuln & 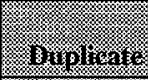 & 11.9. \\
\hline Sivines 1018 & kest & (3) & 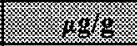 & 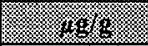 & 1.488 \\
\hline S97T001199 & Core 188 & Solid composite & $<14.3$ & $<14$ & $<14.2$ \\
\hline
\end{tabular}

Table B2-200. Tank 241-T-204 Analytical Results: Neodymium (ICP).

\begin{tabular}{|c|c|c|c|c|c|}
\hline Sumble: & 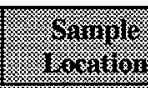 & $\begin{array}{l}\text { Sample } \\
\text { romion }\end{array}$ & Iresinis: & Pripilicure & 1 \\
\hline \multicolumn{3}{|c|}{ 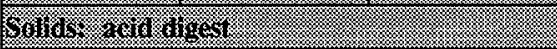 } & $2=0.88 .8$ & 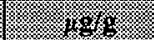 & 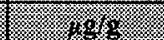 \\
\hline S97T001199 & Core 188 & Solid composite & $<28.6$ & $<28.1$ & $<28.4$ \\
\hline
\end{tabular}

Table B2-201. Tank 241-T-204 Analytical Results: Nickel (ICP).

\begin{tabular}{|c|c|c|c|c|c|}
\hline Shanje- & 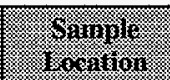 & 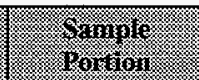 & Resil & Buyjurate & $18 \mathrm{ro}$ \\
\hline \multicolumn{2}{|c|}{ 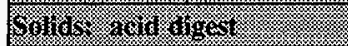 } & 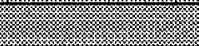 & 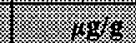 & (2) & 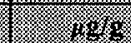 \\
\hline S97T001199 & Core 188 & Solid composite & 242 & 241 & 242 \\
\hline
\end{tabular}

Table B2-202. Tank 241-T-204 Analytical Results: Phosphorus (ICP).

\begin{tabular}{|c|c|c|c|c|c|}
\hline S Samilo: & S Sornille & Samiglo & Resili: & Bableare & Mern: \\
\hline 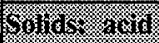 & 3get & (5) & I. & (x) & 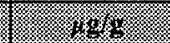 \\
\hline S97T001199 & Core 188 & Solid composite & 2,630 & 2,660 & 2,650 \\
\hline
\end{tabular}


Table B2-203. Tank 241-T-204 Analytical Results: Potassium (ICP).

\begin{tabular}{|c|c|c|c|c|c|}
\hline STanple: & Sarinole & $\begin{array}{l}\text { Sininle } \\
\text { Porrinin }\end{array}$ & mexill & 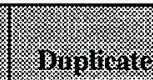 & 13020 \\
\hline Soinds sict & g8t & & (1. & 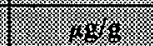 & $1=\sqrt{1.968}$ \\
\hline S97T001199 & Core 188 & Solid composite & 6,090 & 6,150 & $6,120^{\text {eC:d }}$ \\
\hline
\end{tabular}

Table B2-204. Tank 241-T-204 Analytical Results: Samarium (ICP).

\begin{tabular}{|c|c|c|c|c|c|}
\hline 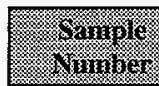 & 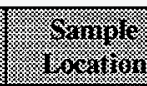 & Pornule & 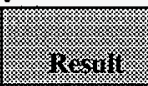 & Euplinar: & Vin \\
\hline 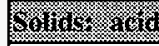 & 65 & (I) & 1 & 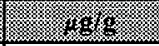 & 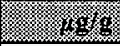 \\
\hline S97T001199 & Core 188 & Solid composite & $<28.6$ & $<28.1$ & $<28.4$ \\
\hline
\end{tabular}

Table B2-205. Tank 241-T-204 Analytical Results: Silicon (ICP).

\begin{tabular}{|c|c|c|c|c|c|}
\hline $\begin{array}{l}\text { Saminer } \\
\text { Jiminer }\end{array}$ & 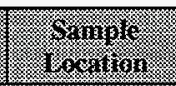 & rominge & nessinin & sipinatix & (1) \\
\hline \multicolumn{3}{|c|}{ 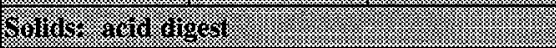 } & 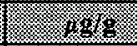 & 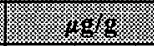 & 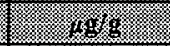 \\
\hline S97T001199 & Core 188 & Solid composite & 1,520 & 1,470 & $1,500^{\mathrm{QC:b}}$ \\
\hline
\end{tabular}

Table B2-206. Tank 241-T-204 Analytical Results: Silver (ICP).

\begin{tabular}{|c|c|c|c|c|c|}
\hline Sampic & Sample & Simplet & Resin: & irpliti:at & 18. \\
\hline \multicolumn{3}{|c|}{ 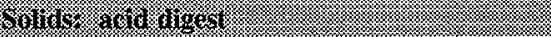 } & 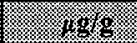 & 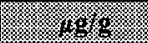 & $(1896$ \\
\hline S97T001199 & Core 188 & Solid composite & $<2.86$ & $<2.81$ & $<2.84^{\mathrm{QC}: \mathrm{d}}$ \\
\hline
\end{tabular}

Table B2-207. Tank 241-T-204 Analytical Results: Sodium (ICP).

\begin{tabular}{|c|c|c|c|c|c|}
\hline Sample: & 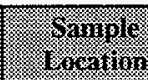 & Sormingle & I. & briplinate & 11:ar \\
\hline Sollos: a aro & 1868 & স্যে & 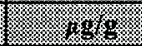 & $18 \mathrm{~g} \%$ & 486 \\
\hline S97T001199 & Core 188 & Solid composite & 31,700 & 31,900 & $31,800^{\mathrm{QC:d}}$ \\
\hline
\end{tabular}


HNF-1501 Rev. 0

Table B2-208. Tank 241-T-204 Analytical Results: Strontium (ICP).

\begin{tabular}{|c|c|c|c|c|c|}
\hline Sampla & Samagle & $\begin{array}{l}\text { Sample } \\
\text { Tomtion }\end{array}$ & resuil: & 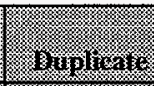 & 1rean \\
\hline \multicolumn{3}{|c|}{ 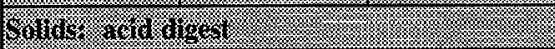 } & 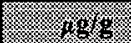 & (1) & 14848 \\
\hline S97T001199 & Core 188 & Solid composite & 495 & 502 & 499 \\
\hline
\end{tabular}

Table B2-209. Tank 241-T-204 Analytical Results: Sulfur (ICP).

\begin{tabular}{|c|c|c|c|c|c|}
\hline 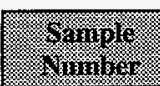 & I) & minil & isesitit & 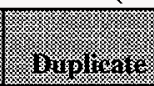 & Me: \\
\hline \multicolumn{3}{|c|}{ 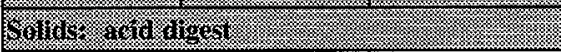 } & 1.36 & 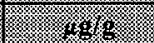 & 10.8 \\
\hline S97T001199 & Core 188 & Solid composite & $<28.6$ & $<28.1$ & $<28.4$ \\
\hline
\end{tabular}

Table B2-210. Tank 241-T-204 Analytical Results: Thallium (ICP).

\begin{tabular}{|c|c|c|c|c|c|}
\hline Saminic & Sorounter & 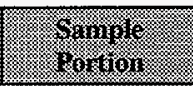 & nesilit & Mingivarte & ten \\
\hline \multicolumn{2}{|c|}{ 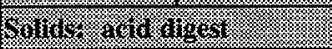 } & & 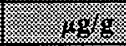 & d 1.96 & (1) \\
\hline S97T001199 & Core 188 & Solid composite & 290 & 308 & 299 \\
\hline
\end{tabular}

Table B2-211. Tank 241-T-204 Analytical Results: Titanium (ICP).

\begin{tabular}{|c|c|c|c|c|c|}
\hline Samples & 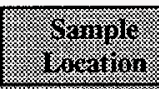 & 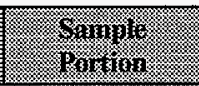 & 3)-3in: & Bingingate & (8) \\
\hline 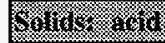 & 46. & 1) & 8.1 .89 & 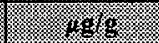 & $8136 \%$ \\
\hline S97T001199 & Core 188 & Solid composite & 3.47 & 3.46 & 3.46 \\
\hline
\end{tabular}

Table B2-212. Tank 241-T-204 Analytical Results: Total Uranium (ICP).

\begin{tabular}{|c|c|c|c|c|c|}
\hline Maringle & Saminge. & 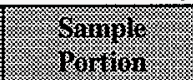 & Resuli & 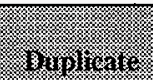 & utern \\
\hline \multicolumn{3}{|c|}{ 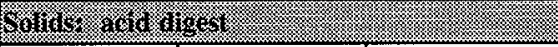 } & 2.838 & 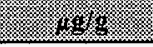 & 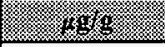 \\
\hline S97T001199 & Core 188 & Solid composite & $<143$ & $<140$ & $<142$ \\
\hline
\end{tabular}


Table B2-213. Tank 241-T-204 Analytical Results: Vanadium (ICP).

\begin{tabular}{|c|c|c|c|c|c|}
\hline 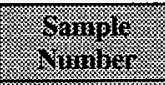 & $\begin{array}{l}\text { Sarinils: } \\
\text { I seation }\end{array}$ & $\begin{array}{l}\text { sample } \\
\text { Iortion }\end{array}$ & resint & minglicas & Vyan \\
\hline $60110 \%$ ardo & yest: & স্যে & 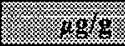 & (2) & 40 \\
\hline S97T001199 & Core 188 & Solid composite & $<14.3$ & $<14$ & $<14.2$ \\
\hline
\end{tabular}

Table B2-214. Tank 241-T-204 Analytical Results: Zinc (ICP).

\begin{tabular}{|c|c|c|c|c|c|}
\hline Sanpier & 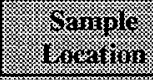 & 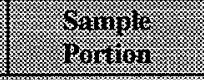 & Resnift & Huplikar: & f \\
\hline \multicolumn{2}{|c|}{ 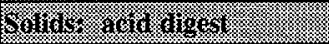 } & $\sqrt{4}$ & $\sqrt{1.868}$ & 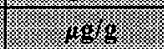 & $1 \times 1=\sqrt{1.968}$ \\
\hline S97T001199 & Core 188 & Solid composite & 54.2 & 71.2 & $62.7^{\text {eC:e }}$ \\
\hline
\end{tabular}

Table B2-215. Tank 241-T-204 Analytical Results: Zirconium (ICP).

\begin{tabular}{|c|c|c|c|c|c|}
\hline Siminer & S Samile & gonvile & Resini: & brinticase & Yean \\
\hline \multicolumn{3}{|c|}{ Wollus acid algest: } & 2.140 & 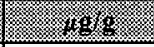 & 8.898 \\
\hline S97T001199 & Core 188 & Solid composite & $<2.86$ & $<2.81$ & $<2.84$ \\
\hline
\end{tabular}

Table B2-216. Tank 241-T-204 Analytical Results: Bromide (IC).

\begin{tabular}{|c|c|c|c|c|c|}
\hline $\begin{array}{l}\text { oamipier } \\
\text { Jiminicer }\end{array}$ & Ginaple & Sumple & Resul & Divoling & $\sqrt{12}$ \\
\hline Nomins vare & ainger: & $x_{3}$ & $4=$ & 4.496 & 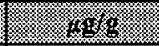 \\
\hline S97T001200 & Core 188 & Solid composite & $<283$ & $<282$ & $<283$ \\
\hline
\end{tabular}

Table B2-217. Tank 241-T-204 Analytical Results: Chloride (IC).

\begin{tabular}{|c|c|c|c|c|c|}
\hline Samiles & Samples & 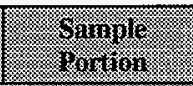 & nesinil: & OH: & Hisin \\
\hline 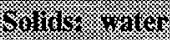 & digest: & স্টে & 3138 & 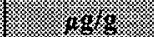 & 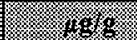 \\
\hline S97T001200 & Core 188 & Solid composite & 694 & 653 & 673 \\
\hline
\end{tabular}


HNF-1501 Rev. 0

Table B2-218. Tank 241-T-204 Analytical Results: Fluoride (IC).

\begin{tabular}{|c|c|c|c|c|c|}
\hline Sinninger & f & $\begin{array}{l}\text { Sample } \\
\text { Rarnitiri }\end{array}$ & 1) & 3.15ing & mer: \\
\hline \multicolumn{2}{|c|}{ 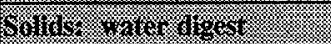 } & & 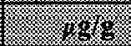 & 48 & 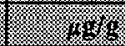 \\
\hline S97T001200 & Core 188 & Solid composite & 6,060 & 5,820 & 5,940 \\
\hline
\end{tabular}

Table B2-219. Tank 241-T-204 Analytical Results: Nitrate (IC).

\begin{tabular}{|c|c|c|c|c|c|}
\hline Sample & $\begin{array}{l}\text { Samingle } \\
\text { Y }\end{array}$ & $\begin{array}{l}\text { Sample } \\
\text { Tontion }\end{array}$ & Resili & Bunglieat & $4\left(4 x_{3}\right)$ \\
\hline 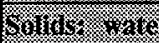 & $118-18=0$ & (1) & 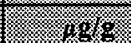 & $4 \sin \mathrm{g}$ & 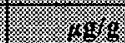 \\
\hline S97T001200 & Core 188 & Solid composite & 57,400 & 52,900 & 55,200 \\
\hline
\end{tabular}

Table B2-220. Tank 241-T-204 Analytical Results: Nitrite (IC).

\begin{tabular}{|c|c|c|c|c|c|}
\hline Suniticy & 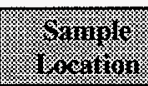 & Timing & Istill & 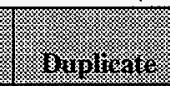 & 81.18 \\
\hline \multicolumn{3}{|c|}{ 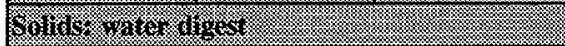 } & 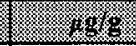 & $1=48 \mathrm{~g}$ & I \\
\hline S97T001200 & Core 188 & Solid composite & 300 & 267 & 284 \\
\hline
\end{tabular}

Table B2-221. Tank 241-T-204 Analytical Results: Phosphate (IC).

\begin{tabular}{|c|c|c|c|c|c|}
\hline Simples & Simile & Somple & resintis & Buplicatio & Meri \\
\hline . & ave a a & (3) & 8.862 & 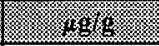 & $f(5)$ \\
\hline $97 \mathrm{~T} 001200$ & Core 188 & Solid composite & 2,550 & 2,360 & 2,460 \\
\hline
\end{tabular}

Table B2-222. Tank 241-T-204 Analytical Results: Sulfate (IC).

\begin{tabular}{|c|c|c|c|c|c|}
\hline Yample & Signifle & Sample & resiul & 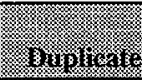 & yesn \\
\hline \multicolumn{3}{|c|}{ 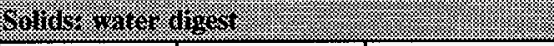 } & 8.88 & $\sqrt{.858}$ & (1) \\
\hline S97T001200 & Core 188 & Solid composite & 414 & $<312$ & $<363^{\mathrm{QC}: 0}$ \\
\hline
\end{tabular}


HNF-1501 Rev. 0

Table B2-223. Tank 241-T-204 Analytical Results: Oxalate (IC).

\begin{tabular}{|c|c|c|c|c|c|}
\hline 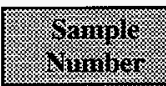 & Simple & $\begin{array}{l}\text { Samile } \\
\text { Irorition. }\end{array}$ & restill & 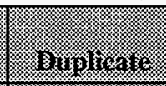 & 14 man \\
\hline borits war & 168ri: & 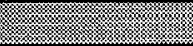 & 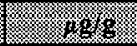 & 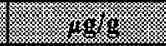 & 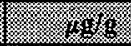 \\
\hline S97T001200 & Core 188 & Solid composite & 1,350 & 1,310 & 1,330 \\
\hline
\end{tabular}

Table B2-224. Tank 241-T-204 Analytical Results: Bulk Density.

\begin{tabular}{|c|c|c|c|c|c|}
\hline $\begin{array}{l}\text { Sampil: } \\
\text { Niminer }\end{array}$ & Samplo & H: samull & resula & 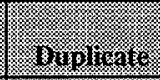 & Vilean \\
\hline 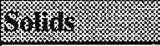 & 2: & 18 & (1. & grais & 98181: \\
\hline S97T000494 & 188: 1 & Lower half & 1.15 & N/A & 1.15 \\
\hline S97T000570 & 188: 2 & Lower half & 1.17 & N/A & 1.17 \\
\hline S97T000571 & 188: 3 & Lower half & 1.16 & N/A & 1.16 \\
\hline S97T000572 & $188: 4$ & Lower half & 1.2 & N/A & 1.2 \\
\hline S97T000609 & $188: 5$ & Lower half & 1.21 & N/A & 1.21 \\
\hline S97T000610 & 188: 6 & Lower half & 1.17 & N/A & 1.17 \\
\hline S97T000611 & 188: 7 & Lower half & 1.18 & N/A & 1.18 \\
\hline S97T000612 & 188: 8 & Lower half & 1.2 & N/A & 1.2 \\
\hline S97T000613 & 188: 9 & Lower half & 1.19 & N/A & 1.19 \\
\hline S97T000614 & 188:10 & Lower half & 1.21 & N/A & 1.21 \\
\hline S97T001191 & Core 188 & Solid composite & 1.21 & N/A & 1.21 \\
\hline
\end{tabular}


HNF-1501 Rev. 0

Table B2-225. Tank 241-T-204 Analytical Results: Percent Water (DSC/TGA).

\begin{tabular}{|c|c|c|c|c|c|}
\hline $\begin{array}{l}\text { Saminile } \\
\text { Vumber }\end{array}$ & Vornition & $\begin{array}{l}\text { Sarnelo } \\
\text { jortion }\end{array}$ & ressing & Hopitos = & Mrain \\
\hline SWolyas & $\sqrt{4}$ & (1) & (1) & $\sqrt{4}$ & $\sqrt{2.3 .}$ \\
\hline S97T000499 & \multirow[t]{2}{*}{ 188: 1} & Upper half & 88.6 & 81 & 84.8 \\
\hline S97T000498 & & Lower half & 79.5 & 78.9 & 79.2 \\
\hline S97T000574 & 188: 2 & Lower half & 75.6 & 79.4 & 77.5 \\
\hline S97T000575 & 188: 3 & Lower half & 79.8 & 79.5 & 79.7 \\
\hline S97T000580 & \multirow[t]{2}{*}{ 188: 4} & Upper half & 72.5 & 71.4 & 71.9 \\
\hline S97T000576 & & Lower half & 74.9 & 74.8 & 74.9 \\
\hline S97T000633 & \multirow[t]{2}{*}{$188: 5$} & Upper half & 76.9 & 76.2 & 76.5 \\
\hline S97T000603 & & Lower half & 75.9 & 74.9 & 75.4 \\
\hline S97T000634 & \multirow[t]{2}{*}{ 188: 6} & Upper half & 75.6 & 78 & 76.8 \\
\hline \$97T000604 & & Lower half & 76.8 & 75.9 & 76.4 \\
\hline \multicolumn{3}{|l|}{ Swlins } & ( & (3) & 28.8 \\
\hline S97T000635 & \multirow[t]{2}{*}{ 188: 7} & Upper half & 72.7 & 72.1 & 72.4 \\
\hline S97T000605 & & Lower half & 76.2 & 74 & 75.1 \\
\hline S97T000636 & \multirow[t]{2}{*}{ 188: 8} & Upper half & 72 & 72.8 & 72.4 \\
\hline S97T000606 & & Lower half & 73.2 & 69.6 & 71.4 \\
\hline S97T000637 & \multirow[t]{2}{*}{ 188: 9} & Upper half & 71 & 75.1 & 73.1 \\
\hline S97T000607 & & Lower half & 74.4 & 76 & 75.2 \\
\hline S97T000638 & \multirow[t]{2}{*}{$188: 10$} & Upper half & 53.7 & 73.3 & $63.5^{\text {QC:e }}$ \\
\hline S97T000608 & & Lower half & 73.9 & 75 & 74.4 \\
\hline S97T001197 & Core 188 & Solid composite & 75.9 & 74.3 & 75.1 \\
\hline
\end{tabular}


HNF-1501 Rev. 0.

Table B2-226. Tank 241-T-204 Analytical Results: Total Alpha (Alpha).

\begin{tabular}{|c|c|c|c|c|c|}
\hline $\begin{array}{l}\text { Saminte: } \\
\text { Suninger }\end{array}$ & $\begin{array}{l}\text { Samule } \\
\text { Voreakin }\end{array}$ & 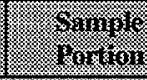 & Imsin: & Bubluminge & (4tes. \\
\hline Soining r (1) & s: & $\sqrt{8}$ & 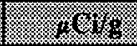 & 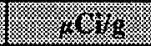 & . \\
\hline S97T000587 & 188: 1 & Lower half & 0.176 & 0.151 & 0.163 \\
\hline S97T000588 & 188: 2 & Lower half & 0.138 & 0.149 & 0.144 \\
\hline S97T000589 & $188: 3$ & Lower half & 0.138 & 0.116 & 0.127 \\
\hline S97T000590 & 188: 4 & Lower half & 0.138 & 0.136 & 0.137 \\
\hline S97T000621 & 188: 5 & Lower $1 / 2$ & 0.127 & 0.165 & $0.146^{\mathrm{QC}: c}$ \\
\hline S97T000622 & 188: 6 & Lower half & 0.129 & 0.138 & 0.134 \\
\hline S97T000623 & 188: 7 & Lower half & $<0.00316$ & 0.146 & $<0.0746$ \\
\hline S97T000624 & 188: 8 & Lower half & 0.184 & 0.158 & 0.171 \\
\hline S97T000625 & 188: 9 & Lower half & 0.208 & 0.189 & 0.199 \\
\hline S97T000626 & $188: 10$ & Lower half & 0.157 & 0.141 & 0.149 \\
\hline
\end{tabular}

Table B2-227. Tank 241-T-204 Analytical Results: Americium-241 (GEA).

\begin{tabular}{|c|c|c|c|c|c|}
\hline Sintir) & 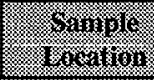 & S S & resing & Bungivarie & y \\
\hline 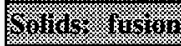 & $\sqrt{3}$ & 40 & 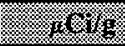 & 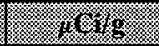 & 要 \\
\hline S97T001198 & Core 188 & Solid composite & 0.0227 & 0.0262 & 0.0244 \\
\hline
\end{tabular}

Table B2-228. Tank 241-T-204 Analytical Results: Cesium-137 (GEA).

\begin{tabular}{|c|c|c|c|c|c|}
\hline Samir & 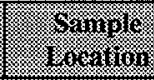 & Samole: & roving & Bungure & 816 and \\
\hline \multicolumn{2}{|c|}{ Soldes - ins inn } & & (m) & 1. (1) & 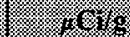 \\
\hline S97T001198 & Core 188 & Solid composite & 0.00775 & 0.00777 & 0.00776 \\
\hline
\end{tabular}


Table B2-229. Tank 241-T-204 Analytical Results: Cobalt-60 (GEA).

\begin{tabular}{|c|c|c|c|c|c|}
\hline Samper & 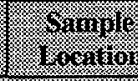 & $\begin{array}{l}\text { Srmple } \\
\text { Orombon }\end{array}$ & nesilli & 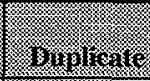 & IItsin \\
\hline bainge trisio & 1. & 18 & 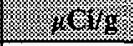 & $1.1 \%$ & 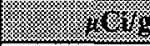 \\
\hline S97T001198 & Core 188 & $\begin{array}{l}\text { Solid } \\
\text { composite }\end{array}$ & $<0.00171$ & $<0.00148$ & $<0.00159$ \\
\hline
\end{tabular}

Table B2-230. Tank 241-T-204 Analytical Results: Europium-154 (GEA).

\begin{tabular}{|c|c|c|c|c|c|}
\hline 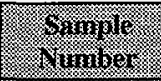 & 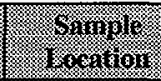 & Sompie & trasil & oringinerte & irsarin \\
\hline Sindo $7 n$ & & 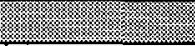 & . & 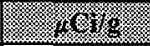 & 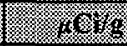 \\
\hline S97T001198 & Core 188 & Solid composite & $<0.00439$ & $<0.00403$ & $<0.00421$ \\
\hline
\end{tabular}

Table B2-231. Tank 241-T-204 Analytical Results: Europium-155 (GEA).

\begin{tabular}{|c|c|c|c|c|c|}
\hline Siminger & f somple & 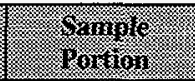 & Resul: & Brivilicite & \\
\hline 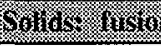 & & & 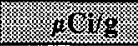 & 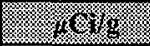 & 169648 \\
\hline S97T001198 & Core 188 & Solid composite & $<0.00353$ & $<0.00329$ & $<0.00341$ \\
\hline
\end{tabular}

Table B2-232. Tank 241-T-204 Analytical Results: Strontium-89/90.

\begin{tabular}{|c|c|c|c|c|c|}
\hline Sumile & $\begin{array}{l}\text { Simpil } \\
\text { Sorearour }\end{array}$ & $\begin{array}{l}\text { Sininile: } \\
\text { rortion }\end{array}$ & Resul: & Jiblinare & Mcur \\
\hline Soluas & & 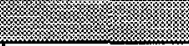 & 1010 & 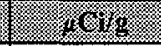 & 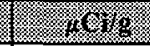 \\
\hline S97T001198 & Core 188 & Solid composite & 0.00346 & 0.00574 & $0.0046^{\text {eC:e, } \mathrm{f}}$ \\
\hline
\end{tabular}

Table B2-233. Tank 241-T-204 Analytical Results: Total Inorganic Carbon.

\begin{tabular}{|c|c|c|c|c|c|}
\hline Sumber & 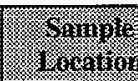 & Siningle & 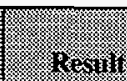 & Hapil Herve & Meri: \\
\hline Ings & 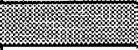 & (1) & 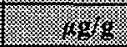 & .1 .96 & 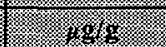 \\
\hline S97T001197 & Core 188 & Solid composite & 1,410 & 1,380 & 1,400 \\
\hline
\end{tabular}


Table B2-234. Tank 241-T-204 Analytical Results: Total Organic Carbon.

\begin{tabular}{|c|c|c|c|c|c|}
\hline Shangle & 9.17ngl & $\begin{array}{l}\text { Sumple } \\
\text { Portion }\end{array}$ & Hesing & Buplicare & Yrom: \\
\hline Soling & (4) & $\sqrt{4}$ & 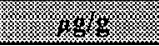 & 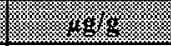 & 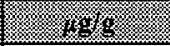 \\
\hline S97T001197 & Core 188 & Solid composite & 346 & 278 & $312^{\mathrm{QC:e}}$ \\
\hline
\end{tabular}




\section{B3.0 ASSESSMENT OF CHARACTERIZATION RESULTS}

This section discusses the overall quality and consistency of the current sampling results for the T-200 tanks. It also evaluates sampling and analysis factors that may impact data interpretation. These factors are used to assess overall data quality and consistency and to identify limitations in data use.

\section{B3.1 FUELD OBSERVATIONS}

Sample recovery from the T-200 series tanks was generally good. Tank 241-T-201 samples were not as complete as expected. There was substantial Drainable liquid present in most samples, where surveillance estimates indicate solid material should be present. This appears to be the result of a localized depression beneath riser 3. Most samples from the other tanks appeared to have nearly complete recoveries. The segments were almost full. The consistency of the waste, gel-like and pliable, was conducive to high sample recoveries. Taking advantage of the available prior information and assuming the similarity observed between the B-200 tanks and the T-200 series tanks is the same, only one core sample was taken from each tank.

The expected depth of the waste ranged from $2.7 \mathrm{~m}$ to $4.9 \mathrm{~m}$ (105 to $194 \mathrm{in}$.) The readings taken at the risers at the time of sampling largely supported these estimates. In-tank photographs showed that the waste in tank 241-T-201 appears different from the other T-200 series tanks. It has a dried, cracked, rust-brown surface. There is a blue-black region that appears to have standing liquid present. The other T-200 series tanks appear to lack any free liquid, and have dried, cracked gray-brown or gray-black surfaces. Although the requirement that vertical profiles of the waste be obtained from two risers was not met, the intent of the safety screening DQO (Dukelow et al. 1995) was met because of the large amount of data collected from the B-200 series tanks and the degree of agreement observed between the two sets of tanks.

\section{B3.2 QUALITY CONTROL ASSESSMENT}

The usual QC assessment includes an evaluation of the appropriate standard recoveries, spike recoveries, duplicate analyses, and blanks that are performed in conjunction with the chemical analyses. All pertinent QC tests were conducted on 1997 core samples, allowing a full assessment of the accuracy and precision of the data. The sampling and analysis plans $(\mathrm{Hu}$ [1997], Bell [1997], Schreiber [1997b], and Winkleman [1997]) established specific criteria for all analytes. Sample and duplicate pairs with one or more QC results outside the specified criteria were identified by footnotes in the data summary tables. 
The standard and spike recovery results provide an estimate of analysis accuracy. If a standard or spike recovery is above or below the given criterion, the analytical results may be biased high or low, respectively. The standard recoveries for the large majority of the analytes examined in the T-200 series samples were within acceptable laboratory operating parameters. Spike recoveries were often invalid for analytes present in very high quantities, such as bismuth, chromium, iron, lanthanum, manganese, and sodium. The spike concentration was often too low with regard to the sample concentration to be distinguished. Post digestion spikes run on the samples would show the results to be acceptable. Some of the total alpha spike recoveries would be outside of the QC thresholds, however, additional assays would attribute this behavior to matrix interference.

The precision is estimated by the relative percent difference (RPD), which is defined as the absolute value of the difference between the primary and duplicate samples, divided by their mean, times 100 . Relative percent differences outside of the specified QC bounds were observed for several analytes in the T-200 series tanks. Total alpha, strontium-90, and phosphate were the analytes most frequently observed having elevated RPDs. Absorbance from alpha solids on the sample mount (self-shielding) was identified as a potential issue for the total alpha measurement, and sample heterogeneity was identified as the cause of the lack of reproducibility for the other analytes. Additionally, analytes near the detection limits are subject to larger RPDs.

Finally, a few samples had results that exceeded the criterion for preparation blanks. The analytes observed were those usually associated with cross contamintation from sample preparation and/or glassware (sodium, calcium, and nitrate). Slightly elevated total alpha measurements were observed in the blanks, however in all cases the concentrations observed were small compared to the sample concentrations. Therefore, contamination was not considered a problem.

In summary, the vast majority of $\mathrm{QC}$ results were within the boundaries specified in the sampling and analysis plans. The discrepancies mentioned here and footnoted in the data summary tables should not impact data validity or use.

\section{B3.3 DATA CONSISTENCY CHECKS}

Comparing different analytical methods is helpful in assessing the consistency and quality of the data. Several comparisons were possible with the data set provided by the core samples: a comparison of phosphorous and sulfur as analyzed by ICP to phosphate and sulfate as analyzed by IC and a comparison of TOC to oxalate. In addition, mass and charge balances were calculated to help assess the overall data consistency. 


\section{B3.3.1 Comparison of Results from Different Analytical Methods}

The following data consistency checks compare the results from two analytical methods. Agreement between the two methods strengthens the credibility of both results, but poor agreement brings the reliability of the data into question. All analytical mean results were taken from Section B2.0 tables. Tables B3-1, B3-2, and B3-3 show comparisons for specific analytes between methods.

Table B3-1. Comparison of Phosphate to Total Phosphorous.

\begin{tabular}{|c|c|c|c|c|}
\hline saini: & 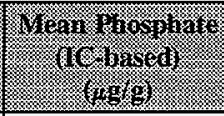 & 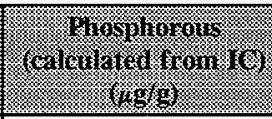 & 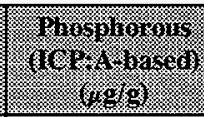 & 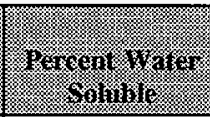 \\
\hline $\mathrm{T}-201$ & 443 & 145 & 4,560 & 3.18 \\
\hline $\mathrm{T}-202$ & 1,840 & 601 & 2,390 & 25.1 \\
\hline $\mathrm{T}-203$ & 2,910 & 951 & 2,390 & 39.8 \\
\hline $\mathrm{T}-204$ & 2,460 & 804 & 2,460 & 32.7 \\
\hline
\end{tabular}

Table B3-2. Comparison of Sufate to Total Sulfur.

\begin{tabular}{|c|c|c|c|}
\hline (15ant & 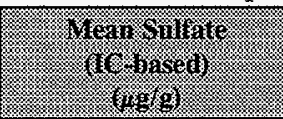 & 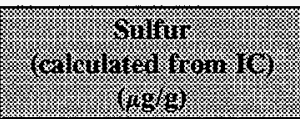 & 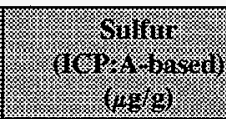 \\
\hline T-201 & $<77.9$ & $<26$ & 92.2 \\
\hline T-202 & 1,110 & 370 & 302 \\
\hline T-203 & $<438$ & $<146$ & 128 \\
\hline $\mathrm{T}-204$ & $<363$ & $<121$ & $<28.4$ \\
\hline
\end{tabular}

Table B3-3. Comparison of Oxalate to Total Organic Carbon.

\begin{tabular}{|c|c|c|c|c|}
\hline Iant: & 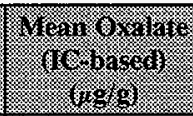 & 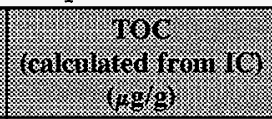 & 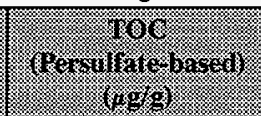 & 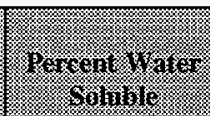 \\
\hline T-201 & 1,140 & 311 & 304 & 100 \\
\hline $\mathrm{T}-202$ & 482 & 131 & 347 & 37.8 \\
\hline $\mathrm{T}-203$ & 1,370 & 373 & No data & NA \\
\hline T-204 & 1,330 & 363 & 312 & 100 \\
\hline
\end{tabular}


The analytical phosphorous mean result as determined by ICP ranged from 2,390 to $4,560 \mu \mathrm{g} / \mathrm{g}$. The phosphate-based phosphorous values (obtained from converting phosphate to phosphorous) ranged from 145 to $951 \mu \mathrm{g} / \mathrm{g}$. This behavior suggests that the phosphate is not water-soluble.

The analytical sulfur mean result as determined by ICP ranged from below detection to $302 \mu \mathrm{g} / \mathrm{g}$. The sulfate-based sulfur values ranged from below detection to $370 \mu \mathrm{g} / \mathrm{g}$. This suggests that there is very little sulfate present, but what sulfate there is, is very water-soluble.

The analytical TOC mean result as determined by persulfate oxidation ranged from 304 to $347 \mu \mathrm{g} / \mathrm{g}$. The oxalate-based TOC values ranged from 131 to $363 \mu \mathrm{g} / \mathrm{g}$. This behavior suggests that most of the TOC present is oxalate.

\section{B3.3.2 Mass and Charge Balance}

The principal objective in performing mass and charge balances is to determine whether the measurements are consistent. In calculating the balances, only the analytes listed in Section B2.0, which were detected at a concentration of $1,000 \mu \mathrm{g} / \mathrm{g}$ or greater, were considered.

Except sodium, potassium, and lanthanum, all cations listed in Tables B3-4, B3-7, B3-10, and B3-13 were assumed to be in their most common hydroxide or oxide form. Sodium and potassium were assumed to be ions, and lanthanum was assumed to be combined as an insoluble fluoride. The concentrations of the assumed species were calculated stoichiometrically. Because precipitates are neutral species, all positive charge was attributed to the cations. The anions listed in Tables B3-5, B3-8, B3-11, and B3-14 were assumed to be present as sodium and potassium salts and were expected to balance the positive charge exhibited by the cations. Phosphorous, as determined by ICP, is assumed to be mostly water insoluble bismuth phosphate and appears only in the cation mass and charge calculations.

The concentrations of cations, anions, and the percent water were ultimately used to calculate the mass balance. The mass balance was calculated from the formula below. The factor 10,000 is the conversion factor from weight percent to $\mu \mathrm{g} / \mathrm{g}$. These calculations are shown in Tables B3-6, B3-9, B3-12, and B3-15.

Tank 241-T-201

Mass balance (Tank T-201) $=\%$ Water $\times 10,000+$ \{Total Analyte Concentration $\}$

$$
\begin{aligned}
= & \% \text { Water }\left(\mathrm{x} \mathrm{10,000)}+\left\{\mathrm{Bi}(\mathrm{OH})_{3}+\mathrm{FeO}(\mathrm{OH})+\right.\right. \\
& \mathrm{MnO}_{2}+\mathrm{Na}^{+}+\mathrm{K}^{+}+\mathrm{Cr}(\mathrm{OH})_{3}+\mathrm{Ca}(\mathrm{OH})_{2} \\
& \left.+\mathrm{Sr}_{(\mathrm{OH}}\right)_{2}+\mathrm{LaF}_{3}+\mathrm{F}^{-}+\mathrm{NO}_{3}^{-}+\mathrm{CO}_{3}^{-2}+\mathrm{Cl}^{-}+ \\
& \left.\mathrm{BiPO}_{4}+\mathrm{C}_{2} \mathrm{O}_{4}^{-2}+\mathrm{SiO}_{2}\right\}
\end{aligned}
$$


The total analyte concentrations calculated from the above equation is $389,000 \mu \mathrm{g} / \mathrm{g}$. The mean weight percent water (obtained from the TGA) is 61.6 percent or $616,000 \mu \mathrm{g} / \mathrm{g}$. The mass balance resulting from adding the percent water to the total analyte concentration is 100.4 percent (see Table B3-6).

The following equations demonstrate the derivation of total cations and total anions; the charge balance is the ratio of these two values.

Total cations $(\mu \mathrm{eq} / \mathrm{g})=\left[\mathrm{Na}^{+}\right] / 23.0+\left[\mathrm{K}^{+}\right] / 39.1=1,527 \mu \mathrm{eq} / \mathrm{g}$

Total anions $(\mu \mathrm{eq} / \mathrm{g})=[\mathrm{F}] / 19.0+\left[\mathrm{NO}_{3}^{-}\right] / 62.0+\left[\mathrm{CO}_{3}^{-2}\right] / 30.0+\left[\mathrm{Cl}^{-}\right] / 35.5+$ $\left[\mathrm{C}_{2} \mathrm{O}_{4}^{-2}\right] / 44.0=1,236 \mu \mathrm{eq} / \mathrm{g}$

The charge balance obtained by dividing the sum of the positive charge by the sum of the negative charge was 1.24 . The net positive charge was $291 \mu \mathrm{eq} / \mathrm{g}$. To balance this net positive charge, an amount of hydroxide equal to the charge imbalance was assumed. Including this term makes the charge balance 1.00 and the mass balance 101 percent, well within the uncertainties associated with the assumptions and measurements made. In summary, the above calculations yield reasonable mass and charge balance values (close to 1.00 for charge balance and 100 percent for mass balance), indicating that the analytical results are consistent.

Table B3-4. 241-T-201 Cation Mass and Charge Data.

\begin{tabular}{|c|c|c|c|c|}
\hline 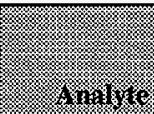 & (4om & 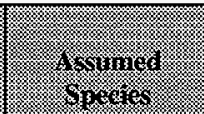 & 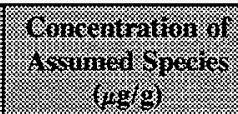 & 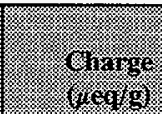 \\
\hline \multirow[t]{2}{*}{$\mathrm{Bi}$} & \multirow[t]{2}{*}{119,000} & \multirow{2}{*}{\begin{tabular}{|l|}
$\mathrm{BiPO}_{4}$ \\
$\mathrm{Bi}(\mathrm{OH})_{3}$
\end{tabular}} & \multirow{2}{*}{$\begin{array}{l}43,800 \\
111,000\end{array}$} & 0.00 \\
\hline & & & & 0.00 \\
\hline $\mathrm{Ca}$ & 1,240 & $\mathrm{Ca}(\mathrm{OH})_{2}$ & 2,290 & 0.00 \\
\hline $\mathrm{Cr}$ & 5,350 & $\mathrm{Cr}(\mathrm{OH})_{3}$ & 10,600 & 0.00 \\
\hline $\mathrm{Fe}$ & 9,860 & $\mathrm{FeO}(\mathrm{OH})$ & 13,900 & 0 \\
\hline $\mathrm{La}$ & 24,900 & $\mathrm{LaF}_{3}$ & 34,800 & 0 \\
\hline $\mathrm{Mn}$ & 44,300 & $\mathrm{MnO}_{2}$ & 70,100 & 0 \\
\hline $\mathrm{K}$ & 4,810 & $\mathrm{~K}^{+}$ & 4,810 & 123 \\
\hline $\mathrm{Na}$ & 32,300 & $\mathrm{Na}^{+}$ & 32,300 & 1,404 \\
\hline $\mathrm{Sr}$ & 1,210 & $\mathrm{Sr}(\mathrm{OH})_{2}$ & 1,530 & 0 \\
\hline & & Total & 325,000 & 1,527 \\
\hline
\end{tabular}


Table B3-5. 241-T-201 Anion Mass and Charge Data.

\begin{tabular}{|c|c|c|c|c|}
\hline . rnalye & 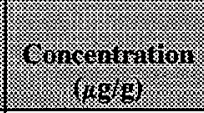 & Szsuried: & (N) & F \\
\hline $\mathrm{Cl}$ & 1,080 & $\mathrm{Cl}^{-}$ & 1,080 & $(30)$ \\
\hline TIC & 810 & $\mathrm{CO}_{3}^{-2}$ & 4,050 & (135) \\
\hline$F$ & 5,080 & $F^{-}$ & 5,080 & (267) \\
\hline $\mathrm{NO3}$ & 48,300 & $\mathrm{NO}_{3-}$ & 48,300 & $(779)$ \\
\hline TOC & 304 & $\mathrm{C}_{2} \mathrm{O}_{4}^{-2}$ & 1,120 & $(25)$ \\
\hline $\mathrm{P}$ & 4,560 & $\mathrm{PO}_{4}^{-3}$ & $\mathrm{As} \mathrm{BiPO}_{4}$ & 0 \\
\hline $\mathbf{S i}$ & 1,860 & $\mathrm{SiO}_{2}$ & 3,980 & 0 \\
\hline \multicolumn{4}{|c|}{ Total $\mid 63,600$} & $(1,236)$ \\
\hline
\end{tabular}

Table B3-6. Mass and Charge Balance Totals for Tank 241-T-201.

\begin{tabular}{|c|c|c|}
\hline I. & 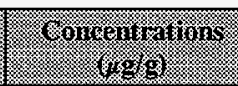 & (1) \\
\hline Total from Table B3-2 (cations) & 325,000 & 1,527 \\
\hline Total from Table B3-3 (anions) & 63,600 & $(1,236)$ \\
\hline Water percent & 616,000 & 0 \\
\hline Subtotal & $1,004,600$ & +291 \\
\hline Added $\mathrm{OH}$ to charge balance & 4,950 & (291) \\
\hline Total & $1,010,000$ & 0 \\
\hline
\end{tabular}

\section{Tank 241-T-202}

The concentrations of cations, anions, and the percent water were ultimately used to calculate the mass balance. The mass balance was calculated from the formula below. The factor 10,000 is the conversion factor from weight percent to $\mu \mathrm{g} / \mathrm{g}$.

Mass balance $($ Tank T-202) $=\quad \%$ Water $\times 10,000+$ TTotal Analyte Concentration $\}$

$$
\begin{array}{ll}
= & \% \text { Water }(\mathrm{x} 10,000)+\left\{\mathrm{Bi}(\mathrm{OH})_{3}+\mathrm{FeO}(\mathrm{OH})+\right. \\
& \mathrm{MnO}_{2}+\mathrm{Na}^{+}+\mathrm{K}^{+}+\mathrm{Cr}(\mathrm{OH})_{3}+\mathrm{SO}_{4}^{-2}+\mathrm{LaF}_{3}+ \\
& \left.\mathrm{F}+\mathrm{NO}_{3}^{-}+\mathrm{CO}_{3}^{-2}+\mathrm{BiPO}_{4}+\mathrm{C}_{2} \mathrm{O}_{4}^{-2}+\mathrm{SiO}_{2}\right\}
\end{array}
$$


The total analyte concentrations calculated from the above equation is $247,000 \mu \mathrm{g} / \mathrm{g}$. The mean weight percent water (obtained from the TGA) is 72.8 percent or $728,000 \mu \mathrm{g} / \mathrm{g}$. The mass balance resulting from adding the percent water to the total analyte concentration is 97.5 percent (see Table B3-9).

The following equations demonstrate the derivation of total cations and total anions; the charge balance is the ratio of these two values.

Total cations $(\mu \mathrm{eq} / \mathrm{g})=\left[\mathrm{Na}^{+}\right] / 23.0+\left[\mathrm{K}^{+}\right] / 39.1=1,744 \mu \mathrm{eq} / \mathrm{g}$

Total anions $(\mu \mathrm{eq} / \mathrm{g})=[\mathrm{F}] / 19.0+\left[\mathrm{NO}_{3}^{-}\right] / 62.0+\left[\mathrm{CO}_{3}^{-2}\right] / 30.0+\left[\mathrm{SO}_{4}^{-2}\right] / 48.0$ $\left[\mathrm{C}_{2} \mathrm{O}_{4}^{-2}\right] / 44.0=1,802 \mu \mathrm{eq} / \mathrm{g}$

The charge balance obtained by dividing the sum of the positive charge by the sum of the negative charge was 0.97 . The net negative charge was $58 \mu \mathrm{eq} / \mathrm{g}$. To balance this net negative charge, an amount of sodium equal to the charge imbalance could be assumed. This would represent approximately $1,330 \mu \mathrm{g} \mathrm{Na} / \mathrm{g}$ waste. The uncertainty associated with the sodium measurement is approximately $1,090 \mu \mathrm{g} \mathrm{Na} / \mathrm{g}$ waste. Because of the relative closeness of the mass and charge balance, and the size of the potential correction with respect to the uncertainty, no futher adjustments are made. The mass and charge balance results are well within the uncertainties associated with the assumptions and measurements made. In summary, the above calculations yield reasonable mass and charge balance values (close to 1.00 for charge balance and 100 percent for mass balance), indicating that the analytical results are consistent.

Table B3-7. 241-T-202 Cation Mass and Charge Data.

\begin{tabular}{|c|c|c|c|c|}
\hline Al. & 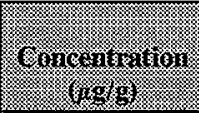 & 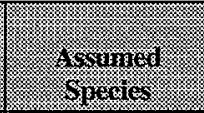 & 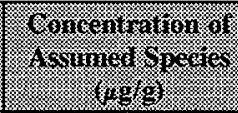 & 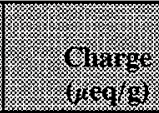 \\
\hline \multirow[t]{2}{*}{$\overline{\mathrm{Bi}}$} & \multirow[t]{2}{*}{41,000} & \multirow{2}{*}{\begin{tabular}{|l}
$\mathrm{BiPO}_{4}$ \\
$\mathrm{Bi}(\mathrm{OH})_{3}$
\end{tabular}} & \multirow{2}{*}{$\begin{array}{l}23,400 \\
30,900\end{array}$} & 0.00 \\
\hline & & & & 0.00 \\
\hline $\mathrm{CI}$ & 3,760 & $\mathrm{Cr}(\mathrm{OH})_{3}$ & 7,450 & 0.00 \\
\hline $\mathrm{Fe}$ & 7,620 & $\mathrm{FeO}(\mathrm{OH})$ & 12,100 & 0 \\
\hline $\mathrm{La}$ & 12,600 & $\mathrm{LaF}_{3}$ & 17,800 & 0 \\
\hline $\mathrm{Mn}$ & 14,800 & $\mathrm{MnO}_{2}$ & 23,400 & 0 \\
\hline $\mathrm{K}$ & 7,140 & $\mathbf{K}^{+}$ & 7,140 & 183 \\
\hline \multirow[t]{2}{*}{$\mathrm{Na}$} & 35,900 & $\mathrm{Na}^{+}$ & 35,900 & 1,561 \\
\hline & \multicolumn{2}{|r|}{ Total } & 158,100 & 1,744 \\
\hline
\end{tabular}


Table B3-8. 241-T-202 Anion Mass and Charge Data.

\begin{tabular}{|c|c|c|c|c|}
\hline 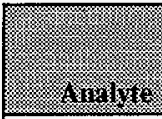 & 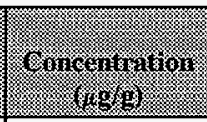 & Isimini: & 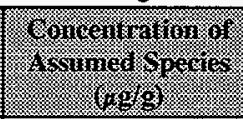 & 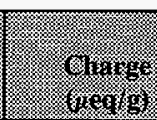 \\
\hline TIC & 2,080 & $\mathrm{CO}_{3}^{-2}$ & 10,400 & $(347)$ \\
\hline$F$ & 6,560 & $F$ & 6,560 & $(345)$ \\
\hline $\mathrm{NO}_{3}^{-}$ & 65,600 & $\mathrm{NO}_{3}^{-}$ & 65,600 & $(1,058)$ \\
\hline TOC & 347 & $\mathrm{C}_{2} \mathrm{O}_{4}^{-2}$ & 1,270 & $(29)$ \\
\hline $\mathrm{P}$ & 2,390 & $\mathrm{PO}_{4}^{-3}$ & As $\mathrm{BiPO}_{4}$ & 0 \\
\hline $\mathrm{SO}_{4}^{-2}$ & 1,110 & $\mathrm{SO}_{4}^{-2}$ & 1,110 & (23) \\
\hline \multirow[t]{2}{*}{$\mathrm{Si}$} & 1,870 & $\mathrm{SiO}_{2}$ & 4,000 & 0 \\
\hline & & Total & 88,900 & $(1,802)$ \\
\hline
\end{tabular}

Table B3-9. Mass and Charge Balance Totals for Tank 241-T-202.

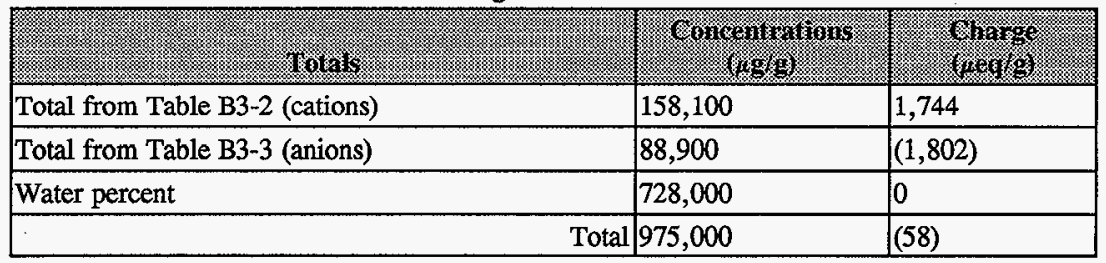

\section{Tank 241-T-203}

The concentrations of cations, anions, and the percent water were ultimately used to calculate the mass balance. The mass balance was calculated from the formula below. The factor 10,000 is the conversion factor from weight percent to $\mu \mathrm{g} / \mathrm{g}$.

Mass balance $($ Tank T-203) $=\%$ Water $\times 10,000+$ \{Total Analyte Concentration $\}$

$$
\begin{array}{ll}
=\quad \% \text { Water }(\times 10,000)+\left\{\mathrm{Bi}(\mathrm{OH})_{3}+\mathrm{FeO}(\mathrm{OH})+\right. \\
\\
\mathrm{MnO}_{2}+\mathrm{Na}^{+}+\mathrm{K}^{+}+\mathrm{Cr}(\mathrm{OH})_{3}+\mathrm{SO}_{4}^{-2}+\mathrm{LaF}_{3}+ \\
\left.\mathrm{F}+\mathrm{NO}_{3}^{-}+\mathrm{CO}_{3}^{-2}+\mathrm{BiPO}_{4}+\mathrm{C}_{2} \mathrm{O}_{4}^{-2}+\mathrm{SiO}_{2}\right\}
\end{array}
$$


The total analyte concentrations calculated from the above equation is $250,000 \mu \mathrm{g} / \mathrm{g}$. The mean weight percent water (obtained from the TGA) is 71.0 percent or $710,000 \mu \mathrm{g} / \mathrm{g}$. The mass balance resulting from adding the percent water to the total analyte concentration is approximately 96.0 percent (see Table B3-12).

The following equations demonstrate the derivation of total cations and total anions; the charge balance is the ratio of these two values.

Total cations $(\mu \mathrm{eq} / \mathrm{g})=\left[\mathrm{Na}^{+}\right] / 23.0+\left[\mathrm{K}^{+}\right] / 39.1=1,681 \mu \mathrm{eq} / \mathrm{g}$

Total anions $(\mu \mathrm{eq} / \mathrm{g})=[\mathrm{F}] / 19.0+\left[\mathrm{NO}_{3}^{-}\right] / 62.0+\left[\mathrm{CO}_{3}^{-2}\right] / 30.0+$

$$
\left[\mathrm{C}_{2} \mathrm{O}_{4}^{-2}\right] / 44.0=1,662 \mu \mathrm{eq} / \mathrm{g}
$$

The charge balance obtained by dividing the sum of the positive charge by the sum of the negative charge was 1.01 . The net positive charge was $19 \mu \mathrm{eq} / \mathrm{g}$. To balance this net positive charge, an amount of hydroxide equal to the charge imbalance was assumed. Including this term makes the charge balance 1.00 and the mass balance 96 percent, well within the uncertainties associated with the assumptions and measurements made. In summary, the above calculations yield reasonable mass and charge balance values (close to 1.00 for charge balance and 100 percent for mass balance), indicating that the analytical results are consistent.

Table B3-10. 241-T-203 Cation Mass and Charge Data.

\begin{tabular}{|c|c|c|c|c|}
\hline (: & 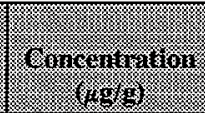 & - 1 isminiod: & 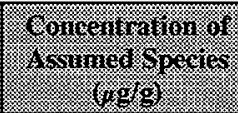 & 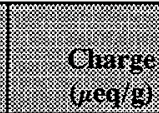 \\
\hline \multirow[t]{2}{*}{$\mathrm{Bi}$} & \multirow[t]{2}{*}{48,700} & \multirow{2}{*}{$\begin{array}{l}\mathrm{BiPO}_{4} \\
\mathrm{Bi}(\mathrm{OH})_{3}\end{array}$} & \multirow{2}{*}{$\begin{array}{l}23,400 \\
40,600\end{array}$} & 0.00 \\
\hline & & & & 0.00 \\
\hline $\mathrm{Cr}$ & 3,790 & $\mathrm{Cr}(\mathrm{OH})_{3}$ & 7,500 & 0.00 \\
\hline $\mathrm{Fe}$ & 6,810 & $\mathrm{FeO}(\mathrm{OH})$ & 10,800 & 0 \\
\hline $\mathrm{La}$ & 11,700 & $\mathrm{LaF}_{3}$ & 16,500 & 0 \\
\hline $\mathrm{Mn}$ & 16,100 & $\mathrm{MnO}_{2}$ & 25,400 & 0 \\
\hline $\mathbf{K}$ & 6,850 & $\mathbf{K}^{+}$ & 6,850 & 168 \\
\hline $\mathrm{Na}$ & 34,800 & $\mathrm{Na}^{+}$ & 34,800 & 1,513 \\
\hline & & Total & 166,000 & 1,681 \\
\hline
\end{tabular}


Table B3-11. 241-T-203 Anion Mass and Charge Data.

\begin{tabular}{|c|c|c|c|c|}
\hline ( & 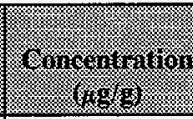 & $\begin{array}{l}f \\
f\end{array}$ & 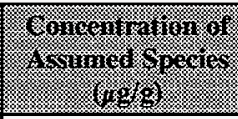 & 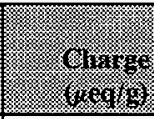 \\
\hline TIC & 1,580 & $\mathrm{CO}_{3}^{-2}$ & 7,900 & $(263)$ \\
\hline $\mathrm{F}$ & 6,340 & $\mathrm{~F}^{-}$ & 6,340 & (334) \\
\hline $\mathrm{NO}_{3}^{-}$ & 64,400 & $\mathrm{NO}_{3}^{-}$ & 64,400 & $(1,030)$ \\
\hline TOC & 420 & $\mathrm{C}_{2} \mathrm{O}_{4}^{-2}$ & 1,540 & (35) \\
\hline $\mathrm{P}$ & 2,390 & $\mathrm{PO}_{4}^{-3}$ & $\mathrm{As} \mathrm{BiPO}_{4}$ & 0 \\
\hline $\mathrm{Si}$ & 1,600 & $\mathrm{SiO}_{2}$ & 3,420 & 0 \\
\hline \multicolumn{4}{|c|}{ Total 83,600} & $(1,662)$ \\
\hline
\end{tabular}

Table B3-12. Mass and Charge Balance Totals for Tank 241-T-203.

\begin{tabular}{|l|l|l|}
\hline & & (1) \\
\hline Total from Table B3-2 (cations) & 166,000 & 1,681 \\
\hline Total from Table B3-3 (anions) & 83,600 & $(1,662)$ \\
\hline Water percent & 710,000 & 0 \\
\hline Added OH to charge balance & 323 & 19 \\
\hline Total & 960,000 & $(19)$ \\
\hline & 0 \\
\hline
\end{tabular}

\section{Tank 241-T-204}

The concentrations of cations, anions, and the percent water were ultimately used to calculate the mass balance. The mass balance was calculated from the formula below. The factor 10,000 is the conversion factor from weight percent to $\mu \mathrm{g} / \mathrm{g}$.

Mass balance (Tank T-204) $=\%$ Water $\times 10,000+$ \{Total Analyte Concentration $\}$ $=\%$ Water $\left(\mathrm{x} \mathrm{10,000)}+\left\{\mathrm{Bi}(\mathrm{OH})_{3}+\mathrm{FeO}(\mathrm{OH})+\right.\right.$ $\mathrm{MnO}_{2}+\mathrm{Na}^{+}+\mathrm{K}^{+}+\mathrm{Cr}(\mathrm{OH})_{3}+\mathrm{SO}_{4}^{-2}+\mathrm{LaF}_{3}+$ $\left.\mathrm{F}+\mathrm{NO}_{3}^{-}+\mathrm{CO}_{3}^{-2}+\mathrm{BiPO}_{4}+\mathrm{C}_{2} \mathrm{O}_{4}^{-2}+\mathrm{SiO}_{2}\right\}$ 
The total analyte concentrations calculated from the above equation is about $232,000 \mu \mathrm{g} / \mathrm{g}$. The mean weight percent water (obtained from the TGA) is 75.1 percent or $751,000 \mu \mathrm{g} / \mathrm{g}$. The mass balance resulting from adding the percent water to the total analyte concentration is approximately 98.3 percent (see Table B3-15).

The following equations demonstrate the derivation of total cations and total anions; the charge balance is the ratio of these two values.

Total cations $(\mu \mathrm{eq} / \mathrm{g})=\left[\mathrm{Na}^{+}\right] / 23.0+\left[\mathrm{K}^{+}\right] / 39.1=1,539 \mu \mathrm{eq} / \mathrm{g}$

Total anions $(\mu \mathrm{eq} / \mathrm{g})=[\mathrm{F}] / 19.0+\left[\mathrm{NO}_{3}\right] / 62.0+\left[\mathrm{CO}_{3}^{-2}\right] / 30.0+$ $\left[\mathrm{C}_{2} \mathrm{O}_{4}^{-2}\right] / 44.0=1,461 \mu \mathrm{eq} / \mathrm{g}$

The charge balance obtained by dividing the sum of the positive charge by the sum of the negative charge was 1.05 . The net positive charge was $78 \mu \mathrm{eq} / \mathrm{g}$. To balance this net positive charge, an amount of hydroxide equal to the charge imbalance was assumed. Including this term makes the charge balance 1.00 and the mass balance 98.4 percent, well within the uncertainties associated with the assumptions and measurements made. In summary, the above calculations yield reasonable mass and charge balance values (close to 1.00 for charge balance and 100 percent for mass balance), indicating that the analytical results are consistent.

Table B3-13. 241-T-204 Cation Mass and Charge Data.

\begin{tabular}{|c|c|c|c|c|}
\hline : & Poncentantion & 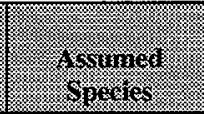 & 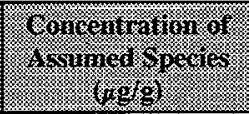 & 1) \\
\hline \multirow[t]{2}{*}{$\mathrm{Bi}$} & \multirow[t]{2}{*}{51,500} & \multirow{2}{*}{$\begin{array}{l}\mathrm{BiPO}_{4} \\
\mathrm{Bi}(\mathrm{OH})_{3}\end{array}$} & \multirow{2}{*}{24,000} & 0.00 \\
\hline & & & & 0.00 \\
\hline $\mathrm{Cr}$ & 4,490 & $\mathrm{Cr}(\mathrm{OH})_{3}$ & 8,890 & 0.00 \\
\hline $\mathrm{Fe}$ & 4,040 & $\mathrm{FeO}(\mathrm{OH})$ & 6,420 & 0 \\
\hline $\mathrm{La}$ & 11,500 & $\mathrm{LaF}_{3}$ & 16,200 & 0 \\
\hline $\mathrm{Mn}$ & 14,100 & $\mathrm{MnO}_{2}$ & 22,300 & 0 \\
\hline $\mathbf{K}$ & 6,120 & $\mathrm{~K}^{+}$ & 6,120 & 156 \\
\hline $\mathrm{Na}$ & 31,800 & $\mathrm{Na}^{+}$ & 31,800 & 1,383 \\
\hline & & Total & 159,000 & 1,539 \\
\hline
\end{tabular}


Table B3-14. 241-T-204 Anion Mass and Charge Data.

\begin{tabular}{|c|c|c|c|c|}
\hline 8067.18 .18 & S & 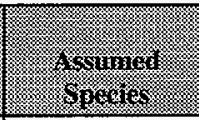 & 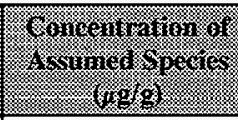 & (aringe \\
\hline TIC & 1,400 & $\mathrm{CO}_{3}^{-2}$ & 7,000 & $(233)$ \\
\hline $\mathrm{F}$ & 5,940 & $F$ & 5,940 & $(312)$ \\
\hline $\mathrm{NO}_{3}^{-}$ & 55,200 & $\mathrm{NO}_{3-}^{-}$ & 55,200 & $(890)$ \\
\hline TOC & 312 & $\mathrm{C}_{2} \mathrm{O}_{4}^{-2}$ & 1,150 & (26) \\
\hline$P$ & 2,650 & $\mathrm{PO}_{4}^{-3}$ & As $\mathrm{BiPO}_{4}$ & 0 \\
\hline $\mathbf{S i}$ & 1,500 & $\mathrm{SiO}_{2}$ & 3,210 & 0 \\
\hline \multicolumn{4}{|c|}{ Total 72,500} & $(1,461)$ \\
\hline
\end{tabular}

Table B3-15. Mass and Charge Balance Totals for Tank 241-T-204.

\begin{tabular}{|c|c|c|}
\hline (1) & $\mathrm{H}$ : & 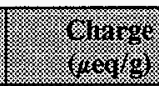 \\
\hline Total from Table B3-2 (cations) & 159,000 & 1,539 \\
\hline Total from Table B3-3 (anions) & 72,500 & $(1,461)$ \\
\hline Water percent & 751,000 & 0 \\
\hline Subtotal & 982,500 & 78 \\
\hline Added $\mathrm{OH}$ to charge balance & 1,330 & $(78)$ \\
\hline Total & 984,000 & 0 \\
\hline
\end{tabular}

\section{B3.4 MEAN CONCENTRATIONS AND CONFIDENCE INTERVALS}

The following statistical evaluation was performed using the analytical data generated from the solid portion of one core composite sample from each of tanks 241-T-201, -T-202, -T-203, and -T-204. From the data set from each of these tanks, a mean concentration was calculated for each analyte. These mean concentrations are denoted by $\hat{\mu}(T 201), \hat{\mu}(T 202), \hat{\mu}(T 203)$, and $\hat{\mu}(T 204)$ respectively. Because only one core sample was obtained from the T-200 tanks, an estimate of the variance of the mean, with both spatial and analytical variability, cannot be computed. 
To obtain an estimate of the variance of the mean (standard deviation of the mean), similar sets of data from tanks 241-B-201, -B-203, and -B-204 were used. There were two core samples from each of these three tanks. However, the analytical data from tank 241-B-201 were based on a chemical analysis of core composite samples, and the data from tanks 241-B-203 and -B-204 were based on core-segment samples. For each of these tanks, the mean for each of the two core samples was computed, and the variance (standard deviation) of the two means was computed. The three standard deviations are denoted by $\hat{\sigma}(\mathrm{B} 201), \hat{\sigma}(\mathrm{B} 203)$, and $\hat{\sigma}(\mathrm{B} 204)$ respectively.

The three standard deviations, $\hat{\sigma}(\mathrm{B} 201)$, $\hat{\sigma}(\mathrm{B} 203)$, and $\hat{\sigma}(\mathrm{B} 204)$, each have one degree of freedom. These three estimates were used as estimates of the variability associated with the means from the four T-200 tanks. The estimate of the standard deviation of $\hat{\mu}(T 201)$ was taken to be $\hat{\sigma}(\mathrm{B} 201)$, with one degree of freedom. For the other three means, $\hat{\mu}(\mathrm{T} 202)$, $\hat{\mu}(\mathrm{T} 203)$, and $\hat{\mu}(\mathrm{T} 204)$, the two standard deviations were pooled into a combined standard deviation, denoted by $\hat{\sigma}$ (pooled). The pooled standard deviation has two degrees of freedom. The method used to obtain the pooled estimate is described in Snedecor and Cochran (1980)

It is appropriate to use a pooled estimate of the standard deviation whenever the variances in the two tanks, 241-B-203 and -B-204, are not significantly different from each other. The equality of variances was tested using an F-test with one degree of freedom for the numerator and denominator. The variances were not significantly different except for bromide, copper, lithium, silver and zinc.

In tank 241-B-204, a majority of the bromide and copper observations were below the instrument detection limits. Consequently, the estimate of the standard deviation for these analytes is ô(B203), with one degree of freedom. In tank 241-B-203, a majority of the lithium and silver observations were below the instrument detection limits. For these two analytes, the estimate of the standard deviation is $\hat{\sigma}(\mathrm{B} 204)$, with one degree of freedom. For zinc, observations were available from both tanks. The magnitude of the data from tank 241-B-203 was closer to that observed in tanks 241-T-202, -T-203, and -T-204. For this analyte, the standard deviation $\hat{\sigma}(\mathrm{B} 203)$, with one degree of freedom, was used. 
A two-sided 95 percent confidence interval for the mean concentration in tanks 241-T-201, -T-202, -T-203 and -T204 was calculated using the standard deviations described above. This was done for each analyte. The confidence interval takes into account the sampling and analytical uncertainties. The upper and lower limits (UL and LL) of a two-sided 95 percent confidence interval for the mean are as follows:

$$
\begin{array}{ll}
\text { Tank T-201: } & \hat{\mu}(\mathrm{T} 201) \pm \mathrm{t}_{(\mathrm{df}=1,0.025)} \times \hat{\sigma}(\mathrm{B} 201) \\
\text { Tank } \mathrm{T}-202: & \hat{\mu}(\mathrm{T} 202) \pm \mathrm{t}_{(\mathrm{df}=2,0.025)} \times \hat{\sigma}(\text { pooled }) \\
\text { Tank T-203: } & \hat{\mu}(\mathrm{T} 203) \pm \mathrm{t}_{(\mathrm{df}=2,0.025)} \times \hat{\sigma}(\text { pooled }) \\
\text { Tank T-204: } & \hat{\mu}(\mathrm{T} 204) \pm \mathrm{t}_{(\mathrm{df}=2,0.025)} \times \hat{\sigma}(\text { pooled })
\end{array}
$$

In these equations, $\hat{\mu}$ is the estimate of the mean concentration from each of the T-200 tanks, $\hat{\sigma}$ is the estimate of the standard deviation from the B-200 tanks, and $\mathrm{t}_{(\mathrm{df}, 0.025)}$ is the quantile from Student's $t$ distribution with $d f=1$ or 2 degrees of freedom for a two-sided 95 percent confidence interval. Bromide, copper, lithium, silver, and zinc are special cases, each with one degree of freedom.

The confidence intervals were computed when at least 50 percent of the observations were above the detection limit. If more that 50 percent of the observations were below the detection limit only the mean of the observations is reported. A concentration is a positive number, consequently, whenever the lower limit to the confidence interval was negative it was truncated at zero.

For each analyte and tank, Tables B3-16 to B3-19 report the relevant summary statistics. The tables give the estimate of the mean concentration $(\hat{\mu})$, the standard deviation of the mean ( $\hat{\sigma})$, and the lower (LL) and upper limits (UL) to the 95 percent confidence interval on the mean. 
Table B3-16. Summary Statistics for Tank 241-T-201.

(Standard Deviation of the Mean is from Tank 241-B-201) (2 sheets)

\begin{tabular}{|c|c|c|c|c|c|c|}
\hline (2) & 1rot & Unin: & 16 & (1) & . & 16 \\
\hline Aluminum & ICP:A & $\mu \mathrm{g} / \mathrm{g}$. & $1.01 \mathrm{E}+02$ & $3.59 \mathrm{E}+03$ & $0.00 \mathrm{E}+00$ & $4.57 \mathrm{E}+04$ \\
\hline Americium-241 & GEA:F & $\mu \mathrm{Ci} / \mathrm{g}$ & $<3.19 \mathrm{E}-02$ & $6.10 \mathrm{E}-03$ & $0.00 \mathrm{E}+00$ & $1.09 \mathrm{E}-01$ \\
\hline Antimony & ICP:A & $\mu \mathrm{g} / \mathrm{g}$ & $<4.59 \mathrm{E}+01$ & NA & NA & NA \\
\hline Arsenic & ICP:A & $\mu \mathrm{g} / \mathrm{g}$ & $<7.66 \mathrm{E}+01$ & NA & NA & NA \\
\hline Barium $^{1}$ & ICP:A & $\mu \mathrm{g} / \mathrm{g}$ & $5.64 \mathrm{E}+01$ & $4.69 \mathrm{E}+01$ & $0.00 \mathrm{E}+00$ & $6.53 \mathrm{E}+02$ \\
\hline Beryllium & ICP:A & $\mu \mathrm{g} / \mathrm{g}$ & $<3.83 \mathrm{E}+00$ & NA & NA & NA \\
\hline Bismuth & ICP:A & $\mu \mathrm{g} / \mathrm{g}$ & $1.19 \mathrm{E}+05$ & $1.82 \mathrm{E}+04$ & $0.00 \mathrm{E}+00$ & $3.50 \mathrm{E}+05$ \\
\hline Boron & ICP:A & $\mu \mathrm{g} / \mathrm{g}$ & $1.72 \mathrm{E}+02$ & $3.47 \mathrm{E}+01$ & $0.00 \mathrm{E}+00$ & $6.13 \mathrm{E}+02$ \\
\hline Bromide & IC:W & $\mu \mathrm{g} / \mathrm{g}$ & $<2.78 \mathrm{E}+02$ & NA & NA & $\mathrm{NA}$ \\
\hline Bulk density & $\begin{array}{l}\text { Bulk } \\
\text { density }\end{array}$ & $\mathrm{g} / \mathrm{mL}$ & $1.27 \mathrm{E}+00$ & NA & NA & NA \\
\hline Cadmium & ICP:A & $\mu \mathrm{g} / \mathrm{g}$ & $<3.83 \mathrm{E}+00$ & NA & NA & NA \\
\hline Calcium & ICP:A & $\mu \mathrm{g} / \mathrm{g}$ & $1.24 \mathrm{E}+03$ & $1.00 \mathrm{E}+04$ & $0.00 \mathrm{E}+00$ & $1.28 \mathrm{E}+05$ \\
\hline Cerium & ICP:A & $\mu \mathrm{g} / \mathrm{g}$ & $<8.43 E+01$ & $\mathrm{NA}$ & NA & NA \\
\hline Cesium-137 & GEA:F & $\mu \mathrm{Ci} / \mathrm{g}$ & $4.57 \mathrm{E}-02$ & $1.80 \mathrm{E}+01$ & $0.00 \mathrm{E}+00$ & $2.29 \mathrm{E}+02$ \\
\hline Chloride & IC:W & $\mu \mathrm{g} / \mathrm{g}$ & $1.08 \mathrm{E}+03$ & $2.12 \mathrm{E}+02$ & $0.00 \mathrm{E}+00$ & $3.77 \mathrm{E}+03$ \\
\hline Chromium & ICP:A & $\mu \mathrm{g} / \mathrm{g}$ & $5.35 \mathrm{E}+03$ & $2.09 \mathrm{E}+02$ & $2.69 \mathrm{E}+03$ & $8.01 \mathrm{E}+03$ \\
\hline Cobalt- 60 & GEA:F & $\mu \mathrm{Ci} / \mathrm{g}$ & $<6.00 \mathrm{E}-03$ & NA & $0.00 \mathrm{E}+00$ & $3.50 \mathrm{E}-01$ \\
\hline Cobalt & ICP:A & $\mu \mathrm{g} / \mathrm{g}$ & $<1.53 \mathrm{E}+01$ & $\mathrm{NA}$ & NA & $\mathrm{NA}$ \\
\hline Copper & ICP:A & $\mu \mathrm{g} / \mathrm{g}$ & $<7.66 \mathrm{E}+00$ & $\mathrm{NA}$ & $0.00 \mathrm{E}+00$ & $5.87 \mathrm{E}+02$ \\
\hline Europium-154 & GEA:F & $\mu \mathrm{Ci} / \mathrm{g}$ & $<1.84 \mathrm{E}-02$ & NA & NA & NA \\
\hline Europium-155 & GEA:F & $\mu \mathrm{Ci} / \mathrm{g}$ & $<1.43 \mathrm{E}-02$ & NA & NA & NA \\
\hline Fluoride & IC:W & $\mu \mathrm{g} / \mathrm{g}$ & $5.08 \mathrm{E}+03$ & $1.41 \mathrm{E}+02$ & $4.18 \mathrm{E}+03$ & $5.97 \mathrm{E}+03$ \\
\hline Iron & ICP:A & $\mu \mathrm{g} / \mathrm{g}$ & $9.86 \mathrm{E}+03$ & $6.55 \mathrm{E}+03$ & $3.28 \mathrm{E}+03$ & $6.87 \mathrm{E}+03$ \\
\hline Lanthanum & ICP:A & $\mu \mathrm{g} / \mathrm{g}$ & $2.49 \mathrm{E}+04$ & $2.17 \mathrm{E}+03$ & $0.00 \mathrm{E}+00$ & $9.31 \mathrm{E}+04$ \\
\hline Lead & ICP:A & $\mu \mathrm{g} / \mathrm{g}$ & $2.12 \mathrm{E}+02$ & $1.81 \mathrm{E}+02$ & $0.00 \mathrm{E}+00$ & $5.24 \mathrm{E}+04$ \\
\hline Lithium & ICP:A & $\mu \mathrm{g} / \mathrm{g}$ & $<7.66 \mathrm{E}+00$ & NA & $0.00 \mathrm{E}+00$ & $2.51 \mathrm{E}+03$ \\
\hline Magnesium & ICP:A & $\mu \mathrm{g} / \mathrm{g}$ & $3.23 \mathrm{E}+02$ & $1.25 \mathrm{E}+03$ & NA & $\mathrm{NA}$ \\
\hline Manganese & ICP:A & $\mu \mathrm{g} / \mathrm{g}$ & $4.43 \mathrm{E}+04$ & $2.00 \mathrm{E}+03$ & $0.00 \mathrm{E}+00$ & $1.62 \mathrm{E}+04$ \\
\hline Molybdenum & ICP:A & $\mu \mathrm{g} / \mathrm{g}$ & $<3.83 \mathrm{E}+01$ & $\mathrm{NA}$ & $1.89 \mathrm{E}+04$ & $6.96 \mathrm{E}+04$ \\
\hline Neodymium & ICP:A & $\mu \mathrm{g} / \mathrm{g}$ & $<7.66 \mathrm{E}+01$ & NA & NA & NA \\
\hline
\end{tabular}


HNF-1501 Rev. 0

Table B3-16. Summary Statistics for Tank 241-T-201.

(Standard Deviation of the Mean is from Tank 241-B-201) (2 sheets)

\begin{tabular}{|c|c|c|c|c|c|c|}
\hline . & 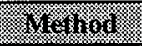 & Ymins: & i & 3. & (1) & (3) \\
\hline Nickel & ICP:A & $\mu \mathrm{g} / \mathrm{g}$ & $6.30 \mathrm{E}+02$ & $1.39 \mathrm{E}+01$ & NA & NA \\
\hline Nitrate & IC:W & $\mu \mathrm{g} / \mathrm{g}$ & $4.83 \mathrm{E}+04$ & $1.77 \mathrm{E}+03$ & $4.53 \mathrm{E}+02$ & $8.06 \mathrm{E}+02$ \\
\hline Nitrite & IC:W & $\mu \mathrm{g} / \mathrm{g}$ & $3.14 \mathrm{E}+02$ & $1.68 \mathrm{E}+02$ & $2.58 \mathrm{E}+04$ & $7.08 \mathrm{E}+04$ \\
\hline Oxalate & IC:W & $\mu \mathrm{g} / \mathrm{g}$ & $1.14 \mathrm{E}+03$ & NA & $0.00 \mathrm{E}+00$ & $2.45 \mathrm{E}+03$ \\
\hline Percent water & DSC/TGA & $\%$ & $6.16 \mathrm{E}+01$ & NA & NA & NA \\
\hline Phosphate & IC:W & $\mu \mathrm{g} / \mathrm{g}$ & $4.43 \mathrm{E}+02$ & $2.09 \mathrm{E}+02$ & NA & $\mathrm{NA}$ \\
\hline Phosphorus & ICP:A & $\mu \mathrm{g} / \mathrm{g}$ & $4.56 \mathrm{E}+03$ & $1.68 \mathrm{E}+03$ & $0.00 \mathrm{E}+00$ & $3.09 \mathrm{E}+03$ \\
\hline Potassium $^{1}$ & ICP:A & $\mu \mathrm{g} / \mathrm{g}$ & $4.81 \mathrm{E}+03$ & $1.07 \mathrm{E}+03$ & $0.00 \mathrm{E}+00$ & $2.59 \mathrm{E}+04$ \\
\hline Samarium & ICP:A & $\mu \mathrm{g} / \mathrm{g}$ & $<7.66 \mathrm{E}+01$ & NA & $0.00 \mathrm{E}+00$ & $1.83 \mathrm{E} .+04$ \\
\hline Selenium & ICP:A & $\mu \mathrm{g} / \mathrm{g}$ & $<7.66 \mathrm{E}+01$ & NA & NA & NA \\
\hline Silicon & ICP:A & $\mu \mathrm{g} / \mathrm{g}$ & $1.86 \mathrm{E}+03$ & $7.10 \mathrm{E}+02$ & NA & NA \\
\hline Silver & ICP:A & $\mu \mathrm{g} / \mathrm{g}$ & $<7.66 \mathrm{E}+00$ & NA & $0.00 \mathrm{E}+00$ & $1.09 \mathrm{E}+04$ \\
\hline Sodium & ICP:A & $\mu \mathrm{g} / \mathrm{g}$ & $3.23 \mathrm{E}+04$ & $4.77 \mathrm{E}+03$ & NA & $\mathrm{NA}$ \\
\hline Strontium-89/90 & Sr:F & $\mu \mathrm{Ci} / \mathrm{g}$ & 1.41E-01 & NA & $0.00 \mathrm{E}+00$ & $9.0 \mathrm{E}+03$ \\
\hline Strontium & ICP:A & $\mu \mathrm{g} / \mathrm{g}$ & $1.12 \mathrm{E}+03$ & $6.46 \mathrm{E}+01$ & NA & NA \\
\hline Sulfate & IC:W & $\mu \mathrm{g} / \mathrm{g}$ & $<7.79 \mathrm{E}+02$ & NA & $2.99 \mathrm{E}+02$ & $1.94 \mathrm{E}+03$ \\
\hline Sulfur & ICP:A & $\mu \mathrm{g} / \mathrm{g}$ & $9.22 \mathrm{E}+01$ & NA & $0.00 \mathrm{E}+00$ & $3.52 \mathrm{E}+03$ \\
\hline Thallium & ICP:A & $\mu \mathrm{g} / \mathrm{g}$ & $<1.53 \mathrm{E}+02$ & NA & NA & $\mathrm{NA}$ \\
\hline Titanium & ICP:A & $\mu \mathrm{g} / \mathrm{g}$ & $9.92 \mathrm{E}+00$ & $3.03 E+02$ & NA & NA \\
\hline $\begin{array}{l}\text { Total inorganic } \\
\text { carbon }\end{array}$ & TIC/TOC & $\mu \mathrm{g} / \mathrm{g}$ & $8.10 \mathrm{E}+02$ & $1.53 \mathrm{E}+03$ & $0.00 \mathrm{E}+00$ & $3.86 \mathrm{E}+03$ \\
\hline $\begin{array}{l}\text { Total organic } \\
\text { carbon }\end{array}$ & TIC/TOC & $\mu \mathrm{g} / \mathrm{g}$ & $3.04 \mathrm{E}+02$ & NA & $0.00 \mathrm{E}+00$ & $2.03 E+04$ \\
\hline Uranium & ICP:A & $\mu \mathrm{g} / \mathrm{g}$ & $<3.83 \mathrm{E}+02$ & NA & NA & NA \\
\hline Vanadium & ICP:A & $\mu \mathrm{g} / \mathrm{g}$ & $<3.83 \mathrm{E}+01$ & NA & NA & NA \\
\hline Zinc & ICP:A & $\mu \mathrm{g} / \mathrm{g}$ & $9.45 \mathrm{E}+01$ & $1.44 \mathrm{E}+01$ & NA & NA \\
\hline Zirconium & ICP:A & $\mu \mathrm{g} / \mathrm{g}$ & $<7.66 \mathrm{E}+00$ & NA & $0.00 \mathrm{E}+00$ & $2.78 \mathrm{E}+02$ \\
\hline
\end{tabular}

Notes:

$\begin{array}{lll}\text { NA } & = & \text { not available } \\ < & = & \text { at least } 50 \text { percent of tank } 241-\mathrm{T}-201 \text { data is below the detection limit. }\end{array}$

${ }^{1}$ Less than detection limit values used in the standard deviation 
Table B3-17. Summary Statistics for Tank 241-T-202. (2 sheets)

\begin{tabular}{|c|c|c|c|c|c|c|c|}
\hline . & Mathor & vanits: & 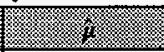 & 3. & 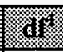 & 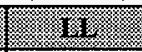 & 18 \\
\hline Aluminum & ICP:A & $\mu \mathrm{g} / \mathrm{g}$ & $7.22 \mathrm{E}+01$ & $1.78 \mathrm{E}+01$ & 2 & $0.00 \mathrm{E}+00$ & $1.49 \mathrm{E}+02$ \\
\hline Americium-241 & GEA:F & $\mu \mathrm{Ci} / \mathrm{g}$ & $<1.14 \mathrm{E}-01$ & NA & NA & NA & NA \\
\hline Antimony & ICP:A & $\mu \mathrm{g} / \mathrm{g}$ & $<1.21 \mathrm{E}+01$ & NA & NA & NA & NA \\
\hline Arsenic & ICP:A & $\mu \mathrm{g} / \mathrm{g}$ & $<2.02 \mathrm{E}+01$ & NA & NA & NA & NA \\
\hline Barium & ICP:A & $\mu \mathrm{g} / \mathrm{g}$ & $1.27 \mathrm{E}+03$ & NA & NA & NA & NA \\
\hline Beryllium & ICP:A & $\mu \mathrm{g} / \mathrm{g}$ & $<1.01 \mathrm{E}+00$ & NA & NA & NA & NA \\
\hline Bismuth & ICP:A & $\mu \mathrm{g} / \mathrm{g}$ & $4.10 \mathrm{E}+04$ & $3.67 \mathrm{E}+03$ & 2 & $2.52 \mathrm{E}+04$ & $5.67 \mathrm{E}+04$ \\
\hline Boron & ICP:A & $\mu \mathrm{g} / \mathrm{g}$ & $1.58 \mathrm{E}+02$ & $2.02 \mathrm{E}+01$ & 2 & $7.13 \mathrm{E}+01$ & $2.45 \mathrm{E}+02$ \\
\hline Bromide & IC:W & $\mu \mathrm{g} / \mathrm{g}$ & $<3.03 \mathrm{E}+02$ & NA & NA & NA & NA \\
\hline Bulk density & $\begin{array}{l}\text { Bulk } \\
\text { density }\end{array}$ & $\mathrm{g} / \mathrm{mL}$ & $1.24 \mathrm{E}+00$ & NA & NA & NA & NA \\
\hline Cadmium & ICP:A & $\mu \mathrm{g} / \mathrm{g}$ & $<1.01 \mathrm{E}+00$ & NA & NA & NA & NA \\
\hline Calcium & ICP:A & $\mu \mathrm{g} / \mathrm{g}$ & $3.10 \mathrm{E}+02$ & $8.38 \mathrm{E}+01$ & 2 & $0.00 \mathrm{E}+00$ & $6.71 \mathrm{E}+02$ \\
\hline Cerium & ICP:A & $\mu \mathrm{g} / \mathrm{g}$ & $5.37 \mathrm{E}+01$ & $7.63 \mathrm{E}+00$ & 2 & $2.09 \mathrm{E}+01$ & $8.65 \mathrm{E}+01$ \\
\hline Cesium-137 & GEA:F & $\mu \mathrm{Ci} / \mathrm{g}$ & $<2.82 \mathrm{E}-02$ & NA & NA & NA & NA \\
\hline Chloride & IC:W & $\mu \mathrm{g} / \mathrm{g}$ & $6.93 \mathrm{E}+02$ & $1.49 \mathrm{E}+02$ & 2 & $4.97 \mathrm{E}+01$ & $1.34 \mathrm{E}+03$ \\
\hline Chromium & ICP:A & $\mu \mathrm{g} / \mathrm{g}$ & $3.76 \mathrm{E}+03$ & $1.02 \mathrm{E}+02$ & 2 & $3.32 \mathrm{E}+03$ & $4.19 \mathrm{E}+03$ \\
\hline Cobalt- 60 & GEA:F & $\mu \mathrm{Ci} / \mathrm{g}$ & $<1.67 \mathrm{E}-02$ & NA & NA & NA & NA \\
\hline Cobalt & ICP:A & $\mu \mathrm{g} / \mathrm{g}$ & $<4.04 \mathrm{E}+00$ & NA & NA & NA & NA \\
\hline Copper & ICP:A & $\mu \mathrm{g} / \mathrm{g}$ & $<2.02 \mathrm{E}+00$ & NA & $\mathrm{NA}$ & $\mathrm{NA}$ & NA \\
\hline Europium-154 & GEA:F & $\mu \mathrm{Ci} / \mathrm{g}$ & $<4.13 \mathrm{E}-02$ & $\mathrm{NA}$ & NA & $\mathrm{NA}$ & NA \\
\hline Europium-155 & GEA:F & $\mu \mathrm{Ci} / \mathrm{g}$ & $<3.96 \mathrm{E}-02$ & $\mathrm{NA}$ & NA & NA & $\mathrm{NA}$ \\
\hline Fluoride & IC:W & $\mu \mathrm{g} / \mathrm{g}$ & $6.56 \mathrm{E}+03$ & $1.45 \mathrm{E}+03$ & 2 & $3.04 \mathrm{E}+02$ & $1.28 \mathrm{E}+04$ \\
\hline Iron & ICP:A & $\mu \mathrm{g} / \mathrm{g}$ & $7.62 \mathrm{E}+03$ & $5.29 \mathrm{E}+02$ & 2 & $5.34 \mathrm{E}+03$ & $9.89 \mathrm{E}+03$ \\
\hline Lanthanum & ICP:A & $\mu \mathrm{g} / \mathrm{g}$ & $1.26 \mathrm{E}+04$ & $3.87 \mathrm{E}+02$ & 2 & $1.09 \mathrm{E}+04$ & $1.42 \mathrm{E}+04$ \\
\hline Lead & ICP:A & $\mu \mathrm{g} / \mathrm{g}$ & $5.80 \mathrm{E}+01$ & $\mathrm{NA}$ & NA & NA & NA \\
\hline Lithium & ICP:A & $\mu \mathrm{g} / \mathrm{g}$ & $<2.02 \mathrm{E}+00$ & NA & NA & NA & NA \\
\hline Magnesium & ICP:A & $\mu \mathrm{g} / \mathrm{g}$ & $8.88 \mathrm{E}+01$ & $3.86 \mathrm{E}+00$ & 2 & $7.22 \mathrm{E}+01$ & $1.05 \mathrm{E}+02$ \\
\hline Manganese & ICP:A & $\mu \mathrm{g} / \mathrm{g}$ & $1.48 \mathrm{E}+04$ & $6.41 \mathrm{E}+02$ & 2 & $1.20 \mathrm{E}+04$ & $1.76 \mathrm{E}+04$ \\
\hline Molybdenum & ICP:A & $\mu \mathrm{g} / \mathrm{g}$ & $<1.01 \mathrm{E}+01$ & NA & NA & NA & NA \\
\hline Neodymium & ICP:A & $\mu \mathrm{g} / \mathrm{g}$ & $<2.02 \mathrm{E}+01$ & NA & NA & NA & NA \\
\hline Nickel & ICP:A & $\mu \mathrm{g} / \mathrm{g}$ & $1.32 \mathrm{E}+02$ & $1.76 \mathrm{E}+01$ & 2 & $5.64 \mathrm{E}+01$ & $2.08 \mathrm{E}+02$ \\
\hline
\end{tabular}


Table B3-17. Summary Statistics for Tank 241-T-202. (2 sheets)

\begin{tabular}{|c|c|c|c|c|c|c|c|}
\hline S malle & Methor: & Vinix & $1 \%$ & 18 & 811 & 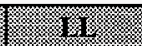 & (1) \\
\hline Nitrate & IC:W & $\mu \mathrm{g} / \mathrm{g}$ & $6.56 \mathrm{E}+04$ & $1.18 \mathrm{E}+04$ & 2 & $1.48 \mathrm{E}+04$ & $1.16 \mathrm{E}+05$ \\
\hline Nitrite & IC:W & $\mu \mathrm{g} / \mathrm{g}$ & $5.25 \mathrm{E}+02$ & $6.22 \mathrm{E}+01$ & 2 & $2.57 \mathrm{E}+02$ & $7.92 \mathrm{E}+02$ \\
\hline Oxalate & IC:W & $\mu \mathrm{g} / \mathrm{g}$ & $4.82 \mathrm{E}+02$ & $4.19 \mathrm{E}+02$ & 2 & $0.00 \mathrm{E}+00$ & $2.29 \mathrm{E}+03$ \\
\hline Percent water & DSC/TGA & $\%$ & $7.28 \mathrm{E}+01$ & $1.00 \mathrm{E}+00$ & 2 & $6.85 \mathrm{E}+01$ & $7.71 \mathrm{E}+01$ \\
\hline Phosphate & IC:W & $\mu \mathrm{g} / \mathrm{g}$ & $1.84 \mathrm{E}+03$ & $6.65 \mathrm{E}+02$ & 2 & $0.00 \mathrm{E}+00$ & $4.70 \mathrm{E}+03$ \\
\hline Phosphorus & ICP:A & $\mu \mathrm{g} / \mathrm{g}$ & $2.39 \mathrm{E}+03$ & $2.20 \mathrm{E}+01$ & 2 & $2.30 \mathrm{E}+03$ & $2.48 \mathrm{E}+03$ \\
\hline Potassium & ICP:A & $\mu \mathrm{g} / \mathrm{g}$ & $7.14 \mathrm{E}+03$ & $2.39 \mathrm{E}+02$ & 2 & $6.11 \mathrm{E}+03$ & $8.16 \mathrm{E}+03$ \\
\hline Samarium & ICP:A & $\mu \mathrm{g} / \mathrm{g}$ & $<2.02 \mathrm{E}+01$ & NA & NA & NA & NA \\
\hline Selenium & ICP:A & $\mu \mathrm{g} / \mathrm{g}$ & $<2.02 \mathrm{E}+01$ & NA & NA & NA & NA \\
\hline Silicon & ICP:A & $\mu \mathrm{g} / \mathrm{g}$ & $1.87 \mathrm{E}+03$ & $2.59 \mathrm{E}+02$ & 2 & $7.56 \mathrm{E}+02$ & $2.98 \mathrm{E}+03$ \\
\hline Silver & ICP:A & $\mu \mathrm{g} / \mathrm{g}$ & $<2.24 \mathrm{E}+00$ & NA & NA & NA & NA \\
\hline Sodium & ICP:A & $\mu \mathrm{g} / \mathrm{g}$ & $3.59 \mathrm{E}+04$ & $1.09 \mathrm{E}+03$ & 2 & $3.11 \mathrm{E}+04$ & $4.06 \mathrm{E}+04$ \\
\hline Strontium-89/90 & Sr:F & $\mu \mathrm{Ci} / \mathrm{g}$ & $2.49 \mathrm{E}-03$ & NA & NA & NA & NA \\
\hline Strontium & ICP:A & $\mu \mathrm{g} / \mathrm{g}$ & $4.97 \mathrm{E}+02$ & $1.57 \mathrm{E}+01$ & 2 & $4.30 \mathrm{E}+02$ & $5.64 \mathrm{E}+02$ \\
\hline Sulfate & IC:W & $\mu \mathrm{g} / \mathrm{g}$ & $1.11 \mathrm{E}+03$ & $1.39 \mathrm{E}+02$ & 2 & $5.07 \mathrm{E}+02$ & $1.70 \mathrm{E}+03$ \\
\hline Sulfur & ICP:A & $\mu \mathrm{g} / \mathrm{g}$ & $3.02 \mathrm{E}+02$ & $1.45 \mathrm{E}+01$ & 2 & $2.39 \mathrm{E}+02$ & $3.64 \mathrm{E}+02$ \\
\hline Thallium & ICP:A & $\mu \mathrm{g} / \mathrm{g}$ & $<4.04 \mathrm{E}+01$ & NA & NA & NA & NA \\
\hline Titanium & ICP:A & $\mu \mathrm{g} / \mathrm{g}$ & $4.68 \mathrm{E}+00$ & NA & NA & NA & NA \\
\hline $\begin{array}{l}\text { Total inorganic } \\
\text { carbon }\end{array}$ & $\mathrm{TIC} / \mathrm{TOC}$ & $\mu \mathrm{g} / \mathrm{g}$ & $2.08 \mathrm{E}+03$ & NA & NA & NA & NA \\
\hline \begin{tabular}{|l|}
$\begin{array}{l}\text { Total organic } \\
\text { carbon }\end{array}$ \\
\end{tabular} & TIC/TOC & $\mu \mathrm{g} / \mathrm{g}$ & $3.47 \mathrm{E}+02$ & NA & NA & $\mathrm{NA}$ & NA \\
\hline Uranium & ICP:A & $\mu \mathrm{g} / \mathrm{g}$ & $<1.02 \mathrm{E}+02$ & NA & NA & NA & NA \\
\hline Vanadium & ICP:A & $\mu \mathrm{g} / \mathrm{g}$ & $<1.01 \mathrm{E}+01$ & $\mathrm{NA}$ & $\mathrm{NA}$ & $\mathrm{NA}$ & NA \\
\hline Zinc & ICP:A & $\mu \mathrm{g} / \mathrm{g}$ & $4.41 \mathrm{E}+01$ & $2.15 \mathrm{E}+01$ & 1 & $0.00 \mathrm{E}+00$ & $3.17 \mathrm{E}+02$ \\
\hline Zirconium & ICP:A & $\mu \mathrm{g} / \mathrm{g}$ & $<2.02 \mathrm{E}+00$ & NA & $\mathrm{NA}$ & NA & NA \\
\hline
\end{tabular}

Notes:

$<=$ at least 50 percent of tank 241-T-202 data is below the detection limit.

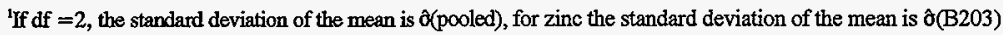


Table B3-18. Summary Statistics for Tank 241-T-203. (2 sheets)

\begin{tabular}{|c|c|c|c|c|c|c|c|}
\hline . & Melios & Uan & 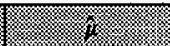 & 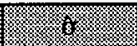 & (1) & 18 & 011 \\
\hline Aluminum & ICP:A & $\mu \mathrm{g} / \mathrm{g}$ & $5.63 \mathrm{E}+01$ & $1.78 \mathrm{E}+01$ & 2 & $0.00 \mathrm{E}+00$ & $1.33 \mathrm{E}+02$ \\
\hline Americium-241 & GEA:F & $\mu \mathrm{Ci} / \mathrm{g}$ & $3.59 \mathrm{E}-02$ & NA & NA & NA & NA \\
\hline Antimony & ICP:A & $\mu \mathrm{g} / \mathrm{g}$ & $<1.20 \mathrm{E}+01$ & NA & NA & NA & NA \\
\hline Arsenic & ICP:A & $\mu \mathrm{g} / \mathrm{g}$ & $<2.00 \mathrm{E}+01$ & NA & NA & NA & NA \\
\hline Barium & ICP:A & $\mu \mathrm{g} / \mathrm{g}$ & $7.48 \mathrm{E}+01$ & NA & NA & NA & NA \\
\hline Beryllium & ICP:A & $\mu \mathrm{g} / \mathrm{g}$ & $<9.98 \mathrm{E}-01$ & NA & NA & NA & NA \\
\hline Bismuth & ICP:A & $\mu \mathrm{g} / \mathrm{g}$ & $4.87 \mathrm{E}+04$ & $3.67 \mathrm{E}+03$ & 2 & $3.29 \mathrm{E}+04$ & $6.44 \mathrm{E}+04$ \\
\hline Boron & ICP:A & $\mu \mathrm{g} / \mathrm{g}$ & $9.31 \mathrm{E}+01$ & $2.02 \mathrm{E}+01$ & 2 & $6.36 \mathrm{E}+00$ & $1.80 \mathrm{E}+02$ \\
\hline Bromide & IC:W & $\mu \mathrm{g} / \mathrm{g}$ & $<2.97 \mathrm{E}+02$ & $8.96 \mathrm{E}+01$ & 2 & NA & NA \\
\hline Bulk density & $\begin{array}{l}\text { Bulk } \\
\text { density }\end{array}$ & $\mathrm{g} / \mathrm{mL}$ & $1.23 \mathrm{E}+00$ & NA & NA & NA & NA \\
\hline Cadmium & ICP:A & $\mu \mathrm{g} / \mathrm{g}$ & $<9.98 \mathrm{E}-01$ & $\mathrm{NA}$ & NA & NA & NA \\
\hline Calcium & ICP:A & $\mu \mathrm{g} / \mathrm{g}$ & $3.46 \mathrm{E}+02$ & $8.38 \mathrm{E}+01$ & 2 & $0.00 \mathrm{E}+00$ & $7.07 \mathrm{E}+02$ \\
\hline Cerium & ICP:A & $\mu \mathrm{g} / \mathrm{g}$ & $5.53 \mathrm{E}+01$ & $7.63 \mathrm{E}+00$ & 2 & $2.24 \mathrm{E}+01$ & $8.81 \mathrm{E}+01$ \\
\hline Cesium-137 & GEA:F & $\mu \mathrm{Ci} / \mathrm{g}$ & $<1.81 \mathrm{E}-02$ & NA & NA & $\mathrm{NA}$ & NA \\
\hline Chloride & IC:W & $\mu \mathrm{g} / \mathrm{g}$ & $6.58 \mathrm{E}+02$ & $1.49 \mathrm{E}+02$ & 2 & $1.49 \mathrm{E}+01$ & $1.30 \mathrm{E}+03$ \\
\hline Chromium & ICP:A & $\mu \mathrm{g} / \mathrm{g}$ & $3.79 \mathrm{E}+03$ & $1.02 \mathrm{E}+02$ & 2 & $3.35 \mathrm{E}+03$ & $4.23 \mathrm{E}+03$ \\
\hline Cobalt- 60 & GEA:F & $\mu \mathrm{Ci} / \mathrm{g}$ & $<1.26 \mathrm{E}-02$ & NA & NA & NA & NA \\
\hline Cobalt & ICP:A & $\mu \mathrm{g} / \mathrm{g}$ & $<3.99 \mathrm{E}+00$ & NA & NA & $\mathrm{NA}$ & NA \\
\hline Copper & ICP:A & $\mu \mathrm{g} / \mathrm{g}$ & $<2.00 \mathrm{E}+00$ & $8.63 \mathrm{E}-01$ & 1 & NA & NA \\
\hline $\begin{array}{l}\text { Endotherm- } \\
\text { transition 1 } \\
\end{array}$ & \begin{tabular}{|l}
$\mathrm{DSC} /$ \\
$\mathrm{TGA}$ \\
\end{tabular} & $\mathrm{J} / \mathrm{g}$ & NA & NA & NA & NA & NA \\
\hline Europium-154 & GEA:F & $\mu \mathrm{Ci} / \mathrm{g}$ & $<4.12 \mathrm{E}-02$ & NA & NA & NA & NA \\
\hline Europium-155 & GEA:F & $\mu \mathrm{Ci} / \mathrm{g}$ & $<3.36 \mathrm{E}-02$ & NA & NA & NA & NA \\
\hline Fluoride & IC:W & $\mu \mathrm{g} / \mathrm{g}$ & $6.34 \mathrm{E}+03$ & $1.45 \mathrm{E}+03$ & 2 & $7.79 \mathrm{E}+01$ & $1.26 \mathrm{E}+04$ \\
\hline Iron & ICP:A & $\mu \mathrm{g} / \mathrm{g}$ & $6.81 \mathrm{E}+03$ & $5.29 \mathrm{E}+02$ & 2 & $4.53 \mathrm{E}+03$ & $9.09 \mathrm{E}+03$ \\
\hline Lanthanum & ICP:A & $\mu \mathrm{g} / \mathrm{g}$ & $1.17 \mathrm{E}+04$ & $3.87 \mathrm{E}+02$ & 2 & $1.00 \mathrm{E}+04$ & $1.34 \mathrm{E}+04$ \\
\hline Lead & ICP:A & $\mu \mathrm{g} / \mathrm{g}$ & $<2.08 \mathrm{E}+01$ & $\mathrm{NA}$ & NA & NA & NA \\
\hline Lithium & ICP:A & $\mu \mathrm{g} / \mathrm{g}$ & $<2.00 \mathrm{E}+00$ & $\mathrm{NA}$ & NA & NA & NA \\
\hline Magnesium & ICP:A & $\mu \mathrm{g} / \mathrm{g}$ & $7.90 \mathrm{E}+01$ & $3.86 \mathrm{E}+00$ & 2 & $6.24 \mathrm{E}+01$ & $9.56 \mathrm{E}+01$ \\
\hline Manganese & ICP:A & $\mu \mathrm{g} / \mathrm{g}$ & $1.61 \mathrm{E}+04$ & $6.41 \mathrm{E}+02$ & 2 & $1.33 \mathrm{E}+04$ & $1.88 \mathrm{E}+04$ \\
\hline Molybdenum & ICP:A & $\mu \mathrm{g} / \mathrm{g}$ & $<9.98 \mathrm{E}+00$ & $\mathrm{NA}$ & NA & NA & NA \\
\hline
\end{tabular}


Table B3-18. Summary Statistics for Tank 241-T-203. (2 sheets)

\begin{tabular}{|c|c|c|c|c|c|c|c|}
\hline 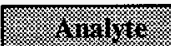 & Yreenor & $8 \mathrm{~min}$ & 1. & 8 & (if & 13 & 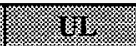 \\
\hline Neodymium & ICP:A & $\mu \mathrm{g} / \mathrm{g}$ & $<2.00 \mathrm{E}+01$ & NA & NA & NA & NA \\
\hline Nickel & ICP:A & $\mu \mathrm{g} / \mathrm{g}$ & $1.48 \mathrm{E}+02$ & $1.76 \mathrm{E}+01$ & 2 & $7.19 \mathrm{E}+01$ & $2.23 \mathrm{E}+02$ \\
\hline Nitrate & IC:W & $\mu \mathrm{g} / \mathrm{g}$ & $6.44 \mathrm{E}+04$ & $1.18 \mathrm{E}+04$ & 2 & $1.37 \mathrm{E}+04$ & $1.15 \mathrm{E}+05$ \\
\hline Nitrite & IC:W & $\mu \mathrm{g} / \mathrm{g}$ & $2.95 \mathrm{E}+02$ & $6.22 \mathrm{E}+01$ & 2 & $2.68 \mathrm{E}+01$ & $5.62 \mathrm{E}+02$ \\
\hline Oxalate & IC:W & $\mu \mathrm{g} / \mathrm{g}$ & $1.37 \mathrm{E}+03$ & $4.19 \mathrm{E}+02$ & 2 & $0.00 \mathrm{E}+00$ & $3.17 \mathrm{E}+03$ \\
\hline Percent water & $\begin{array}{l}\mathrm{DSC} / \mathrm{TG} \\
\mathrm{A}\end{array}$ & $\%$ & $7.10 \mathrm{E}+01$ & $1.00 \mathrm{E}+00$ & 2 & $6.67 \mathrm{E}+01$ & $7.53 \mathrm{E}+01$ \\
\hline Phosphate & IC:W & $\mu \mathrm{g} / \mathrm{g}$ & $2.91 \mathrm{E}+03$ & $6.65 \mathrm{E}+02$ & 2 & $4.89 \mathrm{E}+01$ & $5.77 \mathrm{E}+03$ \\
\hline Phosphorus & ICP:A & $\mu \mathrm{g} / \mathrm{g}$ & $2.39 \mathrm{E}+03$ & $2.20 \mathrm{E}+01$ & 2 & $2.29 \mathrm{E}+03$ & $2.48 \mathrm{E}+03$ \\
\hline Potassium & ICP:A & $\mu \mathrm{g} / \mathrm{g}$ & $6.85 \mathrm{E}+03$ & $2.39 \mathrm{E}+02$ & 2 & $5.82 \mathrm{E}+03$ & $7.88 \mathrm{E}+03$ \\
\hline Samarium & ICP:A & $\mu \mathrm{g} / \mathrm{g}$ & $<2.00 \mathrm{E}+01$ & NA & NA & NA & $\mathrm{NA}$ \\
\hline Silicon & ICP:A & $\mu \mathrm{g} / \mathrm{g}$ & $1.60 \mathrm{E}+03$ & $2.59 \mathrm{E}+02$ & 2 & $4.86 \mathrm{E}+02$ & $2.71 \mathrm{E}+03$ \\
\hline Silver & ICP:A & $\mu \mathrm{g} / \mathrm{g}$ & $<2.21 \mathrm{E}+00$ & NA & NA & NA & NA \\
\hline Sodium & ICP:A & $\mu \mathrm{g} / \mathrm{g}$ & $3.48 \mathrm{E}+04$ & $1.09 \mathrm{E}+03$ & 2 & $3.01 \mathrm{E}+04$ & $3.95 \mathrm{E}+04$ \\
\hline Strontium & ICP:A & $\mu \mathrm{g} / \mathrm{g}$ & $5.57 \mathrm{E}+02$ & $1.57 \mathrm{E}+01$ & 2 & $4.90 \mathrm{E}+02$ & $6.24 \mathrm{E}+02$ \\
\hline Sulfate & IC:W & $\mu \mathrm{g} / \mathrm{g}$ & $<4.38 \mathrm{E}+02$ & NA & $\mathrm{NA}$ & NA & NA \\
\hline Sulfur & ICP:A & $\mu \mathrm{g} / \mathrm{g}$ & $1.28 \mathrm{E}+02$ & $1.45 \mathrm{E}+01$ & 2 & $6.52 \mathrm{E}+01$ & $1.90 \mathrm{E}+02$ \\
\hline Thallium & ICP:A & $\mu \mathrm{g} / \mathrm{g}$ & $<3.99 \mathrm{E}+01$ & NA & NA & NA & NA \\
\hline Titanium & ICP:A & $\mu \mathrm{g} / \mathrm{g}$ & $3.27 \mathrm{E}+00$ & $\mathrm{NA}$ & $\mathrm{NA}$ & NA & $\mathrm{NA}$ \\
\hline Uranium & ICP:A & $\mu \mathrm{g} / \mathrm{g}$ & $<9.98 \mathrm{E}+01$ & NA & NA & NA & NA \\
\hline Vanadium & ICP:A & $\mu \mathrm{g} / \mathrm{g}$ & $<9.98 \mathrm{E}+00$ & NA & NA & NA & NA \\
\hline Zinc & ICP:A & $\mu \mathrm{g} / \mathrm{g}$ & $2.06 \mathrm{E}+01$ & $2.15 \mathrm{E}+01$ & 1 & $0.00 \mathrm{E}+00$ & $2.94 \mathrm{E}+02$ \\
\hline Zirconium & ICP:A & $\mu \mathrm{g} / \mathrm{g}$ & $<2.00 \mathrm{E}+00$ & NA & NA & NA & NA \\
\hline
\end{tabular}

Notes:

$<=$ at least 50 percent of tank 241-T-203 data is below the detection limit.

1 If $\mathrm{df}=2$, the standard deviation of the mean is $\partial$ (pooled); for copper $\mathrm{df}=1$ and the standard deviation of the mean is $\partial(B 203)$; for zinc $d f=1$ and the standard deviation of the mean is $\partial(B 203)$ 
Table B3-19. Summary Statistics for Tank 241-T-204. (2 sheets)

\begin{tabular}{|c|c|c|c|c|c|c|c|}
\hline 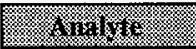 & 13: & Sinis & : & (2) & 8 & (3) & (3) \\
\hline Aluminum & ICP:A & $\mu \mathrm{g} / \mathrm{g}$ & $5.36 \mathrm{E}+01$ & $1.78 \mathrm{E}+01$ & 2 & $0.00 \mathrm{E}+00$ & $1.30 \mathrm{E}+02$ \\
\hline Americium-241 & GEA:F & $\mu \mathrm{Ci} / \mathrm{g}$ & $2.44 \mathrm{E}-02$ & NA & NA & NA & NA \\
\hline Antimony & ICP:A & $\mu \mathrm{g} / \mathrm{g}$ & 3.37E+01 & NA & NA & NA & NA \\
\hline Arsenic & ICP:A & $\mu \mathrm{g} / \mathrm{g}$ & $2.08 \mathrm{E}+02$ & NA & NA & NA & NA \\
\hline Barium & ICP:A & $\mu \mathrm{g} / \mathrm{g}$ & $<1.42 \mathrm{E}+01$ & NA & NA & NA & NA \\
\hline Beryllium & ICP:A & $\mu \mathrm{g} / \mathrm{g}$ & $<1.42 \mathrm{E}+00$ & NA & NA & NA & NA \\
\hline Bismuth & ICP:A & $\mathrm{g} / \mathrm{g}$ & $5.15 \mathrm{E}+04$ & $3.67 \mathrm{E}+03$ & 2 & $3.57 \mathrm{E}+04$ & $6.73 \mathrm{E}+04$ \\
\hline Boron & ICP:A & $\mu \mathrm{g} / \mathrm{g}$ & $9.51 \mathrm{E}+01$ & $2.02 \mathrm{E}+01$ & 2 & $8.36 \mathrm{E}+00$ & $1.82 \mathrm{E}+02$ \\
\hline Bromide & IC:W & $\mu \mathrm{g} / \mathrm{g}$ & $<2.83 E+02$ & $8.96 \mathrm{E}+01$ & 2 & $\mathrm{NA}$ & NA \\
\hline Bulk density & $\begin{array}{l}\text { Bulk } \\
\text { density }\end{array}$ & $\mathrm{g} / \mathrm{mL}$ & $1.21 \mathrm{E}+00$ & NA & $\mathrm{NA}$ & NA & NA \\
\hline Cadmium & ICP:A & $\mu \mathrm{g} / \mathrm{g}$ & $<1.42 \mathrm{E}+00$ & NA & NA & NA. & NA \\
\hline Calcium & ICP:A & $\mu \mathrm{g} / \mathrm{g}$ & $2.06 \mathrm{E}+02$ & $8.38 \mathrm{E}+01$ & 2 & $0.00 \mathrm{E}+00$ & $3.62 \mathrm{E}+02$ \\
\hline Cerium & ICP:A & $\mu \mathrm{g} / \mathrm{g}$ & $6.30 \mathrm{E}+01$ & $7.63 \mathrm{E}+00$ & 2 & $1.73 \mathrm{E}+02$ & $2.39 \mathrm{E}+02$ \\
\hline Cesium-137 & GEA:F & $\mu \mathrm{Ci} / \mathrm{g}$ & $7.76 \mathrm{E}-03$ & NA & NA & NA & NA \\
\hline Chloride & $\mathrm{IC}: \mathrm{W}$ & $\mu \mathrm{g} / \mathrm{g}$ & $6.73 \mathrm{E}+02$ & $1.49 \mathrm{E}+02$ & 2 & $0.00 \mathrm{E}+00$ & $6.43 E+02$ \\
\hline Chromium & ICP:A & $\mu \mathrm{g} / \mathrm{g}$ & $4.49 \mathrm{E}+03$ & $1.02 \mathrm{E}+02$ & 2 & $2.34 \mathrm{E}+02$ & $1.11 \mathrm{E}+03$ \\
\hline Cobalt- 60 & GEA:F & $\mu \mathrm{Ci} / \mathrm{g}$ & $<1.59 \mathrm{E}-03$ & $\mathrm{NA}$ & NA & NA & NA \\
\hline Cobalt & ICP:A & $\mu \mathrm{g} / \mathrm{g}$ & $6.52 \mathrm{E}+00$ & $\mathrm{NA}$ & NA & NA & NA \\
\hline Copper & ICP:A & $\mu \mathrm{g} / \mathrm{g}$ & $6.84 \mathrm{E}+00$ & 8.63E-01 & 1 & $0.00 \mathrm{E}+00$ & $1.75 \mathrm{E}+01$ \\
\hline $\begin{array}{l}\text { Endotherm - } \\
\text { transition } 1\end{array}$ & $\begin{array}{l}\text { DSC/ } \\
\text { TGA }\end{array}$ & $\mathrm{J} / \mathrm{g}$ & NA & NA & NA & NA & NA \\
\hline Europium-154 & GEA:F & $\mu \mathrm{Ci} / \mathrm{g}$ & $<4.21 \mathrm{E}-03$ & NA & $\mathrm{NA}$ & NA & NA \\
\hline Europium-155 & GEA:F & $\mu \mathrm{Ci} / \mathrm{g}$ & $<3.41 \mathrm{E}-03$ & NA & NA & NA & NA \\
\hline Fluoride & IC:W & $\mu \mathrm{g} / \mathrm{g}$ & $5.94 \mathrm{E}+03$ & $1.45 \mathrm{E}+03$ & 2 & $0.00 \mathrm{E}+00$ & $1.22 \mathrm{E}+04$ \\
\hline Iron & ICP:A & $\mu \mathrm{g} / \mathrm{g}$ & $4.04 \mathrm{E}+03$ & $5.29 \mathrm{E}+02$ & 2 & $9.22 \mathrm{E}+03$ & $1.38 \mathrm{E}+04$ \\
\hline Lanthanum & ICP:A & $\mu \mathrm{g} / \mathrm{g}$ & $1.15 \mathrm{E}+04$ & $3.87 \mathrm{E}+02$ & 2 & $0.00 \mathrm{E}+00$ & $1.98 \mathrm{E}+03$ \\
\hline Lead & ICP:A & $\mu \mathrm{g} / \mathrm{g}$ & $3.10 \mathrm{E}+02$ & NA & NA & NA & NA \\
\hline Lithium & ICP:A & $\mu \mathrm{g} / \mathrm{g}$ & $<2.84 \mathrm{E}+00$ & NA & NA & $\mathrm{NA}$ & NA \\
\hline Magnesium & ICP:A & $\mu \mathrm{g} / \mathrm{g}$ & $3.46 \mathrm{E}+01$ & $3.86 \mathrm{E}+00$ & 2 & $1.40 \mathrm{E}+04$ & $1.41 \mathrm{E}+04$ \\
\hline Manganese & ICP:A & $\mu \mathrm{g} / \mathrm{g}$ & $1.41 \mathrm{E}+04$ & $6.41 \mathrm{E}+02$ & 2 & $0.00 \mathrm{E}+00$ & $2.77 \mathrm{E}+03$ \\
\hline Molybdenum & ICP:A & $\mu \mathrm{g} / \mathrm{g}$ & $<1.42 \mathrm{E}+01$ & NA & NA & NA & $\mathrm{NA}$ \\
\hline
\end{tabular}


Table B3-19. Summary Statistics for Tank 241-T-204. (2 sheets)

\begin{tabular}{|c|c|c|c|c|c|c|c|}
\hline . & 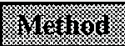 & Shilis & $\sqrt{3}$ & 3 & (1) & 81. & 1 \\
\hline Neodymium & ICP:A & $\mu \mathrm{g} / \mathrm{g}$ & $<2.84 \mathrm{E}+01$ & NA & $\mathrm{NA}$ & NA & NA \\
\hline Nickel & ICP:A & $\mu \mathrm{g} / \mathrm{g}$ & $2.42 \mathrm{E}+02$ & $1.76 \mathrm{E}+01$ & 2 & $5.51 \mathrm{E}+04$ & $5.52 \mathrm{E}+04$ \\
\hline Nitrate & IC:W & $\mu \mathrm{g} / \mathrm{g}$ & $5.52 \mathrm{E}+04$ & $1.18 \mathrm{E}+04$ & 2 & $0.00 \mathrm{E}+00$ & $5.10 \mathrm{E}+04$ \\
\hline Nitrite & IC:W & $\mu \mathrm{g} / \mathrm{g}$ & $2.84 \mathrm{E}+02$ & $6.22 \mathrm{E}+01$ & 2 & $1.06 \mathrm{E}+03$ & $1.60 \mathrm{E}+03$ \\
\hline Oxalate & IC:W & $\mu \mathrm{g} / \mathrm{g}$ & $1.33 \mathrm{E}+03$ & $4.19 \mathrm{E}+02$ & 2 & $0.00 \mathrm{E}+00$ & $1.88 \mathrm{E}+03$ \\
\hline Percent water & $\begin{array}{l}\text { DSCl } \\
\text { TGA }\end{array}$ & $\%$ & $7.51 \mathrm{E}+01$ & $1.00 \mathrm{E}+00$ & 2 & $2.45 \mathrm{E}+03$ & $2.46 \mathrm{E}+03$ \\
\hline Phosphate & IC:W & $\mu \mathrm{g} / \mathrm{g}$ & $2.46 \mathrm{E}+03$ & $6.65 \mathrm{E}+02$ & 2 & $3.26 \mathrm{E}+03$ & $8.98 \mathrm{E}+03$ \\
\hline Phosphorus & ICP:A & $\mu \mathrm{g} / \mathrm{g}$ & $2.65 \mathrm{E}+03$ & $2.20 \mathrm{E}+01$ & 2 & $0.00 \mathrm{E}+00$ & $1.23 \mathrm{E}+02$ \\
\hline Potassium & ICP:A & $\mu \mathrm{g} / \mathrm{g}$ & $6.12 \mathrm{E}+03$ & $2.39 \mathrm{E}+02$ & 2 & $4.66 \mathrm{E}+02$ & $2.52 \mathrm{E}+03$ \\
\hline Samarium & ICP:A & $\mu \mathrm{g} / \mathrm{g}$ & $<2.84 \mathrm{E}+01$ & NA & NA & NA & NA \\
\hline Silicon & ICP:A & $\mu \mathrm{g} / \mathrm{g}$ & $1.50 \mathrm{E}+03$ & $2.59 \mathrm{E}+02$ & 2 & $0.00 \mathrm{E}+00$ & $1.11 \mathrm{E}+03$ \\
\hline Silver & ICP:A & $\mu \mathrm{g} / \mathrm{g}$ & $<2.84 \mathrm{E}+00$ & NA & $\mathrm{NA}$ & NA & NA \\
\hline Sodium & ICP:A & $\mu \mathrm{g} / \mathrm{g}$ & $3.18 \mathrm{E}+04$ & $1.09 \mathrm{E}+03$ & 2 & $0.00 \mathrm{E}+00$ & $5.07 \mathrm{E}+03$ \\
\hline Strontium-89/90 & Sr:F & $\mu \mathrm{Ci} / \mathrm{g}$ & $4.60 \mathrm{E}-03$ & NA & NA & NA & NA \\
\hline Strontium & ICP:A & $\mu \mathrm{g} / \mathrm{g}$ & $4.99 \mathrm{E}+02$ & $1.57 \mathrm{E}+01$ & 2 & $0.00 \mathrm{E}+00$ & $9.58 \mathrm{E}+01$ \\
\hline Sulfate & IC:W & $\mu \mathrm{g} / \mathrm{g}$ & $<3.63 \mathrm{E}+02$ & $\mathrm{NA}$ & $\mathrm{NA}$ & $\mathrm{NA}$ & NA \\
\hline Sulfur & ICP:A & $\mu \mathrm{g} / \mathrm{g}$ & $<2.84 \mathrm{E}+01$ & $\mathrm{NA}$ & NA & NA & NA \\
\hline Thallium & ICP:A & $\mu \mathrm{g} / \mathrm{g}$ & $2.99 \mathrm{E}+02$ & NA & NA & NA & NA \\
\hline Titanium & ICP:A & $\mu \mathrm{g} / \mathrm{g}$ & $3.47 \mathrm{E}+00$ & NA & NA & NA & NA \\
\hline $\begin{array}{l}\text { Total inorganic } \\
\text { carbon }\end{array}$ & TIC/TOC & $\mu \mathrm{g} / \mathrm{g}$ & $1.40 \mathrm{E}+03$ & & & NA & NA \\
\hline $\begin{array}{l}\text { Total organic } \\
\text { carbon. }\end{array}$ & TIC/TOC & $\mu \mathrm{g} / \mathrm{g}$ & $3.12 \mathrm{E}+02$ & & & NA & NA \\
\hline Uranium & ICP:A & $\mu \mathrm{g} / \mathrm{g}$ & $<1.42 \mathrm{E}+02$ & NA & NA & NA & NA \\
\hline Vanadium & ICP:A & $\mu \mathrm{g} / \mathrm{g}$ & $<1.42 \mathrm{E}+01$ & NA & NA & NA & NA \\
\hline Zinc & ICP:A & $\mu \mathrm{g} / \mathrm{g}$ & $6.27 \mathrm{E}+01$ & $2.15 \mathrm{E}+01$ & 1 & $0.00 \mathrm{E}+00$ & $3.36 \mathrm{E}+02$ \\
\hline Zirconium & ICP:A & $\mu \mathrm{g} / \mathrm{g}$ & $<2.84 \mathrm{E}+00$ & NA & NA & NA & NA \\
\hline
\end{tabular}

Notes:

$<=$ at least 50 percent of tank $241-\mathrm{T}-204$ data is below the detection limit.

'If $\mathbf{d f}=2$, the standard deviation of the mean is $\hat{\sigma}$ (pooled); for copper $\mathrm{df}=1$ and the standard deviation of the mean is $\hat{\sigma}(\mathrm{B} 203)$; for zinc $\mathrm{df}=1$ and the standard deviation of the mean is $\hat{\sigma}(\mathrm{B} 203)$ 


\section{B4.0 APPENDIX B REFERENCES}

Bell, K. E., 1997, Tank 241-T-202 Push Core Sampling and Analysis Plan, HNF-SD-WM-TSAP-121, Rev. 0, Lockheed Martin Hanford Corp. for Fluor Daniel Hanford, Inc., Richland, Washington.

DeLorenzo, D. J, A. T. DiCenso, D. B. Hiller, K. W. Johnson, J. H. Rutherford, B. C. Simpson, and D. J. Smith, 1994, Tank Characterization Reference Guide, WHC-SD-WM-TI-648, Rev. 0, Westinghouse Hanford Company, Richland, Washington.

Dukelow, G. T., J. W. Hunt, H. Babad, and J. E. Meacham, 1995, Tank Safety Screening Data Quality Objective, WHC-SD-WM-SP-004, Rev. 2, Westinghouse Hanford Company, Richland, Washington.

Esch, R. A., 1997, Tank 241-T-202, Core 191, Analytical Results for the Final Report, HNF-SD-WM-DP-253, Rev. 0, Waste Management Federal Services of Hanford, Inc. for Fluor Daniel Hanford, Inc., Richland, Washington.

Hall, K. M., 1997a, Additional Core Composite Sample from Drainable Liquid Samples for Tank 241-T-201, (letter 74620-97-199 to D. A. Rice, May 29), Lockheed Martin Hanford Corp. for Fluor Daniel Hanford, Inc., Richland, Washington.

Hall, K. M., 1997b, Letter of Instruction for Core Samples Analysis of Tanks 241-T-201, 241-T-202, 241-T-203, and 241-T-204, (letter 74620-97-195 to A. D. Rice, May 8), Lockheed Martin Hanford Corp. for Fluor Daniel Hanford, Inc., Richland, Washington.

Horton, J. E., 1978, Analysis of Tanks 011-BXR; 201-C; and 204-T, (letter 60120-78-132J to J. E. Mirabella, December 4), Rockwell Hanford Operations, Richland, Washington.

Hu, T. A., 1997, Tank 24l-T-201 Push Core Sampling and Analysis Plan, HNF-SD-WM-TSAP-130, Rev. 0, Lockheed Martin Hanford Corp. for Fluor Daniel Hanford, Inc., Richland, Washington.

Meacham, J. E., D. L. Banning, M. R. Allen, and L. D. Muhlestein, 1997, Data Quality Objective to Support Resolution of the Organic Solvent Safety Issue, HNF-SD-WM-DQO-026, Rev. 0, Duke Engineering \& Services, Inc. for Fluor Daniel Hanford, Inc., Richland, Washington.

Nuzum, J. L., 1997a, Tank 241-T-201, Core 192, Analytical Results for the Final Report, HNF-SD-WM-DP-254, Rev. 0, Waste Management Federal Services of Hanford, Inc. for Fluor Daniel Hanford, Inc, Richland, Washington. 
Nuzum, J. L., 1997b, Tank 241-T-204, Core 188, Analytical Results for the Final Report, HNF-SD-WM-DP-255, Rev. 0, Waste Management Federal Services of Hanford, Inc. for Fluor Daniel Hanford, Inc., Richland, Washington.

Osborne, J. W. and L. L. Buckley, 1995, Data Quality Objectives for Tank Hazardous Vapor Safety Screening, WHC-SD-WM-DQO-002, Rev. 2, Westinghouse Hanford Company, Richland, Washington.

Schreiber, R. D., 1997a, Memoradum of Understanding for the Organic Complexant Safety Issue Data Requirements, HNF-SD-WM-RD-060, Rev. 0, Lockheed Martin Hanford Corp. for Fluor Daniel Hanford, Inc., Richland, Washington.

Schreiber, R. D., 1997b, Tank 241-T-203 Push Mode Core Sampling and Analysis Plan, WHC-SD-WM-TSAP-118, Rev. 0A, Lockheed Martin Hanford Corp. for Fluor Daniel Hanford, Inc., Richland, Washington.

Snedecor, G. W., and W. G. Cochran, 1980, Statistical Methods, 7th Edition, Iowa State University Press, Ames, Iowa.

Steen, F. H., 1997, Tank 241-T-203, Core 190, Analytical Results for the Final Report, HNF-SD-WM-DP-247, Rev. 0, Waste Management Federal Services of Hanford, Inc. for Fluor Daniel Hanford, Inc., Richland, Washington.

WHC, 1992, Fire Protection Engineering Surveys Instructions, WHC-IP-0030, Westinghouse Hanford Company, Richland, Washington.

Winkleman, W. D., 1997, Tank 241-T-204 Push Core Sampling and Analysis Plan, HNF-SD-WM-TSAP-119, Rev. 0, Lockheed Martin Hanford Corp. for Fluor Daniel Hanford, Inc., Richland, Washington. 
HNF-1501 Rev. 0

APPENDIX C

STATISTICAL ANALYSIS FOR ISSUE RESOLUTION

C-1 
HNF-1501 Rev. 0

This page intentionally left blank. 


\section{APPENDIX C}

\section{STATISTICAL ANALYSIS FOR ISSUE RESOLUTION}

Appendix $\mathrm{C}$ documents the results of the analyses and statistical and numerical manipulations required by the DQOs applicable for the T-200 series tanks. The analyses required are reported as follows:

- Section C1.0: Statistical analysis and numerical manipulations supporting the safety screening DQO (Dukelow et al. 1995)

- Section C2.0: Appendix C References

\section{C1.0 STATISTICS FOR THE SAFETY SCREENING DATA QUALITY OBJECTIVE}

The safety screening DQO (Dukelow et al. 1995) defines acceptable decision confidence limits in terms of one-sided 95 percent confidence intervals. In this appendix, one-sided confidence limits supporting the safety screening DQO are calculated for the T-200 series tanks. All data in this section are from the final laboratory data packages for the 1997 core sampling events (Nuzum 1997a, Esch 1997, Steen 1997, and Nuzum 1997b).

Confidence intervals were computed for the analytical data associated with each solid sample number from tanks 241-T-201, T-202, T-203, and T-204 using existing statistical software (Statistical Science 1993). The sample numbers and confidence intervals are in Table C1-1 for alpha. Drainable liquid alpha measurements were sufficiently low that confidence interval calculations were not necessary. Typically, there would also be data associated with the DSC results. However, there were almost no exotherms observed in the waste samples tested. What few exotherms were observed had extremely low values; therefore, no confidence interval calculation was performed on the DSCs.

The upper limit (UL) of a one-sided 95 percent confidence interval on the mean is

$$
\hat{\mu}+t_{(d f, 0.05)} * \hat{\sigma}_{\hat{\mu}} .
$$

In this equation, $\hat{\mu}$ is the arithmetic mean of the data, $\hat{\sigma}_{\hat{\mu}}$ is the estimate of the standard deviation of the mean, and $t_{(d f, 0.0)}$ is the quantile from Student's $t$ distribution with df degrees of freedom for a one-sided 95 percent confidence interval. For each T-200 series tank dataset (per sample number), df equals the number of observations minus one; i.e., $d f=1$. 
Table C1-1 lists the upper limit of the 95 percent confidence interval for each sample number based on alpha data. Each confidence interval can be used to make the following statement. If the upper limit is less than $41 \mu \mathrm{Ci} / \mathrm{g}$, reject the null hypothesis that the alpha is greater than or equal to $41 \mu \mathrm{Ci} / \mathrm{g}$ at the 0.05 level of significance. The upper limit to the 95 percent confidence interval is less than $41 \mu \mathrm{Ci} / \mathrm{g}$ for all 22 intervals in Table C1-1. This means that the null hypothesis that the alpha concentration is greater than or equal to $41 \mu \mathrm{Ci} / \mathrm{g}$, cannot be rejected. Therefore, for these tanks, criticality is not a concern.

Table C1-1. 95 Percent Confidence Interval Upper Limits for Alpha for Tanks 241-T-201, $-\mathrm{T}-202,-\mathrm{T}-203$, and $-\mathrm{T}-204$ (Units are $\mu \mathrm{Ci} / \mathrm{g}$ ). (2 sheets)

\begin{tabular}{|c|c|c|c|c|c|}
\hline (3) & 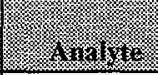 & (3) & 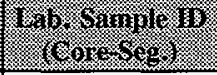 & 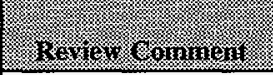 & 1. \\
\hline $241-\mathrm{T}-201$ & Gross alpha & $\mu \mathrm{Ci} / \mathrm{g}$ & $\begin{array}{l}\text { S97T000901F } \\
(192-06)\end{array}$ & & $6.30 \mathrm{E}-01$ \\
\hline $241-\mathrm{T}-201$ & Gross alpha & $\mu \mathrm{Ci} / \mathrm{g}$ & $\begin{array}{l}\text { S97T000919F } \\
(192-07)\end{array}$ & & $7.06 \mathrm{E}-01$ \\
\hline 241-T-201 & Gross alpha & $\mu \mathrm{Ci} / \mathrm{g}$ & $\begin{array}{l}\text { S97T000920F } \\
(192-08)\end{array}$ & Possible outlier (high) & $1.63 \mathrm{E}+00$ \\
\hline $241-\mathrm{T}-202$ & Gross alpha & $\mu \mathrm{Ci} / \mathrm{g}$ & $\begin{array}{l}\text { S97T000939F } \\
(191-01)\end{array}$ & & $2.57 \mathrm{E}-01$ \\
\hline $241-\mathrm{T}-202$ & Gross alpha & $\mu \mathrm{Ci} / \mathrm{g}$ & $\begin{array}{l}\text { S97T000940F } \\
(191-02)\end{array}$ & & $2.90 \mathrm{E}-01$ \\
\hline $241-\mathrm{T}-202$ & Gross alpha & $\mu \mathrm{Ci} / \mathrm{g}$ & $\begin{array}{l}\text { S97T000941F } \\
(191-03)\end{array}$ & & 2.37E-01 \\
\hline $241-\mathrm{T}-202$ & Gross alpha & $\mu \mathrm{Ci} / \mathrm{g}$ & $\begin{array}{l}\text { S97T000942F } \\
(191-04)\end{array}$ & RPD greater than $20 \%$ & $4.09 \mathrm{E}-01$ \\
\hline $241-\mathrm{T}-202$ & Gross alpha & $\mu \mathrm{Ci} / \mathrm{g}$ & $\begin{array}{l}\text { S97T000943F } \\
(191-05)\end{array}$ & & $2.70 \mathrm{E}-01$ \\
\hline $241-\mathrm{T}-203$ & Gross alpha & $\mu \mathrm{Ci} / \mathrm{g}$ & $\begin{array}{l}\text { S97T000759F } \\
(190-01)\end{array}$ & & $3.03 \mathrm{E}-01$ \\
\hline $241-\mathrm{T}-203$ & Gross alpha & $\mu \mathrm{Ci} / \mathrm{g}$ & $\begin{array}{l}\text { S97T000760F } \\
(190-01 R)\end{array}$ & & $2.07 \mathrm{E}-01$ \\
\hline $241-\mathrm{T}-203$ & Gross alpha & $\mu \mathrm{Ci} / \mathrm{g}$ & $\begin{array}{l}\text { S97T000757F } \\
(190-02)\end{array}$ & & $3.56 \mathrm{E}-01$ \\
\hline $241-\mathrm{T}-203$ & Gross alpha & $\mu \mathrm{Ci} / \mathrm{g}$ & $\begin{array}{l}\text { S97T000761F } \\
(190-03)\end{array}$ & RPD greater than $20 \%$ & $4.69 \mathrm{E}-01$ \\
\hline
\end{tabular}


Table C1-1. 95 Percent Confidence Interval Upper Limits for Alpha for Tanks 241-T-201, $-\mathrm{T}-202,-\mathrm{T}-203$, and $-\mathrm{T}-204$ (Units are $\mu \mathrm{Ci} / \mathrm{g}$ ). (2 sheets)

\begin{tabular}{|c|c|c|c|c|c|}
\hline (12ais: & anuve & Units & 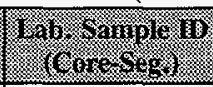 & ie next & 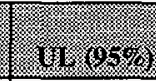 \\
\hline $241-\mathrm{T}-203$ & Gross alpha & $\mu \mathrm{Ci} / \mathrm{g}$ & $\begin{array}{l}\text { S97T000762F } \\
(190-04)\end{array}$ & & $2.53 \mathrm{E}-01$ \\
\hline $241-\mathrm{T}-203$ & Gross alpha & $\mu \mathrm{Ci} / \mathrm{g}$ & $\begin{array}{l}\text { S97T000763F } \\
(190-05)\end{array}$ & & $2.80 \mathrm{E}-01$ \\
\hline 241-T-203 & Gross alpha & $\mu \mathrm{Ci} / \mathrm{g}$ & $\begin{array}{l}\text { S97T000764F } \\
(190-06)\end{array}$ & & $1.30 \mathrm{E}-01$ \\
\hline $241-\mathrm{T}-203$ & Gross alpha & $\mu \mathrm{Ci} / \mathrm{g}$ & $\begin{array}{l}\text { S97T000765F } \\
(190-07)\end{array}$ & & $1.93 \mathrm{E}-01$ \\
\hline $241-T-203$ & Gross alpha & $\mu \mathrm{Ci} / \mathrm{g}$ & $\begin{array}{l}\text { S97T000766F } \\
(190-08)\end{array}$ & & $2.60 \mathrm{E}-01$ \\
\hline $241-T-203$ & Gross alpha & $\mu \mathrm{Ci} / \mathrm{g}$ & $\begin{array}{l}\text { S97T000767F } \\
(190-09) \\
\end{array}$ & RPD greater than $20 \%$ & $2.66 \mathrm{E}-01$ \\
\hline \multicolumn{6}{|c|}{ (1) } \\
\hline 241-T-204 & Gross alpha & $\mu \mathrm{Ci} / \mathrm{g}$ & $\begin{array}{l}\text { S97T000587F } \\
(188-01)\end{array}$ & & $2.60 \mathrm{E}-01$ \\
\hline 241-T-204 & Gross alpha & $\mu \mathrm{Ci} / \mathrm{g}$ & $\begin{array}{l}\text { S97T000588F } \\
(188-02)\end{array}$ & & $1.77 \mathrm{E}-01$ \\
\hline 241-T-204 & Gross alpha & $\mu \mathrm{Ci} / \mathrm{g}$ & $\begin{array}{l}\text { S97T000589F } \\
(188-03)\end{array}$ & & $1.93 \mathrm{E}-01$ \\
\hline 241-T-204 & Gross alpha & $\mu \mathrm{Ci} / \mathrm{g}$ & $\begin{array}{l}\text { S97T000590F } \\
(188-04)\end{array}$ & & $1.40 \mathrm{E}-01$ \\
\hline $241-T-204$ & Gross alpha & $\mu \mathrm{Ci} / \mathrm{g}$ & $\begin{array}{l}\text { S97T000621F } \\
(188-05)\end{array}$ & RPD greater than $20 \%$ & $2.76 \mathrm{E}-01$ \\
\hline $241-T-204$ & Gross alpha & $\mu \mathrm{Ci} / \mathrm{g}$ & \begin{tabular}{|l|} 
S97T000622F \\
$(188-06)$
\end{tabular} & & $1.67 \mathrm{E}-01$ \\
\hline 241-T-204 & Gross alpha & $\mu \mathrm{Ci} / \mathrm{g}$ & $\begin{array}{l}\text { S97T000623F } \\
(188-07)\end{array}$ & RPD greater than $20 \%$ & $5.49 \mathrm{E}-01$ \\
\hline $241-T-204$ & Gross alpha & $\mu \mathrm{Ci} / \mathrm{g}$ & $\begin{array}{l}\text { S97T000624F } \\
(188-08)\end{array}$ & & $2.33 \mathrm{E}-01$ \\
\hline $241-T-204$ & Gross alpha & $\mu \mathrm{Ci} / \mathrm{g}$ & \begin{tabular}{|l|} 
S97T000625F \\
$(188-09)$
\end{tabular} & & 2.63E-01 \\
\hline 241-T-204 & Gross alpha & $\mu \mathrm{Ci} / \mathrm{g}$ & $\begin{array}{l}\text { S97T000626F } \\
(188-10)\end{array}$ & & $2.13 \mathrm{E}-01$ \\
\hline
\end{tabular}




\section{C2.0 APPENDIX C REFERENCES}

Dukelow, G. T., J. W. Hunt, H. Babad, and J. E. Meacham, 1995, Tank Safety Screening Data Quality Objective, WHC-SD-WM-SP-004, Rev. 2, Westinghouse Hanford Company, Richland, Washington.

Esch, R. A., 1997, Tank 241-T-202, Core 191, Analytical Results for the Final Report, HNF-SD-WM-DP-253, Rev. 0, Waste Management Federal Services of Hanford, Inc. for Fluor Daniel Hanford, Inc., Richland, Washington.

Nuzum, J. L., 1997a, Tank 241-T-201, Core 192, Analytical Results for the Final Report, HNF-SD-WM-DP-254, Rev. 0, Waste Management Federal Services of Hanford, Inc. for Fluor Daniel Hanford, Inc., Richland, Washington.

Nuzum, J. L., 1997b, Tank 241-T-204, Core 188, Analytical Results for the Final Report, HNF-SD-WM-DP-255, Rev. 0, Waste Management Federal Services of Hanford, Inc. for Fluor Daniel Hanford, Inc., Richland, Washington.

Steen, F. H., 1997, Tank 241-T-203, Core 190, Analytical Results for the Final Report, HNF-SD-WM-DP-247, Rev. 0, Waste Management Federal Services of Hanford, Inc. for Fluor Daniel, Hanford, Inc., Richland, Washington.

Statistical Science, 1993, S-PLUS Reference Manual, Version 3.2, Statistical Sciences, a division of MathSoft, Inc., Seattle, Washington. 
HNF-1501 Rev. 0

\section{APPENDIX D}

EVALUATION TO ESTABLISH BEST-BASIS INVENTORY FOR THE T-200 SERIES SINGLE-SHELL TANKS

(241-T-201, 241-T-202, 241-T-203, AND 241-T-204) 
HNF-1501 Rev. 0

This page intentionally left blank. 


\section{APPENDIX D}

\section{EVALUATION TO ESTABLISH BEST-BASIS INVENTORY FOR THE T-200 SERIES SINGLE-SHELL TANKS (241-T-201, 241-T-202, 241-T-203, AND 241-T-204)}

An effort is underway to provide waste inventory estimates that will serve as standard characterization source terms for waste management activities (Hodgson and LeClair 1996). As part of this effort, an evaluation of available information for single-shell tanks 241-T-201, $-\mathrm{T}-202,-\mathrm{T}-203$, and -T-204 was performed, and a best-basis inventory was established for each tank. This work, detailed in the following sections, follows the methodology that was established by the standard inventory task.

\section{D1.0 CHEMICAL INFORMATION SOURCES}

The data for the T-200 series tanks (Nuzum [1997a], Esch [1997], Steen [1997], and Nuzum [1997b]) provide characterization results from the most recent sampling events for these tanks. One full-depth core sample was obtained from each tank in March and April 1997. Each core was obtained from the same location on each tank, riser 3. The sample based inventories calculated in this report uses the core composite analytical results. The waste volumes and densities used are particular to each tank and are based on the most current analytical or surveillance data.

Before sampling data was available, inventories were calculated using the data provided in Conner et al. (1997), Dougherty et al. (1997), Jo et al. (1997), and Sasaki et al. (1997). Process history suggested that characterization results for tanks 241-B-201, -B-202, -B-203, and -B-204 could be used to characterize tanks 241-T-201, -T-202, -T-203, and -T-204 because they contain the same type of wastes.

The HDW model (Agnew et al. 1997a) provides tank content estimates in terms of component concentrations and inventories. A projected inventory for selected waste components in the T-200 series tanks has been prepared based on process flowsheets, production records, and waste volume records. 


\section{D2.0 COMPARISON OF COMPONENT INVENTORY VALUES}

The engineering-based inventories listed in Tables D2-1 and D2-2 were calculated by multiplying the sample-based inventories for B-200 series tanks (such as 241-B-201 [Conner et al. 1997]) by the ratio of the waste volume in tank 241-T-201 to the waste volume in tank 241-B-201 (1.00). (The chemical species are reported without charge designation per the best-basis inventory convention.)

Table D2-1. Engineering and Hanford Defined Waste-Based Inventory Estimates for Tank 241-T-201 Nonradioactive Components.

\begin{tabular}{|c|c|c|c|c|c|}
\hline simare & (6) & 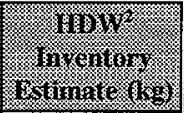 & 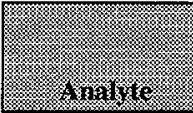 & 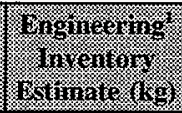 & 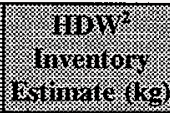 \\
\hline $\mathrm{Al}$ & 473 & 0.00 & $\mathrm{Na}$ & 5,250 & 10,300 \\
\hline $\mathrm{Bi}$ & 13,000 & 1,230 & $\mathrm{Ni}$. & 65.9 & 8.85 \\
\hline $\mathrm{Ca}$ & 1,680 & 1,040 & $\mathrm{NO}_{2}$ & 121 & 15.6 \\
\hline $\mathrm{Ce}$ & 9.57 & $n / r$ & $\mathrm{NO}_{3}$ & 6,780 & 8,050 \\
\hline $\mathrm{Cl}$ & 227 & 89.1 & oxalate & $\mathrm{n} / \mathrm{r}$ & 9,900 \\
\hline $\mathrm{Cr}$ & 459 & 33.3 & $\mathrm{P}$ as $\mathrm{PO}_{4}$ & 2,300 & 846 \\
\hline $\mathrm{Cu}$ & 6.63 & $n / r$ & $\mathrm{Si}$ & 2,780 & 0 \\
\hline $\mathbf{F}$ & 802 & 2,030 & $\mathrm{SO}_{4}$ & 47.9 & 27.2 \\
\hline $\mathrm{Fe}$ & 1,840 & 2,150 & $\mathrm{Sr}$ & 127 & 0 \\
\hline $\mathrm{K}$ & 799 & 851 & $\mathrm{TIC}$ as $\mathrm{CO}_{3}$ & $\mathrm{n} / \mathrm{r}$ & 1,550 \\
\hline $\mathrm{La}$ & 2,080 & 49.8 & $\mathrm{Zn}$ & 29.8 & $\mathrm{n} / \mathrm{r}$ \\
\hline $\mathrm{Mg}$ & 208 & $\mathrm{n} / \mathrm{r}$ & $\mathrm{H}_{2} \mathrm{O}(\mathrm{wt} \%)$ & 60.7 & 69.5 \\
\hline $\mathrm{Mn}$ & 2,640 & 26.4 & Density $(\mathrm{g} / \mathrm{mL})$ & 1.25 & 1.20 \\
\hline
\end{tabular}

Notes:

$\mathbf{n} / \mathbf{r}=$ not reported

'Conner et al. (1997) ratioed to the volume of tank 241-T-201

${ }^{2}$ Agnew et al. (1997a) 
Table D2-2. Engineering and Hanford Defined Waste-based Inventory Estimates for Radioactive Components for Tank 241-T-201 (Decayed to January 1, 1994).

\begin{tabular}{|c|c|c|c|c|c|}
\hline 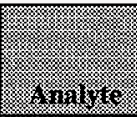 & 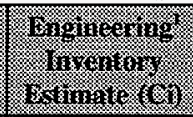 & 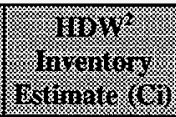 & 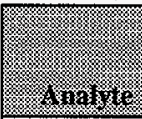 & 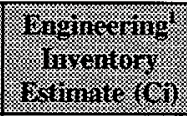 & Wising \\
\hline${ }^{14} \mathrm{C}$ & 0.0435 & $1.75 \mathrm{E}-04$ & ${ }^{239 / 240} \mathrm{Pu}$ & 155 & 0.0505 \\
\hline${ }^{90} \mathrm{Sr}$ & 275 & 18.2 & ${ }^{241} \mathrm{Am}$ & 4.25 & 3.80E-04 \\
\hline${ }^{137} \mathrm{Cs}$ & 105 & 20.7 & Total $\alpha$ & 180 & $n / r$ \\
\hline${ }^{154} \mathrm{Eu}$ & 0.512 & 8.99E-04 & & & \\
\hline
\end{tabular}

Notes:

${ }^{1}$ Conner et al. (1997) decayed to January 1, 1994

${ }^{2}$ Agnew et al. (1997a) decayed to January 1, 1994

Tank 241-T-201 is reported to contain $110 \mathrm{~kL}$ (29 kgal) of waste, and tank 241-B-201 is reported to contain $110 \mathrm{~kL}$ (29 kgal) (Hanlon 1997). Similarly, tanks 241-T-202, -T-203, and $-\mathrm{T}-204$ contain $79 \mathrm{~kL}$ ( $21 \mathrm{kgal}), 132 \mathrm{~kL}$ ( $35 \mathrm{kgal}$ ), and $144 \mathrm{~kL}$ (38 kgal), respectively. In calculating the initial inventory estimates for the T-200 series tanks, the wastes in the corresponding B-200 and T-200 tanks were assumed to be the same. An inventory based on a volume ratio between the tanks was derived using the B-200 sampling information as a basis. The HDW model (Agnew et al. 1997a) inventory also is derived using these same waste volumes and similar density values. Those estimates are given in Tables D2-1 through D2-6. Sampling information has been obtained recently from the T-200 series tanks, and estimates based on that information will be developed and presented later in this section.

Table D2-3. Engineering and Hanford Defined Waste-based Inventory Estimates for Tank 241-T-202 Nonradioactive Components. (2 sheets)

\begin{tabular}{|c|c|c|c|c|c|}
\hline initif & (2) & 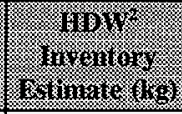 & Inario & 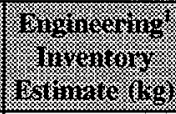 & 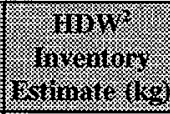 \\
\hline $\mathrm{Al}$ & 93.0 & 0.00 & $\mathrm{Na}$ & 3,530 & 7,740 \\
\hline $\mathrm{Bi}$ & 3,110 & 923 & $\mathrm{Ni}$ & 19.1 & 6.64 \\
\hline $\mathrm{Ca}$ & 149 & 779 & $\mathrm{NO}_{2}$ & 52.0 & 11.7 \\
\hline $\mathrm{Ce}$ & 10.0 & $n / r$ & $\mathrm{NO}_{3}$ & 6,020 & 6,040 \\
\hline $\mathrm{Cl}$ & 79.0 & 66.8 & $\mathrm{~Pb}$ & 60.0 & $n / r$ \\
\hline $\mathrm{Cr}$ & 230 & 25.0 & $\mathrm{P}$ as $\mathrm{PO}_{4}$ & 856 & 634 \\
\hline $\mathrm{Cu}$ & 25.0 & $\mathrm{n} / \mathrm{r}$ & $\mathbf{S i}$ & 311 & 0 \\
\hline
\end{tabular}


Table D2-3. Engineering and Hanford Defined Waste-based Inventory Estimates for Tank 241-T-202 Nonradioactive Components. (2 sheets)

\begin{tabular}{|c|c|c|c|c|c|}
\hline 4 . & 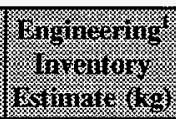 & (1301) & , minge & 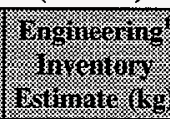 & 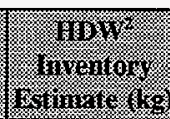 \\
\hline$F$ & 593 & 1,530 & $\mathrm{SO}_{4}$ & 134 & 20.4 \\
\hline $\mathrm{Fe}$ & 622 & 1,610 & $\mathrm{Sr}$ & 55.0 & 0 \\
\hline $\mathrm{K}$ & 631 & 638 & $\mathrm{TIC}$ as $\mathrm{CO}_{3}$ & 172 & 1,170 \\
\hline $\mathrm{La}$ & 1,250 & 37.3 & $\mathrm{Zn}$ & 52.0 & $\mathbf{n} / \mathbf{r}$ \\
\hline $\mathrm{Mg}$ & 23.0 & $\mathrm{n} / \mathbf{r}$ & $\mathrm{H}_{2} \mathrm{O}(\mathrm{wt} \%)$ & 75.8 & 68.6 \\
\hline $\mathrm{Mn}$ & 1,250 & 19.8 & Density $(\mathrm{g} / \mathrm{mL})$ & 1.21 & 1.21 \\
\hline
\end{tabular}

Notes:

'Dougherty et al. (1997) multiplied by 0.78

${ }^{2}$ Agnew et al. (1997a)

Table D2-4. Engineering and Hanford Defined Waste-based Inventory Estimates for Radioactive Components in Tank 241-T-202.

\begin{tabular}{|c|c|c|c|c|c|}
\hline Ninati & 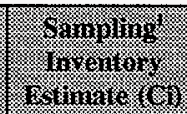 & 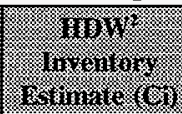 & inatike & 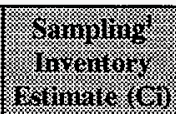 & 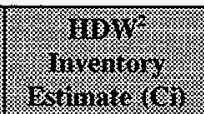 \\
\hline${ }^{90} \mathrm{Sr}$ & 349 & 13.7 & ${ }^{239 / 240} \mathrm{Pu}$ & 19 & 0.0379 \\
\hline${ }^{137} \mathrm{Cs}$ & 2.4 & 15.5 & ${ }^{241} \mathrm{Am}$ & 6.14 & $2.85 \mathrm{E}-04$ \\
\hline
\end{tabular}

Notes:

${ }^{1}$ Dougherty et al. (1997) multiplied by 0.78

${ }^{2}$ Agnew et al. (1997a) decayed to January 1, 1994 
Table D2-5. Engineering and Hanford Defined Waste-based Inventory Estimates for Tank 241-T-203 Nonradioactive Components.

\begin{tabular}{|c|c|c|c|c|c|}
\hline . & 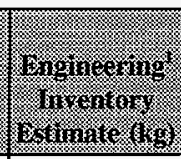 & 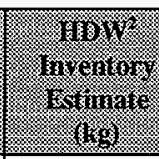 & 2 initits & 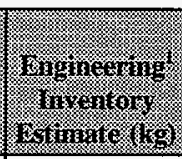 & for \\
\hline $\mathrm{Al}$ & 8.03 & 0.00 & $\mathrm{Na}$ & 4,570 & 12,900 \\
\hline $\mathrm{Bi}$ & 6,430 & 1,540 & $\mathrm{Ni}$ & 28.2 & 11.1 \\
\hline $\mathrm{Ca}$ & 34.3 & 1,300 & $\mathrm{NO}_{2}$ & 117 & 19.5 \\
\hline $\mathrm{Ce}$ & 7.82 & $n / r$ & $\mathrm{NO}_{3}$ & 10,000 & 10,100 \\
\hline $\mathrm{Cl}$ & 135 & 111 & oxalate & 312 & 12,400 \\
\hline $\mathrm{Cr}$ & 476 & 41.7 & $\mathrm{P}$ as $\mathrm{PO}_{4}$ & 1,040 & 1,060 \\
\hline $\mathrm{Cu}$ & 108 & $n / r$ & \begin{tabular}{|l}
$\mathrm{Si}$ \\
\end{tabular} & 142 & 0 \\
\hline $\mathbf{F}$ & 1,210 & 2,540 & $\mathrm{SO}_{4}$ & 110 & 34.0 \\
\hline $\mathrm{Fe}$ & 681 & 2,680 & $\mathrm{Sr}$ & 76.1 & 0 \\
\hline $\mathbf{K}$ & 803 & 1,060 & $\mathrm{TIC}$ as $\mathrm{CO}_{3}$ & 528 & 1,940 \\
\hline $\mathrm{La}$ & 1,610 & 62.2 & $\mathrm{Zn}$ & 9.33 & $\mathrm{n} / \mathrm{r}$ \\
\hline $\mathrm{Mg}$ & 8.16 & $\mathrm{n} / \mathrm{r}$ & $\mathrm{H}_{2} \mathrm{O}(\mathrm{wt} \%)$ & 75.8 & 68.6 \\
\hline $\mathrm{Mn}$ & 2,180 & 33.0 & Density $(\mathrm{g} / \mathrm{mL})$ & 1.19 & 1.21 \\
\hline
\end{tabular}

Notes:

${ }^{1} \mathrm{~J}$ o et al. (1997) multiplied by 0.686

${ }^{2}$ Agnew et al. (1997a)

Table D2-6. Engineering Assessment- and Hanford Defined Waste-Based Inventory Estimates for Tank 241-T-204 Nonradioactive Components. (2 sheets)

\begin{tabular}{|c|c|c|c|c|c|}
\hline 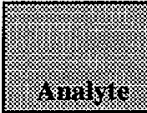 & 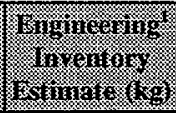 & 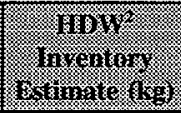 & 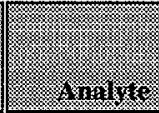 & 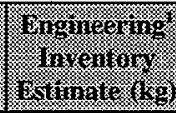 & 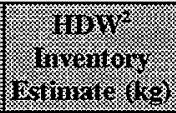 \\
\hline $\mathrm{Al}$ & 10.9 & 0 & $\mathrm{Ni}$ & 39.1 & 12.0 \\
\hline $\mathrm{Bi}$ & 8,130 & 1,670 & $\mathrm{NO}_{2}$ & 122 & 21.2 \\
\hline $\mathrm{Ca}$ & 51.1 & 1,410 & $\mathrm{NO}_{3}$ & 8,970 & 10,900 \\
\hline $\mathrm{Ce}$ & 9.35 & $\mathrm{n} / \mathrm{r}$ & $\mathrm{OH}$ & $n / \mathbf{r}$ & 2,690 \\
\hline $\mathrm{Cl}$ & 118 & 121 & oxalate & 286 & 13,400 \\
\hline $\mathrm{Cr}$ & 543 & 45.2 & $\mathrm{P}$ as $\mathrm{PO}_{4}$ & 1,200 & 1,150 \\
\hline $\mathrm{Cu}$ & 3.71 & $\mathrm{n} / \mathrm{r}$ & Si & 179 & 0 \\
\hline
\end{tabular}


Table D2-6. Engineering Assessment- and Hanford Defined Waste-Based Inventory Estimates for Tank 241-T-204 Nonradioactive Components. (2 sheets)

\begin{tabular}{|c|c|c|c|c|c|}
\hline (.). & 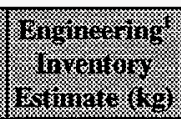 & (19O1) & 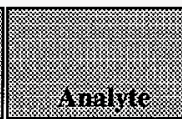 & 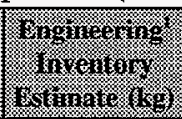 & 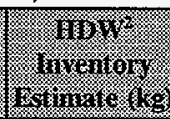 \\
\hline $\mathrm{F}$ & 1,200 & 2,760 & $\mathrm{SO}_{4}$ & 111 & 36.9 \\
\hline $\mathrm{Fe}$ & 638 & 2,910 & $\mathrm{Sr}$ & 65.4 & 0 \\
\hline $\mathrm{K}$ & 980 & 1,150 & $\mathrm{IIC}$ as $\mathrm{CO}_{3}$ & $n / r$ & 2,110 \\
\hline $\mathrm{La}$ & 1,740 & 67.5 & $\mathrm{Zn}$ & 8.97 & $\mathrm{n} / \mathrm{r}$ \\
\hline $\mathrm{Mg}$ & 13.9 & $n / r$ & $\mathrm{H}_{2} \mathrm{O}(\mathrm{wt} \%)$ & 77.1 & 68.6 \\
\hline $\mathrm{Mn}$ & 2,480 & 35.8 & density $(\mathrm{g} / \mathrm{mL})$ & 1.19 & 1.21 \\
\hline $\mathrm{Na}$ & 4,470 & 14,000 & & & \\
\hline
\end{tabular}

Notes:

${ }^{1}$ Sasaki et al. (1997) multiplied by 0.76

${ }^{2}$ Agnew et al. (1997a)

\section{D3.0 COMPONENT INVENTORY EVALUATION}

The following evaluation provides a best-basis inventory estimate for chemical and radionuclide components for tanks 241-T-201, -T-202, -T-203, and -T-204.

\section{D3.1 CONTRIBUTING WASTE TYPES}

The following abbreviations were used to designate waste types:

$224=$ Final plutonium decontamination and concentration waste from the $\mathrm{BiPO}_{4}$ process using $\mathrm{LaF}_{3}$

Agnew et al. (1997b) shows waste in the 200 series tanks in 1952 for B and T Tank Farms and in 1956 for U Tank Farm. However, Borsheim (1994) reports that 224 wastes were routed to the $6.1-\mathrm{m}$ (20-ft)-diameter concrete settling tank (241-361) and then overflowed to a dry well. The dry well was replaced with a crib by June 1945 . 
Cell drainage ( 5 to 6 waste) also was routed to the 241-361 tank. High-activity cell drainage was supposed to be routed to tanks $241-\mathrm{B}-107$ and 241-T-107 in the 1C waste cascades. Borsheim also notes that each 200 series tanks had two inlet lines, was not cascaded, and had no overflow lines. Experiments (as of November 1944) indicated that 224 wastes should contain three percent solids by volume.

Borsheim notes that Hanford Works Monthly Reports show a plan to provide a separate crib for the B Plant cell drainage. At the time the cell drainage was disposed of to tank 241-B-201 along with the 224 waste. Tank 241-B-201 and the $T$ tanks were in service as sludge settling tanks for 224-B and $\mathrm{T}$ wastes, respectively. The remaining 200 series tanks (241-T-202, -T-203, and -T-204) were being excavated and piped in series to increase settling capacity.

Borsheim reports that by July 1950, tank 241-B-204, which had been in service since

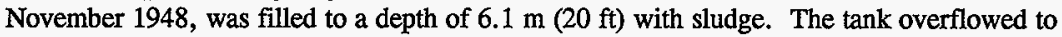
tank 241-B-203 which had received $10.2 \mathrm{~cm}$ (4 in.) of sludge by that time. This suggests tanks 241-B-201 and 241-T-201 received 224 waste before other B-200 and T-200 series tanks. When other B-200 series tanks received 224 waste, it overflowed from tank 241-B-204 to tanks $241-\mathrm{B}-203$ and -B-202. The T-200 series tanks received 224 waste in a similar fashion.

\section{Expected Types of Solids in the Waste}

Hill et al. (1995): 224

Agnew et al. (1997a): $\quad 224$

\section{D3.2 EVALUATION OF FLOWSHEET INFORMATION}

Table D3-1 shows the technical flowsheet information (Kupfer et al. 1997) for 224 streams and the comparative Los Alamos National Laboratory-defined waste streams. 
Table D3-1. Technical Flowsheet and Hanford Defined Waste Streams.

\begin{tabular}{|c|c|c|}
\hline . & 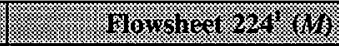 & 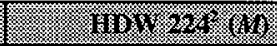 \\
\hline $\mathrm{Bi}$ & 0.00595 & 0.006 \\
\hline $\mathrm{C}_{2} \mathrm{O}_{4}$ & 0.0458 & 0.046 \\
\hline $\mathrm{Cr}$ & 0.00362 & 0.0068 \\
\hline $\mathbf{F}$ & 0.272 & 0.27 \\
\hline $\mathbf{K}$ & 0.223 & 0.231 \\
\hline $\mathrm{La}$ & 0.00376 & 0.0038 \\
\hline $\mathrm{Mn}$ & 0.00514 & 0.0051 \\
\hline $\mathrm{Na}$ & 1.62 & 1.60 \\
\hline $\mathrm{NO}_{3}$ & 1.06 & 1.38 \\
\hline $\mathrm{PO}_{4}$ & 0.0322 & 0.038 \\
\hline $\mathrm{SO}_{4}$ & 0.00140 & 0.003 \\
\hline$\overline{\mathrm{NH}_{4}}$ & $\mathrm{n} / \mathrm{r}$ & $\mathrm{n} / \mathrm{r}$ \\
\hline
\end{tabular}

Notes:

$M=$ moles per liter

${ }^{1}$ Appendix C of Kupfer et al. (1997); see Bismuth Phosphate Process Flowsheet

${ }^{2}$ Agnew et al. (1997a)

\section{D3.3 ASSUMPTIONS FOR RECONCILING WASTE INVENTORIES}

Reference inventories in the T-200 series tanks were estimated using an engineering assessment based on the results of a sampling event from the B-200 series tanks. Tanks in $T$ farm were assumed to have nearly identical process histories and contain the same waste type as the corresponding tank in B farm (for example, 241-B-201 and 241-T-201). Current inventories were calculated using recently obtained sampling data. These inventories were then compared with the HDW model inventories.

The assumptions and observations for the engineering assessment were based on best technical judgment pertaining to input information that can significantly influence tank inventories. This includes the following: 1) correct prediction of contributing waste types and correct relative proportions of the waste types; 2) accurate predictions of flowsheet conditions, fuel processed, and waste volumes; 3) accurate predictions of partitioning of components; and 4) accurate predictions of physical parameters such as density, percent solids, etc. By using this evaluation, the assumptions can be modified as necessary to provide a basis for identifying 
potential errors and/or missing information that could influence the sample- and model-based inventories. The simplified assumptions and observations used for the evaluation are as follows.

- Tank waste mass is calculated using the measured density and the tank volume listed in Hanlon (1997). Engineering assessment-based, sample-based, and model-based inventories are derived using this volume. As a result, inventory comparisons are made on the same volume basis.

- Only the 224 waste stream contributed to solids formation. It is assumed that tanks with the same waste type will have the same concentrations of individual analytes.

- Bulk component (chemical species) information is sufficient for comparing the computed data sets. This information can be obtained from technical flowsheets, model bases, and sample data.

\section{D3.4 BASIS FOR CALCULATIONS USED IN THE ENGINEERING EVALUATION AND SAMPLE-BASED ESTIMATES}

The best-basis evaluations for the B-200 tanks (Appendix D of Conner et al. [1997], Dougherty et al. [1997], Jo et al. [1997], and Sasaki et al. [1997]) compares sample data to flowsheet predictions for 224 waste and shows good agreement between them. Because tanks 241-B-201 and 241-T-201 received the same waste in the same time period and are located in the same position in their respective tank farms, it is considered appropriate to use the tank 241-B-201 sample results to estimate the inventories for tank 241-T-201 (Field and Winward 1997a). The other T-200 series tanks were assessed in a similar fashion (for example, tanks 241-B-202 and 241-T-202 [Field and Winward 1997b], tanks 241-B-203 and 241-T-203 [Field and Winward 1997c] and tanks 241-B-204 and 241-T-204 [Field and Winward 1997d]).

The initial inventories for the T-200 tanks were estimated using the inventories for the B-200 tanks and adjusting them by a factor equal to the ratio of the volumes of waste in the corresponding tank. Estimated component inventories from this engineering evaluation are compared with the HDW-based and sample-based inventories in Table D3-2.

The sample-based inventories were derived using the mean core composite sample analyses in Appendix B, measured tank waste volumes, and densities. The volumes used in calculating inventories for tanks 241-T-201, -T-202, -T-203, and -T-204 are $110 \mathrm{~kL}$ (29 kgal), $79 \mathrm{~kL}$ (21 kgal), $132 \mathrm{~kL}$ ( $35 \mathrm{kgal}$ ), and $144 \mathrm{~kL}$ (38 kgal), respectively. The densities measured in each tank were $1.27 \mathrm{~g} / \mathrm{mL}$ (-T-201), $1.24 \mathrm{~g} / \mathrm{mL}(-\mathrm{T}-202), 1.23 \mathrm{~g} / \mathrm{mL}$ (-T-203), and $1.21 \mathrm{~g} / \mathrm{mL}$ (-T-204). Observations regarding these inventories are noted by component. 
HNF-1501 Rev. 0

Table D3-2. Comparison of Selected Component Inventory Estimates for T-200 Series Tanks. ( 2 sheets)

\begin{tabular}{|c|c|c|c|}
\hline 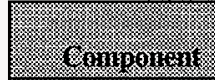 & 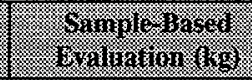 & 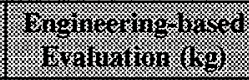 & 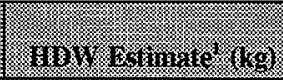 \\
\hline \multicolumn{4}{|c|}{ 1969, } \\
\hline $\mathrm{Bi}$ & 16,600 & 13,000 & 1,230 \\
\hline $\mathrm{K}$ & 671 & 799 & 851 \\
\hline $\mathrm{La}$ & 3,470 & 2,080 & 49.8 \\
\hline $\mathrm{NO}_{3}$ & 6,730 & 6,780 & 8,050 \\
\hline $\mathrm{Mn}$ & 6,180 & 2,640 & 26.4 \\
\hline $\mathrm{SO}_{4}$ & 38.6 & 47.9 & 27.2 \\
\hline $\mathrm{Cr}$ & 746 & 459 & 33.3 \\
\hline $\mathrm{PO}_{4}$ & 1,940 & 2,300 & 846 \\
\hline $\mathrm{F}$ & 708 & 802 & 2,030 \\
\hline $\mathrm{Na}$ & 4,500 & 5,250 & 10,300 \\
\hline $\mathrm{H}_{2} \mathrm{O} \%$ & 61.6 & 60.7 & 69.5 \\
\hline \multicolumn{4}{|c|}{1202} \\
\hline $\mathrm{Bi}$ & 4,040 & 3,110 & 923 \\
\hline $\mathbf{K}$ & 704 & 631 & 638 \\
\hline $\mathrm{La}$ & 1,240 & 1,250 & 37.3 \\
\hline $\mathrm{NO}_{3}$ & 6,470 & 6,020 & 6,040 \\
\hline $\mathrm{Mn}$ & 1,460 & 1,250 & 19.8 \\
\hline $\mathrm{SO}_{4}$ & 109 & 134 & 20.4 \\
\hline $\mathrm{Cr}$ & 371 & 230 & 25.0 \\
\hline $\mathrm{PO}_{4}$ & 720 & 856 & 634 \\
\hline$F$ & 647 & 593 & 1,530 \\
\hline $\mathrm{Na}$ & 3,540 & 3,530 & 7,740 \\
\hline $\mathrm{H}_{2} \mathrm{O} \%$ & 72.8 & 75.8 & 68.6 \\
\hline
\end{tabular}


HNF-1501 Rev. 0

Table D3-2. Comparison of Selected Component Inventory Estimates for T-200 Series Tanks. (2 sheets)

\begin{tabular}{|c|c|c|c|}
\hline Momponent: & 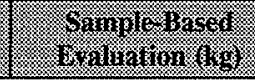 & 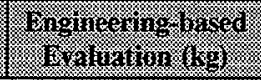 & 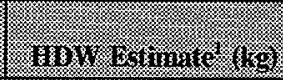 \\
\hline \multicolumn{4}{|l|}{$12,38=$} \\
\hline $\mathrm{K}$ & 1,120 & 803 & 1,060 \\
\hline $\mathrm{La}$ & 1,910 & 1,610 & 62.2 \\
\hline $\mathrm{NO}_{3}$ & 10,500 & 10,000 & 10,100 \\
\hline $\mathrm{Mn}$ & 2,620 & 2,180 & 33.0 \\
\hline $\mathrm{SO}_{4}$ & 71.4 & 110 & 34.0 \\
\hline $\mathrm{Cr}$ & 618 & 476 & 41.7 \\
\hline $\mathrm{PO}_{4}$ & 1,190 & 1,040 & 1,060 \\
\hline$F$ & 1,030 & 1,210 & 2,540 \\
\hline $\mathrm{Na}$ & 5,670 & 4,570 & 12,900 \\
\hline $\mathrm{H}_{2} \mathrm{O} \%$ & 71.0 & 75.8 & 68.6 \\
\hline \multicolumn{4}{|l|}{16.212} \\
\hline $\mathrm{Bi}$ & 8,960 & 8,130 & 1,670 \\
\hline $\mathbf{K}$ & 1,070 & 980 & 1,150 \\
\hline $\mathrm{La}$ & 2,000 & 1,740 & 67.5 \\
\hline $\mathrm{NO}_{3}$ & 9,610 & 8,970 & 10,900 \\
\hline $\mathrm{Mn}$ & 2,450 & 2,480 & 35.8 \\
\hline $\mathrm{SO}_{4}$ & 63.2 & 111 & 36.9 \\
\hline $\mathrm{Cr}$ & 781 & 543 & 45.2 \\
\hline $\mathrm{PO}_{4}$ & 1,310 & 1,200 & 1,150 \\
\hline$F$ & 1,030 & 1,200 & 2,760 \\
\hline $\mathrm{Na}$ & 5,530 & 4,470 & 14,000 \\
\hline $\mathrm{H}_{2} \mathrm{O} \%$ & 75.1 & 77.1 & 68.6 \\
\hline
\end{tabular}


Bismuth. The HDW (Agnew et al. 1997a) estimate is between 5 to 10 times lower than both the sample data and engineering inventory estimates. This appears to be caused by the incorrect assumption in the HDW that bismuth is partially soluble.

Nitrate. The HDW estimate, the engineering assessment result, and the sample data are relatively close. The HDW-estimated inventory is derived from the HDW model defined 224 waste stream in which the nitrate concentration is about 30 percent higher than Appendix C of Kupfer et al. (1997).

Sulfate. The HDW-estimated inventory is generally smaller than the sample data or engineering assessment-based inventories. However, sulfate was not a substantial process chemical in the 224 waste.

Chromium. The HDW-estimated inventory is considerably lower (approximately 10 to 20 percent) than the engineering-based assessment or sample-based inventories. The data for tank 241-B-201 (see Appendix D of Conner et al. 1997) suggests that about 24 percent of the chromium precipitated; the HDW model assumes a much smaller percent.

Phosphate. Although the engineering-based assessment and sample-based values agree closely, no trend can be established between these values and the HDW estimate. The HDW model-defined waste stream phosphate concentration is approximately three times the flowsheet value used.

Fluoride. The sample-based inventories and engineering-based assessments are based on water soluble fluoride only. Insoluble fluoride, such as that associated with $\mathrm{LaF}_{3}$, is not accounted for in the chemical analyses. The assessment value is about 2.5 times lower than the HDW value. Until a sample is analyzed by a methodology that measures total fluoride, these differences cannot be reconciled.

Sodium. The HDW value is approximately twice the value from the sample-based and engineering-assessment evaluations.

Potassium. The HDW and sampling values for potassium agree closely.

Lanthanum. Based on the tank 241-B-201 (see Appendix D of Conner et al. 1997) data, lanthanum appears to partition between the phases in the tank. The HDW (Agnew et al. 1997a) estimate is over 100 times lower than both the sample data and engineering inventory estimates. This appears to be caused by the incorrect assumption in the HDW that lanthanum is partially soluble.

Manganese. Similar to bismuth and lanthanum, the value from this evaluation is much larger than that predicted by Agnew et al. (1997a). The HDW model treats manganese as highly soluble for the B and T-200 series tanks and predicts much less manganese in the waste. 
Total Fydroxide. Once the best-basis inventories were determined, the hydroxide inventory was calculated by performing a charge balance with the valences of other analytes. In some cases, this approach requires that other analyte (for example, sodium or nitrate) inventories be adjusted to achieve the charge balance. During such adjustments, the number of significant figures is not increased. This charge balance approach was consistent with that used by Agnew et al. (1997a). The calculated total hydroxide inventories based on engineering-based assessments and HDW model estimates were $14,600 \mathrm{~kg}, 3,640 \mathrm{~kg}, 6,690 \mathrm{~kg}$ and $6,640 \mathrm{~kg}$, respectively, for tanks 241-T-201, -T-202, -T-203, and -T-204.

\section{D4.0 DEFINE THE BEST-BASIS AND ESTABLISH COMPONENT INVENTORIES}

Information about chemical, radiological, and/or physical properties is used to perform safety analyses, engineering evaluations, and risk assessment associated with waste management activities and to address regulatory issues. These activities include overseeing tank farm operations and identifying, monitoring, and resolving safety issues associated with these operations and with the tank wastes. Disposal activities include designing equipment, processes, and facilities for retrieving wastes; and processing them into a form suitable for long-term storage/disposal.

Chemical and radiological inventory information are generally derived using three approaches: 1) component inventories are estimated using the results of sample analyses, 2) component inventories are predicted using the HDW model based on process knowledge and historical information, or 3) a tank-specific process estimate is made based on process flowsheets, reactor fuel data, essential material usage, and other operating data. The information derived from these different approaches is often inconsistent.

As part of this effort, an evaluation of available chemical information for the T-200 series tanks was performed, including the following:

- Data from core samples of tank 241-B-201, -B-202, -B-203, -B-204 (Shaver [1993], Pool [1994], Jo [1996], and Sasaki [1996])

- Data from the 1997 core sample of tanks 241-T-201, -T-202, -T-203, and -T-204 (Nuzum 1997a, Esch 1997, Steen 1997, and Nuzum 1997b)

- An inventory estimate generated by the HDW model (Agnew et al. 1997a).

The calculations based on information determined from the B- 200 tanks have been compared to the T-200 analytical data of Engel et al. (1997) and the HDW model (Agnew et al. 1997a). These calculations compare well with the analytical data and, in some cases, with the HDW model. Given current resources, the best source of inventory data appears to be the analytical 
data which was obtained during the 1997 core sampling and analysis events. One analyte, for which the analytical data is suspect, is fluoride. Only the water soluble forms of fluoride are reported in the analytical data because water insoluble fluoride was not measured.

Tables D4-1 and D4-2 present the best-basis inventory estimates for the nonradioactive and radioactive waste components. For the most current inventory values, refer to the Tank Characterization Database.

Best-basis tank inventory values are derived for 46 key radionuclides (as defined in Section 3.1 of Kupfer et al. 1997), all decayed to a common report date of January 1, 1994. Often, waste sample analyses have only reported ${ }^{90} \mathrm{Sr},{ }^{137} \mathrm{Cs},{ }^{239 / 240} \mathrm{Pu}$, and total uranium (or total beta and total alpha), while other key radionuclides such as ${ }^{60} \mathrm{Co},{ }^{99} \mathrm{Tc},{ }^{129} \mathrm{I},{ }^{154} \mathrm{Eu},{ }^{155} \mathrm{Eu}$, and ${ }^{241} \mathrm{Am}$, have been infrequently reported. For this reason, it has been necessary to derive most of the 46 key radionuclides by computer models. These models estimate radionuclide activity in batches of reactor fuel, account for the split of radionuclides to separations plant waste streams, and track their movement with tank waste transactions. (These computer models are described in Kupfer et al. 1997, Section 6.1 and in Watrous and Wootan 1997.) Model generated values for radionuclides in any of 177 tanks are reported in the HDW Rev. 4 model results (Agnew et al. 1997a). The best-basis value for any one analyte may be either a model result or a sample-based or engineering assessment-based result if available. (No attempt has been made to ratio or normalize model results for all 46 radionuclides when values for measured radionuclides disagree with the model.) For a discussion of typical error between model derived values and sample derived values, see Kupfer et al. 1997, Section 6.1.10. The radionuclide inventories shown in Table D4-1 are based primarily on Agnew et al. (1997a) HDW model estimates for the T-200 series tanks.

Table D4-1. Best-Basis Inventory Estimates for Nonradioactive Components in T-200 Series Tanks (Effective May 31, 1997). (2 sheets)

\begin{tabular}{|c|c|c|c|c|c|c|}
\hline \multirow[b]{2}{*}{ (1) } & \multicolumn{3}{|c|}{ 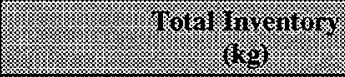 } & & \multirow{2}{*}{ fin } & \multirow[b]{2}{*}{ ( } \\
\hline & 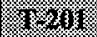 & $8: 212$ & IIs:208 & $18 \times 204$ & & \\
\hline $\mathrm{Al}$ & 14.0 & 7.12 & 9.17 & 9.33 & $S$ & \\
\hline $\mathrm{Bi}$ & 16,600 & 4,040 & 7,940 & 8,960 & $S$ & \\
\hline $\mathrm{Ca}$ & 173 & 30.6 & 56.4 & 35.9 & $s$ & \\
\hline $\mathrm{Cl}$ & 151 & 68.3 & 107 & 117 & $S$ & \\
\hline $\mathrm{TIC}$ as $\mathrm{CO}_{3}$ & 564 & 1,025 & 1,290 & 1,220 & $S$ & \\
\hline $\mathrm{Cr}$ & 746 & 371 & 618 & 781 & $S$ & \\
\hline $\mathrm{F}$ & 708 & 647 & 1,030 & 1,030 & $S$ & \\
\hline $\mathrm{Fe}$ & 1,380 & 751 & 1,110 & 703 & $S$ & \\
\hline $\mathrm{Hg}$ & 0 & 0 & 0 & 0 & $\mathbf{M}$ & \\
\hline
\end{tabular}


Table D4-1. Best-Basis Inventory Estimates for Nonradioactive Components in T-200 Series Tanks (Effective May 31, 1997). (2 sheets)

\begin{tabular}{|c|c|c|c|c|c|c|}
\hline \multirow[b]{2}{*}{ 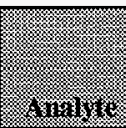 } & \multicolumn{4}{|c|}{ 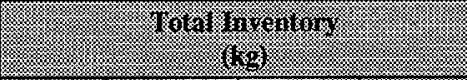 } & \multirow{2}{*}{ ( } & \multirow[b]{2}{*}{ Y onimulin } \\
\hline & 8211 & . & 1.268 & I: 2131 & & \\
\hline $\mathrm{K}$ & 671 & 704 & 1,120 & 1,070 & $S$ & \\
\hline $\mathrm{La}$ & 3,470 & 1,240 & 1,910 & 2,000 & $S$ & \\
\hline Mn & 6,180 & 1,460 & 2,620 & 2,450 & $S$ & \\
\hline $\mathrm{Na}$ & 4,500 & 3,540 & 5,670 & 5,530 & $S$ & \\
\hline $\mathrm{Ni}$ & 87.8 & 13.0 & 24.1 & 42.0 & $S$ & \\
\hline $\mathrm{NO}_{2}$ & 43.8 & 51.7 & 48.1 & 49.4 & $S$ & \\
\hline $\mathrm{NO}_{3}$ & 6,730 & 6,470 & 10,500 & 9,610 & $S$ & \\
\hline $\mathrm{OH}$ & 14,600 & 3,640 & 6,690 & 6,640 & $\mathrm{C}$ & \\
\hline $\mathrm{Pb}$ & 29.6 & 5.72 & 3.39 & 54.0 & $S$ & \\
\hline $\mathrm{PO}_{4}$ & 1,940 & 721 & 1,190 & 1,310 & $s$ & \\
\hline $\mathrm{Si}$ & 259 & 184 & 261 & 261 & $S$ & \\
\hline $\mathrm{SO}_{4}$ & 38.6 & 109 & 71.4 & 63.2 & $S$ & \\
\hline $\mathbf{S r}$ & 156 & 50.0 & 90.8 & 87.0 & $S$ & \\
\hline TOC & 42.4 & 34.2 & 68.4 & 54.3 & $S$ & \\
\hline $\mathrm{U}_{\text {TOTAL }}$ & 8.12 & 10.1 & 10.2 & 11.0 & $\mathrm{M}, \mathrm{S}, \mathrm{M}, \mathrm{M}$ & \\
\hline $\mathrm{Zr}$ & 0 & 0.2 & 0 & 0 & $S$ & \\
\hline
\end{tabular}

Note:

${ }^{1} \mathrm{~S}=$ Sample-based, $\mathrm{M}=$ Hanford Defined Waste model-based, $\mathrm{E}=$ Engineering assessment-based, $\mathrm{C}=$ Calculated by charge balance; includes oxides as hydroxides, not including $\mathrm{CO}_{3}, \mathrm{NO}_{2}, \mathrm{NO}_{3}, \mathrm{PO}_{4}, \mathrm{SO}_{4}$, and $\mathrm{SiO}_{3}$. 
HNF-1501 Rev. 0

Table D4-2. Best-Basis Inventory Estimate for Radioactive Components in T-200 Series

Tanks Decayed to January 1, 1994 (Effective May 31, 1997). (2 sheets)

\begin{tabular}{|c|c|c|c|c|c|c|}
\hline & 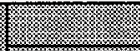 & 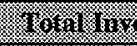 & 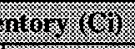 & & 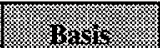 & (n) \\
\hline . & moln & $3 \%$ & 326 & 3 & (6) & (461) \\
\hline${ }^{3} \mathrm{H}$ & $2.86 \mathrm{E}+00$ & $2.02 \mathrm{E}+00$ & $3.34 \mathrm{E}+00$ & $3.57 \mathrm{E}+00$ & E & Based on B-201 \\
\hline${ }^{14} \mathrm{C}$ & $4.41 \mathrm{E}-02$ & $3.11 \mathrm{E}-02$ & $5.15 \mathrm{E}-02$ & $5.50 \mathrm{E}-02$ & $\mathbf{E}$ & Based on B-201 \\
\hline${ }^{59} \mathrm{Ni}$ & $9.56 \mathrm{E}-04$ & $6.76 \mathrm{E}-04$ & 1.12E-03 & 1.19E-03 & $\mathbf{E}$ & Based on B-201 \\
\hline${ }^{60} \mathrm{Co}$ & $2.73 \mathrm{E}-01$ & $1.93 \mathrm{E}-01$ & 3.19E-01 & $3.41 \mathrm{E}-01$ & $E$ & Based on B-201 \\
\hline${ }^{63} \mathrm{Ni}$ & $2.62 \mathrm{E}-02$ & $1.85 \mathrm{E}-02$ & $3.06 \mathrm{E}-02$ & $3.27 \mathrm{E}-02$ & $\mathrm{E}$ & Based on B-201 \\
\hline${ }^{79} \mathrm{Se}$ & $3.68 \mathrm{E}-05$ & $2.76 \mathrm{E}-05$ & $4.60 \mathrm{E}-05$ & $5.00 \mathrm{E}-05$ & $M$ & \\
\hline${ }^{90} \mathrm{Sr}$ & $2.17 \mathrm{E}+01$ & $2.70 \mathrm{E}-01$ & $4.61 \mathrm{E}-01$ & $8.82 \mathrm{E}-01$ & $S$ & \\
\hline${ }^{90} \mathrm{Y}$ & $2.17 \mathrm{E}+01$ & $2.70 \mathrm{E}-01$ & $4.61 \mathrm{E}-01$ & 8.82E-01 & $\mathbf{S}$ & Based on ${ }^{90} \mathrm{Sr}$ \\
\hline${ }^{93 \mathrm{~m}} \mathrm{Nb}$ & $1.45 \mathrm{E}-04$ & $1.08 \mathrm{E}-04$ & $1.81 \mathrm{E}-04$ & $1.96 \mathrm{E}-04$ & $\mathbf{M}$ & \\
\hline${ }^{93} \mathrm{Zr}$ & $1.75 \mathrm{E}-04$ & $1.31 \mathrm{E}-04$ & $2.18 \mathrm{E}-04$ & $2.37 \mathrm{E}-04$ & $\mathbf{M}$ & \\
\hline${ }^{99} \mathrm{Tc}$ & $1.21 \mathrm{E}-03$ & $9.09 \mathrm{E}-04$ & $1.51 \mathrm{E}-03$ & $1.64 \mathrm{E}-03$ & $\mathrm{M}$ & \\
\hline${ }^{106} \mathrm{Ru}$ & $4.20 \mathrm{E}-11$ & $3.15 \mathrm{E}-11$ & $5.25 \mathrm{E}-11$ & $5.70 \mathrm{E}-11$ & $\mathbf{M}$ & \\
\hline${ }^{113 m} \mathrm{Cd}$ & $4.89 \mathrm{E}-04$ & $3.67 \mathrm{E}-04$ & $6.12 \mathrm{E}-04$ & $6.64 \mathrm{E}-04$ & $\mathrm{M}$ & \\
\hline${ }^{125} \mathrm{Sb}$ & $6.46 \mathrm{E}-05$ & $4.84 \mathrm{E}-05$ & $8.07 E-05$ & 8.77E-05 & $\mathbf{M}$ & \\
\hline${ }^{126} \mathrm{Sn}$ & $5.55 \mathrm{E}-05$ & $4.16 \mathrm{E}-05$ & $6.94 \mathrm{E}-05$ & $7.53 \mathrm{E}-05$ & M & \\
\hline${ }^{129} \mathrm{I}$ & $2.29 \mathrm{E}-06$ & $1.72 \mathrm{E}-06$ & $2.86 \mathrm{E}-06$ & $3.10 \mathrm{E}-06$ & $\mathbf{M}$ & \\
\hline${ }^{134} \mathrm{Cs}$ & $3.32 \mathrm{E}-01$ & $2.35 \mathrm{E}-01$ & $3.88 \mathrm{E}-01$ & $4.14 \mathrm{E}-01$ & $E$ & Based on B-201 \\
\hline${ }^{137 m} \mathrm{Ba}$ & $6.60 \mathrm{E}+00$ & $2.88 \mathrm{E}+00$ & $3.05 E+00$ & $1.40 \mathrm{E}+00$ & $S$ & Based on ${ }^{137} \mathrm{Cs}$ \\
\hline${ }^{137} \mathrm{Cs}$ & $6.98 \mathrm{E}+00$ & $3.04 \mathrm{E}+00$ & $3.22 \mathrm{E}+00$ & $1.48 \mathrm{E}+00$ & $S$ & \\
\hline${ }^{151} \mathrm{Sm}$ & 0.139 & 0.104 & 0.174 & 0.189 & $\mathbf{M}$ & \\
\hline${ }^{152} \mathrm{Eu}$ & $1.82 \mathrm{E}-04$ & $1.37 \mathrm{E}-04$ & $2.28 \mathrm{E}-04$ & $2.47 \mathrm{E}-04$ & $\mathbf{M}$ & \\
\hline${ }^{154} \mathrm{Eu}$ & $6.11 \mathrm{E}-01$ & $4.32 \mathrm{E}-01$ & $7.14 \mathrm{E}-01$ & $7.62 \mathrm{E}-01$ & $\mathrm{E}$ & Based on B-201 \\
\hline${ }^{155} \mathrm{Eu}$ & 4.57E-01 & $3.23 \mathrm{E}-01$ & $5.34 \mathrm{E}-01$ & 5.71E-01 & $E$ & Based on B-201 \\
\hline${ }^{226} \mathrm{Ra}$ & 8.22E-09 & $6.16 \mathrm{E}-09$ & $1.03 \mathrm{E}-08$ & $1.12 \mathrm{E}-08$ & $\mathbf{M}$ & \\
\hline${ }^{227} \mathrm{Ac}$ & $4.34 \mathrm{E}-08$ & $3.25 \mathrm{E}-08$ & $5.42 \mathrm{E}-08$ & $5.89 \mathrm{E}-08$ & $\mathrm{M}$ & \\
\hline${ }^{228} \mathrm{Ra}$ & $5.28 \mathrm{E}-13$ & $3.96 \mathrm{E}-13$ & $6.61 \mathrm{E}-13$ & $7.17 \mathrm{E}-13$ & $\mathbf{M}$ & \\
\hline${ }^{229} \mathrm{Th}$ & $1.02 \mathrm{E}-10$ & $7.67 \mathrm{E}-11$ & $1.28 \mathrm{E}-10$ & $1.39 \mathrm{E}-10$ & $\mathbf{M}$ & \\
\hline${ }^{231} \mathrm{~Pa}$ & $1.00 \mathrm{E}-07$ & 7.51E-08 & $1.25 \mathrm{E}-07$ & $1.36 \mathrm{E}-07$ & $\mathbf{M}$ & \\
\hline${ }^{232} \mathrm{Th}$ & $4.62 \mathrm{E}-14$ & $3.46 \mathrm{E}-14$ & $5.77 \mathrm{E}-14$ & $6.27 \mathrm{E}-14$ & $\mathbf{M}$ & \\
\hline${ }^{232} \mathrm{U}$ & $5.36 \mathrm{E}-08$ & $4.02 \mathrm{E}-08$ & $6.70 \mathrm{E}-08$ & $7.27 \mathrm{E}-08$ & $\mathbf{M}$ & \\
\hline
\end{tabular}


Table D4-2. Best-Basis Inventory Estimate for Radioactive Components in T-200 Series Tanks Decayed to January 1, 1994 (Effective May 31, 1997). (2 sheets)

\begin{tabular}{|c|c|c|c|c|c|c|}
\hline & & 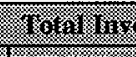 & groul $(1)$ & & & \\
\hline 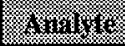 & (1) & $13=20$ & 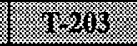 & (3) & $(8.7): 0.19)$ & 6.1minger) \\
\hline${ }^{233} \mathrm{U}$ & $2.45 \mathrm{E}-09$ & $1.83 \mathrm{E}-09$ & $3.06 \mathrm{E}-09$ & $3.32 \mathrm{E}-09$ & $\mathbf{M}$ & \\
\hline${ }^{234} \mathrm{U}$ & 2.67E-03 & $2.00 \mathrm{E}-03$ & $3.34 \mathrm{E}-03$ & $3.63 \mathrm{E}-03$ & $\mathbf{M}$ & \\
\hline${ }^{235} \mathrm{U}$ & $1.19 \mathrm{E}-04$ & $8.92 \mathrm{E}-05$ & 1.49E-04 & $1.61 \mathrm{E}-04$ & $\mathbf{M}$ & \\
\hline${ }^{236} \mathrm{U}$ & 2.33E-05 & $1.75 \mathrm{E}-05$ & $2.91 \mathrm{E}-05$ & $3.16 \mathrm{E}-05$ & $M$ & \\
\hline${ }^{237} \mathrm{~Np}$ & 7.51E-06 & $5.63 \mathrm{E}-06$ & $9.39 \mathrm{E}-06$ & $1.02 \mathrm{E}-05$ & $\mathbf{M}$ & \\
\hline${ }^{238} \mathrm{Pu}$ & $4.85 \mathrm{E}-01$ & 3.43E-01 & $5.67 \mathrm{E}-01$ & $6.06 \mathrm{E}-01$ & E & Based on B-201 \\
\hline${ }^{238} \mathrm{U}$ & 2.71E-03 & 3.30E-03 & 3.39E-03 & $3.68 \mathrm{E}-03$ & $\mathrm{M}, \mathrm{S}, \mathrm{M}, \mathrm{M}$ & \\
\hline${ }^{239} \mathrm{Pu}$ & $1.06 \mathrm{E}+02$ & $2.20 \mathrm{E}+01$ & $3.24 \mathrm{E}+01$ & $2.51 \mathrm{E}+01$ & $\mathrm{E}$ & Based on alpha ${ }^{2}$ \\
\hline${ }^{240} \mathrm{Pu}$ & $4.07 \mathrm{E}-03$ & $3.06 \mathrm{E}-03$ & $5.09 \mathrm{E}-03$ & $5.53 \mathrm{E}-03$ & $E$ & Based on alpha ${ }^{2}$ \\
\hline${ }^{241} \mathrm{Am}$ & $4.32 \mathrm{E}+00$ & $3.06 \mathrm{E}+00$ & $5.85 \mathrm{E}+00$ & $4.25 \mathrm{E}+00$ & $E, E, S, S$ & "E" based on B-201 \\
\hline${ }^{241} \mathrm{Pu}$ & $1.35 \mathrm{E}-02$ & $1.01 \mathrm{E}-02$ & $1.68 \mathrm{E}-02$ & $1.83 \mathrm{E}-02$ & $M$ & \\
\hline${ }^{242} \mathrm{Cm}$ & $3.70 \mathrm{E}-06$ & 2.78E-06 & 4.63E-06 & $5.03 \mathrm{E}-06$ & $\mathbf{M}$ & \\
\hline${ }^{242} \mathrm{Pu}$ & $6.23 \mathrm{E}-08$ & $4.67 \mathrm{E}-08$ & 7.79E-08 & $8.45 \mathrm{E}-08$ & $\mathbf{M}$ & \\
\hline${ }^{243} \mathrm{Am}$ & 3.08E-09 & 2.31E-09 & $3.86 \mathrm{E}-09$ & 4.19E-09 & $\mathrm{M}$ & \\
\hline${ }^{243} \mathrm{Cm}$ & 7.98E-08 & 5.99E-08 & $9.98 \mathrm{E}-08$ & $1.08 \mathrm{E}-07$ & $\mathrm{M}$ & \\
\hline${ }^{244} \mathrm{Cm}$ & 7.84E-08 & $5.88 \mathrm{E}-08$ & $9.80 \mathrm{E}-08$ & $1.06 \mathrm{E}-07$ & $\mathbf{M}$ & \\
\hline
\end{tabular}

Notes

${ }^{1} \mathrm{~S}=$ Sample-based, $\mathrm{M}=$ Hanford Defined Waste model-based, $\mathrm{E}=$ Engineering assessment-based

${ }^{2}$ Assumed total alpha was phutonium-based, and the ratio of ${ }^{239} \mathrm{PU}$ to ${ }^{240} \mathrm{Pu}$ was $94 \%$ to $6 \%$. The other plutonium contributors estimated by the HDW model fell within the uncertainty of the measurement. 


\section{D5.0 APPENDIX D REFERENCES}

Agnew, S. F., J. Boyer, R. A. Corbin, T. B. Duran, J. R. FitzPatrick, K. A. Jurgensen, T. P. Ortiz, and B. L. Young, 1997a, Hanford Tank Chemical and Radionuclide Inventories: HDW Model Rev. 4, LA-UR-96-3860, Los Alamos National Laboratory, Los Alamos, New Mexico.

Agnew, S. F., R. A. Corbin, T. B. Duran, K. A. Jurgensen, T. P. Ortiz, and B. L. Young, 199.7b, Waste Status and Transaction Record Summary (WSTRS Rev. 4), LA-UR-97-311, Rev. 0, Los Alamos National Laboratory, Los Alamos, New Mexico.

Borsheim, G. L., 1994, Bismuth Phosphate 224 Building Waste Data, (memoandum to Distribution), Westinghouse Hanford Company, Richland, Washington.

Conner, J. M., K. M. Hodgson, L. C. Amato, J. L. Stroup, S. R. Wilmarth, and R. T. Winward, 1997, Tank Characterization Report for Single-Shell Tank 241-B-201, HNF-SD-WM-ER-550, Rev. 1, Lockheed Martin Hanford Corp. for Fluor Daniel Hanford, Inc., Richland, Washington.

Dougherty, L. F., J. G. Field, K. M. Hodgson, and R. T. Winward, 1997, Tank Characterization Report for Single-Shell Tank 241-B-202, HNF-SD-WM-ER-371, Rev. 0A, Lockheed Martin Hanford Corp. for Fluor Daniel Hanford, Inc., Richland, Washington.

Engel, D. W., T. A. Ferryman, K. M. Remund, D. S. Daly, G. Chen, S. A. Hartley, and B. C. Simpson, 1997, T-200 Series Tank Concentration Predictions, PNNL-11550, Pacific Northwest National Laboratory, Richland, Washington.

Esch, R. A., 1997, Tank 241-T-202, Core 191, Analytical Results for the Final Report, HNF-SD-WM-DP-253, Rev. 0, Waste Management Federal Services of Hanford Inc., for Fluor Daniel Hanford, Inc., Richland, Washington.

Field, J. G., and R. T. Winward, 1997a, Preliminary Tank Characterization Report for Single-Shell Tank 241-T-201, HNF-SD-WM-ER-726 Rev. 0, Lockheed Martin Hanford Corp. for Fluor Daniel Hanford, Inc., Richland, Washington.

Field, J. G., and R. T. Winward, 1997b, Preliminary Tank Characterization Report for Single-Shell Tank 241-T-202, HNF-SD-WM-ER-727 Rev. 0, Lockheed Martin Hanford Corp. for Fluor Daniel Hanford, Inc., Richland, Washington.

Field, J. G., and R. T. Winward, 1997c, Preliminary Tank Characterization Report for Single-Shell Tank 241-T-203, HNF-SD-WM-ER-728 Rev. 0, Lockheed Martin Hanford Corp. for Fluor Daniel Hanford, Inc., Richland, Washington. 
Field, J. G., and R. T. Winward, 1997d, Preliminary Tank Characterization Report for Single-Shell Tank 241-T-204, HNF-SD-WM-ER-729 Rev. 0, Lockheed Martin Hanford Corp. for Fluor Daniel Hanford, Inc., Richland Washington.

Hanlon, B. M., 1997, Waste Tank Summary Report for Month Ending March 31, 1997, WHC-EP-0182-108, Lockheed Martin Hanford Corp. for Fluor Daniel Hanford, Inc., Richland, Washington.

Hill, J. G., G. S. Anderson, and B. C. Simpson, 1995, The Sort on Radioactive waste Type Model: A Method to Sort Single-Shell Tanks into Characteristic Groups, PNL-9814, Rev. 2, Pacific Northwest Laboratory, Richland, Washington.

Hodgson, K. M., and M. D. LeClair, 1996, Work Plan for Defining a Standard Inventory Estimate for Wastes Stored in Hanford Site Underground Tanks, WHC-SD-WM-WP-311, Rev. 1, Lockheed Martin Hanford Corp. for Fluor Daniel Hanford, Inc., Richland, Washington.

Jo, J., J. G. Field, K. M. Hodgson, and R. T. Winward, 1997, Tank Characterization Report for Single-Shell Tank 241-B-203, HNF-SD-WM-ER-587, Rev. 0A, Lockheed Martin Hanford Corp. for Fluor Daniel Hanford, Inc., Richland, Washington.

Kupfer, M. J., A. L. Boldt, B. A. Higley, K. M. Hodgson, L. W. Shelton, B. C. Simpson, R. A. Watrous, S. L. Lambert, D. E. Place, R. M. Orme, G. L. Borsheim, N. G. Colton, M. D. LeClair, R. T. Winward, and W. W. Schulz, 1997, Standard Inventories of Chemicals and Radionuclides in Hanford Site Tank Wastes, HINF-SD-WM-TI-740, Rev. 0A, Lockheed Martin Hanford Corp. for Fluor Daniel Hanford, Inc., Richland, Washington.

Nuzum, J. L. , 1997a, Tank 241-T-201, Core 192, Analytical Results for the Final Report, HNF-SD-WM-DP-254, Rev. 0, Waste Management Federal Services of Hanford, Inc. for Fluor Daniel Hanford, Inc., Richland, Washington.

Nuzum, J. L. , 1997b, Tank 241-T-204, Core 188, Analytical Results for the Final Report, HNF-SD-WM-DP-255, Rev. 0, Waste Management Federal Services of Hanford, Inc. for Fluor Daniel Hanford, Inc., Richland, Washington.

Sasaki, L. M., J. G. Field, K. M. Hodgson, and R. T. Winward, 1997, Tank Characterization Report for Single-Shell Tank 241-B-204, HNF-SD-WM-ER-581, Rev. 0A, Lockheed Martin Hanford Corp. for Fluor Daniel Hanford, Inc., Richland, Washington.

Steen, F. H. , 1997, Tank 241-T-203, Core 190, Analytical Results for the Final Report, HNF-SD-WM-DP-247, Rev. 0, Waste Management Federal Services of Hanford, Inc. for Fluor Daniel, Hanford, Inc., Richland, Washington. 
Watrous, R. A., and D. W. Wootan, 1997, Activity of Fuel Batches Processed Through Hanford Separations Plants, 1944 Through 1989, HNF-SD-WM-TI-794, Rev. 0, Lockheed Martin Hanford Corp. for Fluor Daniel Hanford, Inc., Richland, Washington. 
HNF-1501 Rev. 0

\section{APPENDIX E}

BIBLIOGRAPHY FOR TANKS 241-T-201, 241-T-202, 241-T-203, AND 241-T-204 
HNF-1501 Rev. 0

This page intentionally left blank. 


\section{APPENDIX E}

BIBLIOGRAPHY FOR TANKS 241-T-201, 241-T-202, 241-T-203, AND 241-T-204

Appendix $\mathrm{E}$ is a bibliography that supports the characterization of the T-200 series tanks. This bibliography represents an in-depth literature search of all known information sources that provide sampling, analysis, surveillance, modeling information, and processing occurrences associated with these tanks and their respective waste types.

The references in this bibliography are separated into three categories containing references broken down into subgroups. These categories and their subgroups are listed below.

\section{NON-ANALYTICAL DATA}

Ia. Models/Waste Type Inventories/Campaign Information

Ib. Fill History/Waste Transfer Records

Ic. Surveillance/Tank Configuration

Id. Sample Planning/Tank Prioritization

Ie. Data Quality Objectives/Customers of Characterization Data

\section{ANALYTICAL DATA - SAMPLING OF TANK WASTE AND WASTE TYPES}

IIa. Sampling of tanks 241-T-201, -T-202, -T-203, and -T-204

IIb. Sampling and Analysis of 224 Waste

\section{COMBINED ANALYTICAL/NON-ANALYTICAL DATA}

IIIa. Inventories using both Campaign and Analytical Information

$\mathrm{IIIb}$. Compendium of Existing Physical and Chemical Documented Data Sources

The bibliography is broken down into the appropriate sections of material with an annotation at the end of each reference describing the information source. Most information listed below is available in the Lockheed Martin Hanford Corporation Tank Characterization and Safety Resource Center. 


\section{NON-ANALYTICAL DATA}

Ia. Models/Waste Type Inventories/Campaign Information

Agnew, S. F., J. Boyer, R. A. Corbin, T. B. Duran, J. R. Fitzpatrick, K. A. Jurgensen, T. P. Ortiz, and B. L. Young, 1997, Hanford Tank Chemical and Radionuclide Inventories: HDW Model Rev. 4, LA-UR-96-3860, Rev. 0, Los Alamos National Laboratory, Los Alamos, New Mexico.

- Contains waste type summaries and primary chemical compound/analyte and radionuclide estimates for sludge, supernatant, and solids.

Hill, J. G., G. S. Anderson, and B. C. Simpson, 1995, The Sort on Radioactive Waste Type Model: A Method to Sort Single-Shell Tanks into Characteristic Groups, PNL-9814, Rev. 2, Pacific Northwest Laboratory, Richland, Washington.

- Contains a qualitative grouping scheme based on waste types and an assessment of the process histories for the tanks

Watrous, R. A., and D. W. Wootan, 1997, Activity of Fuel Batches Processed Through Hanford Separations Plants, 1944 Through 1989, HNF-SD-WM-TI-794, Rev. 0, Lockheed Martin Hanford Corp. for Fluor Daniel Hanford, Inc., Richland, Washington.

- Contains estimates of the overall production of radionuclides at the Hanford Site using ORIGEN2.

\section{Ib. Fill History/Waste Transfer Records}

Agnew, S. F., R. A. Corbin, T. B. Duran, K. A. Jurgensen, T. P. Ortiz, and B. L. Young, 1997, Waste Status and Transaction Record Summary (WSTRS) Rev. 4, LA-UR-97-311, Rev. 0, Los Alamos National Laboratory, Los Alamos, New Mexico.

- Contains spreadsheets showing all available data on tank additions and transfers. 
Anderson, J. D., 1990, A History of the 200 Area Tank Farms, WHC-MR-0132, Westinghouse Hanford Company, Richland, Washington.

- Contains single-shell tank fill history and primary campaign and waste information to 1981 .

Borsheim, G. L., 1994, Bismuth Phosphate 224 Building Waste Data, (memorandum to Distribution), Westinghouse Hanford Company, Richland, Washington.

- Contains an assessment of the 224 separation process and waste products.

\section{Ic. Surveillance/Tank Configuration}

Alstad, A. T., 1993, Riser Configuration Document for Single-Shell Waste Tanks, WHC-SD-RE-TI-053, Rev. 9, Westinghouse Hanford Company, Richland, Washington.

- Shows tank riser locations in relation to a tank aerial view and a description of risers and their contents.

Lipnicki, J., 1997, Waste Tank Risers Available for Sampling, WHC-SD-RE-TI-710, Rev. 4, Numatec Hanford Corporation for Fluor Daniel Hanford, Inc., Richland, Washington.

- Assesses riser locations for each tank, however, not all tanks are included or completed. A estimate of the risers available for sampling is also included.

Tran, T. T, 1993, Thermocouple Status Single-Shell \& Double-Shell Waste Tanks, WHC-SD-WM-TI-553, Rev. 0, Westinghouse Hanford Company, Richland, Washington.

- Contains riser and thermocouple information for Hanford Site waste tanks.

Brevick, C. H., J. L. Stroup, J. W. Funk, 1997, Supporting Document for the Historical Tank Content Estimate for T-Tank Farm, HNF-SD-WM-ER-320, Rev. 1, Fluor Daniel Northwest, Inc. for Fluor Daniel Hanford, Inc., Richland, Washington. 
Brevick, C. H., J. L. Stroup, J. W. Funk, 1997, Historical Tank Content Estimate for the Northwest Quadrant of the Hanford 200 West Area, HNF-SD-WM-ER-351, Rev. 1, Fluor Daniel Northwest, Inc. for Fluor Daniel Hanford, Inc., Richland, Washington.

- Contains consolidated historical, physical, and chemical information on the high-level waste tanks at Hanford.

Smith, D. A., 1986, Single-Shell Tank Isolation Safety Analysis Report, WHC-SD-WM-SAR-006, Rev. 2, Westinghouse Hanford Company, Richland, Washington.

- Contains information regarding the accepted operating thresholds for the single-shell tanks.

\section{Id. Sample Planning/Tank Prioritization}

Brown, T. M., J. W. Hunt, and L. J. Fergestrom, 1997, Tank Characterization Technical Sampling Basis, HNF-SD-WM-TA-164, Rev. 3, Lockheed Martin Hanford Corp. for Fluor Daniel Hanford, Inc., Richland, Washington.

- Summarizes the technical basis for characterizing tank waste and assigns a priority number to each tank.

Hu, T. A., 1997, Tank 241-T-201 Push Mode Core Sampling and Analysis Plan, HNF-SD-WM-TSAP-130, Rev. 0, Lockheed Martin Hanford Corp. for Fluor Daniel Hanford, Inc., Richland, Washington.

Bell, K. E., 1997, Tank 241-T-202 Push Mode Core Sampling and Analysis Plan, HNF-SD-WM-TSAP-121, Rev. 0, Lockheed Martin Hanford Corp. for Fluor Daniel Hanford, Inc., Richland, Washington.

Schreiber, R. D., 1997, Tank 241-T-203 Push Mode Core Sampling and Analysis Plan, WHC-SD-WM-TSAP-118, Rev. 0A, Lockheed Martin Hanford Corp. for Fluor Daniel Hanford, Inc., Richland, Washington.

Winkleman, W. D., 1997, Tank 241-T-204 Push Core Sampling and Analysis Plan, HNF-SD-WM-TSAP-119, Rev. 0, Lockheed Martin Hanford Corp. for Fluor Hanford, Inc., Richland, Washington.

- Contains sampling and analysis requirements for each specified tank based on applicable DQOs. 
Winkelman, W. D., M. R. Adams, T. M. Brown, J. W. Hunt, D. J. McCain, and L. S. Fergestrom, 1997, Fiscal Year 1997-1998 Waste Information Requirements Document, HNF-SD-WM-PLN-126, Rev. 0A, Lockheed Martin Hanford Corp. for Fluor Daniel Hanford, Inc., Richland, Washington.

- Contains Tri-Party Agreement (Ecology et al. 1997) requirement-driven TWRS Characterization Program information.

Stanton, G. A., 1997, Baseline Sampling Schedule, Change 97-03 (internal letter 75610-97-004 to Distribution, October 8), Lockheed Martin Hanford Corp. for Fluor Daniel Hanford, Inc., Richland, Washington.

- Contains the baseline operation schedule for sampling.

Ie. Data Quality Objectives (DQO) and Customers of Characterization Data

DOE-RL, 1996, Recommendation 93-5 Implementation Plan, DOE/RL-94-0001, Rev. 1, U.S. Department of Energy, Richland Operations Office, Richland, Washington.

- Describes the organic solvents issue and other tank issues.

Dukelow, G. T., J. W. Hunt, H. Babad, and J. E. Meacham, 1995, Tank Safety Screening Data Quality Objective, WHC-SD-WM-SP-004, Rev. 2, Westinghouse Hanford Company, Richland, Washington.

- Determines whether tanks are under safe operating conditions.

Osborne, J. W., and L. L. Buckley, 1995, Data Quality Objectives for Tank Hazardous. Vapor Safety Screening, WHC-SD-WM-DQO-002, Rev. 2, Westinghouse Hanford Company, Richland, Washington.

- Contains requirements for addressing hazardous vapor issues.

Hall, K. M., 1997, Letter of Instruction for Core Samples Analysis of Tanks 241-T-201, 241-T-202, 241-T-203, and 241-T-204, (letter 74620-97-195 to A. D. Rice, May 8), Lockheed Martin Hanford Corp. for Fluor Daniel Hanford, Inc., Richland, Washington. 
Hall, K. M., 1997, Additional Core Composite Sample from Drainable Liquid Samples for Tank 241-T-201, (letter 74620-97-199 to A. D. Rice, May 29), Lockheed Martin Hanford Corp. for Fluor Daniel Hanford, Inc., Richland, Washington.

- Contains additional sampling and analysis requirements for each specified tank.

Hewitt, E. R., 1996, Tank Waste Remediation System Resolution of Potentially Hazardous Vapor Issues, WHC-SD-TWR-RPT-001, Rev. 0, Westinghouse Hanford Company, Richland, Washington.

- Describes disposition and resolution of the hazardous vapor issue.

Meacham, J. E., 1996, Implementation Change Concerning Organic DQO, Rev. 2, (internal memorandum 2N160-96-006 to Distribution, December 2), Duke Engineering and Services, Inc. for Fluor Daniel Hanford, Inc., Richland, Washington.

Schreiber, R. D., 1997, Memorandum of Understanding for the Organic Complexant Safety Issue Data Requirements, HNF-SD-WM-RD-060, Rev. 0, Lockheed Martin Hanford Corp. for Fluor Daniel Hanford Inc., Richland, Washington.

Turner, D. A., H. Babad, L. L. Buckley, and J. E. Meacham, 1995, Data Quality Objective to Support Resolution of the Organic Complexant Safety Issue, WHC-SD-WM-DQO-006, Rev. 2, Westinghouse Hanford Company, Richland, Washington.

- Contains requirements for addressing the organic complexant issue.

Wilkins, N. E., 1996, Flammable Gas Data Review for Tanks 241-T-201, 241-T-202, and 24I-T-204, (internal letter 74A10-96-133 to J. H. Wicks, October 22), Lockheed Martin Hanford Corp. for Fluor Daniel Hanford, Inc., Richland, Washington.

- Contains flammable gas evaluation of tanks using surveillance and vapor analysis information. 


\section{ANALYTICAL DATA - SAMPLING OF TANK WASTE AND WASTE TYPES}

\section{IIa. Sampling of T-200 Tanks}

Esch, R. A., 1997, Tank 241-T-202, Core 191, Analytical Results for the Final Report, HNF-SD-WM-DP-253, Rev. 0, Waste Management Federal Services of Hanford, Inc. for Fluor Daniel Hanford, Inc., Richland, Washington.

Nuzum, J. L., 1997, Tank 241-T-201, Core 192, Analytical Results for the Final Report, HNF-SD-WM-DP-254, Rev. 0, Waste Management Federal Services of Hanford, Inc. for Fluor Daniel Hanford, Inc., Richland, Washington.

Nuzum, J. L., 1997, Tank 241-T-204, Core 188, Analytical Results for the Final Report, HNF-SD-WM-DP-255, Rev. 0, Waste Management Federal Services of Hanford, Inc. for Fluor Daniel Hanford Inc., Richland, Washington.

Steen, F. H., 1997, Tank 241-T-203, Core 190, Analytical Results for the Final Report, HNF-SD-WM-DP-247, Rev. 0, Waste Management Federal Services of Hanford, Inc. for Fluor Daniel Hanford, Inc., Richland, Washington.

- Contains results for 1997 sample analyses for each tank.

Horton, J. E., 1978, Analysis of Tanks 011-BXR; 201-C; and 204-T, (letter 60120-78-132J to J. E. Mirabella, December 4), Rockwell Hanford Operations, Richland, Washington.

- Contains results for 1978 sample analyses from tank 241-T-204.

IIb. Sampling and Analysis of 224 Waste

Conner, J. M., K. M. Hodgson, L. C. Amato, J. L. Stroup, S. R. Wilmarth, and R. T. Winward, 1997, Tank Characterization Report for Single-Shell Tank 241-B-201, HNF-SD-WM-ER-550, Rev. 1, Lockheed Martin Hanford Corp. for Fluor Daniel Hanford, Inc., Richland, Washington.

Dougherty, L. F., J. G. Field, S. M. Hodgson, and R. T. Winward, 1997, Tank Characterization Report for Single-Shell Tank 241-B-202, HNF-SD-WM-ER-371, Rev. 0A, Lockheed Martin Hanford Corp.for Fluor Daniel Hanford, Inc., Richland, Washington. 
Jo, J., J. G. Field, S. M. Hodgson, and R. T. Winward, 1997, Tank Characterization Report for Single-Shell Tank 241-B-203, HNF-SD-WM-ER-587, Rev. 0A, Lockheed Martin Hanford Corp. for Fluor Daniel Hanford, Inc., Richland, Washington.

Sasaki, L. M., J. G. Field, S. M. Hodgson, and R. T. Winward, 1997, Tank Characterization Report for Single-Shell Tank 241-B-204, HNF-SD-WM-ER-581, Rev. 0A, Lockheed Martin Hanford Corp. for Fluor Daniel Hanford, Inc., Richland, Washington.

- Contains the results of 224 waste analysis from B-200 series tanks.

Kupfer, M. J., A. L. Boldt, B. A. Higley, K. M. Hodgson, L. W. Shelton, B. C. Simpson, S. L. Lambert, D. E. Place, R. M. Orme, G. L. Borsheim, N. G. Colton, M. D. LeClair, R. T. Winward, and W. W. Schulz, 1997, Standard Inventories of Chemicals and Radionuclides in Hanford Site Tank Wastes, HNF-SD-WM-TI-740, Rev. 0A, Lockheed Martin Hanford Corp. for Fluor Daniel Hanford, Inc., Richland, Washington.

- Contains the method for deriving a single-point inventory estimate for a selected number of chemicals and radionuclides for all Hanford high-level waste tanks.

Engel, D. W., T. A. Ferryman, K. M. Remund, D. S. Daly, G. Chen, S. A. Hartley, and B. C. Simpson, 1997, T-200 Series Tank Concentration Predictions, PNNL-11550, Pacific Northwest National Laboratory, Richland, Washington.

- Contains the results of a statistical study to define the 224 waste composition using existing data.

\section{COMBINED ANALYTICAL/NON-ANALYTICAL DATA}

\section{IIIa. Inventories from Campaign and Analytical Information}

Allen, G. K., 1976, Estimated Inventory of Chemicals Added to Underground Waste Tanks, 1944 -1975, ARH-CD-601B, Rev. 0, Atlantic Richfield Hanford Company, Richland, Washington.

- Contains major components for waste types and some assumptions. Purchase records are used to estimate chemical inventories. 
Allen, G. K., 1975, Hanford Liquid Waste Inventory as of September 30, 1974, ARH-CD-229, Rev. 0, Atlantic Richfield Company, Richland, Washington.

- Contains major components for waste types and some assumptions.

Schmittroth, F. A., 1995, Inventories for Low-Level Tank Waste, WHC-SD-WM-RPT-164, Rev. 0, Westinghouse Hanford Company, Richland, Washington.

- Contains a global inventory based on process knowledge and radioactive decay estimations using ORIGEN2. Pu and $\mathrm{U}$ waste contributions are taken at one percent of the amount used in processes. Also compares information on Tc- 99 from both ORIGEN2 and analytical data.

Agnew, S. F., and J. G. Watkin, 1994, Estimation of Limiting Solubilities for Ionic Species in Hanford Waste Tank Supernates, LA-UR-94-3590, Los Alamos National Laboratory, Los Alamos, New Mexico.

- Gives solubility ranges for key chemical and radionuclide components based on supernatant sample analyses.

Field, J. G., and R. T. Winward, 199, Preliminary Tank Characterization Report for Single-Shell Tank 241-T-201, HNF-SD-WM-ER-726, Rev. 0, Lockheed Martin Hanford Corp. for Fluor Daniel Hanford, Inc., Richland, Washington.

Field, J. G., and R. T. Winward, 1997, Preliminary Tank Characterization Report for Single-Shell Tank 241-T-202, HNF-SD-WM-ER-727, Rev. 0, Lockheed Martin Hanford Corp. for Fluor Daniel Hanford, Inc., Richland, Washington.

Field, J. G., and R. T. Winward, 1997, Preliminary Tank Characterization Report for Single-Shell Tank 241-T-203, HNF-SD-WM-ER-728, Rev. 0, Lockheed Martin Hanford Corp. for Fluor Daniel Hanford, Inc., Richland, Washington.

Field, J. G., and R. T. Winward, 1997, Preliminary Tank Characterization Report for Single-Shell Tank 241-T-204, HNF-SD-WM-ER-729, Rev. 0, Lockheed Martin Hanford Corp. for Fluor Daniel Hanford, Inc., Richland, Washington.

- Contain initial inventory estimates for the T-200 series tanks. 


\section{IIb. Compendium of Data from Other Physical and Chemical Sources}

Hanlon, B. M., 1997, Waste Tank Summary Report for Month Ending March 31, 1997, HNF-EP-0182-108, Lockheed Martin Hanford Corp. for Fluor Daniel Hanford, Inc., Richland, Washington.

- Contains a monthly summary of the following: fill volumes, Watch List tanks, occurrences, integrity information, equipment readings, equipment status, tank location, and other miscellaneous tank information.

Husa, E. I., 1993, Hanford Site Waste Storage Tank Information Notebook, WHC-EP-0625, Westinghouse Hanford Company, Richland, Washington.

- Contains in-tank photographs and summaries of the tank descriptions, leak detection systems, and tank status.

Husa, E. I., 1995, Hanford Waste Tank Preliminary Dryness Evaluation, WHC-SD-WM-TI-703, Rev. 0, Westinghouse Hanford Company, Richland, Washington.

- Assesses relative dryness between tanks.

Klem, M. J., 1990, Total Organic Carbon Concentration of Single-Shell Tank Waste, (internal letter 82316-90-032 to R. E. Raymond, April 27), Westinghouse Hanford Company, Richland, Washington.

- Assesses total organic carbon content in single-shell tanks.

Kummerer, M., 1995, Topical Report on Heat Removal Characteristics of Waste Storage Tanks, WHC-SD-WM-SARR-010, Rev. 1, Westinghouse Hanford Company, Richland, Washington.

- Assesses heat load using a heat transfer model and dome space temperature information.

Shelton, L. W., 1996, Chemical and Radionuclide Inventory for Single- and Double-Shell Tanks, (internal memorandum 74A20-96-30 to D. J. Washenfelder, February 28), Westinghouse Hanford Company, Richland, Washington.

- Contains a tank inventory estimate based on analytical information. 
Van Vleet, R. J., 1993, Radionuclide and Chemical Inventories for the Single-Shell Tanks, WHC-SD-WM-TI-565, Rev. 1, Westinghouse Hanford Company, Richland, Washington.

- Contains selected sample analysis tables prior to 1993 for single-shell tanks.

TWINS: Tank Waste Information Network System, PNNL, 1997, In: SYBASE version 4. Available: Hanford Local Area Network (HLAN), Fluor Daniel Hanford Inc. or TCP/IP access at http://twins.pnl.gov:8001/ htbin/TCD/main.html

- Contains physical and analytical data for each of the 177 Hanford Site waste tanks. 
HNF-1501 Rev. 0

This page intentionally left blank. 


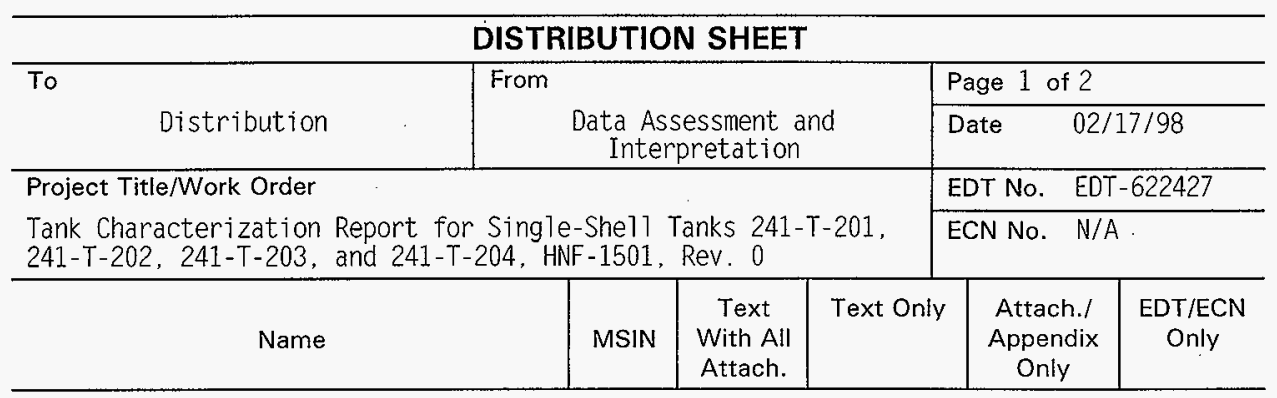

\section{OFFSITE}

Sandia National Laboratory

P.0. Box 5800

MS-0744, Dept. 6404

Albuquerque, NM 87815

D. Powers

Nuclear Consulting Services Inc.

P. 0. Box 29151

Columbus, OH 43229-01051

J. L. Kovach

X

Chemical Reaction Sub-TAP

P.0. Box 271

Lindsborg, KS 67456

B. C. Hudson

$X$

SAIC

555 Quince Orchard Rd., Suite 500

Gaithersburg, MD 20878-1437

H. Sutter

$X$

Los Alamos Laboratory

CST-14 MS-J586

P. 0. Box 1663

Los Alamos, NM 87545

S. F. Agnew

$X$

Tank Advisory Panel

102 windham Road

Oak Ridge, TN 37830

D. 0. Campbe1]

$X$ 


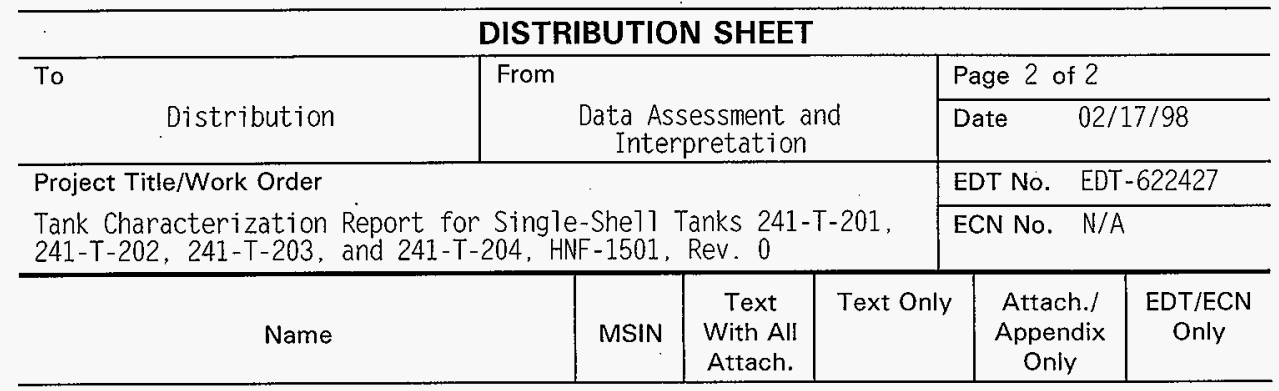

\section{ONSITE}

Department of Energy - Richland Operations

J. F. Thompson

W. S. Liou

J. A. Poppiti

$\begin{array}{ll}57-54 & X \\ 57-54 & x \\ 57-54 & x\end{array}$

DE\&S Hanford, Inc.

R. J. Cash

W. L. Cow7ey

G. L. Dunford

G. D. Johnson

J. E. Meacham

S7-14

R2-54

A2-34

S7-14

\$7-14

$x$
$X$
$X$

Fluor Daniel Northwest

E. D. Johnson

E6-08

$x$
$x$
$x$
$x$
$x$

Lockheed Martin Hanford, Corp.

K. M. Hodgson

T. J. Kelley

L. M. Sasaki

B. C. Simpson

R. R. Thompson

L. R. Webb

ERC (Environmental Resource Center)

T.C.S.R.C.

$\mathrm{HO}-34$

S7 - 21

R2-12

R2-12

R2- 12

$\mathrm{R} 2-12$

R1-51

R1-10

Lockheed Martin Services, Inc.

B. G. Lauzon

Central Files

R1-08

B1-07

H6-08

EDMC

$\begin{array}{ll}\text { H5- }-49 & X \\ \text { T6-07 } & X \\ H 5-61 & X \\ H 5-61 & X\end{array}$

Numatec Hanford Corporation

J. S. Garfield

D. L. Herting

J. S. Hertze1

D. L. Lamberd

H5-61

Pacific Northwest National Laboratory A. F. Noonan

K9-91. $\quad x$ 